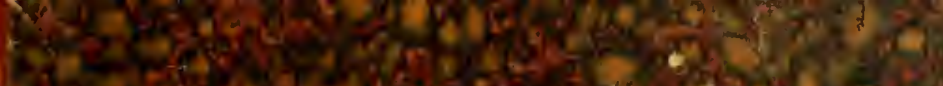

H.

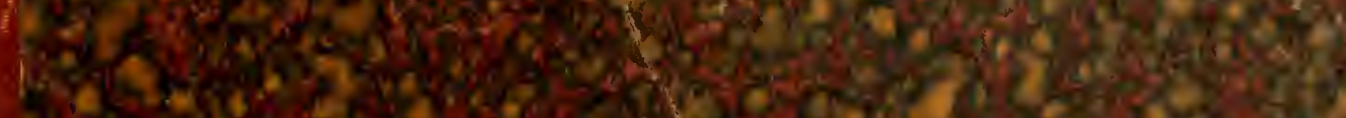

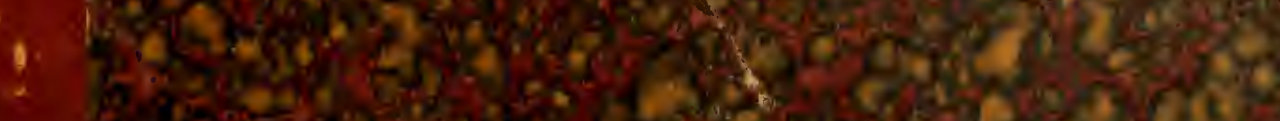

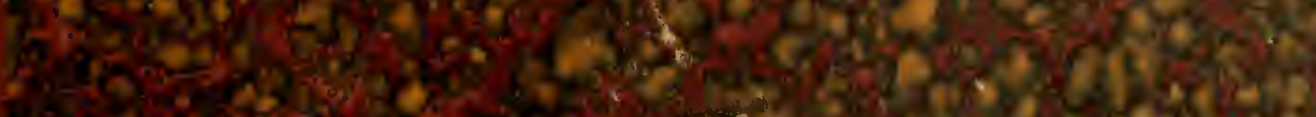

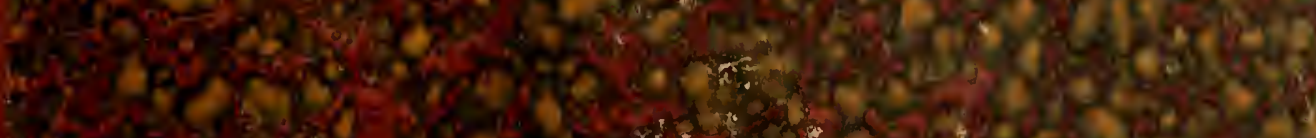

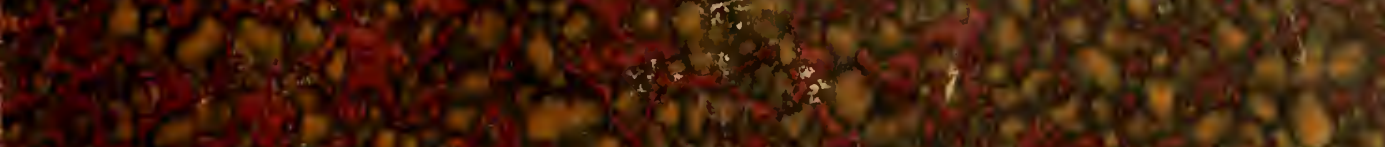

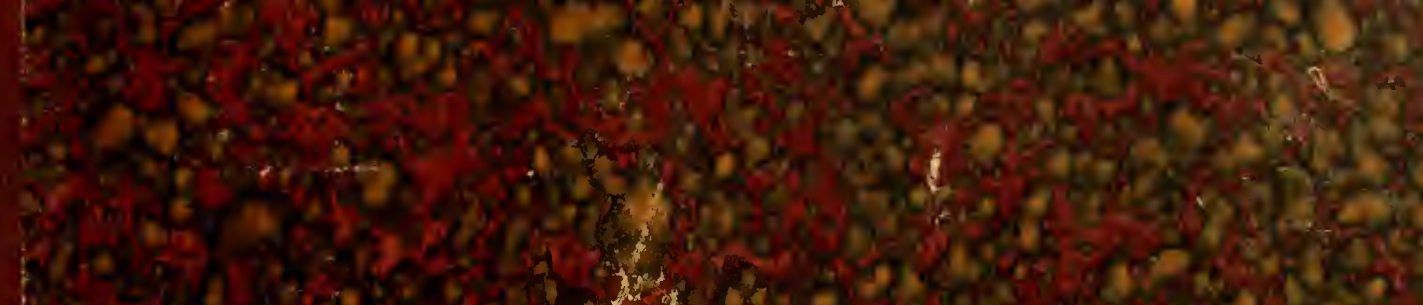

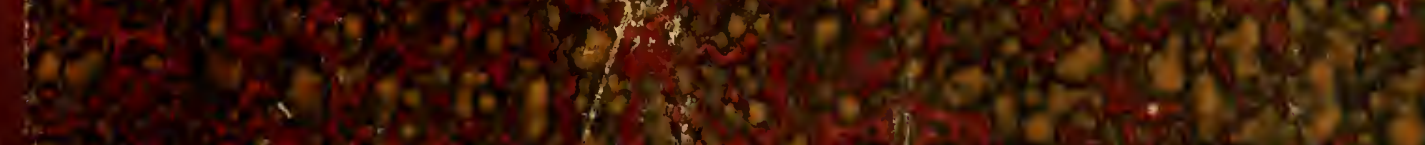

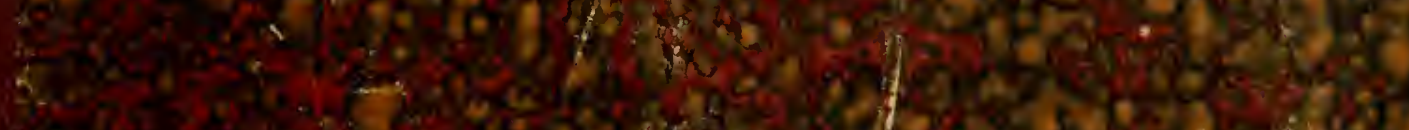

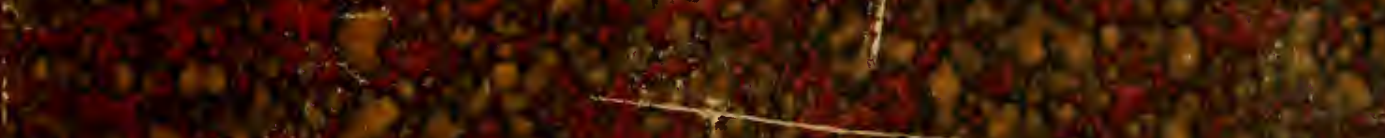
1.

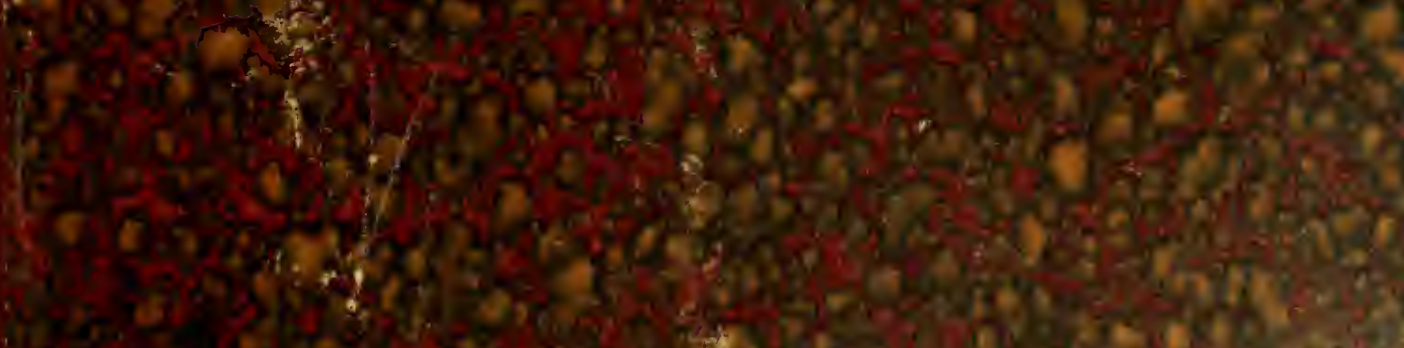
$(7)$

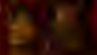

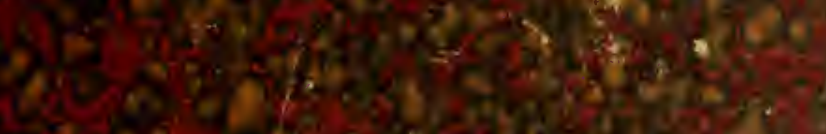

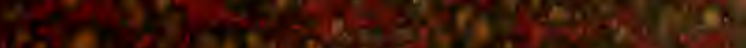




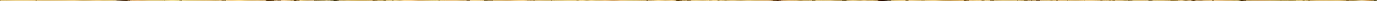




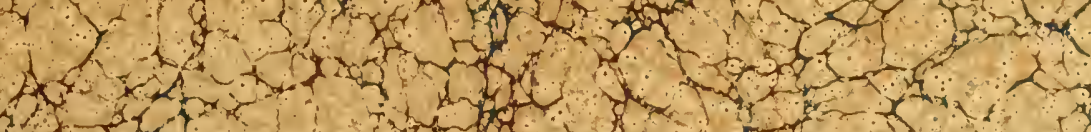

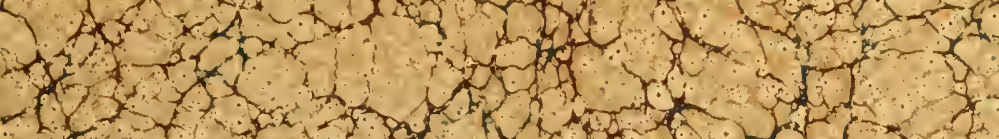

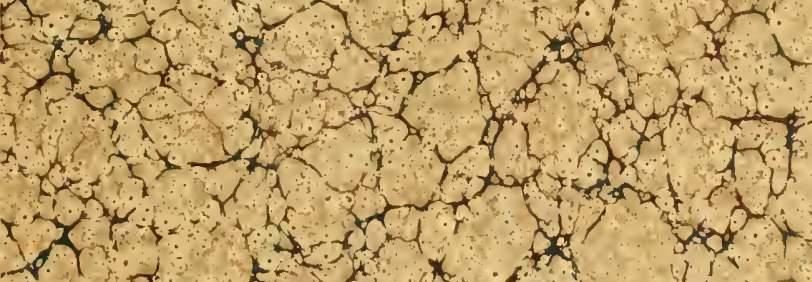

$x+2 \times$ $+2+x^{2}$ 





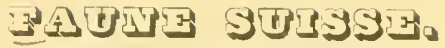

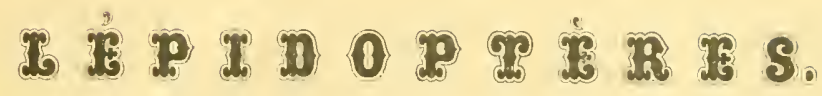

IV. Partie. Phalénides.

(Geometra. Lin.)

Par

7. $\mathfrak{C}$. Ae la Ggarpe, I). 



\section{PRÉFACE.}

La famille des Phalénites n’est point aussi complétement étudiée yu’on pourrait le croire en parcourant les ouvrages spéciaux qui traitent des Lépidoptères. La structure et la contiguration exacte des organes, chez l'insecte parfait, laisse encore des points à éclaircir ou à compléter. Il ne laut pas ètre surpris dès-lors si lès classifications proposées manqunent de stabilité. L'étude de la chenille est bien moins avancée que celle du papillon; c'est à peine si lon connaît passablement la moitié des larves' des phalènes. Dans certains genres les chenilles sont à-peu-près toutes inconnues.

Il eut été fort intéressant de rassembler sous un comp-d'oeil général ce que nous savons et ce que nous ne savons point encore sur ces divers points; mais loccasion ne s'y prètait pas, puisque nous devons nous borner ici à compléter l'énumération d'une fort petite portion de la Faune helvétique. On trouvera d'ailleurs éparses, sous forme de notes, un grand nombre d'ohservations sur la structure des organes et sur la distribution des genres el des espèces. Nous y renvoyons les entomologistes.

Cn point, généralement peu appréeié, doit cependant nous arrèter un instant, si nous voulons ètre compris du lecteur; nous voulons parler de la nervation des ailes ou de la disposition de leurs nervures venae). L'aile est l'organe le plus essentiel à étudier chez le papillon. Sa structure et la comparaison des deux paires entrelles fournissent des données de première importance pour la distinction des tribus et des ordres. L'aile est 
pour le lépidoptère ce que les pattes sont chez le coléoptère. Pour hien apprécier les caractères fournis par cet organe, it ne laut pas se borner à y signaler des handes, des raies, des taches ou des points, encore moins des couleurs ou de simples dessins: il faut surtont connaìtre son anatomie et, dans celle-ci son squelette. C'est là ce qu'a parfaitement compris HerrichSchäffer lorsquil publia dans sa Révision du grand ouvage de Hübner une série de talılean organographiques destinés à poser les bases d'une nouvelle elassification des lépidoptìres. C'est en suivant les traces de cet labile observateur que l'on parviendra un jour ì élever l'étude de ces insectes au niveau de celle des classes roisines, et particulierement des coléoptères.

Le syuelette de l'aile est tout entier dans ses norvures; c'e sont elles qui représentent le nieux les analogies et les diflérences de forne et de structure. Leur distribution, leurs anastomoses, leurs rapports réciproques détcrminent la position des lignes et des traits qui serrent de base an dessin. celle des angles et des sinuosités d'où résulte la forme générale. Il existe des relations constantes entre toutes les parties du dessin et les nervures. Pour énoncer ces rélations, il fallait créer une nomenclature spéciale des nervures et des lignes essentielles dans le dessin; e’est là ce que Herrich-Sclı̈̈fer a tenté de mettre à exécution en désignant les nerıures prises à leur extrémité marginale par la série des chiffres 1 à 11 , en partant de l'angle anal. Cette méthode de classification a plus d'un inconvénient. Elie se fixe difficilenent dans l'esprit; elle devient obscure dès qu'une mème nervure principale angmente ou diminue ses subdivisions. En comptant les nervures à leur extrémité elle fait oublier la structure typique de la racine de l'aile et celle du réseau central. Enfin, et c'est iei son principal défaut, elle n’est comparative que pour les lépidoptères, en sorte qu'en passant aux diptères, aux hyménoptères ou aux coléoptères, il faut l'abandonner.

La distribution ptérographique proposée par O. Heer est exempte de ces inconvénients; aussi lui avons-rous donné la préférence dans nos notes. Cet entonologiste reconnail dans tout aile d'insecte six nerrures fondamen- 
tales. En partant du bord antérieur on de la còte de l'aile, il énumère: $1^{\circ}$. La nervure costale (vena marginalis s. costalis. No. 11. Herr.-Schr:) $2^{\circ}$. La nerure médiastine (vena mediastina. No $^{\circ}$ 10. H.-S.). Ces deun nervures chez le papillon s'accolent liune à l'autre, restent indistinctes el ne se subdivisent pas dans leur cours, sauf à leur extrémité où elles forment sourent un petit éventail au sommet de l'aile. Ce sont elles yui par leur réunion domnent à la côte la fixité dont elle a besoin pour le vol. Elles sont beaucoup plus développées à l'aile supérieure qu'à l’inférieure. 3. La nervure scapulaire (venal scapularis. No. 9. H.-S.). Celle-ci naìt tantòt isolée, tantòt rapprochée de la suivante; dans ee dernier cas on la prendrait pour son rameau antérieur; nais il est aisé de se cunvainere qu'elles ne sont alors qu'accolées et non confondues. Cette nervure ne se subdivise pas dans les Géomètres. - $4^{\circ}$. La nervure moyenne externe (ven a externo-media. $\mathbf{N}^{\circ}$. 7 et 8 . H.-S. . Elle forme la cloison antérienre de la cellule et se subdivise en deux branches. Près de sa bifurcation, rarement plus en dehors qu'elle, sourent plus en dedans, se détache le filet anastomotique transversal qui, se dirigeant vers la nerture suivante, clòt la cellule en dehors. Ce dernier filet reçoit la petite nervure centrale de la cellule: le point de jonction des deux on le noeud, correspond au point discoïdal des Phalènes. - 5\%. La nervure moyenne-interne vena internomedia. $\mathrm{N}^{\circ} . \mathbf{2}, 3,4$ et 5. H.-S.) nait toujours isolée de la précédente et forme la cloison postérieure de la cellule; elle se contourne ordinairement un peu sur elle-mêne à mesure qu'elle se subdivise en :3 ou 4 rameaux et se rapproche du lilet anastomotique. La nervure $\mathbf{N}^{\circ}$. 5 de Her.-Schf. fait-elle ou non partie de cette nervure, ou appartient-elle an filet celiulaire central, c'est ce que nous n'examinerons point ici. Les deux nervures moyennes circonscrivent la cellule (area externo-media) qui dans les phalènes est divisée dlans sa longuenr en 2 parties plus on moins égales par un filet parti de la noyenne interne et qu'on peut nommer nervire cellulaire. Celle-ci ne se prolonge pas toujours jusqu'à la marge de l'aile. $6^{\circ}$. La nervure anale (rena analis. $\mathrm{N}^{\circ}$. I et 2 H.-S.) longe le bord interne de Yaile: près de son origine elle énet, dans presque toutes les 
phalènes, un rameau qui circonscrit entre lui et la moyenne-interne un espace que 0 . Heer nomme area interno-media.

Tout autour et au-devant du réseau vasculaire dont nous venons de désigner les troncs et les rameaux, se disposent d'une manière constante et symétrique les points, les yeux, les angles saillants et rentrants des lignes, des bandes et des bords; les échancrures et les dentelures des ailes, les intersections de la frange, etc. Pour donner à ce système de nomenelature une fixité compléte, et pour établir nettement ses rapports avec le dessin, nous avous adopté dans la description de l'aile de beaucoup de phalénes des dénominations arrètées que nous devons aussi faire connaître. Nous avons dù le faire pour éviter les confusions qui règnent dans la plupart dès auteurs sur ce point. Dans la majeure partie de la subdivision des Dendrométrides l'aile supérieure se divise transversalement en 3 espaces, séparés l'un de l'autre par deux rubans, lesquels sont ordinairement encore partagés par 2 ou $\mathbf{3}$ ligues. Si l'on part de la base de l'aile d'une Cidaria p. ex. on trouve d'abord un espace basilaire qui somvent se suhdivise en 2 parties. A cet espace succède un premier ruban fréquemment marqué de stries ou raies parallèles. Après le prenier ruban vient le $2^{\text {me }}$ espace ou bande moyenne (transverse). Cette bande porte vers son milien du còté de la côte le point discoïdal et se tronve aussi sonvent marqué de lignes transverses plus ou moins sinueuses ou anguleuses. Le deuxième ruban semblable au premier suit le $2^{\text {eme }}$ espace. Le $\mathbf{3}^{\text {eme }}$ espace ou bande marginale termine l'aile; il est partagé par la ligne ondulée ou lulgurale (lorsqu'elle existe) et limité par les points ou les stries qui bordent la frange. En donnant à ces expressions le mème sens dans toutes les descriptions, quelles que soient les teintes et les dessins qui recouvrent l'aile, il n'arrivera plus que l'un décrive comme fond ce que l'autre représente comme un dessin superposé, ou que l'un appelle bande ce qui est espace ou raie chez un autre observateur.

Notons encore que dans la description comparative de la nervation, il fant autant que possible prendre des individus du mème sexe; quoique les dillerences entre les deux sexes soient généralement petites, elles n'en existent pas moins. Nous avons choisi de préférence l'aile inférieure soit 
parce que les nervures y sont plus faciles à découvrir, soit aussi parce qu'elle varie davantage que la supérieure et fournit dès-lors des points de comparaison plus saillants.

Nous n'avons apporté ancun changement à la circonscription de la lámille des phalénides telle que l'on entendue les entomologistes depuis Linné. Ce groupe est si naturel, qu'il ne serait possible ni de le subdiviser, ni de le réunir à quelque autre sans méconnaître des analogies qui se révèlent au premier coup-d'oeil; il est en mème-temps si compacte et si fortement serré dans sa texture par l'entrecroisement des relations spécifiques, que l'on est presque forcé d'en revenir à la pensée dı grand naturaliste suédois et de n'en firire qu'un seul grand genre subdivisé tant bien que mal en sous-genres dilliciles à caractériser. - Nous n'avons exclu des géomètres qu'une seule espèce suisse, Aventia flexularia Bdv, qui par la chenille et les caractères de l’insecte parfait se place plutòt dans la tribu des Noctuo-phalénites (Boisdl. Index), ou, si l'on préfère, non loin des Herminies.

Entre toutes les méthodes de classification proposées nous avous préféré, non sans hésitations cependant, celle de Herrich-Schäffer (Hübner's Revision), ce n'est pas qu'elle fut à nos yeux exempte de délauts, mais la série qu'elle établissait conservait assez bien les rapports généraux des groupes. L'ayant adoptée, nous oserons mieux en faire la critique. Son premier défaut se trouve dans la subdivision de la famille entière en deux tribus, désignées sous le noms de Dendrométrides et de Phytométrides. A ne prendre que les formes extrèmes, cette distinction paraît fonlée en raisons; mais dès que l'on cherche des caractères positil's de structure ou que l'on veut tenir compte des espèces intermédiaires, on ne sail plus où s'arrêter. Le caractère que Her,-Schäffer tire de l'insértion de la nervure scapulaire sur la nervure moyenne-externe dans un point plus ou moins rapproché de l'angle antérieur de la cellule, est tout-à-fait mal cloisi, puisqu’il n'est qu'un plus ou un noins passablement variable. La différence de développement entre les deux paires d'ailes eut offert des caractères plus solides, sans ètre netter ent tranchés, il est vrai; la série qui en résulte diffère peu de celle de notre auteur. mais elle est mieux assise. En partant des 
groupes qui ont la paire postérieure aussi complétement organisée que l'antérieure (Acidalia, Ephyra, Boarmia, Gnophos, etc.), on passe insensiblement à ceux où les postérieures sont moins développées que les antérieures (Cidaria, Eupithecia, Lobophora).

Les grompes formant les genres de Her.-Schälfer sont tantòt trop restreints, tantòt trop étendıs. Là où quelque modification des nerrures on de la marge des ailes donnait prise à des distinctions, il n'a guères man¡ué de les faire valoir; là ò̀ au contraire le facies parlait seul, il a admis des genres très étendus. Ici, comme partout ailleur, on peut voir comment la manière, dont lobservateur saisit la valeur des caractères, détermine seule les coupures qu'il établit. L'homme de cahinet divise et subdivise à l'infini; l'observateur pratique applique plus volontier la synthèse. Il nous eut élé facile de modifier plusieurs des genres adoptés dans la Révision de Hubner; mais une pensée nous a sans cesse arrèté: s'il n'est pas prudent de construire des groupes avec les seules espèces européennes, combien plus cette tentative est-elle téméraire lorsquil s'agit d'une Faune très limitée. La manie de construire des classifications et de créer des genres dans tous les catalogues, a fait un grand mal à la science; c'est elle qui a éleré ces échaflaudages qui, tombés successivement, embarrassent aujourd'hui le terrain de leurs décombres.

Un troisième dèfaut de la Révision de $\mathrm{Hubner}$ fut d'établir par dich otomie la série des espèces dans les genres un peu nombreux Acidalia, Eupithecia, Larentia), il en est résulté que, sous espoir de rendre la science plus accessible aux amateurs, il a souvent détruit les analogies et placé deux espèces très voisines aux deux extrémités du genre.

Nous ne parlerons pas ici des autres méthodes de classification, parce qu'elles sont toutes plus vicieuses que celle de Her.-Schäffer. La meilIeure d'entr'elle est encore sans contredit celle de Treitschke, mais elle est aussi la moins systématique.

Nous arons évité autant que possible de créer des noms spécifiques nouveaux. La dénomination la plus répandue en Allemagne nous a toujours paru préférable. La question de priorité nous touche fort peu; les descrip- 
tions ou les figures, qui ne laissent pas de doute, devant senles faire règle.

La Faune de la Suisse se montre aussi riche en Phalènes, qu'elle l'est eu égard à d'autres animaux mieux connus. En général, aucun pays sur le globe ne présente, entre des limites aussi étroites, une aussi grande variété d'espèces, Le dernier catalogue de Heydenreich (1851) énumère 7222 espéces européennes. Si de ce nombre on écarte 60 à 70 numéros employés pour des variétés ou pour des espèces douteuses, on comptera 660 espèces en Europe. Notre catalogue indique 329 espèces, ou environ la moitié de celles qui habitent le continent.

Ce fait trouve son explication dans la position géographique de la Suisse au centre de l'Europe, ainsi que dans la variété d'exposition solaire et de constitution géologique de son sol. Voiei du reste, comment nos 329 espèces se distribuent géographiquement. - Un tiers environ, soit 112 espèces. se trouvent communément répandues sur toute la surface de l'Europe, i l'exception peut-être des points les plus extrèmes de la chaleur ou du froid. Un nombre un peu plus considérable, soit 130 espèces à-peu-près, sont réputées rares et ne se recontrent guères qu'isolées. La plupart d'entre ces dernières (environ 80) ne se présentent pas exclusivement dans une localité limitée, tandis que les $\mathbf{4 0}$ autres n'ont été jusqu’iei recueillies que sur un point particulier. 11 de celles-ei n’ont pas été, jusqu’à présent, saisies hors de la Suisse.

Eu égard à la latitude ou plutôt à l'exposition du sol ou an elimat qui en est la conséquence, nos Phalènes comptent 48 espèces purement alpines: plusieurs d'entr'elles reparaissent dans le nord de l'Europe. 16 espèces particulières aux sous-alpes et aux montagnes existent presque toutes dans le nord de l'Allemagne.

La Suisse méridionale ou chande compte 25 espèces du midi: celles-ci ne se prennent guères que sur les bords de nos grands laes, ou dans les vallées chaudes. Les espèces septentrionales et qui ne fréquentent pas les montagnes, ne vont pas au-delà de 20.

Il eut été fort intéressant de distribuer nos Phalènes d'après les chaìnes 
de montagnes, les bassins on les rersants, auxquels elles appartiennent; maais cette distribution exige des observations bien plus nombreuses que celles dont nous avons pu faire usage. Les observateurs en état de les recueillir sont clairement semés sur le sol helvétique. Il n'est pas aisé de rassembler toutes les espèces d'une contrée même limitée, encore moins d'arriver à leur détermination exacte. La plupart des collecteurs laissent de côté une classe d'insectes qui leur paraît inabordable: ceux qui ont plus de persévérance se procurent très difficilement les espèces rares. Les marchands d'insectes sont encore moins disposés à récueillir ces animaux fragiles et peu recherchés.

Les renseignements quelque peu complets qui nous sont parvenus, émanent de deux sources: de Mons. Meyer-Dür à Burgdorf et de Mons. Rothenbach père, à Schüpfen près d'Aarberg. Ces deux zélés entomologistes ont exploré avec le plus grand soin les lieux qu'ils habitent et les Alpes de la Suisse centrale. Mons. Bremi-Wolf à Zurich nous a livré aussi des renseignements précieux sur la Faune du canton de Zurich et des Grisons. C'est surtout à l'obligeance inépuisable des deux premiers collecteurs que je dois la connaissance des espèces les plus rares. J'ai eu d'ailleurs sous les yeux les collections de Mons. le Dr. Cliavannes de Lausanne et de Mons. Zeller, fabricant de soieries, près de Zurich. Mons. Kriechbaumer et Mons. l'ingénieur Mengold à Coire m'ont encore fait parvenir quelques renseignements. Sans l'empressement avec lequel ces Messieurs ont bien voulu concourir à mon travail, je n’eusse sans doute jamais pu le présenter comme une portion de la Faune helvétique.

Les désignations spécifiques méritent, j’ose le dire, toute confiance: chaque espèce a été déterminée avec soin, soit au mojen des auteurs fondamentaux, soit par des échantillons sûrs. Là où les doutes n'ont pas pu être entièrement levés, il en a été fait mention. Partout où mes observations pouraient éclairer un diagnostic difficile, ou rectifier quelque erreur, je les ai énoncées.

La détermination des époques d'apparition a été spécialement soignée; ce point est important dans une faune. Lorsque ces époques sont exprimées 
en dates fixes, il va sans dire qu'il ne faut point leur accorder pour cela une valeur mathématique.

Je n'ai obtenu presqu'aucun renseignement sur les chenilles de nos Phalènes. Les difficultés dont est hérisséc cette partic de leur histoire rendent ee genre d'observations extrèmement difficile.

Les Phalènes n'offrent pas un très grand intérèt au cultivateur. Deus de leurs larves ont seules jusqu'ici attiré l'attention par leurs ravages sur les arbres fruitiers; encore l'une d'entr'elles (Hibernia defoliaria) cause-t-elle fort rarement quelque dommage. Il ne reste dès-lors qu'une seule espèce (Idea brumaria, Treit.) à classer parmi les insectes vraiment nuisibles. Chose bizarre, les femelles de ces deux espèces sont destituées d'ailes; mais l'abondance de leurs oeufs supplée à la privation du vol.

Le Bulletin de la Société vaudoise des sciences naturelles a publié dans soll $\mathbf{n}^{\circ} \mathbf{2} 2$ (tome III) un catalogue des phalènes suisses, destiné à préparer la publication de celui que nous mettons aujourd'hui sous les yeux du public. Ce catalogue devrait subir aujourd'hui quelques modifications. Le nombre des phalènes suisses s'est augmenté dès-lors de neuf espèces; mais en revanche deux espèces, les $\mathrm{n}^{\text {os }} \mathbf{1 7 5}$ et $\mathbf{2 8 0}$, doivent être retranchées. II laut en outre y corriger la désignation des deux grandes sections des géonètres: Ies phytométrides doivent se nommer dendrométrides et vice-versà. Le $n^{\circ} 113$, serotinaria, doit porter le synonyme de Hub. 147, et non celui de Treit. - Le $n^{\circ} 114$ est une espèce inédite, appellée mendicaria, par Her--Schf. Le $\mathbf{n}^{\mathbf{0}} \mathbf{1 7 5}$ doit appartenir à tibialaria. Hub. Les espèces qui doivent ètre ajoutées sont: $116^{\text {b }}$ Zelleraria. H.-S. - $151^{\mathrm{b}}$ glaeraria. W. V. - 188 lariciaria. Fr. $-205^{b}$ inturbaria. Hub. $-211^{b}$ pumilaria. Hub. $-217^{b}$ riguaria. Hub. $-243^{\text {b }}$ chalybearia. Hub. $-302^{\mathrm{b}}$ perfuscaria. Haworth.

Lausanne, ce $1^{\mathrm{r}}$ Janvier 1852. 


\section{Phalénites. Latr.}

(Phalenides. Dup. - Geometra. Lin. - Phalaena. Fab. - Geometra. Bdv. - Geometriden. Hub. Rev.)

Inse ete parfait. - Ailes larges, étalées dans le sommeil; inférieures presque aussi déreloppées que les supérieures, ne se reployant pas sur elles-mémes, dans le repos. Pattes faibles. - Antennes fliformes, recourertes d'éeailles à la partie supérieure; villeuses en dessous; sourent barbues ou ciliées sur les bords, ehez le male. - Palpes ordinaires, simples, courts, droits ou légèrement recourbés en dessous, arrondis. - Corps gréle.

Larve. - Alongée, lisse ou rugueuse, rarement velue, arpenteuse. - Paltes: 2 paires thoraciques pointues; 2 paires rapprochées de lamus; 1 paire anale, forte.

\section{A. Phytométrides. Hub. Reris.}

G. I. Geometra. Tr.

Nota. Ce genre fournit des espèces aux Gen.: Geometra, Hemithea, Phorodesma de Boisd. et Chlorochroma de Dup., aux G. Ilipparchus, Cleora et Chlorissa de Steph., et enfin à Macaria Curt. - On peut en faire 6 ou 7 groupes, si l'on veut. La nervation des ailes offre quelques variations.

1. Papilionaria. Lin.

Hub. 6. - Treit. - Dup. - Bdv. 1415. - Her.-Sch. I. 1. Wood 19,494. - Heydenr. Cat. 54.

Larva. Hub. G. I. Ampl. B a. f. I.

Nulle part fréquente. Les bosquets d'aune; à Rolle (Chvn.), Burgdorf (Meyer). Prise quelquefois à Lansanne (Lah.). Langnau et Schüpfen du 12 juillet au 5 aoùt; rare (Rothb.). A Zurich sur l'Albis et le Hörnli; rare (Bremi). Berne (Renk). N'a pas èté encore trouvée dans le Doubs (Bruand). 
Vole le soir à la nuit tombante. Dans la fig. de Hub. le bord externe des ailes supérieures n'est pas assez arrondi, ce qui fait paraitre le sommet trop aigu.

2. Bajularia. Esp.

W. V. - Hub. 3. - Treit. - Dup. - Bdv. 1417. - Wood, 19,498. - Fr. b. 36, 1. - Her.-Schf. I. 3. - Heydenr. Cat. 50.

Ditaria. F.

Pustularia. Panz. Knoch.

L a rv. Hub. G. I. A mpl. A. b. f. 1.

Plus rare que papilionaria. Zurich: les bords des bois; l'Uto; rare (Bremi). Berne (Meyer). Burgdorf (Heuser). Lausanne, une fois (Lah.). Tris rare dans le département du Doubs (Bruand).

3. Aestivaria. Esp.

Hub. 9. - Treit. - Dup. - Bdv. 1428. - H.-Seh. I. ‡. Heydenr. Cat. 59.

Fimbriata. Götz.

Strigata. Vill.

Thymiaria. W. V. Wood 26,736 .

Vernaria. F. Esp.

Larv. Hub. G. I. Ampl. B. a. b. f. 2 et B. b. f. 1.

Commune dans les bois taillis au commencement de juillet (Lah.). Schüpfen; du 27 juin au 13 juillet; pas rare (Rthb.); très rare à Burgdorf et dans l'Emmenthal (Mey. Jamais en mai chez nous, oủ l'on n'a observé jusqu'ici qu'une seule apparition.

4. Buplevraria. W. V.

Hub. 8. - Treit. - Dup. - Bdv. 1429. - Her.-S'ch. I. 8. Heydenr. Cat. 61.

Thymiaria. Lin. - F. - Bork.

Fimbrialis. Scop.

Larv. G. I. Ampl. B. a. b. f. 3.

Les lieux sers exposés au soleil, dès le milieu de juillet au commencement d'aoút; 
près de Lausanne sur les collines qui couronnent Lavaux; a Montcherand près d'Orbe; en Valais (Lah.). A Salgetsch près Sierre, le 10 août. - Berne, Burgdorf sur les collines arides le 8 juillet (Meyer). Les escarpements de l'Uto près Zurich (Bremi). Au pied du Jura; Lys, Nidau, du 10 au 16 juillet, Rothb.). Cette espèce est partout assez rare.

La femelle, phus grande que le mâle, a le fond des ailes comme aestivaria; le màle prend souvent une teinte rougeâtre sur un fond plus pâle.

5. Virida ria. Lin.

IV. V. - Hub. 11. - Treit. - Dup. - Bdv. 1423. - H.-Schf.

I. 9. - Wood 26,734. - Heydenr. Cat. 62.

Cloraria. Steph. (non Hub. 352.)

Commune dans les prés bumides du Joral. Je ne l'ai pas encore prise dans les alpes (Lah.). Elle parait en mai le long du lac et dans les lieux chauds; en juin sur les coteaux élcvés. Reparaît quelquefois dans les premiers licux à la fin d'aoùt (Lah.). L'Uto, l'Albis, le Zürichberg; fréquemment (Bremi). - Commune à Schüpfen et à Burgdorf du 10 mai au 8 juin (Rothb. Meyer).

Varie pour la grandeur, l'intensité du vert et les ondulations des stries blanches.

6. Putalaria. Lin.

$$
\text { W. V. - Hub. 10. - Bdv.? 1127. - Dup.? - H.-Schf. }
$$

1, 11. - Wood 26,737. - Heydenr. Cat. 67.

Lacte a ri a. Scop. ent. Carn. (Treit.)

Pas rare à la fin de mai et au commencement de juin, dans les bois élevés et ombrés où croît le myrtille, au-dessus de Lausanne; elle y précéde aeruginaria de peu de jours (Lah.). Assez communc sur le bord des forèts près de Schüpfen et de Burgdorf, du 18 mai au 8 juin (Rothb. Meyer).

Les synonyıes de Dup. et de Bdv. me paraissent incertains, parce qu'en France aeruginaria porte ordinairement le nom de putataria; celle-ci ne parait pas y avoir été trouvée jusqu'ici. De là vient que Dup. affirme que dans aeruginaria l'angle de la ligne médiane est plus prononcé aux ailes inférieures que dans putataria; tandis que le contraire a lieu. Curtis cite aeruginaria, Hub. comme synonyme de son Hipp. putatarius. 
7. Aeruginaria. W. V.

Ilub. 46. - Treit. et Sup. - Dup.? - Bdv. 1426? Fr. b.

30, 2. - H.-Schf. I. 12 . Heydenr. Cat. 68.

Lacte a ria. Lin. (Treit.)

Larv. G. I. Ampl. B. b. f. 2.

Très commune dans les bois et les taillis entremélés de bouleaux des euvirons de Lausanne; descend plus près du lac que putataria, et craint moins le soleil et la chaleur. Apparait à la fin de mai et au commencement de juin (Lah.). - Sur les hauteurs des environs de Zurich, dans les bois (Bremi.) - Commune du 13 mai au 21 juin dans les bois du canton de Berne (Rothb.), à Burgdorf dans les mèmes lieux que la précédente, mais beaucoup plus commune. (Meyer)

Duponchel, en reproduisant la mauvaise fig. de llub., ne s'est pas aperçu qu'elle représentait la phalène qui est partout reçue en France sous le nom de putataria et qui $y$ est commune.

8. Vernaria. Lin.

W. V. - Hub. 7. - Dup. - Bdv. 142\%. - H.-Schf. 1. 13. Wood 19,495. - Heydenr. Cat. 70 .

Aeruginaria. Bork.

Chrysoprasaria. Esp.

Larv. Hub. G. I. Amplis. B. a. f. 2.

Assez commune dans les taillis des environs de Zürich; sur l'Uto, l'Albis, le Zurichberg (Bremi). Peu fréquente antour de Lausanne; dans les lieux humides et chauds, en mai; plusieurs fojs près de Crissier (Vand), vers la fin de juillet Lah.). - Rare dans le canton de Berne (Meyer) et dans le Doubs (Bruand).

\section{G. II. Pseudoterpna. Hub. Verz.}

Nota. Dès qu'on ne subdivise pas le gen. préeédent, il n'y a pas de raison suftisante pour en sẻparer celui-ei. Curtis réunit cythisaria à papillonaria. La nerration des inférieures est la même que dans viridaria. Les tarses postérieurs ont une gaine poilue ainsi qu'aestivaria. 
9. Cylhisaria. W. V.

Hub. 2. - Dup. catal. - Bdv. 1417, - Her.-Schf. I. 14.

Wood 19,497. - Heydenr. Cat. 71.

Prasinaria. F, Devill.

Genistaria. Devill.

Coronillaria var. et agrestaria. Dup. hist.

Pruinata. Götz.

Larv. Hub. Geom. I. Ampliss. B. a. f. 1. et B. a. b. f. I.

Les taillis de pins près de Wangen (Zurich); assez fréquente (Bremi). - Les bois et les taillis du Jorat au-dessus de Lausanne; le pied du Jura près d'Orbe; peu rare; juillet et aoủt (Lah.) - Environs de Bienne du 10 juillet au 2.2 août; rare (Rothb.)

Je l'ai prise à la même époque sur les bords du lac Majeur. La var. agresıaria Dup. se prend quelquefois, mais rarement, en juin, dans les haies près de Lausanne.

\section{G. III. Acidalia. Bdv.}

Nota. Guères mieux circonscrit que les deux précédents et peut aussi fournir plusieurs groupes. La série de Her.-Sichf. n’est pas naturelle. - Curtis réunil vibicaria, amataria, a piciaria et vespertaria dans son G. Timandra; imitaria, emutaria et emarginaria dans Macaria et les autres dans Ptychopoda. Steph. - Dosythea Dup. ne saurait être séparé d'Acidalia. Idaea et Acidalia. Treit. se confondent en plusieurs points. - Acidaliar. Boisdv. est mieux caractèrisé.

Amataria tient le milieu entre Acidalia et Ephyra, qnoique mieux placée dans ce dernier: si ces 2 genres n’ètaient pas déjả trop voisins, on pourrait admettre entr'eux Timandra. Bdv. - Vibicaria a plus de rapport avec Geometra, par sa nervation, qu'avec Acidalia. - En ayant égard à ce dernier caraetère les $\Lambda \mathrm{e}$ idalia se divisent en deux groupes. Le premier, comprenant les $n^{05} 13$ a $32,36,41,48,49$ et 51 , a le prolongement de la nervure moyenne-externe des postérieures, bifurqué au-delà de la cellule; le second, formé des $n^{o s} 33$ à 35,37 à 40,42 à 47 , présente la bifurcation à la pointe de la cellule. La plupart des espèces ont les tarses de la paire postérieure chez le màle, ou épaissi, ou dilaté, ou enveloppé de pinceaux de poils, ou simplement aminci; avec l'extrémité de la patte d'autant plus atrophièe que le tarse est plus gros. Les antennes sont fréquemment ciliẻes ou pennicillées. 
10. Amataria. Lin.

W. V. - Hub. 52. 524 ct 525 (var.) - Treit. - Dup. - Bdv. 1918. - Fr. B. 60, 1. - Her.-Schf. I. 18. - Wood 20,533. - Heydenr. Cat. 637.

Larv. Hub. G. I. Ampl. J. b. f. 1.

Commune, une première fois en mai et une seconde à la fin de juillet et en aoùı, le long des murs, dans les lieux secs et les buissons des bords du Lèman. La seconde apparition est ordinairement plus abondante que la première. (Lah.) - Du $2 t$ mai au 20 juin et du 23 juillet au 24 août, dans les environs de Schüpfen: commune Rothb.). - Lieux secs et exposès au soleil des environs de Zurich; pas rare (Bremi). - Espéce commune dans toute l'Europe.

11. Vibicaria. Lin.

$$
\text { W. V. - Hub. 50. - Treit. - Dup. - Bdv. 1479. - Her.-Schf. }
$$

I, 19. - Wood 20,538. - - Heydenr. Cat. 635. Pellonia Dup.

Rubrociliata. Götz.

Larv. Borkb. t. 5. p. 503. (descript.

Les côtes arrides et exposées au soleil des bords du Léman: le pied des Alpes, dans la vallée du Rhône, à la fin de juin et au commencement de juillet; le pied du Jura en juillet: assez rare (Lah.). - Dans les mêmes expositions, sur les bords du lac de Zuricb; l'Uto (Bremi). - Assez commune dans les environs de Bicnne, du 9 juin au 2 aoùt (Rothb.).

J'ai reçu du pied du Jura vaudois un exemplaire portant l'intervalle des deux raies entièrement rouge, comme dans calabraria; mais les deux raies restent toujours bien marquées et plus foncèes, ce qui n'a pas lieu dans celle-ci. Les deux espèces se distinguent d'ailleurs encore par la structure des antennes chez le màle. - Vibicaria est propre à l'Europe centrale.

12. Calabraria. Esp.

Hub. 495 365 (var.), 546, 547. - Treit. - Dup. - Bdv. 1480.

- Her.-Schf. I. 20. - Heydenr. Cat. 632. Hellonia Dup.

Var.? Tacniaria? Fr. n. B. 131,3. - Bdv. 1458. (teste. Iler.-Schf.)

Les coteaux tres chauds et secs des bords du Léman et de la vallée du Rhône. Le 
Valais, à la même époque que Vibicaria; mais plus rare (Lah.). - Assez commune au pied du mont Salève près de Veirier (Meyer). - Sur l'Irchel, près Zurich; pas très rare (Bremi). Probablement plus frèquente dans la Suisse transalpine. - Espéce méridionale.

Cette espèce et la précédente agitent leurs ailes après s'être posées de la même manière que Palumbaria.

13. Microsaria. Bdv. 1846.

Dup. cat. - Her.-Sehf. I. 22.

Pusillaria. Hub. 99. - F. v. R. 1. 61. - Dup. hist. - Hub. Verz. 3015.

Treit. - Heydenr. Cat. $347^{\mathrm{a}}$.

La rv. Fisch. v. R. p. 163. t. 61.

Pas très rare dans les environs de Zurich oì elle a été prise par MM Breni et Zeller. Ce dernier l'a aussi trouvée près de Misocco, vallée de ce nom (Lah.).

14. Laevigaria. (ta.) W. V.

Hub. 74. 331. - Treit. - Dup. - Bdv. 1853. - Iler.-Schf. 1. 23. - Heydenr. Cat. 705.

Molliliata. Bork.

Au bord dn lac de Constance (Leiner). - Très rare en Suisse.

15. Scutularia. II. V.

Hul. 72. - Dup. hist. t. 210. - Bdv. 1850. - Her,-Schf.

I. 25. - Heydenr. Cat. 706 .

Lividata. Lin. ? - Wood 25,715. - Steph. - Westw.

Trigeminala? Steph.

Dimidiata. Götz.

L a rv. Annal. de la Sc. entom. de France, I. série. t. 3. p. 417. pl. IX. f. $1-5$.

Pas rare dans les haies garnies d'orties, depuis la mojuillet jusqu'à la mi-aoùt, dans le voisinage de Lausanne et le long du Léman (Lah.). - Rare autuor de Burgdorf. A la Chartreuse près de Thoune (Meyer). - Commune près de Schüpfen, du 11 au 25 juillet. (Rothb.) - De même autour de Zurich (Bremi). 
Espèce europẻenne qui préfère les lieux chauds et abrités. Mr. Audoin a décrit et figurè avec soin la chenille et ses métamorphoses; elle vit sur les plantes basses. La figure qu'il donne du papillon est à peine reconnaissable.

16. Reversaria. Treit.

Dup. hist. 1. 173. f. 3. - Her.-Schf. 1. 26. - Heydenr. Cat. 715.

Scutularia? Hub. 73 . -

Bisetaria, var. Bdv. 1851. - Steph.

Fimbriolata? Westw. - Steph.

Les bois taillis au-dessus de Lausanne en juin et en juillet (Lah.). - Le 21 juillet dans les bosquets Schönbübl, près de Burgdorf (Meyer). Rare en Suisse.

Ordinairement confondue avec bisetaria, dont elle se distingue par les caractères indiqués par Treits. el par Her.-Schf. Son apparition est plus hative; elle prèfère les bois, où l'on ne trouve guères bisetaria; elle est aussi pour l'orlinaire un pen plus grande qu'elle.

17. Incanaria. W. V.

Hub. 106. - Treit. - Dup. hist. 1. 173. f. 7. - Bdv. 1841. - Her.-Schf. I. 27. - Heydenr. Cat. 694.

Dilutaria Hub. 589 ?? (non 100).

Virgularia Hub. 104 (var. \$). - Steph.

Cinerata Stepb.? - Wood 25,714.

Scutularia Dup. hist. t. 174. f. 2.

Commune partout en juin, juillet et aoùt, dans les jardins et les haies; s'introduit volontiers dans les maisons (Lah.). - De mème à Burgdorf (Meyer). - Commune près de Schüpfen, du 19 juin au 28 juillet et mème jusqu'au 25 août (Rothb.)

Femelle ordinairement plus foncee et plus grosse, surtout dans les endroits chauds: de là vient virgularia Hub. La même différence s'observe dans d'autres Acidalia praLa ria, mutataria, etc.) - Il en existe une varièté plus petite et plus blanche qui pourrait bien être une espèce distincte, quoique je n'aie pu encore lui découvrir de caractères suffisants. Chez celle-ci la femelle ne diffère pas du mâle, et l'èpoque de l'apparition est double (ce qui n'est pas pronvé pour la forme ordinaire), d'abord en mai, puis à la 
fin de l'èté, en août et en septembre. On l'a confondue avec calceearia Kol.; mais celle-ci a toujours une teinte crétacèe, très prononcėe. - Cette variété n'est pas rare certaines années, dans les haies au-dessous de Lausanne. Mr. Bruand l'a aussi recoltẻe a Besançon. J'ai lieu de croire que c'est sur elle qu'a èté prise la fig. 7 (t. 173) de Dup.; seulement la base de l'aile n'est pas ombrée de gris comme dans cette fig. Dilutaria Hub. 589. peut mieux s'appliquer à calce aria (Her.-Schf. 437.) ou à sodaliaria (Zell.) qu'à incanaria; en tout cas pas à interjectaria. - Ces espèces difficiles à caractériser ont besoin d'être revues au moyen d'un grand nombre d'exemplaires frais.

\section{B ischoffaria. Hub. G. 586-588.}

Dup. Cat. - Heydenr. Cat. 216 (Psodos).

Le seul individu (mâle) de cette espèce que je possède fut pris en juin 1848 dans une maison d'Aigle, oủ il était appliqué contre un mur à la manière d’incanaria. Cette particularité n'est pas le seul trait qui la classe parmi les Acidalia. Les tarses postėrieurs renflès se retrouvent cbez plusicurs espèces voisines, quoique ici le renflement ne s'accompagne pas d'un pinceau de poils comme dans bisetaria. L'éclaircie médiane blanchâtre qui existe sur les ailes supérieures et la ligne ondulèe marginale, brisèe vers son milieu, se retrouvent dans les Acidalia. Du reste l'aspect général et la coupe des ailes confirment ce rapprochement.

Duponchel ne fit attention qu'à la couleur lorsqu'il rangea cette phalène parmi les Psodos. Sa taille fit croire sans doute à Her.-Schf. qu'elle devait appartenir aux Eupithecia.

Lindividu que j’ai sous les yeux tient le nilieu entre les fig. 586 et 588 de Hub. Les figures citées sont parfaitement exactes.

19. Straminaria (ta).

Treit. sup. II. 205. - Bdv. 1880. - Dup. Cat. - Her.-Schf.

I. 28. fig. 82, 83. - Heydenr. Cat. 665 .

Sylvestraria. Hub. 94 .

Grammicaria. Bdv. 1893. - v. Tisch.

Dilutaria? Steph.

Cette espèce, très rare, a èté prise une fois près de Burgdorf par Mr. Heuser, et près d'Aarberg, le 4 août, par Mr. Rothenbach. 
La fig. de Hubn. sur l'exemplaire que j'ai sous les yeux) est tellement chargée de gris-violet, que l'on a de la peine à admettre son identité avec les fig. de Her-Scbf. Sur un autre exemplaire de Hub., que j'ai pu voir, la couleur est beaucoup moins foncée et lient le milieu entre la première et la seconde. - II n'est done pas étonnant que Treit. n'ait pas reconnu la sylvestraria (Hub. 94) dans sa stra minata, et que Bdv. ne voyant que la fig. de Hub. en ait fait 2 espéces distinetes.

20. Bisetaria. Bork.

$$
\begin{aligned}
& \text { W. V. (ta). - Treit. - Dup. hist. 173, 4. - Bdv. 1851. (exel. } \\
& \text { synon.) - Wood 714. - Westw.? - Her.-Schf. 1. } 29 . \\
& \text { f. 116. - Heydenr. Cat. 714. }
\end{aligned}
$$

Assez commune dans les haies et sur les bords des bois en juillet et au commencement d'aoút; elle fuit moins les hauteurs que seutularia (Lah.). - Environs de Soleure, de Meyringen, Burgdorf etc. (Meyer). Du 11 juillet au 7 aoút; commune prés de Schüpfen (Rothb.). -

B is etaria (Westw.) pourrait bien être une especce anglaise et dilutaria (Wood 712) se rapporter à reversaria. La sylvestraria $\sigma^{*}$ (Dup. 177, 7) est encore indècise; sa figure est trop imparfaite.

21. Aversaria (ta). Lin.

$$
\begin{aligned}
& \text { W. V. - Treit. - Dup. - Bdv. 1910. - Hub. 56. - Her.- } \\
& \text { Schf. 1. 33. - Fr. n. B. 36, 1. - Wood. - Heydenr. Cat. } \\
& 682-684 \text {. } \\
& \text { Remutata. Bork. - Wood 729. - Hub. 389? (ria). } \\
& \text { Larv. Schw. Reitr. t. 16. f. 1-10. }
\end{aligned}
$$
el dans tout l'Emmenthal (Meyer). 
22. Deversaria. Treit. et F. v. R., in litter.

Her.-Schf. f. 305-308, 314? I. 34. - Heydenr. Cat. 686

Je confondais cette espèce aver suffusaria avant de l'avoir reçue de Vienne; elle lui ressemble en effet beaucoup. Je l'ai prise au milieu de juillet près de Lausanne et au-dessus d'Aigle, au pied de la montagne. - Assez fréquente près de Bienne, du 29 juin au 11 juillet. - Près de Vevey (Rothb.). - Salgetsch, près Sierre, Haut-Valais, le 10 aoùt (Meyer).

Le mâłe a les tarses postérieurs fortement culottés. Ses antennes filiformes ont les tubercules carrés, peu proéminents et pubescents. - La tête est d'un blanc pur et le collier roux. - Chez la femelle les tarses sont de la longueur des tibias, tandis que dans suffusaria ils sont plus courts qu'eux.

Devers a ria se distingue, du reste: $1^{\circ}$ par la troisieme ligne onduleuse, formant un lèger angle vers la côte; dans suffusaria elle s'arrondit en s'approcbant de la cóte; cetle différence est surtout marquée en dessous; $2^{\circ}$ par les points du centre des supérieures placés en dehors de la deuxiẻme ligne et non en dedans; $3^{\circ}$ par la première ligne très ondulée et moins arrondie: $4^{0}$ par les points de la frange placés en dehors de la ligne de démarcation, ce qui fait paraitre celle-là dentelée.

Son vol est pesant; elle s'alıat à terre. Son corps est un peu ramassé et son thorax épais et carrè.

\section{Suffusaria. Treit.}

Bdv. 1908 - Her.-Schf. I. 35. f. 309. - Heydenr. Cat. 685. Inornata. Steph. - Haw. - Wood.

Je n'ai pris qu'une senle fois cette espèce dans les environs de Lausame; elle ressemble tellement à la précédente qu'elle est ordinairement confondue avec elle. Peut-être même le seul èchantillon que je possède et qui n'est pas très bon, appartientil encore à deversaria (Lah.).

24. Ossearia (ta). F.

W. V. - Treit. - Hub. 102. - Steph. - Dup. - Bdv. 1877.

Her.-Sichf. 1. 36. - Heydenr. Cat. 660.

Bimaculata. Westw.

Commune partout, excepté dans les bois sombres, en juillet et en aoùt (Lah.). - 
Très commune au pied du Jura, près de Soleure, vers la lin de juillet (Meyer). - Fréquente autour de Zurich (Bremi). - Commune près de Scbüpfen dès le 17 juin au 6 août. - Les exemplaires recueillis en juin appartiennent probablement á holosericearia. Cette espèce varie pour la taille et la couleur; dans les lieux secs et arrides elle diminue de grandeur et de coloration. Les femelles sont volontiers plus grosses et plus colorèes que les mâles. Les 3 points noirs, marquant à la côte l'origine des 3 lignes, sont plus au moins saillants comme dans interjectaria. La couleur rouille de la côte devient quelquefois à peine visible, tant elle pâlit. Le filet noir qui limite la frange peut disparaitre en dessus, janajs en dessous. Les points discoïdaux sont toujours visibles. Le dessous est plus ou moins teintė de gris violet, plus marqué aux supèrieures et vers la base. Les 2 lignes moyennes sont plus saillantes en dessous, parfois celle de la marge et même celle de la base se dessinent en dessous: dans ce dernier cas la teinte grise y est très faible. Les atômes noirs s'accumulent sur la côte et sur les deux lignes mèdianes. - Espèce très répandue.

25. Interjectaria. Bdv. 1879.

Her.-Schf. f. 78, 79. 1. 37. - Heydenr. Cal. 661.

Dilutaria. Huh. 100 (non 589).

II a rginepunct a ta? Steph.

Dès le milieu de juin à la mi-juillet, dans les haies à l'orient et au sud de Lausanne; frèquente. A Bellinzona le 22 juillet (Lah.). - Fort rare sur la pente d'une colline arride exposée au soleil et couverte de Thymus serpillum, a la Ziegelmatte, près Burgdorf; 22 juillet (Mcyer).

La côte est souvent grisâtre et plus foncèe, mais jamais brunâtre comme dans osse aria. Les ailes ont un aspect luisant et les stries sont fortement anguleuses et ondulees. Les points noirs de la côte sont très saillants. On la confond aisément avec la précédente. Ses moeurs la distinguent entièrement d'ossearia; elle parait avant elle et disparaît lorsque celle-ci commence; elle se plaît dans les fourrés de ronces. - Se trouve aussi dans les forêts (?) du Doubs. Bruand Cat. n $\mathrm{n}^{\circ}$ 2:1.)

26. Holosericearia. F. v. R.

Dup. sup. - Her.-Schf. f. 80, 81. 1. 41. - Heydenr. Cat. 658. Var.? Praeustaria. Mann, manuscr.

Pas très rare dans les haies et les taillis sur les bords du Leman et dans toute la 
vallée du Rhône en juin et en juillet (Lah.). — Pied du Jura, en juillet (Rothb.). Le 24 juin au pied du Jura, près de Soleure (Meyer).

La distinction entre holosericearia et os searia n'est pas toujours facile a faire. Les points discoïdaux constants dans ossearia ne manquent pas toujours dans sa voisine, seulement sont-ils alors fort peu distincts. Le dessous est aussi plus ou moins teinté de violet dans holosericearia; mais les raies sont toutes plus prononcées et plus larges. La disposition de ces raies est un peu differente dans les deux. L'origine des raies n'est jamais marquée en noir à la côte dans holosericearia. La côte peut être si peu colorée dans certaines ossearia, qu'on la confond alors avec l'autre. La strie noirâtre qui limite la frange, disparaît quelquefois en dessus dans ossearia: dans holosericearia on la trouve souvent indiquèe en dessous, jamais en dessus.

Pracustaria du catalogue de Mann (1849) ne diffère d'bolosericearia que par un peu plus de gris violace vers la pointe des supérieures en dessus et en dessous.

\section{Pallidaria. W. V.}

Bork. - Hub. 96 ठ์? - 'Dup. - Bdv. 1865. - Wood 26,741? - Heydenr. Cat. 656. - Her.-Schf. i. 110, 111. హ. 112 , 113. 9 - I. 42.

7 Byssinata. Fr. B. 60, 2. - Treit. - Bdv. 1886. - Dup. eat.

Paraît fort rare eu Suisse; je l’ai prise près de Bellinzona le 24 juillet, et aussi près de Lausanne à la même époque. Mr. Bremi l'a dit assez fréquente dans les lieux sees et boisés des environs de Zurich; mais il fait probablement erreur; car je ne l'ai pas vue dans les collections de Zurich (Lah.).

Cette espèce est encore l'une de celle que l'on confond souvent, comme l'observe Treit. Les individus que j’ai sous les yeux répondent à la description de Treit. et aux fig. de Her.-Schf.; mais non à la description abrégèe de ce dernier. Cet entomologiste dit de pallidaria mohue Glanz"; or ceux que je possède sont au contraire presque aussi luissants que Botys byalinalis. ‘. - Quant à la fig. de Hub., elle est tellement mauvaise que je la croirais prise sur une autre espèce et probablement sur une varièté de sylvestraria ou de rufaria.

Ma pallidaria se distingue par ses ailes bien arrondies, sa frange luisante, sans trace de points; par ses raies parallèles et ondulées, les extérieures plus larges. Les tarses posterieurs du mảle sont très raccourcis, épais, engainés. Les antennes sont surtout caractèristiques; leur dos est d'un blane jaunàtre, pur, lisse; leur partie inférieure 
finenent couverte de poils courts, blonds. Les ségments qui les composent sont très courts, rapprochés; les tubercules sont peu saillants, imbriqués, à peine distincts les uns des autres, de telle sorte que l'antenne elle-nème en est raccourcie d'un tiers.

28. Perochraria. F. v. R. 49. (descr. p. 125.) Tiscb. - Her.-Schf. I. 43. - Heydenr. Cat. 3ł2.

Ochrearia. Hub. 110 ? - Fr. n. B. 66, 3. non 1, 2).

Fréquente dans tous les environs de Lausanne en juillet et en aoùt, sur les prés et dans les ctairières des hois sees, parmi les bruyères; préfère les montagnes (Lah.). Commune à Burgdorf sur les prairies bumides (Meyer). - Voir ochrearia. -

Avee quelque persévèrence on parvient très bien à distinguer perochraria d'ochrearia, malgré leur extrême ressenblance. La couleur et la taille disent peu de cboses, quoique l'on rencontre plus souvent de petits individus de perochraria, surtout parmi les femelles. La position dn point discoïdal peut indnire en erreur. Ochrearia le porte en dedans de la ligne iuterne aux inférieures, et perochraria entre les 2 lignes; mais on observe des individus des 2 espèces oủ le point, se rapprochant de la ligne interne, vient a la toucher et alors la confusion est facile. Un caractère plus sür se tire de la troisième ligne (en partant de la base); dans perochraria elle est plus large et plus dentelée en dehors, tandis que dans sa voisine elle ressemble plutôt à un trait denticulé du côtè de la marge. Les 2 caractères les plus constants sont eupruntés aux antennes et aux tarses des pattes postérieures chez les mâles. Ochrearia a les tubercules des antennes peu saillants, carrés et couverts de poils blonds; tandis que perochraria les porte très coniques, alongés, noirs et surmontés d'un pinceau de poils foncés. La première présente denx éperons aux tarses postèrieurs, la seconde n'ell a point.

29. Rufaria. Hub. 112.

Treit. et sup. - Dup. - Bdv. 1864. - Fr. n. B. 66. 4, 5. F. v. R. 50, 2. - Her.-Schf. 1. 44. - Heydenr. Cat. 34s.

Les haies et les prés des environs de Lausanne, rapproches du lac; assez fréquente dès le commencement de juillet. Au pied du Jura prẻs d'Orbe. Abondante dass la Suisse transalpine et en Valais (Lah.). - Environs de Zurich (Bremi). - Près de Bienue, le $\mathbf{2}$ aoùt; fort rare (Rothb.). 
Cette espèce prèfère les lieux chauds, aussi est-elle rare dans la Suisse centrale. Le papillon très frais a une teinte olivâtre. - Je conçois difficilenent sa coufusion avec ochrearia et perochraria lorsque les ecbantillons sont en bon état.

30. Ochrearia. W. V.

Treit. et sup.? - Dup.? - Bdv. 1863? - Fr. 11. B. 66, 1, 2 (non 3). - Her.-Schf. I. 45. - Fisch. v. R. p. 12厅. t. 49. - Heydenr. Cat. 343.

Moins frequente que perochraria et parait un peu plus tôt; dès la fin de juin et juillet, jusqu'en aoùt (Lah.). - Du 12 mai au 27 juin, puis du 8 juillet au 28 août: commune (Rothb.). - Les prairies sèches des environs de Zurich; commune (Bremi). Dans les deux derniéres citations oehrearia est certainement confondue avec perochraria. Mr. Meyer n'a jamais trouvé ochrearia dans le canton de Berne.

Les synonymes de W. V., Treit., Dup. et Bdv. sont incertains par suite de la confusion des espèces citees. - La var. plus pâle à frange gris de fer, dont parle Treit. dans sun supplément I1. p. 202 et qui habite les Alpes, n'est autre que flaveolaria; mais conme cet auteur place celle-ci dans ses Idaea et celle-lả dans les Acidalia, on comprend que la confusion devenait plus facile. - Pour la distinction entre ochrearia et perochraria, voir à cette derniere.

Fisch. v. R. rattache à celte espèce la fig. 110 de Hubner et Her.-Schef. y voit perochraria.

31. Moniliaria. F.

W. V. - Hob. 59. - Hub. Beitr. - Treit. - Dup. - Bdv. 1847. - Her.-Schf. I. 46. - Heydenr. Cat. 711.

Ca et là, toujours rarement, sur les bords du Léman et en Valais, dans les lieux herbeux, à la fin de juin et en aoùt (Lah.). - Rare dans les pâturages des bois près de Zurich (Bremi). - Espèce méridionale peu répandue et qui ne varie pas.

32. Rusticaria. IV. V.

Hub. 241. - Treit. -- Dup. - Bdv. 1849. - Her.-Sehf. I. 49.

- Wood 703. - Heydenr. Cat. 375.

Var. Vulpin aria. Mann cat. - Heydenr. Cat. 376.

Pas rare dans les baies et les taillis au-dessous de Lausanne; commune en Valais, 
en juillet et au commencement l'aoùt. Fréquente au pied méridional des Alpes, à la mêne époque (Lab.). - Quelquefois prẻs de Meyringen (Meyer). En Valais du 13 au 20 juillet (Rothb.).

La variété à hande rouillie nommée vulpinaria par Mann, ne se destingue que par la couleur de sa bande. On trouve des excmplaires de rusticaria oủ la bande n'est rouillée qu'à la côte. Vulpinaria est plus commune dans la Suisse transalpine; je l'ai prise à Gondo (route du Simplon). - Mr. Heuser l'a aussi recueillie dans l'Oherhasli, au-delà de Meyringen.

3:3. Commutaria. Treit. sup.

Fr. n. B. 77, 4. - Bdv. 1906. - Dup. cat. - Her.-Schef.

f. 91-96. I. 53. (non Hub. 505). - Heydenr. Cat. 647.

Ternata. Schr.

Fumat a. Steph. - Wood 730.

S altuata. Speyer. Isis).

Adjunctaria? Bdv. 1896. - Bruand cat. $n^{\circ} 633$ ?

Commune dans les Alpes du 12 juillet au 3 août (Rothb.). - Meyringen en juillet: assez rare. - Alpes de Chamouny (Chavannes). - La Furca du cóté de Rẻalp, le 25 juillet, dèjà à demi passée (Lah.).

Il est surprenant qu'on l'ait confondue avec remutaria. Le mảle est assez diffèrent de la femelle; celle-ci est plus petite, plus claire, a les ailes moins chargées d'atómes et plus aigües au sommet (comme plusieurs autres femelles de ce genre). Le mâle est très chargè d'atômes; ses tarses postérieurs ne sont ni renflés, ni poilus et ont deux paires d'éperons.

Speyer est dans l'erreur lorsquil distingue commutaria saltuata) de remutaria par son front noir; l'une et l'autre l'ont noir ou brun foncé, ainsi que plusieurs espèces voisines. - Les palpes de la première sont petits, roux, avec le dernier article effite; ceux de la seconde sont plus gros, bruns ou noirâtres en dehors; leur dernier article est épaissi, presque sécuriforme. La frange de remutaria est marquee d'une ligne de points noirs; celle de commutaria d'une ligne brune peu marquée, surtout en dessus. Les figures de Her.-Schf. sont un peu pâles, surtout pour les mâles.

Jai quelque lieu de croire que a djunctaria (Bds. 1896, a été prise sur le male de commutaria; quoique Bdv. dise malae omnes sublus nitidiores.l Dans commutaria les atomes ne paraissent plus nombrenx en dessous que paree que le fond 
est plus blanc. Ce qu'il ajoute nanticae lineis duabus, posticae tribus signatae, " est trop contraire à tout ce qui s'observe chez les phalènes pour ne pas croire à une erreur typographique. Du reste, com mutaria esl plus voisine de strigaria que d'aucune autre.

34. Rubricaria. IV. $Y$.

$$
\text { Bork. - Hub. 111, 9. 487, \%. - Treit. - Dup. - Steph. }
$$

Wood 722. - Bdv. 1866. - Her.-Schf. 1. 54. - Heydenr. Ca. 346.

Rubiginala. Goetze.

I la fin de juillet et en aoùt sur les près arides et chauds des environs de Lansanne; pas rare (Lah.). Mèmes localités près de Zurich; lUto; pas rare (Bremi). - Sur les bords du lac de Constance (Leiner). - Près d'Aarberg, du 4 au 5 aoùt; rare Rothb.).

La variété à bande moyenne pâle est plus fréquente près de Lausanne.

35. Remutaria (ta). Lin.

W. V. - Hub. 98. - Treit. - Dup. - Fr. n. B. 77, 1. Bdv, 1907. - Her.-Schf. I. 55. f. 86-90 (non Bork). - Heydenr. Cat. 646.

Lactata et floslactata. Wood 731, 732 .

Centrata. Steph.

L a rv. Hub. G. I. Ampliss. (). a.

Commune vers la fin de mai et en juin dans les bois et les haies (Lah.). - Les bois de bètre du canton de Zurich; commune (Bremi). - De mème près de Schüpfen du 14 nai au 19 juin. - A Burgdorf en grande abondance (Meyer).

Espèce europèenne; varie souvent pour la nelteté et la force des raies centrales. La teinte charbonnée dn dessous varie aussi; parfois elle se répand un peu sur le dessus des supérieures. Lorsque cette phalène est très fraîche elle a un aspect soyeux.

36. Nemoraria. Hub. 89.

Her. Schf. f. 101, 102. 1. 56 (non Bork.). - Heydenr. Cat. 321. Aliata. W. V.

Punctata? Scop.

J'ai pris un seul exemplaire daus les environs de Lausanne en juillet (je crois) (Lab.). 
Celte espice tres rare est fort distincte de toutes les voisines. La fig. de Hubner la caracterise parfaitement el devail sulfire pour prévenir toute confusion. Je possède une paire provenant des environs de Darmstadt. Nemoraria de Borh. est strigillata Treit. , prataria Boisd. (vide cernsaria).

37. Sylvestraria (1a). Bork.

$$
\text { Hub. 97. - Treit.? - Fr. n. B. f. 77, 2, 3. - Wup.? - Bdr. }
$$
1895? - Her.-Schf. 1. 57. f. 103-105. - Heydenr. Cat. 645.

Immulala. Wood 721 .

Des la mi-juillet à la mi-aoùt, à Lausanne; rare (Lah.. Assez comnune dans le Jura et aux environs de Schüpfen du 30 mai an 22 juin; puis du 8 juillet au 8 aoùt (Fothb.). - Les bois des environs de Zuricb; IUto; pas commune (Bremi).

Les exemplaires pris en mai et en juin appartiennent probablement a une autre espece. La synonymie de sylvestraria est très confuse et les errenrs fréquentes. Je doute que punctata Devill. et Treit. appartienne à cette espece, quoique Her.-Schl. affirme le contraire. - La sylvestrata Bork. est plutôt une espèce allemande, tandis que punctata Devill. se prend volontier en France et en Suisse. - La var. f. 106 et 107. Her.-Schf. ne peut être punctata Devill.; car elle n'a pas de points marginaux. Elle n'a pas encore été ohservèe en Suisse; j'en possède un èchantillon (si c'est bien la même espèce) venant de Bourgogne et que je dois à l'obligeance de Mr. T. Bruand. La disposition des raies, la teinte plus foncéc des ailes, les dentelures irrégulières de la marge, la disposition en quinconce des points de la frange des inférienres /comme dans compararia), la situation du point central en dedans de la première ligne aux inférieures, enfin les antennes presque pectinées, à poils noirs, en pinceau chez le mâle, en font une espece distincte.

Il me parait que c'est à cette derniere espèce. encore indéterminée, qu'il faut rapporter la fig. \& pl. 177 de Dup. - Observons encore ici que sylvestraria est figurèe par Frey. In. Beitr.) à sa tab. 77 el non 113: celle-ci represente Cab. strigillaria. Caespitaria (Bdv. 1897) repose done sur quelque erreur.

Il serait plus aisè de confondre sylvestraria avec des individus pâles (surtout les 9 ) de rufaria; mais elle ne porte pas à la seconde ligne (à partir de la base) des inférieures, près du bord anterieur, le coude prononcé faisant un angle saillant en delıors, qui fait l'un des caractères de celle-ci. On observe à celte mème place une simple sinuosité. 
Dans la vraie sylvestraria les ailes sont assez arrondies, les points discoïdaux généralement bien dessinés, surtout aux inférieures; les points de la marge manquent souvent et sont au nombre de 3 à 5 (Jorsqu'ils existent); les inférieures sont lègèrenent anguleuses; les antennes sont fortement ciliées, de poils blanchàtres, simples, avee des tubercules peu élevés, arrondis. Le mâle est blanc-roux et la femelle blane-sale. Les tarses postérieurs sont très épaissis et engainés chez le mảle. Mr. Bruand /Cat. n 632 et 634) a probablement copié Boisd.

\section{Cerusaria mihi).}

Subpunctaria. Her.-Schf. 1. 58. f. $311,312,5$. 313. \&. non 415.

- Heydenr. Cat. 648.

Punclala. Bork. - Devil. - Treit. V1. p. 305. - ria Bdv. 1898. Dup. cat.

\section{Maritimaria? Bru. cat, $n^{0} 641$.}

Cetle espèce est fréquente dans les baies des horils du Léman et de la vallée du Rhóne, dès le milien de juillet Lah.). - Bienne, Frainvilliers; les hords du lac de Tboune du 24 juin an 22 juillet; rare Rothb.).

J'ai beancoup de peine à admettre encore ici les indications d'apparitions antérieures à la mi-juillet; dans les lieux les plus précoces de la vallèe d'Aigle, je n'ai rien observé de semblable.

Varie beaucoup pour l'intensite des raies: les petits points de la marge s'effacent très vîte. Treit. avait bien cette espèce sous les yeux puisqu'il parle de 7 points noirs sur la marge des supérieures et de quelques antres semblables aux inférieures; aucune autre espéce ne présente ce caractère anssi tranché. La fig. 3 tab. 177 de Dup. ne peut être rapportée qu'à celte espece.

Le nou de punctaria, déjà appliqué par Bdr. a son $n^{\circ} 1823$, doit en eflet disparaitre ici; mais on we peut lui substituer sulponctaria, sans admettre une sorte d'analogie avec Cabera punctaria. Her.-Schf: d'ailleurs dèsigne de la mème manière une espèce tres voisine de trilinearia (et par conséquent de punctaria) figuree au $n^{0} 415$.

Treitschke a commis une autre inadvertance en confondant nemoraria de Hub. avec sa punctata; celte méprise a été reproduite par Boisd. et par Duponchel. (Vide nemoraria.) 
le soupconne encore que maritimata (Guén. Bru.) se rapporte ici: en la comparaut à commutaria, Bruand dit nlignis valde obscurioribusa, ce qui n'est pas comprébensible, puisque celle-ci a les lignes à peine visibles. C'est du reste souvent le ras chez cernssaria.

Gy paria (Bdr. 190:) pourrait bien a'être que cerussaria; cet auteur, guidé par la fig. 89 de Hub., n’aura pas recounu daus sa nouvelle espèce la p u uctat a de Devill. et Treit. Si cela ètait, on pourrait échanger la dénomination que j'ai choisic contre relle de Boisduval, quoique en cas pareil le nom d'un eatalogue, ou une phrase, ne puissent étre admis pour motiver une priorité, si l'on veut sortir du dédale.

Les fig. de Her.-Schf. ne reproduissent pas complétement cerussaria tel qu'elle se présente en Suisse. Le sommet des supérieures y est trop aigu, la taille un peu forte et l'angle du milieu des inferieures trop prononce. Y aurait-il ici quelque confusion avec com pararia? Je ne parle pas de la couleur, car cerussaria est tellement de la couleur du papier qu'il est difficile de la peindre sur du blanc sans la faire trop coloréc.

39. Mutat aria. Treit.

Bdv. 1915. - Dup. -- Fr. n. B. 54, 3. - Her.-Schf. II. 62. f. 98,99 , ๖े. 100 , ธ. -- Heydenr. Cat 688 .

Suhsericeata. Wood.

Demutaria. Bruand catal. no 614. - (ta) Guén.

Les Alpes, dès la fin de juiu, à la fin de juillet. Descend dans les bois et les taillis jusques au pied des montagnes: assez fréquente (Lah.). - Les Alpes et le Jura du 10 au 22 juillet (Rotbb.). - LOberhasli (Meyer).

Her.-Schf. pense que le synonyme de Rösel pourrait fort bien se rapporter à inımutaria. Il met en doute celui de Bdv., parce que ce savant place cette espèce partui les acidalia à ailes inféricures anguleuses. Si Bdv. avait sous les yeux un individu femelle, ce doute ne serait pas fondé, car souvent chez celle-ci les ailes inférieures ont un angle prononcé. La méme chose s'observe dans r emutaria.

La femelle de wutataria est plus foncée et souvent un peu plus petite yue le mâle. Demutaria (Bruand, catal. Guen.) est une mutataria un peu pâle. Je ne me suis pas arrèté dans la synonymie à distinguer mutaria de mutataria. 
40. Immutaria. W. V.

Hub. 108. - Treit. - Dup. - Fr. n. B. 54, 4, 5 et 180.1.

- Bdv. 1838. - Iler'-Schf. 101. S. I. 63. (non Westw.). Heydenr. Cat. 690.

Contiguaria. Steph.?

Puellaria. Bdv. 1839.?

Degenerata. Wood 719 .

Pas rare des la fin de juin jusqu'en aoùt, et mème en septembre (le 8 , dans les endroits chauds, au hord des vignes, au-dessous de Lausaune (Lah.) - Rare dans le Canton de Berne (Meyer). - Le pied du Jura; les bords du lac de Thoune: envirens de Vevey etc. du 22 juillet à la lin d'aout ; rare (Kothb.). - Les bords des bois exposés au soleil, dans les environs de Zurich, peu rare (Bremi).

Cette phalène préfère les lieux chauds; aussi est-elle plus rare en Allemagne. J'ai lieu de croire aver: Treitschke qu'elle a deux apparitions (je ne dis pas générations) dans l'annee. II est difficile de la prendre fraiche; dans ce dernier etat elle offre une teinte jaunâtre, que Huboer a exagérée dans sa fiğ. 108 .

Ne se trouve jamais dans les forèts, comme le dit M. Bruand (Catal. $\mathrm{n}^{\circ}$ 613.).

41. Contiguaria. Hub. 105.

Treit. suppl. - Bdv. 1840. - Dup. cat. - Her.-Schf. 1. 65. Heydenr. Cat. 691.

Degeneraria (fig.) Wood?

Cette espèce, tris distincte d'immutaria et de submutaria, est fort rare; jen ai pris deux exemplaires au milieu de juillet 1847 dans les taillis près de St. Nicolas, vallée de la Viège, en Valais (Lah.). - Mr. Anderegg l'a recueillie près de Brigue. Le synonyme de Wood me paraît douteux.

Se place à coté de microsaria, a laquelle elle ressemble à tous ègards. 
42. Ornataria. W. V.

Hub. 70. - Treit. - Dup. - Bdv. 1835. - Steph. - Her.Schf. 1. 66. - Heydenr. Cat. 720.

Vestita. Huffng.

Paludata. Devill.

Violata. Thunb.

Apparait deux fois l'an, d'ahord en mai, puis dès la fin de juillet jusqu'en septembre, dans les prairies de toute la Suisse. Ne se montre pas dans les .Itpes (Lah.). - Commune dans le canton de Berne du 4 mai au 22 juillet, du 29 juillet au 18 septembre Rothb.). - Commune dans les taillis du canton de Zurich (Bremi).

43. Decoraria (ta). W. V.

Hub. 71. - Bork. - Treit. - Dup. - Bdv. 1836. - Her.Schf. 1. 67. - Heydeur. Cat. 719.

Cinera ta F. - Devill.

J'ai pris cette espèce, rare en Suisse, près de Martigny en Valais, au milieu de juillet; elle ètait alors très fraiche (Lah.). - Plusieurs exemplaires, mâle et femelle, pris par Mr. Meyer sur un plateau près de Sierre (Siders), le 9 aoùt. - Diffèrent de ceux d'Autricbe par une teinte blanche plus crètacée, moins jaunàtre. (Effet du soleil?

44. Immoraria. Lin.

$$
\begin{aligned}
& \text { Esp. - Hub. 133. - Treit. - Dup. - Bdv. 1891. - Fr. B. } \\
& \text { 90. O. - Her.-Schf. I. 68. - Heydenr. Cat. } 246 .
\end{aligned}
$$

Fréquente près de Lausanne, surtout dans le vallon de la Paudèze: paraît à deux epoques; en mai et en juin d'abord, puis en juillet et en août. Vole toujours près des champs d'esparcette [Hedysarum onobrychis] (Lah.). - Messieurs Meyer et Rothenbach ont fait la même observation dans le canton de Berne et au pied du Jura près de Soleure. - Du 21 avril au 10 juin et dn 11 au 17 juillet (Rothb.). Mr. Meyer l'a trouvée en grande abondance le 9 août dans les prés au-dessus de Naters (Haut-Valais).

La variété tesselaria n’a point encore èté prise en Suisse; mais je possėde quelques individus mâles qui s'en rapprochent beaucoup. - La femelle est plus petite. 
J'iguore pourquoi Bdv. place cette phalène dans les lieux plantės de bruyère, si ce n'est parce que Freyer a éleré la chenille sur la bruyère. - Les èditeurs du Catal. de Vienne la placent sur le chẻvre-feuille. En Suisse, elle ne prèfère ecrtainement ni l'une ni l'autre plante.

45. Strigaria. Hub. 95.

Treit. - Dup. - Bdv. 1894. - Her.-Schf. f. 114. 115. I. 69. Heydenr. Cat. 644.

Virgulata. W. V.

Sylvestraria. Dup. 1. 177. f. 7.?

Sulcaria. Hul, Verz.

Larv. Hub. Gen. I. Amplis. P. b. f. 1. a. b.

Espèce assez rare partout et propre à l'Europe centrale. Les lienx herheux, secs et chauds des environs de Lansanne et de la vallée d'Aigle, une première fois à la fin de juin, puis une seconde fois à la fin de juillet et en aoñt Lah.). — Salgetsch, près Sierre, Haut-Valais, le 10 aoùt (Heyer).

46. Compararia (ta). F. v. R.

Her.-Schf. I. 70. f. 299,300 , o. 301,302, \&. - Heydenr. Cat. 641.

Umbellaria ta). Hub. 437. 438? - Bdv. 1916? - Dup. cat?

Cette phalène n'est point rare dans les taillis et les clairieres abritees des bois près de Lausanne, dés Ie 28 mai au milieu de juin ; Aigle (Lah.). - Ca et là dans les environs de Burgdorf en juin (Meyer). - Du 13 juin an 10 juillet entre Aarberg et les bains de Worben (hothb.). - Les Grisons, près de Coire. (Kriechbm.).

J'ai eité avec doute le synonyme de Hub. parce que Mr. Bruand (in litter.) aflirme qu'umbellaria appartient à une autre espèce. On trouve aisément de grands individus qui correspondent entièrement aux fig. de llubn. Je n'ai pas encore pu me proenrer des exemplaires venus de France pour les comparer.

On distingue aisément cette espéce des voisines par la disposition des points de la frange qui sont rangès en quinconce. Aux ailes inférieures, cette distribution plus marquée fait parâtre la frange dentelée. La mêue cbose se reproduit plus nettement dans submutaria. Elle se reconnait encore à son collier brun. - On ne saurait la confondre avec strigillaria (Fidonia). 


\section{Prataria. Bdv. 1917.}

Dup. eat. - Her.-Schf. I. 71. f. 108, 109. - Heydeur. Cat. 640. Strigillaria. Jub. 109. - Dup. hist.

Strigil at a. Treit. et suppl. - W. V. - Fr. n. B. 114, 1.

Nemorata. Bork.

Variegata. Scop. - Wood 739.

Var. Catenaria. Bruand, catal.

Commune en juin et en juillet dans les haies, le long des champs, aux environs de Lausanne (Lah.). - Du 22 juin au 12 août, communement, dans le Cauton de Berne (Rothb.). - Jusques dans les Alpies (Neyer).

Ou ne peut guères coufondre cette espèce qu'arec mutataria. Le dessous des ailes est fort diffèrent dans tes deux. La fig. 109, Hub., est très manvaise L'angle de la ligne moyenne sur le centre des inférieures n'existe ni dans prataria, ni dans aucun autre $\Lambda$ cidalia. L'angle formé par l'avant-dernière raie, près de la côte, est très prononcé et les fig. d'Hub. 75. suppl. ne le font pas sentir suffisamment. La var. catenaria Bruand, ne diffère que par la ligne ondulée mieux dessinée.

48. Aureolaria. Fab.

Hub. 62. - Treit. - Dup. - Bdv. 1862. - Her.-Scbf. I. 74. Heydenr. Cat. 339.

Trilinearia. Hub. Beitr.

Bicinctaria. Devill.

Les pentes chaudes et sèches des environs de Zurich: rare; près de Frauenfeld Bremi). - En Valais ca et là; au pied du Jura, près d'Yverdon, dans les lieux ehauds.

Espèce rare qui prèfère les montagnes mèridionales. Filacearia (Iler.-Schf. 392, 393.) espéce du Tyrol, très rare, n’a pas encore été observée daus les Alpes suisses.

19. Flaveolaria. Hub. 311.

Treit. sup. - Dup. - Bdv, 1861. - Her.-Schf. I. 76. - Heydenr. Cat. 340.

Ochrearia var. Treit.

Les Alpes du Valais; vallée de St. Nicolas; le Simplon cte.; sur les prairies et le long des bois; fréquente en juillet (Lah.). - Très commune dans les Alpes rhètiennes (Meyer). 
Les prairies sèches des montagnes de la Suisse orientale: assez commune (Bremi). Les bains de Louësebe, du 11 au 27 juillet; commune (Rothb.); le 11 aoùt (Meyer).

Varie du jaune eitron au jaune orange fonce. Les deux bandes, peu saillantes en dessus, sont très prononeées en dessous. La frange est toujours gris-noir. Les femelles sont beaucoup plus difficiles à découvrir que les mâles.

50. A uroraria. Hub. 6:3.

Treit. - Dup. - Bdv. 1860. - Her.-Schf. 1. 77. - Wood 685.

- Jleydenr. Cat. 222.

A uroralis (Pyralis). W. V.

Variegata. Fab.

Cette espèce a étẻ observée en Suisse par le pasteur Rordorf, près de Seen, en Thurgovie Bremi) et par Mr. Zeller, près de Viège, en Valais (Lah.). - Très rare.

51. Emarginaria. Lin.

$$
\begin{aligned}
& \text { Hub. 107. - Treit. - Dup. - Wood 749. - Bdv. } 1911 .- \\
& \text { Her.-Sehf. I. 79. - Heydenr. Cat. } 13 . \\
& \text { Dem a n d a ta. Fab. }
\end{aligned}
$$

Ca et là, toujours rarement, sur les hords des bois, en juillet, près de Lausanne; au commencement d'août près de Paudex (Lah.). - Les mêmes localités qu'aversaria, mais plus tard; dans les environs de Zurich: rare (Bremi). - Près d'Aarberg, le 29 juillet; très rare (Rothb.).

\section{G. IV. Ephyra. Dup. Bdv. Steph. Curt. \\ (Caberae Treit.)}

Nota. La nervation des ailes est identique chez toutes; la cellule des infërieures coupée carrément, porte à ehaque angle, la bifurcation des deux nervures qui la limitent.

52. Trilinearia. Bork.

Treit. - Dup. - Bdv, 1822. - Wood 532. - Her.-Schf. 1. 82. (non Hub.) - Heydenr. Cat. 329.

\section{Linearia. Hub. 68.}

Une première fois en mai (dès le 12) et en juin; puis à la fin de juillet et en août, dans les bois et les taillis au-dessus de Lausanne; fréquente (Lab.). - Sur le Balgrist, 
près Zurich (Zeller). - Fréquente près de Schüpfen du 8 mai au 27 juin et du 23 juillet au 15 août (Rothb.). - Très commune certaines années près de Burgdorf où la chenille se trouve en abondance sur les hêtres (Meyer).

On rencontre quelquefois des individus portant les o micron aux deux ailes. Ceux des inférieures sont alors plus visibles et plus souvent cernés de brun. Ils sont tantôt oblongs, tantót triangulaires. Aux supérieures ils se placent entre la première et la seconde ligne; aux inférieures ils se rapprochent de la seconde ligne et même la tonchent. Lorsqu'ils ne sont pas cernés, ils sont très peu visibles, comme dans gyraria.

La ligne de la frange est tantôt formée d'une série de stries noirâtres séparèes par les nervures, tantot les stries se touchent et la ligne est étranglée au niveau des nervures.

Les trois lignes se voient quelquefois en dessous; la première et la troisième sont toujours plus marquées. Le nombre et la couleur des atomes varient aussi, et avec eux la couleur des lignes. Les noirâtres sont mieux marquées que les rougeâtres. La troisième ligne est tantót ponctuèe, tantót dentelèe, tantôt ondulée (surtout aux inférieures). - Les femelles sont plus pâles que les màles.

Une femelle élevée de chenille a le fond couleur d'ocre, rougeàtre (Collect. Meyer).

5:3. Punctaria. Lin.

$$
\begin{aligned}
& \text { Bork. - Hub. 574. - Treit. - Frey. B. 54, 1. - Dup. - } \\
& \text { Wood 531. - Bdv. 1823. - Her.-Schf. 1. 83. - Heydenr. } \\
& \text { Cat. } 326 .
\end{aligned}
$$

Commune dans les mêmes lieux et à la même époque que trilinearia (Lab.). Daus le Canton de Perne, fréquente do 8 mai au 26 juin et du 26 juillet au 26 août (Rothb.). Dans les environs de Burgdorf, elle parait moins fréquente que $t r i l i n e$ aria (Meyer). - Sur le Balgrist, près Zurich (Zeller).

Quelques rares exemplaires portent l'omicron, mais il est peu visible. La tache rosée de l'angle interne est souvent absente; d'autrefois on en trouve une seconde vers le milieu tu bord marginal.

54. Omicronaria. W. V.

$$
\begin{aligned}
& \text { Hub. 65. - Treit. - Wood 527. - Dup. - Bdv. 1831. - } \\
& \text { Her.-Schf. I. 85. - IIeydenr. Cat. } 333 .
\end{aligned}
$$

Anularia. Fab.

Fréquente dans les haies de noisettier en mai et en juillet; le long du Léman (Lah.). 
Les bords des bois; pas rare dans les environs de Zurich (Bremi). - Prés de Langnau, de Burgdorf et d'Aarberg; toujours rare. N'est pas dans les environs de Schüpfen (Rothb.). - Commure en Valais (Meyer).

5.5. Pendularia. Lin.

IV. V. - Hub. 66. - Fr. B. 54, 2. - Treit. - Wood 528. Dup. - Bdv. 1829. - Her.-Schf. I. 86. - Heydenr. Cat. 334. V a r. Her.-Schf. 357 .

L a r v. Hub. G. I. Ampl. L. a. b. fig. 1.

Assez fréquente dans le bois de hêtre en mai; reparait quelquefois en aoùt (Lah.). - Prês de Berne; (le 11 aoùt) assez commune (Rothb.). - Très rare dans les environs de Burgdorf (Meyer). - Sur l'Uto, près Zurich; environs de Coire, très rare, en mai Bremi).

Cette espece est rarement prise parce qu'elle ne descend pas près de terre et parce que, la chrysalide etant fixée aux feuilles, le papillon naîl et vil sur les arbres élevés.

56. Orbicularia. Hub. 60.

Treit. - Dup. - Wood 529. - Bdv. 1830. - Her.-Schf. I. 87.

Sur le Balgrist, près Zurich (Zeller). - Trouvée une fois près de Burgdorf (Heuser). Du 3 au 18 juin près de Schüpfen; rare (Rothb.). - Espèce européenne; partout rare.

57. Poraria. Lin.

F. - W. V. - Treit. - Wood 530. - Dup. - Bdv. 1825. Her.-Schif. I. 88. - Heydenr. Cat. 330.

Punctaria. Hub. 67.

Larv.? Hub. G. I. Ampl. L. a. b. f. 1. f.

Rare autour de liausanne; parait un peu plus tôt que puuctaria; je l'ai prise au milieu de mai (Lah.). - Sur le Balgrist, près Zurich (Zeller). - Du 8 au 17 juin, puis vers le 6 aoùt, à Schüpfen; rare Rothb.). - Ràre dans le Doubs (Bruand, catal. 608.).

Poraria, gyraria et punctaria sont très faciles à confondre. Plusieurs des caractères distinctifs indiqués par les auteurs sont trompeurs. Les lignes varient de couleur de position, de forme et de netteté. Les lunules centrales tautôt existent, lantôt manqueut. Les atômes varient aussi en nombre et en couleur; mais moins dans leurs dimensions. 
58. Gyraria. Hub. 434 (non 69 et 543).

Treit.? - Dup.? - Bdv. 1827? - Her.-Schf. 1. 89. - Heydenr.

Cat. 326 ?

Pupillaria? Bru. Catal. n $\mathrm{n}^{\circ}$ 610. - Zeller Isis 1847. p. 498.

Pas rare dans les taillis et les bois de luêtre des environs de Lausanne, à la méme époque que trilinearia et punctaria (Lah.). -- Près de Sehupfen du 15 mai au 10 juillet et même au 15, aoùt: rare (Rothb.). Sur le Balgrist, près Zurich (Zeller). La ehenille vit sur les plantes basses (Lab.).

Voisine de punctaria, mais s'en distingue bientôt par ses atômes plus fins, par l'absence de pointillé rouge-vil', par sa couleur uniforme d'un gris-roux, allant jusqu'au rougeâtre et surtout par un léger liseré rosé qui divise la frange en deux et prend une teinte violete plus ou moins prononcée vers la pointe de l'aile en dessous.

Gyraria est souvent plus grande que punctaria. Je possède des individus de moitié plus petits, résultant sans doute d'un défaut d'alimentation chez la ebenille.

L'omicron existe constamment; souvent trés peu visible, parce qu'il est rarement cernè de roux: il est plns visible aux inférieures.

On ne peut réunir cette espèce à pupillaria, comme le pense Her.-Schf. Les fig. 69 et 434 de Hub. représentent bien l'une et lautre. La dernière est, il est vrai, trop foncée; mais elle porte la seconde ligne (moyenne) très nette et bien prononcée, ce qui n'arrive jamais dans pupillaria. Il existe d'ailleurs d'autres différences. (Voir pupillaria.

La synonymie de cette espèce est encore incertaine; on ne peut l'ètablir qu'en comparant plusieurs individus de localités différentes. - Heydenreich par exemple applique la fig. 543. de Hub. à albipunetaria, Mann à gyraria.

59. Pupillaria. Hub. 69.

Treit. et supp.? - Dup. - Bdv. 1826? - Her.-Schf. I. 90.

Heydenr. Cat. 331 ??

Var.?? Nolaria et gyraria. Hub. 327 und 543.

J'ai pris eette espèce méridionale le 21 juillet au bord du lac majeur Lah.).

Je ne conçois pas pour quels motifs Treitschke réunit nolaria et gy raria (Hub. 327 et 543 à pupillaria. Il faut, s'il a raison, que celle-ei varie incroyablement ou que les fig. des Hub. soient des plus trompeuses. Lne simple variation de teinte ne peut 
expliquer ce rapprochement. J'ai vu pupillaria, prise dans le midi de la France, se montrer aussi foncée que la fig. 69 de Hub. (à part la teinte violette); je la possède plus pâle que gyraria, sans qu'il y ail analogie entre nolaria et elle.

La teinte gènérale violette ou rosée de la frange, surtout en dessous, et la finesse des atómes disposés en stries transversales comme dans onoraria, fait aisément reconnattre pupillaria. Il se pourrait cependant qu'on la confondit avec des individus rougeàtres de gyraria; mas le trait noir qui limite la frange, manique toujours daus pupillaria, quoique Treit. affirme le contraire. Il y a done plusieurs doutes à éclaircir. Je soupçone que pupillaria Treit. est notre gyraria, que notre pupillaria appartient à une espéce qui lui était inconnue (pupillaria Zel.?), tandis que Hub. 327 et 543 forment une autre espèce, ètrangère a la Suisse (albipunctaria. Mann??

$$
\begin{aligned}
& \text { G. V. Emmiltis. Hub. Verz. } \\
& \text { (Acidalia. Tr. - Bdv. - Dup.) }
\end{aligned}
$$

Nota. La construction de la cellule des inférieures se rapproche infiniment de celle des Acidalia de la première division. Les antennes de sericearia sont finement cilièes à la marge; celles de parrularia sont pectinèes. - Ce genre est mal assis.

60. Sericearia. Hub. 404 .

Treit. - Dup. - Bdv. 1888. - Her.-Schf. I. 91. - Heydenr. Catal. 355 .

Pas rare en Valais sur les colines arides et chaudes; Martigny; Viège; juillet (Lah.). - Commune près de Naters et Brigue; 8 aoùt (Meyer). - Espèce méridionale.

61. Parvularia. Bdr. 1857 .

Dup. cat. - Heydenr. Cat. $347^{\mathrm{b}}$.

Pygmaearia. Hub. 335, 336. - Treit. - Dup. hist. - Her.-Schf. I. 92.

Cette espèce méridionale et propre à l'ttalie septentrionale a probablement été prise dans la Suisse italienne. Bdv. l'indique en Suisse Lah.).

Her.-Schf. aurait dú conserver la dénomination de Bdv. puisqu'il enrégistre une Eupithecia pygmaearia. - Je la possède venant de Livourne où elle paraît en mars; elle a donc deux apparitions. 
G. VI. Aplasta. Hub. Verz.

(Cabera. Tr. - Bdv. Fidonia. Dup.)

Nota. Très rapproché d'Ephyra dont il a la nervation.

62. Ononaria. Fuess.

$$
\text { Bork. - Hub. 93. - Treit. - Dup. - Bdv. 1820. - Her.-Schf. }
$$

I. 96 . - Heydenr. Cat. 324.

Sudalaria et faecalaria. Hub. 492 et 503.

Nulle part comnune en Suisse: habite les collines sèches et chaudes; fin de juillet et août. Près de Lausanne, le pont de Belmont \{Lab.); - de Wangen (Zurich\}; de Baden en Argovie (Bremi) - et d'Aarberg (Rothb.). - Espèce mérídionale.

\section{G. VII. Botrtobia. Boisd.}

(Gnophos: Treit.)

Nota. Les palpes alongés, les antennes cilièes sur les bords, denticulèes et la nervation de la cellule des inférieures caractérisent suffisamment ce genre, indépendamment de sa larve.

63. Carbonaria. Lin. f. suec.

W. V. - F. - Hub. $151,548,549 .-$ Treit. - Dup. - Bdv 1596. - Her.-Schf. I. 101. - Heydenr. Cat. 176.

La rv. Hub. Geom. I. Amplis. X. b.

Espèce européenne, nulle part fréquente. Lausanne (Lah.). - Burgdorf (Meyer). Berne; 25 juillet (Rothb.) - Les taillis (?) de l'Uto; Vögeli, près Zurich (Bremi'.

G. VIII Metrocampa. Latr. Boisd. Dup. Curt.

(Ellopia. Steph. - Treit.)

Nota. La nervation des ailes inférieures est celle de Geometra. Les caracteres tirés de la larve sont-ils suffisants? Dans margaritaria la paire de pattes surnuméraires est raccuurcie et reste sans usage. 
64. Fasciaria. Lin.

Hub. 446, 447. - Treit. el sup. Dup. - Bdv 1431. Her.Sehf. 1. 104. - Heydenr. Cat. 44.

lar. Prasinaria. Hub. 4. - Treit. - Dup. - Heydenr. Cat. 45.

Lar v. Hub. G. I. Ampliss. A. a. b.

Prasinaria est commune dans les bois de sapin du Jorat, dès le milieu de juin au milieu de juillet: fasciaria est beaucoup plus rare surtout à l'étar frais Lah.. Il en est de mème dans les environs de Zurich; sur les bêtres [?] (Bremi). - Moins abondante dans le centre du canton de Berne que dans les bautes vallees de l'Oberbasle, ou elle se trouve frègnemment sur les sapins. Entre l'Urweid et Guttannen 6 aout Meyer/. - Commune du 14 juin au 15 juillet, près de Schüpfen (Rothb.) - A la mème epoque au pied do Jura près Soleure (Meyer). - Jamais en mai et toujours dans le voisinage des sapins (Lah.). - Une seule apparition en Suisse.

L'exposition de prasinaria à la moindre vapeur acide la transforme en fasciaria. Pourquoi ne pas adopter prasinaria pour désiguer l'espèce, puisque fasciaria n'est qu'une variètè accidentelle? On ne peut être esclave de la prioritè à ce point.

Les caractères tirés de la chenille éloigneraient fasciaria de margaritaria, puisqu'elle n'est ni catocaliforme, ni munie de 12 pattes vraies.

6.). Margaritaria. Lin.

Hub. 13. - Treit. - Dup. Bdv. 1432. - Her.-Sebf. I. 105. Heydenr. Cat. 42.

Bomb. sequistriataria. Esp.

Larv. Hut. G. I. Ampliss. A. b

Commune en juin et juillet dans les bois des euvirous de Lausanne; sur les hêtres. Jamais prise en Suisse, ni en arril, ni en mai (Lah.). - Les taillis, sur l'Uto, près de Zurich: peu commune (Bremi). - 19u 12 juin an 11 juillet, dans les bois du cauton de Berne; commune (Rotlals.) - Espèce européenne; très répandue; rare cependant dans le Doubs Bruand.

Les auteurs indiquent souvent des doubles apparitions là oú on n'en olserve point dans la nature, parce qu'ils tiennent compte des eclosions en chambre; celles-ci correspoudent rarement aux spontanées, surtout thez les phalénes. II faut se souvenir de ce fait en lisant Freyer. 
66. Honoraria. W. V.

Hub. 16. \&. 17. б. - Treit. - Dup. - Bdv. 1433. - Her.-Schf. I. 106. - Heydenr. Cat. 41.

Excisaria et bonorifica. Esp.

Idsociaria. Bork.

Cette espèce partout rare, n’a ete trouvée jusquïci en Suisse que dans les Grisons par Mr. Neugold.

Chenille à 12 pattes parfaites; marche en arpentant; frange de poils le long des bords du ventre (Esp.).

G. IX. Eugonia. Hub. Verz.

(Geometra. Steph. - Ennomos. Treit. Boisd. Dup. Curt.)

Nota. La nervure scapulaire des inféricures s'accole pendant un moment à la moyenneexterne et s'en sépare vers le milieu de la cellule. La cellule alongée rapprocbe ce genre de Crocallis.

67. Tiliaria. Hub. 23.

Treit. - Dup. - Bdv. 1454. - Her.-Schf. I. 107. - Heydenr. Cat. 35.

Canaria. Hub. Beitr. - Wood.

Rare aux environs de Lausanne; je ne l'ai tronvée qu'une fois en août Lah.'. De même près de Zurich (Brenii). - Eclos à la fin de l'été.

68. Alniaria. Lin.

Hub. 26. - Fuessly. - Treit. - Dup. - Bdv. 1455. -- Her.-Schf.

I. 108. - Ileydenr. Cat. 36.

Larv. Hub. G. I. Amplis. D. b. f. I.

Rare à la fin de l'èté dans les forêts de hêtre des environs de Lausanne (Lah.). Les mèmes localités sur le Zurichberg et l'Uto; le Siblwald, dans les environs de Zurich: pas rare (Bremi). 
69. Quercinaria. Bork.

Hub. 24. - Treit. et suppl. - Bdv. 1452. -- Fr. 1. B. 716. Her.-Scbf. f. 74, 75. I. 110. - Heydenr. Cat. 32.

Erosaria. Wood 476 .

N'a été trouvée jusqu'ici en Suisse que dans les Grisons, par Mr. Mengold (Bremi). Cette espéce paraît appartenir à la vallée du Rhin.

70. Erosaria. W. V.

Hul. 25. 440. - Treit. et supp. - Dup. - Bdv. 1451. - Hub. Verz. - Her.-Sclıf. f. 72,73 . I. 111. - Heydenr. Cat. 31.

Crassaria. Fab.

Ochraria. Wood 475.

Larv. Hub. G. I. Amplis. D. a. f. 2.

Pas rare dans les bois de hêtre près de Lausanue, à la fin de juiltet et en aoùt (Lah.). - Le Burghölzli et le Zurichberg, près de Zurich; peu fréquente (Bremi). Rare dans le canton de Berne (Rothb.). - Espèce européenne, assez rare partout.

71. Angularia. W. V.

Esp. - Hub. 22. - Treit. el sup. - Dup. - Bdv. 1450. - Her.-Schf. f. 76, 77. I. 112. - Heydenr. Cat. 28.

Dentaria. Devill.

Carpinaria. Wood 480.

Var. Carpinaria. Hub. 27. -

Q uercinaria. Wood 477 .

L a r v. Hub. G. I. Amplis. U. a. f. I

Cette espèce commune dans toute l'Europe, l'est aussi en Suisse; en juillet et en août dans tous les bois feuillés (Lah.). - Dans le canton de Berne dès le 12 juillet au 13 septenbre; commune (Rothb.). - Parait moins commune dans le canton de Zurich (Brewi).

Carpinaria. Ilub. mérite à peine le nom de variẹté. 


\section{G. X Crocallis. Tr. Boisd. Steph. Curt.}

(Colotois. Hub. Verz.)

Nolit La nervure scapulaire ne s'anastomose point avec la mediane-externe, mais la suit cóte à côte jusques vers le milieu de la cellule, ce qui se voit surtout distinetensent dans aglossaria.

72. Elinguaria. Lin.

Hub. 20. - Treit. - Dup. - Bdv. 1462. - Her,-Sclıf. 1. 116.

- Wood 471. - Heydenr. Wat. 115.

L a r v. Hub. G. I. Amplis. C. c. fig. 1.

Assez rare dans les taillis, le long des haies, des environs de Lausame; en juillet (Lalt.). Plus fréquente dans les environs de Zurich; l'Uto, le Zurichberg, Dubendorf (Bremi). - Gadmenthal, au cauton de Berwe, fin aoùt (Mleyer). - Langnau, Burgdorf, Schüpfen; rare; du 19 au 25 juillet (Rothb.).

Jamais trouvée en septembre.

G. XI. Odontoptera. Steph.

(Ennomos. Boisd. - Gonodontis. Hub. Verz. - Crocallis. Curt.

Nota. Les dentelures de la marge ne paraissent pas suffisantes pour séparer dentaria des Cracallis. La nervation est la mème. Cr. aglossaria présente déjá des deutelures assez sensibles.

73. Dentaria. Esp.

Hub. 12. - Treit. et sup. - Dup. - Bdv. 1456. - Fr. ı. B. 156. - IIer--Schf. I. 118. - Heydenr. Cat. 38

Bidentata. Lin. - Fab. (ria). - Wood 472.

L a rv. Bdv. Chen. d'Eur. pl. 1. -

"Hub. Geom. I. Ampl. C. c. f. 2.

En mai et juin dans les bois du Jorat; pas très rare. La chenille se métamorphose dans la mousse au pied des hìtres (Lah.). - Enrirons de Burgdorf, très commune le soir le long des forèts de hètre (Meyer). - Mêmes localités dans les environs de Zurich: assez rare (Bremi). - Rare à Schüpfen, du 19 mai au 16 juin (Rothb.). - Ne varie pas. 
G. XII. Himera. Dup. Boisd. Curt.

Colotoïs. Hub. Verz. - Metra. Steph. - Crocallis. Tr.

- Nola. Nervation des inférieures comme dans Crocallis. - Le genre de Tr. pouvait fort bien être conservé. Les palpes de extimaria sont parfaitement semblables a ceux de pennaria: le deruier article n'est petit el grêle que dans elinguaria seulement. La chenille de Irapezaria porte une pointe à l'arantdernier segment.

74. Pennaria. Lin.

Hub. 14. - Treit. - Dup. - Fr. B. 78. - Bdv. 1459. Her.Schf. 1. 119. - Wood 470. Heydenr. Cat. 117 Larv. Hub. G. I. Amplis. C. b.

Quoique répandue dans toute lEurope, celte espèce n'est pas lréquente en Suisse; on tronve le papillon en automne appliqué contre le tronc des tilleuls el des ormes sur les promenades publiques (Lab.). - Les bois des environs de Zurich; peu rare Bremi. - Dans les Grisous (Mengold). - Rare aux environs de Burgdorf Meyer); - rare à Schüpfen, mais fréquente près de Berne; le $\mathbf{1} 6$ octobre (Rotbl.). N'a jamais èté trouvèe en Suisse au priulemps.

G. XIII. Selenia. Hub. Verz.

Linnomos. Tr. Bdv. Curt. - Geometra. Leach. - Pericallia. Steph. Curt.)

Nota. La nervation de la cellule rapproche ce genre de Geometra. Syringaria ne peut en être séparé, quoique privé de lunule transparente.

75. Illustraria. Hub. 35.

Treit. - Dup. - Bdv. 1449. - Fr. n. B. 222. 1. - Her.-Schf.

I. 120. - Wood 487. - Heydenr. Cat. 25.

Luuaria, rar. Bork. - W. V. - Hub. Beitr.

Sublunaria. Wood 486 .

La rv. Hul. G. I. Anplis. F. E.

Issez rare dans les environs de Lausanne en mai: pas rare a la fin de l'èté (Lah.). - Rare dans le canton de Rerne; le $\mathbf{1 3}$ mai, puis le 26 juillet, à Schüpfen (Rotbb.) 
Parait moins rare dans les environs de Zurich Bremi). - Promenade du Schönbuhl près Burgdorf Heuser).

76. Lunaria. IV. V.

Hub. 33, 3. 451, \&. - Treit. - Hub. Beitr. - Bdv. 1446. Fr. n. B. 234 . - Ifer.-Schf. 1. 121. - (non Dup. hist.). Dup. cat. - Heydenr. Cat. 23.

Lunularia. Hub. Beitr.

Lars. Hub. G. I. Amplis. F. a. b. f. 1. et F. b. - Wiener Verz. t. I. a. fig. 4 .

Un peu plus fréquente près de Lausanne quillustraria, ans mẻmes époques (conmencement de juin) et dans les mènes localités. La seconde apparition, en aoút, est rare surtout dans les annèes et les localités moins chaudes (Lah.). - Très rare dans le canton de Berne; Schüpfen, du 23 juin au 12 juillet (Rothb.) - Très rare à Burgdorf Meyer): un peu moins dans le canton de Zurich (Breni).

77. Delunaria. Steph.

$$
\begin{aligned}
& \text { Hub. 34, \&. - Bdv. 1447. - Dup. cal - Her.-Schf. 1. } 122 . \\
& \text { Wood 484. - Heydenr. Cat. 2\%. }
\end{aligned}
$$

Lunaria. Dop. hist.

Peu rare (?) dans les environs de Zurich (Bremi). - Une fois, prés de Lausanne, parmi les aunes, en juin. Plus petile que lunaria et d'une coulear plus rosée (Lah.. - Ordinairement confondue avec Iunaria.

78. Il hunaria. Hub. 36, 37.

Treit. - Dup. - Fr. n. B. 228. - Her.-Schf. I 123. - Wood 481. - Heydenr. Cat. 26

Lunaria, var. W. V.

Juliaria. Wood 482 .

Var? minor (aestivalis) Dup

L a r v. Hub. G. I. Amplis. F. b.

Assez commune dans toute la Suisse. A Lansanne en mai et quelquefois en septembre (Lah.). - Fréquente a Burgdorf (Meyer). - Moins commune à Zurich (Bremi). - 
Commune a Schüpfen du $1^{\text {r }}$ au 22 mai, puis du 4 aoúl au 13 septembre (Rothb.). - La varièté Minor n'est pas propre à l'été, conme le pense Duponchel; elle est née chez moi au mois de mars 1848. J'ai tout lieu de croire qu'elle est uniquement due au dépérissement des chenilles élevées en captivité, comme on l'observe chez d'autres Géomètres.

79. Syringaria. Lin.

Hub. 29. - Treit. - Dup. - Bdv. 1437, - Her.-Selif. I. 124.

- Wood 488. - Heydenr. Cat. 22.

La rv. Hub. G. J. Anplis. G. a.

Assez rare dans les haies des environs de Lausanne, en mai et en juin (Lah.). Moins commune dans te canton de lierne, da 19 juin au 24 juillet, près de Schüpfen Rolbb.). - Fort rare près de Zurich (Bremi); Seen (Rohdf.).

On n'a pas observe de seconde apparition en Snisse, chez liudividu en liberté.

\section{G. XIV. Epione. Steph. Dup.}

Euchlena. IHub. Verz. - Ennomos. Tr. Bdv. - Timandra. Curt.

Nota. La cellule est coupèe carèment comme dans Ephyra.

80. I drenaria. Esp.

Hnb. 45. - Hub. Beitr. - Treit. - Dup. - Bdv. 1444.

Fr. B. 120. - Wood $536($ non 748). - Her.-Schif. I. 126.

Heydenr. Cat. 18.

Très conınune dans les taillis de hètre, aux environs de Lausanne: fin de mai et commencement de juin (Lah.). - De mème dans le canton de Berne; Schüpfen; du 27 mai au 21 juin (Rothb.). - Burgdorf; du $1^{\mathrm{r}}$ au 12 jnin en abondance dans les perits bois (Meyer). - Fréquente dans les bois des environs de Zurieb (Bremil. - Espèce propre à l'Europe centrale.

81. Apiciaria. W. V.

Esp. - Hub. 47. - Hub. Beitr. - Treit. et sup. - Dup. -

I3dv. 14i2. - Fr. B. 102 ; n. B. 198, 2 (var.) - Her.-Schf.

I. 128. - Wood 534. - Heyilenr. Cal. 17.

Parail une premiere fois dans le milien de l'èté; puis une seconcie en automne. dans 
les haies et les taillis. Pas commune dans les environs de Lansanne Lah.. - Frèquente dans le canton de Berne. Du 9 juillet au 14 aoủt et du 6 septembre an 4 octobre, dans les environs de Schüpfen (Rothb.). -- De même dans le canton de Zurich (Bremi). Parallellaria n’a pas encore èté trouvée en Suisse.

G. IV. Therapis. Hub. Verz.

(Ennomos, Aspilates, Eubolia. Auclor.)

Nota. Artesiaria cst trop rapprochée de gilvaria par sa nervation pour étre placéc dans un autre genre qu'elle.

82. Artesiaria. W: V.

Hub. 15. - Treil. - Dup. - Bdv. 1603. - Fr. B. 35, 2. Her -Schf. I. 130. - Heydenr. Cat. 96.

Festucaria. Hub. Beitr.

Distrigaria. Wood 505.

Cette espece a été prise une fois dans les environs de Lausanne, en juillet, par Mr. G. Leresche. - Rare partout (Lah.)

G. XVi. Macaria. Curt. (partim). Boisd.

(Philobia. Dup. - Ennomos. Treil.)

Nola. La nervation est celle de Epione; mais les antennes denticulees avec des tubercules proeminents, en distinguent II a c a ria: a estimaria forme la transition.

8:3. Nolataria. Lin.

$$
\text { Bork, - Hub. 53, 316. - Treit. et sup. - Dup. - Bdv. } 1471 .
$$

- Fr. n. B. 53, 1. - Her.-Schf. I. 132. - Ileydenr. Cat. 6. Lar v. Hub. G. I. Amplis. J.

Prise une fois en Valais; mi-juillet Lah). - Très rare dans les environs de Zurich: l'Uto Bremi'. - Pas commune dans le Doulss Bruand.

81. Ilternaria. W. V.

Hub. 315. - Treit, et sup. - Dup. - Bdv. 1472. - Fr. B. 138. - Her.-Schf. I. 133. - Wood 743. - Heydenr. Cat. 7.

Pas tres rare dans les bois du Jorat au-dessus de Lausanne, a la fin de juin et au 
commencement de juillet (Lah.). - Assez frèquente près de Burgdorf et plus encore au pied du Jura, près de Soleure, parmi les sapins (Meyer). - Du 7 juin an 7 juillet près de Schüpfen; assez rare (Rothb.). - Bois de bêtre des environs de Zurich; l'Uto; rare (Bremi).

Pas de double apparition en Suisse. - Chenille sur les plantes basses et non sur le sapin (Her.-Schf.).

85. Signaria. Hub. 313 .

Treit. et sup. - Dup. - Bdv. 1474. - Fr. B. 42, 2. - Her.Sclif. I. 135. - Iteydenr Cat. 9.

La rv. Hub. G. I. Amplis. I. a. b. f. 1. c. d.

Commune dans le canton de Berne; Schüpfen dn 2 juin au 12 juillet (Rothh.). Assez fréquente dans les bois de sapin du Jorat; en juin et en juillet (Lah.). - Plus rare près de Zurich; Waldgruss; l'Uto (Bremi). - Prés de Soleure, au pied du Jura; abondante a la fin de juin (Meyer).

Une seconde génération est fort peu probable en Suisse.

86. Lituraria. Lin.

W. V. - Hub. 54, 314 (errore 315). - Hub. Beitr. - Treil. et sup. - Dup. - Bdv. 1473. - Fr. n. B. 53, 2. - Her.Sehf. I. 136. - Heydenr. Cat. 10.

Larv. Hub. G. I. Amplis, J. a. b. f. 2.

Moins fréquente que signaria el alternaria; se trouve dans les mèmes localités et a la mème époque (Lah.). - Dans le canton de Berne, à Schüpfen, du 30 mai au 3 juillet (Rotbb.). - Mr. Meyer l'a trouvé communément en mai, aux environs de Burgdorf. - Dans les bois de hêtre (?) des environs de Zurich; pas rare (Bremi).

Je doute qu'on l'observe en août comme l'indique Boisduval; car elle ne fait en Suisse qu'une apparition par an.

Lituraria et signaria ne sont pas énumérées dans le Catalogue des Lépidoptères du Doubs, par Mr. Bruand.

G. XVII. Venilia. Dup. Steph. Curt.

(Melanippe. Bdv.)

Nota. Macularia ne peut guères se séparer de clathraria et surtout de wa waria dont elle a la nervation. 
87. Macularia. Lin.

F. - W. V. - Huh. 135 (non Hub. Beitr. texte). - Treit. Dup. - Fr. B. 558. - Bdv. 1779. - Her.-Sel.. I. 139. Wood 710. - Heydenr. Cat. 608.

Var. Brunnea. Dup.

"Quadrimacularia. Haw. 3ł3. - Heydenr. Cat. 609.

Larv. Hub. G. I. Amplis. V. b. c.

Très commune partout en mai et en juin: apparaît souvent à la fin de juillet. Pas sur les hautes Alpes (Lah.). - De même dans les eantons de Berne et de Zurich et sur le Jura: du 15 mai an 13 juin; puis du 23 juillet au 8 aoút (Rothb., Meyer, Rremi). Mr. Zeller a pris nne fois la variété brune au Burghölzli près Zurich. - Quadrimacularia a été trouvée près de Zurich par Mr. Bremi.

G. XVIII. Urapteryx. Kirby. Steph. Dup. Curt. Bdv.

(Acaena. Treit.)

Nota. A quelques rapports èloignés avee margaritaria: la nervation est un peu différente.

88. S ambucaria. Lin.

IIul. 28. - Treit. - Dup. - Bdv. 1435. - Her.-Schif. I. 141.

- Wood 491. - Heydenr. Cat. 39.

Larv. IIub' G. I. Amplis. K. a.

Communc dans les haies durant l'été; juillet (Lah.). - A Schüpfen du 26 juin au 26 juillet (Rothb.). - Vole à l'entrée de la nuit. La chenille passe l'hiver dans les mousses.

G. XIX. Rumia. Steph. Dup. Curl. Bdr.

(Ennomos. Treit.)

Nota. Nervation comme Geometra: la lunule v'est pas trausparente comme daus Selenia. 
89. Cralaegaria. Lin.

Hub. 32. - Treit. - Dup. - Bdv. 1436. - Her.-Schf. I. 142.

- Wood 490. - Heydenr. Cat. 49.

Larv. G. I. Aupl. F.•a. et F, a, b. fig. ?2.

Espece commune dans toute l'Europe; préfére les bois taillis. Deux apparitions, l'une en arril et en mai, l'autre en juillet et en aoùt (Lah.). - Du 1.2 mai au 17 juin, puis au 13 juillet, a Scluïpfen (Rothb.). - La chenille porte denx paires de pattes ventrales incompletes.

Les individus du Riesengebjrge portent 4 points bruns, très marqués, sur la frange des ailes posterieures, que je n’ai pas encore observés sur ceux de nos contrées (Meyer).

G. XX. Eurymene. Steph. Dup. Curt.

Pharmacis. Ifub. Verz. - Ennomos. Treit. Bdv.)

90. Dolabraria. Lin.

Hub. 42. - Treit. - Dup. - Bdv. 1438. - Fr. n. B. 414. Her.-Schf. I. 143. - Wood 537. - Heydenr. Cat. 19.

La rv. Hub. G. I. Amplis. G. b. f. 1 .

Ca et là dans les bois taillis; nulle part fréquente; Sauvabelin, Rovéréaz, prés de Lausanne; fin de mai et juin (Lab.). - Le Burghölzli, prẻs Zurich (Vögeli, Zeller). Du 13 mai au 26 juin; assez rare, près de Schüpfen (Rothb.). - A Burgdorf (Meger). On n'a point obserié de seconde apparition en Suisse.

\section{G. XXI. Ploseria. Bdv.}

(Numeria. Dup. - Fidonia. Treit.)

91. Diversaria ta'. Bork.

Hub. 202. 5. - W. V. - Treit et sup. - Dup. - Bdv. 1499.

Her.-Schf. I. 147. - Heydenr. Cat. $23 \%$.

Aurantiata. Fab.

l'ulverata. Thunb. - Zetler.

Cette l,elle espèce n’a ètè prise jusqu'ici en Suisse que dans les taillis de I'Uto, près de Zurich, au mois de mars. (Collectiou Bremi). 
G. XXII. Plıasiane. Dup.

(Lilrentia. Bdy. - Aspilates. Treit. - Lozogramma. Steph. Curt.)

Noti. Ce que nous arons dit d'artesiaria (Therapis) s'applique á petraria et, ce nous semble, á peltaria; soit qu'on les réunisse sous $\Lambda$ spilates ou sous Phasiane.

92. Petraria. Esp.

Hub. 113. - Treit. - Dup. - Bdv. 1659. - Her.-Sichf. I. 148.

Wood 617. - Heydenr. Cat. 100.

Virgaria. Bork.

Chlorosata. Scop.

Recueillie plusieurs fois près de Burgdorf par Mr. Meyer, et prés de Langnau par Mr. Rothenlsach; partout rare. - Espèce de l'Allemagne méridionale.

G. XXIII. Hibernia. Latr. Dup. Bds. Steph.

Lampetia. Steph. olim. - Erannis Schf. d. Ins. - Fidonia. Tr.)

Notah Rupicapraria a la cellule coupée carrément et lermèe au point de bifurcation des nervures qui la circonserivent; dans toutes les autres l'anastomose de elôture dépasse le point de bifurcation. - Dans defoliaria la cellule est coupée obliquement. L'avortement des ailes chez la femelle n'est pas exclusif à ce geure, il se retrouve dans Gnophos, Nyssia (Bdv.) et Cheimatobia. (Steph.) Comment Curtis a-t-il pu placer brumaria dans Hiberuia et borearia dans Electra. (Steph.)?

9:3. Rupicapraria. W. V.

Hub. 222. ‡. 512. ค. - Treit. - Dup. - Bdv. 1527. - IIer.Sclif. I. 149. - Wood 641, - Heydenr. Cat. 292.

Larv. Hub. G. II. Aequiv. B. a. b. f. 1.

Cette espéce rare a étẻ prise près de Schüpfen, le 9 février (Rothb.) et dans la seconde quinzaine de février près de Lausanne, par Mr. G. Leresche (Lah.j. Elevée souvent par MM. Heuser et Meyer à Burgdorf.

Paraît en mẻme-temps que leucoplua aria. 
94. Defoliaria. Lin.

Esp. - W. V. - Hub. 182, 510. 9. - Dup. - Treit. - Bdv. 1530. - Her.-Schf. I. 150. - Wood 464. - Heydenr. Cat. 287. Lar v. Hub. G. Il. Aequiv. B. a. f. 1. a. b.

Assez commune, certaines années, dans les vergers, à la fin d'octobre et jusques aux premiers froids de la mi-novembre. Reparait en arril (Lah.). - Rare dans le canton de Berne. - Très fréquente dans les vergers du nord de la Suisse (Bremi). - Près de Berne et de Burgdorf, uniquement sur le Sebünbühl, où elle est commune; en avril (Meyer). -

Dans les environs de Lausanne elle paraît plus fréquente en automne qu'au printemps. (Voir: Denkschriften der Schweiz. naturf. Gesellsch. Vol. I. deuxieme livraison p. 94.)

Varie beaucoup.

95. Aurantiaria. Esp.

Hal. 184. - Treit. - Dup. - Bdv. 1528. - Her.-Schf. f. 351. S.

I. 151. - Ileydenr. Cat. 285.

Prosapiaria. Wood 463.

Larv. Hub. G. II. Aequiv. B. a. f. 1. e. d.

Les.bois de chẻne, mi-novembre; quelquefois au mois de mars; Sauvabelin près de Lausanne: rare (Lah.). — Près de Berne; très rare (Rothb.); - de mème près de Burgdorf (Heuser). Moins rare dans les environs de Zurich (Bremi).

96. Progemmaria. Hub. 183.

Treit. - Dup. - Bdv. 1529. - Her.-Schf. f. 346, 347, \&. I. 152.

- Jeydenr. Cat. 286.

Connectaria. Wood 462.

Capreolaria. Esp. - Wood 461.

L arv. Hub. G. II. Aequiv. B. a. f. 2.

Fréquente parmi les feuilles, au pied des arbres, aux envirous de Lausanne, tantót en novenshre, tantôt en mars en mème temps que les premiers exemplaires de lobularia Lah.). - Sur le Balgrist près Zurich (Zell.). - Du 18 mars au 8 avril dans le canton de Berne; assez commune (Rothb.). 
97. Leucaphoearia. W. V.

$$
\begin{aligned}
& \text { Hub. 195. - Treit. - Dup. - Bdv. 1531. - Her.-Schf. } 350 \% \text {. } \\
& \text { 1. 153. - Wood 459. - Heydenr. 290. }
\end{aligned}
$$

Var. Sordidaria. Hub. 334? - Heydenr. Cat. 289.

"Nigricaria. Ilub. 181. - Fr. n. B. 258. 2. - Heydeur. Cat. 291. Marmorinaria. Esp.

Pas rare dans les bois et les taillis, à la fin de férrier et en mars: Sauvabelin, près Lausanne (Lah.). - Du 12 mars au 21 arril, pres de Schüpfen; assez rare (Rothb.) De même sur l'Uio, près Zurich (Bremi). Pas rare à Burgdorl. En èlevant la chenille. le papillon èclos en chambre à la mi-février (Neyer et Heuser).

La variété nigricaria se trouve uèlée avec l'espece ordinaire. C'est la première hibernie qui se montre après l'liver.

98. Ba jaria.

IIUb. 19 4. 594. 8. - Treit et sup. - Dup. - Bdv. 1532. Her-Schf. 354 S. I. 154. - Ileydenr. Cat. 288.

Aerugaria. W. V.

Sericearia. Bork. - Esp.

Cette espèce a èté trouvée dans les Grisons par Mr. Mengold (Bremi). - Mr. Bruand l'a prise en abondance dans le Doubs, pres de St. Vit, en octobre et en novembre (Bruand, Catal. $n^{\circ} 511$ ). - Je possède un individu $\sigma^{\alpha}$ pris en mars 1851 , contre le tronc d'un bouleau, an-dessus de Lausanne (Lah.).

Malgré l'autorité de Treit., je ne puis admettre sordidaria Hub, au nombre des variétés de bajaria.

\section{G. XXIT. Scoria. Steph.}

(Siona. Bdv. - Idaea. Tr. Curt.)

Nota. La nerration de albaria est très voisine de celle de fumidaria, d'aspersaria et de conspersaria. Les genres IIypoplectis et Scodiona Her.-Schf. devraient ètre peut-être réunis a Scoria. La différence des antennes est insuffisante pour fonder uı genre. 
99. Dealbaria. Lin.

W. V. - F. - Hub. 214, 528-31. - Dup. - Bdv. 1923. -

Fr. n. B. 282, 4. - Her.-Sclif. I. 155. -- Wood 598. Heydeur. Cat. 629.

Lineata. Scop.

Cette phalène répandue dans toute l'Europe, se trouve partout communément dans le canton de Vaud en juin et à la fin de mai; elle aime les prés humides et se cache parmi les joncs. Ne s'élève pas sur les Alpes (Lah.). - Prairies humides du canton de Zurich Bremij, - A Schüpfen du 12 mai au 19 juin (Rothb.). - En grande ahondance à Burgdorf (Meyer.

Les individus pris sur If Jura sont beaucoup plus petits (Meyer).

G. XXV. Cleogene. Dup. Steph. Bds.

(Ninoa. Treit. - Angerona. Curt.

No a. Par sa nervation ce genre se rapproche extrêmement de P'sodos; mais il n'en a pas les épaules larges.

100. Tinctaria. Hub. 121 .

Dup. - Bdv. 1411. - Her.-Schf. 361 F. I. 156. - Wood 63.

- Heydenr. Cat. 619.

Lutearia. Esp. - F. - Treit. - (non Devill.).

Cette espèce est commune dans presque toutes les Alpes de la Suisse depuis la fin de mai jusques à la fin de juillet; elle ne descend guères au-dessous de 2000 pieds, à la limite des hois de hètre. Ne s'èlère guères sur les hantes $\Lambda$ lpes el préfère les pâturages subalpins. Ne paraît pas exister dans le Jura. Rare dans les Alpes du nord de la Suisse. Très commune dans tout le Valais (Lah.). Prise quelquefois dans les montagues près de la ville de Berne (Meyer) - Obserrèe encore le 3 août sur la Gemmi (Rothb.).

Le mâle vole au soleil avec assez de vivacité, cherchant la femelle. Celle-ci a des ailes plus courtes, coupées carrèment et aigües au sommet; elle vole peu et pesamment; se cache au pied des rocs et parmi les pierres où on l'aperẹoit difficilement. Sa couleur est aussi plus pảle. 
101. Illibaria. W. V.

Hub. 207. - Treit. - Dup. - Bdv. 1412. - Her-Schf. f. 331, 332. I. 157. - Heydenr. Cat. 624.

Niveata. Scop. carn.

Cette espèce très rare et propre aux Alpes méridionales, a éte prise une seule fois en Suisse sur le revers méridional du St. Gothard, au-dessus d'Airolo, par Mr. Bremi.

Cet entomologiste zèlé n'en saisit qu'un seul exemplaire mâle, le 25 juillet 1837 , en conplagnie de corvaria.

\section{G. XXV. Angerona. Dup. Steph. Curt.}

Crocata. Hub. Verz. - Ennomos. Tr. Bdv.

Nota. Nervation comme Geometra. La nervure scapulaire se détache de la moyenneexterne très près de la racine de l'aile. Pattes postérieures chez le mâle, raccourcies, avec les tarses épaissis. Ces caractères se retrouvent dans Geometra et Ietrocampa. - La base de la nervure moyenne-externe des inférieures offre en dessus un renflement léger analoque à celui des Argynes.

102. Prunaria. Lin.

W. V. - Hub. 122, 123, 556. - Treit. - Dup. - Bdv. 1458. Her.-Schf. I. 159. - Wood 489. - Heydenr. Cat. 20.

Var. Corylaria. Esp. - Heydenr. Cat. 21.

Larv. Hub. G. I. Amplis. H. a.

L'une et l'antre sont communes dans les bois et les taillis, durant le mois de juin. pres de Lausanne (Lah.) - La varieté brune paraît fort rare près de Zurich; elle n'a èté prise que par le pasteur Rohrdorf, près de Seen (Bremi). - Du 3 juin all 11 juillet près de Schüpfen; fréquente (Rothh.).

Corylaria compte des individus des deux sexes. - Prunaria fraiche varie du jaune serin all jaune orange.

G. XXVII. Zerene. Treit. (non Curt.).

(Abraxas. Leach. Steph. Curt.)

Nota. Marginaria n’est pas mieux placée ici qu'à côte de maeularia ct de trista- 
ria. Ses tarses postèrieurs renflés, avec le métatarse atrophié, ainsi que la structure de la cellule la rapprochent des Acidalia et spécialement d'ornataria. La nervure scapulaire s'accole à la médiane-externe jusques vers le milieu de la cellule.

103. Melanaria. Lin.

Hub. 86. - Bork. - Treit. - Dup. - Bdv. 1513. - Her.Schf. I. 160. - Wood 62. - Heydenr. Cat. 232.

Prise près de Zurich, sur l'Uto, dans les bois de pins, par le pasteur Rohrdorf (Bremi). - Partout très rare.

104. Grossularia (ta). Lin.

W. V. - Hub. 81, 82. - Treit. - Dup. - Bdv. 1804. Fr. n. B. 84, 3-125, 2, 3 (var.) - Her. Schf. I. 161. - Wood 601. - Heydenr. Cat. 610.

L a rv. Hub. G. 1. Ampliss. O. a. b. f. 2.

Commune dans les haies des environs de Lausanne depuis le commencement de juillet jusqu'au milieu d'aoùt (Lah.). - Mèmes localités dans toute la Suisse. - Zurich ; fréquente (Bremi). - Du 25 juin au 29 juillet, à Schüpfen et dans le voișinage (Rothb.).

105. UImaria. Fab.

Hub. 85, 391, 392, - Treit. - Dup. - Bdv. 1805. - Fr. n. B. 125, 1. - Her.-Schf. I. 162. - Wood 599. - Heydenr. Cat. 611.

Pantherata. Bork. - Hub. Beitr.

Sylvata. Devill.

Lar v. Hub. G. I. Amplis. O. a. b. f. 1.

Cette espèce préfère les montagnes du nord de la Suisse. - Dans le Sihlwald et l'Allein près Zurich; assez rare (Bremi). - Existe aussi dans le Doubs (Bruand).

106. Pantaria. Lin.

Hub. 34. - W. V. - Treit. - Dup. - Bdv. 1806. - Her.Schf. I. 163. - Wood 600. - Heydenr. Cat. 612.

Espèce méridionale prise près de Zurich, dans le Siblwald, par Mr. Bremi. - Près Pontarlier (Bruand). - Très rare en Suisse. 
107. Marginaria. Lin.

W. V. - Bork - Hub. 80, 544. - Treit. - Dup. - Bdv. 1780.

- Her.-Schf. I. 164. - Wood 733.

Pollutaria. H. 77.

Naevaria. H. 79. - Hub. Beitr. (Nachträge).

Larv. Hub. G. I. Amplis. N. b.

Se montre surtout dans le mois de juin et de juillet; mais on la prènd de boune beure au printemps et dans l'automne. Commune dans les prés, les taillis et le long des ruisseaux daus toute la Suisse, jusques sur les Alpes (Lah.). - De même à Zurich (Bremi). - A Schüpfen du 15 mai au 13 juin et du 23 juillet au 8 aoùt (Rothb.) La forme naevaria Hb. est la plus frèquente. Marginaria (Hub. 80) se trouve volontiers sur les montagnes; je l'ai prise sur les pâturages des Alpes. - Pollutaria (Hub. 77) est la plus rare; elle a ètẻ prise près de Lausanne et près de Burgdorf; Sommerhauswald (Meyer). On rencontre des intermédiaires nombreux.

G. XXVIII. Numeria. Steph. Boisd. Curt. Dup.

(Fidonia. 'Treit.)

Nota. Je ne trouve pas des caractères suffisants pour séparer ce genre de Fidonia. Pulveraria se rapproche extrêmement de plumaria; la nervation et le limbe des inférieures sont semblables.

108. Pulveraria. Lin.

W. V. - Bork. - Hub. 203. - Treit. - Dup. - Bdv. 1500. Her.-Schf. I. 171. - Wood 523. - IIeydenr. Cat. 274.

L arv. Hub. G. I. Amplis. G. b. f. 2.

Assez frèquente dans quelques bois-taillis humides des montagnes; Sauvabelin près de Lausanne, etc., fin de nai et juin (Lah.). - - Du 8 au 19 juin près de Schüpfen; assez rare (Rotbl.). - Sur le Randen près Schaffhouse (Seiler). - Au Sommerhanswald, près Burgdorf, fort commune certaines annèes (Meyer).

Je donte de l'apparition en juillet, en tout cas il n'en existe pas deux par an. 
109. Capreolaria. W. V.

$$
\text { F. - Hub. 204, 205. - Treit. - Dup. - lids. 1501. - Iler.- }
$$
Schf. 1. 172. - Heydenr. Cat. 272.

Pas rare dans les bois de sapin du Jorat et des Alpes vaudoises en juillet et en août; tonjours dans le voisinage des sapins (Lah.). - Les Grisons (Mengotd). - Bois du Gournigel [Berne] (Meyer). - Près de Schüpfen depuis le 21 juillet jusqu'au 11 septembre; commune (Rothb.).

La femelle est ordinairement plus pâle que le màle. Quoique cette phalène se prenn r quelquefois en septembre, je ne pense pas qu'elle ait deux générations par an.

L'époque où elle se prend fréquemment est la fin de juillet; des lors elle ne parait plus que rarement. Dans les bois élevés elle éclos en août.

\section{G. XXIX. Bapta. Steph. Curt.}

Cahera et Acidalia. Bdv. - Lomographa. Hub. Verz. - Corycia et Ephyra. Dup. - Zerene. Treit.)

Nota. Si l'on place pusaria et exanthemaria dans les Fidonia, taminaria et temeraria ne peuvent guères en être éloignées: la nervation est identique de part et d'autre. Pictaria se rapproche de capreolaria. Les antennes n'offrent pas ici, mieux qu'ailleurs, un caractère gènérique.

110. Taminaria (la). W. V.

Bork. - Hub. Beitr. - Hub. 90. - Treit. - Dup. - Bds. 1808. - Fr. n. B. 84, 2. - Her.-Schf. I. 174. - Heydenr. Cat. 614 .

Bimaculata. Fab. - Wood.

Jusqu'iei je n'ai pris cette espèce dans le canton de Vaud qu'auprès du village de Panex (montagnes d'Aigle), dans les taillis de Coudrier; au milieu de juin (Lah.). Près de Schüpfen elle n'est pas rare, du 1. mai au 12 juin (Rothb.). - Conmune près de Burgdorf, dans les haies, à la même èpoque; l'Oberhasli (Meyer). - Pas rare aux environs de Zurich; le Zurichberg (Bremi). 
111. Temeraria. IV. V.

Hul. 91, 376, 377. - Hub. Beitr. (suppl.) - Treit. - Dup.

Bdv. 1832. - Fr. n. B. 84, 1. - Her.-Schf. 1. 175. - Heydenr. Cat. 615. -

sylvestrata. Hub. Beitr. (texte).

Punctata. F. - Wood 689.

Pas rare dans les bojs de bètre aux environs de Lausaune en snai et juin, puis en 10ùt Lah.). - Fréquente près de Burgdorf dans les mêmes localités que la précédente (Neyer). - Commune près de Schüpfen, du 11 mai au 20 juin (Rothb.) - Dans les mêmes eirconstances, près de Zurich (Bremi).

Probablement deux générations, dans les lieux plus tempèris.

112. Pictaria. Curt. 417.

Bdv, 1821. - Her.-Schf. f. 40. I. 176. - Wood 503, - Ileydenr. Cat. 178.

Une femelle prise près de Gamsen, dans le Hant-Valais, par Mr. Anderegg. - Un mâle, en arril, au-dessous de Lausanne (Lab.).

Elle a plus de rapport avec capreolaria, qu'avec temeraria et taminaria.

\section{G. XXX. Gnophos. Treit. Dup.}

Charissa. Curt. Steph. - Gnophos et Ellophos. Boisd.)

Nola. Ce genre qui n’est guères caractérisé que par son facies, n'est pas moins très naturel. Rien ne le différentie de Boarmia d'une manière positive. La nervation donne cinq groupes différents. a. Mendicaria, dont la cellule coupée carrement se ferme un peu au-dela de la bifurcation. b. Dilucidaria et glaucinaria où la cellule, scublable à celle de la précédente, est fermée au-delà de la bifurcation du cóté interne seulement. c. Spurcaria, qui ne diffẻre de b que par la nervure scapulaire détachée plus près de la base de l'aile. d. Operaria ressemble à spurcaria, mais sa cellule est fermée par un angle rentrant. e. Serotinaria, furvaria, pullaria, obscuraria, limosaria et Andereggaria, ont la cellule comme b, mais fermée en biseau. Les antennes sont pectinèes, semi-pectinées et filifarmes dans les espèces les plus voisines, par 
exemple: Meyeraria et dilucidaria; limosaria et Andereggaria. Les tarses des pattes postéricures sont tnméfiés chez les unes et non chez les autres. Le contour des ailes n'offre pas de caractères plus certains; on trouve tous les interwédiaires depuis Zelleraria dont la marge est entière, jusqu'à glaucinaria et obseuraria avec leurs ailes festonées.

\section{Serotinaria. Hub. 147.}

Her.-Schf. f. 328. I. 182. (cetera Synon. incerta).

Prise en juillet dans la vallée Léventine, près de Faïdo, au pied des rochers. Je l'ai reçue de Meyringen où elle n'est pas rare (Lah.). — Rosenlauï et Breitbodenalp au mois de juillet (Meyer).

Le dessin est celui de mendicaria; mais la taille est un peu superieure. Le fond, d'un jaune roux, est convert d'un grand nombre de grossiers atômes gris-bruns. Les points de la marge sont petits. Le sommet des snpérieures fait un angle assez vif. Les lunules sont bien marquèes; celles des inférieures touchent la raie voisine. La troisième raie (marginale) est rapprochée de la seconde et marquée par une lègère ombre. Le dessous est d'un gris soyeux, roux, plus elair aux inférieures. Le front est brun vers les palpes et blanc-jaunâtre au-dessus. Les antennes sont également pectinèes jusques près de leur sommet; leors barbillons sont courts et spatulés. Les tarses postérieurs dı màle sont épaissis et armés de deux paires d'éperons.

J'ai vu plusieurs mâles, mais je n'ai pu encore me procurer une seule femelle. Mr. Mann m'assure que l'échantillon, sur lequel Hub. a pris sa fig. 147, est bien celle inserite ici; quant à la fig. de Her.-Sebf. l'identité est évidente. Cette espẻce ayant ètè confondue avec la suivante, les synonymes des auteurs restent incertains. Freyer et Treitschke me paraissent avoir eu l'autre espèce sous les yeux. Je l'avais nommée laberularia avant de connaitre mendiearia. H. S.

\section{Mendicaria. Her.-Schf. (Mann, in litter.)}

$$
\begin{aligned}
& \text { Serotinaria? WV. V. - Treit. - Fr. n. B. 353, 354. - Wood } 629 . \\
& \text { - Heydenr. Cat. } 125 .
\end{aligned}
$$

Sur le Breitbodenalp, près de Rosenlauï (Oberhasli). Une fois près de Burgdorf (11 juin 1848) fraîche éclose, posée sur la bruyère (Meyer). - Environs de Langnan et pied du Jura (Rothb.) 
Le màle est d'un gris un peu luisant, mais moins qu'op erari a. La femelle a souvent une teinte pâle. Les points marginaux sont gros; les inférieures sont à peine crénelées et bien arrondies; le sommet des supérieures est arrondi, les atômes sont bruns et petits.

Mendicaria habite les basses Alpes, tandis que serotinaria préfère le pied des hautes Alpes. Mr. Mann a fait la même observation en Autriche.

Les indications de Boisd. et de Dup. sont insignifiantes puisqu'ils ont suivi Treit. - Her.-Schf. n'a point encore, que je sache, décrit ou figuré cette espèce.

115. Dilucidaria. W. V.

$$
\begin{aligned}
& \text { Hub. 143. - Treit. et sup. - Dup. - Bdv. 1578. - Fr. B. } \\
& \text { 125, 2? (pullaria). - Her.-Schf. f. 71. I. 183. (non Bru. catal. } \\
& \text { n }^{\circ} \text { 714.) - Wood 627. - Heydenr. Cat. } 128 .
\end{aligned}
$$

Pas rare en Suisse, dès le pied des Alpes, jusques sur leurs sommités; parmi les rochers; en juillet (Lah.). - Fréquente dans l'Oberland hernois du 13 au 28 juillet (Rotłb.). - Sous-Alpes, vallée de Maderan; pas rare (Bremi). - Commune près de Meyringen, en juillet (Meyer). Une femelle a été prise au-dessus de Guttannen le 6 août.

Dilucidaria présente deux formes, l'une a le fond bleuâtre semé de nombreux atómes gris; e'est celle qu'a représentéc Hub., en foręant un peu les teintes. - L'autre que Her.-Schf. paraît avoir eue sous les yeux, a le fond blanc-terne, les atómes moins nombreux et un peu plus foncés.

Her.-Schf. figure le dessous des supérienres d'un blane sale; dans les échantillons que j'ai vus, il est plus on moins noiràtre, surtont dans la varièté bleuâtre. La femelle est ordinairement plus foncée que le mâle.

Cette espèce est souvent confondue avec serotinaria; je l'ai reçue de Besançon sous le nom d'operaria; elle se trouve done dans le Jura.

116. Spurcaria. Nob. fig. 1 .

Cette espèce, dont je n'ai vu que le mâle tient le milieu entre Zelleraria (Frey.) et operaria (Hub.). - Je l'ai prise dans les hauts rochers au pied du Mont-Rose, en juillet 1847.

Sa taille est un peu au-dessous de celle d'operaria. Les ailes sont d'un gris jaunâtre et recouvertes de nombreux atômes, gris, grossiers et groupés par taches, surtout le long 
de la cóte et sur la marge. - Les deux raies medianes sont à peine marquees: leur origine est indiqué à la côte par deux taclıes noiràtres, qui divisent celle-ci en trois portions ègales. La bande marginale est marquée par une ombre, parallèle à la marge, sans dentelures ni points, ni taches. Un point noir, non pupillé et peu prononce, se trouve au centre de chaque aile. - Frange de la couleur du fond, sans raje ni points, très entière: Sommet des supérieures: arrondi. La raie des inlérieures est à peine visible et non dentelée.

Dessous d'un blanc sale, semé d'atômes gris-clair. - Une tache plus foncée part du sommet des supérieures et se prolonge un peu le long de la frange. Da côté de la racine cette tache est suivie d'une éclaircie qui se perd sur le disque.

Antennes pectinées: barbillons courts et spatulés. - Front gris sale. - Dessous de l'abdoinen enfumè. - Tarses postérieurs non tuméfiès, avec deux paires d'éperons.

On peut mieus la confondre avec Zelleraria qu'avec operaria; mais la taille est plus forte que chez celle-lá, le sommet des ailes supérieures est moins arrondi et la bande noire de la marge, en dessous, ne s'ètend qu’à une partie des supérieures el nullement aux inférieures.

117. Zelleraria. Fr. n. B. t. 192, 2. 3.

Her.-Schf. f. 57, 58. I. 181 - Bdx. $1580 . \quad$ Dup. sup.

Heydenr. Cat. 126.

\section{Calcaria. Stenz.}

Un individu fané, quoique très reconnaissable, a èté trouvé par Mr. Zeller, andessus de St. Moritz (Grisons), au milicu d'aoǹt.

118. Operaria. Hub. 9359.37.

Dup. - Bdv. 1581. - Treit. sup. - Her.-Sichf. 1. 184. - Woud 630? - Heydenr. Cat. 129.

Je ne mentionne cette espèce que sur l'autoritè de Treitschke qui dit l'avoir recue des Alpes de la Suisse. Elle préfère les Alpes méridionales. - Mr. Rothenbach l'a cite comme trouvèe a Meyringen. En tout cas elle ne se trouve pas dans le Jura comme l'indique le Catal. de Mr. Bruand $\left(n^{\circ} 570\right)$. Voir a dilucidaria.

La femelle est aptère (Manu). 
119. Furvaria. Fab.

Hub. 144. - Treit. - Hub. Beitr. - Dup. Bdv. 1584. -

Her.-Schf. I. 185. - Heydenr. Cat. 119.

Larv. Hub. G. I. Amplis. X. a. lig. 1.

Ca et là dans les alpes et les montagnes; les lieux escarpès, en juillet. Une fois prés de Lausanne, au Signal (Lab.). - Sur l'Uto, près de Zurich; rare Bremi). - Près de Bienne, du 9 juillet au 22 aoùt; assez rare Rothb.).

120. Pullaria. Hub. 145.

Treit. - Dup. - Bdv. 1588. - Her.-Scht. f. 70. I. 188. Heydenr. Cat. 131.

Dilucidaria. Esp. - Fr. B. 125, 2. ?

Vallée de l'Oberbasli, très rare; juillet (Meyer). - Vallèe de Maderan (Grisons), très rare (Bremi).

121. Meyeraria 1). Nob. fig. 2.

Dilucidata? Bru. Catal. $n^{0} 714$.

Grisearia. Mann (in litter.).

Cette espèce a la taille de pullaria et le dessin de dilucidaria, mais plus efface.

Le fond est hlanc sale, couvert de nombreux atômes brun-foncés; ceux-ci sont plus nombreux vers la marge et à la base des inférieures. Les deux lignes transverses des supérieures sont marquées par des points brun-foncés sur les nervures; l'interne droite est à peine visible. L'angle de l'externe, prẻs de la côte, est indéterminé. La ligne des inferieures est presque droite, denticulèe, légèrement ombrée de brun en dedans chez la femelle. - Les quatre lunules sont arrondies, bien dessinèes, d'un blane de lait pur, cernees de brun. - Entre la marge et la seconde ligne on observe, en partant de celle-ci, d'abord une nuance plus claire, puis une foncée sous forme de bande formé d'atômes et faisant deux ondulations parallèles à la ligne médiane, sauf vers l'ang̣le interne où elle s'en èloigne. A cette bande foncers, plus ou moins visible, en sncerde

1) En souschic de mon ami Mr. Meyer-Dur, de Burgdorf, entomologiste el collecleur cele. 
une troisième claire el étroite; après celle-ci le fond se courre plus ou moins d'atômes jusques près de la frange. Celle-ci est d'un blanc sale, entiere aux supérieures, érodè au milieu des inférieures. Des points noirs, bien dessinés, limitent la frange; deux d'entreux, rapprochès et rentrants', sont placés ris-ả-vis de l'écosion des infeirieures.

Le dessous des supéricures est d'un gris roux, uniforme, sur lequel se dessinent en clair les nervures, une lègère bande centrale et la côte. Les inférieures sont d'un blanc jaunâtre, semées d'atômes bruns.

Les quatre lunules y sont représentées par autant de points bruns foncés. Les points de la marge y sont moins marqués qu'en dessus.

Les antennes du mâle sont filiformes, tomenteuses en dessous. Le frunt est blauc sale; les palpes bruns et courts. Les pattes sont rousses: les tarses des postérieures, chez le mâle, sont forts, non èpaissis, arniés de deux paires d'éperons. - Le ventre est roux vers l'anus. - Le pinceau de l'anus du mâle est court et gris.

Meyeraria a èté prise par Mr. Rothenbach au pied du Jura, dans les environs de Bienne et d'Arbourg. J'ai vu deux femelles et un mâle semblables entr'eux. Si je n'arais pu voir le màle, j’aurais pris la fenselle pour une variété plus petite et plus pâle de dilucidaria. (Collect. Rothenbach.)

J'ai tout lieu de croire que la dilucidata à antennes filiformes, chez le mâle, du Catal. de Mr. Bruand, est notre espèce. - Mr. Mann l'a prise en certain nombre dans les Alpes autrichiennes, il y a quelques annèes; il l'avait appelée grisearia, mais ce nom est dejà employé ailleurs.

122. Obscuraria. W. V.

Hub. 146. - Treit. - Dup. - Bdv. 1589. - Her.-Schef. I. 190. - Wood 626. - Heydenr. Cat. 124.

Lividata. Fab.

A nthracinaria. Esp. (non fig.)

L arv. Hub. G. I. Amplis. X. a. f. 2.

Sur l'Albis, près Zurich; très rare (Bremi). - Au pied du Jura, près de Bienne, du 14 au 22 juillet; rare (Rotbb.). 
123. Glaucinaria. Hub. 150.

Treit. - Dup. - Bdv. 1591. - Fr. B. 125, 1. - Her.-Selif. I. 68, 69-66, 67 (errore sartaria). I. 192. - Heydenr. Cat. 132.

Var. Falconaria. - Fr. n. B. 277, 3. - Dup. sup. - Heydenr, Cat. 133.

A u pied des Alpes et du Jura daus les lieux exposés au soleil et rocailleux; Aigle, fiu juin; Faïdo, 24 juillet (Lab.) - Du 27 juin au 13 juillet; pas rare (Rothb.). - La vallé de Maderan (Bremi). - Oberhasli; Meyringen, en juin et juillet (Meyer). - N'est point rare dans le Doubs (Bruand). - Mr. G. Leresche a pris à Lausanue une femelle le 21 septemlbre et Mr. Ileyer un màle, au Schönbühl, prẻs Burgdorf, le 25 aoùt. Cette phalene aurait donc une seconde apparition? - Varie souvent dans les Alpes.

Falcuuaria (Frey.) est tout au plus une varièe (Hann).

127. Limosaria. Hub. 360 .

Dup. sup. - Bdv. 1583.

Canaria. Hul. 3it. - Fr. 1. B. $377,1,2$.

Obfuscaria Hu's. 142. ๑. - Treit. - Dup. - Bdv. 1582. - Her.Scbf. 1. 195. - Wood 626. - Heydenr. Cat. 122.

Les Alpes bernoises, 25 juillet; pas rare (Rothb.). - Les forèts (?) des Alpes (Bremi), le Balgrist (Zeller). - L'Oberhasli, la Grindelalp, Rosenlaui, entre 5-6000 pieds (Meyer). - Les Alpes savoyardes Chavannes). - Le St. Gothard, 24 juillet (Lah.)

Jai préféré la dénomination de limasaria parce que canaria dans Huls. est identique a limosaria et que obscuraria, choisi par Treit. et Her.-Schf., s'applique dans Hul. à la femelle. Cette derniere est si diffẻrente du mâle, qu'on a dû aisément l'envisager comme espèce distincte. La fig. 142 de Hul, a été prise sur une femelle trís pâle el 360 sur un mâle très foncé.

\section{Andereggaria. Nob. figr. :3 et 4.}

Cetle espece a èlé decouverte daus le Vant-Valais, près de Brigg, par le pretit-its de Mr. Anderegg, marehaud naturaliste bien connu. anqquel je l'ai dédièe. Elle apparait vers te milien de juillet. La fruelle est plus fréquente que le mảle, car jai vu plusieurs fenelles et un seul mâle. Jaes denx sexes sont trés différents l'un de l'autre. Le mảle ressemble tellement à celui de limosaria que, sans ses antennes fortement pectinées, on Ir prendrait pour une variete foncée et teintée de jaunc-orange. La teinte orangée est 
repandue surtout le long des nervures. Les dentelures de la banle médiane sont irès prononcées et aigües. Le thorax est revêtu de longs poils gris, mêlès de fauves. L'abdomen plus court que le bord interne des infërieures, est terminé par des poils roux et annelé de fauve elair. Les palpes sont recourbès au-devant du front, et couverts en dessous de longs poils grisâtres. Les barbillons des antennes sont un peu élargis à leur extrèmité et roux. La frange des inféricures est formée de longs poils fauves, surtout vers le bord interne.

la femelle est beaucoup plus petite que le mâje et ressemble, pour le dessin et pour la taille, à carbonaria. Le fond de ses ailes est d'un blane sale; les atômes nombreux qui le couvrent sont noirâtres et très grossiers: le jaune-orange y est remplacè par une très légère teinte fauve répandue sur la frange et sur les bords de la bande médiane: celle-ci est à peine prononcée et ne se distingue que par ses dentelures fauves bordées en dehors de nombreux atômes, et par son centre plus clair. La racine des ailes est très chargèe de noir-bleuâtre. Les ailes inférieures sont étroites et semblables aux superieures. Le dessous est d'un gris-roux clair, couvert d'atómes gris-noirs, moins nombreux qu'en dessus. Le corps est gris-noirâtre, piquetè de blanc-sale.

\section{G. XXXI. Bo a rmia. Treil.}

(Boarmia et Tephrosia. Bdv. - Boarmia, Alcis, Cleora. Curt. el IIemerophylla. Stph.).

Ce genre est difficile à earacteriser nettement et ses subdivisions ne sont pas tranches. Ni la nervation, ni les antennes, ni le dessin, ni les ehenilles ne fournissent de caractères suffisants. En gónéral l'anastomose de clôture de la cellule des inférieures se place un peu au-dela de la bifureation, et celle-ci est fort ouverte; mais il y a de nuances ombnreuses. La nervure scapulaire, si importante pour Her-Schäf, n'est pas celle qui varie le moins; son point de jonction avec la mediane externe peut être très rapproché de l'origine do l'aile comme dans perversaria, et très voisine de la bifureation comme dans consonnaria et surtont lividaria.

Adustaria n'appartient pas plus à ce genre qu’à Cidaria ou à Larentia; sa place naturelle est à côté de Temeraria, dont elle a la nervation. 
126. Nyclemeraria. Huh. 561-6.

Dup. - Bdv. 1566. - Fr. n. B. 78,1 . - IJer.-Schf. I. 198. Heydenr. Cat. 183.

Var. Petrific ariae. Treit.

Decouverte et élevée ehaque anuée prar Mr. Andereggr. Une superbe femelle, le 9 aoùl, contre un rocher, entre Mœrel et Naters (Ilaut-Valais) (Meyer).

127. Perversaria. Bdv. 155:3.

Dup. sup. - Her.-Schf. 35,36 ค. - 425, 426 ठั. I. 200.

Hcydeur. Cat. 160.

Sabinaria. Auderegg, Catalog.

Cette espéce, découverte par Mr. Anderegg en Valais, u'a ètè retrouvée jusqu'iri qu'en Turquie; il est cependaut probable qu'elle existe partout oủ croît la Sabine dont la chenille se nourrit exclusivement. Elle éclos en juin. On ne pent la confondre avec Rhomboïdaria, comme l'a démontré Her.-Schf.

128. Abielaria. W. Y. -

$$
\text { Hub. 160. - Treit. - Dup. - Bdv. 1557. - Fr. 1. B. } 264 \text { - }
$$$$
\text { Her.-Sclıf. I.202. - Heydenr. Cat.. } 152 .
$$

Genmaria. Esp.

Var. Tota brunnea.

Pas rare dans les hois de sapin du Jorat, au-dessus de Lausanne, ell juillet. Je doute très fort qu'en Suisse elle apparaisse en avril, ni mème en juin; il est probable que les èclosions printanières ont èté uhtenues artificiellement. Elle est née chez moi à la fin de juin (Lah.). Dans le canton de Berne Mr. Rothenbach l'a prise près de Schüpfen et de Nidau, dans les forêts, du 1 . juillet au 27 aoùt; clle y est assez rare. A la même époque près de Burgdorl (Mcyer). - Mẻmes localilés dans le canton de Zurich. oủ elle n'est pas rare (Bremi).

La variété complétement brune, sans aucun dessin, existe dans la collection de Mr. De la Chaumette et a été prise prés de Lausanne.

Taeniolaria (Fidonia. Bdr. a été trouvée dans le Doubs, sur la haute montague +?. Catalog. Nr. 559). 
129. Secundaria. W. V. -

Hub. 156 - Treit. - Dup. - Bdv. 1558. - Fr. Is. B. 276. -

Her.-Sclf. f. 247. 1. 206. - Heydenr. Cat. 162.

Larv. Hub. G. I. Amglis. Y. b. c. fig." 2.

Cette espèce, particulière aux forêts de l'Allemagne, se trouve ẹà et là en Suisse, dans les bois de sapin des montagnes. Elle a été prise au-dessus de Lausanne par Mr. G. Leresche en juillet (Lah.). - Près de Schüpfen; assez rare; du $\mathbf{1 0}$ juillet au 30 aoùt (Rothb.). - De nuême près de Burgdorf. - Le mâle est pluns rare (Meyer). - Près de Seen (Rordorf); sur le Ralgrist (Zeller); dans les Grisons (Mengold, Bremi).

130. Cinctaria. W. V. -

Hub. 166. - Treit. - Dup. - Bdv. 1559. - Her.-Schf. I. 207.

- Fr. 11. B.498. - Wood. 502, - Heydenr. Cat. 144.

Paseuaria. Esp. - Bork.

Commune dans les bois de chêne au-dessus de Lausanne, d'abord ả la fin d'arril et au commencement de mai; puis une seconde fois en juillet. Elle s'appliq̨ue contre les murs et le tronc des gros arbres (Lah.). - Niemes lucalites anx environs de Zurich; Uto: pas rommune; plus fréquente dans les Grisons (Bremi); une seule fuis dans nue forèt de sapin et de chèue près Burgdorf (Heyer). - N'a pas étè prise dans le canton de Berne par Mr. Rothenbach. Cette espèce craint à ce quill paraitt les règions froides. Varie beaucoup.

183. Consortaria. F.

$$
\begin{aligned}
& \text { Esp. - Hul. } 168 . \text { - Treit. - Dup. - Pdv. 1551. - Fr. 11. B. } \\
& \text { 270. - Her.-Schf. I. 208. - Wood. 509. - Heydenr. Cat. } 151 .
\end{aligned}
$$

Larr. Hub. G. I. Ampliss. Y. a. fig. 2.

Pas commune dans les environs de Lausanne, en mai et juin. Je ne l'ai point prise en juillet; je ne crois pas qu'elle aie en Suisse denx apparitions (Lah.). - Prés de Schüffen, du 8 mai au 26 juin; rare Rothb.). - Sur le Balgrist (Zeller). - Pas très rare dans les environs de Berne ei de Burgdorf. - Dans les hasses Alpes de la vallée de l'Oberhasli; Rosenlaui, sous les toits des ehalets, en août (Mleyer).

Baraît avant Roboraria. D'après Her.-Sehf.: Consobrinaria. Hul. 15z: ąppartient à Roboraria et nou á Cousorlaria. 
1:32. Rlomboidaria. W. V.

Hub. 154, 170, 488. - Treit. - Dup. - Bdv. 1554. - Fr.

n. B. 288. -- Her.-Schf. fig 420. I. 210. - Wood. 511. Heyd. Cat. 158.

Gemmaria. Bork.

La plus conmune de toutes aux environs de Lausanne, si l'on excepte Repandaria. Parait en juillet dans les bois de chêne. Jamais prise en septembre (Lah.). - Beaucoup plus rare dans le canton de Berne; près de Burgdorf (Meyer); près de Schüpfen, de Twann (lac de Bienne); du 9 juillet au 20 aoùt (Rothb.). - Les bois entremêlés; assez rare; près de Zurich (Bremi). Les individus de Croatie diffèrent des nôtres par une teinte plus foncée et le dessin plus marqué (Meyer).

133. Roboraria F.

W. V. - Hub. 169. - Treit. - Dup. - Bdv. 1548. - Fr. n. B. 270. § - Her.-Schf. 1. 211. - Wood. 510. - Heydenr. Cat. 150.

Consobrinaria. Ilnb. 152. --

L arv. Hub. G. I. Ampliss. Y. a. f. 1.

Dés le commencement de juin jusqu’à la mi-juillet; au-dessus de Lansanne, dans les bois de chêne; pas très rare. Je ne l'ai jamais prise en avril, ni Mr. Bruand non plus (Catal.), quoique la femelle soit née chez moi au premier printemps (Lah.). Assez. rare près de Schüpfen, du 24 mai au 17 juin (Rothb.). - Plus fréquente prés de Burgdorf el de Berne (Meyer), et de Zurich (Bretui) - voir Consortaria).

134. Viduaria. W. V.

Hub. 165, 364. - Treit. - Dup. - Bdv. 1569. - Her.-Sclif.

I. 213. - Wood. 500. - Heyd. Cat. 175.

Angularia. Thunb.

Assez frequente dans les bois des environs de Lausanne, en juin. En chambre elle éclos déjà en avril. La cbrysalide gî dans la mousse au pied des chènes. Jamais prise en juillet. Une seconde génération chez nous est plus que douteuse (Lah.). - Du 27 mai au 26 juin a Schupfen; rare (Rothb.). - Rare près de Burgdorf, en juin (Meyer). Pas commune dans le Doubs (Bruand). 
1:55. Glabraria. Hub. $162,8339$.

Treil. - Dup. - Bdv. 1568. - Her.-Schf. I. 214. - Heyd. Cal. 174.

Teneraria. Hub. 348. - Wood. 501.

Fort rare, en juillet, sur les bords des bois, au-dessus de Lausanne. Préfère les pays montagneux; le St. Golthard (Lah.). Les alpes du Vałais à la même époque (A. Chavannes). Du 5 au 17 juillet près de Scbüpfen; rare (Rothb.). - De mème près de Burgdorf (Meyer). -

Les individus du nord de l'Allemagne ont un fond gris comme pullaria; les nôtres sont blanc-jaunâtres (Meyer).

136. Repandaria ta). Lin.

W. V. - Hub. 161. - Treit. et sup. - Dup. - Bdv. 1547.

Fr. n. B. 252. - Her.-Schf. I. 21.5. - Wood. 504. - Heyd. Cat. 153.

Conversaria. Huh. 321,393 . - Dup. hist.

Luridaria. Fr. n. B 444, 3. - Heydenr. Cat. 155.

Larv. Hub. G. I. Ampliss. Y b. c. fig. 1.

Assez fréquente dans les bois, les taillis et les vergers des environs de Lausanne; en juin et en juillet; non en mai (Lah.). - Dans le canton de Berne (Langnau, Schüpfen, Burgdorf) commune depuis le 13 juin à la fin de juillet (Rothb. - Mey.). Les bois de sapin (?) des environs de Zurich; commune (Bremi). - Les Grisons (Mengold, Kriechlsaumer).

Cette espèce varie beaucoup: Mr. Meyer a recueilli une varièté qui porte une bande médiane brun-foncée sur un fond gris-clair. - La chenille que j’ai élevée offrait la couleur jaune d'ocre que lui donne Huls. fig. 1. b. (loc. c.).

137. Lichenaria. W. V.

Ilub. 164. - Treit. - Dup. - Bdv. 1570. - Fr. n. B. 71, 1.

- Her.-Schif. I. 216. - Wood. 499. - Heydenr. Cat. 173.

Cineraria. Fab. - Bork.

Cette espèce, rare en Suisse, a èté prise par Mr. G. Leresche dans les bois de sapin, au-dessus de Lansanne, en juillet (Lah.). - Du 14 juillet au 4 aoủ près de Schüpfen; rare (Rothb.). - Prés de Berne (Meyer). - Buis de sapin près de Zürich; l'Uto; très rare (Bremi). 
138. Lividaria. Hub. 141.

Treil. - Dup. sup. - Bdv. 1565. - Her.-Schf. f. 37, S I 218 (non Lin.). - Heydenr. Cat. 185.

Celte espèce n'a èté jusqu'ici trouvée en Suisse que près de Zurich, sur l'Uto, par Nlr. Schulthess (Bremi). - J'en ai vu un exemplaire pris aus environs de Zurich par Mr. Zeller.

139. Crepuscularia. W. V.

Götze. - Hub. 158. - Treit. - Dup. - Bdv. 1571. - Fr. n.

B. 246 . - Her.-Schf. 389 (var). I. 219 . - Wood. 517.

Heydenr. Cat. 147.

Consonuaria. Hub. 157. o (Frey.). - Wood. 518.

Biundularia. Esp. - Bork.

Similaria. Götz.

Abielaria. Var. Wood. 516.

- Var. Tota nigra. vob.

Larv. Hub. G. I. Ampliss. Y. c. fig. 1.

Cette espèce, commune au premier printemps (8 avril), reparaît plus rare en juillet et en aoủt. Les bauteurs des euvirons de Lausanne. Le Gütsch (Lucerne), le 8 août Lah.). - Les basses alpes, le Righi; assez rare (Bremi). - Les Grisons (Mengold). Commune dans le canton de Berne, Burgdorf, Schüpfen, Meyringen etc. (Mejer). - Du 20 avril au 5 juin (Rotbb.).

En rattachant à Crepuscularia la fig. 157 de Hub., Freyer justifie l'opinion de Treit. qui ne voil dans Consonnaria qu'une var. de la première espèce. Pour inu a la vraie Consonnaria sous les yeux, le doute cesse et Treit. est évidemment dans l'erreur.

La varièté noire a èté trouvée près de Coire (in der Au) par le Prof. Kriechbaumer, au mois de juin (?). Si je n’avais pas pu distinguer la ligne dentelée sur les nervures, je l'aurais prise pour une espéce distincte. Le dessus est d'un gris-noir foncé, presque uni, ct le dessons d'un gris-roux. Le mâle et la femelle sont semblables. 
140. Consonnaria. Hub. 157. ?

Dup. - Bdv. 1572. - Her.-Sehl. 246. \& I. 220. - Heydenr. Cat. 146.

Tetragonaria. Wood. 515.

Crepuscularia. Var. Treit.

Larv. Hub. G. I. Ampliss. Y. c. fig, 2.

Cette espèce est presque aussi commune que la prècedeute, dans les bois de bètre au-dessus de Lausanne, en avril et en mai. Varie assez peu (Lah.). - Dans tous les petits bois d'aune autour de Burgdorf; fréquente et bien plus commune que la précédente: Avril et Juillet (Meyer).

141. Punctularia. W. V.

Hub. 317. (Var.?) 545. - Hub. Beitr. - Treil et sup. - Dup. Bdv. 1574. - Wood. 521. - Her.-Schf. 228, 229, 333, (var.), I. 221.

Larv. Hub. G. I. Ampliss. U. b. fig. 1.

Fréquente dans les taillis d'aume au-dessus de Lausanne, en mai (Lah.). - Commune près de Burgdorf dans un bois d'aune; toujours à 8 ou 10 pieds de bauteur, avec les ailes étalées et romme colèes sur l'éeorce des arbres. Nos individus sont en général plus gris que ceux de la Silésie et du nord de l'Allemagne (Meyer). Prise en mai et en septembre par Mr. Heuser. Commune près de Schüplen, du 6 mai au 7 juin (Rothb.)

Ne parait jamais en Suisse dans le mois d'avril, moins encore dans celui de mars. Une seconde éclosion existe, quoique rarement, en automne.

142. Extersaria. Hub. 159.

Treit. - Dup. - Bdv. 1573. - Her.-Schf. I. 222. - Wood. 520. - Heydenr. Cat. 163.

Quelquefois au bois de Sauvabelin, au-dessus de Lausanne, en mai. La chrysalide gît dans la mousse au pied des chênes (Lah.). - Trouvée près de Berne par Mr. Renk. - Du 19 mai au 17 juin; rare; près de Schüpfen (Rothb.). - Rare aussi près de Burgdorf (Meyer). 
143. A duslaria. Bork.

W. V. - Fab. - IJub. 75. - Treit. - Fr. B. 102. - Dup. -

Bdv. 1802. - Wood. 605. - Her.-Schf. I. 223.

I,arr. Hub. G. II. Aequir. L. a. fig. 1.

Pas rare; une première fois à la fin de mai et en juin, puis une seconde lois ell aøût et en septembre; dans les haies des environs de Lausanne (Lah.). - Les bords des bois dans les environs de Zurich; assez fréquente (Bremi). - Aarber et Schüpfen, du 17 mai au 25 juin; puis du 11 au 22 aoùt (Rothb.). Assez commune dans tous les taillis autour de Burgdorf (Meyer).

\section{G. XXXII. Fidonia. Treit.}

Fidoniae. Caherae. Speranza. Treit. Bdv. - Macaria. Curt.

Nota. Ce geure se compose de plusieurs groupes rapprochès tant bien que mal. Le petit nombre d'especes suisses "fu'il renferme ne me permet pas de les caracté riser. Pusaria et Exanthemaria seraient mieux placés a cote des liapta 'Ju'ici. Strigillaria fait a elle seule un groupe; la nervure scapulaire s'mit a la médiane voisine justjue prés de la elòture de la cellule, comme dans Boar. lividaria. Wawaria est fort roisine des Macaria; Picearia la suit hien plutôt qu'Atomaria. Les autres ont la nervation des Fidonia.

11. Pusaria. Lin.

W. V. - Hub. 87. - Treit. et sup. - Dup. - Wood. 524. Fr. B. 144. - Bdv. 1809. - Her.-Schf. I. 224.

Var. rotundaria. Haw. - Stph - Wood. 525. - Heyd. Cat. 313.

Var. confinaria. Fr. n. B. 60, 2. - Heyd. Cat. 312 ,

Larr. Huls. G. I. Ampliss. P. b. fig. 1. d. e. f.

Commune. En mai, sur les bords du lac, dans les haies; en juin, dans les bois au-dessus de Lausanne; reparaîı en juillet et en août (Lah.). - A Schüpfen du 8 mai au 27 juin: du 11 au 17 aoùt; commune (Rotbb.). - Partout dans les taillis et les baies prés de Zurich (Bremi). - S’èlève sur les alpes; trouvée près du Gournigel, à 3800 pieds de hauteur, par Mr. Meyer. 
145. Exanthemaria. W. V.

Esp. - Bork. - Treit. et sup. - Dup. - Bdv. 1811. - Her.Schf. I. 225. - Wood. 526. - Heydenr. Cat. 315.

Striaria. Hub. $88,506$.

Larv. Hub. G. I. A. P. b. fig. 1. (strigaria).

Commune dans les bois, les taillis et les haies des environs de Lausanne, en mai, juin et juillet (Lah.). - Fréquente à Schüpfen du 16 mai au 25 juin; puis au milieu de juillet (Rothh.). - Les bords des bois; sur les pentes de l'Uto; pas fréquente anx environs de Zurich (Bremi). - Sur le Balgrist (Zeller). - A Burgdorf et le long du Jura en ahondance, dans les bois d'aune et dans les haies, le long des forêts de hêtre Meyer). - Une double apparition dans les lieux chauds est très probable.

146. Strigillaria. Esp.

Hub. Beitr. - Hub. 540, 541. - Treit. et sup. - Dup. Bdv. 1812. - Fr. n. B. 113. - Her.-Schf. I. 229. - Heydenr. Cat. $91^{\mathrm{b}}$.

Respersaria. Hub. 125. - Wood. 542.

Var. cretaria. Eversm. - Her.-Schf. 540, 541.

Cette espèce n’a èté trouvée ni par Mr. Rothenbach, ni par Mr. Meyer, ni par moi; Mr. Bremi la dit fréquente dans les taillis des environs de Zurich; mais j’ai quelque lieu de croire qu'il l'a confondue arec Strigillata. Hub. (Acid. prataria Bds.). J'ai vu à Zurich un seul exemplaire de la vraie Strigillaria Esp. pris sur l'Uetliberg. Mr. Rothenbach en possède aussi un exemplaire dans sa collection; mais il ignore sa provenance Lab.). - Mr. Bruand la prend dans les bois du département du Douhs Cat. Nr. 603).

147. PIumaria. W. V.

Hub. 124. - Treit. et sup. - Dup. - Bdv. 1507. - Her. -Schf. I. 230. - Fr. B. 354. - Heydenr. Cat. 266.

Roraria. Fab.

Vespertaria. Esp.

Var. pennulataria. Hub. 507, 508.

Larv. Hub. Geom. I. Ampliss. U. b. fig. 2.

Cette espèce rare a été prise dans les environs de Bienne, au pied du Jura, par 
Mr. Rothenbach; elle est difficile à saisir et se pose sur les rochers et les pierres. Du 14 au 30 août; assez fréquente dans la localité qu'elle habite. Mr. Bremi en a pris un exemplaire sur l'Alhis. - Un individu 5 presque blanc et sans dessin sur tout un cóté, se (rouve dans la collection de Mr. Rothenbach.

118. Wawaria. Lin.

W. V. - Bork. - Hub. 55. - Dup. - Bdv. 1477. - Her.Schf. I. 240. - Wood. 522. - Heydenr. Cat. 256.

Larv. Hub. G. I. Ampliss. K. a.

Pas très commune dans les jardins et les vergers le long du lac Léınan; en juillet. On ue la reucontre ni dans les montagnes, ni sur les alpes; elle préfère les lieux chauds Lah.). - Les mêmes localités sur les bords du lac de Zurich; fréquente 〈Brenì. - Du $2 \bar{i}$ juin au 13 juillet dans la vallée de l'Aar, au-dessous de Berne (Rothb.). - Fréquente à Burgdorf (Meyer). - Varie fort peu.

149. Roraria. Esp. (text).

Bdv. 1522. - Dup. Cat. - Her.-Sclff. I. 241. - Heydenr. Cat. 324. Spartiaria. Treit. et sup. - Dup. hist. - Fr. n. B. 59, I. Spartariaria. Hub. 116.

Cette espèce, fort rare en Suisse, a été prise près de Seen par le pasteur Rohrdorf Bremì.

150. Conspicuaria. Esp.

Bork. - W. V. - Hub. 117, 118. - Hub. Beitr. (Nachtr.).

Treit. - Bdv. 1521. - Dup. Cat. - Fr. n. B. 59, 2. - Her.-

Sclif. 1. 242. - Heydenr. Cat. 225.

Limbaria. Fab. - Dup. hist. - Wood 452.

Auroraria. Hub. Beit.

N'a èté prise jusquici que dans les environs de Schaffhausen, sur le Randen, par Mr. Seiler Rremi). Dans le Doubs, au pied du Jura, frontière suisse (lỉruand. Cat. Nr. 571). 
151. Pinetaria. Hub. $130, \%$.

Treit. - Her.-Schr. I. 243. - Heydenr. Cat. 223.

Quinquaria. Huh. 516, 517. ऊ. - Dup. - Bdv. 1519 , Flammataria. 'Zett.

Brunneat a. Wood. 451 .

Mons. Meyer prend cette espèce toutes les annėes en juin dans les bois du MeyenMoos, près Burgdorf. - Les taillis de l'Uto, près de Zurich; très rare (Bremi). Commune près de Langnau, du 8 juillet an 3 aoùt. Se prend aussi dans les alpes (Rothb.). - Vallée de l'Oberhasli près de Guttanucn, dans les hois; très fraiche le 26 juillet (Lah.). - Ia femelle est très rare.

152. Clathraria. Lin.

Fah. - W. V. - Fuess. - Ilub. 132. - Treit. - Dup. Bdv. 1919. - Her.-Sehf. I. 245. - Wood. 709. - Heydenr. Cat. 248.

Var. e a n cellaria. Hul. 322. - Dup. - Heydenr. Cat. 249.

Var. Her.-Schif. 356.

Var. roscidaria? Ilub. 128, 332.

Commune dans les champs de luzcrne en mai, puis à la fin de juillet ef en aoùt. On la rencontre du reste égrénée partout oủ croit l'hedysarum; pendant tout l'été (Lah.). - Mêmes localités dans toute la Suisse Bremi, Heyer, Rothh.). - A Schüpfen. du 12 arril an 30 mai, puis vers is milieu de juillet.

Qunique cette espèce lienne ses ailes relevées dans le repos, est-il certain qu'elle les conserve dans cette position la nuit, durant son sommeil? Brumaria releve ses ailes dans le repos $\mathrm{et}$ les abaisse dans le sommeil, durant le jour. Les individus de la seconde apparition ont en général une teinte mélangée de jaune, snrtout les femclles.

153. Glarearia. W. V.

Bork. - Hub. 131. - Treit. -- Bdv. 1890. - Dup. - Her.-

Sehf. I. 246. - Heydenr. Cat Nr. 245.

Var. alomariae. Fab.

Une femelle a été prise dans les environs de Bàle par Mons. le doctenr Imboof, je ne sais à quelle époque. J'ai pu la voir dans sa collection. 
154. Piniaria. Lin.

IV. V. - Fuess. - Hub. 119, 120, 469, 470. - Treit. - Dup.

- Bdv. 1510. - Her.-Sehf. 1. 247. - Wood. 453. - Heydenr. Cat. 227.

Tiliaria. $\&$ Lin. f. suec.

Cette espèce assez fréquente dans le nord de la Suisse, l'est beaueoup moins dans les eantons méridionaux. Elle disparait et reparait plus abondante certaines annees; en mai et juin au-dessus de Lausanne (Lah.). - Du 10 mai au 29 juin près de Sehüpfen; assez commune (Rothb.). - I la Rysi près Soleure; forèt de Bremgarten, près Berne; commune sur la promenade du Schönbühl, près Burgdorf: dans les bosquets de pin Meyer). - Commune près de Zuricb (Bremi).

155. Plumistaria Esp.

Hub 127, 417-20. - Treit. - Dup. - Bdv. 1514. - Her.Sehf. I. 250. - Heydenr. Cat. 230.

Var. a uritaria. Hub. 416 .

Très rare en Suisse: près de Seen (Rohrdorf); sur le Balgrist - Zurich - (Zeller); en Valais (Anderegg). - Varie beaucoup. - Fréquente dans le midi de la France.

156. Picearia. Hub. 552-55.

Fr. B. 66, 3. - Treit. sup. - Dup. sup. - Bdv: 1517. - Her.Sehf. 1. 251. - Heydenr. Cat. 242.

Roscidaria. Hub. 128, 332. - Heyd. cat. 243.

Amnieularia. Zetterst.

Le Ilaut-Valais (Anderegg/. - Les Grisons (Treil.).

Les exemplaires venus du nord, plus pàles que ceux de nos alpes, fournissent la forme roscid aria Hub. 
157. A tomaria. Lin.

$$
\begin{aligned}
& \text { F. - Esp. - W. V. - Fuess. n. mag. - Hub. 136, 526, } 527 . \\
& \text { - Dup. - Treit. - Bdv. 1515. - Her.-Schf. 322, 323 (var.) } \\
& \text { I. } 252 .- \text { Wood. 454. - Heydenr. Cat. } 239 .
\end{aligned}
$$

Artemisiaria. Fuess. arch. - Lang.

Carbonaria. Wood. 455.

Lars. Hub. G. I. Ampliss. V. b.

Trés commune daus les prairies, les taillis et les bords des bois, d'abord en avril et en mai, puis en juillet et en août. S'èlève sur les alpes au-dessus des forèts et y apparaît nombreuse en juin et juillet (Lah.). - Très commune dans les environs de Zurich et partout en Suisse (Bremi). - A Schüpfen, première apparition dès le 12 avril, seconde, des le 17 juillet (Rothb.). - Les individus du Haut-Valais, du glacier du Rhòne (Meyenwand) et des alpes bernoises sont plus petits et plus sombres que ceux des vallées et de la plaine (Meyer).

\section{G. XXXIII. Mniophila. Boisd. \\ (Boarmia. Treit.)}

Nota. Rapelle à quelques égards le G. Lemmatophylla (Tineïtes) de Treit.

158. Corticaria. Hub. 167.

Dop. - Bdv. 1595. - Her.-Schf. 211, 212, 213. 1. 257. Heydenr. Cat. 181.

Cremiaria. Fr. o. B. $258,1$.

Larv. Hul. Geom. I. Ampliss. Y. d. fig. 1.

On la prend de temps en temps le soir à la lumière des reverbères de Lausanne; en juillet. - Cremiaria, reçue de Hanoover, est parfaitement semblable Lah.). - Press de Burgdorf, de temps en temps, contre les murs (Meyer).

Cineraria, trouvee dans le Doubs par Mr. Bruand (Catal. Nr. 565), n'a pas encore été observée en Suisse. 


\section{G. XXXIV. Aspilates. Treit. Steph. Curt.}

Nota. Ce genre est bien restreint dans IIcr.-Schf. - Sacraria ne pcut guères ètre éloiguée de Gilvaria.

159. Gilvaria. Bork.

W. V. - Fuess. a. mag. - Hub. 201, 534, 535. - Treit. -

Dup. - Bdv. 1492. - Her.-Schf. I. 259. - Wood 541.

Heydenr. Cat. 86.

Purpuraria. Wood 539 .

Larv. Hub. G. Aequi. C. b. fig. 2.

En août sur les collines sẻehes et chaudes, parmi les chaumes: Moulin de Belmont, près de Lausanne; rare (Lah.). - Près de Mandach (Bossard); - Meyringen, Oberstein; aoùt et septembre; rare (Mleyer). - Commune près de Bienne du 14 au 30 aoùt (Rothb.).

\section{G. XXXY. Amphidasis. Treit.}

(Eubyia, Lycia, Apocheima, Ithysia, Hub. Verz. - Nyssia, Curt. Dup. Boisd. Pligalia, Dup. Steph. - Biston, Leach. Curt.)

Nota. L'absence d'ailes ebez les femelles n'est pas suffisante pour motiver le genre Nyssia. Cellule longue, ètroite, coupée earrẻment; nervation très caractéristique.

160. Zonaria. W. V.

Fucss. n. mag. - Hub. 179, o; 511, §. - Treit. - Dup. Bdv. 1537. - Her.-Schf. I. 269. - Wood. 1674. - Heydenr. Cat. 200.

La rv. Hub. G. II. Aequiv. A. c. fig. 1. a. b. c.

Cette espéce, partout rare hors de l'Allemagne, n'a été prise jusqu'ici que sur quelques points de la chaine du Jura. Dans le canton de Schaffhausen (Bremi). Dans celui de Neuchàtel; au Locle; à la Neuveville; moins rare (Rothb.). - Dans le Jura français (Bruand). - Parait en avril. 
161. Bombycaria. Bdr. 15036. fig. อ̃.

\section{Dup. eat.}

Ce qu'en dit Bdw. suffit pour la faire distinguer de toutes ses visines. Elle se rapproche extrêmement de $\mathrm{Z}$ onaria. La figure que j'en donne me dispense de toute deseription. - Haut-Valais (Anderegg). - Excessivement rare.

162. Alpinaria. Scrib.

Bork. - Ilub. 178, 513 9. - Treit. - Dup. - Bdv. 1535. -

Her.-Sehf. f. 12, ₹ ; 438. I. 270. - Heydenr. Cat. 196.

Larv. Huh. G. II. Aequiv. A. c. fig. 1. d. e.

Mr. Rothenbacb l'a obtenue des Alpes, où elle est très rare; elle l'est moins dans les alpes d'Italie et de Dalmatie.

16:3. Pilosaria. Bork.

W. V. - Huh. 176, 594 \&. - Treit. - Dup. - Bdv. 1533. -

Her.-Seluf. f. 13, 9. I. 274. - Wood 465. - Heydenr. Cat. 194.

Plumaria. Esp.

Pedaria. Bork. - Fab.

H y emaria. Bork.

Larv. Hub. Geom. II. Aequiv. A. d. fig. 2.

Espece rare et trouvée isolèment dans les bois; près de Lausanue (De la Chaumette); de Berne (Renk.); au Zurichberg (Bremi); à Sehüpfen, le 17 mars (Rothb.). - N'apparait guères en Suisse qu'en wars.

167. Hirtaria. Lin.

W. V. - Hub. 175. - Fuess. n. mag. - Treit. - Dup. Bdv. 1542. - Her.-Schf. I. 275. - Wood 469. - Heydenr. Cat. 190.

Contiguaria. Bork. (copie).

Var. congenerariı. Hub. 174.

- lavaria. Wood $\mathbf{1 6 7 5}$.

Hyemaria ?. Bork. (Treit.) ?

Larv. Hub. G. II. Amplis. A. b. c. fig. 1.

Sur les promenades publiques, dans les jardins, en mars et en avril; pas rare a 
Lausanue certaines annèes (Lah.). - $\mathbf{A}$ Burgdorf, id. (Meyer). - Burghölzli, près Zurich; assez fréquente (Bremi). Près de Schüpfen, le 6 avril; rare (Rothb.)

La var. congeneraria a été recueillie dans les Grisons par M. Mengold.

Mr. Rothenbach possède une var. ? entièrement noire.

16.5. Prodromaria. Fab.

IV. V. - IInb. 172. - Fuess. n. mag. - Treit. - Dup.

Bdv. 1544. - Her.-Schf. I. 276. - Wood 467. - Heydenr. Cat. 188.

M armoraria. Esp. - Fuess. a. mag.

Larv. Hub. G, II. Mequiv. A. a.

Les environs de Lausanne dans le bois et les taillis, assez fréquemment; en mars et en avril (Lah.). - Mèmes localités près de Zurich (Bremi). - Près de Berne; rare; née de chenille le $\mathbf{2 9}$ mars (Rothb.). - Très rare près de Burgdorf; un exemplaire dans la collection Heuser (Meyer).

Se pose sur les troncs des peupliers, tilleuls, bouleaux ete.

166. Betularia. Lin.

IV. V. - Fuess. - Bork. - Hub. 173. - Treit. - Jup. -

Bdv. 1543. - Her.-Schf. I. 277. - Wood 468. - Heydenr. Cat. 187.

Var. ulmaria. Bork.

Larv. Hul.. G. II. Aequiv. A. b.

Cette espèce est assez fréquente dans les environs de Lausamne, autour de la ville; mai et juin (Lah.). - Du 1. mai au 16 juin; assez commune près de Schüpfen (Rothb.); de Burgdorf (Meyer). Prise une fois dans le Gaduenthal, en septembre (Meyer). - Mêmes localitès et tout aussi fréquente près de Zurich Bremi). - Les Grisons Mengold, Kriechbaumer). - Le Valais.

La chenille varie souvent.

G. IXYVI. P'sodos. Treit.

(Pygmaena et Cleogene. Bdv. - Parascolia, Orphna et Schida. Iluh. Vor\%.) Nota. Venetiaria a quelque rapport avec Corticaria. Torvaria se rapproche beaucoup de Gnoph. Zelleraria. Lloridaria et trepidaria rappellent les 
Hercyna, Treit. (Pyralides) par la forme de la còte. Les poils longs des palpes se retrouvent dans la plupart des especes des hautes alpes.

167. Venetiaria. Hub. 329.

Treit. - Dup. - Bdv. 1939. - Her.-Schf. I. 278. lig. 444. Heydenr. Cat. $21 \%$.

Trepidaria. Wood 449.

Cette petite espece vole en essaims au soleil, sur les prairies des hautes alpes, au bord des glaciers, le long de quelque filet d'eau. Zermatten; Enzcindaz etc.; en juillet et en août (Lah.). - Grindelalp (Meyer). - Commune (?) sur les hautes alpes bernoises du 10 au 28 juillet (Rothb.).

On prendrait facilement cette espèce pour une Psyche à son vol, à sa taille et à sa couleur.

168. Torvaria. Hub. $366-69$.

Treit. - Dup. - Bdv. 1414. - Her.-Schf. I. 279. - Heydenr. Cat. 209.

Tenebraria. Esp.

Horridaria ?. Hub. 149.

Trepidaria. Wood 449.

Assez fréquente dans les hautes alpes en juillet et en août; se pose de préférence parmi les pierres. - Zermatten; Enzeindaz; la Grimsel; le St. Gotthard etc. (Lah.). La Gemmi (Rothb.). - Oberhasli, Grindelalp, Hohenstollen (7960 $)$. - Wengernalp etc. (Meyer). - Varie peu. Vole isolée. Préfère le voisinage des glaciers. Je l'ai prise très fraiche le 24 juillet.

169. Horridaria. W. V.

Hub. 312, 590, 591. - Treit. - Dup. - Bdv. 1936. - Her.Schf. I. 280.

Commune sur les hautes alpes. Fréquente les pentes exposées au soleil; à la fin de juillet et au commencement d'août (Lah.). - Commune sur les alpes bernoises, du 10 juillet au 3 août (Rothb.). 
170. Trepidaria. Hub. 3133.

Treit. et sup. - Dup. - Bdv. 1937. - Her.-Schf. I. 281. Heydenr. Cat. 211.

Chaonaria. Frey. B. 36,3. - Bdv. 1938. - Heydenr. Cat. 212.

Commune sur les hautes alpes, parmi les pierres et sur les pentes exposées au suleil, à la fiu de juillet et au commencement d'aoùt (Lah.). - Du 10 au 28 juillet (Rothb.).

Le reflet des ailes varie. Les exemplaires un peu usés sont noirs ou grisâtres. Les individus très frais ont un reflet tantot brun, tantôt noir luisant. Les ailes sont cbez les uns semées d'atômes brun-jaunâtres, chez d'autres d'écailles blanchâtres. Ces derniers unt fourni a Freyer sa $c h a o n a r i a$, qui n'est pas même une variétè. Le dessous varie encore plus que le dessus pour la netteté des ombres et le luisant des intervalles. Les espices sablécs de gris portent un pinceau de poils blanchàtres sur la tête, celles qui le sont de brum-clair en ont un de même couleur et à peine distinct.

G. XXXVII. Torula. Bdv.

(Baptria. Hub. Verz. - Psychophora. Kïb. Curt.)

Nota. Ne peut être réunie à chaerophyllaria.

171. Equestraria. Esp.

Götze. - Bork. - Bdv. 1935. - Her.-Schl1. I. 282. - Dup. cat. - Heydenr. Cat. 203.

Alpinata. W. V. - Hub. 197. - Treit. - Dup. hist. - Wood 450. Quadrifaria Götze.

Commune sur les prairies des alpes moyennes dès Je milieu de juin, en juillet (Lah.). - Du 10 au 25 juillet (Rothb.). -

Le papillon frais a une couleur d'un brun-noir qui devient plus claire par l'effet du soleil. Les individus du Riesengebirge ont les bandes plus larges que ceux de nos alpes (Neyer . 


\section{B. Dendrometrides. Hub. Revis.}

G. I. Inisopteryx. Steph. Bdv.

(Ilibernia. Dup. Curt. - Fidonia. Treit. - Alsophilla. Deutsche Ins.)

Nota. La nervation de la cellule se rapproche de celle des Larentia.

172. Aescularia. W. V.

Hub. 189 3. - Treit. - Dup. - Bdv. 1524. - Her.-Scht. II. 1. - Wood 460. - Heydenr. Cat. 293.

Murinaria. Bork. - Esp.

Capracolaria. Wood?

Partout assez rare en Suisse. Environs de Lausanne, en novembre, et plus souvent en mars (Lah.). - Prés de Schüpfen, du 11 mars an 21 avril Rothb.). - Prise une fois a Burgdorf par Mr. Heuser (Meyer). - Elle parait plus frequente près de Zurich (Bremi).

Se prend de préférence dans les jardins el près des habitations; vole le soir lorsyu'il fait humide et doux.

Barbes des antennes fortement pénicillées.

173. Aceraria. W. Y.

Hub. 185, o ; 514, 9. - Treit. - Dup. - Bdv. 1526. - Her.Schf. 348, 349. II. 2. - Heydenr. Cat. 254.

Quadripunctaria. Esp.

La rv. Hub. Geom. Il. Aequiv. B. a. b. fig. 2.

Tres rare en Suisse. Prise une fois en novembre, dans le bois de Sauvabeliu, audessus de Lausanne, par Mr. De la Chaumetle (Lah.) - Le 27 novembre a Schüpfen Rothb.). - Observèe dans les bois du Zurichberg, par Mr. Vögeli (Bremi) et près de Zitzers, dans les Grisons, te 19 fèvrier 1846, par Mr. Kíriechlsaunıer.

\section{G. II. Lythria. Hub. Verz.}

(Aspilates. Treit. Bdv. Curt.)

Nota. La cellule courte, conpée ohliquement, bien en-decá de la bifurcation de la 
nervure nédiane-externe rappelle plusieurs especes du G. Acidalia. Ce genre, ainsi que les deux suivants, appartiennent plutôt aux Phytometrides.

174. Purpuraria. Lin.

W. V. - IIul. 198, 199. - Wood 64.? - Treit. et sup.

Dup. - Bdv. 1481. - Frey. n. B. 60,1. - Her.-Schf. II. 3. Sordidaria. Zetterst.

La rv. Hub. G. Aequiv. C b. fig. 1.

Les collines arides du Valais, en juillet et août (Lah.). - Les mèmes localités dans les enviruns de Zurich (Bremi). -

Cette espèce, commune dans presque toute l'Europe, est rare en Suisse. Ses variètés sont nombreuses; Her.-Schf. les range sous 5 chefs, suivant la disposition des haudes.

Mr. Th. Bruand l'a prise quelquelois au printemps et admet deux génèrations par an; cette explication me parait plus plausible que celle de Her.-Sebf. qui croit à une hiveruation. Je doute que celle-ci existe pour aucune géomêtre (comp. Treit. sup. II. p. 179.

17.). Rheticaria. nob.

Her.-Schf. II. 4. (non Bdv. 1940.).

Plumularia. Fr. n. B. 36,3 .

Je ne counais que le dessin de Freyer. Her.-Schf. croit que cette espèce, originaire des Grisons, est l'une des nombreuses varietes de Purpuraria. - Mr. Meyer, qui la possède, l'envisage comme bonne espèce. En la conservant, on doit changer son non pour éviter de la confondre avec le Nr. 1940 de Boisdv.

\section{G. III. Minoa. Curt. Stph. Treit. Bdv.}

Nota. La cellule d'Euphorbiaria a le plus grand rapport avec celle des Acidalia du premier groupe, celle de Tibialaria est construite comme dans Chaerophyllaria, dont on ne peut la sẻparer. L'accolement de la nervure seapulaire à la médiane voisine sur une ètendue plus ou moins longue, n'est point un caractère de première inportance; car il varie dans le méme genre, comme tant d'autres. 
176. Euphorbiaria (ta). W. V.

Hub. 78. - Wood 687. - Treit. - Dup. - Bdv. 1941. Her.-Schf. 11. 10. - Heydenr. l'at. 617.

Unicolorata. Hub. Beitr.

Murinata, Vill.

Var. griseata. Schrk. - Mann, in litt.

Var. monochroaria. Her.-Schf. fig. 391. - Heydenr. Cal. 616.

Commune une première fois en mai et en juin, puis une seconde en aoùt. S'èlève sur les alpes et s'y montre en juin et en juillet (Lah.). - N'a pas été trouvée dans le centre du canton de Berne; elle préfère le Jura (Meyer). - Près d'Aarberg du 24 au 27 mai, puis du 10 juillet au 18 aoút Rothb.). - Commune dans les environs de Zurich (Bremi).

Cette espèce disparait certaines annèes: tantôt elle est plus abondante en juin, tantốt en aoùt. - La taille varie souvent; les màles sont voluntiers plus grands que les lemelles.

Ia teinte gẻnérale varie beancoup aussi; elle passe du gris-noiràtre au blanc-sale et du roux-clair au rouge-brique. J'ai observè toutes les nuances intermédiaires. La var. rouge-brique (monochroaria) se prend dans les taillis secs au-dessus de Lausanue; les individus reçus de Vienne sont identiqnes. La var. blanche ou gris-claire est propre aux alpes. J'ai vu cette dernière, près de Bellinzone, offrir deux légères zônes aux inférieures; je ne les ai pas observeees dans d'autres localités. Un grand individu ô, pris sur le Simplon, avait les inférieures plus blanches que les supérieures. - Sur les individus rouges la racine des ailes en-dessous est ronge-brun; chez les gris-pâles elle est noirâtre. - Du reste, malgré les recherches les plus ninutieuses, je n’ai pu dícouvrir deux ou trois espèces diffẻrentes dans Euphorbiaria. Celle que javais d'abord admise sous le nom d'amylaria n'est que la variété pâle (voir Bulletin de la Soc vaud. des se. nat. T. 3. Nr. 22).

177. Tibialaria (la). Hub. 210, 2 ii.

Dup. - Treit. - Bdv. 1934. - Her.-Scht. II. 11. - Heydenr. Cat. 204.

Dimidiata. Hub. Beitr.

Eversmannaria? Her.-Schf. (H. S.) fig. 443.

Je n'ai vu qu'un seul individu femelle de cette espèce, pris par un jeune amateur 
dans la vallée d'Ormonds-dessus, près de l'èglise, vers le milieu de juillet 1851. L'extrémité postérieure de la baude blanche n'est point terminèe en pointe alongée, comme dans la lig. de Hub.; peut-être cela tient-il au sexe? Les inférieures ont la tache blanclıe bien marquée et en forue l'are en-dessus; mais plus encore en-dessous: du côté interne cette dernière tache est dentelée et parait limiter un espace basilaire.

Eversmannaria II. S. f. 443 reproduit encore mieux l'individu que je possède que les fig. de Hub.

Tibialaria se rencontre quelquefois, mais très rarement, dans les alpes d'Autriche: elle est beancoup plus fréquente en Gallicic Mann)

\section{G. IV. Hydrellia. Hub. Verz.}

(Acidalia. Bdv. - Emmelesia. Steph. Curt.)

Nola. Le G. Emmelesia dans Curtis renferme plusieurs Acidalia. Nervation de la cellule comme dans les deux genres précédents; mêmes observations.

178. Candidaria (la). W. V.

$$
\text { Hub. 101. - Treit. -- Dup. - Bdv. 1885. - Her.-Schf. II. } 12 .
$$$$
\text { - Wood 706. - Heydenr. Cat. } 353 .
$$

I mmutata. F.

Albulata. Götze.

Var.? bassiaria. Feisth. Ann. Soc. entom. - Pdv. 1672, var.

Commune dans les laaies, les taillis et sur les bords des bois, en mai et en juin; repraraît à la fin de juillet et en aoùt. Je l'ai prise le 4 avril et à la fin d'août près de Lausaune (Lah.). - Les collines couvertes de balliers aux environs de Zurich; commune (Bremi). - A Schüpfen, du 17 mai au 14 juin; puis du 23 juillet au 12 aoùt; fréquente (Rothb.). - En graude abondance du 25 au 30 mai, le long du Sommerhaus* wald, près Burgdorf, dans les liaies de coudrier (Meyer).

Varie pour la disposition des raies; celles-ci circonscrivent quelquefois une sorte de bande médiane.

179. Sylvaria. W. V.

Hub. 231. - Treit. -- Dup. -- Bdv. 1884. - Her.-Srlıf. II. 13.

- Wood. 231. - Heydenr. Cat. 356.

Larv. Hub. G. II. Aequir. G. b.

Rare; dans les bois de sapin du Jorat, au-dessus de Lausanne, des la mi-juin au 
commencemeut de juillet (hah.). - Près d'Aarberg, le 27 juin; rare Rothb.). - Sommerlauswald, près Burgdorf (Meyer). - Meyringen (Ott). -

IJubner a placé la chenille sur l'aune (alnus viridis) et non sur le betre comme l'a cru Treitschke. Le papillon ne se prend guères ailleurs que dans les hois de sapin humides où croissent des aunes.

180. Lutearia. F.

W. V. - Hub. '103. - Treit. - Dup. - Bdv. 1881. - Fr. n.

13. 83, 3. - Her.-Sehf. II. 14. - Wood. 707. - Heydenr.

Cat. 351.

Centrata. F.

Les bois de bouleau et d'aune; Saurabelin, près de Lausanne; à la fin de wai et au commencement de juin; pas frèquente (Lah.). - Sur l'Uto, près de Zurieh (Bremi). - Les taillis d'aune près de Schüpfen; pas rare, du 24 mai an 23 juin (Rothl).).

181. Hepararia. W. Y.

Hnb. 58. - Treit. el sup. - Dup. - Bdv. 15:0. - Her.-Sch1.

II. 15. - Wood 708 .

Strigata. Thumb.

Obliteraria. Hufn. - Jeydenr. Cat. 221

Larv. Hub. G. I. Amplis. K. b.

Commune d'abord en mai (ct en juin; puis en juillet et en août, dans les taillis et les haies le long des chemins, aux environs de Lausanne (Lah.). - Mêmes lucalités et fréquente du 4 mai au 29 juin, puis dn 1 juillet au 2 aoùt, près de Schüpfen (Rothb.). - Le long de l'Emme; dans les bois d'anne; tout l'élé (Meyer). - Au Katzensee; sur le Balgrist; près de Zurich; pas commune (Bremil. - Rare dans le Doubs (Bruand). Le papillon se pose avec les ailes èlevees, comme les Diurnes.

182. Erutaria. Bdv. 1624.

Dup. sup. (fig. mala). - Her.-Sclif. 258, 259. II. 16. - Heydenr. Cat. 357 .

Nebulosaria. Fr. 13. B. C. 88. Tab. 528. fig. 5. et C. 91. Tab. 546. lig. 2, 3.

Cette espèce, très rare, a été prise dans les forêts de sapin du Gournigel, par Mr. Meyer, le 10 juillet 1848. 
Se rapproche de $\$$ ylvaria et rapelle aussi Vespertaria et Bupestraria.

Les individus du Gonrnigel ont le fond plus blanchàtre que celui que Mr. Standfuss m'a envoyé du Riesengebirge et que Frcyer a figuré. Dn reste ils sont complétement semblables (Meyer.

G. V. Eupithecia. Curt. Steph. Bdv.

(Larentiae. Treit.)

Nota. La nervure scapulaire s'accole à la médiane jusque vers l'angle antérieur de la cellule; celle-ci est conrte et coupée obliquement, mais carrément: l'anastomose de cloture tombe plus ou moins pres du point où la nervure scapulaire se détache ensorte qu'elle se tronve plus on moins èloignéc de la bifureation de la mediane externe. Dans Togaria la cellule est plns alongèe et cette bifurcation se place près de ia cellule. Ce genre est très compacte tet les subdivisions y sont fort arbitraires et difficiles.

18:3. Coronaria Hub. 372, 373.

Fr. n. B. 306,2 . - Dup. sup. - Her.-Schf. II. i8. - Heydenr. Cat. 45 !.

V - ata. Wood 655 . corticulata ef undata. Fr. u. B. 323.

Var. rectangulariae. Bdv. 1;24.

Les environs de Lausanne; rare, en mai. Une fois à la fin l'avril, au bois de Sauvabelin, appliquèe contre un tronc (G. Leresche). Dans la collection de Mr. De li Chaumette (Lah..). - Près de Schüpfen; fort rare (Rothb.).

184. Centaurearia. F.

$$
\text { Bork. - W. v. - Hub. 240, 452. - Dup. - Bdv. } 1694 .
$$

Wood 679. - Fr. B. 6, 2. - Her.-Schf. II. 20. - Heydenr. Cal. 453.

Larv. Hub. Geom. II. Aeqquiv. H. b. lig. 2.

Rarement autour de Lausanne, d'abord à la lin de mai, puis une seconde fois à la fin de juillet. Le long des haies et sur les collines sèches (Lah.). - Rare: près de Bienne, le 30 mai (Rothb.). - Le Zuricbberg; octobre; rare (Bremi). - Près de Rorschach, assez fréquente (Isenring). - Très rare autour de Burgdorf. Un seul individu a èté trouvé le 5 mai 1850 (Meyer). 
185. Lin aria. Bdv. 1720.

Dup. catal.

Lin ariaria. Her.-Sehf. II. 21. (non Bork.). - Heydenr. Cat. 454.

Linariata. F. - W. V. - Hub. 242. - Treit. - Dup. - Wood 649. Pulchellata. Wood 650 .

Mr. Bruand l'a prise dans le Doubs et Mr. Rothenbach croit l'avoir reconnue pres de Scbüpfen en juin. Mr. Heuser l'a prise une fois près de Burgdorf. Partout très rare. Linariaria. Bork. vide ferrugaria.

186. Hospitaria (ta). Treit. et sup.

Dup. cat. - Bdv. 1701. - Fr. n. B. 119, 2. - Her.-Schf. II. 22. - Ileydenr. Cat. 455.

Succenturiata. Hub. 236. -

Subfasciata. Wood 665 .

Espece très rare, trouvèe près de Schüplen, du 20 arril au 12 mai, par Mr. Rothenbach. - Jamais en octobre (Treit.).

187. Venosaria (ta). F.

Bork. - Hub. 244. - Treit. - Dup. - Bdv. 1703. - Fr. n.

B. 204,1 . - Her.-Schf. II. 23. - Wood 683. - Heyd. Cat. 456.

Jnsignata. Hub. Beitr. I. B.

Larv. Ilub. G. II. Aequiv. H. b. (2 planches).

Au Schönbühl près de Burgdorf. Chenille dans les capsules de Cucubalus behen; commune (Meyer). - Languau; très rare (Rothb.). - Le Burghölzli, près de Zurich; élevée de chenille (Zeller).

188. Consignaria (ta). Bork.

Hub. 245. - Treit. - Dup. - Bdv. 1702. - Fr. n. B. 204, 2.

- Her.-Schf. II. 24. - Wood 682. - Heydenr. Cat. 457.

Insigniata. Hub. Beitr. II. B.

Près de Dubendorf, canton de Zurich (Bremi). 
189. Sobrinaria (ta). Hub. 465.

Treit. et sup. - Dup. - Bdv. 1736. - Fr. n. B. 90. - Her.-

Schf. 1I. 25. - Heydenr. Cat. 466.

Laevigata, Abbreviata et Exiguata. Wood. 658, 659 et 660.

Schüpfen, du 26 juillet au 13 septembre: rare (Rothb.). - Près de Coire, le 18 decembre (Prf. Zimmer). - Quelquefois en juillet au-dessus de Lausanne, dans les bois ctairs Lah.). - Commune prés de Burgdorf (Meyer).

Espèce tardive et non du mois de juin, comme le dit Bdv. - Cheuille sur geniévrier (Meyer).

190. Succenturiaria (ta). Lin.

Hub. 459. - W. V. - Treit. - Dup. - Bdv. 1693. - Her-

Schf. 11. 28. - Wood 677. - Heydenr. Cat. 461.

Var. oxydata. Treit. - (ria) Bdv. 1692. - Dup. cat.

Disparata. Ilub. $246,247$.

Ferruginata? Dup. sup.

Subfulvata? et eognata. Wood 676.

Larv. ? Hub. Geom. II. Aequiv. H. b. c. fig. 22.

Press de Sebüpfen; fort rare (Rothb.).

Cette espèce fournit à Her.-Sehf. 3 variètés distinctes: une brune (Hub. 247); une grise marquee de rouille, et une grise sans stries rouillees (Hub. 459, 246). - Fischer v. R. arait déjà réuni oxydata, Treit. à suceenturiata. - Varie beaueoup.

191. Lariciaria (ta). Fr. n. B. IV. pag. 135. Tab. 366 .

Her.-Schf. II. 29. f. 170-174. - Heydenr. Cal. 465.

Scopariata, Ericeata et Ligustieata. Feisth. ann. cutom, de Fr.

Rare. Le long des forêts de sapin, autour de Burgdorf, au mois d'avril. Les exemplaires de Mr. Meyer sont parfaitement semblables à ceux reẹus de Mr. Freyer. Un autre, dans la mème collection, provenaut des alpes de Styrie (Mann), a la teinte plus foncée et les bandes plus marquees.

Cette espèce ressemble tellement à silenata Standf. qu'on pourrait la regarder comme telle, si les ehenilles u'ètaient très différentes. Celle de silenata se trouve au mois de juillet dans les eapsules de Cueubalus behen., et eelle de lariciata au 
mois de septembre sur le melèze. L'apparition des papillons est la même (mars et avril) (Meyer). - Près de Lausanne, une fois, à la même époque (Lah.).

192. Modicaria (ta). Hub. 361 .

Dup. cat. - Bdv. 1678. - Iler.-Schf. f. 178. II. 30. - Heydenr. Cat. 463.

Var. impurata. Hnb. 347. - Bdv. 1679. - Dup.?

Minorata. Her.-Schf. synops.

Cauchyata? Dup. - Bdv. 1683.

Assez fréquente autour de Guttannen dans la vallèc d'Oberlasli (auùt): paraî avoil deux apparitionș (Meyer). - Je l'ai recue du Haut-Valais et de Meyringen, et l'ai prise une fois a Lausanne à la fin de juin (Lah.). - Les echantillons que j'ai rus, provenant de l'Oberhasli, se rapportaient à impurata Hub. L'individu pris à Lausanne se rapprochait plutôt de la fig. 361; mais l'une et l'autre (surtout la dernière) sont très mal figurées dans Hub. De nouvelles figures sont à désirer.

19:3. Innotaria (ta). Knoch.

Bork. - Hub. 441, 442 . - W. V. - Treit. - Dup. - Bds. 1699. - Her.-Schf. f. 274 (var.) II. 32. - Heydenr. Car. 472. Tamarisciata. Fr. n. B. 192, 1 (non Mann, eat.). - Bdv. 1712. - Dup. cat.

Rare en Suisse. A Schüpfen du 14 juiı an 3 aoùt (Rothb.). - Environs de Burgdorf: dans les jardins (Meyer).

La chenille vit sur l'absynthe (artemisia) (Mann).

194. Tenuiaria (ta). Hub. 394.

F. v. R. - Her.-Sehf. fig. 168,169 . II. 34. - Heydenr. Cat. 472 . Valerianariae var. Buv. 1710.

Subumbrata? Wood.

Une fois près de Lausanne en juillet (Lah.): - Près de Dübendorf (Bremi).

19.). Veratraria. F. v. R.

- Her.-Schf. f. 152. II. 36. - Heydenr. Cat. 476.

Satyraria (ta). Her.-Schf. fig. 147, 148 (non descrip.). - Deuts. insec. A us terata. Evers.

Cette espèce des Alpes a èté recueillie près de Dübendorf par Mr. Bremi. Sa che- 
nille vit daus les capsules du Veratrum album. Très voisine de Satyraria Huh. el probablement souvent confondue avec elle.

196. Satyraria (ta). Huh. 4399.

Treit. - Dup. cal. - Bdv. 1689. - Fr, n. B. 294, 1. - Her.Sclıf. f. 149-51 et 448 (var.) 11. 37. - Heydenr. Cat. 477. Castigata. Her.-Schf. d. ins.

Près de Schüpfen le 17 août; rare (Rothb.). - - Près de Dühendorf (Bremi; - - Commune dans l'Oberhasli, 26-29 juin (Meyer).

197. Helveticaria. Anderegg.

Bdv. 1687. - Dup. sup. - Her.-Schf. 130-33. H. 38. - Heyd. Cat. 479. Caliginata? Treit. - Dup. sup. - Bdv. 1705.

Cette espèce n’a èté jusqu'ici trouvée qu'en Valais, par Mr. Anderegg; sa chenille vil sur la Sabine et le papillon naît en octobre.

198. Arceutharia. Fr. n. B. $27: 3$.

$$
\text { Her.-Schf. f. 134-37. II. 39. - Heydenr. Cat. } 480 \text {. }
$$

Cette espece unit Helveticaria à Satyraria. - Prise le 1. juillet sur les Iuontagues au-dessus de Montreus, et vers le milieu de juin dans les bois de sapin au-dessus d'Yvorne Lah.). - La vallec d Oberhasli, parmi les rochers; en juin (Mejer.

199. A bsynthiaria (ta). Lin.

Ilub. 453. - Wood. 669. - Her.-Schf. II. 41. - Heyd. Cat. 483. Minutata. Huls. 454. - W. V. - Treit. - Dup. - Bdv. 1718 ria. - (non Fab.) - Evers.

Isuotata, Notata, Elongata et Subfulrata. Wood. 668, 670, 671 et 675 .

Schüpfen, du 11 mai an 20 juin; pas rare (Rothb.). - Pres de Zurich Bremi. Environs de Lausanne; pas lrès rare; sous ses deux formes (Lah. 
200. Trisignaria. Her.-Schf. f. 175, 176. Il. 41.

Heydenr. Cat. 485.

Cette espèce a élé trouvéc près de Zurich sur le Hottingerberg par Mr. Bremi, el èlevèe de chenille; cellc-ci vit en sociétè sur l'Heracleum sphondilium. J'en possède un exemplaire femelle, pris aux cnvirons de Lausanne, cn juillet; celui-ci est plus gros que ceux de Zurich, probablement parce qu'il n’avait pas été élevé en captivité.

Très voisine de Castigaria; elle s'en distingue et par ses trois points vers la côte et par la ligne ondulée de la marge, beaucoup moins distincte, surtout en-dessous..

201. Scriptaria. Her--Schf. fig. 123. II. 45.

Heydenr. Cat. 486.

Sur le Simplon, près des neiges, le long de la route; le 20 juillet, assez effaréc Lab.).

202. Singularia. Her.-Schf. f. 141, 142. II. 46.

Heydenr. Cat. 484.

Begrandaria? Dup. sup. (non Bdv.)

J'en possède deux excmplaires, pris en juillet, dans les haies des environs de Lausanne (Lah.).

Voisine de Pygmaearia et surtout de Begrandaria Dup. avec laquelle je l'ai confonduc au premier moment.

2083. Castigaria (ta). Hub. 456 .

Bdv. 1715. - Fr. n. B. 294, 2. - Dup. sup, - Her.-Sehf. fig. 164-67. II. 48. - Heydenr. Cat. 475.

Minutata. Hub. 237.

Austerata. Hub. 457? (non Treit.)

'impinellata. Her.-Schf. synops. - Fr. n. B. 300, 2. - Zeller (ins.).

Dimidiala. Her.-Scbf. synops.

Residuata, Evers.

Albipunctata et Nebulata. Wood 663,662 .

Impurata? Dup. sup.

Rarc en Snisse. Près de Schüpfen, du 4 mai au 10 juin (Rothb.). - Près de Constance (Leiner), - de Burgdorf et de Meyringen (Meyer). - Le Zurichberğ (Prf. Frei). 
Cette espèce est l'une de celles que l'on confond le plus. Fisch. v. R. Ia considère comme identique avec la pimpinellata de Treit. - Celui-ci en fait une variété d’austerata. Dans la collection de Hub. l’idendité des deux especes est, dit-on, évidente, cependant Fisch. v. R. persiste à croire que les deux fig. de Hub. $(456,457)$ appartiennent à deux espèces différentes.

\section{Valerianaria (ta). Hub. 395 .}

Treit. - Dup. - Bdv. 1710. - Her.-Schf. II. 51. - Heydenr. Cat. 497.

Larv. Hub. Geom. II. Aequiv. H. b. fig. 1.

Parait commune près de Zurich, sur les prairies humides? (Bremi). - Indiquée comme douteuse près de Schüpfen, par Mr. Rothenbacb. - $\mathbf{\Lambda u}$ bas des rochers, près du Reichenbach, Meyringen (Meyer).

205. Is ogrammaria (ta). Treit.

Bdv. 1728. - F. v. R. - Her.-Schf. fig. 188. II. 53. -- Heydenr. Cat. 496.

J'en possède un exemplaire pris dans les environs de Lausanne, en juillet, sur une colline aride (Lab.). - Très voisine de Begrandaria.9F. v. R. (C. 6.) la regardait comme une varieté de Valerianata.

206. Pusillaria (ta). W. V.

$$
\begin{aligned}
& \text { Bork. - Hub. } 378 .- \text { Treit. - Dup. - Bdv. 1708. Fr. I. } \\
& \text { B. } 119,3 \text { ? - Her.-Schf. f. 158, 159. II. 57. - Heyd. Cat. } 500 .
\end{aligned}
$$

Subumbrata. Mub. 233. - W. V. - Treit. - Dup. cat. - Bdv. 1707.

L a rv. Hub. G. II. Aequiv. H. a. b. fig. 1.

La plus commune du genre. Sur les sapins, en mai el en juin (Lab.). - I Sctrupfen du 24 avril au 14 juin (Rothb.). -

La forme subumbrata est assez rare. - Les indications diverses des auteurs au sujet des plantes qui servent d'aliment à la chenille, sont à vèrifier. II est certain que le papillon ne se trouve jamais que sur les pins et les sapins, ou dans leur voisinage. 
207. Manniaria. F. v. R.

Her.-Schf. fig. 156. Il. 58. - Heydenr. Cat. 501.

J'ai vu un individu, trouvé sur le Zurichberg par Mr. le Prf. Frei.

208. Inturbaria (ta). Hub. 461 et coll.

Treit. et sup. - Bdv. 1730. - Dup. - Her.-Sehf. II. 60. Heydenr. Cat. 502.

Neglecta. Schf. synops. Eupithec.

Trouvée en certain nombre près de Burgdorf, te 25 juillet, par Mir. Meyer.

209. Exiguaria (ta). Hub. 379.

Treit. - Dup. - Bdv. 1697. - Fr. n. B. 119, 1. - Her.-Schf. II. 59. - Heydenr. Cat. 504.

Singulariata. Wood 661 .

Larv. Hub. G. Il. Aequiv. H. a. b. fig. 2.

Près de Schüpfen, du 24 mai au 7 juin; espèce rare (Rotbb.). - Sur l'Uto, près de Zurich (Bremi). - Pas très rare dans les haies des environs de Lausanne, en juin et en juillet (Lab.).

210. Grapharia (ta). Kind.

Treit. - Bdv 1682. - Her.-Schf. fig. $179,180,189$. H. 62 . Dup. cat. - Heydenr. Cat. 512.

Trouvée dans le Haut-Valais par Mr. Anderegg. - Ressemble beaucoup à modicaria, avec laquelle il est facile de la confondre.

211. Rectangularia (ta). Lin.

F. - Bork. - W. V. - Hub. 235. - Treit. - Dup. - Bdv.

1724. - Her.-Schf. fig. 275, 276. H. 64. - Wood 651. Heydenr. Cat. 509.

Cydoniata. Bork. - Treit. - Bdv. 1729. - Dup. cat.

Subaerata. Hub. 463. - Wood 652. - Bdv. 1725.

Nanata. Treit. (non Ilub.)

Nigrosericeata? Wood. 654 .

Espèce assez fréquente aux environs de Lausanne dans les jardins et les vergers; 
en juin et en juillet (Lah.). - Commune à Schüpfen du 3 juin au 14 juillet (Rothb.). De même aux environs de Zurich (Bremi). - En grande quantité dans les environs de Burgdorf, contre les planches et les murs des jardins (Meyer).

212. Debiliaria (1a). Hub. $462,466$.

Treit. et sup. - Bdv. 1726. - Dup. cat. - Her.-Schf. f. 277.

II. 65. - Heydenr. Cat. 510.

Nigropunctata. Wood 653 .

Près de Constance (Leiner). - Environs de Burgdorf, en juillet; très rare; babite les mêmes localités que rectangularia (Meyer).

213. Pimpinellaria (la). Hub. 443, 444.

Treit. - Dup, - Bdv. 1688. - Fr. B. 309. - Her.-Schf. fig. 153. II. 66. - Heydenr. Cat. 473.

Denotata. Hub. 455 et coll. - Bdv. 1719. - Dup.?

Larv. Hub. Geom. II. Aequiv. H. b. c. fig. 1.

Près de Meyringen et de Burgdorf (Meyer). - Environs de Zurich (Bremi). Une fois près de Iaausanne à la fin d'avril, dans un bosquet (Lah.). - Très rare.

214. Strobilaria (ta). Bork.

Degeer. - Hub. 449, 450. - Treit. - Dup. - Bdv. 1723. Her.-Schf. II. 68. - Heydenr. Cat. 513.

Residuata. Hub. 467. - Treit.? - Dup. cat. - Bdv. 1690.

Subumbrata. Treil. (partim). - (non Hub.)

Rufifasciata? Wood 657 .

A bietaria. Götze.

Assez fréquente près de Schüpfen, du 15 mai au 9 juillet (Rothb.). - Sur le Balgrist et l'Uto (Zeller). - Bois de bêtre et de sapin près de Burgdorf, à la fin de mai (Meyer). - De méme près de Lausanne (Lah.).

215. Togaria (1a). Hub. 461.

Dup, cat. - Bdv. 1722. - Her.-Schf. II. 69. - Ileydenr. Cat. $51 /$.

Jai reçu cette phalène de Mr. Rothenbach qui l'avait prise dans les environs de Schüpfen; je l'ai recucillie aussi en juin, près de Lausanne (Lah.).

Ne peut être réunic à strobilaria. 


\section{Pumilaria. Hub. 388.}

Bdv. 1713. - Dup. sup.? - Her.-Schf. f. 127. II. 72. - Heyd. Cat. 506.

Improbata. Treit. in litt. - Zell. Isis.

Sirobilata. Wood 656.

Un individu près de Lausanne au milieu d'avril. Il ne diffère de ceux de Mann, provenant de l'Italie, que par des couleurs un peu moins vives et un fond plus pâle (Lah.).

\section{G. VI. L a rentia. Her,-Schf.}

(Eubolia, Cidaria, Larentia, Anaitis. Bdv. Dup. - Clresias et Acidalia. Treit. Zeryntluia, Zérene, Electra, Ypsipetes, Larissa, Lozogramma, Aplocera, Emmelesia, Steph. et Curt.)

Nota. Ce genre est plus qu'aucun autre la pierre d'achoppement des entomologistes. Quelque distribution que l'on tente, on lıeurte à des difficultés; il ı'y a pas nn caractère qui, pris isolèment, ne froisse des analogies évidentes. Le facies est des plus trompeur et dilficile à saisir. La nervation de la cellule des inférieures offre de bons caractères; mais ils ne sont pas partout tranches; ils doivent ètre pris sur un seul sexe; j'ai prélèrè la femelle qui exprime plus nettement ici le caractère spécifique. La nervation fournit les coupes suivantes:

a) Cellule très inégalement divisée par sa nervure moyeıne, terminée du côté interne par une anse portant quatre nervures marginales disposées en éventail. La nervure cellulaire mojenne ne continue pas au-delà du noeud (point discoïdal) et sa remplaçante part de l'anse de clôture. L'anastomose s'unit à la médiane externe très en arrière de la bifurcation, près du poínt de séparation de la scapulaire. Les espèces se groupent comme suit:

1. A cidalia
badiaria
dubitaria
montivagaria
sabaudiaria
dilutaria
certaria.

rbamnaria
vetularia
undularia
2. Larentia $\mathrm{Tr}$.
turbaria
kollariaria
nobiliaria

caesiaria flavicinctaria tophacearia cyanaria

3. Cidaria Tr. vallesiaria aquearia 


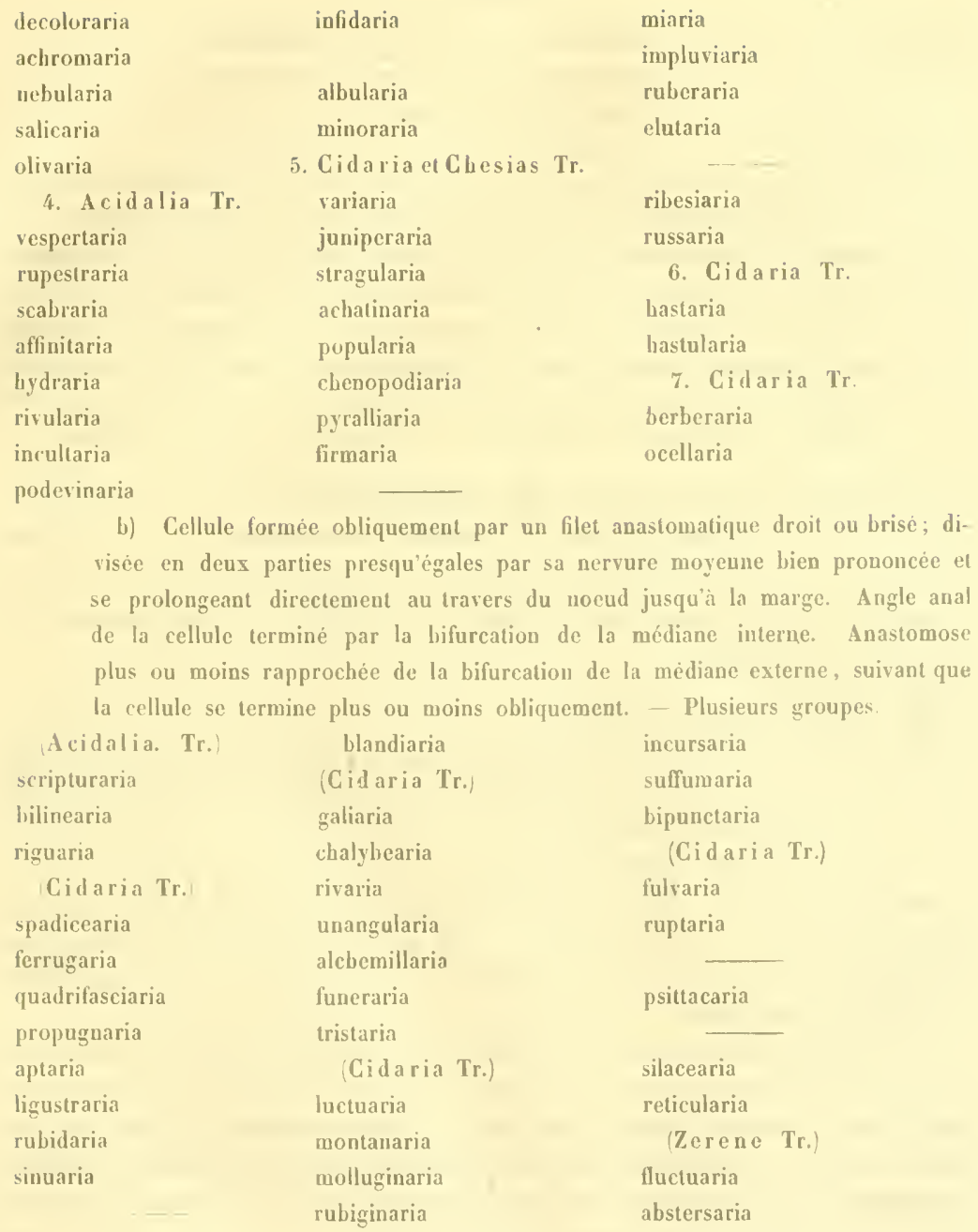




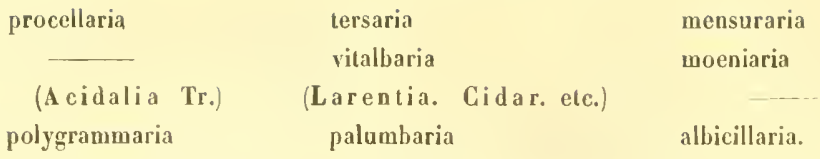

c) Cellule courte, coupée carrément au niveau de la séparation de la nervure scapulaire et très en arrière de la bifurcation de la médiane externe; terminée à son angle anal par une pointe effilée portant la bifurcation de la médiane interne. - 2 espèces. - A cidalia Tr. - Fluviaria et gemmaria.

d) Cellule très large, coupée carrẻment entre la bifurcation et la séparation de la scapulaire, portant à son eôté anal 3 nervures écartées, dont la plus interne est fort courte. Nervure anale enveloppée dans le bord interne de l'aile, invisible. 3 espèces. - Anaïtis Bdv. - Plagiaria, cassiaria, sororaria.

217. Sparsaria (ta). Hub. 398. (non 326, 327).

Treit. - Dup. - Bdv. 1685. - Her.-Seh. II. 74. - Heydear. Cat. 517.

Du 10 juin au 23 juillet, près de Schüpfen; rare (Rothb,). - Près de Burgdorf et dans le Haut-Valais (Meyer).

218. Dubitaria (ta). Lin.

IV. V. - Hub. 265. - Dup. - Bdv. 1637. - Fr. n. B. 402, 426. - Her.-Schf. II. 75. - Wood 622. - Heyd. Cat. 411. Cinerata? Wood 623.

Larv. Hub. G. II. Aequiv. K. b. f. 1. a. b.

Paraît deux fois l'an, au mois d'avril et de mai, puis à la fin de juillet et en aoùt. Toujours cachée sous les gazons creux, le long des chemins (Lah.) - De même près de Schüpfen, le $\mathbf{5}$ avril et le $\mathbf{2 5}$ août (Rothb.). - S'introduit dans les maisons (Bremi).

- Les Grisons (Mengold).

Mr. Bruand pense que Dubitaria passe l'hiver dans les cavernes et qu'elle en sort au printemps. Je ne puis partager cette opinion. - Je ne l'ai jamais trouvée avant l'hiver, ni au premier printemps, dans les lieux où je la prends en abondauce, soit en arril, soit en août, quoique j'y aie pris plusieurs autres papillons tardifs ou printaniers. D'ailleurs, au printemps elle ne parait que 3 ou 4 semaines après les autres hivernès. Je doute fort enfin qu'aucune phalène puisse passer l'hiver sans périr. 
Je possède une variété plus petite et foncée dont le dessin des inférieures a presque disparu sous une teinte noirâtre uniforme; à peine aperçoit-on le point blanc de l'angle anal des supérieures. Serait-ce la einerata, Wood?

219. Montivagaria. Bdv. 1639 .

Dop. - Her.-Schf. f, 203, 204. 1I. 76. - Heydenr. Cat. 414.

J'ai pris cette espèce dans tne haie de sapin, au-dessus de Zermatten (Valais) sur le chemin de Tzmutt, dans le milicu de juillet 1847. Mr. Zeller l'a trouvée dans la vallèe de Conches (Valais).

Elle se eache dans les taillis sombres. Son vol est rapide, semblable à celui de dubitaria.

220. Certaria (ta). Hub. 573.

Hub. Verz. - Treit. - Dup. - Bdv. 1638. - Fr. 396. - Her.Sebf. II. 77. - Heydenr. Cat. 413.

Cervinata. Hub. 266. - Wood 621.

Larv. Hub. G. II. Aequiv. K. a. b. fig. 1.

Les collines rocailleuses et les bords des chemins secs, près de Lausanne (Betusy), de Chexbres, une première fois en avril et une seconde fois en juillet, mais non dans les mèmes localités. En juin dans les sous-alpes. Assez rare (Lab.). - Très rare près de Langnau Rotbb.). - De mème près de Burgdorf (Mleyer). - Les taillis de l'Uto: pas fréquente (Bremi). - L'Oherbasli (Meyer).

Varie pour la teinte depuis le roux clair au brun foncé.

221. Scripturaria $\mathbf{W}$. V.

Hub. 274. - Treit. - Dup. - Bdv. 1662. - Her.-Sehf. II. 78. - Heydenr. Cat. 379.

Miata. Sebwarz.

Pas rare près de Balm, vallée de Meyringen; en juillet (Meyer). - Çà et là dans les alpes: montagnes du Bas-Valais; vallèe de Salvan (Chavannes). - Près de Glaris dans un bois de bêtres, au milieu des rochers, à la fin de juillet (Lah.). - Quelquefois dans le Jura. 
222. Bilinearia (ta). Lin.

$$
\begin{aligned}
& \text { F. - Hub. } 264,- \text { W. V. - Treit. - Dup. - Bdv. 1647. - } \\
& \text { Fr. n. B. } 83,1,2 \text { (var.) - Her.-Sehf. II. 79. - Wood } 624 . \\
& \text { - Heydenr. Cat. } 393 .
\end{aligned}
$$

La plus commune de toutes les phalènes; elle peuple les haies et les taillis durant tout l'èté Lah.). - Du 27 mai au 7 août; mais plus spécialement du 9 au 27 juin, puis du 4 au 29 juillet (Rotbb.).

223. Riguaria (ta). Hub, 35̃8.

Treit. - Dup. - Her.-Schf. II. 80. - Heydenr. Cat. 389.

Trouvee en Valais par Mr. Meyer, le 9 août, au-dessus de Gamsen, appliquee contre un mur. Espèce mèridionale.

224. Vetularia (ta). W. V.

Hub. 263 - Treit. - Dup. - Bdv. 1642. - Her.-Schf. II. 81. - Wood 619. - Heydenr. Cat. 391.

L a rv. Hub. G. II. Aequiv. K. a. b. fig. 2.

Paraît deux fois l'an, près de Lausanne; une fois au commencement de mai, une seconde fois à la fin de juillet. Se retire volontiers sous les gazons et les racines; pas très fréquente (Lah.). - Près de Schüpfen et d'Aarberg du 23 juin au 14 juillet, pas rare (Rothb.). - Très fréquente près de Zurich, dans les haies (Bremi). A Burgdorf parmi les aunes; assez rare (Meyer).

225. Sabaudiaria. Dup.

Hub. 584, 585. - Bdv.'1636. - Fr. n. B. 390, 2. - Her.-Schf. II. 82. - Heydenr. Cat. 399.

Je l'ai reçue Je Mr. Anderegg; je doute cependant qu'elle se trouve en Valais (Lah.). - Au Locle, canton de Neuchàtel (Rothb.) - Mr. Bruand la prend dans le Jura français. - Mr. Bugnion l'a prise au pied du Jura, près de Grandson.

Espece propre au Jura. 
226. Undularia. Lin.

$$
\begin{aligned}
& \text { F. - W. V. - Hub. 262, 436. - Wood 625. - Dup. - Bds. } \\
& \text { 1643. - Her.-Scbf. II. 83. - Ileydenr, Cat. 392. }
\end{aligned}
$$

Cá et la dans les haies des vallées des alpes, mais surtout sur les basses alpes; Rosenlaui; Gournigel, etc. (Meyer). - Montagnes d'Aigle, en juillet (Lab.). - Une fois à Zurich et une fois an pied de l'Albis (Breuii). - $\Lambda$ Schüpfen du 17 juin au 16 juillet; rare (Rothl.). - Commune à Burgdorf (Meyer).

227. Polygrammaria (la). Bork.

Hul. 277, - Treit. - Dup. - Bdv. 1655. - Her.-Schf. II. 84.

-- Woud 1676. - Heydeur. Cat. 409.

Cetle espece, propre à l'Europe méridionale, existe dans la collection de Mr. Rothenbach, prise près de Vevey. - Fort rare en Suisse.

228. Rhamnaria (1a). W. V.

Hub. 271, 400. - Treit. - Dup. - Bdv. 1641. - Her.-Schl.

II. 87. - Wood 618. - Heydenr. Cat. 410.

La rv. IJub. G. II. Aequiv. K. b. fig. 1. c. d. e.

Partout assez rare; j'en possède un individu des environs de Lausanne (Lah.). Prese du lac de Bienne et près de Verey, le 22. juin (Rothb.). - Le Zurichberg, l'Uto Bremi). Loberhasli; rare (Meyer).

229. B adiaria (ta). W. V.

Hub. 291. - Treit. - Dup. - Bdv. 1758. - Her.-Schf. II. 88 (non Bork. - Frey.). - Wood 58\%. - Heydenr. Cat. 41 t.

Larv, Hub. G. 11. Aequiv. L. a. b. fig. 2.

Environs de Lausanne; dans les creux des murs et des rochers; avril; pas rare. Le papillon m'est nè le 22 mars, quoiqu'au froid (Lah.). - Le 5 avril à Schüpfen; très rare (Rothb).) - Le Zurichberg; pas rare (Bremi). - Près de Berne [Renk).

Cette espéce a probablement deux éclosions en Autriche, puisque la chenille y passe l'hiver pour se chrysalider au printemps et donner le papillon en juin et en juillet. Boisduval et Duponchel ont ici, comme à l'ordinaire, copié l'entomologiste de Vienne. En Suisse la chenille se transforme en automne et le prapillon éclos au premier printemps, 
pour ne donner quine fois dans l'annee. Mr. Bruand a fait la même observation dans le Donbs (catal. $n^{\prime \prime}$ 691). Ce fait nous montre combien le climat influe sur les moeurs des phalines.

La chenille que jai trourée sur le rosier, comme toujours, est d'un lorun rinlel, avec des points jaunes sur lesflanes; sa tele est brunc. IJubner la figure verte avee la tête runge et des points blancs sur les anneans. Varierait-elle a ce point?

2:30. Berberaria (ta) F.

$$
\begin{aligned}
& \text { W. Y. - IHub. 287. - Bork. - Treit. - Dup. - Bdv. } 1759 . \\
& \quad \text { - Her.-Schl. II. 89. - Wood 585. - Heydenr. Cat. 537. }
\end{aligned}
$$

Larr. Hub. G. II. Aequiv. I. c.

Issez fréquente partout durant l'èté; prise dans les alpes de Chamonny piar Mr. Chavannes (Lah.). - Pas rare près de Scbüpfen, du 5 juin au 19 aoút (Rotbl.). - Environs de Zurich Bremi. - Partout commune dans le canton de Berne (Meyer). - Na pas encore èté tronvée, que je sache, en mai, comme l'indique Boisduval..

2:31. Rubidaria (ta). W. Y.

Bork. - Hub. 290. - Treit. - Lup. - Bdv. 1757. - Fr. B. 18, 2. - Her.-Schf. II. 90. - Wood 586. - Heyd. Cat. 536.

Plus rare que Berberaria, dans les mémes lienx "t à la même époque Lab.). A Schüpfen assez. fréquente, du 2 juin an 11 juillet Rothb.). - Commune à Meyringeu et antour de Burgdorl (Mryer). - Parait rare à Zurich; pres de Secu (Rordorf).

232. Turbaria. Hub. 235.

Treit. - Dup. - Bds. 1784. - Her.-Schf. II. 92 - (non Woud). Rivinaria. F, v. R.

Cette espèce n'est pas rare dans les bois élevès des Alpes; mais elle y parait de honne heure: montagnes d'Aigle en juin (Lah.). - Oberhasli; Kaltenbrumen; commune du milieu à la fin de juin; se pose sur les sapins (Nleyer). - Mr. Rothenbach l'a prise encore en juillet. Les alpes de Glaris (Pfr. Heer).

Doit être rapprocbée de caesiaria, flavicinctaria, Kollariaria cte. Turbaria Wood appartient à affinitaria. 
203:3. Ocella ria (ta). Lin.

W. V. - Bork. - Hub. 252. - Treit. - Dup. Bdv. 179\%.

- Her.-Schl. Il. 93. - Wood 562.

Lyuceit a. F.

Fasciata. Devil.

Commune partout dan's les haies, d'aborl en mai, puis en aoùt (Lah.) - A Schüpfen, du 24 mai au 28 juin, puis du 13 juillet au 23 aoùt Rothb,). - De même dans les environs de Zurich (Bremi). - Parait plus rarement dans le canton de Berne (Meyer;

2:34. Propugnaria (la). F.

W. V. - Hub. 286. - Treit. - Dup. -- Bdv. 1771. - Her.Sichf. II. 94. - Wood 560. - Heydenr. Cat. 540.

Cette espèce de l'Europe septentrionale est partout très rare en Suisse; elle a èté Irouvée jusqu'ici à Langnau, à Aarberg et près de Schüpfen par Hr. Rothenbach; mais toujours rarement. Du 19 mai au 8 juin et du 4 au 7 aoủt. - Elle se trouve aussi daus le Doubs (Bruand).

2:35. Ligustraria (ta). W. V.

Hub. 282. - Treit. - Dup. - Bdv. 1630. - Fr. B. 114, 1.

Her.-Schf. II. 95. - Heydenr. Cat. 570.

Quadrifasciala. Wood 5.52 .

Larv. Ilub. Geom. II. Aequiv. L. a. fig. 2.

Cá et liz dans les haies aux environs de Lausanne, en mai et en juin; puis eu aout et en septembre. Pas commune (Lah.). - Les vergers des environs de Zurich; plus fréquente (Bremi). - Du 12 juin au 2 aoùt, à Schüpfen; rare (Rotbb.). - Assez commune, contre tes troncs des peupliers et dans les haies autour de Burgdorf. Varie à bandes noires, larges el bien tranchées (Meyer). 
236. Quadrifasciaria la. F.

W. V. - Hub. 284. - Bork. - Treil. - Dup.? - Bdv. 1629?

- Her.-Schf. I1. 97. - Heydenr. Cat. 572.

Badiata. Bork.

Unilobata. Wood 564 (var.).

Quadriannulata. Wood 565.

Larv. Hub. Geom. II. Aequiv. L. a. fig. 1.

Pas rare dans les environs de Schüpfen, dn 23 avril au 18 mai, puis du 9 juin au 2 août (Rothb.). - Meyringen; assez fréquente (0t). - Près de Seen; assez rare (Rordorf). - Burgdorf; le long des forêts de hêtre et dans les haies, en juillet; pas commune (Meyer). - N'a pas èté prise dans les environs de Lausanne ( $\boldsymbol{L}_{\text {ah. }}$.).

237. Olivaria. W. V.

Bork. - Hub. 307. - Treit. - Bdv. 1775. - Dup. sup. Her.-Sehf. II. 98. - Wood 557. - heydenr. Cat. 561.

A ptaria? Bruand Catal. $n^{\circ} 595$.

Cette espèce n'est pas rare dans les cantons de Berne et de Vaud; quelquefois en juin; plus souvent en juillet et en aoùt. Les taillis et les haies (Lah.). - Commune du 13 juillet au 12 août, prés de Schüpfen (Rothb.). - Pas observée dans le canton de Zurich. - Forèts des environs de Burgdorf (Meyer). - N'a pas été prise, que je sache, dans les alpes.

Vit volontiers en famille. On prend quelquefois les exemplaires effaces d'olivaria pour aptaria.

2:38. Aptaria. Hub. 349.

Treit. - Bdv. 1778. - Dup. cat. - Her.-Schf. II. 99. - Heyd. Cat. 560 .

Suplata? Fr. n. B. 323,3 .

Descend quelquefois dans les forèts du canton de Berne; près de Burgdorf, en aoùt (Meyer), - de Schüpfen (Rotbb.); - mais toujours rare el égrenèe. Plus fréquente dans les forèts de sapin des alpes; Oberstein près de Meyringen, à la fin de juin; commune (Meyer). - Dans les montagnes d'Aigle; sur le Simplon etc; mi-juillet (Lab.). 
239. Ferrugaria. W. V.

Bork. - Hub. 285? - Dup.? - Bdv. 1628? - Her.-Schf. II. 100. - Heydeur. Cat. 565.

Linariaria. Bork.

Salicala. Wood 555.

Pontissalaria.? Br. catal. $n^{\circ} 596$.

Je nai pris qu'une seule fois cette espèce autour de Lausanne, au mois de mai (Lal.) - Tout l'èté prés de Sehüpfen, du 28 avril au 30 mai, du 9 au 20 juin, du 12 an 20 juillet jusqu’au 19 aoút (Rothb.)? - De même près de Zurich (Bremi)? - Il est fàcbeux que dans ces indications il $\mathbf{y}$ ail confusion avec $\mathrm{Spadicearia.}$

La distinction entre ferrugaria et cette dernière n'est pas aisée. La première varic moins, est aussi moins bariolèe. Sa bande est à peine striee, plus large à sa moitic supérieure. L'angle de la bande est plus saillant, non bifide. L'espace marginal dés la bande médiane à la frange et dans sa moitiè interne, est lavé de gris. assez uniforme; on $\mathrm{y}$ distingue à peine le second ruban et la ligne ondulée.

Du reste je n’ai séparé ces deux espèces que sur l'autorite de Her.-Schf. et faute d'avoir pu examiner un nombre suffisant de ferrugaria. On ne peut guères ici se fier anx noms des marchands d'insectes. - Treit. a commis une erreur typographique en citant Hub.; la figure de celui-ci est 285 et non 258.

210. Spadicearia. Bork.

W. V. - Her.-Schf. H. 101. - Heydenr. Cat. 566.

Ferrugaria (partim) Borls. - Hub. 460. - Dup.? - Bdv. 1628.? Wood 553.

Spadicearia est très fréquente autour de Lausanne, au premier printemps (avril); elle continue à paraître dés lors çà et là, jusqu'en juillet. Se rencontre aussi dans les lasses alpes (Lab.). - La plupart des indications de ferrugaria se rapportent sans doute à spadicearia. - Voir la première pour la distinction à établir entr'elles. La bande mediane passe du gris-roux clair, traversé par plusieurs lignes, soit au noir, soil au rouge-brique où toutes les raies disparaissent. Je ne lai jamais trouvée rougeamaranthe comme ferrugaria. 
2it. Kollariaria. Mann cat.

Her.-Schf. fig. 243, 214. II. 103. - Heydenr. Cat. 432. Var.? laete viridis. - fig. 6.

Cette belle espèce, découverte par Mr. Mann dans les alpes d'Autriche, a èté prise Jans les Grisons (Bremi). - Mr. Heyer l'a observee le 12 juin sur la sommite du Jura.

La var. verte a été prise dans l'Oberhasli par Mr. Oll, sur le Jura par MIlr. Heuser el Meyer et par moi au pied des monts d'Arvel, derrière Villeneuve, an commencement de juin. Celle-ci ne différe de la premiere qu'en ce que les bandes brunes y sont d'un beau vert clair. En est-il de celte variété comme de celle d'impluviaria, ou le vert passe au brun par l'effet d'influences atmosphériques? J'ai quelque lieu d'en douter.

L's antennes du mâle (var. verte) sont pectinées, comme dans mont anaria, dont elle se rapproche à plus d'un égard. Leur pointe est blanchàtre, leur dos ragé de hrun et de blanc sale et leurs plumnles noirâtres. Son apparition précoce la fait écbapper jusqu'ici aux recherches des naturalistes.

202. Galiaria (ta). iv. V.

Hub. 272. - Treit. - Dup. - Bdv. 179i. - Her.-Schf. II. 101. - Wood 563. - Heydenr. Cat. 576.

Bicolorata? Bork.

Assez rare dans le Jorat au-dessus de Lausanne, en juin, sur le bord des bois Lah.). - Sur le Jura, le 28 juin; très rare Rothl... - Noins rare à Meyringen; rare à Burgdorf; en juin (Meyer).

\section{0: Alpicolaria.? F. v. R.}

Heydenr. Cat. 592.

Abstersaria. Her.-Schf. d. Ins. 165, 2 non H. S. 119, 120, ... Her.Schf. H. S. texte II. 104.

Prise près de Lausanne, en juillet (Lah.). .

C'est avec quelque hésitation que j’introduis ici cette especce. Le seul individa \{femelle? que j’ai sous les yeux a quelque ressemblance avec fluctuaria et ne peut étre confondu arec aucun autre. Il en diffère premièrement par sa bande médiane plus large, non interrompue et fortement anguleuse du cóté de la base de l'aile: (dans lltuctuaria elle est très arrondie et légèrement sinueuse); 2) par la tache noirâtre, plus petite et 
itroite, de l'extremite des ssipéricures; 3) par l'absence des denx points noirs du bord de la ligne sinuèe; 4) par les ailes inférieures d'un gris de fumée unilorme, sur lequel se dessinent tries légérement les bandes blanches à peine ondées.

Je ne trouve pas du reste que l'angle externe des superieures soit plus arrondi que dans fluctuaria. Serait-ce une variété plus foncée de fluetuaria, a bande plus larọce et entière? En tout cas ce nंest pas abstersaria. H. S. sup. lig. 119, 120.

214. Sinuaria (ta). IV. V.

Hul. 288. - Treit. - Dup. - Bdv. 1756. - Fr. B. 6, 1. -

Her.-Schf. II. 105. - Wood 587. - Heydenr. Cat. 603.

Celte espéce, rare partout, a été trouvèe près de Soleure, de Langnau et de Vevey Mothh.); pres de Burgdorf et de Meyringen; en juin (Meyer). - Dans la vallec de Maderan (Bremil. - Une fois près de Lausanne en juin (Leresche).

245. Rivaria (1a). Hub. 449.

Treit. - Dup. - Pdr. 1787. - Mer.-Kiclif. II. 107. - F. v. R. p. 96. - Heydenr. Cat. 579.

Sylratica. Wood 568.

Galiata. Bork. F. v. B.)

Les haies des environs de Lausanne; fin de juin et juillet; pas commune (Lah.. - be mème dans le canton de Berne (Meyer). - Plus commune près de Schüpfen; du 2 ¿ avril an 16 juin Rothb.). - Peut-être y a-t-il ici quelque confusion avec alehemillaria.

ha synonymie de cette espece et dalchemillaria a ète suffisamment établie par I. r. R.

246. Inangularia (1a). Wood 519.

Her.-Schf. II. 166. f. 338. Heydenr. Cat. 573. Amniculata. IIub. 386. - Bdv. 1789 (ria). - Dup. cat.

Bicolorala. Bork. - F. v. R. p. 98.

Jai pris cette espèce rare le 23 juin dans les taillis au-dessus de Lausanme. J'en ai vu un second exemplaire pris à Meyringen Lab.).

Elle est extrêmement voisine de rivaria et proballement partout confondne arec elle. Elle en differe eependant au premier coup-d'oeil par le blanc pur de ses deux rubans. Alehemillaria est tonjours plus couverte de gris et de noir. 
Ses caractères les plus saillants sunt: 1) la bande médiane d'un brun rougeâtre, gris-foncèe a son milieu, marquée vers son bord postèricur d'un trait noir très ondulé. souvent lisèré de blanc; 2) le $\mathbf{2}^{\mathrm{e}}$ ruban blane de lail, un peu jannàtre du cóté de la marge; 3) la ligne ondulée bien marquée sur la marge; 4) un large espace vers le milien de celle-ei; 5) une grosse tache brune au-dessus du milieu de la marge, aux inférieures; 6) la frange noirâtre dès le sonmet jusqu'au milieu des supérieures; blanche et entrecoupée de noir au-dessous du milien; blanche et tachetée de gris aux infërieures; 7) la frange bordee de petits traits, brisés en chevrons, au milieu des superieures et aux inférieures; celles-ci en paraissent dentelèes.

La figure de Hob. est très inexacte. Ceile de Her.-Schf. a le trai oblique du bout de l'aile supérieure trop prononcé et les marbrures de l'aile inferieure, près de l'angle antérieur, pas assez marquees.

\section{Alchemillaria (1a). Lin.}

$$
\text { F. - W. V. - Hub. 261, 370. - Dup. - Bdv. 178s. - F. }
$$
v. R. P. 100. - Her.-Schf. Il. 108. - Heydenr. Cat. 580. Sociata. Bork. (F. v. R.).

Substriata. Wood 567 .

Commune dans les haies et les vergers des environs de Lausamne; parail deja en avril (le 25 le long du lae, en mai sur les collines; reparaît en juillet et en aoùt Lah.). - Dn 20 arril an 18 juin, puis du 3 an 28 juillet, à Schüpfen Rothb.). - Mèmes localites dans les environs de Zurich (Bremi).

Cette espece varie beacoup; elle reste ecpendant toujours plus petite et plus couverte de gris et de noir que rivaria.

218. Molluginaria. Hub. 371.

Treit. - Dup. - Bdv. 1663. - Fisch. v. R. p. 101. - Her.Sehf. II. 109. - Heydenr, Cat. 582.

Larv. Hub. Geom. II. Aequiv. M. a. b. lig. 1. c. d.

Sur les pâturages des alpes. Jaman; montagnes d'Aigle ete.; peu comınue (Lah.). - Les alpes moyennes; le Righi (Bremi). - Alpes de l'Emmenthal (Blaser). - Assez fréquente sur le Jura, du 19 juin au 12 juillet (Rothb.).

En tout cas elle ne parait pas en mai dans les alpes. 
219. Montanaria. W. V.

Hub. 248. - Bork. - Treit. - Wood 558, - Dup. - Bdv. 1790. - Fr. B. 18. - Her.-Sehf. II. 110. - Heyd. Cat. 555. Ocellata. Fab.

Très commune, jusqu'à la heuteur de 6000 pieds, sur toutes les alpes et les montagnes, où elle se cache parmi les herbes. Elle descend dans les bois elairs et les taillis; frèquente sur le Jorat. Près de Lausanne elle se montre déjà au commeneement de mai et y reparaiı à la fin de juillet. Dans les lieux montueux et sombres elle paraît en juin et dans les alpes en juillet (Lah.). - En grande quantité le long des forêts de bêtre, près de Burgdorf (Meyer). - Commune autour de Langnau et de Schüpfen, du 29 mai au 28 juin (Rothb.). - Au Burgbölzli, près de Zurich, fréquente (Bremi).

Des individus, pris auprès dı glacier du Rhône, le 6 août, sont beaucoup plus pâles (Meyer) et se rapprochent ainsi de ceux de Laponie qui ont la bande à peine marquée (Lah.).

250. Chalybearia (ta). Hub. 350. ?.

Heydenr. Cat. 577.

Var. galiariae. Treit. et auctor. sequent.

Le premier individu (une femelle) que j'ai vu avait été pris dans le Haut-Valais par Ir. Anderegg; j'en vis un second au milieu de juin près de Villeneuve, au pied de la montagne. J'en ai pris un troisième (mâle) au-dessus de Lausanne, au milieu d'un bois, à la fin de juillet; ce dernier était déjà quelque peu effacè.

Cette espèce ne peut être confondue avee aucune autre; elle se rapproche beaucoup de galiaria; mais la teinte fauve du fond, l'absence de bande marginale et surtout les. antennes très pectinèes du mâle la font distinguer à l'instant. Elle a du rapport avee montanaria; mais sa large bande brun-fonece, se détachant sur un fond noisette elair, nuancẻ de blanc sale, la earactérisent suffisamment. Les antennes du mâle sont d'ailleurs plus pectinées que celles de montanaria $\sigma^{*}$. - La figure de Hub. est très exacte et je m'ètonne qu'elle n'ait pas tout au moins fait naitre des doutes sur le parentage avec galiaria

Je ne puis croire que Treit. ait jamais vu chalybearia en nature; en tout cas il n'a pas vu le mâle; sans cela il n'eût pas écrit ces paroles en parlant de galiata: whierher, als eine mit mehr Blau und weniger Braun auf den Vorderlügeln ausge- 
fülte Varietät, gehört unbezweifelt Hübners chalybeata. " - C'est précisément le contraire qui a lieu. Les autcurs qui ont sucrédé, ont simplement copié Treit. sur ce point.

Les deux sexes sont semblables.

251. Scabraria. Hub. 229. 9.

Treit. - Dup. - Fr. n. B. 137; 1, 2. - Bdv. 1623. - IIer.Schf. fig. 359, 360. II. 114. - Heydenr. Cat. 358.

Alpestrata? Hub. 320. o. - Treit.? - (ria). Dup. cat. - Bdv. 1772. Didymata. Thunb. - Wood 549.

Clairière d'un bois sur la Gysnau et ailleurs, près de Burgdorf; rare: août (Meyer). - Près de Langnau, en aoùt; rare (Rothb.). - Dans le Jura (Bruand). - Trouvée en abondance le 5 août (1850) dans les prés de Guttannen (valléc d'Oberhasli) à 3300'; mais pas une seule femelle. Cette dernière se tient probablement cachèe dans les herbes. Les mâles volaient bas et semblaient être à la recherche de la femelle (Meyer).

Malgrè l'affirmation de Her.-Schf,, je n'admets alpestrata (Hub. Treit.) comme synonyme du mâle, qu'avec doute. La fig. de Hub. ne peut être prise en considèration, tant elle est mauvaise. Treit. affirme que les antennes dn mâle sont filiformes; or on ne peut croire que cet habile entomolngiste ait pris une $\mathcal{P}$ pour un $\delta$. J'ai vu une pbalène, venant du Wurtemberg, qui répondait entièrement à la description de Treit. Le dessin et la taille étaient ceux de Podevinaria, avec un fond brun et une bande verdâtre, foncée. Je ne puis y voir que la vraie scabraria (alpestrata de Treit.) avec ses antennes filiformes. - Dup. et Boisdv. ne sauraient faire autorité parce qu'ils ont copié Treit.

252. Minoraria (ta). Treit.

Dup. sup. - Bdv. 1680. - Her.-Schf. figg. 118. II. 115. - Heydenr. Cat. 559.

Ericetata. Wood 696. - Stph.

Commune sur les Alpes et le Jura durant l'ètẻ (surtout en juillet). Vole au soleil sur les påturages. Se pose volontiers contre les rochers (Lah.). - Du 12 juillet au 3 aoùt (Rothb.). - L'Oberhasli, le Jura ete.; commune (Mleyer).

Cette espèce n'a point été figurée par Hub.; il l'a probablement confonduc avec sa voisine, blandiaria. Si Boisduval les eut vues l'une à côté de l'autre, il n’eut jamais placé minoraria dans ses Enpithecia, et hlandiaria dans ses Melanthia.

Certains individus se courrent d'une teinte brunatre qui augmente dans les collections. 
253. Blandiaria (ta). W. V.

Hub. 258. - Treit. - Dup. - Bdv. 1796. - Her.-Schf. fig. 290, 291. II. 116. - Wood 697. - Heydenr. Cat. 599.

Adaequata. Bork.

Trigonata. Wood 699 .

Albidata. Eversm.

Cà̀ et là sur les montagnes et dans la plaine; une première fois au printemps et une seconde à la fin de l'été. Iu milieu de juin dans les alpes (Lah.). - Le long du Sommerhauswald, près de Burgdorf; en mai (Meyer). - A Schupfen du 12 juin an 27 juillet; du 7 aoùt au 12 septembre; pas rare (Rotbb.).

Jueundaria Bdv. 1797 n'est probablement qu'une variétè. On trouve des exemplaires de blandiaria dont le blane prend une couleur de chair fort pàle.

Blandiaria, minoraria et albularia sont très voisines.

254. Fluctuaria (ta). Lin.

Bork. - W. V. - Hub. 249. - Treil. - Dup. - Bdv. 1793.

- Her.-Schf. 11. 118. - Wood 559. - Heydenr. Cat. 597. Var. stragulat a. Treit. sup. - Her.-Schf. fig. 296-298. - (non Hub.)

Commune dans les taillis et les baies, une première fois en mai et en juin, puis une seconde en juillet et en aoùt (Lah.). - Du 24 avril au 12 mai; puis du 6 juin au 29 aoủt; à Schüpfen. Commune (Rothb).) - Nêmes localitẻs: fréquente à Zuricb (Bremi).

- De méme dans tout le canton de Berue (Meyer).

Stragulata de Treit., figuree dans Her.-Schf. comme variéte de fluctuaria, lui appartient en effet; celle de Hub., trés voisine de variaria et probablement la même que telle de Bdv., appartient à une autre espèce. (voir stragularia).

250. Albicillaria (a). Lin.

$$
\begin{aligned}
& \text { F. - Bork. - W. V. - Hub. 76. - Treit. - Dup. - Bdv. } \\
& \text { 1803. - Fr. B. 96. - Her.-Schf. II. 119. - Wood 603. } \\
& \text { Heydenr. Cat. } 604 .
\end{aligned}
$$

La rv. Hub. G. II. Aequiv. L. a. fig. 2.

Fréquente dans les bois taillis et les haies, en juin et quelquefois en juillet (Lah.). Aarberg, Sehüpfen; assez commune du 27 mai au 26 juillet (Rothb.). - Nèues lo- 
calitès dans les environs de Zurich (Bremi). - Une des espèces les plus communes autour de Burgdorf, sans y prèsenter de variètés (Meyer).

256. Derivaria (ta). W. V.

$$
\text { Bork. - Hub. 289. - Treit. - Dup. - Bdv. 1760. - Her.- }
$$

Schf. II. 120. - Wood 588. - Heydenr. Cat. 538.

Nigrostrigata. Devil.

Dans les bois de hêtre et de chêne, en mai et en juin; contre les trones. Rare anx environs de Lausanne (Lah.). - Langnau, Schüpfen, très rare; milieu de mai (Rothb.). - Les bois de hêtre du canton de Zurich; pas fréquente (Bremi). - Les bois des environs de Burgdorf; très rare; dès le milieu de mai à la fin de juin (Meyer).

Jamais en Suisse elle ne parut en avril. - Voisine de berberaria.

257. Rubiginaria (ta). Fab.

Bork. - W. V. - Huh. 250. - Treit. - Dup. - Bdv. 1800.

Wood 606. - Fr. n. B. 102, 1. - Her.-Sehf. II. 121. Heydenr. Cat. 600 .

Albata. Devil.

Espèce de l'Europe septentrionale; assez rare partout; les bois d'aune des montagnes et des alpes; en juillet. Rare dans le Jorat (Lah.). - Plus commune près de Burgdorf (Meyer). - De même près d'Aarberg et de Schüpfen, du 25 juin au 12 uillet (Rothb.). - Sur l'Uto, près de Zurich; pas très rare (Bremi).

258. Procellaria (ta). F.

W. V. - Hub. 251. - Treit. - Dup. - Bdv. 1801. - Wood 604. - Her.-Sehf. II. 123. - Heydenr. Cat. 591.

Pas rare dans les haies en mai et en juin autour de Lausanne (Lah.). - Burgdorf et Aarberg; assez fréquente; plus rare à Schüpfen; du 25 mai au 25 juin. Reparaît çà et là an milieu de septembre (Rothb.).

Cette espèce préfère les expositions tempèrées. 
259. Hastaria (ta). Lin.

$$
\begin{aligned}
& \text { F. - Bork. - W. V. - Hub. 256. - Dup. - Bdv. } 1781 . \\
& \text { Her.-Schf. II. 124. - Wood 602. - Heydenr. Cat. } 586 .
\end{aligned}
$$

Propre aux régions froides. Je ne l'ai jamais trouvè aux environs de Lausanne (Lab.). - Rare dans les environs de Sebüpfen; en mai. Dans les Alpes, en juin (Rothb.). - Au commencement de juin ęà el là dans le canton de Rerne; Meyringen. Partout rare (Meyer). - Les bois de sapin du Zurichberg; rare (Bremi).

Celte espèce varie assez par le dessin plus on moins chargè de noir; cependant elle a toujours un aspect qui lui est propre; le noir est disposé par laches et non par bandes; son aspeet la fait deja distinguer de hastularia (voir hastularia).

H astaria est assez isolée dans sa place et rapelle à quelques égards marginaria.

260. Hastularia (ta). Hub. 356 bis. (non Hub. Beitr.). Hasıaria var. auetor. - Heydenr. Cat. 587.

Ce n'est qu'après beavcoup de recherehes que je me suis déeidé à conserver cette espèce de Hub., malgré l'autorité de tous les entomologistes postérieurs à lui. - Elle tient le milieu entre hastaria ef Iristaria (Hub. 254), quoique plus près de la seconde. Un peu plus petite que bastaria, elle s'en rapproche pour le dessin; mais le noir y est distribue par bandes el raies. Plus grande que tristaria, elle n'a pas ses angles aigus et saillants de la bande médiane. l.a fig. de Hub. est prise sur un individu très chargé de noir; je n'en ai point vu de semblable; elle est exacte et suffit pour faire saisir la diffèrence entre hastularia et ses voisines, quoiqu'elle ne fasse pas assez ressortir les angles arrondis de la bande mediane.

H astularia est fort rare el paraît exclusive à la Suisse. Elle a été prise dans l'Oberland, près de Mleyringen, au Kaltenbrunnenalp, en juin, par Mr. Olt. - Le 6 août ęà et là, dans les bois de sapin, sur la route de la Grimsel, entre Guttanmen et la Handeck. Dans le Haut-Valais entre le glacier du Rhóne et le village d'Obergestelen. A la mi-juillet dans les bois du Gourniguel. Partout rare et isolèc (Meyer).

Cette phalène était bien faite pour mettre la confusion entre hastaria, tristaria et funeraria. Si l'on n'a pas vu un certain nombre d'individus, on la fait servir d'intermédiaire entre les deux premières, et la troisième reste isolée, comme cela se voit dans Treit. A cette erreur on en joint une seconde, celle de transporter á funerata Hub. les synonymes de sa tristata. 
Elle diffère à première vue de hastaria par sa bande toujours entière, à bords arrondis, plus large vers le bord interne. La base des ailes est noire, sablée de blanc; même aux inférieures. Le dessous du corps est gris-jaunảtre. Le corselet a fort peu de blanc. Sur les iufeerieures la bande médiane est bien sèparée de la base. A ces caractẻres, qui la différencient de hastaria, on peut encore ajouter que dans celle-ci le dessous des ailes inférieures porte, au licu de bande, deux raies dont la plus éloignèe de la racine correspond au milieu de la bande', en sorte que le point discoïdai noir se trouve placè sur cette deuxième raie. Dans hastularia cette mème raie est suivie d'une troisième (l'interne peut manquer), et le point est placé sur l'avant-dernière ligne.

La quantité de blane répandu sur la bande moyenne et sur la marge varie dans les trois espèces; mais il est répandu différemment dans chacune d'elles. Il en est de même pour le dessous. Ces différences ne pourraient être rendues sensibles que par plusieurs figures (voir tristaria et hastaria).

261. Tristaria (ta). Hub. 254.

$$
\begin{aligned}
& \text { Bork. - Dup. - Bdv. 1782.? - Her.-Schf. 11. 125.? - Heyd. } \\
& \text { Cat. 584. }
\end{aligned}
$$

Luetuata. Hub. Beitr. I. 1. - Hastulata id. ib. (Naclıträge).

Près de Lausanne, au hord du bois de Sauvabelin, dans le voisinage des aunes, du 13 au 20 mai (Lah.). - Un exemplaire a ètė pris sur la Gysnau, près de Burgdorf, par Mr. Meyer. - Pas encore trouvee ailleurs en Suisse. - Je l'ai aussi reçue des bord́s du Rhin. - Duponchel l'a prise près de Paris. - Très rare.

Les figures de Hub. Geom. 254 et Beitr. I. 1. IV. Y. ne laissent rien à désirer. Celle de Dup. peut s’appliquer à tristaria et à funeraria; sa description est faite sur tristaria. - Bdv. reste incerlain. - Treit. parât n'avoir eu devant lui que funeraria. - Her.-Schf. décrit la vraie Iristaria tout en la confóndant avec funeraria. - Les auteurs anglais, Linné et Fabr. n'ont connu que cette derniere sous le nom de tristata. La premiere est en effet commune dans le Nord. - Borkh. parle de la chenille de trislata rivant sur l'aune, et Westwood copie Treit. sur ce point, lui qui n'avait en vue que la chenille de funerata vivant sur le galium confr.: Bulletin de la Société vaudoise des sciences naturelles $\mathrm{n}^{0}$ 201.

Hastaria, hastularia et tristaria sont constamment d'un noir foncè pur fraiches); funeraria est brune, tachee de noir et d'un peu gris (voir hastnlaria et funerariat. 
262. Funeraria (ta). Hub. 260.

Heydenr. Cat. 583.

Tristata. Lin. - Dup. hist.? - W. V. - Haw. - Stph. - Westw. Pupillata? Thunb. - Bork.

Commune sur les påturages des alpes, en juin et en juillet; vole parmi les herbes a la manière de clathraria (Lah.). - Sur toutes les alpes du 19 juin au 24 juillet; pas rare (Rotlib.).

Se trouve dans presque toutes les collections sous le nom de tristaria. La figure de Hub. est méconnaissable; celle de Westw. est un peu meilleure. Je ne connais pas d'autres à consulter, car Dup., en copiant Hub. comme à l'ordinaire, a cherché à y faire rentrer tristaria quil avait sous les yeux (voir tristaria).

Tristaria a les bandes d'un noir pur, le blanc un peu jaunâtre et les angles de la bande mediane fort saillants, surtout aux inférieures. Funeraria a les bandes brunes tachées de gris et de noir, le blane un peu grisâtre et l'angle de la bande médiane à peine saillant aux inférieures. Sa taille est toujours un peu plus forte.

Plus voisine d'alehemillaria que de tristaria.

263. Luctuaria (ta). W. V.

Treit. - Bork. - Hub. 253. - Dup. - Bdv. 1783. - Her.-

Schf. f. 321 II. 127. - Heydenr. Cat. 588.

Au-dessus de Lausanne et sur le Jorat, en mai et en juin; sous-alpes d'Aigle; rare. Lab.). - Du 15 mai au 21 juin près łle Schüpfen; rare (Rothb.). - Les forêts de hêtre et de chêne sur l'Uto; rare (Bremi). - Forêts de chêne dans les environs de Burg̣dorl. - Vole haut el dans les arbres, ce qui la rend difficile à saisir (Meyer).

261. Rupestraria (ta). F.

W. V. - Hub. 192. - Treit. - Dup. sup. (fig. mala). - Bds.

1672. - Fr. n. B. 137, 3, 4. - Her.-Schf. fig. 192. II. 128

- Wood 645. - Heydenr. Cat. 446.

Bassiaria 2 Feist. Ann. de la Soc. entom.

Très comnune sur tous les pâturages des alpes: elle ne s̈èlève sur les sapins que pour y cliercher un refuge; car on ne la trouve bien fraiche que dans les herbes; juillet et aoùt (Lah.). - Les alpes, du 11 juillet au 1 aoùt; commune (Rothb.). 
Mr. Anderegg a trouvé la chenille sur la grande gentiane (gentiana lutea) et l'a élevée par son moyen. Plusieurs chenilles vivent sur cette plante.

La femelle est plus petite, plus pâle et moins bien dessinée que le mâle.

265. Albularia (ta). F.

Bork. - W. V. - Hub. 257. - Treit. - Dup. - Bdv. 1883.

Her.-Schf. II. 129. - IVood 698. - Heydenr. Cat. 349.

\section{Ablutata. Eversm.}

Très commune à la fin de mai et au commencement de juin sur les près et dans les bois des montagnes et du Jorat. S'élève jusques à la dernière limite des bois sur Jes alpes et y apparaît en juin. Vole volontiers au moment du coucher du soleil (Lah.). - Aarberg; Schüpfen; du 30 mai au 21 juin; fréquente (Rothb.).

Varie pour la couleur et la largeur de la bande médiane; ses stries passent du jaune nankin clair au gris noirâtre avec des points de même couleur sur les nervures. La coloration des ailes inférieures varie aussi souvent.

266. Decoloraria (ta). Hub. 243.

Treit. - Dup. -- Bdv. 1882. - Wood 243. - Her.-Schf. II. 130. - Heydenr. Cat. 348.

Près de Burgdorf dans les taillis; surtout le long du Plecrwald; du 5 au 15 juillet. Pas fréquente (Meyer).

267. Hydraria F. v. R.

Treit. sup. - Fr. n. B. 54,1. - Bdv. 1786. - Dup. cat. Her.-Schf. fig. 400. II. 131. - Heydenr. Cat. 373.

Espèce fort rare. N'a été trouvéc jusqu'ici qu'au Sommerhauswald, près de Burgdorf, par MM. Heuser et Meyer; elle est assez fréquente dans cette localité et y paraît au milieu de juin.

268. Rivularia (ta). W. V.

Hub. 259. - Treit. - Dup. - Bdv. 1785. - Her.-Schf. f. 289.

Nassat a. F.

II. 132. - Wood 695. - Heydenr. Cat. 374.

Larv. Hub. Geom. II. Aequiv. H. b. fig. 2.

Les haies humides et berbenses sur le Jorat; au-dessus de Lausanne; assez rare, en 
juin d'abord, puis en août (Lalı.). - Du 25 juin au 24 juillel; assez rare près de Schüpfen (Rothb.). - Nèmes localités dans les environs de Zurich; pas commune (Bremi). - En aboudance autour de Burgdorf, le long des forêts de sapin entrentêlées de hêtres. surtout le soir à la tombèe de la nuit (Meyer).

269. Affinitaria. Wood 693. o.

Her.-Schf. fig. 271,272 . б. et 319,320 . \%. II. 133. - Frey, n.

B. Taf. 510. f. 2. - Heydenr. Cat. 590.

2. Turbaria. Wood 694 .

J'ai vu trois exemplaires de cette phalène pris en Suisse, l'un le 12 juin, sur le sommet du Jura, près du Weissenstein, par Mr. Heuser; les deux autres près de Sehüplen, par Mr. Rothenbach. Sa ressemblance avec rivularia la faisait prendre pour de grands individus de cette dernière. Le mâle diffère sensiblement de la femelle, d'où les deux espèces de Wood.

270. Incursaria. Hub. 351 .

Bdv. 1619. - Dup. cat. - Her.-Sehf. fig. 255-57. II. 135. Heydenr. Cat. 436.

Decrepitaria. Zetterst. - Heydenr. Cat. 435.

Polygrapharia. Keferst. - Bdv. 1620? - Dup. cat. ? - Heyd. Cat. 384.?

Alpes centrales (Rothb.). - Rosenlauï; pied du Wetterhorn, au mois de juin. Trés rare (Meyer).

\section{Lotaria. Bdv. 1625 .}

Heydenr. Cat. 443. - Dup. eat.

Aquaria. Treit. et sup.

A que a ri a. Her.-Schf. fig. $50,121,122$. II. 134. - (ta). IIub. 353.

Dans les alpes, du 10 au 24 juillet (Rothb.). - Près de Meyringen, contre les roeliers, en septembre. Rare. Le Jura; 12 juin (Meyer). - Deux apparitions annuelles.

Facies de tophacearia effacee, mais plus petite. Intennes fortement pectinées; harbillons spatulès, très velus. Front blane pur. Palpes noirs, blancs à l'extrémité, assez saillants, moins cependant que dans to phacearia. Le dessin du dessus se répète au-dessous, mais très effacé et sous forne d'éclaircies. Les bandes dn dessus ont une teinte grise légèrement verdàtre. Bord externe des inférieures d'un gris plus foncè. 
272. Podevina ria. Her.-Schf. fig. 250. II. 1336.

Heydenr. Cat. 382.

$$
\text { Salicaria? Hub. 273. - Dup. - Bdv. } 1622 .
$$

Podevinaria, salicaria, ablutaria Her.-Schf. et probaria Zell. sont tres difficites à distinguer les unes des autres. Dans l'impossibilité oủ je me trouve de mettre de l'ordre en ce point, je ne fais mention que de ce que je puis affirmer.

Podevinaria a été prise sur divers points de la Saisse et partout inscrite sons la dénomination de salicaria. Mr. Rothenbach la signale dans les environs de lierne et de Langnau, vers la fin de mai; sur le Jura (13 juin). Mr. Meyer près de Meyringen, 25 mai au 29 juin. Mr. Bremi ęa et là sur les alpes. J'ai pris un individu $\&$ au borr du Léman, parmi les saules, en septembre. Elle aurait donc deux apparitions et se trouverait un peu partout, isolément et toujours rare.

Her.-Schf. affirme qu'elle diffère au premier aspect de salicaria par l'absence de teinte janne. J'ai sous les yeux un individu légèrement nuancé de jaune sur les bords de la bande médiane. La teinte générale passe du gris-blanc au gris-de-souris. La bande prend dans ce dernier cas une teinte gris-de-fer foncée.

Elle se distingue des voisines 1) par une ligne de points blanes isolés, remplaçant la ligne ondèe marginale; les plus gros points sont vers l'angle interne; 2) par de petits points blancs semés sur les nervures des supérieures, et surtout nombreux sur la bande blanche qui borde la médiane en debors; 3) par ses ailes inférieures gènéralement grises et funcees, traversées par une double bande plus claire, peu marquée; 4) par l'angle saillant et alongé, quoique arrondi à son extrémité, que forme la base obscure sur le disque des infërieures.

273. Salicaria. W. V.?

Her.-Schf. fig. 207, 208. II. 137. - Wood 555.? -- Heydenr. Cat. 385. - (non Hub.).

Ruficinctaria? Bruand, Catal. dẹ lépid, du Doubs, $n^{0} 586$.

Je n'ai vu qu'un individu, pris en Suisse, dans les jardins de Zurich, au mois de mars, par Mr. Bremi. Les taches d'un jaune vif qui ornaient les bords de la bande médiane, Ini donnaient quelque ressemblance avec une petite flavicinctaria Hub.

Les individus que Mr. Meyer et moi ont reçus de Mr. Mann sous le nom de sali- 
caria W. V. n'ont aucune nuance jaune et appartiennent peut-être à des espéces voisines, mal caractérisées encore.

Les collections renferment sous le nom de salicaria deux ou trois especes, ordinairement mélangèes.

\section{Vallesiaria nobis fig. 7.}

J'ai reeu la femelle de Mr. Anderegg et le mâle de Mr. Ott, a Meyringen. Cette espèce habite donc les alpes centrales de la Suisse.

Elle est très voisine de nobiliaria, d'achromaria, de lotaria, d'austriacaria et d'adumbraria (Zcll.). Le dessin est celui du groupe caesiaria, cyanaria, salicaria, etc. Le fond est gris-fauve. La bande mediane ei la marge sont d'un roux-brunatre, sale. La taille est au-dessous de caesiaria. Elle se distingue de nobiliaria par l'absence de jaunatre est de gris noirâtre et par sa ligne ondulée, à peine visible; d'achromaria par le sommet des supérienres arrondi, par les bords de la bande médiane à sinuosités arrondies et non anguleuses, et par les points geminés de la marge, moins distincts et figurant une ligne ondulee; de lotaria par ses antennes non peetinees et son Iront blanc sale; d'austriacaria (qui lui ressemble beaucoup) par les mèmes caractères que pour achromaria et par la ligne ondèe presqu'invisible. - Adum braria (Zell.) a le sommet des supérieures très aigu, le fond plus clair et plus marqué de lignes transverses. - Vallesiaria est surtout caractérisèe par sa marge des supérieures uniformément foncée, sur laquelle se voit à peine, vers le sommet, la ligne ondulée; puis par la rangée marginale de doubles traits, simulant une ligne brune fincment ondulée.

La femelle est plus foncée et un peu plus grande que le mâle. - Les antennes sont filiformes et pubescentes en dessous. Les palpes sont très courts.

275. Dilutaria (ta). W. V.

Hub. 188. - Treit. - Dup. - Bdv. 1669. - Fr. n. 13. 408. - Her.-Scbf. II. 140. - Wood 639. - Heydenr. Cat. 363. Var. quadrifasciata. Bork.

Affiniata. Bork.

Larv. Hub. G. II. A equiv. B. b.

Pas rare, en octobre, dans les bois et les taillis des environs de Lausanne (Lah.. - 
Fréquente dans les environs de Schüpfen, du 23 septembre au 22 octobre (Rothb.). En grande quantité dans toutes les forêts autour de Burgdorf et dans tout l'Emmenthal (Meyer).

Dilutaria Hub. 100 est interjectaria.

276. Nobiliaria. Mann, Calal.

Her.-Sehf. fig. 449, 453, 454. - Heydenr. Cat. 441.

Cette espèce, découverte par Mr. Mann dans les alpes d'Autriche, a aussi èté trouvée en Suisse, dans l'Oberhasli (Meyer); - sur la Gemmi Rothb.). - Je n'ai vu que le mâle.

Le dessin est celui de caesiaria sur uu fond gris-jaunat(re, soyeux, presque brillant. Taille un peu au-dessous de caesiaria. - Très voisine d’austriacaria Mann; elle en diffère 1) par le sommet des supérieures moins aigu; 2) par les ondulations arrondies de la bande et des rubans; 3) par la teinte olive répandue sur les points elairs des supérieures; 4) par l'angle plus obtus et plus arrondi de l'espace basilaire, sur le centre des inférieures; 5) par l'éelat soyeux et blanc du dessous; 6) par l'aspect lisse, soyeux, pâle verdâtre des ailes en-dessus, à-peu-près comme dans lotaria; 7) par les ailes postérieures plus arrondies, moins alongées.

Tête et anus blancs. Antennes filiformes, annelèes de gris et de blanc, à poils très courts. Palpes à pointe blanche. Frange soyeuse, presque blanche, sans lignes, ni points. Aucun point sur le disque. Inférieures à peine dessinèes. Côte arquée. La teinte paléacée est répandue sur les clairs comme dans cyanaria, et non sur les foncés.

277. Caesiaria W. V.

Hub. 275. - Treit. - Dup. - Bdv. 1665. - Wood 690. Her.-Schf. II. 142. - Bruand cat. $n^{\circ}$ 707? - Heyd. Cat. 433.

Peu rare dans les hautes alpes centrales, le long des rochers humides (Lah.). Commune dans les alpes du 26 juin au 3 aoùt (Rothb.). - En grande quantice au commencement de juillet, dans les forêts de sapin, au-dessus des bains du Gourniguel à $3700^{\prime}-4000^{\prime}$; en compagnie de montanaria. Elle se eache pendant le jour dans les bruyères et s'envole rapidement, dès qu'on l'approche, pour se poser eontre les trones de sapin, les ailes à demi étalées (Meyer).

Mr. Meyer affirme qu'elle n'existe pas dans le Jura; un èchantillon, venu de cette 
localite et que j'ai sous les yeux, ne lui appartient pas et ne peut ère qu'une cyanari a pâle et effacée.

C'est à tort que l'on réunit cette espèce à la suivante (voir flavicinctaria), Cyabaria, flavicinctaria et caesiaria se distinguent encore les unes des autres par la cellule des inférieures. Dans la première la cellule se termine presqu'en biscau, la bilurcation de la médiane interne est peı écartée et la nervure cellulaire se détache fort près du noeud (point discoïdal). Flavicinetaria a la cellule terminée en polygone et la nervure cellulaire insérèe assez près du noeud. Dans caesiaria la cellule se termine en forme d'anse arrondie et le point de detachement de la nervure cellulaire se trouve éloigné du noeud; la bifurcation de la médiane interne est écartéc. Ce caractère m’a sorti d'embarras chez les individus douteux.

\section{Flavicinctaria Hub. 354.}

Treit. - Frey. n. B. 390,3 (non 4 et 504?). - Wood 691. (non Dup. hist. - Bruand cat.). - Heydenr. Cat. 434.

Var. caesiaria e. Treit. sup. - Bdv, 1665? - Her.-Schf. - Dup. cat. ?

Espéce rare; parmi les rocailles ę les grandes herbes des bautes alpes. Tour d'Aï, etc. - juillet (Lah.). - Sur le Jura? et les Alpes, vers le milieu de juillet (Rothb.).

Quoique celte espèce soit très roisine de caesiaria, elle doit en rester sèparée; pour s'en convaincre, il importe de comparer entr'eux une série d'échautillons. - Voiei les principales differences: 1) flavicinetaria a un contour plus arrondi que caesiaria; aussi le sommet des ailes supérieures de celle-ci est-il plus aigu, la côte plus droite et les infèrieures sont-elles plus alongèes. Les figures de Hub., quoique mauvaises, expriment cette diffèrence; 2) la bande marginale des supèrieures, dans la première, est plus claire à l'extrême bord; quelquefois elle est tachetée de noir vers le sommet et le centre; mais jamais on n'y voit de traits bien nets et bien aigus comme dans la seconde. Celte même bande devient très claire à l'angle anal dans flavicinctaria; 3) les zigzags de la ligne fulgurale sont arrondis dans celle-ci et aigus dans caesiaria; 4) le second ruban est de méme couleur que la marge dans flavicinctaria et se fond avec elle vers l'angle anal; il est plus clair dass caesiaria et en reste bien séparé; 5 la teinte jaune du bord externe de la bande médiaue de flavicinctaria est séparée de l'espace cenIral par un lisèré gris; la teinte fauve qui se voit souvent dans eaesiaria va jusqu'à l'espace clair; 6 la base des supérieures porte des ombres orangées prononcées dans la première; dans la seconde il n'y a pas de fauve sur ce point; 7) les inféricures sont plus 
colorées dans celte-là, avec une tache blanche à l'angle anal et rarement un petit point central; dans celle-ci le dessin s'efface et le point central est très marqué; 8) la frange est claire dans flavicinctaria et tachée de noir; elle reste de la couleur du fond et entrecoupée de gris dans sa voisine; celle-ci a de plus la frange divisée par un Irait fiu; 9) le dos de la première n'est pas marqué de deux points noirs réunis, à chaque anneau, comme dans la seconde.

Le symonyme de Wood se rapporte bien à cette espèce, au dire de Curtis.

Les individus attribués au Jura sont fort douteux. La figure de Frey. pl. 504 pourrait bien s'appliquer à salicaria $\mathrm{H}$. S.

279. C y ana ria. Hub. 319.

Treit. - Dup. - Bdv. 1664. - Her.-Schf. II. 143. - Heydenr. Cat. 439.

Peu répandue dans les alpes. Je l'ai reçue de Chamouny. Prise une fois sur le Jura au Weissenstein, par Mr. Heuser. L'époque exacte de son apparition m'est inconnue (Lal.).

Cette espece ne peut pas plus ètre confondue avee caesiaria que flavicinctaria; elle est plus voisine de la seconde que de la première. La finesse de ses atômes bleuàtres suffit déjà pour la caractériser. Lorsque cyanaria est très fraîche, elle offre sur les parties claires (et non sur les foncées) des supérieures une nuance d'un jaune sale, très différente de celle de caesiaria et de flaviciuctaria. La première bande, à la racine de l'aile, est très étroite, saillante et séparée de la racine d'un blane sale, par une raie d'un gris presque noir. Les antennes du mâle sont alternativement gris foncé et blanc pur.

Paraît préférer tes alpes occidentales, qu'elle habite jusques près de Grenoble. Varie beaucoup, sans perdre ses caractères spécifiques (Meyer).

280. Infidaria. nob.

Flavicinctaria. Dup. hist. t. 8. $1^{\mathrm{e}}$ part. p. 400. pl. 199. - Br. cat. n ${ }^{\circ}$ 706. - Fr. n. B. Tab. 390. fig. 4.

Les bois de sapin sombres des montagnes et même de la plaine; Jorat de Lausanne; el juillet (le 7); pas très rare (Lah.). - Dans l'Oberhasli; mèmes localités; fréquente (Meyer). - Bois du Jura (Rothb.).

Duponchel, qui le premier a bien distingué cette espèce, l'a derechef confondue 
avec caesiaria dans son Catalogue, entrainé par l'autorité de Boisd. qui lui-même copiait Treit. - Le caractère saillant, noté par Dup., devait cependant suffire. Le sinus profond du bord interne de la bande médiane est Irès caractéristique.

Les atomes jaunes sont distribues comme dans la vraie flavicinctaria. Le sommet des supérieures est encore plus aigu que dans caesiaria. Le ruban blanc (1 ${ }^{\text {er }}$ quui précede la bande médiane est large comme dans caesiaria et presquie privé de jaune.

Les individus gris sont des exemplaires ternis; jamais je n'ai pu m'en procurer un frais.

281. Tophacearia. W. V.

Hub. 309. - Treit. - Dup. cat. - Bdv. 1776. - Her.-Sehf. I. 198, 199. II. 114. - Heydenr. Cat. 438.

Propre aux vallèes des bautes alpes, oủ elle n'est pas raré. Près de Wasen, au pied du Gotthard (Bremi). - Les alpes; assez commune, vers le 25 juillet (Rothb.). Commune près de Meyringen; les sous-alpes (Meyer). - Je doute fort qu'elle existe près de Besançon (Bruand, cat. $n^{\circ} 699$ ).

\section{Nebularia Treit.?}

$$
\text { Fr. B. 66, 2. - Hub. 571. - Dup. sup.? - Bdv. 1617? }
$$

Her.-Schf. fig. 370,371 . II. 146. - Heydenr. Cat. 386.

Cette espèce fort rare est encore douteuse pour la Suisse; elle a été trouvée à Meyringen, par Mr. Ott (Meyer). - (Collection Meyer). - Dans le Doubs (Bruand).

Les figures de Freyer, de Hubner et de Duponchel sont presque méconnaissables. Facies de salicaria pảe et sans teinte jaune. Antennes filiformes.

283. Incultaria. Her.-Schf. fig. 372. II. 147.

$$
\text { I gnobiliaria. Mann cat. - Zell. - Heydenr. Cat. } 444 .
$$

Fréquente dans l'Oberhasli (Meyer). - Les alpes vaudoises; la Furca; le St. Gotthard, ete.; à la fin de juin et en juiltet (Lab.). - On la prend rarement bien fraiche. Varie assez. Les rubans blancs sunt parfois ètroits et couverts de taches grises; d'autrefois ils sont larges et d'un blanc laiteux, la bande médiane est alors lavée de blanc sale. Les inférieures sont aussi plus ou moins grises avec une bande plus claire, ou bien d'un blane sale et sans bande. Les antennes du mâle sont filiformes, tomenteuses en 
dessous. Les femelles paraissent plus fréquentes que les mâles. Dans les échantillons trẻs frais le dessin est gris, taché de verdàtre.

Sans l'indieation de Mr. Mann je n'eusse pas reconnu son ignobiliaria dans l'ineultaria de H. S.; je m'ètonne que le premier nom n'ait pas été conservé.

Cette espéce appartient au groupe rivularia, bydraria, ete. Elle présente les mêmes chevrons sur les nervures que podevinaria. On pourrait aussi bien lui rapporter la figure 273 de Hub. qu’á cette dernière.

281. Achromaria. nobis fig. 8 .

Celte espèce n’a été prise jusqu'ici qu’a Meyringen, par Mr. Ott. Le mâle que j'ai figurè existe dans la collection de Mr. Meyer; je possède deux femelles, un peu passées.

Elle ressemble beaucoup à lotaria pour le dessin et la taille; mais les antennes du mảle sont filiformes. La couleur générale est le fauve pále; le fond est couvert de beaucoup d'atômes fins, brunâtres, qui lui donnent un aspect grisàtre. Le dessin est très peu saillant. La base, la lande médiane et la marge sont d'un gris-roux, séparées par deux rubans plus elairs, recouverts eux-mêmes de lignes grisâtres qui les rendent indistincts. La bande médiane est plus foncée vers le bord interne et offre une éelaircie à son milieu vers la eôte; sur l'èclaircie se voit à peine un point gris. - Le bord externe de la bande est très sinuè et très dentelè; il forme trois angles saillants. séparés par autant d'enfoncements. Le second ruban (bistriẻ) est plus visible que le premier; les lignes qui le couvreut le confondent avec la marge. La ligne ondulée n'est indiquée que vers le sommet. Celui-ei est assez aigu et présente deux taches plus foncées. La frange est faure-pâle, entrecoupée de gris, borlèe d'une série de doubles-points bien visibles.

Les infẻrieures sont fort pâles. Leur base est grisâtre, marquẻe par un angle presque droit sur le centre de l'aile. Vient ensuite une bande plus claire, mal limitée. La marge est derechef enfumée, et la ligne ondulée y apparail sous forme de points plus clairs.

Abdomen annelé de fauve. Dessous d'un blane roux, sali de gris sur les bords et la base. - Quatre points distincts. - Paltes enfumées. Tubercules des antennes carrés, peu saillants et pubescents chex le màle. - Palpes très courts. - Femelle plus pâle que le mâle. La nervation de la cellule des inlérieures est trés différente de celle des espèces voisines et la rapproche de cyanaria. 
285. Palumbaria (ta). F.

W. V. - Hub. 221. - Dup. - Bdv. 1606. - Treit. - Her.-

Schf. 11. 154. - Heydenr. Cat. 109.

Luridata. Götze. - Brahm.

Mucronata. Devill. - Scop.

Plumbaria. Esp. - Wood 543.

Commune dans les bois taillis secs des environs de Lausanne. Parait une premiere fois en mai (le 7), peu nombreuse; puis une seconde fois en août (Lah.). - Rare dans la Suisse centrale; près de Bienne du 21 juin au 3 juillet. Une seule apparition (Rotbl). - Meyer). -- Assez fréquente dans les environs de Zurich (Bremi).

Cette espèce préfère les expositions méridionales.

286. Plagiaria (ta). Lin.

W. V. - Hub. 220. - Treit. - Dup. - Bdv. 1633. - Her.-

Schf. II. 156. - Wood 596. - Heydenr. Cat. 420.

Duplicata. F.

Cá et là dans les vergers et les haies, le long du Léman; pas rare à la fin de mai et en juin; puis à la fin de juillet et en août (Lah.). - Commune sur les collines sèches des environs de Zurich, I'Uto etc. (Bremi). - Près de Schüpfen du 10 mai au 14 juin, puis du 20 aoùt au 18 septembre. Assez fréquente (Rothb.). - Fort commune près de Burgdorf sur les pentes exposées au grand soleil. Vole de jour; fin mai et miseptembre (Meyer). - Deux apparitions; la première ne se montre guère que sur les collines exposées au soleil; la seconde est plus répandue, suivant les années. Une variété plus pảle et plus petite sur les prairies élevées du Jorat, en août (Dup. hist. 1. 8. pl. 195. f. 3.). L'apparition du printemps a surtout lieu le long du lac, et celle d'automne sur les collines du Jorat (Lah.).

287. Cassiaria (ta). Treit.

Her.-Schf. Il. 157. - Fr. n. B. 528. - Heydenr. Cat. 419.

Praeformaria. Hub. 532, 533. - Dup. - Bdv. 1634.

Performata. Wood 597.

Pas fort rare dans les bois et les taillis humides des sous-alpes et des montagnes: les Ormonts; une fois à Lausanne même, en juillet (Lah.). - Rare dans les Alpes, le 
13 juillet (Rothb.). - L'Oberhasli. Assez commune au mois d'août sur les pâturages de la Grindelalp: 6000 pieds s. m., oủ l'on trouve de très grands exemplaires. Vine seule fois prise a Burgdorf sur les pentes de l'Oberthal (Meyer). - Les bords des bois; assez $\operatorname{rare}_{e}$ (Bremi).

288. Sororaria (riala). Hub. 35.5.

Treit. - Dup. - Bdv. 1632. - Her,-Schf. II. 158. - Fr. n. B. 528. - Heydenr. Cat. 424.

Imbutata. Hub. 403. - Bdv. 1631. - Wood 595.

Paludala. Thunb.

Treitschke indique cette espèce en Suisse. Boisduval l'a copiè; je l'ai reçue de Mr. Anderegg; elle est plutôt originaire des montagnes de l'Allemagne et du Nord.

289. Moeniaria (ta). IV. V.

Hub. 298. - Bork. - Treit. - Dup. - Bdv. 1609. - Her.Schf. 1I. 160. - Heydenr. Cat. 525.

Unidentata. Wood 551 .

Prise une première fois au commencement de juin et une seconde fois en août, dans un bois près de Lausanne, au milieu des bruyères (Lah.). - Commune près de Bienne du 14 au 30 aov̂t (Rothb.). - Très rare près de Zurich; les taillis de l'Uto (Bremi).

290. Chenopodiaria (la). Lin.

W. V. - Bork. - Hub. 299. - Dup. Bdv. 1746. - Her.Schf. II. 161. - Heydenr. Cat. 530.

Comilata. Hlub. Verz. - Wood 589.

L a rv. Hub. Geom. 1I. Aequiv. M. b.

Rare dans les environs de Lausanne. Parmi les orties, dans quelques haies; en juillet et en aoùt (Lah.). - Pas rare près de Zurich; au bord des bois; le Zurichberg (Bremi). Une femelle près des moraines du glacier du Rhône à la hauteur d'environ 6000 pieds, le 7 août 1850 (Meyer). 
291. Mensuraria. W. V.

Hub. 193. - Treit. - Dup. - Bdv. 1607. Her.-Scht. II. 162. - Heydenr. Cat. 110.

Chenopodiaria. Esp. - Wood 545.

Très comıune dans les taillis et les bois au milieu de l'ètè. S'élève jusque dans les hautes alpes; elle fourmille sur les pâturages des montagnes vaudoises en juillet et en août (Lah.). - Commune près de Schüplén du 10 juillet au 28 août (Rothb.). Mèmes loealités dans le canton de Zurich (Bremi).

Varie beaucoup dans l'intensité du brun et dans la nettete des bandes.. Cervinaria Treit. n'existe pas en Suisse; on ne la prend que dans le Riesengebirge. Jignore oủ Mr. Bruand a pris l'indication de son habitation sur le Jura; il y a là certainement une erreur (eatal. $n^{\circ} 5-7$ ).

292. Miaria (ta. W. V.

$$
\text { Bork. - Treit. -- Dup. - Bdv. 1627. - Hub. 292. - Her.- }
$$
Scbf. II, 167. - Wood 556. - Heydenr. Cat. 578.

Viridaria. lin. - F.

Deleataria. Thunb.

$$
\text { Pectinaria. Knoch. }
$$

Pas rare dans les bois de hêtre, vers la fin de juin (Lah.). - A Schüpfen du 4 juin au 2 juillet; commune (Rothb.). - Les jardins (?) des environs de Zurich; assez fréquente (Bremi). - Fort commune dans la région sousalpine; vallèes d'Oberlasli, Gadmen, etc. (Mleyer).

293. B ipunctaria. Bork.

W. V. - Hub. 276. - Treit. - Dup. - Bdv. 1616. - Her.-

Schf. II. 169. - Wood 547. - Heydenr. Cat. 111.

Undulata. Scop.

Commnne le long des murs et des rochers exposés au soleil, contre lesquels elle se pose; juillet et aoùt (Lah.). - A Schüpfen dès le 27 juin à la fin d'aoút; fréquente (Rothb.). - Mèmes localités près de Zurich; dans les Grisons, etc. (Bremi, Mengold). - Manque totalement dans le Bas-Emmenthal, tandis qu'elle est fort commune dans tes 
montagnes ealcaires autour d'Interlaken et sur les pentes rocailleuses du Seitenberg, à Böningen, près de Meyringen, etc. (Meyer).

Je possède des exemplaires du Valais, d'un gris presque noir. Cette espèce bat des ailes en se posant, comme mensuraria et palumbaria.

291. Vespertaria. Lin.

Bork. - W. V. - Hub. 226. - Bdv. 1618. - Fr. B. 107, 2.

Dup. eat. -- Her.-Sehf. II. 170. - Heydenr. Cat. 101.

Brunnearia. Devil.

Quelquefois dans les haies des environs de Lausanne; fin d'aoủt (Lab.). - Les haies a la fin d'aoůt, dans les environs de hurgdorf, de Meyringen, etc.; pas rare (Meyer). - Sur les rochers du St. Gotthard (an rupestraria?) (Bremi). - Du 22 aoùt au 13 septembre, près de Schüpfen; assez fréquente.

La figure de Hub. est mèconnaissable.

29.5. Impluviaria ta. W. V.

Hul. 223. - Treit. - Dup. - Bdv. 1767. - Her.-Sehf. f. 193 (var.) II. 171. - Wood 610. - Heydenr. Cat. 361.

Trifaseiata. Bork.

L arv. Hub. Geom. II. Aequiv. F. a. b. fig. 1.

Les bois au-dessus de Lausanne en juin et juillet; pas rare; quelques individus égreués le long du Léman, dans les vergers, à la fin de mai. S'èlève sur les sous-alpes, etc., y parait à la fin de juin (Lah.). - Près d'Aarberg et de Schüpfen du 20 juin au 12 juillet; peu rare (Rothb.). - Sur le Righi au milieu des rochers, dans le mois d'aoút, fréquente; dans les Grisons (Bremi. Mengold). - Très commune dans le canton de Berne (Meyer).

Quoiqu'elle paraisse dès la fin de mai jusqu'en août, je doute très fort qu'elle fasse deux apparitions; ees diffẻrences se lient à la diversité des localités; car on la voit successivement paraitre en mai et en juin dans les lieux plus chauds; en juin et juillet sur les montagnes, et en aoùt dans les alpes plus élevées. Ce fait se reproduit ehez la plupart des phalènes qui habitent à des hauteurs différentes. Lorsqu'il s'agit d'une seconde génération dans l'annèe, il importe de savoir si elle a lieu dans la même localitẻ que la première. 
296. Ruberaria (ta). Fr. n. B. 36,2

Her.-Schf. fig. 209, 210. - II. 172. - Heydenr. Cat. 360.

Cette espèce, recueillie dans les Grisons, près de Coire, y a été prise par Mr. Bredow, dans l'été. Je ne l'ai pas encore vue en nature (Lah.). - Her,-Sehf. lui donne deux caractères distinctifs: 1) la bande claire moyenne, plus couverte d’atómes que dans impluviaria el presque de la couleur du fond; 2) les deux filets rougeâtres avec les taches de même coulenr; les premiers sur les bords de la bande médiane; les secondes vers la marge. De ces deux earacteres le premier seul me parait avoir quelque valeur; mais je l'ai retrouve sur des exemplaires d'impluviaria, provenant des Alpes. Quant au second, il pourrait bien u'être qu'une modification de certaines teintes verdàtres, analogue à celle que présente elutaria, aeruginaria, etc. Il faut ici de nouvelles observations $($ Lah.). - Freyer la compare avec elutata et lui donne d'autres caractères: 1) les ailes supérieures plus alongées; 2) et sur lenr dessons une seule bande, raceourcie derrière le point, tandis qu'elutata en a deux. Ces deux caractères sont très vagues. En tout cas ruberata est plus voisine d'impluviaria que d'elutaria. Je crois que tous les individus, provenant de Meyringen et de la région sousalpine, lui appartiennent, car ils ont les ailes alongées, qu'ils aient ou non au-dessous des ailes supérieures une seconde bande, assez visible (Meyer).

297. Elutaria (ta). W. V.

Hub. 224, 381 - 385. - Treit. - Dup. - Bdv. 1766. - Her.Schf. II. 173. - Wood 611. - Heydenr. Cat. 359.

L arv. Hub. Geom. II. Aequiv. F. a b. fig. 2.

Fréquente dans tous les bois sombres et frais où croît le myrtile, en juin et juillet (Lah.). - Gadmentbal; Burgdorf, etc.; commune: en juin (Meyer). - De même dans le canton de Zurich (Bremi). - Commune près de Schüpfen et de Langnan, du 24 juin an 7 aoùt (Rothb.).

La variété brune est un accident postérieur à l'èelosion et qui résulte de l'exposition à quelque vapeur légèrement acide; on la produil à volonté. - Varie souvent en grandeur et plus encore dans les dessins et les couleurs. 
298. Suffumaria (la). W. V.

Hub. 306. - Treil. Bdv. 1761. - Dup. sup. - Her.-Sehf.

II. 174. - Wood 582. - Heydenr. Cat. 546.

Pic ata? Bruand catal. $n^{\circ} 698$.

A la lin de mai et au commeneement de juin, dans quelques localites restreintes des forèts de sapin du Jorat, au-dessus de Lausanne. Très rare (Lah.). - Au commencement de juin près de Burgdorf; fort rare (Ileyer). - Les taillis de l'Uto; moins rare près de Zurich (Bremi). - Très rare dans le canton de Berne; Langnan; Berne; Schüpfen; 23 mai (Rothb.).

Un amateur de lierne l'élève toutes les années de chenille.

299. Ribesiaria. Bdv. 1762.

Dup. cat - Her.-Sehf. II. 175. - Heydenr. Cat. 547.

IPrunata. Lin. - W. V. - Hub. 304. - Bork. - Dup. hist. - Wood j81.

Peu rare au milieu de l'été, partout (I,ab.). - Aarberg; Schüpfen, du 10 juillet au 2 septembre (Rothb.). - Se trouve le plus souvent au plafond des pavillons de jardin et vole volontiers le soir dans les chambres éclairèes. - Conmune à Burgdorf (Neyer).

300. Silacearia ta). W. V.

Hub. 303, 477, 478. - Treit. - Dup. - Bdv. 1763. - Her.Schf. II. 177. - Wood 571. - IJeydenr. Cat. 549.

L arv. Hub. Geom. II. Aequiv. M. b. fig. 1 .

Prés de Schüpfen, assez fréquente, du 21 mai au 26 juin; puis du 17 an 31 aoủt; deux apparitions (Rothb.). - Près de Burgdorf (Meyer). - Les pentes de l'Uto; assez rare (Bremi). - Prise une fois près de Lausanne, au milieu de mai, une seconde fois en juin et une troisième a la fin de juillet, par Mr. Leresehe. Je l'ai prise dans les bois des sous-alpes d'Aigle, en juin (Lah.).

Varie beaucoup dans la forme de la bande médiane et présente souvent des variétès méconnaissables. L'une des plus belles est celle, oủ le bord extérieur de la bande forme une série de longues pointes (Meyer). 
301. Reticularia (ta). W. V.

Hub. 308. - Treit. - Bdv. 1764. - Dup. sup. - Her.-Schf. II. 178. - Heydenr. Cat. 551.

Prés de Meyringen en juillet (Meyer). - Les Alpes. Environs de Sehüplen; très rare (Rothb.). - LUto près de Zurich; très rare (Bremi). - Près de Seen Rordorf).

Trouvee par Mr. Heuser, le 12 juin, sur le Jura, près de l'hôtel du Weissenstein, à 4000 pieds (Meyer).

302. Psittacaria (ta). F.

W. V. - Hub. 227. - Treit. - Dup. - Bdv. 1667. - Her.Schf. II. 179. - Wood 609. - Heydenr. Cat. 430.

Coraciata. Hub. 278. - Treit. - Dup. - Bdv. 1668. - Heydenr. Cat. 431.

Pas Iréquente dans les environs de Lausanne; en mai; puis en automne (septembre) Lah.). - Près de Schüpfen du 9 mai au 14 juin; puis du 20 août au 1 octobre; pas rare (Rothb.). - Le 6 septembre 1848 en grande quantité et par vols au bord du Sommerbauswald, près Burgdorf, sous les chênes. Ces vols se répètèrent trois soirs de suite quelques minutes après le coucher du soleil. Femelie rare (Meyer). - Les vergers des environs de Zurich; pas rare (Bremi). - Près de Coire; mèmes localités (Kriesehb.).

Lidentité de psittaearia et de coraciaria ne me parait pas suffisament établie, quoiqu'en dise Her-Sehf. Ce point reste à éclaircir. Je n'ai vu qu'un seul mauvais échantillon de la dernière. Mr. Bremi indique celle-ci sur les alpes; au Rhigi. (?)

303. Firmaria. Treit. sup.

Hub. 515. - Bdv. 1755. - Her.-Schf. f. 237-239. II. 180. Heydenr. Cat. 309.

Simulata. Wood 634 .

Cette espèce très rare a été trouvèe près de Schüpfen par Mr. Rothenbach. 
304. Fulvaria (ta). IV. V.

$$
\text { Bork. - Hub. 297. - Treit. - Dup. - Hub. Beitr. Bdv. }
$$

1747. - Her.-Schf. H. 183. - Wood 561. - Heyd. Cat. 528.

Sociata. F.

Cuspidata. Thunb.

Larv. Hub. Greom. II. Aequiv. M. a.

Assez fréquente, dans les haies des environs de Lausanne, en juillet (Lah.). -. Du 22 juin au 21 juillet près de Schüpfen; assez commune (Rothb.). - De même près de Zurich (Bremi). - A Burgdorf (Meyer).

La chenille vit sur les rosiers; MN. Bremi et Rothenhach l'ont élevée sur le premier rosier venu.

305. Popularia (ta). Lin.

$$
\begin{aligned}
& \text { F. - W. V. - Treit. - Dup. - Bdv. 1743. - Hub. } 300 . \\
& \text { Her.-Schf. Il. 185. - Wood 590. - Heydenr. Cat. } 529 .
\end{aligned}
$$

Très commune en èté sur les prairies des alpes; moins fréquente dans la plaine (Lah.). - Les haies sur le Zurichberg; pas rare (Bremi). -- Da 21 juin an 30 aoùt, dans les contrées alpines du canton de Berne; assez fréquente (Rotbb.).

La chenille ne peut vivre uniquement sur le tremble, car on trouve le papillon en abondance sur les hautes alpes où n'existe aucun peuplier, à peine quelques rares saules. - Paraît avoir deux apparitions. Je l'ai prise sur la Meyenwand á 5000 pieds, d'abord à la mi-juin, puis au mois d'aoủt. Dans ces régions le dessin est bien plus vif (Neyer).

306. Pyraliaria (ta). F.

W. V. - Hub. 302. - Treit. - Dup. - Bdv. 1745. - Her.-

Schf. II. 186. - Fr. .n. B. 444. - Wood 594. - Heydenr. Cat. 532 .

Rare près de Lausanne: les haies touffues; en juillet (Lah.). - Dans le Valais; près des bains de Louësche; fréquente. Dans le Jura, près de Bienne; rare (Rothb.). Près de Seen (Rordorf). - Très rare autour de Burgdorf (Meyer). 
307. A chatinaria (ta). Hub. $301,408$.

Treit. - Dnp. - Fr. n. B. 198, 1. - Bdv. 1742. - Her.-Sehf.

II. 187. - Wood 593.

M a ru orata? Bruand Catal. no 687.

Testata. Wood 492.

Une fois sur le mont de la Tour de Gourze au commencement de juillet; une seconde fois dans les bois du Jorat à la fin d'août (Lah.). - A Schüpfen do 6 au 8 octobre; rare (Rollib.).

Il est probable que cette espèce a deux apparitions dans nos contrées. - La figure de Dup. est mauvaise.

Marmorata appartient à l'Allemagne orientale; elle y est fort rare.

308. Russaria (ta). W. V.

Hub. 305 (non 445). - Bork. - Treil. - Bdv. 1765. - Dup.

Fr. B. 18, 1. - Her.-Schf. II. 189. - Heydenr. Cat. 553.

Centumnotata. Fabr. - Wood 578.

Immanata, amoenata, marmorata, concinnata, commanotata et saturata.

Wood 573-577. Heydenr.).

Peu fréquente dans les environs de Lausanne; une première fois en mai; une seconde en août (Lah.). - Commune près de Schüpfen à la fin de mai, puis du 3 juillet au 14 aoùt (Rothb.). - De mêue près de Zurich (Bremi) - et de Burgdorf (Mleyer).

309. Perfuscaria (ta). Haworth, (teste Curtis ipse).

Stph. 6577. - Curt. Cat. - Wood s80.

Russata. Hub. 445 ? - Treit. (var.)

Acutata? Guèn. - Broand Catal. nº 689.

Après avoir vn plusieurs échantillons de cette espèce, je suis demeuré convaincu que les auteurs anglais avaient raison de la séparer de la précédente. Je n'ai point observé d'individus intermédiaires; l'espèce varie fort peu.

Elle a été prise par Mr. Leresche dans les environs de Lausanne en juillet, et autour de Zurich, par MM. Bremi et Zeller, à la même époqne. - Je l'ai prise près de Lausanne au milieu de septembre (Lah.).

La femelle ressemble entièrement au mâle. Les caractères qui la distingnent de 
russaria sont: 1) une taille constamment plus petite; 2) le dessin de la base des supérieures peu marqué et moins découpé; 3 la bande moyenne entièrement d'un grisroux foncé pointillé, sans stries festonnèes du côté de la racine de l'aile; 4) la ligne ondulée de la marge moins saillante, surtout vers son milieu, avec des dentelures plus aigaes; 5) les ailes inférieures plus alongèes, avee un angle saillant sur le disque, sans taches le long de la marge; 6) l'angle externe de la bande médiane des supérieures prolongé et aigu. Chez une femelle je l'ai vu moins saillant, mais toujours plus qüil n'est représenté dans la figure de Hub. - Quant à cette dernière, elle peut se rapporter mieux à l'une des espèees de Wood, qu'à la nôtre. Ses ailes arrondies, ses teintes foncées, sa bande arrondie sur le bord postèrieur, ne se rapportent ni à russaria, ni à perfusearia.

310. Ruptaria (ta). Hub. 295.

Treit. et sup. - Dup. - Bdv. 1750. - Fr. n. B. 24, 1. Her.-Schf. II. 190. - Heydenr. Cat. 554.

Corylata. Wood 572.

La rv. Hub. Geom. II. Aequiv. M. a.

Cette espèce peu commune se trouve ca et là dans toute la Suisse; elle habite les taillis et les bois fenillés, en mai et en juin. Sanvabelin près de Lausanne (Lah.). Pas rare, à Schüpfen du 11 mai au 29 juin (Rothb.). - Les bois de l'Uto; rare (Bremi). - Près de Meyringen, de Burgdorf (Sommerhauswald); commune dès le milicu de mai au milieu de juin (Meyer). - Varie beaucoup.

311. Variaria (ta). IV. V.

Hub. 293. - Treit. et sup. - Dup. bist. (descript.). - Bdv. 1749. - Her.-Schf. II. 192. - Heyd. Cat. 306. - IVood 635. Juniperata. Dup. hist. (fig.). - Fulvata Fab. - Pinetata Bork.

Var. vitiosata. Hub. 380 (non 337) - (an Her.-Schf. fig. 296-298)? Heydenr. Cat. 307.

Var, obeliseata. Hub. 296. - Treit. - Dup. hist. (deseript. non fig.). - Her.-Schf. fig. 240- 242. - Heydenr. Cat. 308.

Larv. Hub. Geom. II. Aequiv. $\xi$. b. fig. 1.

Très commune dans les bois de sapin dès la fin de mai jusqu'au commencement de juillet; reparaît an commeneement de l'autonne, mais en petit nombre, sur le baut du 
Jorat (Lah.). - Du 16 mai an 21 jnin; du 8 août au 23 septembre, à Schüpfen; très coiomnne (Rothb.). - De même près de Zurich (Bremi). - S'élève sur les alpes jusqu’à la limite des bois, et s'y montre à la fin de juin et en juillet. En grande abondance du 8 an 13 juin dans toutes les forêts de sapin, au pied du Jura, près de Soleure (Meyer).

La femelle est tonjours plus grande et moins colorée que le mâle. - Dup. unit à variaria la figure de juniperata avec des couleurs exagérees, et sous le nom de juniperata male il représente variaria femelle (pl. 206, fig. 3 et 4).

Obeliscata est fort rare; elle a èté prise te 8 juin prẻs de Schüpfen, par Mr. Rotbenbach. Les figures $380 \mathrm{Hub}$. et 60,3 de Fr. n. B. sont tellement imparfaites quil est difficile de dire si elles se rapportent à une variété de fluetuaria, de variaria, on méme de stragularia. Prises sur des individus altérés, elles ne peuvent servir.

Si Obeliscata Hub. n'est réellement qu'une variète de variaria, il pourrait bien en être de même de simularia. Malgré l'autorité de Treit. la question de ces deux variètés ne me parait point jugée dèfinitivement. J'ai recueilli un très grand nombre de formes, mâles et femelles, de rariaria dans des localitès très diffèrentes, depuis la lin de mai jusqu'en septembre, et je u'ai jamais rencontré obeliscata, ni rien qui en approche. Un individa, provenant de Mr. Rothenbach, a les nervures saillantes et noiràtres, ce que je n’ai jamais vu dans aucuue variaria où le contraire a plutố lien. Les mêmes confusions, signalées à l'oceasion de bastaria, de galiaria et de leurs variétès, pourraient bien se reproduire ici.

312. Stragularia (ta). Hub. 3:37.

Bdv. 1794? - „non Treit.)

Vitiosata? Fr. I. B. $60,3$.

Variariae var.? Her.-Sehf. fig. 296-298. - Hub. 384?

Rare dans les bois des environs de Lausanne; on la prend difficilement fraîche. Jus. qu'ici je n’ai po saisir que des màles. MI. Bremi et Rothenbach possèdent chacun une femelle (Lal.).

Malgré l'autorite de Her.-Schf., je ne puis encore admeltre que stragulata Hulb. ne soit qu'une varièté de variaria. Voiei les differences que j’ai constatees: 1 le sommet des superieures est plus arrondi dans stragularia que dans variaria; 2, le commeneement de la bande médiane de la première n'offre aucun angle saillant du cóté du sonmet de l'aile, là où la seconde en présente un très prononcé; 3 l'angle central 
formé par la bande, sur les inférieures, est plus alongé dans stragularia, et la ligne ondulèe s'y dessine micux sur un fond plus foncé; 4) la femelle de la première est parfaitement semblable au mâle, dans la scconde elle est constamment différente de lui; 5) il n'existe pas d'individus faisant passage d'une espèce à l'autre. Les exemplaires de variaria qui ont une teinte jaunàtre autour de la bande médiane sont précisément ceux qui s'éloignent le plus de stragularia par leur bande très prononcée; 6) je n'aj jamais vu variaria fraiche présenter la teinte gris-noire de la bande médiane de stragularia; 7) la ligne ondulée de variaria a les dentelures plus aigües; 8) la cellule des inférieures de celle-ci est plus alongèe.

La figure de Hub. 337 est trop vivement coloriee. Il est probable que Bdv. avait notre stragularia sous les yeux. Les synonymes de Freyer et de Her.-Schf, ainsi que la figure de Hub. 380, sont incertains. Stragulata Hub. (vitiosata Fr.) se trouve placèe, dans le musée de Vienne, tout à côtẻ de fluctuaria; elle est spécifiquement distincte de variata, tandis qu'obeliscata ne l'est pas (Mann).

313. Juniperaria (ta). Lin.

W. V. - Bork. - Hub. 294. - Treit et sup. - Dup. idescript. non fig.) - Fr. n. B. 108, 1. - Bdv. 1748. - Her.-Schf. II. 193. - Wood 638. - IIeydenr. Cat. 304.

L arv. Hub. G. IJ. Aequiv. J. b. fig. 2.

Cette espèce, propre aux régions septentrionales, descend quelquefois jusqu'au pied des Alpes, dans les forêts de sapin; elle s'èlève sur les hautes Alpes avec le genièrrier, dont la chenille se nourrit. Montagnes d'Aigle en septembre et en octobre; assez fréquente (Lah.). - Meyringen, etc. (Ott). - A Schüpfen dans le milieu d'octobre; rare (Rothb.))

314. Tersaria (ta). W. V.

Hub. 268. - Treit. - Dup. - Bdv. 1652. - Her.-Schf. II. 198.

- Wood 612. - Heydẹnr. Cat. 403.

A enulata. Hub. 448.

Larv. Hub. G. III. A. I. a-d (Aemulata).

Pas fréquente dans les environs de Lausanne, en juin; pẻnêtre dans les maisous; s'applique contre les murs et les clotures Lah.). - De même près de Burgdorf (Meyer). 
- Parait plus rare dans la Suisse septentrionale; vallèe de Maderan (Bremi). - Aarberg; Moudon, le 9 juin; rare (Rothb.).

Cette espèce varie souvent.

Tersaria a deux apparitions par an. Mes individus sont pris le 21 avril, le 8 mai et le 9 juillet. Ceux de la seconde génération ont une teinte moins brunatre et le dessin plus effacé (Meyer).

315. Vitalbaria (ta). W. V.

Hub. 269. - Treit. - Dup. - Her.-Schf. II. 200. - Wood 613. - Heydenr. Cat. 184.

Pas rare dans les haies des environs de Lausanne, d'abord en juin, puis une seconde fois en août (Lah.j. - Près d'Aarberg, au mois d'août; rare (Rothb.).

Espèce omise dans Boisduval.

316. Fluviaria (ta). Hub. $280,281$.

Treit. - Dup. cat. - Bdv. 1645. - Her.-Schf. II. 202. - Heydenr. Cat. 397.

Cette espèce, très rare, a èté prise deux fois dans les environs de Lausanne, au mois de juillet, par Mr. Leresche (Lah.). - (Collection du musée de Lausanne.

317. Gemmaria (ta). Hub. 283.

Treit. sup. - Bdv. 1644. - Dup. sup. - Her.-Scbf. fig. 345.

II. 204 (non Bork.).

Cette espèce a èté prise par Mr. Bruand sur la frontière suisse de Pontarlier: j’ai vu un individu, provenant de cette localité. Mr. Zeller l'a trouvée aussi près des hains de Louësche (Valais) - (Lah.).

Gemmaria Bork. est rhomboïlaria.

G. VII. Clieimatobia. Steph. Dup.

(Operobia. Stph. - Operothera. Deuts. ins. - Acidalia. Treit. Larentia. Bdv. - Hybernia. Curt.)

Nota. Brumaria ne peut se séparer de dilutaria. La nervation est celle de la première division du genre précédent. Ni les antennes péunicillées du mâle, ni l'al,sence d'aile chez la femelle, ne peuvent motiver une séparation. 
318. B rumaria. Lin.

$$
\begin{aligned}
& \text { Esp. - W. V. - Hub. 191, 415, } 509 \text { \&. - Dup. - Bdv. } \\
& \text { 1670. - Her.-Schf. fig. 352. \&. II. 206. - Wood 640. - } \\
& \text { Heydenr. Cat. 297. - Denkschriften der schweiz. naturforsch. } \\
& \text { Gesellschaft: 1. Bd., 2. Abth. pag. } 88 .
\end{aligned}
$$

Larv. Hub. G. II. A. B. b. fig. 2.

Très commune partout ou il y a des arbres fruitiers dans le mois de novembre, surtout alors que la température est plurieuse. Lorsque novembre est très froid, elle retarde son apparition jusqu'en décembre, en profitant d'un dégel. Abonde surtout du 1 an 20 novembre dans les environs de Lausanne (Lab.). - A Schüpfen du 19 octobre au 1 novembre (Rothb.).

Dans le repos complet, durant le jour, le papillen tient ses ailes abaissées comme toutes les phalènes; ce n'est que le soir, lorsqu'il est en quête de la femelle, qu'il les a relevées quoique pasé.

Les antennes sont fortement crénelées en-dessous et garnies de deux rangees de poils, assez longs et pennicilles, comme dans plusieurs acidalia.

319. Borearia (ta). Hub. 113, 414.

Treit. sup. - Bdv. 1671. -- Dup. sup. - Her.-Schf. fig. 353.

9. HI. 20\%. - IJeydenr. Cat. 298.

J'ai pris deux fois cette espèce dans les bois des environs de Lausanne, en mème temps que brumaria. Sa taille est un peu au-dessus de celle de cette dernière et la cellule des inférieures plus alongée.

\section{G. VIII. Lobophora. Curt. Bdv. Dup. (Acidalia. Treit.)}

Nota. Le plus artificiel de tous les genres, quoique fondé sur un caractère unique et saillant. Les ailerons surnuméraires résultent d'un reploiement du bord interne des inférieures, avec séparation par fissure de la nervure anale. Dans Anaïtis Búv. la même nervure avorte sans se reployer. La nervation varie beaucoup. Polycommaria, viretaria el sexalaria l'ont semblable à la première division des Larentia. La première se place à cóté de variaria, la seconde et la troisième non loin de dilutaria. - Lobularia et sabinaria ont la cel- 
lule construite sur un plan particulier qui n'existe nulle parı dans les phalènes; la scapulaire se détache de l'angle extérieur de la cellule et le ramean interne de la médiane externe se eonfond avec l'anastomose et la suit pendant un instant, pour s'en séparer un peu avant le noeud; le reste se rapproche de la forme $b$ de Larentia. Hexapteraria reproduit la forme de la cellule d'Anaïtis; mais l'anastomose s'insère au-delà de la bifurcation et uou avant elle.

\section{A ppendicularia. Bdv. 1673 .}

Dup. cat. - Her.-Sehf. fig. 366-369. II. 208.

Sertata. Hub. 489, 490. - Treit. - Dup. hist.

Ir. Bremi l'a prise dans les bois du Zurichberg, au mois de mai. - Prés de Berne, une fois (Rothb.).

321. Poly commaria (ta). Hub. 190.

IV. V. - Treit. et sup. - Dup. (descript. non fig.) - Bdv. 1674. - Her.-Sehf. II. 209. - Fr. n. B. 522. - Wood 642. Heydenr. Cat. 366.

Larv. Hub. Geom. II. Aequiv. G. a. b. fig. 2.

Quelquefois dans les environs de Lansanne, en juillet; les bois (Lah.). - Sur l'Uto, près de Zurich; rare (Bremi).

Je doute que eette phalène habite les Alpes. Elle parait avoir deux apparitions, une en avril, l'autre en juillet. Dans la figure $6 \mathrm{pl}$. 206, Dup. donne une mauvaise copie de obeliseata Hub., sous le nom de polycommata et vice-versâ.

322. L obularia (ta). Hub. 362.

Treit. - Dup. - Bdv. 1675. - Her.-Schf. II. 210.

Dentistrigata. Wood 643.

Larv. Hub. Geom. II. Aequiv, G. a. b. fig. 1. e.

Cette espèce, peu rare dans certaines années en avril, disparaît entièrement pendant plusieurs autres. Autour de Lausanne dans les bois de chêne (Lah.). - Près de Schüpfen, du 31 mars au 9 mai; très rare (Rothb.). - Dans un bois d'aune à Burgdorf (21 avril); appliquéc sur les troncs d'arbre (Mcyer).

Se pose fréquemment à terre. 
323. Viretaria (ta). Hub. 2:30.

Treil. - Dup. - Bdv. 1773. - Her.-Schf. II. 212. - Wood 646. - Heydenr. Cat. 371.

Larv. Hub. G. II. Aequiv. G. b.

Rare dans toute la Suisse. Schüpfen; du 20 mai au 9 juin; très rare Rothb.). Près de Seen. Environs de Zurich, dans les bois; rare (Bremi). - Sommerhauswald, près de Burgdorf, à la fin de mai (IIcyer). - Une fois au-dessus de Lausanne au commencement de juin. Dans les alpes en juillet (Lah.).

Le mâle est plus petit que la femelle.

321. Sabinaria (ta). Anderegg.

llub. 550, 551. - Treil. sup. - Bdv. 1737. - 1)up. cat. et sup. - Her.-Schf. II. 213. - Heydenr. Cat. 303.

Espèce propre au Valais, où sa chenille vit sur la sabine. Mr. Anderegg l'élève chaque annẻe. - Les Grisons (Treit.).

\section{Hexapteraria (ta). F.}

W. V. - Hub. 232. - Treit. - Dup. - Bdv. 1676. - Her.Schf. fig. 245 9. II. 214. - Wood 647. - Heyd. Cat. 369.

Assez fréquente dans les bois de hètre, en mai (le 3); Sauvabelin près de Lausanne (Lah.). - Du 23 arril au 14 juin, près de Schüpfen; peu rare (Rothb.). - De même près de Burgdorf 19 juillet); s'élève dans les régions subalpines jusquà $\mathbf{4 0 0 0}$ pieds; Jura, Gadmenthal (Meyer). - Deux générations dans la plaine.

326. Sexalaria (ta). Bork.

Treit. - Dup. sup. - Bdv. 1677. - Her.-Schf. 11. $215 .-$ Fr. n. B. 6.

Sezalata. Hub. 228 .

Sexal is a la. Hub. Beitr. - Wood 648 .

Larv. Llub. Geom. II. Aequiv. G. a. b. fig. 1. a. b.

Çà et là au pied des alpes; partout rare; montagnes d'Aigle, en juin et en juillet Lah.). - Sur l'Ćto, prẻs de Zurich; assez rare (Bremi). - Près de Burgdorf (Meyer). - Schüpfen, du 8 mai á 11 juillet; assez rare Rotlıb.). 


\section{G. IX. Chesias. Treit. Steph. Curt. Bdv. Dup.}

Nota. Spartiaria a la cellule construite sur le plan du G. Anaïtis Bdr. - Chaerophyllaria doil former avec tibialaria un genre a part, dans les Phytométrides, quoique la scapulaire s'accole à la médiane externe jusqu'à l'angle de la cellule.

3927. Obliquaria. Bork.

W. V. - Hub. 225, 423. - Treil. - Dup. - Bdv. 1739.

Iler.-Schf. 1I. 217. - Wood 633. - Heydenr. Cat. 300.

Rufata, F.

Bombycala. Hub. Beitr.

Citee en Suisse par Treitseble; elle se tronve plutôt dans la Suisse orientale, le long du Rbin.

328. Spartiaria (ta). F.

Bork. - W. V. - Hub. 187. - Treil. - Dup. - Bdv. 1738.

- Wood 632. - Heydenr. Cat. 299. - Her.-Schf. II. 218.

Tin. legatella. W. V.

Larr. Hub. G. II. Aequiv. F. a.

Près de Langnau; très rare (Rotbb.). - Suisse orientale (Treit.). - Il ne faut pas la coufondre avee la Spartiaria de Treit. qui est la roraria d'Esp. et de Bdv., et la spartariaria de Hub. 116.

329. Cha erophyllaria (Ia). Lin.

Bork. - W. V. - Hub. 196. - Dup. - Bdv. 1933. - Her.Sehf. 11. 228. - Wood 686. - Heydenr. Cat. 207.

Atrat a. Lin. f. suce.

Très commune sur les pàturages des montagnes, surtout à leur pied; en juin et en juillet. Pas sur le Jorat. A Villenenve, au pied de la montagne, au bord des marais et du lac (Lah.). - Dans les Alpes, du 12 mai au 29 juin; quelquefois méme près de Schüpen Rothb.). - Les pâturages montagneux du canton de Zurich (Bremil. En énorme quantitè sur quelques pâturages bumides, entourés de collines boisées, autour de Burgdorf, en juin (Neyer). 


\section{Premier Supplément.}

Depuis que ce Catalogue a èté livrẻ à l'impression, plusieurs faits nouveaux rn'ont èté communiqués. Leur nombre et leur importance ne me permettent pas d'attendre pour les publier l'époque indéterminée où un supplèment séparé sera nécessaire. En les plaçant à la fin de cette publieation, ils pourront rentrer dans une table des matières unique.

Les renseignements obtenus portent le nombre des Phalénites suisses, déduction faite des espèces retranchées, à 339, pour la fin de 1852. Tous les doutes ne sont pas complétement levés à mes yeux, en publiant cet appendice; mais ceux qui restent sont en fort petit nombre.

Les planches que nécessitent les dernières observations pourrout paraître, s'il y a lieu, avec le dernier supplèment.

Je regrette extrêmement de n'avoir pas eu connaissance, avant la rédaction de mon catalogue, des longues et persévérantes recherehes de Mr. L. Couleru de la Neuveville. Elles m'auraient procurè un grand nombre de renseignements nouveaux sur les ebenilles de Geomètres, à la poursuite desquelles cet observateur s'est particuliẻrement voué. Je ferai mon possible pour qu'ils ne restent pas ignorés. Mr. Freyer a publiè une partie de ses observations; mais il en existe encore beaueoup en porte-feuille.

En terminant, je dois expliquer quelques citations abrégées dont on ne pourrait pas comprendre la signification:

H er. -Schf. se rapporte à la Révision de l'ouvrage de Hubner sur les papillons d'Europe, - planches et texte - publiè à Regensbourg par Mr. Herrich-Schäffer. Le teste est intitulé: Systematisebe Bearbeitung der Schmetterlinge von Europa. - Le cbiffre romain (I. II.) indique la première ou la seconde des deux grandes divisions des phalènites adnises par l'auteur. Le chiffre arabe désigne le $n^{\circ}$ de l'espèce.

Bruand, Cat.: ou Catalogue des lépidoptères dn département du Doubs, publie à Besançon dans les Mémoires de la Société libre d'émulation, par Mr. Th. Bruand.

Curt.: - Guide 10 a natural arrangement of british insects. - 1 édit. Hubd. 1829.

Heydenr. Cat. - Lepidopterorum europaeorum catalogus methodicus, v. Dr. Heydenreich. - 3. Aufl. - Leipzig 1851. 


\section{$\mathrm{N}^{\circ}$ 17. Incanaria W. V. - (page 19.)}

Var. calcearia. Koll. - Hub. G. fig. 589. (dilutaria). - Her.-Schf. fig. 437. - Ileydenr. Cal. 696.

J'ai reçu calcearia de Mr. Oll à Meyringen; elle ne diffère en rien des individus fournis par Mr. Mann, et provenant de Croatie. A part sa couleur d'un blane-jaunâtre (et non blenâtre), je n'ai pu découvrir ancun earactẻre qui la distinguât dinca naria.

II faut eependant avouer que si celle-ei varie beaucoup en grosseur, surtout chez les máles, elle ne fait pas de même quant à la couleur qui reste toujours le blanc plus ou moins grisàtre ou bleuâtre. Calcearia est d'un blanc mat, crétacé et un peu jaunâtre, ce qui tient à la couleur brune des atômes. La seule diffèrence dans le dessin, et qui même n'est pas bien sensible, est une grande largeur de l'espace moyen dans calcearia, mais il faut l'attribuer à ce que le dessin de la marge est plus ètroit. Heydenr. s'est mépris en cet endroit, sur l'application des synonymes de Hub.; la fig. 100 appartient évidemment à interjectaria et nullement à incanaria, tandis que la fig. 589 ne peut s'appliquer quà ineanaria, var. calcearia. Cette figure est tetlement exacte que je ne conçois pas comment Her,-Schf. ne l'a pas reconnue et ait cru devoir la reproduire de nouveau.

\section{No 18. Bischoffaria Hub. G. - (page 20.)}

Deux femelles observèes cette année (1852), à la fin de juin, et deux mâles pris à Meyringen, justifient entièrement la place que j’ai assignée à cette phalène. Ils reproduisent exactement la fig. 588 de Hub. Les deux femeltes furent prises dans tes environs de Lausanne, près des habitations.

Chez la femelle l'analogie avec inceanaria est plus frappante que chez le mâle; on serait mème tenté de l'envisager comme une simple rarièté de celle-ci, si des caractères positils ne l'en distinguaient. Le mâle de Biscboflaria est aussi foneé que la femelle, ce qui n'a pas lieu dans incanaria; la première a le fond des ailes gris-de-fer, seme d'écailles blanches; la seconde l'a banchàtre, semé d'atômes noiràtres. Celle-ci porte sur la frange une série de points noirs, outre la ligne de stries noires qui limitent te bord de l'aile; chez celle-là ees points n'existent pas et les stries sont à peine marquées.

l,es fig. de Ilub. ont peut-être contribuè à faire méconnaître cette espéce. La ligne ondulée de la marge est tout-ả-fait semblable à celle dincarnaria et nullement à la ligne fulgurale des Larentia, comme elle est représentée. - Le màle (fig. 586 Hub.) 
est très mal fait; au lieu de reproduire les deux lignes obliques des Acidalia, il est dessinè à la façon des Cidaria, à-peu-près comme albularia, ee qui le rapprocherait en effet un peu des Eupithecia, si le dessin était exact. - Cinerata Stph. pourrait bien appartenir à cette espèce-ei.

\section{Yo 32 Confinaria Fisch. v. R. -- (page 27.)}

Her.-Schf. fig. 3t5-317. I. 51. - Heydenr. Cat. 698 (non Freyer).

J'ai pris un mâle de cette espèce au pied des rochers exposés au soleil, dans la vallée d'Aigle, à la fin de jnin 1852. Les exemplaires de Croatie, reçus de Mr. Mann sous le nóm d'infirmaria, n’en diffèrent que par une teinte un peu plus foncée; lidentité tie peut être douteuse. Je suis un peu moins sûr de la désignation de Her.-Schf,, ear ses figures sont quelque peu différentes. Y aurait-il une faute d'impression dans le catalogue de Mann (1852), et confinaria serail-elle prise ici pour confirmaria F. v. B. que je ne connais pas? - Quoi qu'il en soit, notre espèce est très voisine d'immutaria. Ses antennes fortement eiliées, presque pennées, et ses tarses postérieurs dépourvus de fourreau et de poils, l'en distinguent suffisamment.

Il est fảcheux quïnfirmaria ne puisse être conservé, puisqu'il appartient déjà à une espéce décrite par Rambur et voisine d'ineanaria. Confinaria est mal ehoisi; Frejer a déjà employé cette dénomination pour une variété de pusaria.

\section{No 33. Commutaria (ta). Treit. - (page 27.)}

Cette espèce varie beaucoup, ainsi que ia fait voir Her.-Schf. dans ses planches (Hub. révis.). La femelle est plus pâle el ses raies sont mieux marquèes. J'ai reçu dereebef quelques exemplaires ( $\sigma$ et $\$$ ) provenant du pied des alpes centrales. - J'ai toujours plus lieu de croire quadjunctaria (Bdv.) a été prise sur un mâle un peu roux, dont les lignes étaient peu colorées. - Les antennes du màle som hordées de poils fins, rapprochés deux à deux; leurs tubercules sont peu saillants.

\section{No 147. Plumaria W. V. - (page 76.)}

J'ai pris la femelle en Valais, près de Sion, au milieu d'août; cette espèce n'est donc pas, en Suisse, exclusive au Jura, 


\section{G. XXXIV bis Sthanelia. Bdv. - (page 81.)}

(Alsophila. IInb. Verz. - Chesias. Treit. - Pachinemia. Stplı.)

Nota. Ce genre doit-il réellement rester sépare de Mniophila? - La nervation de corticaria ne differe de celle l'hipocastanaria qu'en ce que dans la première la nervure médiane externe se bifurque après la cellule et dans la spconde avant elle. En tout cas Sthanelia n'a aucun rapport avec Chesias Treit.

$\mathrm{N}^{\circ} 159^{\mathrm{h}}$ Hipocastanaria (ta). Hub. 186.

Dup. - Treit. - Bdv. 1932. - Her.-Schf. I. 261, lig. 330.

Wood 631. - Heydenr. Cat. 301.

Degenerata. Hub. 405 .

Cette espèce a èté receuillie dans les environs de Burgdorf Berne) par Mr. Heuser, auquel nous devons plusieurs captures très intéressantes pour la Faune suisse.

\section{No 182. Erutaria. Bdv. - (page 90.)}

Mr. Couleru a pris quelquefois cette espece dans les bois de sapin du Jura, sur le Chasseral, etc.; mais elle y est toujours rare.

No 186. Hospitaria. Treit. - (page 92.)

Je l'ai prise sur les sapins près de Lausanne, au milieu d'avril.

$\mathrm{N}^{\circ} 198^{\text {b. }}$ Majoraria. nob. - (page 95.)

C'est encore à Mr. Heuser de Burgdorf que je dois la comaissance de cette espèce. Sa taille surpasse celle de toutes ses congénères: elle a presque les dimensions d'alchemillaria. Je n'ai pu voir que deux femelles.

Ia teinte des quatre ailes est le gris jaunatre; le gris est réparti sur le pourtour en forme de large liséré, et le jaune sur le centre et la base. Les deux tiers internes sont parcourus par un grand nombre de stries grisâtres, pâles, au milieu desquuclles se voient les guatre points centraux, peu prononcés. Le large liséré du bord externe est d'un gris roux, pâle, sur lequel se dessine en blane terne la ligne ondulèe très dentelée, surtont anx inférieures. Vers l'angle anal des antérieures la ligne ondulée se termine par une tache blanche. La côte grise est marquée d'un grand nombre 9 a 10 te taches jau- 
nâtres. L'angle du second ruban, placè près du sommet des supérieures, est très prononeé, presque droit, mais arrondi à son sommet. La frange est grise, légèrement tachée de jaune et limitée par une ligne brune, fine, coupèe par un point blane sur les nervures. Aux inférieures la frange est plus claire et les intersections plus saillantes.

Le dessous ressemble au dessus, mais le gris y est plus brunatre, plus répanda, et la base des ailes en est fortement pointillée. Le ligne ondulée de la marge et les quatre points y sont peu visibles. Le corps entier est d'un gris-de-fer bleuâtre, entremêlè de blanc sale. Le premier anneau de l'abdomen est tout entier de cette dernière couleur. le front est blane.

Jignore l'époque de l'apparition. Cette espéce se place à côté d'absynthiaria, pour le dessin seulement.

$\left.\mathrm{N}^{\circ} \mathbf{2 0 0}\right)^{\mathrm{h}}$ Dist inctaria. Her,-Schf. II. 42. f. 162, 163. - (page 96.) T a mariciata. Her.-Schf. deut. Ins. - (non Fr. 11. B. - Bdv. 1712.

C'est avec doute que je rattache ì cette espèce de Her.-Schf. les trois exemplaires que j'ai sous les yeux et provenant des environs de Burgdorf, où les a recueillis Mr. Heuser. Je ne puis la comparer quà eastigaria parce que toute autre espèce est aisée à distinguer. Le fond de distinctaria est plus uniformément gris-noiràtre; le blanc, tirant sur le roux, n'y apparait que par stries et vers la base des inférieures. La coupe des ailes et la taille est la mêne dans les deux espèces. La disposition de la frange est ce qui les distingue le mieux. Dans notre espece la frange est d'un gris un peu luisant et roussâtre, légèrenent plus elair que le bord voisin gris-foncé et uni. Les dentelures sont à peine marquées. La ligne ondulèe est peu distincte et ne porte pas de tache blanche à son milieu, ni vers l'angle anal. Un trait fin, brun-foncé, limite la frange; il est suivi d'une ligne d'un roux assez elair.

Le corps entier est d'un gris foncé, presque noir. Le front porte quelques écailles blanches.

No 208. Castigaria. Hub. - page 96.)

J'ai pris trois individus femelles sur les montagnes d'Aigle, le 10 juin, à 5000 pieds d'elévation, an milieu des buissons de genièvrier, de rbododendron et d'aune vert. Son vol est assez rapide. Un exemplaire, recu de Mr. Rothenbach, esl parfaitement identique.

Les individus jaunis par le temps ont le plus grand rapport avec ceux de pusillaria dont le fond est chargé de brun. Castigaria se distingue sûrement 1) par 
la ligne ondulée de la marge, formant des zigzags aigus et marquée de 2 tacbes blanches, l'une à son milieu, l'autre vers l'angle anal; 2) par l'espèce de bande circulaire qui règne sur toute la périphẻrie des ailes, oủ le gris est moins mélangé de points blancs. Ce caractère est surtout frappant sur les individns dont le disque est fortement pointille et strié de blanc; 3) par sa frange, nettement entrecoupée de gris et de blanc sâle, sèparée de la marge par une série de petits traits noirs, entre lesquels des points blanes. placès sur les nervures et s'avançant sur la frange, se distinguent très nettement. Cette disposition est particulièrement visible aux inférieures. Les quatre points discoïdaux sollt bien marqués; ceux des supérieures sont moins gros que dans pusillaria, L'angle des bandes et des rubans, vers le sommet des supérieures, est très marqué, sans ètre alongé. - La taille et la coupe des ailes est la même que dans pusillaria, sanf pour les ailes infërieures qui sont un peu mienx développées. - Les palpes sont gris-blanes, le dernier article d'un blanc pur; le front est gris, pointille de blanc.

\section{$\mathrm{N}^{\circ}$ 20:3. Indigaria (ta). Hub. 399. - (page 97.) \\ Bdv. 1716. - Dup. cat. - Her.-Schf. II. 49. fig. 183-186.}

Heydenr. Cat. 490.

Conterminata. Zell. Isis. 1846.

Mes a ta. Tiscb.

Cette espèce rare a ètè prise le $\mathbf{9}$ mai près de Burgdorf, dans le Lissachwald, par Mr. Heuser. J'en possède aussi an exemplaire des environs de Lausanne.

\section{$\mathrm{N}^{\circ}$ 205. Begrandaria. Bdv. 1727. - (page 97.)}

Dup. sup. t. 4 p. 530 ? - Her.-Schf. H. 54. fig. $128,129$.

Pygmae ata. Her.-Schf. Synops.

Valerianata. F. v. R. - Zell. Isis.

Subumbrata, pusillata, plumbeolata et niveata. Wood. (teste Her.-Schf.)

J'ai trouré cette jolie espéce dans te Jorat, prés de Montpreveyres, vers la fin de mai, et le 20 juin, près de Panex, dans les montagnes d'Aigle. - Elle vole sur les pâtnrages au soleil et paraît préfèrer les lieux bumides. Si les individus ne sont pas très frais, le dessin est insaisissable, tant il est peu marqué. On n'aperçoit guères sur un fond gris et finement ondulè, qu'une partie du second rnban se détachant en clair.

J'avais déjà pris cette espèce sur les pâturages du Jorat de Lausanne, mais endô. 
magee. - On pent aisement la confondre avec singularia, comme parait l'avoir fait Dup.; mais celle-ci est plus petite et d'une couleur fauve.

$\mathrm{N}^{0} 205^{\text {c. A }}$ rgillacearia. Her.-Schf. II. 55 fig. 143, 144. - (page 97.)

Kous devons encore la decouverte en Suisse de celle espece à Mr. Heuser de Burgdorf, qui m'en a adressé un exemplaire. Son facies est très particulier; on dirait, à premiere vue, avoir devant les yeux une Psyche bombycella o゙. Le dessin est celui de Begrandaria; mais ses ailes sont plus alongées. Le sommet des supérieures est très arrondi. La frange est d'uu gris plus foncé, légèrement entrecoupee. La teinte de l'individu \& que je possède est plus jannàtre que la fig. de Her-Schf. Les quatre points centraux manquent dessus et dessous.

No 230. Berberaria. F. - (page 106.)

Mr. Zeller de Zollikon (Zurich) a pris, dans le Ilaut-Valais, une bizarre variete, dans laquelle il ne reste de la bande mediane que deux raies noires, l'une, du côte de la base, presque directement transversale, l'autre, du cóté de la usarge, se réunissant à la preniere, sur la côte, et se dirigeant de la vers le bord interne en décrivant un cercle, puis un sinus et enfin quelques ondulations (Collection Zeller).

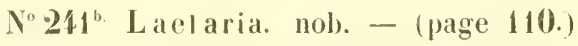

Var. kollariariac, lacle-viridis. nob. fig. 6.

La variete verte de Kollariaria que j’ai figuréce appartient certainement à une espéce listincte. N'ayast eu d'abord qu'un exemplaire de chaque espèce sous les yeux et pendant peu de temps, je dus m'en rapporter au jugement d'un ani micux placé que mor pour ohserver. Aujourd'hui, eurichi de plusieurs exemplaires de l'une et de l'autre espèce, je puis me prononcer plus hardiment et rendre à la variéte le nom de la etaria que je lui avais donne prècédemment. Cette espece a été prise cette annèe (1852) sur plusicurs points des montagnes de la Suisse par MM. Meyer, Heuser, Ott et par moi. Elle apparait partout en juin et se montre surtout fréquente près de Meyringen.

Laetaria diffère de Kollariaria 1) par sa taille constamment plus petite; elle tient le milieu entre caesiaria et aptaria; 2) par ses contours moins arrondis, surtum aus superieures; 3 par sa bande mediane (second espace) beaucoup plus sinueuse, surtout en dehors. Un sinus profond se dessine entre la pointe centrale et la dent placese vers la côte; 4) par le retrécissement de cette même bande, surtout près de la côte; 5 par une trace blanche qui part du sommet des supérieures et s'avance 
en s'éteignant jusques au second ruban; 6) par une ligne blancbe qui divise la frange en long et qui est précédée d’un trait noir, interrompu par les nervures. Dans Kollariaria, cette ligne est brun-foncée, bien marquée et séparèe par un trait blanc-sale d'une ligne de doubles points, limitant la frange; 7) Iaetaria, enfin, a les points discoïdaux plus gros aux supérieures. - La coloration des ailes est du reste constamment très diffërente dans les deux especces. - Les antennes sont semblables, les palpes de Kollariaria sont légèrement plus courts.

No 246. Unangularia. Wood. - (page 111.)

Des observations multiplièes me font douter toujours plus de la réalite de cette espèce. Telle que la caractèrise Her.-Schf. et telle que j’ai pu l'observer, elle ne diffère pas suffisamment de rivaria, dont elle serait tout au plus une forme. - J'ai de la peine à admettre que la fig. 386 de Hub. appartienne à l'espèce de Wood; ne s'appliquerait-elle pas plus aisément à alchemillaria? Peut-être est-ce encore une de ces espèces de Hub. que tôt ou tard on retrouvera?

\section{No 253". Jucundaria Bdv. 1797. - (page 115.)}

\section{Dup. cat. - Heydenr. Cat. 596.}

C'est avec doute que je rattache à l'insecte que j'ai sous les yeux la courte et inparfaite description qu'en donne Boisduval. It paraît que cet entomologiste n’a eu devant lui que la femelle, car outre son analogie avec blandiaria, il n'eut pas manqué de citer les antennes pectinées chez le mâle; peut-être même, en les voyant, eut-il placé jucundaria á cóté de ferrugaria.

Elle se distingue encore de blandiaria par sa taille un peu plus forte, par la teinte ferrugineuse de toutes les parties brunes, qui se répand même sur le premier ruban, et par la couleur noiràtre du corps entier, y compris la tête. - La bande médiane est aussi moins ondulée sur ses deux bords et également colorée sur toute son étendue.

Jucundaria ressemble quelque peu, pour le facies, à quadrifasciaria; mais la bande médiane n'a pas d'augle saillant en dehors, et le liseré foncé de la marge des infërieures est chez elle plus prononce. - Les points du centre des ailes ne se voient qu'en dessous.

Cette espèce a été prise dans la vallèe Leventine, en juillet, par Mr. Heuser de Burgdorf. - Son dessin viendra plus tard, s'il y a lieu. 


\section{No 303. Firmaria. Treit. - (page 135.)}

Mr. Couleru de la Neuverille a élevé cette espèce; il trouve la chenille sur le pin sylvestre en mai; le papillon éclos à la fin de juin et an commencement de juillet, en même temps que variaria.

No 309. Perfuscaria. Haw. - (page 137.)

Aux synonymes donnés à cette espèce il faut encore ajouter passeraria Fr. Beitr. 486. - Heydenr. Catal. 552. - Je dois ce renseignement à l'obligeance de Mr. Couleru de la Neuveville. Cot infatigable observateur a élevé la chenille de cette espèce et a fait parvenir l'insecte à Mr. Freyer. La larve, m’apprend-il, vit sur le saule marceau et s'y trouve en juillet; le papillon est éclos le 6 aoùt, en chanbre et vers le 13 , au haut du Chasseral où avait été prise la ehenille. - La désignation de Hawortb doit être conservèe comme plus ancienne. Les exemplaires de Mr. Couleru ont le dessin encore plus vif que ceux que j’ai pu voir; mais ils restent tout-à-fait différents de russaria.

\section{No 312. Stragularia. Hub. - (page 139.)}

De nouveaux individus, très frais, pris récemment, n'ont fait que dèmontrer toujours mieux à mes yeux la validitè de l'espèce admise par Hub. Non seulement la coloration et la forme générale restent diffẻrentes, mais encore le mâle est constamment semblable à la femelle, ce qui n’a jamais lieu dans variaria. La ligne ondulée de la marge est aussi lègèrement ondulée, à ondulations arrondies dans la première, tandis que ces ondulations sont aigües et bien prononcées dans la seconde.

Les exemplaires de variaria dont la bande moyenne est avortée dans sa moitié interne, ne sont pas pour cela des stragularia et en restent fort èloignés. On ne peut citer sans méprise la fig. 337 de Hul. comme var. de stragularia, ainsi que le fait Heydenreich (Cat. 598. v.)

Quant à obeliscata Hub., il doit rester comme variéte de variaria. J'ai vu plusieurs individus élevés par Mr. Couleru à la Neuveville, d'autres, pris par Mr. Rothenbach; il est aisé d'étahlir une transition entr'eux, depuis variaria la mieux dessinée, jusqu'à obeliscata dans laquelle le desssin disparait presqu'en entier sous une large bande rouillée, confondue sur ses bords avec le fond. - Obeliscata parait n'exister en Suisse qu'au pied du Jura et toujours en compagnic de variaria. 


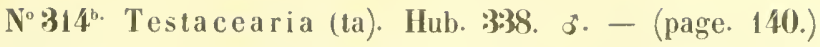

Bdv. 1651. v. - Dup. cat. - Her.-Schf. II. 198 - Ileydenr. Cat. 401.

9. Aemulata. Hub. 443. - Bdv. 1653. - Her.-Sebf. II. 196.

Var. tersata e. Treit. - Dup. cat. - Heydenr. Cat.

Duponchel (cat.) avait déjá entrevu l'erreur commise par Treit., en réunissant aemulata Hub. à tersata; s'il ent en pour guide d'antres moyens que les figures malfaites de Hub., il eut bientôt levé tous les doutes. J'ai vu quatre exemplaires de testacearia, dont trois femelles et un måle, et je puis affirmer que les fig. 338 et 443 appartiennent à la même espèce. La fig. 338 est très reconnaissable; seulement le somnet des supérieures y est mal à propos arqué, tandis qu'il devrait être figurè comme dans tersaria; la même faute, mais moins prononcée, existe aussi dans la manvaise figure de cette dernière (263).

Testacearia a ète prise dans les environs de Meyringen, par Mr. Ott, et autuor de Burgdorf, par Mr. Heuser, dans la prenière moitiè de l'été. - Elle se distingue facilement de tersaria par sa taille un peu inférieure et par l'aspect pointillè et tacheté de ses quatre ailes. Cet aspect est dû au grand nombre de stries et de petites taches qui les reconvrent et leur donnent presque le facies de vetularia. La bande mediane porte deux angles, l'un rentrant, vers la cote, comme celui de tersaria, l'autre obtus et saillant, vers le centre, qui n'existe pas dans cette dernière. - Le màle et la femelle sont semblables et plus ou moins nettement dessinés.

\section{Er ratat.}

Page 87. - Les synonymes de rheticaria s'appliquent à plumularia Fr. 


\section{Table des noms spécifiques.}

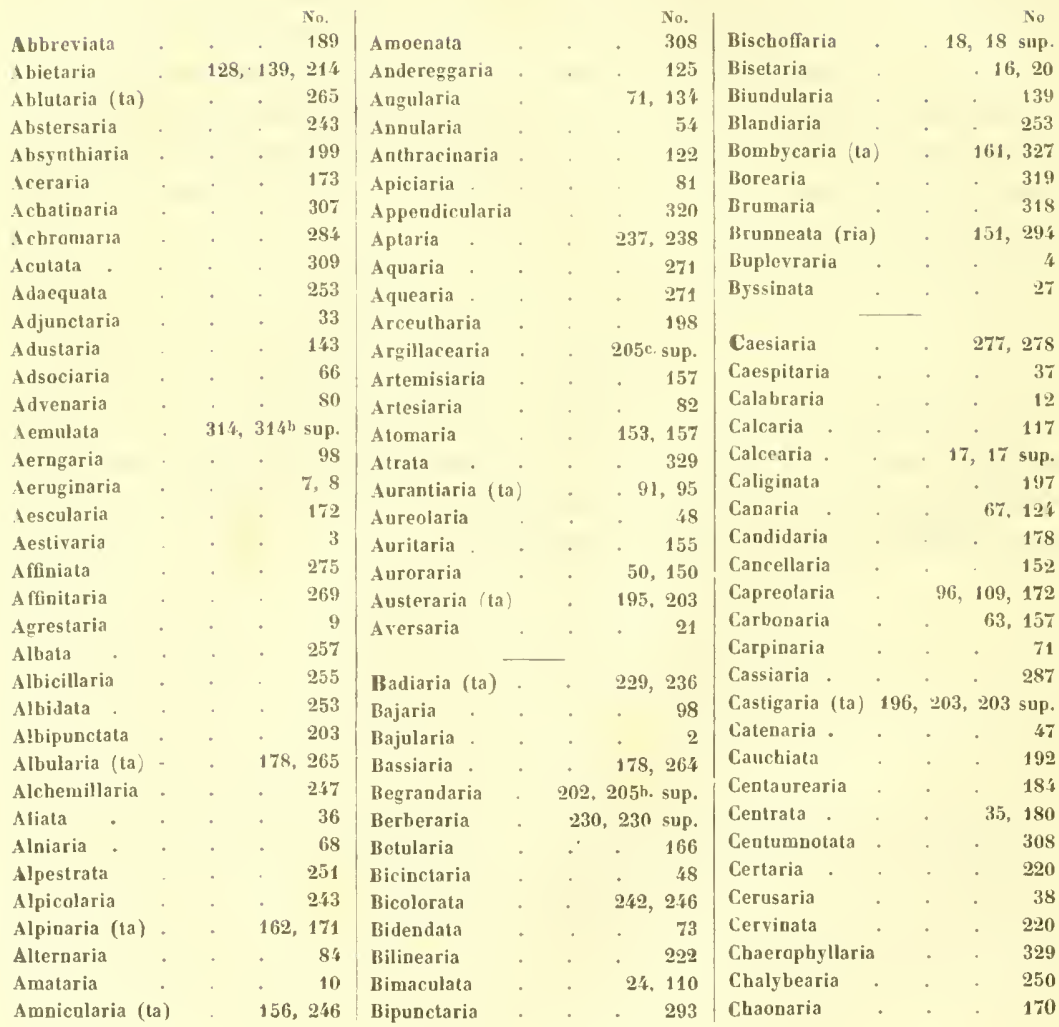




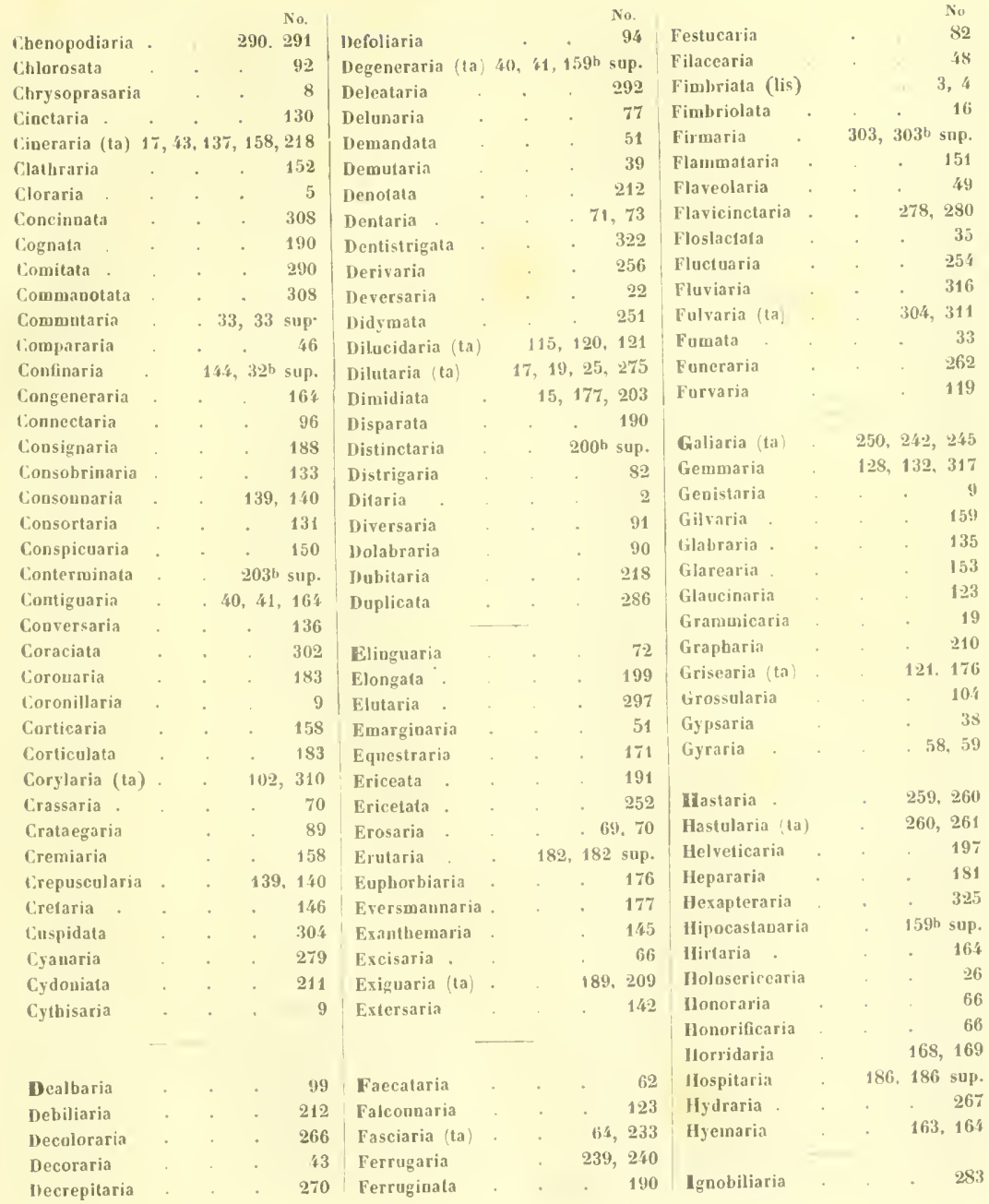




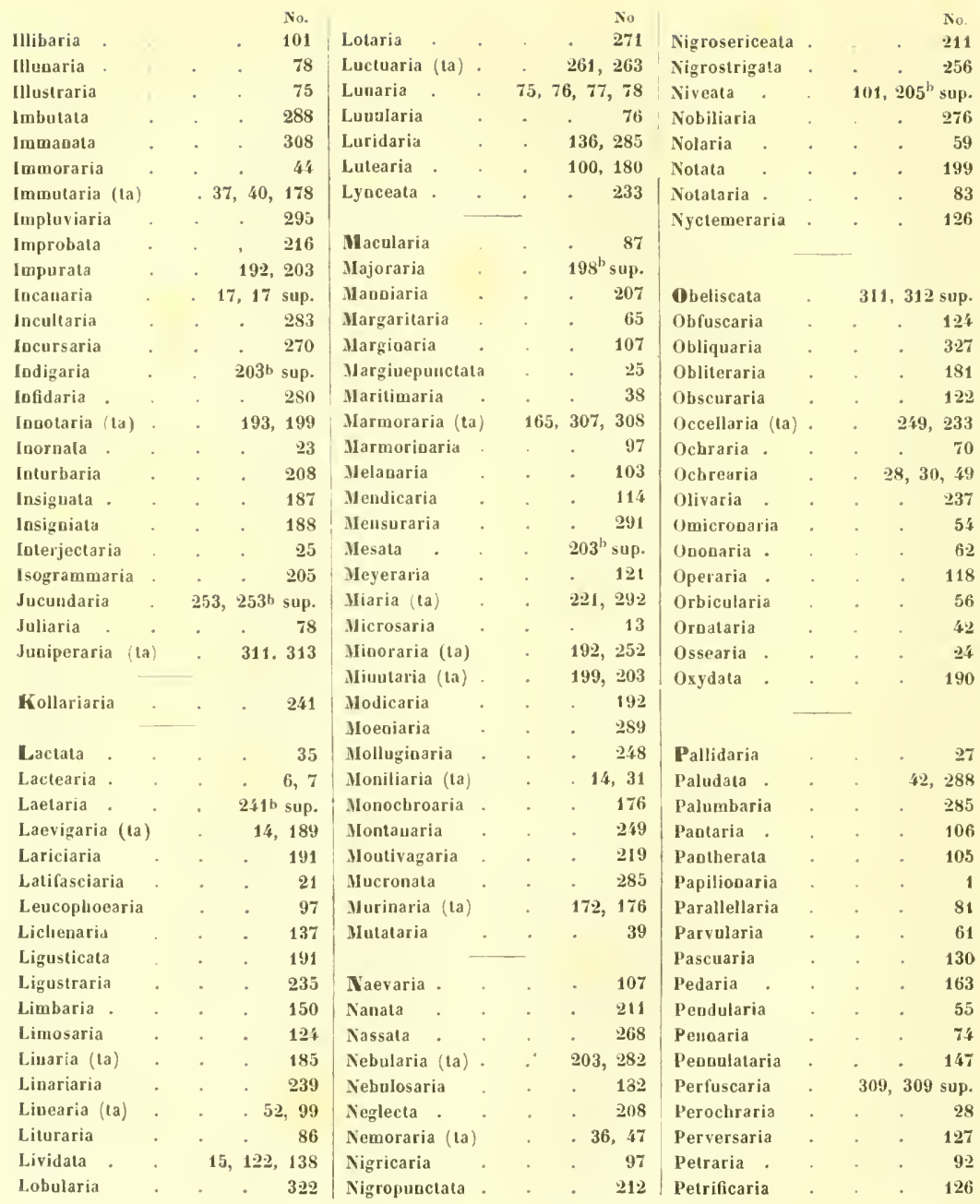




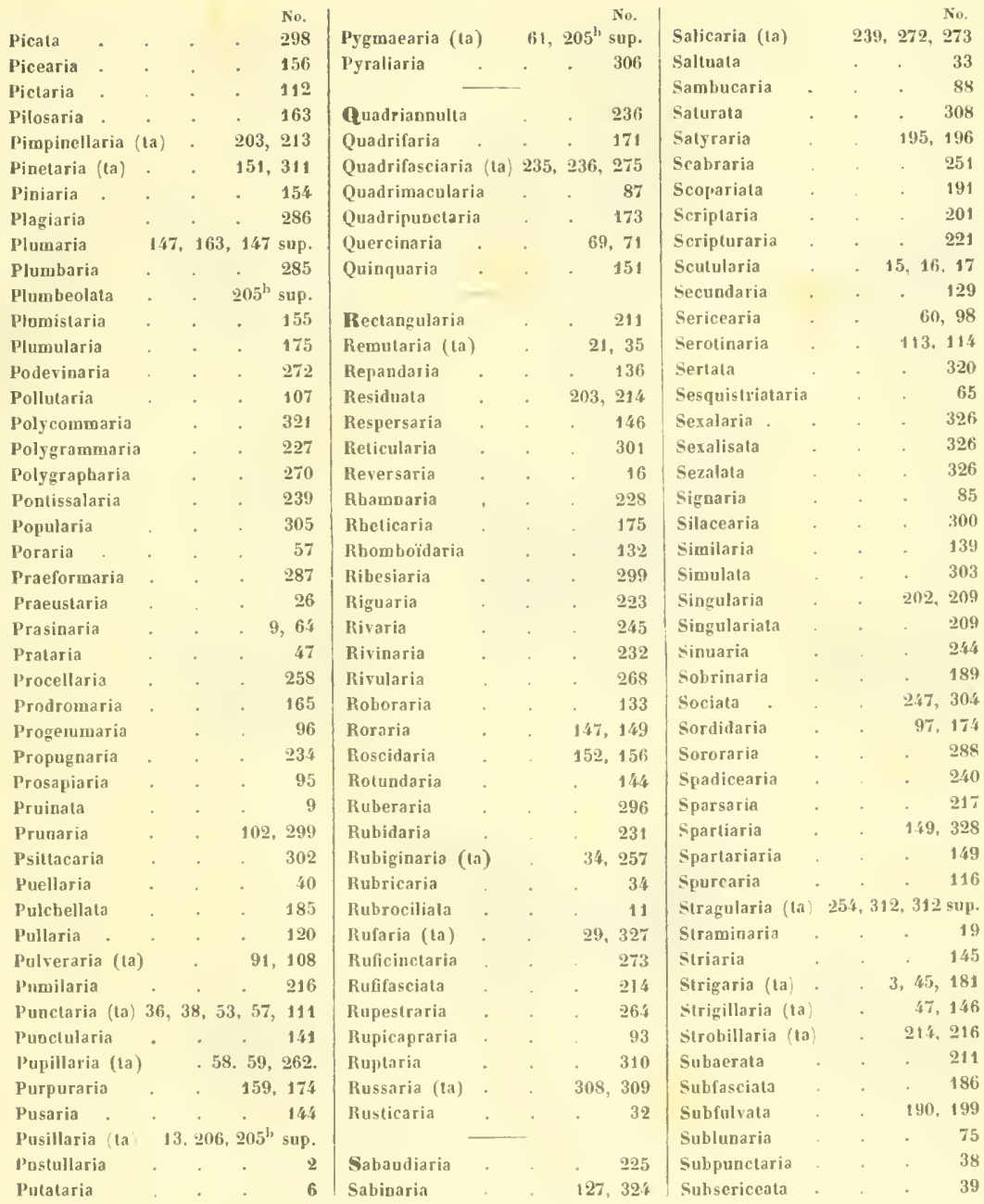




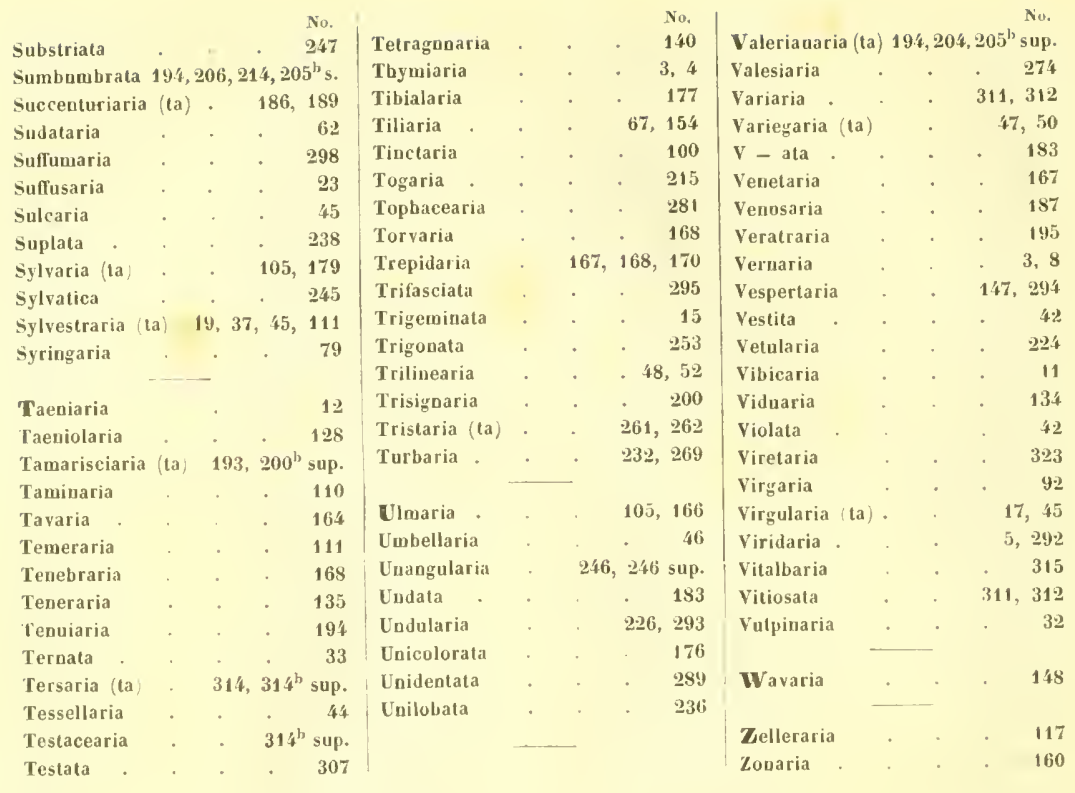



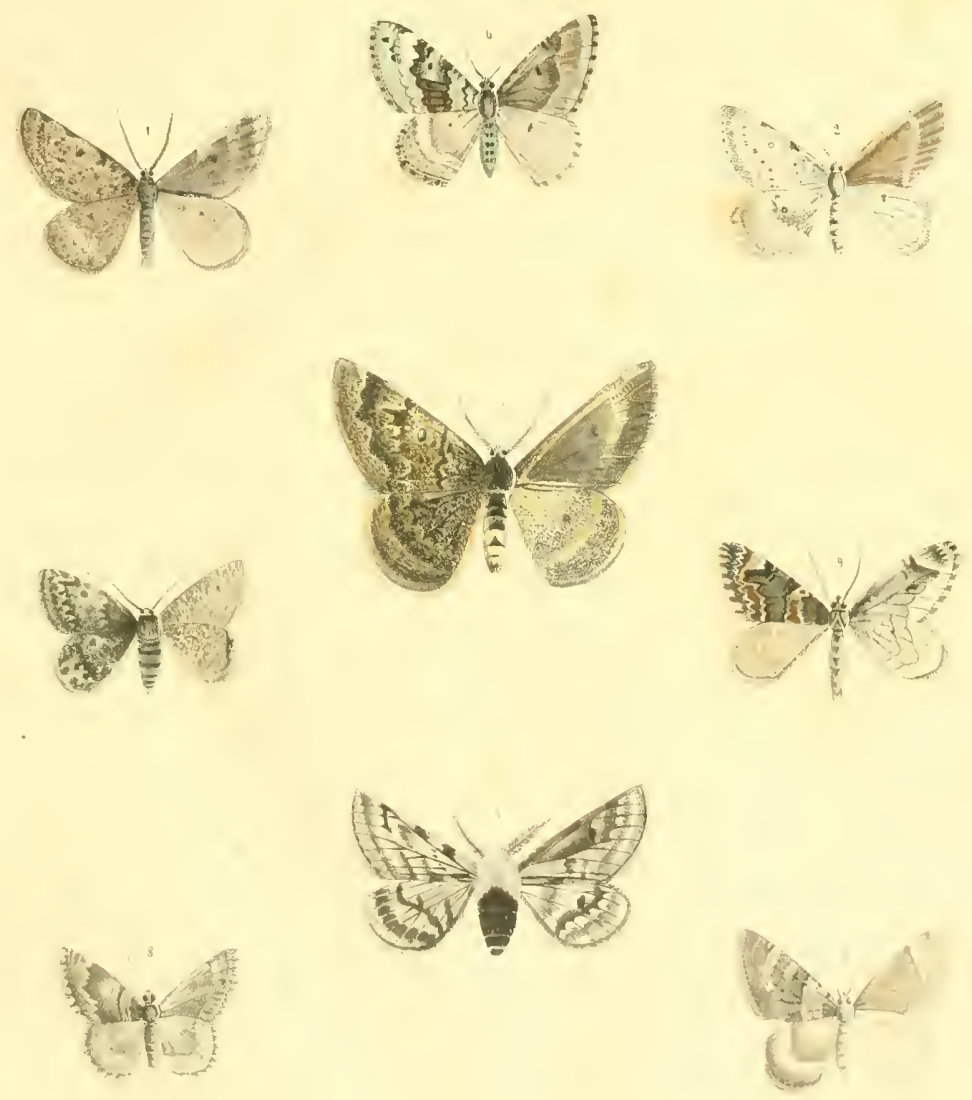



\title{
SECONI) SUPPLÉLENT
}

$\mathrm{IIX}$

\section{phalénides de la haune suisse.}

\author{
P.IR
}

I. $\mathfrak{C}$. 刃e la

Voir : Jouseaur Memoires de la Société helvétique des sciences naturelles. Vol. XIII, p. 1hti. 


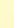


Tross années se sont écoulóns depuis la publication des Plialénilles de la Faune suisse el déja je suis obligé de faire paraitre un second supplément, plus volumineux que le premier. L'addition de quelques especes nouvelles pour notre pays motive bien moins ae dernior travail que l'obligation de rectifier et de compléter bon nombre de laits énoncés. Il est fâcheux de s'exposer il ces rectifications; mais dans un travail de cette nature, une vie d'homme n'est pas toujours suffisante pour tout enrégistrer et pour le faire sims rrreur.

J'ai placé à la fin de ce second supplément, un catalogue systématique des Phalènes unisses tel qu'il résulte de mes dernières observations. Il servira à indiquer, pour chaque esprice, la distribution des notes qui la concerne dans la faune et dans ces deus suppléments. Ce catalogue élève le nombre des epices ì 346.

LAUSANNE, Je $1^{\mathrm{er}}$ Janvier 1855.

\section{Phalenides.}

No 2. B B а jul a ria, Esp.

M. Rothenbach la prise aussi une fois près de Schïpfen dans une forèt de hètre.

$\mathrm{N}^{\circ}$ 18. B is choffaria. Hub. ( ${ }^{\mathrm{er}}$ Supplément.)

Jai reçu de Meyringen quelques individus moins foncés et qui semblent former le passage ì in canaria. Its conservent rependant les caractères signalés; car les points d'intersection de la frange n'existent pas chez eux, de mème que le pointillé de la troisième ligne. Il fant avoir sous les yeux plusienrs individus pour bien saisir ces caractìres.

1) Les numéros indiqués correspondent a ceus de la faune suisse: Phalenides Voir Mruoires de la societe helvelique; $2^{\mathrm{e}}$ sèrie. Vol. X111, 1853. 


\section{N 23. Sulfusaria (ta). Treit.}

N'a pas été réellement recueillie en Suisse jusqu’à ce jour. Tous les individus que j’ai cru lui appartenir étaient des deversaria.

S̀ lfusaria est d'un janne plus orangé, sa lrange est limitée par un trait fin, surtout marquée en dessous. Les rayes transierses sont fauves et à peine visibles. L'avant-dernière rourt droit ì la rîte. Les antennes du mâle sont fortement dentellées et bordées de faisceau de plpoils courts.

N 27". Renularia. Hub. 33:31.

Bdv. 1854. - Heyden. Cat. 709.

II. Bruand (Catal.) signale la présence de cette espèce aux frontières de la Suisse, dans le département du Doubs. Diss lors M. Couleru l'a récoltée au pied oriental du Jura, prìs Ie la Newverille. L'échantillon que jai sons les yeux ne laisse aucun doute à cet égard, il ne differe de la figure de lluh. que par la couleur générale qui est le gris-pâle, dans Hub., tandis qu'elle est jaune-nanquin dans la nature. Cette espèce est extrèmement voisine de ex larsaria Koll. (eflloraria Zell.). L'ombre de l'espace marginal (mal reproduit dans Hub.) est ondulée et fait, vers son milieu, un angle tout semblable ì celui que l'on olsserro dans toutes les Acidalia voisines d'incanaria, de bisetaria, ete. Renularia differe d'extarsaria par l'absence d'ombre noiràtre entre les deus lignes transterses et par relle des points noirs plarés sur la frange en dehors des petits traits marginanx.

Il est probable que llub. arait reçu cette espire, ainsi que int e rject a ria, non d'Espagne, mais de la Suisse.

No 3:3. Commutaria. Treit. sup.

Ajoutez aux synonymes indiqués:

Duplexaria s. Bruand, Cat.

N $: 37^{\text {t }}$. Caricaria. Her.-Schf. (Mnscpt.)

Phlearia. C. Reutti; Fanne badoise (1853, p. 113.

Je dois à ['obligeance de M. C. Zeller, de Zurich, davoir pu examiner cette jolie espèce. déerite pour la première fois par M. Reutti et à laquelle M. Iler.-Schf. avait donné le nom de earicaria. Elle se rapproche extromment de sylvestraria 0 , dont elle a la taille et le faries. Sa blancheur est plus jure et le dessous est d'un blanc luisant très-poli. La frange large et luisante, l'absence de points sur la marge et la ligne dentellée noire, du dessous des ailes, la distinguent suffisamment de cerusaria et de sylvestraria. 
Le màle ne differe pas de la femelle. Le front est noir et le rolier brun-clair. Les antennes du màle sont blanchàtres et crénelées en dessous, leurs bords sont garnis de cils assez. longs, distribués par pinceaux de 2 a 3 poils. Les tubereules sont proéminents ot séparés par des sillons. Dans le même sexe, les tarses des pattes postérieures sont rulottés et garnis à leur sommet d'un pinceaux de poils soyeux. L'extrémité de la pate dépasse le fourrean d'un' longueur égale à celui-ei.

Cette espèce a été prise dans les environs de Zurich, en juin. L'éperyue de son apparition peut done encore servir at la distinguer.

No 38. Cerusaria. Nob.

Tracez le synonyme du catalogue dn Doubs. Son auteur ma lait observer la maritimaria, et j’ai pu me convaincre que cette espèce, bien caractérisée, est très-voisine d' a ve rsa ria, et s'en distingue $1^{\circ}$ par l'absence du trait vif qui limite la marge; $2^{\circ}$ par ses deuxième et troisième lignes plus prononcées; $3^{\circ}$ par une ombre d"un roux-clair qui cerne la ligne ondulée dans l'espace marginal.

\section{No 39. Mutataria. Treit.}

J'ai sous les yeux une femelle complétement gris-bleuâtre, chez laquelle la ligne ondulée lorme un ruban blane continu, ondulé en dedans, anguleux en dehors. Les lignes transserses et surtout la deuxième, ressortent en roux sur le fond gris. Les quatre points sont trè̀-prononcés. Le dessous des supérieures est moins fortement enfuné.

No 49. Flaveolaria. Hub.

Se prend aussi sur les Alpes vaudoises, au-dessus de Bex, à la hauteur des derniers sapins.

Je n'ai point encore pu me procurer des individus provenant de Fontainebleau, pour les comparer; je doute quills appartiennent à la même espìce.

\section{N 52. Trilinearia. Bork.}

La variété couleur d'ocre a été nommée c a rnearia par M. Bruand dans son catalogue.

N 58. Schaefferaria. Nob. Fig. 1.

Gy raria. Treit. - Mann. (non IIub.). - Zell. Isis. 1847, p. 497. Faune suisse.

Pupillaria. Zell. Isis I847, pag. 498 (non Hub.).

Givaria. Ilub. 434, n’est décidément pas celle que j’ai enrégistrée. M. HerrichSchaller m 'érrit à son sujet et après l'avoir comparée. que jusquici il avait confondu cette 
espèce aver poraria. "Zeller, “ ajoute-t-il, „fait évidemment mention d'elle dans le jour. nal entomologique, année 1849, p. 209 , disant quil ne peut pras encore la distinguer "spécifiquement de punetaria. J'estime qu'elle en diffère réellenent par la marge des - ailes antérieures moins sinuruse, par la couleur du fond tirant plus sur le ronge que sur . le jaune, par la ligne de séfraration ronge de la frange, par l'absence constante de trinte . rouillée sur la ligne moyenne, jar la présence des points centraux qui nexistent jamais (?) dans punctaria, et enfin par l'absenre de loute tarlue obscure près de l'angle anal des ailıs antérienres. J'ai retrouré 6 exemplaires de rette espère daus la collection de Fisch: 1. RösJ. et 3 dans la mienne, recueillis dans nos environs. Il s'agit maintenant de saroir si cette espèce peut porter le nom de gy raria. La figure $\$ 34$ de Hubner doit dérider la question. Chez votre gy raria les points centraux ne sont point entourés d'un cercle noir, la ligne moyenne est nettement marquée et atteint le bord antérieur de l'aile, la frange n’est pas d'un jaune rif, mais elle est ravée de rose vif, rouleur qui s'étend mìme, . a l'état très-frais, sur tonte la frange; l'abdomen enfun n'a pas de taches dorsales; caractères de la figure de Hubner qui se rapportent tous au contraire à l'espèce que Zeller a décrite. Jans le journal cité $\left(n^{\circ} 60\right)$, sous le nom de pupillaria Mann. et que jai reçue du même entomologiste eomme provenant de Toseane. C'est done cette pujpillaria liı , qui seule mérite le nom de gygaria Hub. 434. Treitsehke déerit la rotre sous le nom "de gy raria; les collections de Vienne sont daccord sur ce fait. Ilubner ne l'a pas fignrée. Gy raria de Dupouchel s'accorde aree la vôtre par Ja couleur de la frauge et ares celle . de Hubner par les taches enutrales; on ne peut dones'y arrèter: aiusi votre gry aria doit - resevoir un nonvean nom."

Le eatalogne de Heydenseich ne pent servir de gnide pour ces espèces.

$\mathrm{N}^{\circ}$ 59. Pupillaria. Hub. 69.

(Non Zell.)

11. Her--Schí, m’écrit que des exemplaires déterminés par Treitsehke lui-même se rajportent hien à l'espèce de IJubner, upui est aussi celle que j’ai observíe. La p upill a ri a de Zeller Entom. Zeit. loc. eit. - Voil S chaefferaria) est me autre espèce. Fyraria. IIub. 543, et nolaria, Huh. 327, nappartiemnent pas dasantage à pupillaria, Ilub. Zeller ne ronnaissait pas rette dernière, quoiquil en lasse mention à la fin de som article sur pupillaria (Ier.-Sehf.).

$N^{\circ}$ 61. Parvularia. Boist.

II. C. Zeller a pris cette espèee dans la rallée de Misox (Tessin) : elle est donc bien surisse. 


\section{No 69. Quercinaria. Bork.}

II. Rothenbach en a pris un individn près de Schüpfen, le 12 juillet 18.51 .

\section{$\mathrm{Nr}^{\circ}$. 77. Delunaria. Steph.}

M. Her.-Schælfer m’écrit que delun aria a été plusieurs fois ćlerée comme deuxième génération de lu uaria. Celle que j’ai prise, le fut an printemps: je n’ai pas pu men procurer d'antres individus. Jai de la peine à croire à l'identité des -2 espèces, à canse de la taille plus petite de ma delunaria, quni n’a cependant pas été élevée en captivité.

\section{$\mathrm{N}^{\circ} 81^{\mathrm{l}}$. Parallellaria. W. V.}

Hub. 43, 44. - Treit. - Dup. - IIer.-Schf. 1, 127.

- Hub. Beitr. H. - Frey. ält. b. 42. - Ileyden. C. 16. Vespertaria. Fab. - Wood 535.

Affiniaria. Bork.

Re pan daria. Berl. mag.

Cette espèce, rare en Suisse, a été prise dans les environs de Schïpfen, par M. Alfred Rothenbach, le 26 juillet 1853 .

No 92. Petraria. Esp.

M. Rothenbach a aussi pris cette phalène près de Schüpfen, et M. Ott à Meyringen. Elle parait en juin dans les hois de pin.

No8. Bajaria. Hub.

II. Couleru a pris plusicurs fois cette espèce dans un bois de chêne près de la Neuveville.

\section{$\mathrm{N}^{\curvearrowleft}$ 114. Mendicaria. Her--Schif.}

L'indication de II. Rothenbach s'adresse à serotinaria $\left(n^{\circ} 113\right)$, et non à celle-ci.

\section{Y 119. Furvaria. Fab.}

Assez fréquente dans les forêts de sapin et les rocailles du pied des Alpes d'Aigle, en juillet.

No 120. Pullaria. Hub.

Au pied du Weissenstein, au-dessus de Soleure, le 31 juillet (Rothb.). 
No 121. A mbiguaria. (la). Dup. hist. pl. 186.

Boisd. 1575. - IJer.-Sehf. I, 193. f. 379-381.

- Ileyden. C. 140. - Dup. cat.

V eyeraria. Faune suisse.

L'espèce décrite et figurée par Duponchel est presque certaimenent celle que j’ai ajpelée Me y er a ria dans mon premier travail. J'ai longtemps douté de l'identité des deux espèces, parce que Boisduval l'éloignait des Guophos et des Elophos, et que M. Bruand la distinguait de sa dilucidat a. Celle-ci appartient, sans aucun doute, à la mème espèce, tandis que son operaria est la raie dilucidaria, Hub.

II. C. Zeller m’écrit qu il la prend assez fréquemment sur le Balgrist près de Zurich.

$\mathrm{N}^{\circ}$ 122. Obscuraria. $\mathrm{W}$. V.

Habite les rocailles sur lesquelles elle se pose volontiers. Fort difficile à alteindre (Rothb.).

\section{$\mathrm{N}^{\circ}$ 12:3. Glaucinaria. Hub.}

Les ceufs de rette phalène sont d'un jaune pàle au moment de l'éclosion; ils passent hientôt au rouge carnin et conservent cette couleur jusqu'à la fin. La chenille est courte, épaisse, applatie en dessous et d'un gris jaunàtre; elle vit sur diverses plantes basses (Couleru).

On peut aisément confondre les iudividus foncés, et dont le dessin est couvert par la teinte générale, avec supinaria Mann.

\section{$\mathrm{N}^{5} 12 \mathbf{3}^{h}$. Falconaria. Frey. N. B. 277, 3. Duj. cat. - Heid. cat. 133. \\ S a rtaria? Hub. sup. f. $66,67$.}

I. G. Leresche a jris deux exemplaires is appartenant à cette espèce, à Pully, au milieu des rignes, non loin de Lausanne, au mois d'aoùt. M. Couleru de son cóté a recueilli deux exemplaires $\Omega$ dans les environs de la Neuveville Lalı.). Elle n'est jas très rare daus le Jura.

Quoique voisine de gla ucin aria et de s a r taria elle s'en distingue parfaitement. Sa taille est plus forte que celle de la première et mềme de la seconde. Les ailes sont d'un blase-grisìtre légèrement mélangé de bleuàtre surtout sur les bords. Le dessin et le pointillé sont gris. L'extrémité des nervures est marquée sur la marge par des points noirs bien évidents. Les dentelures de la frange des inférieures sont moins profondes et moins festonnées que dans gla u ciuaria. La frange blanchàtre est à preine et très légèrement entrecoupée de 
gris en dessus; tandis qu'en dessous elle est d'un blanc pur. Le front est d'un brun-noisette, tandis que la tête est de la couleur du thorax. Les autres caractères sont moins sûrs et suljets a varier.

Heydenreich rattache sartaria, H. S. it lalconaria; je ne puis partager cette manière de voir, à moins que ses figures n’aient été prises sur des individus élevés en captivité et qui en ont souffert dans lenr taille et dans leur facies. Les caractères que j’ai indiqués suffisent à établir l'espèce.

I 12:3. I I c idaria. Hub." 148.

Frey. ä. B. 125, 3. - Treit. - Dup. - Iler.-Schll. I. 194, f. 266 -268. - Bdv. 1593. - Iteyden. 136. Var. variegaria Dup. hist. 184,185 . - Iler.-Schf. f. 504. Heyd. C. 137.

M. C. Zeller m’annonce qu'il a trousé la var. variegaria dans le Tessin, en 18.53.

N 1:30. Cinctaria. W. V.

M. Rothenbach l'a aussi obtenue de Meyringen.

$\mathrm{N}^{5}$ 140. Consonnaria. Hub.

11. C. Zeller a trouvé la chenille sur les sapins bas.

No 147. Plumaria. W. V.

J'ai dit, par erreur, que cette espèce se pose sur les rochers; elle hante de préférence les taillis herbeux.

$\mathrm{N}^{\circ}$ 158. Corticaria. Hub. 167.

N'a été réellement recueillie que par 11. Meycr dans les environs de Burgdorl et part II. C. Zeller à Engelberg, en juillet. Les autres localités indiquées se rapportent i la suivante.

$\mathrm{N}^{\circ} 158^{\mathrm{b}}$. Cineraria. Hub. 171.

Fab. - Dup. - Bdv. 1594. - Treit. - Her.-Schl. I. 256. - Ileyd. C. 179.

Scpiaria. Berl. Magaz.

C'est cette espèce, et non cortic aria, qui a été prise quelquelois dans le canton de Vaud, au $\mathbf{1}^{\text {er }}$ printemps, puis en juillet. 
$\mathrm{N}^{\circ} \mathbf{1 5 9}$. Hipocastanaria. Hub.

I. Rothenbach l'a prise plusieurs fois dans les environs de Schüpfen, dès le 20 mai jusqu'en septembre.

$\mathrm{N}^{\circ}$ 162. Alpinaria. Scrib.

L'individu obtenu des Alpes, par M. Rothenbach, est bo mbycaria; on doit dèslors tracer alpinaria du nombre des espèces suisses.

$N^{\circ}$ 16:3. Pilos aria. Bork.

Dans les premiers jours de janvier 1853, j’ai reçu une pilosaria qui venait de naître en liberté.

\section{$\mathrm{N}^{\circ}$ 161. Hirtaria. Lin.}

La chenille de la rar. congeneraria vit sur les pruniers et celle d'hirtaria sur les ronces. L'une et l'autre virent dans les environs de Zurich (Zeller).

$\mathrm{N}^{\circ}$ 167. Venetiaria. Hub. 3229 .

La femelle est fort rare, plus pâle et plus petite que le mâle; elle se troure figurée dans Frey. Beitr. t. 125, 4 sous le nom de canitiaria. Les autres synonymes de lespèce sont fuscaria, Zeller, et fusca, Thunb.

\section{$\mathrm{N}^{\circ}$ 188. Consignaria. Bork.}

Aussi recueillie une fois près de Schüpfen, le $\mathbf{9 5}$ mai 1853 (Rothb.).

\section{$N^{0}$ 189. Sobrinaria. Hub.}

II. Couleru a élevé la chenille indifféremment sur le genièvrier et sur les scabieuses. iégétanx sur lesquels on la trouve en liberté, mais il n'a pas remarqué que cette différence de nourriture influât, ni sur la chenille, ni sur le papillon. L'espèce est assez commune.

$\mathrm{N}^{\circ}$ 190. Succenturiaria. Lin.

M. Ileuser l'a prise près de Burgdorf. 


\section{$N^{\circ} 195$. In notaria. Knoch.}

Ajoutez comme synonyme :

Artem is i at ar. Brot.

$\mathrm{N}^{\circ} 193^{\mathrm{b}}$. A usteraria (ta). Fisch. v. R.

Treit. collect. - Tisch. - Frey. n. b, 300. - Dup.? Her.-Sclsf. f. I38-I 40. II. 33. - Hejden. C. 471. - Non IIub. 457, et Boisd.)

$\mathrm{Vulgata}$ et subfuseata. Wood. 666 et 667 .

J'ai reçu cette espèce de Meyringen où elle a été recueillie par $\mathbf{M}$. Ott. Rare. - M. Zeller l'a prise aussi dans les Alpes, et M. Rothenbach dans le Jura, au-dessus de Wan, en mai.

$\mathrm{N}^{\circ}$ 198". Majoraria. nob. fig. 2.

II. Bruand avait déjà recueilli cette espèce dans le Doubs et l'avait appelée Gris e imarginata. Il m'écrit que le mảle ressemble à la femelle; mais quil offre moins de blanc sur le centre ensorte, que la ceinture marginale est moins saillante et que le fond paraît entièrement couvert de stries transverses ondulées.

\section{$\mathrm{N}^{0}$ 200. Trisignaria. Her.-Schf.}

Cette espèce est fort douteuse; il se pourrait que les individus recueillis par M. Bremi ajpartiusent à abs ynthiaria, quoique plus petits que la variété $\mathrm{minutata} \mathrm{Hub.} \mathrm{L'in-}$ secte ayant été élevé en captivité, a pu souffrir dans son déreloppement. La ligne ondulée marginale, quoique très-effacée dans toute son étendue, reste cependant visible. Le point blanc de l'angle anal est aussi couvert de brun.

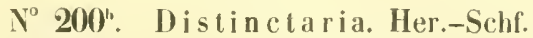

Cette espèce doit être retranchée jusqu'à nouvel ordre; il est plus que frobable qu'il y a ici confusion avec castiga ria.

\section{No 203. Castigaria. Hub.}

De noureaus renseignements m'ont convaincu que cette espèce n'est point rare en Suisse. Elle fréquente les montagnes et les alpes. Je l'ai reçue du Jura et de Mleyringen. Son facies 
larie infinimont. Ce qui en est dit dans le premier Supplément, se rapporte essentiellement ì l'une d'elles qui a quelques rapports aree pusillaria.

\section{$N^{\circ} 204$. Valerianaria. Hub.}

Des individus reçus d'Angleterre par M. Bruand sous le nom de plu mbe olat a Haw. sout rapportés par les auteurs anglais ì vale ria n aria, tandis que IJer.-Schf. fait de celle-ci un synonyme de Begrandaria. Tout me porte à croire en elfet que ces deux dernières espèces sont identiques et qu'il fant effacer l'une des deu.. Les figures de Ilub., quoique peu exactes, ont bien l'air d'avoir été prises sur Begrandaria, un peu pâle. Deux individus reçus de II. C. Zeller, et pris dans les environs de Zurich, répondent parfaitement aux figures de IIubner, et ne se distinguent de notre Begrandaria que par une teinte un peu plus fawe. Pour ériter toute confusion, je conserverai encore l'cspèce de Ilub. jusqu’a preuve du contraire.

Subu mbrata Wood. est d'ailleurs, au dire de MI. Bruand, une espèce distincte de Begrandaria; tandis quil faut rattacher is cette deruière palustrata Doubled.

No $2099^{\prime}$. I rriguaria. Hub. 397.

Treit. - Boisd. 1696. - Dup. - Iler.-Schl. II. 6 I.

Herden. C. 50.5 .

V ariegata. Wood. $68 \mathrm{I}$.

Trouvée près de Schïpfen, par H. Rothenbach, le $1^{\text {er }}$ mai 1849.

No 217. Sparsaria. Hub.

Trouvée aussi près de Zurich, sur le Balgrist, par MII. Zeller.

$\mathrm{X}^{\circ}$ 218. Dubitaria. Lin.

Je dois rétracter ce que j’ai dit de l'hibernation de eette espèce et de sabaudiaria. II. Bruand prend l'une et l'autre au eceur de l'hiver dans les grottes des environs de Bezençon, oì elles se réfugient en automue et restent jusqu'au printemps. J'ai pris un individu $\boldsymbol{\subseteq}$, dans ma chambre, ì la fin de décembre $\mathbf{1 8 5 3 .}$

$\mathrm{X}^{+0}$ 223. Riguaria. Hub.

J’ai omis comme sı nom me, Boisd. 1646. 
No 22.5. Sabaudiaria. Dup.

Les individus provenant de M. Anderrgg lui avaient été adressés de Bezençon par $\mathbf{M .}$ Bruand. Elle a aussi été prise à Meyringen (Rothb.).

$\mathrm{N}^{\circ}$ 232. Turbaria. Hub.

Se prend dans le Jura (Rothb., Couleru).

No 237. Olivaria. W. V.

C'est bien en effet aptata. Bru. Cat.

$N^{\circ}$ 2:38. A ptaria. Hub.

Ajoutez comme synonyme:

Pontissalaria. Bru. Cat..

Effacez-le à ferrugaria.

Je prossède un individu chez lequel la teinte verte a passé au brun, comme dans elut aria.

$\mathrm{Y}^{\circ} \mathbf{2 1 0 ^ { b }}$. Arctaria. Lienig, Isis, Lief. Faun.

Her.-Sclif. II. I02, f. 416.

Cette espèce, fort rare, a été prise à Meyringen par M. Ott, qui l'a communiquée à $\mathbf{H}$. Couleru. - Collection de Lausanne. - La Via-Mala (Rothb.). Sur la Morgetenalp, dans le Simmenthal (Heuser).

$\mathrm{N}^{\circ}$ 211. Kollariaria. Mann.

II. Ott la prend aussi à Meyringen au printemps.

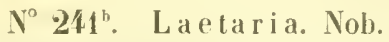

Cette espèce avait déjà été recueillie dans le Jura par M. Bruand qui lui avait donné le nom de larentiaria (Bru. Cat. sup. 1849, $\mathrm{n}^{\circ} 683^{\mathrm{b}}$ ), dénomination que l'on ne peut conserver dans le geure larentia.

J'ai vu plusieurs exemplaires ternis et blanchis, mais aucun d'eux ue passait an brun. Sur les individus très-frais le vert est très-vif; mais jamais muancé de brun.

$\mathrm{N}^{0}$ 214. Sinuaria. W. V.

Effacez les indications de M. Rothenbach. 


\section{$\mathrm{N}^{2}$ 250, Cha lybearia. Hub.}

Après examen réitéré, il ne m’est pas possible de distinguer spécifiquement cette espèce Je galiaria, quoique son facies reste constamment différent. Peut-ètre serai-je plus heureus lorque j'aurai pu me procurer un certain nombre d'exemplaires de galia ria très-frais, dans les bois de notre Jorat. La connaissance de la chenille pourrait aussi décider la question.

J'ai fait erreur en attribuant au mâle des antennes pectinées, trompé que j'étais par un exemplaire fané de ligustraria, dont le dessin était le même que celui de chalybearia.

A la place de cette derniere il faut inscrire:

$\mathrm{N}^{\circ} \mathbf{2 5 0}$. Picaria (ta). Hub. 435.

Treit. - Dıı. - Bdv. 177\%. - Her.-Schf. II, 113. Ileyden. Cat. 545.

\section{Biangulata. Wood. 570 .}

J'ai un dans la collection du Iusée de Neuchàtel un individu appartenant à cette espèce yui avait été pris par II. Coulon, sur le Jura.

$\mathrm{N}^{\circ}$ 25:3 . Lirinaria. Nob. lig. 3.

Jucundaria. Nob. Naune suisse n" $253^{\mathrm{b}}$. $1^{\text {er }}$ Supplément.

L'espèce indiquée par Boisdural (Catal.) sous n"1797, ne peut être celle que j’ai sous les yeux; il est plus que probable que cet entomologiste avait devant lui un individu appartenant à blan Jiaria, ombré de couleur suie, comme on rencontre quelquefois dans le Jura.

Liviuaria a la taille de blandiaria et le facies de quadrifasciaria et d'arctaria; on la confondrait surtout avec cette deruière sans l'absence de tache blanche au rentre du bord externe. Le brun-bistre de la base, de la bande mojenne et de l'espace marginal est gà et là ombré de rouge ferrugineux. Le blane des deus rubans est un pen terne. tandis que celui du trait, qui borde de part et d'autre la bande moyenne, est parfaitement pur. Les inférieures sont divisées en deux par une bande blanchâtre qui sépare deux espaces gris-pâle.

Cette espèce se place par ses antennes pectinées à cóté de spadicearia.

$\mathrm{N}^{\circ}$ 256. Derivaria. W. V.

Se montre quelquefois en avril le long du Léman.

\section{$\mathrm{N}^{\circ}$ 262. Funeraria. Hub.}

Je suis surpris que M. Zeller (Ent. Zeit. 1849, pag. 214) confonde encore tristata 
et funerata, Ilub. - La première varie beaucoup, sans doute, mais ne passe jamais à fun erata. La deuxième varie peu et reste très-distincte pour les mœurs, l'habitat, l'apparition et le dessin.

\section{$\mathrm{N}^{\circ}$ 269. Affinilaria. Wood.}

Recueillie dans la vallée d'Engelberg, par II. Zeller.

Treitschke (sup.) parle d'une femelle de Kirularia plus grande et dont les ailes inférieures sont blanches, qui se rapporte très-probablement à affinitaria.

\section{$N^{\circ}$ 272. Podevinaria. Her.-Schf.}

Commune à Meyringen, en juin. Il est à-peu-près certain qu'olivaria Dup. hist., ou a bl u ta ri a Dup. sup., appartient à cette espèce; Heyden. la rattache à tort, d'après Zeller(?), à albularia; la figure et la description de Dup. ne conviennent en aucune façon à cette dernière. Je ne puis affirmer que a blutari a Ilub. sup. f. 38:2, 383, doive être rattachée à muscosaria Led. comme le fait M. Zeller (Ent. Zeit. 1849, p. 212). Je serais plutôt tenté de la réunir aussi à Po devina ria, puisque Her.-Scht. la trouve trop voisine de S alic a ria et qu'il rattache muscosaria a frustraria Treit.

J'ai reçu ablutaria de M. Millière à Lyon, en sorte que j’ai pu me convaincre de son identité. La figure de Dup. quoique insuffisante, est très reconnaissable, sa description l'est moins. Quant à l'espèce de Boisdural, elle reste indécise.

\section{$\mathrm{N}^{\circ} \mathbf{2 7 4 ^ { b }}$. Multistrigaria. Stph.}

Wood. 548. - Her.-Schf. II. 139, f. 196, 197, 329.

- Heyden. C. 364.

Trouvée près d'Aubonne, au printemps 1853, par M. G. Leresche. - Collection de Lausanne.

$\mathrm{N}^{\circ}$ 277. Caesiaria. W. V.

Ajoutez comme synonyme:

Griseicinctata. Bru. Cat. $706^{3}$.

J'ai vu des individus pris dans le Jura, à Pontarlier, près de la frontière suisse, par $\mathbf{M}$. Th. Bruand, en sorte que cette espèce existe dans le Jura. 
$\mathrm{N}^{\circ}$ 278. Flavicinctaria. Hub.

La vraic flavieinctaria n’a en effet pas été prise jusqu'ici dans le Jura (Rothb.); les individus recueillis sous ce nom appartiennent ì in fidaria, Nob.

$\mathrm{N}^{\circ}$ 279. Cyanaria. Hul.

II faut ajouter aux synonymes :

Ca esia ta (pâle). Bru. Cat.

No 280. Infidaria. Nob.

Le sinus du bord interne de la bande médiane est parfois peu prononcé; dans ce cas on peut établir le diagnostic par l'absence de teinte jaune sur le premier ruban, toujonrs large et plus clair que le reste de l'aile, et par la frange non limitée par des traits noirs, aux inférieures, en dessus.

\section{$\mathrm{N}^{0}$ 282. Nebularia. Treit.}

J'ai pris un individu appartenant à cette espèce dans le Schächenthal, a la fin de juillet: il ressemble parfaitement à ceux que $\mathbf{M}$. Bruand prend dans le l)oubs sous re nom. Les individus de Vienne (Mann) en diffèrent ì quelques égards.

\section{No 281. A chromaria. Nob.}

Ayant pu me procurer quelques individus bien frais appartenant a cette espèce, j’ai ju l'étudier avee plus d'exactitude et ajouter quelques observations à celles que j’ai données sur son compte. - A chromaria se prend assez fréquemment en mai, au pied des $\mathrm{A}_{\text {pres, }}$, près de Meyringen. La teinte grisâtre du fond tire sur le bleuâtre ehez les individus trèsfrais. Le point discoïdal est un peu alongé en forme de trait. Quelques points pâles indiquent la place de la ligne ondulée le long de la marge. Les doubles points qui bordent la frange sont très-risibles sur les denx ailes et bien séparés les uns des autres par un point clair. La femelle est ordinairement un peu plus grande que le mâle et plus rare que lui. L'anus du màle est terminé par un mouchet de poils blanc-jaunâtres.

Cette espèce ne peut guères être confondur quavec u ebularia.

No 288. Sororaria. Hub.

Troucée près de St.-Noritz, dans l'Engadine, par N. C. Zeller, an mois de juillet 18.54 


\section{$\mathrm{N}^{\circ}$ 296. Ruberaria. Frey.}

J'ai reçu de M. Ott, à Meyringen, deux individus, mâle et femelle, de cette belle espèce, provenant très-probablement de la même source qui l'avait fournie, de seconde main, ì II. Freyer. A première vue elle n'est pas une variété d'impluviaria; bien moins encore d'elutaria. Les figures $(\mathbf{2 0 9}, \mathbf{2 1 0})$ de IIer-Schf. ne lui conviennent pas complétement ; tout l'animal est plus foncé et plus marqué de rouge-sang que dans ces figures. M. Bruand in'a fait voir des ruberaria reçues d'Angleterre qui ne sont que des variétés d'el u taria, marbrées du rouge-brun. Les caractères indiqués par Her.-Schf. et par Freyer ne sont pas non plus suffisants. Ruberaria a la taille des plus grands individus d'impluviaria, et la même coupe d’aile qu’elle. Son dessin est à peu des choses près aussi le même. Les ailes supérieures se divisent en quatre champs bien déterminés. L'espace basilaire et le second espace (bande médiane) sont d'un fauve-elair un peu rougeâtre, sablé de gris, surtout sers la côte. Le second champ ( $1^{\text {er }}$ ruban) est d'un brun rouge plus foncé au centre de l'aile, et d'un gris-rougeâtre vers les deux bords. Un trait noir sépare ce champ de l'espace basilaire, une bandelette rouge-sang le limite en dehors. Le quatrième champ (espace marginal) porte de dedans en dehors: $1^{\circ}$ une bandelette rouge-sang, finement bordée de noir en dedans; $2^{\circ}$ un ruban gris-rougeàtre ordinairement cerné par un trait blanchàtre en dedans; $3^{\circ}$ une bandelette rouge, lavée sur ses bords et vers son extrémité interne, marquée sur les nervures de traits noirs; $4^{\circ}$ une bandelette grise entrecoupée par des points noirs, qui eux-mèmes sont divisés par les nervures d'un fauve-rougeâtre, clair.

La frange est d'un gris-rougeâtre, mouchetée de gris-noir, limitée par un trait plus clair du còté de l'aile. Le point discoïdal, qui n'est pas toujours visible, est représenté par un trait en forme de virgule, sapproché du bord interne de la bande moyenne. Deux à trojs traits aigus, très-noirs, se voient vers le sommet de l'aile.

Le dessus des inférieures est d'un gris-rougeâtre, plus foncé vers la marge; on y distingue un point discoïdal brun et une ligne sinueuse de mème couleur peu apparente. Le dessous de's mêmes ailes est d'un gris un peu fauve, luisant, très-sablé de gris, marqué comme le dessus, mais plus distinetement. Le dessous des supérieures est plus luisant encore, lavé de gris-noirâtre, surtout au centre et à la base. Un seul trait gris-foncé, très-sinueux, et le point discoïdal y sont bien visibles. La frange est en dessons de la couleur du fond et séparée de lui par un trait noir, fin. La côte, en dessous, est marquée de denx taches brunes. Le dessous du corps est de la conleur du dessous des ailes. Le dessus de l'abdomen a la couleur des inlérieures en dessus. Le thorax est gris-foncé, mẻlé de rougeâtre. 
Les caractères qui distinguent essentiellement $r$ uberaria d'impluviaria sont : $1^{\circ}$ la bande moyenne et l'espace basilaire d'un gris-fauve, et non bleuâtre ou verdâtre; $2^{\circ}$ le bord externe de cette bande très-dentellé et formant un grand sinus; $3^{\circ}$ la bande moyenne plus large au centre et retrécie vers la côte (pas constant); $4^{\circ}$ les bandelettes rouges, indiquées; $5^{\circ}$ la ligne du dessous lléchie fortement en dedans en s'approchant de la côte: $6^{\circ}$ les inférieures d'un gris plus foncé.

La femelle ne diffère pas du mâle. Le caraetère tiré de la présence d'un seul trait foncé en dessous se retrouve dans quelques exemplaires d'impluviaria.

\section{$\mathrm{N}^{+0}$ 302. Psittacaria. F.}

Commune autour de Lausanne sur le bord des bois de sapin en avril, le long du Léman, dans les lieux abrités et en septembre sur les hauteurs.

J'ai pu voir dans la collection de M. Couleru un individu màle de c oracia la Hub., parfaitement frais, pris dans le Jura et qui n'est éridemment qu'une variété de psitta c aria. On trouve d'ailleurs facilement des passages de l'une à l'autre.

\section{$\mathrm{N}^{\circ}$ 30:3. F i r ma ria. Treit.}

Les individus recueillis par $\mathbf{M}$. Rothenbach appartiennent à la variété obelis c at a de variaria. Je n'ai pas vu ceux de $\mathbf{1 1}$. Couleru, qui affirme l'avoir aussi recueillie au pied du Jura.

$\mathrm{N}^{\circ}$ 309. Perfuscaria. Haw.

M. Bruand a reçu d'Angleterre sous le nom d'i mm a n a ta, Wood. 573, la même espèce à laquelle 11. Curtis lui-même a donné en ma présence celui de perfus cata. Depuis lors j'ai pu me procurer un certain nombre d'individus de cette espèce et de sa voisine, et j'ai pu me eonvaincre que ces espèces anglaises ne sont que des variétés de russaria. A cuta ta Guén. n'est aussi pas autre chose.

Russaria varie extrêmement et je n'hésite pas à admettre parmi ses varićtés la figure 445 de Hubner, quoique la forme générale soit plus ramassée, puisque cet accident peut résulter de l'éducation de la chenille en captivité:

La bande moyenne varie à tous égards extrêmement, plus peut-être que dans v a ri a ria; il en est de même des angles et des courbures des diverses lignes transverses. La coloration des bandes est dans le même cas.

Le mâle offre plus souvent la variété perfusc at a et immanat a que la femelle. Le 
dessin et la teinte des ailes inférieures varie aussi très-souvent. Les entrecoupures de la frange disparaissent parfois; les points de la marge tantôt forment une ligne noire, tantôt restent séparés par des points blancs. La chenille se troure aussi sur divers arbres.

\section{$N^{\circ}$ 314. Tersaria (ta). II. V.}

IIub. 268. - Treit. - Dup. descript. non fig. Bdv. 1652. - Her.-Schf. II. 198. - Wood. 612. - Heyden. Cat. 403.

L'espèce que j'avais sous les yeux en rédigeant mon catalogue, n'appartient point à la véritable tersat a, IIub.; mais à ma radicaria $\left(\mathrm{n}^{\circ} 314^{\mathrm{c}}\right)$ qui a été confondue jusqu'ici avec elle. En examinant la figure 268 de IIubner, javais déjà conçu des doutes à ce sujet; mais celle que Duponchel donne de tersata, se rapportant parfaitement aux individus de ma collection, j’arais admis quelques incorrections dans la figure de Hubner.

La véritable tersaria n'a pas été prise encore dans les environs de Lausanne. Je l'ai reşue plusieurs fois de Meyringen, et l'ai vue dans la collection de M. Bremi à Zurich. Les exemplaires reçus de Vienne sont un peu plus grands que les nôtres, mais identiques. Elle paraît préférer les sous-alpes de la Suisse centrale où elle se montre en juin. M. Heusser l'a prise près de Burgdorf. C'est elle que javais devant moi en décrivant ma test ac e a ria $\left(1^{\text {er }}\right.$ Supplément, $\left.n^{\circ} 314^{\mathrm{h}}\right)$; ensorte que ce que je dis des caractères qui distinguent test acearia de tersaria doit se rapporter entièrement à celle-ci, et la différentier de ma r adicaria, et non de test a ce a ri a (aemularia) qui est une toute autre espece. Tersaria, reçue de M. Mann, est identique avec les nôtres.

$\mathrm{N}^{\circ} 314^{\mathrm{h}}$. A emularia (ta). Hub. 448.

Boisd. 1653. - Her.-Schf. II. 196.

Var. Tersat a. Treit. - Dup. - Heyden. Cat.

C'est à tort que j’ai rapproché (1 ${ }^{\text {er }}$ Supplément) t es t a c eata, Ilub. 338, de a e m ulat a, Hub. 448. Ces deux espèces doivent rester parfaitement distinctes. La première peut bien n'être qu'une variété de tersaria, tandis que la seconde en diffère à plusieurs égards. J'ai reçu deux individus, mâle et femelle, de cette espèce, pris à Meyringen par M. Ott.

La figure de Hubner, trìs-fidèle pour le dessin, ne l'est pas pour le coloris; l'individu sur lequel elle a été prise paraît avoir été un peu eflacé, car la bande médiane n’y est pas visilıle.

La couleur d'a emularia est le brun grisatre, tirant sur le roux vers la racine de 
l'aile. La bande médiane est indiquée par deux raies recourbées et parallèles, d'un gris noirâtre, dont l'externe, très-denticulée, ne porte qu'un léger angle fort prìs de la côte. L'interne porte deux angles, l'un près de la côte, l'autre à son centre; son extrémité abdominale se lléchit assez fortement du côté de la racine de l'aile. La ligne marginale ou fulgurale et les points blancs et noirs des nervures sont tels que les indique la figure de Hubner. La frange est limitée par une série de points noirs séparés par des points blanes. Le premier anneau de l'abdomen est presque blanc. Lo dessous est d'un gris roussâtre, par'nuru par plusieurs lignes ondulées plus prononcées vers la marge, et marqué de quatre points discoïdaux, comme le dessus.

La ligne fulgurale, la teinte brunâtre, l'alsence d'angle au milieu du côté externe de la bande médiane, les points marginaux, la distinguent suffisamment de tersaria. La taille de celle-ci est un peu inférieure à celle d'a emularia. L'exemplaire figuré par Hubner était de petite taille.

$\mathrm{N}^{\circ} 314^{c}$. Radicaria, nobis.

Ters ata. Dup. hist. pl. 197, f. 1 et 2 (an descript. ?). L a pidata. Bdr. 1657 ?

Ce que j'ai dit de l'apparition de tersaria aux environs de Lausanne doit se rapporter à cette espèce ci. La figure de Duponchel lui convient parfaitement, la description qu'il en donne se rapporte plutôt à la vraie tersaria. La figure 324 de Hubuer (lapidata) s'en rapproche à plus d'un égard; ensorte qu'il y a tout lieu de croire qu'il faut rapporter à cette espèce la lapidata que Boisduval a reçue du Midi de la France et de Fontainebleau. Lapida ta figurée dans Hub. sup. f. $\mathbf{2 8 6}, \mathbf{2 8}$, venant de Laponie, en diffère totalement.

Les figures de Duponchel, surtout celle de la femelle, sont suffisantes (quoique imparfaites), mais comme les deux espèces sont très-voisines, je dois ajouter quelques détails.

Radicaria a la taille des grands individus de tersaria. Le bord externe des supérieures est légèrement plus arqué, et celui des inférieures un peu plus anguleux. La teinte générale est le gris mélangé de fauve-clair. Les nervures n'étant entrecoupées que par fort peu de lignes transverses, offrent moins de pointillé que dans tersaria.

Aux supérieures, l'espace basilaire est couleur biche uni; dans tersaria il est trèspointillé de noir. Le premier ruban d'un faure plus clair est uni, deux fois plus large à la côte qu'au bord postérieur. La bande moyenne, étroite à la côte, s'élargit un peu dans son milieu. Son bord interne forme un angle vif près de la côte. L'externe porte un angle profond en regard du précédent, et de ce point la bande descend en festons arrondis et en décrivant 
une courbe donce et uniforme, jusqu'au bord postérieur. T ersaria a la bande mojenne plus large et volontiers coudée en dehors, vers son milieu; chez elle cet espace est plus ou moins couvert d'atomes gris, qui n'existent pas dans radicaria. Le second ruban de cette dernière n'est limité que dn còté de la bande movenne; en dehors il se fond avec l'espace marginal, de là vient qu'il est moins distinct que dans tersaria. quoiqu'il aie une teinte plus claire.

La ligne dentellée marginale est ordinairement plus saillante dans radicaria; vers le sommet de l'aile elle est coupée par un trait oblique noir, très-prononcé, au-delà duquel se voit un ispace uni, d'un fawe clair qui termine l'aile. Tersaria porte le trait oblique peu visible et u’interrompant pas le dessin de la marge.

Le dessin des inférieures est le même dans les deux espèces; il est un peu moins chargé de lignes transverses dans radicaria, et chez celle-ci le second ruban, placé an milieu de l'aile, forme un coude plus brusque en s'approchant du bord antérieur.

Les points marginaux se rapprochent les uns des autres dans tersaria et forment un trait brisé et entrecoupé; dans sa voisine ces points sont plus espacés.

Le dessous des ailes est semblable dans les deux espèces; il en est de mèmes des autres organes.

$\mathrm{N}^{\circ}$ 329. Chaerophillaria. Lin.

J'ai pris aussi cette phalène sur le haut du Jorat, au mois de juillet. 


\section{CATLLOGLE SISTÉIILTHOLE}

IUES

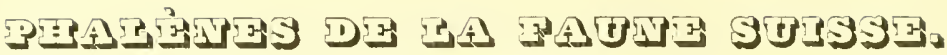

Numéros

\section{Geomerira.}

I. Papillonaria, Lin. - ILub.

2. Bajularia, Esp. -- Ilub.

3. Aestivaria, Esp. - IIub.

4. Buplevaria, W. V. - Hub.

5. Viridaria, Lin. - Hub.

6. Putataria, Lin. - Hub.

7. Aeruginaria, IV. V. - Iub.

8. Vernaria, Lin. - Hub.

\section{Psendoterpua.}

9. Cythisaria, W. V. - Hub.

\section{Icidaliá.}

10. Amataria, Lin. - IIub.

I1. Vihicaria, Lin. - Hub.

12. Calabraria, Lin. - Hub.

13. Nicrosaria, Bdv. - Pusillaria, Hub.

14. Laevigaria, IV. V. - IIub.

15. Scutularia, WV. V. - Ilub.

16. Reversaria, Treit.

17. Iucanaria, IV. V. - IIub.

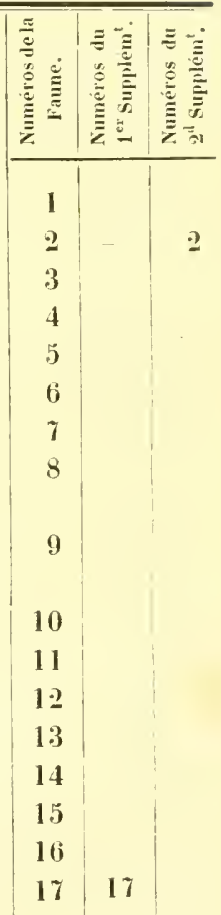


Numeros.

18. Bischollaria, IIub.

19. Straminaria, Treit. - Sylvestraria, IIub. 94.

Grammicaria, Bdr.

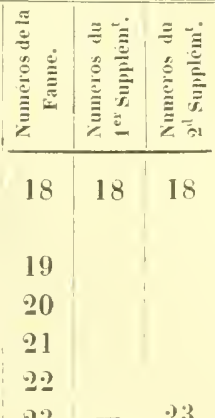

Suffusaria, Treit. - Bdv. (il ellacer) . . 23

23. Ossearia, F. - IV. I.

24

24. Interjectaria, Bdv. - Dilut aria, Ilub. 100.

2.5

25. Holosericearia, Fisch. v. R.

26. Pallidaria, W. V. - Ilub. - B y s s in ari a, Bdv.

27. Renularia, IIub.

28. Perochraria, Fisch. 1. R.

-

29. Rufaria, Hub. - Treit.

28

30. Ochrearia, IV. V.

29

3I. Moniliaria, F. - Hub.

30

32. Rusticaria, W. V. - Hub.

31

33. Confinaria, Fisch. v. R.

34. Commutaria, Treit.

32

35. Rubricaria, IV. V. - Iub.

$-$

33

36. Remutaria, Lin. - Hub.

37. Nemoraria, Hub.

38. Sjlvestraria, Bork. - Hul.

37

39. Caricaria, Her.-Schf. - P hl earia, Reuti

40. Cerusaria, mihi. - Puncta ta, Bork. - Subpunct a ria, Hub. sup.

41. Mutataria, Treit.

42. Immutaria, W. V. - IIub. 
Xивาюm.

43. Contiguaria, Ilub.

44. Ornataria, W. V. - Ilub.

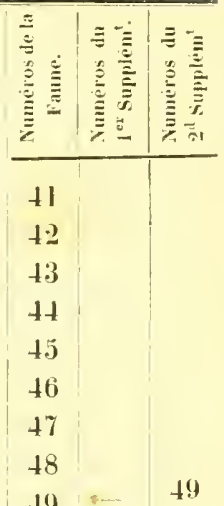

45. Decoraria, W. V. - Llub. . . . . 43

46. Immoraria, Lin. - llub. . . . 44

47. Strigaria, Ilub. - Treit. . . . . 45

48. Compararia, Fisch. v. R. - U inbellaria, llub. . 46

49. Prataria, Bds - Strigillat a, Treit. . . . 47

50. Aureolaria, Fab. - Hub. . . . . . 48

5I. Flareolaria, llub. - Treit. sup. . . 49

52. Auroraria, IIub.

50

53. Marginaria, Liı. - Hub.

51

\section{Epligrai.}

54. Trilinearia, Bork. - Treit. . . . . . 5.

55. Punctaria, Lin. - Ilub.

53

56. Omicronaria, W. V. - Hub.

57. Pendularia, Lin. - Ilub.

55

58. Orbicularia, Hub. . . . . . . 56

59. Poraria, Lin. - Treit. - Punctaria, Itub). . 57

60. Schæfferaria, mibi. - Gyraria, Treit. (non Hub.) Faune hel. 1. 39

61. Pupillaria, Ilub. (non Zell.)

\section{Emmiltis.}

62. Sericearia, Hub. . . . . . . . . 60

63. Parrularia, Bd. - Pygma e aria, Ilub. - Treit. . 61

\section{Aplasta.}

64. Ononaria, Fuess. - Hub. . . . . . 62

Boletobia.

65. Carbonaria, Lin. - Itub. 
Numeros.

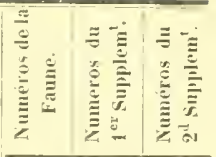

Metrocampa.

66. Fasciaria, Lin. - Hub. . . . . . 64

67. Margaritaria, Lin. - Hub. . . . . . 65

68. Honoraria, IV. V. Hub. . . . 66

Eugonia.

69. Tiliaria, Hub. . . . $\quad 67$

70. Alniaria, Lin. - Hub. . . . . 68

71. Quercinaria, Bork. - Ilub. . . . . . . $69: 69$

72. Erosaria, W. V. - Hub. . . . . 70

73. Angularia, W. V. Hub. . . . . . 71

Crocalis.

\begin{tabular}{l|l|l|} 
74. Elinguaria, Lin. - Hub. . . . . . & 72
\end{tabular}

Odontopterat.

75. Dentaria, Esp. - Hub. . . . . 73

Himera.

76. Pennaria, Lin. - Hub. . . . . 74

\section{Selenia.}

77. Illustraria, Hub. - Treit. . . . . 75

78. Lunaria, W. V. - Hub. . . . . . 76

79. Delunaria, Stph. - Hub. . . . . . 77

80. Illunaria, Hub. - Treit. . . . . . 78

81. Syringaria, Lin. - Hub. . . . . . 79

Epione.

82. Advenaria, Esp. - Hub. . . . . . 80

83. Apiciaria, W. V. - Hub. . . . . . 81

84. Parallelaria, W. V. - Hub. . . . . . - - - $-81^{\text {b }}$

Therapis.

85. Artesiaria, IV. V. - Hub. . . . . 82 
Numeres.

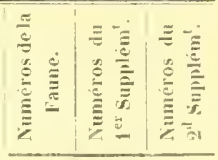

Macaria.

86. Notalaria, Lill. Ilıb. . . . . . 83

87. Alternaria, W. V. Ilub. . . . . 84

88. Signaria, Hub. - Treit. . 85

89. Lituraria, Lin. IIub. . . . . 86

Ienilia.

90. Macularia, Lin. . . . 87

Irapteryx.

91. Sambucaria, Liu.

Rumia.

92. Crataegaria, Lin. .

Eurymene.

93. Dolobraria, Lin.

Ploseria.

94. Niversaria, Bork. - Inub. . . 91

Pluasiane.

95. Petraria, Esp. - Hub. . - . 92

\section{IIiberuia .}

96. Rupicapraria, IV. V. -- Hub.

97. Deloliaria, Lin. Hub. _ . . 94

98. Aurantiaria, Esp. Hub. . . . 95

99. Progemmuaria, IIub. - Treit. . . . 96

100. Leueophaearia, W. V. Ilub. . . . . 97

101. Bajaria, Hub. - Treit. . 98

seoria.

102. Dealbaria, Lin. - Hub. _ . . 99

\section{cleogene.}

103. Tinctaria, Ilub. - Bdv.

I04. Illibaria, W. V. - Hub. . . . 101 
Numeros

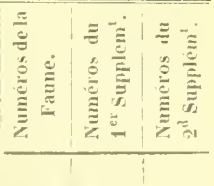

Angeroira.

105. Prunaria, Lin. IIub. . . .

Zerene.

106. Melanaria, Lin. - Hub. 103

107. Grossularia, Lin. . 104

108. Ulmaria, Fab. - 1luh. . . . 10.5

109. Pantaria, Lim. - Iub. . . 106

110. Marginaria, Lin. . . . . 107

Vumeria.

111. Pulseraria, Lin. . . 108

112. Capreolaria, IV. V. Huh. 109

Bapta.

113. Taminaria, W. V. IIub. 110

114. Temeraria, W. V. - Hub. 111

115. Pictaria, Curt. Bds. . . 112

Ginophos.

116. Serotinaria, Hub. - Her.-Schf. . 113

117. Mendicaria, Her.-Schf. 114

114

118. Dilucidaria, IV. V. IInb. . 115

119. Spurcaria, Lah. . . 116

120. Zelleraria, Frey. Bdv. $\quad 117$

121. Operaria, Ilub. - Treit. . 118

122. Furvaria, Fab. - IInb. . $119-119$

123. Pullaria, IIul. Treit. . . 120120

124. Anbiguaria, Dup. Bds. M eycraria, faunchelvét. 121 - 121

125. Obscuraria, W. V. IIub. . . 122 122

126. Glancinaria, IIub. - Treit. . . $123-123$

127. Falconaria, Frey. . . - 12:3" 
Numeros

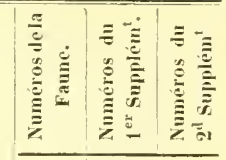

128. Variegaria, Hub. - Treit.

129. Limosaria, Ilub. - Bdv. (O b fus c a ri a, Treit.)

130. Andereggaria, Lah.

124

125

\section{Boal'mia.}

131. Nyctemeraria, Hub. - Bdv. . . . . 126

132. Perversaria, Bdv. - Her.-Schf. . . . . . 127

133. Abietaria, W. V. - Hub. . . . . . 128

134. Secundaria, W. V. - Ilub. . . . . . 129

135. Cinctaria, W. V. - Hub. . . . 130

$-123$

136. Consortaria, Fab. - Ilub.

137. Rhomboidaria, W. V. - Ilub.

131

138. Roboraria, Fab. -.. IIub. . . . . . 133

139. Viduaria, W. V. - Hub. . . . . . 134

I 40. Glabraria, Ilub. Treit. . . . . . 135

141. Repandaria, Lin. - Hub. . . . . . 136

142. Licheuaria, W. V. - Itub. . . . . . 137

143. Lividaria, Ilub. - Treit. . . . . . 138

144. Crepuscularia, W. V. - Ifub. . . . . 139

145. Consonnaria, Bdv. . . . . . 140

146. Punctularia, W. V. - Ilub. . . . . 141

147. Extersaria, Itub. - Treit. . . . . . 142

148. Adustaria, Bork. - IIub. . . . . . 143

Fillonia.

149. Pusaria, Lin. . . . . . . 144

150. Exanthemaria, W. V. I Ilub. . . . . 145

151. Strigillaria, Esp. - Hub. . . . . . 146

152. Plumaria, W. V. - IIub. . . . . . . $147|147| 147$

153. Wawaria, Lin. 
Vumeros.

154. Roraria, Esp. - Bd.

15.5. Conspicuaria, Esp. - Hub.
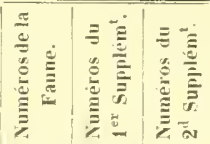

156. Pinetaria, Hub. - Treit.

149

157. Clathraria, Lin.

150

158. Glarearia, IV. V. - Hub.

151

159. Piniaria, Lin. - W. V.

152

160. Plumistaria, Esp. - - Hub.

153

161. Picearia, Hub. - Treit.

154

I62. Atomaria, Lin.

155

156

157

Mniophilas.

163. Corticaria, Hub. - Bdv.

158

164. Cineraria, Hub. - Treit.

$-158^{b}$

Aspilates.

165. Gilvaria, Bork. - Ilub.

159

Stabnelia.

166. Hypocastanaria, Hub.

$-159^{\mathrm{b}}$

Imphidasis.

167. Zonaria. Hub.

160

168. Bombicaria, Bdv.

- Alpinaria, Scrib. (a effacer)

169. Pilosaria, Bork. - Ilub.

161

$162-162$

163 - 163

170. Hirtaria, Lin.

164

164

171. Prodromaria, Fab.

165

172. Betularia, Lin.

166

Psortas.

173. Venetiaria, Hub. - Treit.

$167-167$

174. Torvaria, Hub. - Treit. . . . . . 168

175. Horridaria, W. V. - Hub. . . . . . 169 
Numeres.

176. Trepidaria, IIuh. -- Treit.

17i. Equestraria, Esp. - (Alpinata, Hub.) .

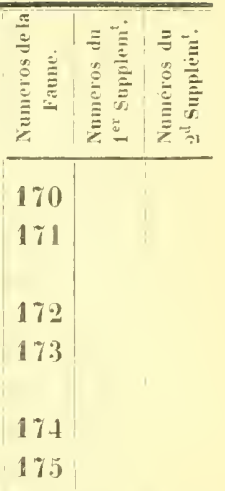

Minoil.

182. Euphorhiaria, W. V. - Hub. . . 176

183. Tibialaria, Ilub. - Treit. . . 179

Hydrellia.

184. Candidaria, W. V. - Hub. . . . 178

185. Syaria, W. V. - Ilub. . . 179

186. Lutearia, Fab. - Ilub. . . 180

187. Itepararia, W. V. - IIub. . 181

188. Erutaria, Bdv. - Her.-Schi. 182

182

Enpitlıscè̃a.

189. Coronaria, IIub. - Frey. . . 183

190. Centaurearia, Fab. - W. V. 184

I9I. Linaria, Bdv. -- IJub. . . . 185

192. Hospitaria, Treit. - Bdv. $\quad 186 \cdot 186$

193. Venosaria, Fab. IIub. . 187

194. Consignaria, Bork. Ilub. . . 188 - 188

195. Sobrinaria, Hlub. - Treit. _ 189-18!

196. Succenturiaria, Lin. - Ilub. . . $190-190$

19\%. Lariciaria, Frey. - IIer.-Schı. . . . . 191

I98. Modicaria, Ifub. - Bdr. . . . . 192 
Vumeros.

199. Innotaria, Knocl. - Treit.

¿00. Austeraria, Fisch. y. R.

201. Tenuiaria, IIub.

202. Veratraria, Fisclı. 1. R.

203. Satyraria, ILub. - Treit.

204. Helseticaria, Anderg. - Bilv.

-05. Arceutharia, Frey.

o66. Majoraria, Lalı.

207. Absyntharia, Lin. - IIul.

208. Trisignaria, Her.-Schf.

D ist inctaria, IIer.-Schf. (à retrancher

209. Scriptiria, Her.-Schf. .

210. Singularia, Her.-Schf.

211. Castigaria, Hub.

21:2. Indigaria, Hub.

213. Valerianaria, Hub.

214. Isogrammaria, Treit. - Bd.

215. Begrandaria, Dıр.

216. Argillacearia, IIer.-Schf.

217. Pusillaria, IV. V. - Hub.

218. Manniaria, Fiscls. r, R.

219. Inturbaria, Hub.

2.20. Exiguaria, Hub.

221. Irriguaria, IIub.

222. Grapharia, Kind. - Treit.

223. Rectangularia, Lin.

22. Debiliaria, Ilub.

2.25. Pimpinellaria, Hub.

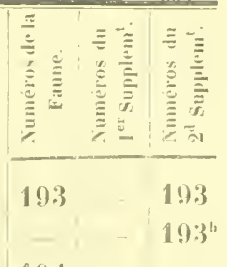

194

19.5

196

197

198

- $198^{\mathrm{b}} 198^{\mathrm{b}}$

199

200

\begin{tabular}{c|c|c}
- & $200^{h}$ & $200^{h}$
\end{tabular}

201

202

$\because 03 \quad 203: 203$

$-203^{b}=$

$204-204$

20.5

- $205^{\mathrm{h}}-$

$-20.5^{c}-$

206

207

208

$20 !$

210

211

212

213 
Nimeros.

226. Strobilaria, Bork. - Hub.

\section{政}

2:7. Togaria, Ilub.

2.28. Pumilaria, Hub.

\section{Larentiat.}

229. Sparsaria, Ilub. 398

230. Dubitaria, Lin.

231. Montivagaria, Boisd.

232. Certaria, Hub.

233. Scripturaria, W. V. - IIub.

234. Bilinearia, Lin.

235. Riguaria, Hub. - Treit.

236. Vetularia, IV. V. - Hub.

237. Sabaudiaria, Dup. - Iub.

38. Undularia, Lin. - W. V.

239. Polygrammaria, Bork. - Hub.

240. Rhamnaria, IV. V. - Ilub.

241. Badiaria, W. V. - Hub. .

242. Berberaria, Fab. - Ilub.

243. Rubidaria, IV. V. - Ilub.

244. Turbaria, Hub. - Treit.

245. Ocellaria, Lin.

246. Propugnaria, Fab. - IIub.

247. Ligustraria, W. V. - IIub.

248. Quadrifasciaria, Fab. - Hub.

249. Olivaria, W. V. - Ilub.

250. Aptaria, Hub. - 'Treit.

251. Ferrugaria, W. V. - Bork.

252. Spadicearia, Bork. - W. V.

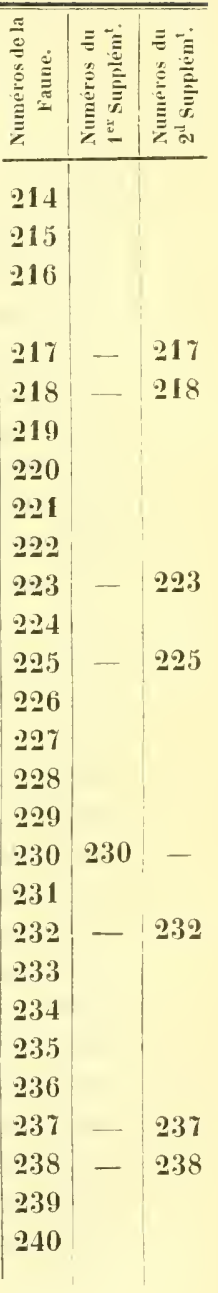


Numéros.

253. Arctaria, Lin. - Her.-Schf.

254. Kollariaria, Mann. - IIer.-Schf.

255. Laetaria, Lah.

256. Galiaria, W. V. - Hub.

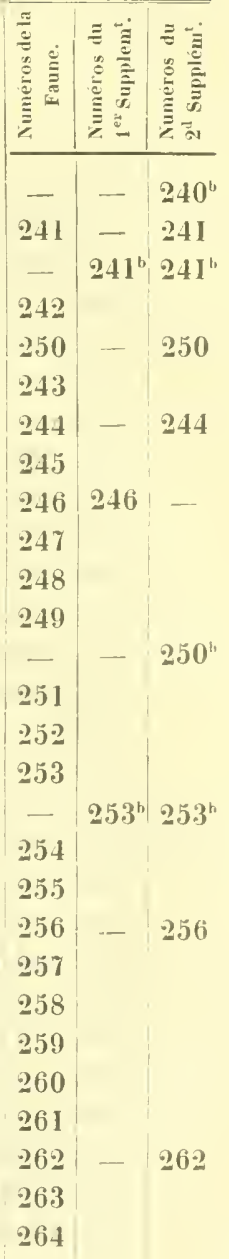

- Var.? Chalybearia, Hub.

257. Alpicolaria ? Fisch. v. R. - A b ster s a ri a, Her.-Schf.

258. Sinuaria, W. V. - IJub.

259. Rivaria, Ilub. - Treit.

260. Unangularia, Wood. - A m niculat a, Ilub.

26 I. Alchemillaria, Lin.

262. Molluginaria, Hub. - Treit.

263. Nontanaria, IV. V.

264. Picaria, Hub. - Treit.

265. Scabraria . Ilub. - Treit.

266. Minoraria, Treit. - Bdv.

267. Blandiaria, W. V. - Hub.

268. Livinaria, Lah. - J u cu n d a ria, Faune helvét.

269. Fluctuaria, Lin.

270. Albicillaria, Lin.

271. Derivaria, W. V. - Hub.

272. Rubiginaria, Fab. -- Hub.

273. Procellaria. Fab. - Hub.

274. Hastaria, Lin.

275. Hastularia, Hub. 365 bis

276. Tristaria, Hub. 254 .

277. Funeraria, Hub. 260 .

278. Luctuaria, IV. V. - IIub.

279. Rupestraria, Fab. - W. V.

264 
Numeros.

280. Albularia, Fab. - W. V.

281. Decoloraria, Ilub. - Treit.

28\%. Ilydraria, Fisch. v. R. - Treit. sup.

283. Rivularia, W. V. - Ilub.

284. Affinitaria, Wood. - IIer.-Schf.

285. Incursaria, Hub. -.. Bds.

286. Lotaria, Bdv. - A quaria, Treit.

28\%. Porlevinaria, Iler.-Schf.

288. Salicaria, W. V.? - IJer.-Schf.

289. Valesiaria, Lah.

290. Multistrigaria, Steph. - Her.-Schf.

291. Dilutaria, W. V. - Hub.

29.2. Nobiliaria, Mann. - ILer.-Schf.

293. Caesiaria, IV. V. - Lub.

294. Flavicinctaria, IIub. - (Non Dup.)

295. Cyanaria, Ifub. - Treit.

296. Infidaria, Lalı - Flavicin c tari a, Dup.

297. Tophacearia, W. V. - Hub.

298. Nebularia, Treit. - Hub.

299. Incultaria, Her.-Schf. - I g n o bili a ri a, Man.

300. Acliromaria, Lah.

301. Palumbaria, Fab.

302. Plagiaria, Lin.

303. Cassiaria, Treit. - Pr a eforma ta, Hub.

304. Sororaria. Ilub. - I m b u t a t a, Hub.

305. Hoeniaria, IV. V. - Hub.

306. Chenopodiaria, Lin.

307. Mensuraria, IV. V. - Hub. .

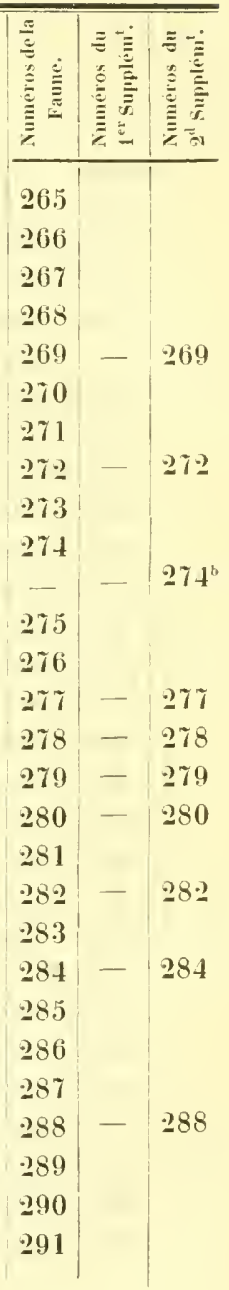


Numèros.

308. Niaria, IV. V. - Ilub.

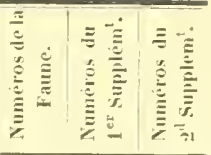

309. Bipunctaria, Bork. - Ilub.

292

310. Vespertaria, Lin. - W. V.

293

311. Impluviaria, W. V. - I Iub.

294

312. Ruberaria, Fres. - Her.-Schl.

295

313. Elutaria, W. V. - Hub.

296

296

314. Suffumaria, W. V. - Hub.

297

315. Ribesiaria, Boisd. - 1ler.-Scht. - Pru u ata. Lin.

298

316. Silacearia, W. V. - Hub.

299

317. Reticularia, W. V. - Ilıb. .

300

318. Psittaccaria, Fab. - Var. Cor a eiat a, Hub. .

301

$302-302$

319. ?Firmaria, Treit. sup. - Ilub.

320. Fulvaria, W. V. - Ilub.

303

303

304

321. Popularia, Lin. .

305

32.2. Pyralliaria, Fab.

306

323. Achatinaria, IIub. - Treit.

307

324. Russaria, W. V. - Hub.

308

Var. Perfuseuta, Haw. - Faune helvét.

309

$309 \quad 309$

325. Ruptaria. Hub. .

310

326. Variaria, IV. V. - IIub.

311

327. Stragularia, Ilub. 337. - (Non Boisd.

$312 \quad 312$

328. Juniperaria, Lin.

313

329. Tersaria, W.V. - Ilub. - T est a ce a ria, Faune helv.

- $314^{\mathrm{h}} \cdot 314$

330. Aemularia, Ilub. 448 (non Fanne helv.) .

$-\quad-314^{\mathrm{b}}$

331. Radicaria, Lah. - Ters aria, Faune helv. - Dup.?

314

$314^{\mathrm{c}}$

332. Vitalbaria, IV. V. - IIub. . . . . 315

333. Fhriaria, Ilub. . . . . . . . 316

334. Gemmaria, llub. . . . . . . 317 
Numeros.

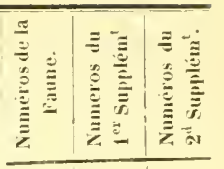

Cheinatobia.

335. Brumaria, Liı. . . . 318

336. Borearia, IHul.. . . 319

\section{Lobophopa.}

337. Appendicularia, Boisd. Sertata, Ilub. . $\quad 320$

338. Polycommaria, Hub. . . . 321

339. Lobularia, 11ub. . . . . . . 322

340. Viretaria, Hlub. . . . . . . . 323

341. Sabinaria, Anderg. - Ilub. . . . . 324

34. Ilexapteraria, Fab. . . . 325

343. Sexalaria, Bork. - Treit. . 326

\section{Chesias.}

344. Obliquaria, Bork. - Hub. . . 327

345. Spartiaria, Fab. - Ilub. 328

346. Chaerophyllaria, Lin. . . . $\quad 329-329$ 


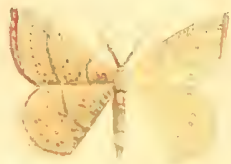

2

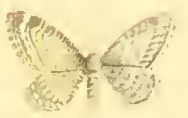

$\therefore$ ind)

6
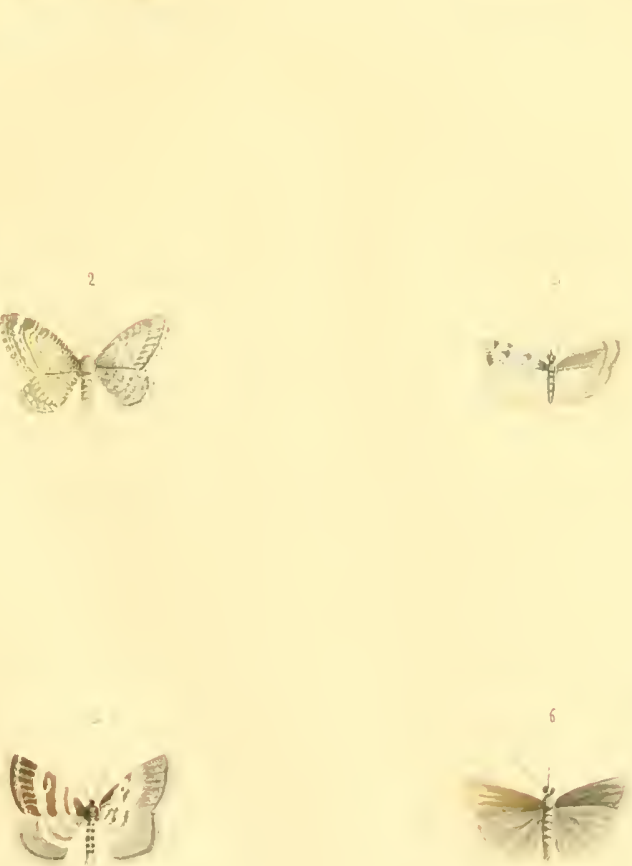

4

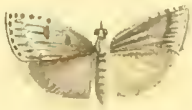

gedir o Ruff \& Hojis

PBrizgier on Zurteh

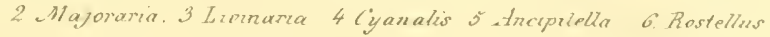




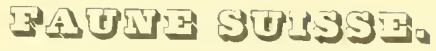

\section{LEIDOP I R R}

V. Partie. Pyrales.

PAR

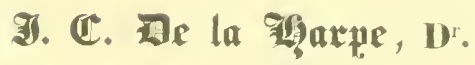





\section{PRÉACE.}

Nors avons suivi dans l'énumération des Pyrales suisses la mème classification que pour les Phalènes (voir Mémoires de la Société helvétique des sciences naturelles, vol. XIII). Si nous hésitàmes quelque peu à l'adopter dans la distribution de cette dernière famille, il n'en a pas été de mème pour celle qui va nous occuper. Les entomlogistes qui ont précédé HerrichSclı ffer ont tous laissé les Pyrales dans un grand état de désordre.

Il n'y a du reste là rien qui doive surprendre. Les Pyrales ne sont point, comne les Phalènes ou les Noctuelles, un groupe compacte et bien limité que l'on ne saurait confondre avec ses voisins. Elles n'offrent pas non plus, comme les Tinéites, cette foule de caractères divers qui facilitent singulièrement tout classement. Chez elles, à còté de petits groupes nettement branchés et isolés, en existent d'autres qu'il est malaisé de déliniter, d'autres encore dont toutes les espèces restent isolées. Les affinités sont obscures; les facies souvent insignifiant, parfois trompeur; les larves très-diverses et mal connues.

L'histoire méthodologique des Pyrales fera mieux comprendre nos assertions. Lixvé avec son coup-d'œil perçant, saisit leurs premiers contours et en forma l'un de ses grands genres destinés à prendre après lui le rang d'ordre, de tribu ou de classe, suivant la nomenclature adoptée. Sa décomposition analytique eut lieu graduellement, au fur et à mesure qu'un nouvel. observateur saisissait uni groupe et le délimitait. Latrellle distingua les $\mathrm{Her-}$ minia, les Botys et les A glosses; Scurank introduisit Hypena, Scopula, Nymphula, Agrotera, Pyrausta; Treitscilke reprit aux Noctuelles son genre Hercyna et établit Eunychia. 
Duponchel et Guenée, poursuivant la dissection tléjà fort avancée entre les mains des auteurs anglais, arrivèrent à un eanevas beaucoup plus serré, mais aussi beaucoup plus éloigné de la nature. Leur tribu de Pyralides se subdivisa en sous-tribus, correspondant à-peu-près aux genres de leurs prédécesseurs. De là surgit une série de nouveaux genres, mal assis pour la plupart. Guenee indroduisit Boreophila, Rlıodaria, Stenia, Pionea, Lemia, Rivula, Udea, Sthenopteryx, Helia; Dopoxcher Orenaia, Threnodes, Odontia, Cledeobia, Sopluronia, Madopa, Nola.

Herricu-Schaffer mil un terme à cette dissémination croissante el jeta les prenières base d'une synthèse méthodique plus naturelle. A-1-il complétement réussi dans ce travail ? un jugement nous sierait fort mal en présence de pareille autorité. Toujours est-il qu'il fit faire un pas immense à la classification en purgeant les Pyrales de genres qui ne leur appartenaient pas, en rapprochant d'elles d'autres groupes rejetés par ses prédécesseurs dans la classe incohérante des Tinéides et en détruisant un bon nombre de distinctions artificielles.

Un premier service rendu par Herkich-Schaffer fut de réunir aux Noctuelles les Herminies et les Hypènes de Treitschke. Il sulfit de parcourir les espèces d'Amérique pour saisir les transitions insensibles par lesquelles ces genres se lient à Ophiusa, Brephos, Agrophilla, Anthophilla, etc. Le genre Rivula, Guén. (Botys sericealis) devait épronver le mème sort. Nola des auteurs anglais (Hercyna B. Treit.), que Duponchel (Catalogue) place, on ne sail pourquoi, dans ses Plat yomides (Tordeuses) fut encore éloigné des Pyrales et réuni aux Lithosies. Cette place est en effet la seule qui lui convienne.

Après l'épuration des Pyrales venait leur reconstruction. A còté des Botys à ailes étroites et alongées se rangeaient tout naturellement les Crambes. Le dessin de Sthenopteryx et d'Eudorea rappelait celui de Botys et des Phycies. La forme de la tète, la position des antennes, la structure des pattes, le développement relatif des ailes, la présence des palpes accessoires, confirmaient ces rapprochements. Il étail dès lors démontré que les genres Phycis, Scirpopliaga et Chilo de Treitschke (Schoenobides et Crambides, Dup.) et Eudorea, Curt., deraient quitter les Tinéides et se réunir aux Pyrales. Cette union indique d'ailleurs assez les rélations qui existent entre les Y ps olophes (Tinéides) et les Pyrales. 
Après avoir reconstitué de la sorte les Pyrales de Linné, Herricu-Scinfarer détermina avec tout antant de bonheur la distribution des genres. Ici encore, comme partout, tantôt il luttait contre les tendances de l'analyse qui, poussées à l'excès, tirent de chaque caractère un genre nouveau, tantòt il heurtait aux inconvénients de rapproclıements synthétịues exagérés. Qui pourrait se flatter de toujours tenir le juste-milieu entre ces deux extrêmes, lorsqu'on est forcé de disposer en série linéaire ce que le Créateur distribua en réseau entrelacé? Les résultats principaux de ce travail furent la disparution des genres Scopula, Pyrausta, Asopia, Rhodaria, Threnodes, Ennychia, Udea réunis à Botys et la dininution du nombre des genres établis par Zeller, Hubner et Guénée parmi les Phycides.

L'entomologiste de Ratisbonne divisa enfin l'ordre des Pyrales ainsi constitué en deux familles; celle des Prandidmes, qui elle-mème se subdivisa en 3 tribus, et celle des Crammines. La première tend de loin la main aux Tordeuses et la seconde touche aux Tinéides. Il nous semble qu'il y aurait eu quelque avantage ì n'en faire que deux subdivisions d'une mème lamille appelée Prralides ou plus simplement Pyrales.

La distribution géographique des Pyrales est peu différente de celle des géomètres. Les espèces qu'elles embrassent appartiennent en plus grand nombre aux régions méridionales. Sur les 176 espèces enrégistrées dans notre Catalogue, nous en comptons 18 des régions septentrionales, 29 pour les méridionales, 38 exclusives aux Alpes et 91 plus ou moins dispersées dans toute l'Europe.

Si nous comparons le chiffre total des espèces suisses à celui des européennes, nous avons un rapport exact de 1 sur 3 , proportion que de nouvelles recherches démontreront sans doute trop faible. Le chiftre total de 529 espèces porté au catalogue de Heydenreich (1851) est beaucoup trop élevé, puisqu’il comprend toutes les espèces énumérées par les collecteurs et renferme par conséquent un grand nombre de doubles emplois.

Les Pyrales embrassent d'ailleurs un groupe nombreux, celui des Phycies, dont l'énumération complète est à-peu-près impossible par snite de la rareté d'un grand nombre d'espèces. On peut estimer qu'un tiers de celles que la Suisse nourrit, restent encore à recueillir. Nous pensons qu'on ne s'écarterait pas sensiblement de la vérité en admettant le rapport de $\mathbf{2}$ à $\mathbf{5}$ 
pour exprimer le chiffre des espèces helvétiques comparé à celui des curopéennes. Avec cette correction le nombre proportionnel de cette famille se trouve encore sensiblement au-dessous de celui des Phalènes.

La distribution géographique indiquée donne cependant, pour les $\mathbf{P y}$ rales, une proportion plus forte d'espèces purement alpines, que pour les Phalènes. Dans celles-ci elle était de 1 sur 7 ; pour celles-là elle s'élève à 1 sur $4 \frac{1}{2}$. Les espèces qui se prennent dans le nord de l'Europe donnent la mème proportion, de 1 sur $\mathbf{9}$, pour les deux familles. Le rapport des espèces méridionales qui est de 1 sur 13 pour les Phalènes, s'élève au double pour les Pyrales. Les espèces qui se retrouvent dans toute l'Europe figurent pour plus de la moitié; mais cette indication est insignifiante parce que un grand nombre d'entr'elles n'ont été trouvées que dans un petit nombre de localités, et y apparaissent rarement.

Les mêmes personnes qui m’ont aidé à rassembler les matériaux nécessaires à mon premier travail, ont continué à m’accorder leur concours bienveillant dans cehni-ci. Je dois à M. Rothenbach, à Schüpfen, les renseignements les plus nombreux. MII. G. et Z. Zeller, de Zurich, et M. Conleru, de la Neuveville, m’ont procuré plusieurs pièces rares; M. Bremi-Wolf et M. le professeur Frey, de Zurich, des indications de localités.

En livrant ce Catalogue à la publicité, je reconnais tout ce qu'il á d'incomplet; mais je ne pouvais faire plus, ni mieux. Les Pyrales sont bien plus difficiles à rassembler que les Phalènes; l'observateur le plus actif pourrait difficilement, dans toute une vie, résoudre les questions encore indécises qui se rattachent à leur étude.

Plusieurs espèces énumérées intéressent l'agriculteur, il n’en est cependant aucune qui cause de réritables ravages. Ce que l'on peut dire sous ce point de vue, ne présente qu'un très-médiocre intérèt économique.

Les insectes qui ont servi de base à ce travail restent déposés au Musée cantonal de Lausanne.

LAUSANNE, ce $1^{\text {er }}$ Novembre 1854. 


\title{
Famille des Pyralidides. Her.-Schf.
}

\author{
(P y ralien. Treit. partim.)
}

Ce grompe ne me parait pas assez distinct des Crambides pour former une lamille à lui seul. Ses caractères sont i-peu-près les mêmes.

I. Gen. Aglossa. Latr. - Stph. - Curt. - Dup. - (Py ralis. Lin. - Treit).

$\mathrm{N}^{\circ}$ 1. Pinguinalis. L.

(Phaliena.) Fab. - W. V. - Hub. 24. - Treit. - Dup.

- Wood. 775. - Heyden. Cat. 32.

P ing u is, (Crambus). Fab.

Espèce européenne, sans apparition fixe: générations successives, non interrompues. Commune dans les maisons, quelquefois anssi dans les haies. A Zurich, M. Bremi a observé qu'elle devient rare étant classée des habitations par la propreté. A Schüpfen, commune (Rothb).). Assez fréquente is Lausanne (Lah..).

$\mathrm{N}^{\circ}$ 2. Cuprealis. Hub. Vrz.

Huh. 153. @ (caprealis, errore). - Treit. - Dup. - IVood. 776. - Hub. Sup. 51, 3. - Heyden. C. 31.

A enealis, Costa.

Espèce partout rare. Prise à Bâle par le Dr. Imhoof.

II. Gen. Asopia. Treit. - Dup. hist. (P yrales. Stph. - Dup. Cat.)

$N^{\circ}$ 3. Farinalis. L.

$$
\text { F. - Hub. 95. - Treit. - Dup. - Wood. 778. - Hejden. }
$$
Cat. 170.

Européenne. Apparât au commencement et à la fin de l'été, dans les maisons. Peu 
commune autour de Lausanne; prise aussi dans la campagne contre le trone des arbres (Lah.). Commune dans les environs de Zurieh (Bremi). Assez rare autour de Schüpfen; dans les écuries (Rothb.)

$\mathrm{N}^{\circ}$ 4. Fimbrialis. W. V.

Hub. 97. 9. - Hub. Beitr. - Treit. - Dup. - Herden. C. 179.

$$
\text { Costalis. Fab. - Götze. - Wood. } 78 \mathrm{I} \text {. }
$$

Espèce peur épandue. Assez fréquente autour des éfuries de rache, à Hottiugen (Zurich. Bremi). Près de Verey (Rotlhb.)

$\mathrm{Y}^{\circ}$ 5. Glaucinalis. Lin. Faun. Suec.

Illig. - Clerk. - Treit. - Dup. et Suj» - Wood. 780. IIeyden. Cat. 173 (non W. V. - IIub.).

Nitidalis. F. - Hub. 98. S.

Espèce très-rare en Suisse. J'en ai vu un échantillon dans la collection de H. Rotheubach, qui avait été pris en Suisse.

\section{Gen. Pyralis. Lin. etc.}

\section{$\mathrm{N}^{\circ}$ 6. Brunnealis. Treit.}

Dup. - Heyden. Cat. 43.

Gla ucin alis. Hub. I26. 5 (err. I:27).

I. Zeller a trouvé cette espèce sur les bords du lar de Como. J'en ai deux exemplaires des envirous de Lausanne: ou la trouve eu juillet sur les pentes gazonnées, exposées au soleil, où elle vole parmi les herbes. Espèce rare partout. A Zurich, ì la fin de juin. Chenille sur ancholie [aquilegia vulgaris] (Frey). Il n'y a pas d'ancholies là où elle se troure près de Lausanne.

N ${ }^{0}$. Ingustalis. W. Y. 5.

Treit. - IIub. 21. 5. - 123. \& (err. 124). - Duр. Wood. 770 . - Heyden. C. 42.

Curtalis. 9. W. V. - Fab, - Götze.

Erigalis. Fab. - Götze.

Commune partout sur les prés un peu marécageux où croit la mousse. Le inàle est heaueonp plus fréquent que la femelle. Deux apparitions autour de Lausanne; la premièr 
en mai et juin; la seconde, plus fréquente, en aontt. - Sur les bords gazonnés du lac de IIofwyl près Berne (Rothb.). Zurich, tourbières; commune (Bremi).

\section{Famille des Crambides. Her.-Schfo}

\section{(Pyralien, partim; Phycis, Chilo, elc. Treit.)}

Insecte parfait. Ailes supérieures triangulaires, plus ou moins alongées, déclines daus le repos. - Inféricures moins développées que les supérieures, plus ou moins reployées sous elles, en éventail, dans le repos. - Téte petite; yeux rapprochés; trompe développée. Imtemes capillaires, arrondies; parfois rentlées à la base. - Pulpes ordinaires simples, alongés, droits ou recourbés; palpes accessoires bien risibles. _- forps grèle, alongé. Paltes longues, fortes, armées de longs éperons.

L arve. Chenilles allongées, attenuées aux deux extrémités, glabres, poilues ou verruqueuses, portant 14 à 16 pattes. Plusieurs ont une plaque écailleuse sur le premier anneau.

Les Crambides touchent aux Tinéides d'une part et aux Lithosies de l'autre. Les rapports arec les Nottuelles s'établissent par le genre Her c y a. Elles se rapprochent des Phalènes (Psodos), d'une manière plus éloignée, par $N_{y} \mathrm{mphul}$ a et des Tordeuses par rertaines If ercyna (helveticalis).

\section{Gen. Cataclysta. Hub. Verz. \\ Nymphulae. Tr. II y droc ampac. Latr.}

$\mathbf{N}^{\circ}$ 1. Lemualis (ta). Lin. f. s.

$$
\text { Fab. - W. V. - IIub. 83, 84. - Treit. - Dup. }
$$

Ileyden. C. I62. - Her.-Schf. n०1.

Uligin ata (Phal.) F. - Wood. 801.

Les fossés le tong du lac de Zurich; a l'Enge; au Greifensee; au Katzensee; le Glattthal, etc. Commune (Bremi). - Les marais de l'Emme, au-dessous de Burgdorf (Mey.). Près de Nidau et de Walperswyl (Rothb.). - Deux apparitions, l'une en juillet, l'autre en septembre (Frey.). 
1I. Gen. Tegostoma. Zell.

Botys. Dup. - Treit.

$\mathrm{N}^{\circ}$ 2. Comparalis. Hub. 127 (err. 126).

Treit. - Ifub. Sup. 5. ऽ, 158. - Heyden. C. 82. Her.-Schf. $n^{\circ}$ 4. - Zell. 1s. 1847.

s Ramalis. Hub. 92. - Treit. - Her.-Scluf. f. 158. - Heyd. C. 236. Trousé près de Zurich par M. C. Zeller (Bremi).

III. Gen. Nymphula. Hub. Verz. - Treit. - Dup.

Yo 3. Straliotalis. W. V.

Lin. (lata). - Hub. 87. 5. - Treit. - Dup.--Heyden. n" 163. - Her.-Schf. n" 7. - Wood. 802.

Pal u d a ta. (Phal.). Fab.

Au Katzensee (Zurich), pas rare (Bremi). Près de Münchenbuchsee; assez rare (Rothb.). En Suisse, la larve doit vire sur d'autres plantes aquatiques que le Stratiotes, qui ne se troure pas là oì se prend le papillon.

No f. Potamogalis. Lin. (la).

F. - Treit. - Dup. - Wood. 799. - Heyden. C. 165.

-.. Ier.-Schf n" 9.

Nymphacalis. W. V. - Hub. 85.

Commune près de Münclrenbuchsee (Rothb.); de même près de Zurich (Bremi). Çà et là autour de Lausanne; dans les haies el les broussailles humides; pendant tout l'été; sur le Jorat en août, quoique le $x_{!} m p$ h a ea ne se trouve nulle part dans les environs (Lah.).

$\mathrm{N}^{\circ}$ j. Nymplatealis. Lin. (la).

$$
\begin{aligned}
& \text { F. -- Tr. - Dup. - Wood. 800. - Heyden. C. } 164 . \\
& \text { Her.-Schf. } n^{\circ} 11 .
\end{aligned}
$$

Souvent près de Zurich, le long de la Glatt (Bremi); près de Münchenbuchsee (Berne), assez rare (Rothb.). 
IV. Gen. Agrotera. Schr. - Hub. Verz.

(A sopia. Tr. - Dup.)

No 6. Nemoralis. Scop.

Hub. 100, 206. - Lub. Beitr. - IV. V. - Treit. - Dup. -

Herden. C. 181. - Iter.-Schf. n" 13.

Erosalis? F.

Les bois de l'Uto (Zurich), pen rare (Bremi, Frey). - Environs de Lausanne: le's taillis chauds exposés au midi, en juillet; une génération en Suisse; rare Lah.). Très-rare près de Schüpfen (Rothb.). Dans le Tessin (Zeller).

V. Gen. Endotricha. Zell.

(A s o pia. Tr. - Dıp.)

$\mathrm{N}^{\circ}$ 7. Flammealis. W. V.

Hub. 99. - Treit. - Wood. 782. - Dup. - Heyden.

C. 180. - Her.-Schf. $\mathrm{n}^{0} 14$.

Fréquente dans les haies antour de Lausanue, à la fin de juillet et en août. Très-commune dans le Valais dès ła mi-juillet (Lah.). - Pas observée dans les environs de Zurich. Près de Rorschach sur les bords du lac de Constance (Breni). - Au pied du Jura, près de Bienne (Rothb.) -.. Cette espèce recherche les expositions chaudes.

VI. Gen. Stenia. Guén.

Nymplula, Treit. - Botys, Dup.

Yo 8 . Carnealis. Tr.

Dup. - Ilub. 187, 189. - Heyden. C. 9.5. - Her.-Schl. $\mathrm{n}^{\circ} 16$, f. 39,40 .

Corsicalis? Dup.

Trouvé près de Constance, par 11. Isenring [?] (Bremi). - Espèce d'Italie.

$\mathrm{N}^{\circ}$ 9. Punctalis. W. V.

Ilub. 140. - Dup. - Treit. (non Lin.). - Her.-Schf. ก 19, f. 24.

A etnalis. Dup. 2223.

Long ipedalis. Wood. 82.5 .

M. Bruand l'a trouvée sur le Jura, au mont Suchet. Je l'ai prise sur les bords da lac Majeur à la fin de juillet, et $\mathrm{j}$ 'en possède un mauvais échantillon des environs de Lausanne (Lah.). 
VII. Gen. Cynaeda. Hub. Ferz

(Odontia, Dup. - Seopula, Treit.)

No IO). Dentalis. IV. V.

Ilub. 25 - Ilub. Beitr. - Treit. - Dup. - Wood 837 - Her.-Schf. $n^{\circ} 21$.

Ram al is (Plial.) F.

Fulminans (Noct.). F.

Près de Seen (Bremi). - Eurirons de Rolle, canton de Vaud (A. Charannes). - Les Alpes; rare (Rothb.). - Sur le Balgrist (Zeller).

VIII. Gen. Hercyna Truit. Dup.

$\mathrm{N}^{\circ}$ 11. Atralis. Hub. 27.

Treit. - Dup. - I Iub. Beitr. - Ileyden Cat. 262.

Her.-Schf. $n^{0} 23$.

Cette espèce est tommune sur les Sous-Alpes, le long des haies et des taillis pierreux exposés au soleil, à la fin de juin et au commencement de juillet. Ne s'élève pas au-dessus de la limite des bois (Lah). Les Alpes; assez commune (Rothb.). - Eu se fanant elle prend une couleur brune.

No 12. Pollinalis. W. Y.

IJub. 29. - Hub. Beitr. - Treil. — Dup. - Heyden. C. 258.

IJer-Schf. $\mathrm{n}^{0} \mathbf{2 6}$.

Bigulta (Noctua), Esp.

Cà et là dans les taillis et les haies des environs de Lausanne en mai et juin. Assez rare. Se pose sur les feuilles et s'y étale au soleil (Lah.). Rare dans le cauton de Berne (Rothb). Assez rare dans les environs de Zurich Zeller).

$\mathbf{N}^{\circ}$ 13. Pyrenaealis. Dup. Sup. 82.

IIer.-Schf. $n^{\circ} \mathbf{2 9}$.

Simplonialis, Anderg. - Her.-Schf. f. 31 - 34. - Heyden. C. ․․9.

Rupestralis, Hub. Verz.

Les hautes Ajpes au-dessus de Brigg. Je l'ai reçue de I. Anderegg. Alpes de l'Engadine (Frey.). Rare. 
La dénomination de py renaelis a été aussi appliquée par Duponchel à une espèce de son genre Pyrausta, elle derrait done céder la place à simplonialis; mais l'une n'est pas mienx choisie que l'autre. Pyr. pyrenaelis pourrait bien avoir reçu ailleurs un autre nom. Dans l'incertitude, conservons le plus ancien.

\section{$\mathrm{N}^{\circ}$ 14. Rupicolalis. Hub. 139, 198-200. \\ Treit. - Dup. Heyden. C. 231. - Her.-Schl. nº 32. \\ Phrygialis. Ifub. 42. - Heyden. C. 232. \\ Seric alis. Hub. 43. Heyden. C. 230.}

Cette espèce est extrêmement commune sur toutes les Alpes, au-dessus de la limite des hois. Elle se pose de préférence sur les chemins et sur les pierres, les ailes étendues au grand soleil. Il n'est pas aisé de l'obtenir très-fraíche; car elle s'agite beaucoup, à la manière des hesperies. Sa teinte normale est le gris blenàtre, très-soyeux et marbré de noir et de verdàtre. La femelle est un pen plus grande que le mále; son vol est plus lonrd. Le mâle s'enfuit rapidement dis qu'on l'approche. Ce papillon, comme plusieurs de cenx des Alpes, se réfugie sous les pierres sitốt que le soleil se cache.

$\mathrm{N}^{\circ}$ 15. Holosericealis. Hub. 112.

$$
\text { Dup. 217. - Treit. - Dup. - Heyden. C. ․ㅡ8. }
$$

Var. Rupicolali s. Her.-Schf. $n^{0} 32$.

Cette espèce, extrêmement voisine de la précédente, ne peut cependant pas ĉtre envisagée comme une simple variété. Le dessin et la forme générale sont, il est vrai, les mêmes de part et d'autre; mais les teintes sont constamment différentes. Les parties gris-bleuâtre dans r $\mathrm{r}$ u pi c ola lis sont brun-rougeatre dans hol os eric e alis. La frange de la première est d'un noir-gris dans sa moitié interne et d'un gris-blanchàtre dans l'externe, en sorte que, mêmes aux inlérieures. la séparation des deux moitiés est mal tranchée. Dans lı o lo se rice a li s la moitié externe est d'un blane roussătre et l'interne d'un noir-brun foneé anx supérieures. Aux inférieures la différence des deux moitiés est très-tranchée, car l'externe y est d'un blanc pur. If olosericealis habite exclusivement la grande chaine des Alpes centrales, tandisque sa congénère se trouve sur toutes les Alpes. Ces deux espèces vivent à côté l'une de l'antre sans se confondre: holosericealis a le vol plus lourd et se laisse plus aisément approcher. Jamais je ne pus observer le moindre passage de l'une des espèces à l'autre. On ne peut admettre ici l'effet d'inlnences locales. 
La question serait sans doute tranchée par l'éducation des chenilles; mais celles-ci passent probablement une grande partie de leur vie sons la neige; car le papillon vole sur les hautes Alpres en juillet et en août, en sorte que l'œul doit être à peine éclos que déjà les neiges surviennent. - Hautes Alpes centrales depuis Chamounix à l'Engadine.

$\mathrm{N}^{\circ}$ 16. Alpestralis. F.

Hub. 135. - Treit. - Dup. - Her.-Schf. n³3.

IJerden. C. 233.

Rupestralis. Hub. $201-203$.

Assez rare sur les hautes Alpes de toute la Suisse; plus fréquente en Valais (Lal. Rothb.). Elle se pose aussi sur les pierres et les sentiers, en plein soleil, comme les précédentes. Son vol est moins vif et moins soutenu; elle est facile à saisir. Lorsqu'elle est fraiche, elle est d'un noir luisant foncé, tacheté de blanc-bleuàtre. Elle ne tarde pas à prendre an soleil une couleur brun-terne.

$\mathrm{N}^{\circ}$ 17. Andereggialis. Leder.

Hul. sup. f. 124-126. - Heyden. Gat. 234.

Scabralis. Eversm.

Cette espèce, extrêmement voisine de la prédécente, n'a été prise jusquu'ici en Suisse que tans les Alpes au-dessus de Brigg par M. Auderegg.

liessemble extrêmement à alpestralis fané, en sorte qu'au premier moment il 'st difficile de l'en distinguer. Cependant les supérienres sont un peu plus étroites. La bande grise de la marge, en dessous, est mal limitée et lavée (disposition mal rendue dans la figure 12.5 de 1I.-S.). Les rayes et les taches, d'un gris olivâtre, sont plus étroites et moins marquées que dans sa roisine.

$\mathrm{N}^{\circ}$ 18. Helvelicalis. Anderegg.

IIub. sup. f. 127, 128.

Cette espèce, extrèmement rare, n’a été trourée jusqu’ici que sur les Alpes voisines de Brigg, en Valais, par M. Anderegg.

Elle ressemble beaucoup à la précédente, mais est d'un tiers plus grande. Le dessin des supérieures est plus confus. Leur fond est d'un gris-hrun, soyeux, tacheté de blanc-bleuàtre. Les inférieures sont presque noires, surtout à la périphérie. La frange est gris-noir, mouche- 
téc de blanc aux supérieures; blancle el lisérée de noir en dedans, aux inférieures. Le dessous est d'un blanc sale, très-soyeux, bordé d'une large bande noire tout autour. Sa frange est limitée par une ligne de points blancs très-visibles aux inférieures. Les pattes sont blanches dessous. Un gros point discoïdal noir, aux supérieures, en dessous.

Cette espèce, ainsi que la précédente, rappelle quelque peu le genre s a rrothripa.

IX. Gen. B ot y s. Latr.

(Ennychia, Pyrausta, Scopula, Botys; auctor.)

Scopula et Botys ne peurent pas former deux genres distincts; il n'en est pas de mème, à mon avis, d'Ennychya et surtout de Pyrausta; mais il est très-difficile de diviser le genre Botys sans arriver à un morcellement plus fàcheus que le groupement que l'on rherche à éviter.

\section{$N^{*}$ 19. Nigralis. F.}

Hub. 26. - Treit. - Dup. - Her.-Sehf. $\mathbf{n}^{0}$ 36. - Heyden. C. 263.

A été prise jusqu'ici à Dättlikon sur l'lrchel, en août [Zurieh] (Bremi); près de Gelterkinden (Dr. Mlenzel); sur une alpe du Weggithal (Schwytz), à la fin de juin, $4500^{\prime}$ (P. Frey.) ; dans la vallée d'Engelberg et les Grisons (C. Zeller).

$\mathrm{N}^{\circ}$ 20. Inguinalis. Hub. 32.

Treit. - Dup. - Wood. 789. - Her.-Schl. 12"39. Ileyden. Cat. 252.

Fascialis. Schrk.

Assez commune partout (Rothb.). Peu rare autour de Lausanne. Se pose souyent sur les chemins et se promène dans l'herbe, en plein soleil, à la fin de mai et en juin. Seconde apparition en août. Se prend aussi dans les Alpes, en juillet (Lah.). Assez rare près de Zurich (P. Frey.).

\section{$\mathrm{N}^{\circ}$ 21. Cingulalis ( $\left.\mathrm{ka}\right)$. Lin.}

W. V. - Hub. 30. - Treit. - Dup. - Wood. 788.

Her.-Schf. 40. - Heyden. C. 251.

Les mèmes lieux et à la même époque que la précédente; mais plus rare (Lah.). - Sur le Zurichberg et l'Uto; assez fréquente (Bremi). Commune autour de Zurich (P. Frey.). Près d'Aarberg; assez rare (Rothb.). A la fin de juillet dans l'Engadine, près Samaden, $5200^{\prime}$ (P. Frey.). 


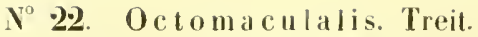

Dup. - Wood. 797 (ta). - Her.-Schf. n" 43. - Heydeu. C. 254.

Octomaculata. Lin. (Noctua.)

fruttalis. W. V. - Ilub. 75. - Curt. - Hub. Beitr.

Atralis. Fab.

Trigutta. Esp.

Les taillis ot les haies dans les endroits montagneux; fréquente daus les Sous-Alpes viudoises (Lah.). - Assez rare dans le canton de Berne et sur le Jura (Rothb.). - Les montagnes et les basses-Alpes des environs de Zurich; I'Uto; I'Irchel; pas rare (Bremi). - Fin de juin et juillet.

M. Bremi m’écrit que M. Künzli a recueilli de cempunctalis Mann sur le Schnebelhorn; n’ayant pas pu voir cette espèce en nature, je suis resté dans le doute.

No23. Punicalis. W. V.

Treit. - Dup. - Zell. Isis 1847. - Her.-Schf. I. I03.

I04, 10 47. - Ileyden. C. 209. - Wood. 794.

\& Porphyralis. Ilub. 36. - Schr.

Punicalis. F. - Devill.

Deux apparitions, l'une en avril et en mai, l'autre en juillet et en aoùt. Pas rare dans toute la Suisse; au bord des bois. Préfère les lieux ombragés et humides, où se trouve la mentha sylvestris. Varie assez peu. Dans quelques exemplaires mâles la racine des inféricures est tachée de jaune. La frange, surtout aux inféricures, est d'un blanc-roux à l'extrémité. Ce caractère s'eflace promptement par le vol.

Dans la femelle la bande jaune des inférieures se termine à une certaine distance des deux bords.

No 24. Porphyralis. W. V.

F. - Treit. - Dup. sup. 82. - Huh. sup. .1 ${ }^{\circ} 48,16101$, 102. - Ileyden. C. 211. - Wood. $\$ 96$.

Coecinalis. Hub. 37 .

Je n'ai trouvé cette espèce qu'une seule fois dans les Mpes vaudoises, à la hauteur de

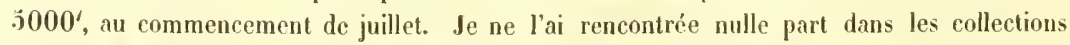
suisses. MII. Frey et C. Zeller l'indiquent, le premier à Zurich, le second dans le Tessin.

Ressemble beaucoup à punicealis, mais s'en distingue immédiatement par son fond violet-foncé, marbré de violet-clair. Les taches jaunes, plus petites que celles de cette der- 
nière, sont aussi plus pàles: l'extrémité de la frange est d'un blanc pur. En dessous les parties claires sont plus larges que dans pu nic a a lis et d'un blanc sale tirant sur le rose. Un petit point d'un blanc roussatre placé vers l'angle postérieur des antérieures, mais jui n'est pas toujours visible, n'existe pas dans les especes voisines.

$\mathrm{X}^{\circ}$ 25. Purpuralis. Lin.

Fab. - Treit. - Dup. - Wood. 793, 794. - Iler.-Schf. $n^{0} 49$, f. 105, 106. - Heyden. C. 206. - Hub. 3.5. Zell. Is. 1847 .

Punicaealis. IIub. 34.

Mo estalis. Dup. 22 4 , f. 9.

Cette espèce varie beaucoup, et presque autant que c aespitalis. La lemelle dillère davantage du mâle que chez les deux précédentes; elle est constamment plus petite, plus brune et moins tachée de jaune. Les individus qui volent se décolorent bientòt et prennent un foud noir.

J'ai recueilli dans le Tessin, au mois de juillet, quelques individus plus grands, d'un rouge plus vif, avec des taches jaunes plus grosses, une raie de mème couleur bien marquée aux quatre ailes, le long de la frange. Celle-ci est bordée de jaune-vif et non de blanc-sâle comme dans les nôtres. Je n’ai pas cru devoir ériger cette variété en espèce; sa femelle ne dillère que par un pen plus de jaune sur le milieu des ailes. Le filet jaune, parallèle à la lrange, se trouve aussi indiqué chez quelques individus pris à Lausanme. La coloration de la frange est probablement un effet du climat.

Il oestalis Dup. n'est qu'une femelle figurée comme un màle.

Deux apparitions dans l'année; l'une en mai, moins abondante; l'autre en juillet et en août. Espèce européenue, partout commune, que je n'ai cependant jamais rencontrée sur les Alpes élerées. Parait 15 jours euviron après pu n i c a ealis; plus commune qu'elle. Préfère les lieux chauds, les bords des fossés où croissent les men tha rot undifolia et hirsu $\mathrm{t}$ a.

0 strinalis est extrêmenent roisine de purpuralis; mais en diffère cependant par des caractères qui me paraissent suffisants pour l'admettre comme espèce. Je ne l'ai rencontrée jusqüici nulle part en Suisse. Phaenice a lis F. v. R. n'y a pas été prise non plus.

No6. Caespitalis. F.

W. V. - Treit. - Dup. - Wood. 791, $7 ! 92$ - Ilub. 39. Zell. Isis 1847. - Her.-Sehf. n 53. - Heyden. C. -213. 


\section{S? S ordidalis. Ilub. 40.}

Var. Interme diali s. Dup. 234. - Iler.-Schf. f. 2.5.

Très-commune partout sur les pâturages et le long des chemins. Dans les lieux chauds elle parait d'abord en avril et en mai, arec les premiers jours du printemps, puis, une seconde fois, dès le milieu de juillet jusqu'a la fin de l'été. L'apparition du printemps est moins abondante que celle de l'été. S'élère sur les plus hautes montagnes où elle est très-alondante à la fin de juin et au commencement de juillet; elle y vole en essaims, contre le rent et en plein soleil.

Limfluence des localités est assez peu maryuée. Les individus pris sur les alpes sont généralement un pen plus petits. Cens du printemps m'ont paru moins nettement dessimés.

L'influence des sexes est très-prononcée. La femelle est constamment plus petite et plus fortement dessinée; les bandes jaunes plus larges, ressortent davantage sur un fond plus foncé. Le màle a un fond plus uni et plus clair; on trouse des individıs qui n'ont aucur dessin aux supérieures, une seule ligne jaune, très-fine, le long de la marge des inlérieures. Les deux lignes foncées qui limitent la frange s'effacent parfois presqu'entièrement, aux supérieures surtout. Le point discoïdal des supérieures disparait quelquefois; tantôt il est noir, tantôt brun, tantòt ocellé.

Le dessous varie encore plus que de dessus. La taille peut atteindre celle de purpuralis.

No 27. Sanguinalis. Lin.

$$
\begin{aligned}
& \text { F. - W. V. - Ulub. 33. - Treit. - Dup. - Zell. } \\
& \text { Isis 1847. - Her.-Schf. 10 55. - Heyden. C. 197. }
\end{aligned}
$$

II a e m a t a lis. Hull. 178?

Cette espèce propre ì l'Europe méridionale se trouse çà et là en Suisse, mais partout très-rare. Je l'ai jrise une fois, en anît, à Paudex près Lausanne (Lah.). Euvirons de Zurich (Bremi) et de Bienne, au pied du Jura (Rotlıb.); Rare. Près d'Aoste (C. Zeller).

$\mathrm{N}^{\circ}$.28. A enealis. W. V.

F. - Hub. 46. - Treit. - Dup. - Wood. 826. IJer.-Schf. $n^{\circ}$ 6 I. - IJeyden. C. 77.

Rufimitralis. Ilub. 120.

Nigralis. Schrk.

Cette espèce n’a été prise jusqu'ici que dans le Jura bernois: le Chasseron (Rothb.). Toujours rare. Les individus reçus d'Autriche ont une couleur plus claire. 
N 29. Rhododendronilis. Dup. 235. f. 3.

Her.-Schf. no 63, f. $112,113$. - Heyden. C. I:31.

In solat a li s. II.-S. deut. ins.

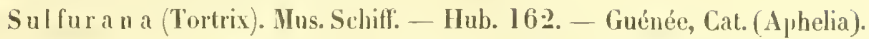

l'as très-rare sur toutes les Alpes élevées; vole sur les pâturages en plein soleil, dès la fin de juin au commencement d'aout. II est fort douteux que la chenille vive sur le rhododendron, car le papillon ne recherehe point cet arbuste. La femelle est plus petite que le màle et a les ailes supéricures coupées plus carrément et plus aigues au sommet; disposition qui se retroure dans plusieurs espèces des Alpes. Je l'ai prise à la fin de juin sur les Alpes raudoises et le $\mathbf{2 3}$ juillet sur la Furea. II. Zeller l'a prise aussi dans le IIaut-Valais et M. Frey dans l'Engadine.

Il est surprenant que Guénée ait rapproché cette espèce de Tor t. Gouana et l'ait placée dans sa tribu des Aphólides. Les figures de Her.-Schf. sont inexactes. Le mâle (II.2) est trop petit, et le sommet des supérieures trop aigu. Les dimensions de la femelle sont bounes, mais l'angle externe des supérieures est trop acuminć. Dans l'un et l'autre sexe, la couleur de l'insecte frais est le jamne-souffre tirant sur le vert.

$\mathrm{N}^{\circ}$ :30. Murinalis. Fisch. v. Rösl. t. 92. f. 3.

Heyden. C. 76. - Iler.-Schf. n 65.

Cette espèce découverte pour la première fois par $\mathbf{M}$. Anderegg, n'a été jusquiici décrite et figurée que par Fischer von Röslerstamm. Je l'ai vue, sans désignation de localité, dans la collection de M. Rothenbach: elle provenait des Alpes. M. Couleru la possédait, aussi saus nom, et l'avait probablement reçue de Jeyringen. L'un des individus portait une petite éclaircie blancheâtre en dehors du nœud des supérieures. On doit la rapprocher d'elutatis, dont efle a le facies et l'ombre du dessin. Je n'ai ru jusqu'ici que des màles. Très rare.

N'31. Literalis. II. V.

$$
\begin{aligned}
& \text { Ilub. 86. - Treit. - Dup. - Wood. 798. - IIer.-Schf: } \\
& \text { " 67. - IIeyden. C. I } 58 .
\end{aligned}
$$

Reticularis. Lin.

Argentalis. F.

Très-commune sur les prairies un peu lıumides; surtout dans les montagnes et sur les Alpes. Dans la pleine elle parait en mai, puis une seconde fois en juillet. Dans les montagnes elle vole en juin (Bremi, Rothb., Lah.). M. Frey la dit rare près de Zurich. 
$N^{\circ} 32$. Urticalis (la). Lin.

Fab. - W. V. - Hub. 78. - Treit. - Dup. - Wood. 805. Her.-Schf. ${ }^{\circ}$ 69. - Heyden. C. 115.

Assez commune, le long des haies, où croit l'ortie; en juin et juillet (Rothb., Lah.).

No 33. Repandalis. W. V.

Her.-Schf. $\mathrm{n}^{0} 70$.

Pallidalis. Hub. 115. - Treit. - Dup. - Wood. 818. - Heyden. Cat. 106.

Pas rare, dans les environs de Lausanne en mai une première fois, puis une seconde en juillet. La chenille vit sur les verbascum thapsus, Iyehnytis, etc., dont elle ronge les fleurs et l'extrémité des tiges, en se logeant sous le duret qui les recourre. Celles qui passent l'hiver restent enveloppées dans une coque mince, sans se transformer jusqu'en arril; alors seulement elles se crysalident et naissent trois semaines plus tard (Lal..).

Parait rare dans le reste de la Suisse.

$\mathrm{N}^{\circ}$ 34. Terrealis. Treit.

Fisch. v. R. t. 75, f. 4. - Frey. 456. - Her.-Schf. $71 .-$ Ileyden. C. 118. - Dup. Cat.

Cineralis? Hub. 66.

Cette espèce, habituellement confondne avec la suivante, se prend şà et là à la mème époque, mais beaucoup plus rarement. Je l'ai prise près de Lausanne et de Villeneuse: je l'ai reçue de M. Rothenbach sous le nom de glabralis; elle a été recueillie près de Burgdorf par M. Heuser. - Les individus de Vienne ne diffèrent pas des nòtres.

Cin eralis Hub. f. 66, reproduit plutòt cette espèce-ci que la suivante. Les femelles que j'ai vues sont aussi grandes, pour le moins, que le mâle; celles de fuscalis sont plus petites que lui.

Certaines individus sont tellement ombrés de brun-noirâtre que le dessin disparait en entier sous lui.

Le vol de terrealis est vif, rapide et très-irrégulier; ce qui la rend difficile à saisir.

No 35. Fuscalis. W. Y.

Illig. - Treit. - Wood. 827. - Her.-Schf. $\mathrm{n}^{0}$ 72. - Heyden. Cat. 104. - Dup. Cat. 
Cinneralis. F. - Dup. hist.

Glabralis. Hub. 65 ?

Espèce très-fréquente sur les montagnes, un peu moins dans la plaine. Se plaît dans les taillis, les grandes herbes et sur les prés humides. En mai et en juin. - Paraît 3 à 4 semaines plus tard dans les montagnes. - Le dessin est parfois à peine visible. - Femelle plus petite que le mâle, avec le sommet des ailes plus arrondi.

No $36 . \quad$ Pulveralis. Hub. 109.

Treit. - Dup. - Curt. - Wood. 826. - Her.-Sehf. n 73.

f. 17, 18. - Ileyden. C. $\mathrm{n}^{\circ} 65$.

Partout rare et isolée. A Paudex, près Lausanne, en juillet (Lah.); près d'Aarberg (Rothb.). Environs de la Neureville (Couleru). Paraît préférer, en Suisse, le lieux chands; vole parmi les taillis herbeux.

$\mathrm{N}^{0}$ 37. Crocealis. Hub. 71 et Verz.

Fisch. v. R. t. 75, f. 2. - Treit. sup. - Dup. Cat. (non Dup. hist.). - Iler.-Sclif. n 74. - Ifeyden. C. 103.

Verb a sealis. Wood. 824 .

Assez rare en Suisse. Je l'ai prise plusieurs fois à Paudex, près Lausanne, en juin et en juillet, dans les lieux ombrés et humides, au milieu des herbes. M. Rothenbach l'a collectéc quelquefois aussi près de Schïpfen. M. Bremi la trouve plus commune le long des fossés humides des bords du lac de Zurich. La chenille, dit-il, vit sur le ve rb a s cum lychnitis. Cette plante n'existe pas dans les localités où elle vit près de Lausanne.

$\mathrm{N}^{0}$ :38. Citralis. Podev. (teste Her.-Schf.).

Lut e alis. Dup. hist. $\mathbf{2 3 3}, 1$. - (Non Hub.)

Var. Fla r ali s. Her.-Schf. text. (non fig. 115). - Heyden. C. n 109.

Flavalis, Dup. hist. 217,7 ?

Assez fréquente au pied des Alpes dans tout le Valais et dans le district d'Aigrle, en juin et en juillet (Lah.). Le pied du Jura dans les lieux chauds (Rothb., Couleru). — Val d'Aoste (Zell.)

Duponchel ayant réuni dans son catalogue lutealis à flavalis, j’ai cru devoir adopter le nom de Podevin, d'autant plus que Ifubner a figuré pascualis Lien. sous le nom de lu te ali s. - La figure de Duponchel est mauvaise, quoique reconnaissable par la teinte charbonnée des inférieures, toujours plus prononcée ehez les lemelles. Duponchel dit que cette 
espece ne porte aucun vestige de lignes en dessus dans les deux sexes. Cela peut être rai pour les individus pris dans le midi; mais n'est plus exaet pour les nótres, qui portent tous, lorsqu'ils sont fruis, une ligne (la seconde) peu marquée, il est rrai, mais cependant bien visible, très-sineuse, sans dentelmes, placée vers les deux tiers externes de l'aile. Les inférieures ont aussi cette mùme ligne, mais plus souvent couverte par la teinte charbonıée répandue sur le fond. La tache réniforme (externe) est aussi souvent indiquée par un petit trait, ou point grisatre. Du reste, Duponchel me parait avoir commis une erreur plus fàehense encore dans sa figure $7, \mathrm{pl} .2 \mathrm{I}$, oì il représente évidemment notre espèce sous le nom de flavalis; quant à la description, il reproduit celle de Treitschke qui ne conuaît que la raie l'lavalis, Hub. 69. Il parait, d'après cela, que cette derniere est rare en France. comme rhez nous, tandis qu'elle l'est moins en Allemagne. Citralis serait an contraire assez commune en Suisse et en Franee, mais fort rare en Illemagne, si mème elle y existe. La confusion qui jusıu'ici a règné entre ces deux espèces, moblige à les différencier plus evactement.

La teinte jaune de eitralis tire un peu sur le verdatre, celle de fla a lis est plus dorée, et un peu orangée sur les individus qui se fanent. Le dessin de la première est peu marqué, quelquefois même presque ellacé, celui de la seconde est très-saillant el se rapproche beancoup de celui de cinctalis. Le dessous de citralis est d'un noir grisàtre tacheté légèrement de jaunàtre; celui de flavalis est plus varié de jaune-roux, rajé et cadrillé de brun foncé. Les ailes inférieures dans fla talis sont en dessus un peu plus pàles que les supérieures et marquées d'une ligne transrerse au milieu et d'une hordure de taches grisàtres entre cette ligne et la frange; cette bordure manque parfois.

Dans citralis ces mêmes parties sont, chez le mâle, d'uı jaune verdâtre ou grisâtre plus foné qu'aux supéricures; chez la femelle, la nuance grise est plus prononcée et eoure somvent presque tout le fond, en sorte que l'on apercoit à peine la ligne transrerse. Celle-ci. lorsug'elle est visible, forme une anse très-marquée à son milieu.

La seconde ligne transwerse de fl a valis est ordinairement fortement dentellée par les nervures et ne forme point.un sinus profond daus son eentre, mais se dirige rers la eóte, plus ou moins oblinuement, en se rapprochant de la base. Cette mème ligne dans eitralis n'est pas dentellée à l'état hien frais, et décrit, en se rapprochant du bord postérieur, un large et profond sinus, en sorte qu'elle fait un angle très-marqué avant de se diriger vers la cóte. Du reste, ces lignes, ainsi que les taches, sont d'un Jrun plus ou moins foncé dans fl a val is, tandis que dans sa voisine alles forment un léger trait grisàtre. Le earactère le plus saillant se tire de la frange: celle-ei est d'un noir rous, à rellets plombés, comme le remarque Treitsehke. 
dans flavalis, tandis que dans citralis elle est d'un gris-ronx à peine plus foucé que le lond. La différence des franges est surtout saillante aux ailes inférieures. Ajoutons enfin que le sommet des supérieures est très-aigu dans fla valis et légèrement olotus dans citralis. ce que je ne puis accorder avec les paroles de IIer.-Schf. (llavalis).

No 39. Flavalis. W. Y.

IIub. 69. - Treit. -- Dup. text. (non fig.). - Wood. 819. Her.-Schf. 7\%. - Hryden. C. 108.

liare en Suisse. Je l'ai prise trois fois dans la vallée du lihône oì elle so rencontre isolément, en juillet et au rommencement d'août. Je l’ai reçue aussi de Meyringen. M. Bremi l'a récoltée au pied du Gothlhard.

Cette espèce est facile à confondre avec pandalis; cependant avec mu peu d'attention. sa frange et la tache ocellée du disque, la font aiséunent reconnaitre. Elle a été jusqu'ici confondue avec citralis (voir cette dernière especc), et parfois avec inctalis.

Elle paraît un mois plus tard que les trois espices que je viens de nommer, et n'a, selon tonte apprarence, qu'ume apparition par année. - Préfère les vallées des Alpes.

$\mathrm{N}^{\circ}$ 40. Hyalinalis. Schr.

Hub. 74. - Treit. - Dup. - Stph. - Wood. 814,811?Her.-Schf. $n^{0}$ 78. - Ileyden. C. 110.

Commune le long des haies et dans les taillis humides, en juin et en juillet; dans pres'jue toute la Suisse. Parait rare autour de Zurich, puisque M. Frey n'en a rectneilli qu'un individu, et M. C. Zeller ancun.

$\mathrm{N}^{\circ}$ 41. Pandalis. Hub. Verz.

Treit. - Zeller, Isis 1847. - \& Dup. sup. - Iler.-Scbf. n"79, f. 88. - Heyden. Cat. 113.

Verba scalis. Ilub. 59 (mala). - Fisch. 1. R. 27,3 ?

o Oblitalis. Dup. et cat.

Très-commune sur tous les prés en mai et en juin; reparaît une scconde fois à la fin de "juillet et en août. Dans tonte la Suisse, saul' les Alpes élevées.

Le male est sourent plus petit que la femelle et moins nuancé qu'elle; ses ailes sont anssi plus aigues au sommet. Les teintes varient beaucoup: le fond est tantôt jaune-paille pur, tantôt arec un rellet violet et tantòt un peu safrané; les lignes transverses sont aussi de lar* geur très-variable et courrent quelquefois une bonne partie du fond. 
Il est difficile de comprendre pourquoi une espèce aussi répandue a donné lieu ì autant de confusion. La figure 59 de Hubner en a sans doute causé plusieurs, et a engagé Duponchel à créer oblitalis; mais comment s'est-il fait que Duponchel dans son catalogue n'ait pas reconnu son erreur et quỉ ait conservé les denx espèces distinctes? - La figure de verbascalis Fisch. 1. R. qui doit représenter arcualis (IIub. 80), conient parfaitement a pandalis et nullement à ar cualis. Y a-t-il là encore une erreur? La figure de Duponchel suppl. est mauraise; celle de Hlubner suppl. est bonne. - Heyden. applique le synonyme de Fisch. v. R. a deux especes pandalis et verbascalis W. V.

$N^{\circ}$ 42. Verticalis. Lin. f. suec.

Hub. 57. - Treit. - Dup. - Wood. 810 . - Her.-Schf. 80. - Heyden. C. 112.

Commune dans les haies de toute la Suisse, là où croit l'urtie a dioica; en juillet et en aoùt. Ne s'élìve pas sur les Alpes avec l'ortie.

No 43. Lancealis. W. V.

Treit. - Bup. - Wood. 809. - Her.-Schf. n" 82. - Heyden. Cat. 8.5 .

Gla bralis. F. - IHub. 11 (non 65).

Pas commune aux environs de Lausame: à Paudex dans les taillis herbeux, à la fin de mai et en juin (Lah.). - Les broussailles de l'Uto; assez rare près de Zurich (Bremi). De mème près de Schüpfen (Rothb.). - L'Uetliberg, en juin (Frey).

$\mathrm{N}^{\circ}$ 44. Sambucalis. W. V

IIub. 81. - Treit. - Dup. - Wood. 803. - Her.-Schf. $n^{\circ} 83$. - Heyden. Cat. 87.

Nulle part en abondance. Les taillis humides, à Paudex, près Lausanne et ailleurs (Lah.). Assez rare près de Schüpfen (Rothb.), et dans les environs de Zurich (Bremi, Zell., Frey).Juin et juillet.

$\mathrm{N}^{\circ}$ 45. Stachydalis. Zinck. Germ.

Her.-Sch. $\mathbf{n}^{\circ} 84$, fig. 114.

Parietarialis. Man. Cat.

Var. S a m bucalis. Dup. Cat.

Că et là sur les prairies sèches, parmi les lrerbes; rare. Lausanne (Lah.). - Burgdorf 
(Mleyer, Heuser). - Le Tessin (Zell.). C'est par erreur que la figure de Her.-Schf. porte le nom de stachytalis.

$\mathrm{N}^{\circ}$ 46. Verbascalis. W. V. (non Hub.).

Treit. - Dup. - Her.-Schf. 85. - Heyden. Cat. 91.

Fisch. v. K. (texte).

A reu alis. IInb. 80.

Ochrealis. Wood. 820.

Les clairières des bois et les pentes sèches exposées au soleil. -- Sauvabelin, près Lausanne, Villeneuve, Aigle, etc.; pas rare (Lah.). Près d'Aarberg, très-rare (Rothb.); le Balgrist (Zell.). Parait une première fois en juin, puis une seconde en aoùt dans les lieux chauds. Rubiginalis n'a pas encore été pris en Suisse; les figures qu'en donne Fisch. v. Rösl. ne peuvent servir à le distinguer de notre verbas calis. La teinte brune de la marge existe quelquefois dans verbasealis. Y aurait-il là aussi une erreur?

$\mathrm{N}^{\circ}$ 17. Silacealis Hub. 116. 9.

Treit. - Dup. - Frey. - Her.-Schf. 87. - Herden. n" 86. Nubilalis. Hub. 94. .

Angustalis, terminalis et glabralis. Wood. 815,816 et 817 .

Rare dans les environs de Lausanne; à Paudex, en juin (Lah.). - De même près de Zurich (Bremi).

Les deux sexes diffèrent tellement l'un de l'autre, qu'on s'y méprend souvent.

La chenille a été trouvée sur la Conyza squarrosa par M. Bremi.

$\mathrm{N}^{\circ}$ 48. Praetextalis. Hub. Verz.

Treit. sup. - Dup. eat. - Frey. - Her.-Schf. n 90. - Heyd. 80. Politalis. Hlub. 61. - Dup. hist.

Pris une fois, près de Lausanne, dans un taillis abrité et chaud, en juillet, par M. G. Leresche. - M. Boll l'a aussi recueilli dans le canton d'Argorie Bremi).

No 49. Alpinalis. W. V.

IJub. 63, 5, 175, 176, \&? - Treit.? - Dup. - Hevden. Cat. 69.

Les hautes Alpes centrales, la Furca, etc. (Lah.). Fréquent dans les Alpes orientales (Zell.). - Juillet et août. - Quelquefois dans le Bas-Valais avec monticolalis. 
Les caractères distinctifs de cette espèce sont: $1^{\circ}$ Une petite tache d'un blanc pur. bien limitée aux supérieures, visible aussi en dessous. $2^{\circ}$ Une grande tache de la même couleur, nettement limitée du côté de la marge, aux inférieures. La base de ces mêmes ailes est enfumée. $3^{\circ}$ La frange des inférieures blanche, surtout en dessous. $4^{\circ}$ Trois à quatre crochets, blanchâtres, peu visibles, sur la moitié externe de la côte des supérieures. $5^{\circ}$ Une rangée de points blancs à la limite de la frange des supérieures, en dessous, séparés par de petits points noirs.

Cette espèce se place à còté d'elutalis W. V. La femelle est plus petite quue le mâle et al le sommet des supérieures aigu.

\section{$\mathrm{N}^{\circ}$ 50. Monticolalis. Kuhlw.}

Man. Cat.

$$
\text { Alpinalis (var.). Heyden. cat. } 71 \text { (rar.). - Hub. sup. f. } \mathbf{i}-9 .
$$

Très-répandue dans les Alpes occidentales en juillet.

La femelle porte très-rarement aux supérieures une petite tache jaunâtre, traversée par les nervures. Ses inférieures sont entièrement d'un gris-noirâtre.

Cette espèce n'est point une variété de la précédente; elle s'en distingue: I" par la couleur jaunâtre de la tache du disque; $2^{\circ}$ par une bande grise, étroite, parallèle an bord postérieur, ,pui traverse le disque gris-clair des inférieures; $3^{\circ}$ par la frange des inférieures grise, plus claire que le fond; $4^{\circ}$ par l'absence constante de crochets à la côte des supérieures.

La variété cin eralis Man. (Heyden. cat. 70) ne pent se rapporter qu’à monticolalis, puisque la taclse centrale ne manque jamais dans alpinalis.

No51. Polygonalis. IT. V.

Hub. 67, 204, 205. - Treit. - Dup. - Wood. 207?

Her.-Schf. $n^{0} 92$. - Hub. sup. f. 91 ? - Heyden. cat. 121 .

$$
\text { Zell. Isis, } 1847 .
$$

Je n’ai su qu’un maurais exemplaire de cettẹ espèce méridionale qui doit avoir été pris en Valais (Collect. Bremi). - M. Zeller l'a prise dans le Tessin. Les entomologistes ne sont pas d'accord sur la distinction de diversalis Hub. 102, comme espèce; je ne puis juger cette question et je me borne à citer arec doute les synonymes de llub. sup. et de Wood. 
$\mathrm{N}^{0}$ 52. Na nualis. Hub. $195-197$.

Treit. - Fres. ä, b , 19, 2. - Dup. - Her.-Schf. no 96. IJeyden. Cat. 222.

Cette espèce, très-rare en Suisse, habite les Alpes du Haut-Valais, où M. Anderegg la recucille.

Yo 53. Nebulalis. Hub. 51, 144.

Treit.? - Dup. sup. - Hub. sup. f. 11, 12. - IIer.-Schl. n $^{\circ} 97$. - Wood. 70.

Var. Alpin alis., Dup. hist.?

Pinet alis. Zetterst.

Squalidalis. F. 1. R. - Nub. 144. - Dup.

Prat a lis. Zell. Isis.

Très-commune dans les Alpes, dìs le commencement de juiliet au milieu d'août (Lah.). Parait plus rare dans le nord de la Suisse que dans le midi; on la prend aussi sur le Jura.

La femelle est un peu plus petite et plus foncée que le mâle; ses ailes inférieures sont enlièrement d'un gris foncé, plus elair vers le bord interne. Chez le màle, ces mêmes ailes sont plus foncées à la marge et plus claires sur le disque et à la base, avec un petit trait noir peu apparent, faisant suite à la ligne externe des supérieures.

L'observation de Treit. (sip. III, p. 16) a lieu d'étonner chez un auteur aussi scrupuleux; je ne comprends pas comment il a pu réunir nebulalis Hub. avec al pinalis. J'ai de l'une et de l'autre un grand nombre d'exemplaires sous les yeux (Lahi).

No 51. A erealis. Hub. 44.

Treit. sup.? - Dup. cat.? - Her.-Schf. no 99 ? - Hub. sup. f. $137-139$ ? - Heyden. cat. 67 .

O p a c a lis. Dup. hist. - Iub. 169,170 ?

Assez fréquente sur toutes les Alpes, à la limite des bois; fin de juin et de juillet (Lah.). M. Zeller m'affirme l'avoir prise aussi près de Zurich.

Je n'hésiterais pas aujourd'hui, si j'avais sous les yeux des individus provenant de Dalmatie, il séparer complétement aerealis, Hub. 44, de Hub. sup. f. 137-139; mais les meilleures figures peuvent induire en erreur. Si mon opinion se trouve confirmée par l'observation, il faut conserver la dénomination d'aerealis à Hub. 44, et I joindre opacalis Dup. list.; et rapporter tous les autres synonymes d'aerealis el d'opacalis il opacalis, Hub. 169 et 170. 
En attendant que la difficulté puisse être résolue, voici les motifs sur lesquels je me londe. $\mathbf{I}^{\circ}$ Herrich-Schæffer dit en parlant de la figure $\mathbf{4 4}$ de Hubner: , ich sah kein Exemplar mit am Vorderrand so gelbem hinteren Querstreif "; puis, en citant Duponchel 215, 6, 7, il ajoute: "schlecht". Or, la ligure citée de Hubner et celles de Duponchel répondent parfaitement à notre aerealis, tandis que celles de IIub. sup. (137-139) sont, ponr moi, presque méconnaissables. 2" Jamais les exemplaires de nos Alpes ne présentèrent les points marginaux figurés dans Hub. sup. et déerit avec soin dans Treitsehke. $3^{\circ}$ La première ligne transverse (basilaire) est chez cux à peine indiquée vers le bord interne, la seconde est beancoup moins sinueuse, ot l'éclaircie jaunatre de la marge est plus large, fondue aver la frange et l'espace brunàtre voisin. $4^{\circ}$ Je ne vis jamais anx supérieures de point central d'aucune espèce. D'après la description de Treitschke, les antennes du mâle doivent encore offrir des différences. Dans notre a ere alis elles sont sétiformes, brunes, et leurs poils, trèscourts, ne sont visibles qu'au moyen d'une très-forte loupe.

La femelle est rare, plus petite que le mâle. Ses ailes supérieures sont coupées carrément et ses couleurs sont un peu plus tranchées que celles du mâle. Elle diffère encore plus des figures $137-139$ de Ifub. sup.

$\mathrm{N}^{\circ}$ 5.5. Margaritalis. W. V.

Hub. Beitr. - Treit. - Dup. - Wood. 831. - Ifer.-Schf. $\mathrm{n}^{\circ}$ 100. - Heyden. Cat. 78.

Erucalis. Hub. f. 5.5.. .

Assez rare en Suisse. Je l'ai regue des environs de Franenfeld (Lah.). - Prise plusieurs lois dans les environs de Zurich par M. Zeller. - Moins rare en Valais, oi la chenille vit sur les $\mathbf{S}$ is ymbrium. Fin de juillet et août.

$\mathrm{N}^{\circ} \mathbf{5 6 .}$ Sticticalis. Lin.

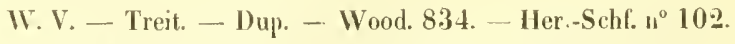
Heyden. cat. 66.

Fuscalis. Hub. 45.

Prise quelquefois dans les environs de Zurich (Zeller); je l'ai vue dans la collection de M. Bremi. - Je l'ai reçue du pied du Jura, où M. Couleru l'a recueillie dans les environs le la Neuveville (Lah.). Très-rare en Suisse. 
No 57. Slramentalis. Hub. Verz.

Treit. - Dup. - Wood. 830. - Her.-Schf. n 105.

Ileyden. cat. 79.

Elutalis. Hub. 62. 5 .

Cette espèce est partout rare. M. Zeller l'a prise sur les bords du lac de Como; M. Rothenhach dans les emvirons de Schïpfen, et H. G. Leresehe près de Lausanne, en juillet.

$\mathrm{N}^{\circ}$ 5̇S. Sophialis. Fab.

IHub. 50. Treit. - Dup. - Her.-Sch. n 107. - Heyden. Cat. 58.

Variegalis. Fab.

Nest point rare dans les vallées des Alpes raudoises, à la fin de juin et au commencement de juillet. Elle préfère les lieux exposés au soleil et se pose contre les rochers, les murs et les maisons. Son vol est rapide et saccadé (Lah.). - On la prend souvent à Meyringen Ott... - Près de Thun, à la caverne de St-Béat (Rothb.). - Canton de Glaris, fin de juillet, $7000^{\prime}$ (Frey).

Les deux sexes sont semblables. Le dessin est le même que dans frumentalis.

$\mathrm{N}^{\circ}$ 59. Forlicalis. Lin.

Hub. 58. - Treit. - Dup. - Wood. 806. - Her.-Schf. $\mathrm{n}^{\circ} 111$. - Heyden. eat. 142.

Commune partout dans les jardins et les plantages; deux apparitions, la première à la fin de mai el en juin, la seconde à la fin de l'été.

$\mathrm{N}^{\circ}$ 60. Cinctalis. Treit.

Dup. - Wood. 812. - Her.-Sehf. n 115. - Ileyden. cat. 107.

Limbalis. Hub. 72, 73 (non W. V.).

Assez fréquente au pied des Alpes et du Jura, sur les prairies sèches, exposées au soleil (Lah., Rothb.). - Commune en Valais (Lah.). Fin de juin et juillet.

La lemelle est un peu plus petite que le mâle; ses teintes orangées sont aussi plus pronoucées. La chenille ne it pas sur le spartium scoparium uniquement, puisque cette plante n'existe pas là où le papillon se prend dans le canton de Vaud.

$\mathrm{N}^{\circ}$ (it. Clathralis. Hub. 168.

Treit. - Dup. 231. - Hub. sup. f. 47, 48. - Her.-Schl. $\mathrm{n}^{\circ} 117 .-$ Heyden. cat. 134.

J'ai reçu cette espèce méridionale de M. Anderegg, qui l'a prise en Valais. 
No 62. Palealis. W. V.

Hub. 70. - Treit. - Dup. - Wood. 813. - Her.-Schf. $n^{\circ} 122 .-$ Heyden. cat. 126.

Var. Selenalis. Inb. 177. - Dup.

Cette espèce méridionale se prend dans la Suisse italienne (Bremi). An pied du Jura, près d'Aarberg, et dans les environs de Vevey (Kotlib.). A la Neuserille, dans les lieux exposés au soleil (Couleru). Partout assez rare. Juillet. Chenille sur spira e a $\mathrm{l}$ maria; pas rare, près Hlottingen [Zurich] (Bremi).

\section{$\mathrm{N}^{\circ}$ 63. Ferrugalis. Hub. 54, 150 .}

Treit. - Dup. - Wood. 822. - Her.-Schf. n" 123. - Heyden. cat. 100.

Commune dans les environs de Lausanne, le long du lac, sur les prairies sèches, parmi les herbes et les broussailles (Lah.). Très-rare près de Schüplen (Rothb.). N'a pas encore été prise ailleurs en Suisse. Août et septembre.

Le mâle est légèrement pluns petit et moins coloré que la femelle.

$N^{\circ}$ 61. Numeralis. Hub. 89.

Dup. 221. - Treit. - Hub. sup. f. 69, 70, 79. - Her.Sehf. $n^{\circ}$ 125. - 1Ieyden. cat. 105 ${ }^{\mathrm{c}}$. - Zell. Isis, 1847.

Prise dans les environs de Zurich par Mn. Zeller.

No 6.5. Prunalis. W. V.

Treit. - Dup. - Wood. 836. - Frey. 114. - Her.-Sehl. n" 128. - Heyden. C. 5.5.

Leucophoealis? Ilub. $7 \%$.

Commune dans les entirons de Lausanne, sur les arbustes et les haies; en juillet (Lah.). De même dans toute la Suisse (Rothb., Bremi). Pas dans les Alpes.

La femelle est um pen plus grande que le mâle et a les ailes antérieures légèrement plus larges. - Cette espèce varie très-peu, et seulement dans sa teinte grise plus ou moins mèlée de brun. Elutalis W. V. n'a pas encore été pris en Suisse; tous les individus reçus sous ce nom, appartenaient ou à prun alis, ou à l'espèce suivante.

$\mathrm{N}^{\circ}$ 66. Cy analis. Lah. fig. 4 .

Point rare dans les environs de Lausanne, dès la fin de mai au commencement de juillet. 
Habite les haies, les taillis et les bois (Lah.). M. Rothenbach l'a prise aussi daus les environs de Schïpfen, où elle est plus rare, et M. Heuser près de Burgdorf.

J'ai regardé assez long-temps cette espèce comme inqu in a talis, Zell.; M. HerrichSchaller m'affirme qu'elles sont différentes l'une de l'autre. Je n'ai pas vu la dernière en naume. On ne peut confondre cyanalis qu'arec prunalis, à laquelle elle ressemble extrênement. J'ai iu un assez grand nombre d'exemplaires tous identiques. Il sulfit pour la carartériser de la différentier de prunalis.

L'apparition de cyanalis précède celle de cette dernière d'environ un mois. Sa taille est aussi constamment un peu plus petite. La plus grande partie des ailes supérieures, ì l'exeption de la base, est saupoudrée d'une teinte blane-bleuastre, toujours beaucoup plus faible dans prunalis. Cette teinte est surtout marquée sur les deux bords de la seconde ligne transierse et le long de la marge. Il en résulte que cette ligne, les denx taches du disque, ainsi que les points noirs de la marge sont plus saillants que dans l'espèce voisine. Les deux taches sont plus visibles; la réniforme est plus carrée, plus grosse. En dessons, ces mêmes taches, très-visibles dans prunalis, le sont peu ou point dans cy analis. La frange est limitée, anx supérieures, par une rangée de points noirs, triangnlaires, suive en delırs d'une ligue grise plus foncée, puis d'une bordure d'un blanc sale. Aux inférieures ces points n'existent pas ou ne s'aperçoivent que vers le sommet de l'aile au nombre de 2 a 3 ; la frange I est d’un blane sâle, mouchetée de gris. Daus prunalis, ces points sont assez visibles: mais ils sont mieux marqués en dessous. Les 4 ou 5 dents de la côte des supérieures sont plus petites dans notre espèce et séparées les unes des autres par la teinte bleuâtre et non paur des taches jaunâtres comme dans prunalis. Les inférieures sont, en dessus, d'un grisnoiràtre foncé, tandis que prunalis les a gris-brun et moins foncées. Le dessous de ces mèmes ailes est blanchâtre, souvent sablé de gris vers le sommet dans cy analis. La raie transierse des mêmes ailes, bien marquée dans prunalis, s'aperçoit rarement et toujours faiblement dans sa voisine; elle est d'ailleurs prarallèle à la marge, dans cette dernicre, tandis qu'elle s'en écarte vers le bord antérieur. Le dessous des supéricures est plus recouvert de noir dans ey a nalis. Les antennes sont enfin plus distinctement annellées de blanc et de noir.

Le mâle ne diffère pas de la femelle. L'espèce ne varie pas. Sa taille et son facies la font aisément distinguer lorsqu'elle est très-fraiche.

Y 67. Pascualis. Lienig. Isis. 1846.

Hub. sup. f. $64-66$. - Her.-Schil. n [34. - IIeyden cat. $105^{\mathrm{a}}$. 
Lutealis. Hub. 145? - Dup. cat. (Scopula).

Institialis. Wood. 829 .

Cette espèce n'est pas très-rare dans les Alpes raudoises; je l'ai prise à Ormond-dessus, à l'Etivaz, dans le Haut-Valais et les Alpes de Bex; en juillet et en août. - Les taillis près d'Engelberg (C. Zeller).

Extrêmement voisine de nebulalis Ilub. (squalidalis F. v. R.) par sa forme, sa taille et son dessin; on ne saurait guères la confondre quarec elle et avec elutalis $\mathbf{W}$. V. Elle diffère de la première par les caractères suivants: $1^{\circ}$ La couleur générale de pas c u alis est le blanc sale tirant m pen sur le roux; celle de nebula lis est le gris plus on moins mểé de blanc sale et de gris plus foncé. 20 Le dernier article des palpes est alongé et effilé dans la première, tandis qüil est court et à peine visible chez la seconde. $3^{\circ}$ La scconde ligne transverse fait, dans paseualis, un sinus profond an-dessons de la tache réniforme et qui la dépasse du côté de la base; chez sa voisine ce sinus est moins profond et s'arrête au niveau interne de la tache. $4^{\circ}$ La tache ronde des supérieures est oblongne dans celle-là, arrondie dans celle-ci. $5^{\circ}$ La frange est limitée dans pascu alis par un trait brun-foncé très-marqué en dessus et en dessous; dans nebulalis on n'observe à sa place que des points rapprochés, visibles seulement en dessous et aux inférienres. $6^{\circ}$ Celles-ci sont blanches des deux cótés, aree un point central, une raie grise transverse et une ombre de mème couleur au sommet. Dans nebulalis, ces mèmes ailes sont enfumées dessus et blanchâtre en dessous, arec une raie pen marquée. $7^{\circ}$ Le dessous des supérieures est gris-foncé dans nebulalis; il est gris-roux, plus ou moins clair et nuancé de blanc-jaune sur les bords dans pascualis. Lorsque cette dernière a ces ailes blanchatre en dessous, on ! distingue les denx taches et la seconde raie du dessus. La bordure brunatre de la marge d' el u$t$ a lis, et l'absence de ligne limitant cette marge, suffisent pour en distinguer pascualis.

La description que Treitsehke dome de suffusalis (Bd. VII, p. 68) sapplique en tout point à pascualis.

La femelle est à peine plus petite que le mâle et ses ailes sont plus aigues au sommet , parce que le bord externe est coujé en ligne droite, comme dans plusieurs femelles du mème genre.

$\mathrm{N}^{\mathrm{n}} 68$. Olivalis. Wien. Verz.

Treit. - Dup. - Wood. 835. - Iler.-Schf. n"135.

Umbralis. Hub. 5:2. - IIeyden. cat. 61.

Rare en Suisse; je ne l'ai tronvée jusqu'ici que dans le Jorat, au-dessus de Lausanne, le long des bois et dans les haies: en juillet. 
Appartient au grompe prunalis, cyanalis, fulvalis, elutalis, etc. La femelle est aussi chez ces dernieres, souvent plus grande que le måle; ses ailes ne sont pas non plus coupées carrément au bord externe.

Decrepitalis F. v. R. n'a pas encore été trourée en Suisse.

X. Gen. Stenopleryx. Guénée.

(Chilo, Treit.)

Ce genre lie parfaitement Botys à Eudorea.

$N^{\circ} 69$. Hybridalis. Hub. 114, 184.

Treit. - Dup. - Wood. 1476. - IIer.-Schf. n० 137. -

Heyden. cat. 116.

Noetuella (Tiir.). IV. V.

Var. In c e rt alis. Hub. Verz.

Très-commune dans toute l'Europe. Je l'ai aussi reçue de l'Amérique du nord où elle parait fréquente. - En Suisse, on la prend tout l'été, depuis le mois de mai jusqu'en octohre; partout, excepté dans les forèts. Elle est surtout commune au printemps et plus commune encore en août et en septembre. Elle abonde sur les pentes arides et herbeuses. Elle s'élève encore sur les Alpes jusqu'au bord des neiges éternelles.

Cette espèce varie pour le dessin et la conleur. On trouve des individus dont le dessin est complétement nul, et le fond d'un brun bistre clair et uniforme. Il en est d'autres qui portent les deux taches (réniforme et ovale) et les deux raies médianes, propres aux Bo tys, se détachant en noir sur un fond brun-foncé. Entre ces deux extrêmes existent une foule de nuances diverses.

XI. Gen. Eudorea. Curt. Dup. Zell.

(Chilo, Treit.)

Les espèces sont dans ce genre tellement semblables les unes anx autres, qu'il ne lant se déterminer à leur donner un nom qu'après avoir comparé un nombre suffisant d'individus bien frais.

$N^{\circ}$ 70. Centuriella. W. V. (Tin.).

Hub. 239. - Treit. (Galleria). - Dup. cat. (Galler.) Hub. sup. 93, 94. - Zell. - Her.-Schf. no 138. - Heyd. cat. 106. 
Borealis. Lefeh. -- Dup. sup. (ella).

Qu adratella. Scop.

If $1 \mathrm{~m}$ e ralis. Zeller.

N'a été prise en Suisse jusquici que dans les hautes Alpes des Grisons. H. Zeller l'a collectée dans la Haute-Engadine. M. Kriechbaumer l'a aussi recueillie dans les mêmes contrées. Très-rare.

\section{$\mathrm{N}^{\circ}$ 71. I mbiguella. Treit. sup. 108.}

Dup. hist. pl. 229, $5^{\mathrm{c}}\left(\right.$ non $\left.5^{a-b}\right)$. - Zell. - Hub. sup. f. 108, 109. - Her.-Sohf. n² 142. - Heyden. cat. 115.

Pas fréquente sur le Jorat, dans les bois de sapin (Lah.) — De mème au pied du Jura (Rothh.). - Emirons de Zurich, en juin (Zell.; Bremi); — de la Neuverille (Couleru).

L'extrémité de la frange des supérieures est blanchâtre; après elle viennent denx lignes grises dont l'interne est plus fine; un trait blanc dentellé en dedans, limite le bord externe de l'aile, il est suivi d'une séric de points noirs se prolongeant en pointes sur les nervures. La tache ell $x$ porte un point gris en dessus et un blanc en dessous. Les deux taches de la base sont noires, l'antérieure en forme de trait, la postérienre en forme de point peu visible.

Lineola, Curt. - Wood. Est très-distincte d'ambiguella.

$\mathrm{N}^{\circ}$ 72. Perplexella. Fisch, v. Rösl.

IIub. sup. f. I10-112. - IIer.-Schf. no 143. - Zell. Heyden. cat. 110.

Recueillie dans les Grisons, par M. Amstein (Bremi). Un individu que ma euvoyé sous ce nom II. C. Zeller, appartient à dubitella.

$\mathrm{N}^{\circ}$ 73. Manifestella. Fisch. V. R.

Hub. sup. f. 104. - Her.-Schf, $n^{0}$ 144. - Heydeı, cat. 109.

Je rattache à cette espèce un individu pris dans les Alpes par $\mathbf{1 1}$. Zeller et qui ne pent se rapporter qu'à la figure $\mathbf{1 0 4}$ de Iler.-Schf. Il. se distingue de toutes les espèces à moi connues par le bord externe des supérjeures coupé carrément. Pour la taille et le facies il ressemble à a mbiguella ef à asphodeliella. Avant d'en faire une espèce distincte, il est nécessaire de l'observer encore et de la comparer. 
$N^{\circ}$ 74. Asphodeliella. Man.

IIeyden. cat. 111.

Deux exemplaires de cette espèce, pris dans les Alpes du Valais (Lah.).

Je n'ai pu la déterminer que d'après un individu qui avait été placé sous les yeux de M. Mann, et que m'a communiqué obligeamment M. Th. Bruand de Bezençon. Elle se rapproche beaucoup d'ambiguella, quoiqu'elle s'en distingue au premier ahord, par une large bande grise, tirant légèrement sur le brun, qui oceupe tout l'espace terminal (troisième espace) des supéricures. La lrange porte deux traits gris-bruns et deux traits hlancs, elle est limitée par unc éclaircie blanc-bleuatre, sur laquelle se dessinent quelques points bruns. Le sommet de l'aile et le bord terminal sont plus arrondis que chez a mbiguella. La frange n'a pas les dentelures blanches de mercurella. L'espace moyen est d'un blanc hleuâtre, plus elair que dans a mbiguella. La tache en $x$ est entourée de fauve-brun. Des deux taches internes, la postéricure seule est bien risible sous forme d'un très-petit trait noir. L'antérieure se confond arec la ligne arrondie qui sépare les deux premiers espaces. Le mâle est semblable à la femelle.

$\mathrm{N}^{\circ}$ 75. Sciaplilella, Lah.

$$
\text { Parella. S. IIuh. sup. f. } 102 .
$$

Cette charmante espèce a été découverte par M. Couleru dans les environs de la Neureville [Berne].

Taille de mercurella; beaucoup plus blanche qu'elle. La couleur du fond des ailes supérieures est le blanc pur, nuancé de gris et de bleuâtre. L'espace basilaire est sablé de points gris-foncés, plus nombreux vers l'articulation. L'espace moyen bande médiane), large is la côte et très-rétréci vers le bord interne, est plus recouvert de pointillé que le précédent, il parait aussi plus foncé que lui; les atomes gris sont surtout acumulés le long de la première raie, vers le bord postérieur et autour de la tache en $x$. Un petit espace blanc-bleuâtre existe en dehors et en dedans de cette même tache. Un point noir nnit cette tache an bord antérieur. Le troisième espace (terminal) est derechef sablé de gris et teinté de bleuâtre sous forme de large tache du côté des bords postérieur et antérieur. La première ligne, légèrement oblique, fait un angle à son milieu; la seconde, après avoir décrit un sinus pour s'approcher de la frange, s'en éloigne beaucoup pour se rapprocher de la première et atteindre le bord interne en dedans du niveau de la tache en $x$. Le sommet de l'aile cst arroudi et le bord terminal coupé presque carrément. Le long de la frange existe une série de points noirs contigus, et sur sou 
milien quelques atomes de mème couleur, faisant un petit angle entouré de blane bleuâtre pur. La frange est blanche, entrecoupée de points gris triangulaires.

Les ailes inférieures sont d’un gris brunâtre, luisant, plus foncé à la péripherie. I'n trait blanc-jaunâtre, suivi d'un trait gris, limite la frange blanche.

Le dessous est d'un gris blanchâtre luisant, mélaugé de jaunâtre le long de la frange et de la eôte, et teinté de gris foncé sur les bords. Un point sur la côte des supérieures, un autre, plus petit, sur le disque des inféricures, et une strie transverse, se détachent en gris sur le foud de l'aile.

La tête et le corselet sont blanes pointillés de gris. Les palpes sont de la même couleur, mouchetés de noir en dehors. Les antennes sont entrecoupées de blane et de noir en dessus. L'abdomen est brunâtre, annelé de blane jaunâtre en dessus. Le dessous du corps et les pattes sout d'un blanc pur.

La femelle m'est incounue, je ı’ai un que deux mâles.

M. IIerrich-Schieffer, auquel j’ai présenté cette espèce, l'a reconnue pour celle qu'il a figurée sous le $1 " 102$ (H.-S.) et l'a adoptée comme bonne especee.

$\mathrm{N}^{\circ}$;6. Mercurella. Lin.

Zinck. - Treit. - Zell. f. 7. - Her.-Sehf. $n^{\circ} 148 .-$ Heyden. cat. 117 (non Dup. eat.).

A m bigualis. Dup. pl. 229, f. $5^{a}$.

Crataegella. Steph.

Cette espirce est commmue dans les vergers et ailleurs, à la fu de juin et en juillet (Lah.). De mème près de Sehüplen et de Znrich (Rothb., Bremi).

Truncieolella. Staint, appartient à une autre espèce, comme j’ai pu m'en assuret par un individu que m'a coufié M. Th. Bruand.

Le bord terminal arrondi sur ses deux angles, la frange entrecoupée et bordée par une lentelure blanche, fine et très-délicate, ta tache en $x$ entourée de roux, distinguent suffisamment cette espece de toutes ses voisines.

La femelle est pour l'ordinaire plus grande que le mâle; son dessin n'est pas différent. Chez quelques individus la bande moyenne se rétrécit beaucoup vers le bord interne.

$N^{\circ}$ 77. Ingratella. Fisch. v. Rösl.

Hub. sup. f. 105, 106. - IIer.-Schf. n ${ }^{\circ}$ 140. - Heyd. cat. 113.

Cette espèce doit avoir été prise à Malans [Grisons] par M. Amstein (Bremi). Des indi- 
vidus euvorés de Zurich sous ce nom, à M. Rothenbach et à moi, appartiennent à dubitella. Sa présence en Suisse est douteuse.

No 78. Parella. Zell.

$$
\begin{aligned}
& \text { D) cat. - IIub. sup. f. } 100,119,120 \text { (non } 101 \text { et 102). } \\
& \text { Iler.-Schf. } \mathrm{n}^{\circ} 146 \text {. - Heyden. cat. } 125 \text {. }
\end{aligned}
$$

Un individu femelle, lans la vallée de Tzermatten, au-dessus du village de ce nom, auprès d'un chalet. Très-rare en Suisse (Lah.).

L'échantillon que j'ai sous les yeux est encore plus chargé d'atomes bruns que la figure 100 de llub. sup., aussi n'aperģoit-on pas sur lui les points de la hase. Il répond du reste parfaitement à la figure citée, mais moins bien aux figures 119 et 120 qui cependant ne me paraissent pas pouvoir en ètre séparées. Quant aux figures 101 et 102 clles appartiennent à d'autres espèces.

\section{No 79. Sudeticella. Dup. sup. pl. 84, f. 7.}

Zell. Isis et Lin. - IIub. sup. 5116, s 117 . - Iler.-Schf. "1 151. - Ilejden. cat. 127.

D u bitalis? Ilub. 207.

Très-commune sur toutes les $\mathrm{Alpes}$ et sous-Alpes, autour des chalets, dans les endroits pierreus et escarpés; juillet (Lah.). M. Rothenbach l'a recueillie aussi dans le Jura. Je ne l'ii jamais observée sur les arbres (Lah..

Cette espèce se reconnaît aisément à sa côte très-droite, à ses ailes coupées obliquement ¿ leur extrémité et terminées par un sommet assez aigu, à sa teinte gris-brun loncée, qui recourre fortement le fond. La tache en $x$ est ombrée de blane sale, devant elle se trouve un pretit espace d'un blane plus clair encore. Les taches internes et surtout l'antérieure sont peu visibles. La bande médiane est très-rétrécie en arrière, et la seconde ligne fort oblique, peu sinueuse.

La femelle est beauconp plus rare que le male. Elle est de moitié plus petite, d'un blane plus pur, en sorte que le dessin et les taehes ressortent mieux. Ses ailes sont aussi plus étroites. Elle a du rapport avec vallesiella et a été prise sans doute plus d'une fois pour elle; la coupe cunéiforme des ailes l'en distingue sur-le-clımp. La bande médiane forme chez elle une pyramide dont la base est siluce à la côte et dont les bords sont nettement linités par un trait d'un blanc pur. 


\section{$N^{\circ} 80 . \quad$ I uranelia (na). Steph.}

Curt. - Wood. 1440.

Parella. IIub. sup. f. 101 ? (non 100, 102, 119, 120).

Je dois à l'obligeance de M. Th. Bruand la connaissance de cette espèce, reçuc par lui d'Angleterre. Je l'ai prise à la fin de juin, sur les rochers chands de la route d'Aigle au Scpay, au milien de juillet à Champéry dans le val d'lliers, à la mìme époque au pied occidental de la Furca; elle ı'est pas rare dans ces diverses localités. Il est probahle qu'clle se rencontre partout au pied des Alpes.

Lorsqu'elle est fraiche elle se reconnaît sans grandes difficultés; mais dìs qu'elle est un pen fancé on la confond avec mercurella et amhiguella dont elle a la taille et la forme. La teinte générale des supérieures est le gris-de-fer, teinté de bleuâtre sur le centre et vers le sommet, de fauve-pàle le long de la còte. Le fond est surchargé d'atomes gris et noirs, plus nombreux à la racine de l'aile. La cóte est un peu arquée vers son extrémité externe et marquéc d'une trace blanche à la naissance de la seconde ligne. La première ligne hlanche est plus ou moins arquée et condée dans son milieu; elle est limitée en dehors par une ligne noire devant laquelle se roient un point antérieur blane cerné de noir et un second, postérieur, noir; l'un et l'autre sont pen visibles sur certains individus. L'espace moyen est assez large, d'un gris uniforme et pour l'ordinaire aussi foncé que le reste de l'aile, un peu rétréci du côté du bord interne. Sur lui, vers les $2 / 3$ de l'ailr se distingue la tache en $x$ entourant, en arant, un point blane bleuâtre pur, et quelquefois un semblable, plus petit, en arrière. Le troisième esparc (terminal) est d'un gris pâle plus ou moins courert d'atomes. Une tache triangulaire, plus petite que ses voisines, grise ou noire, s'appuie sur le centre de la marge. Les deux autres taches se touchent parfois sur le milicu de l'aile; elics sont séparées par deux traits blancs formant un $x$ majuscule. - La frange est limitée par une ligne de points blancs, quelquefois contigus, ordinairement cernés de noir, à laquelle succède un trait limitant la frange, puis un trait blanc, puis un dernier trait noir entrecoupé de blanc. L'extrémité de la frange est blanche entrecoupée de gris pàle. Le bord terminal est coupé un peu obliquement et l'angle antéricur est obtus.

Les inférieures sont d'un gris påle, luisant; leur frange est blanc sale, limitéc par une ligne grise suivie d'une jaunattre.

Le dessous des supérjeures est gris, luisant, arec une tache fauve à la côte. Celui des inféricures est plus pàle.

Tête hlanche plus ou moins tachée de noir. Palpes gros, épais, noirâtres en delors, tachictés de blanc en dessus. Anternes sétiformes, noirâtres. Thoras comme la tète. Abdomen 
d'un blanc roux, annelé de gris-noirâtre et terminé par un court pincean fauve chez le mâle. Dessons dn corps et pattes blanchatres.

La femelle ne diffère pas du mâle.

La figure que Duponchel donne de sa valesialis (pl. 229, f. 3) répond mieux à muranella qu’̀̀ aucune autre espèce; mais la description ue concorde pas aree la figure. Je l'ai reçue de Mann sous le nom de parella.

$\mathrm{N}^{\circ} 81$. Tales iella (alis). Dup. hist. $229, \mathbf{3}$.

Heyden. cat. 121. - Hub. sup. f. 103. - Her.-Schl. n"147. Oeto n ella. Zell. Lin.

Rare; les hautes Alpes du Valais et des Grisons. Je l'ai reçue de M. O. Heer qui l'avait prise sur le Bernina (Lah.). M. Zeller l'a aussi trouvée dans les Grisons et M. Frey à Zermatten.

Cette espèce se distingue an premier abord par ses ailes supérieures étroites, dont les deux bords sont presque parallèles. Les deux points de la base sont très-saillauts; l'antérieur est plus petit. Une tache blanche existe entre la tache en $x$ et la troisième ligne. La frange est blanche, limitée par des points noirs et quelquefois monchetée de gris.

La figure de Duponchel est mauraise et peut servir à plus d'une espèce; sa description est bonne. La frange n'est point entrecoupée d'une manière sensible et les points de la base sont presque toujours an nombre de deux.

$\mathrm{N}^{\circ}$ 82. Oertziella. Mess. - Heyden. C. 108.

O ertzeniella. Hub. sup. \{. 97. - Her.-Schf. $n^{0} 153$.

Pallida. Curt. cat.

Lolusella. Guén.

M. Rothenbach a pris plusieurs exemplaires de cette espèce rare, près de Schüplen, dans une haie, au bord d'un pâturage humide.

Je dois à l'obligeance de M. Th. Bruand la connaissance des synonymes de Curtis et de Guénée. La première ne diffère de la seconde que par son dessin moins marquée.

La taille d'oertziella est au-dessous de dubitella. Le fond est presqu'entièrement d'un blane sale; les raies et les taches sont peu marquées, a l'exeeption des denx points noirs placés à côté de la première ligne.

$\mathrm{N}^{\circ}$ 8:3. Vandaliella. Her.-Schf. (in litter.).

Delunella. Guén. (teste Bruand). - Ileyden. cat. 1:20.

A m bigualis? Dup. hist. pl. $229,5^{\text {th }}$.

Pas très-rare antour de Lausanne, sur le tronc des peupliers et des arbres fruitiers; pro- 
menade de Montbenon; juillet (Lah.). - De mème près de Schüpfen (Rotbh.). - Elle existe aussi en Italic.

Le mâle ne diffère pas de la femelle. La figure de Duponchel est un peu trop brune dans les places claires. Delun ella Guén. que m’a communiquée M. Bruand, ne diffère pas de l'espèce de Herrich-Schæffer. Trìs-voisine de laetella Zell., s'en distingue par les grosses taches noires de la côte.

\section{N" 84. Crataegella. Hub. 231.}

Dup. 229, f. 7. - Frey. ä. b. 168. - IJub. sup. f. 1 13, 1 14.

-. Her.-Sehf. n' 149. - Ileyden. eat. 118. - Curt. cat.

M e r cure lla. Dup. cat.

Pyralella? W. V. - Wood.

Freiguentella? Staint. (fide Bruand).

Var. La e tella, Guén. (fide Bruand). - (non Zell. - IIub. sup.).

Assez commune partout sur le tronc des arbres fruitiers, en juiliet (Lah). - De même près de Schüpfen et de Zurich (Kothb., Bremi).

La femelle ne diffère pas du mảle. La figure de Duponchel est inexacte, beaucoup trop chargée de brus. La première ligne transterse est trop prononcée. Les trois taches de l'espace terminal se touchent presque toujours par leur extrémité.

Frequentella Staint. ne differe de crataegell a que par quelques taches brunes au coté externe de la premiere ligne transierse.

J'ai sous les yeux un individu appartenant à cra 1 a e ge 11 a, qui ressemble parfaitement à l'espèce anglaise.

La variété L a et ell a Guén. (Hub. sup. fig. 113), que m’a communiquée M. Th. Bruand, se rapproche tellement de e rataeg el la que je n'ai pas pu en faire une espèce différente. Elle ne s'en distingue que par le fond des ailes beaucoup moins chargé d'atomes gris, et par les deux points internes plus gros et plus saillants. Dans c rataeg ell a ces points sont souvent entièrement couverts par les atomes gris. Du reste, elle diffère entièrement de Lae tella Zell., layuelle se rapproche beaucoup plus de vandaliella.

J'ai pris un individu mâle de la variété $\mathbf{L}$ a c t e l'a daus les environs de Lausanne; il serait possible, en comparant un grand nombre d'exemplaires, qu'on décourrit des caractères spécifiques suffisants. 
Yo 8.5. Dubilella (alis). Zell.

Zinck. - 5 Hub. 49 ? - Ilub. sup. 107. Treit. - Dup. Heyden. eat. 114. - Iler.Schf. $n^{\circ} 141 . \quad$ Zell. Lin.

OPralella. llub. 167?

Tristrigella. Stpl. - Wood. 144. - Ilub. sup. 98?

Mereurit. Fab.

Assez fréquente dans les taillis et les herbes le long des bords du Léman, en juin. Pas rare dans toute la Suisse. Préfère les lienx chands.

Cette espèce ne varie pas. La femelle ne diffère pas du mảle.

No 86. Ancipitella. Lah. fig. 5.

Prise dans les environs de Meyringen, par M. Ott, en juin, et par moi, dans les bois de sapin du Jorat, en juillet. Environs de Schïpfen (Rothb.).

Elle se distingue de toutes ses congénères par la simplieité de son dessin, et se rapproche sons ce rapport de perplex ella, Her.-Schf. f. 1 I I. La taille est celle d'une petite dubitella. Les supérieures sont en dessus d'un gris perlé presque uni. L'espace basilaire est légèrement plus clair que le reste de l'aile, à peine enfumé à la racinc. L'espace moyen est séparé du précédent par une ligne plus ou moins arquée, laisant dans son milieu deus petits angles aigus, et ombrée de gris-foncé du eôté du limbe. Les deux taches sont petites, noirâtres, confondues aver la première ligne; la tache en $x$ est ombrée de roux et surmout tée d'un petit point. La seconde ligne est presque parallèle an hord postérieur, assez rapprochée de lui et forme un sinus peu profond vers son milien. L'espace terminal est marqué de trois taches grises séparées par la couleur do fond qui est ici presiue blanche. Des trois taches de la marge, l'antériemre et la postérieure, sont très-pren marquées, l'externe, appuyéc sur la frange, est plus foncée. Une série do points noiràtres, peu distincts et séprarés par tle pretites taches roussàtres, limile la frange. Celle-ci est d'un blanc-rous, divisée par une ligne grise. Le sonmet de l'aile sujérieure est obtus et son bord exteme légèrement oblique et arrondi.

Les inférieures sont d'un blanc-grisàtre luisant, ombré de gris sur le bord exteme. Le dessous des quatre ailes est entièrement de la mème couleur, mais ombré de ron luisant aur supérieures.

La tête et le corselet sont de la couleur des ailes supérienres; l'abdomen de ctlle de's inférieures; eelui-ci est terminé, chez le mâle, par un pinceau fanve-elair.

Elle se distingne de perplexella (Iler.-Schf. f. 111) par sa seconde ligne transverse qui suncline vers la base, et non vers la marge, en se rapprochant du bord interne. Je n’ai un que le mâle. 
XII. Gen. Chilo. Zinck.

(Schonohius, Dup.)

$N^{\circ}$ 87. Forficellus. Thunb.

Treit. - Fisclı. 1. R. 1. 17. - Dup. 268. - Stepl. -

Wood. 1523. - Her.-Schf, ॥ 157 . Heyd. rat. 5. -

Guén. cat.

Consortella. Ilub. 2:20.

Consorta. Haw.

C a u d ell us. Wood. 1.528?

Lance olella s. Hub. 296 . - Curt.

Près d'Aarberg et autour de Münchenbudhsee, sur les prairies humides et les marais Rothb.). - Environs de la Neuverille (Couleru).

XIII. Gen. C r a mbus. Fab. - Dup., etc.

Chilo, Treit.

Ce genre est l'un des mieux circonscrits, et cepentant, combien le dessin ne varie-t-il pas? - La plupart des espèces vivent sur terre, parmi les herbes.

$\mathrm{N}^{\circ}$ 88. Pascuellus. Lin.

W. V. - IIub. 3I c. - Treit. - Dup. 269, I. - Wood. 1492. - Zell. - Guén. cat. - Iler.-Schf. ॥ 164.

Ileyden. cat. 28 .

P ascuum. Fab.

Var.? Uliginosellus. Zell. - Heyden. cat. 29.

Espèce commune, en juin et en juillet, sur les prairies du canton de Vaud. Préfère les lieux chauds. Hoins commune que dumetellus et pratellus (Lah.). - De même près de Schuipfen, de Zurich et Jans les Grisons (Rothl., Zell., Bremi, Amstein).

La femelle ne diffère que fort peu du mâle; elle est volontiers un peu plus pàle. Cette espèce varie un peu moins que ses roisines; son vol est aussi diffërent do leur et ne se prolonge pas. La hande centrale, argentée, s’approche plus on moins de la ligne terminale ef ra jusqu" la toucher; elle est aussi plus ou moins large ou effilée à sa terminaison.

J'ai reçu de M. Maun le Cr. uligin o sellus Zell., provenant de Glogau et de II. Zeller lui-même. Cette espèce ne se distingue de pasecuellus que par sa lame argentée médiane 
plus elfilée à l'extrémité et terminée un peu plus loin de la ligne brisée marginale. In pretit śtranglement vers le milieu de la metme lame n'eviste pas dans pas euellus. Les autres diférences, telles que la taille et la teinte ne peurent la caractériser. J'ai pris sur les II osses, platean marécageux des Mpes d'Aigle, le même Crambe, que je ne puis encore admettre comme espèce distincte. La comparaison d'un nombre suffisant d’individus doit déeider la yuestion. L'habitat m'avait surpris; car pasc u e l] us préfère les pâturages claands et seés.

Io 89. Dumelellus. Hub, :389, 390 .

Treit. - Dup. 269, 2. - Zell. - IVood. 149:3. Giuén. rat. - IJer.-Schf. $n^{\circ}$ 165. - Heyden. cat. 2:3.

Prat ell us. Wood. 1495. - Dıp. $269,3^{\text {h? }}$

Pratella. IIub. 29 ?

$S$ altalis. Hul. Verz.

Commune sur les pàturages sers, dès la fin de mai aı milieu de juillet (Lalı.). Commune sur toutes les montagnes (Rothb.). De même dans les environs de Zurich (Bremi, Zeller).

Varie peu dans sa taille et dans sa couleur, davantage dans les détails du dessin. Le màle ne differe pas sensiblement de la femelle. La bande centrale, argentée, varie de longueur, de largeur et de forme; cependant toujours son extrémité est couprée en biseau, et son angle postérieur est marqué d'un trait foncé. Les rayons qui partent de son estrémité sont plus ou moins visibles, plus ou moins plombés ou argentés.

Pratellus, var. Dup. pl. 269, fig. $3^{\text {h}}$, n'est évidemment qu'un dumetellus. La synonymie de cette espèce est encore quelque peu embrouillée.

\section{No 90. Pralellus. Zinck.}

Hub. 401. 9. - Treit. - Dup. pl. 269, I. 3 $3^{\text {a, c }}$ (non ".

Guér. cat. - Heyden. cat. 24.

Pratopum. Fab. - Her.-Sehf. $n^{\circ} 166$. - Zell.

Ericellus. Wood. 1494. - (Non Hub.)

Angustellıs? Wood 1496. - Steph.

- Nemorel]us. IIub. 384. - Dup. cat. - Heyden. cal. 26. - IIer.-

Sehf. n 171. - Guén. cat.

Le plus commun de tous les Crambes; paraît avec le précédent, dès la fin de mai, et se trouve sur toutes les prairies et dans les haies eı abondance. Les pâturages des Mpes en fourmillent (Lah.). De mème dans toute la Suisse (Rothb., Bremi, etc.). 
Cette espèce varie extrêmement; aussi sa șnonymie est-elle riche et obscure. La femelle est presque toujours plus pâle et souvent plus petite que le mâle. On la troure assez fréquemment blanche, et parfaitement semblable à la figure 384 de IIubner. Le mâle va jusqu'au brun-foncé; dans ce cas le dessin disparait en grande partie sous la couleur dı foud. La taille varie aussi beaucoup; elle atterint celle de dumetellus et peut descendre jusquà celle de l'alsellus. La bande longitudiuale et rentrale, argentée, est sourent réduite à un simple trait chez les individus foncés.

No 91. Adipellus. Zinck.

Treit. - Dup. 269, 4. Guén. cat.

Silı ellus. Huls. 369, 370. - Iler.-Schf. $\mathrm{n}^{\circ} 168$. - Ifeyden. cat. 30.

Zeller.

Près de Hofw] et de Nlünchenbuchsee [Berne], sur les prés marécageus; assez fréquent Rothb. Sur le Jorat; mèmes localités; rare (Lah.). Les prés marécageus sur l'Uto Zeller, Frey.). Près de Coire (Kriechbaum).

No 92. Lucellus. Hub. sup. f. 1:35.

Her.-Schf. $\mathbf{n}^{\circ} 189$.

Lathon ie llus? Zinck.

Assez lréquent dans les lieux chauds des bords du lac Léman et de la vallée d'Aigle, à la fin de juin et en juillet (Lah.). Les vallées an midi des Alpes centrales Zell.).

Cette espèce, peu romnue jusqu’ici, se rapproche bien plus de prat allus que d'lu ortisellus. Sa taille dépasse sensiblement celle du premier; son dessin est à peu de chose près le même. Elle s'en distingue essentiellement par la dent postérieure de la bande longitudinale centrale, prolongée en pointe et par la ligne trausverse marginale arrondie en sinus dans son milieu, comme daus hort uell us, et non brisée, ainsi qu'elle l'est daus les espèces précédentes. La figure yu èn dome ILub. sup. n’est pas très-bonne. Nemorellus IIul). 384 ne saurait lıi ètre appliqué. Quant à lathon iellus, Zinck., je ne l'ai pas eu sous les yeus.

La teinte générale est le rous; sur elle se dessine, en blanc terne, nullement argenté, $1^{\circ}$ la bande centrale, très-large, terminée par deux pointes presque obtuses, inégales; sa partie antírieure porte wn large trait brun longitudinal; $2^{\circ}$ six rayons blancs, qui s'appuient sur la ligne transverse terminale. Cette ligne est formée d'un trait roux et d'un trait blanc, qui 
restent distants de la frange et décrivent un large sinus; entr'elle et la frange, l'espace brun est teinté de gris et marqué de 4 à 5 points noirs, séparés par du fauve. La frange des supérienres est presque droite, d'un blanc métallique luisant, limitée par un trait brmn.

Les ailes inféricures sont d'un gris loncé, tirant sur le hrun. Leur lrange est d'un hlanc terne.

Le dessous des supérieures est entièrement d'un roux noirâtre, saul' le bort terminal qui est d'un blanc roux et sur lequel se dessinent à peine les points de la marge. La ligne transverse limite hrusquement les deux teintes. Le sommet est teinté de brun. - Le dessons des inférieures est d'un blanc roux, fortement nuancé de brun, surtout en arant.

La tête et les palpes trìs-alongés sont d’un brun-olise; le thorax est brun, ainsi que l'aludomen à sa partie supérieure. Le dessous est grisàtre.

Le malle ne diffère de la femelle yue par sa taille un peu plus faible.

No 93. Hortuellus. Hub. 46.

Treit. - Dup. - Zell. - Wood. I 497. - Her.-Sch. n 190. Heyden, cat. 3.5. - Guén. cat.

Chrysonuchella. W. V.

Strigella et strigatus. Fab.

Ilontanellus. llood. 1499.

Var. Cespitella. Hub. 4.5. - Heyden. cat. 36.

Var. Montana. Wood. 1498.

Très-commun dans les taillis herbenx et sur les prairies de toute la Suisse; s'élère sur les pentes des Alpes. Dès le commencement de juin à la fin de juillet (Lah.. Rothb., Bremi, etc.).

La variété e spitell a n'a d'autre caractìre à revendiquer que la teinte plus claire des ailes inférieures, surtout. le long de la marge. Il ne me parait pas suffisant pour la séparer d'hortuellus. Guénée affirme que la ligne transverse n'est jamais bordéc de couleur plombée. J'ai sous les yeux un individu où cette teinte existe, mais à un moindre degré.

La taille varie beaucoup. La couleur violette du fond s'efface vit par le vol. La lemelle ne diffère pas du mâle. 
No4. Saxonellus. Zinck.

Dщр. - Fiscl. v. R. 89. - IIer.-Schl'. 1" 180. - Treil. sup. III, p. 170. - Heyden. cat. 76. - Guén. rat.

Chrysellus. Treit. IX, I32.

Partout rare en Suisse. Je l'ai pris quelquefois à Paudex, an bord du lac Léman, solant parmi les herbes et les taillis, avec hortuellus. Juillet. - Collection Rothenbach. Prìs de Burgdorl (Mer.).

No 9.5. I1pinellus. Hub. 3:38.

Trrit. - Dup. - Zell. - Heyden. cat. 17. - Her.-Sicht. n 172 . - Gutén. cal.

Inabite les lieux chauds et arides dı Valais, des bords du Léman el du pied du Jura. A Martigny, auprès de la tour de la Batia; a Cour sur la grève dn lae, sous Lausanne (Lah.): Aurberg (Rothenb.); la Neureville Couleru).

Cette espèce n’est point alpine, car elle recherche toujours les lieus chands et secs.

$\mathrm{N}^{\circ}$ 96. Cerussellus. W. V.

Treit. - Zell. - Dnp. eat. - Her.-Sehf. n" 175. - Gmén. cat. - Heyden. eat. 3i. - Wood. 1502.

; Anriferella. Hub. 6.2. - Steph. - Curt.

Q Quadrellus. W. V. - Dup. hist.

S Barbella. Ilub. 61. - Curt. - Steph.

S Pygmaens. Steph. - Wood. 1503.

Bot. nemausalis. Dup. hist.

A l'embouchure du Flon, près de Lausanne, sur um tertre sablonneus et ser; en juin.

Le màle vole en essains, se posant sur tous les brins d'herbe; la femelle s'y tient ordinairement immobile et se laisse tomber à terre sitòt qu'on l'approche; elle vole peu. L'alpparition ne dure que peu de jours.

\section{$\mathrm{N}^{\circ}$ 97. Inquinatellus. W. V.}

Ilub. 54. - Treit. - Dup. - Zell.

Luteelus. Wood. 1511. - Steph.

Rore a. Haw.

Assez commune sur les coteanx ehauds et arides, en août et septembre (Lah.). De míme 
priss de Zurich Zell.), de Schïpfen (Rothb.), de Malans (Amstein), de la Nenverille (Couleriı).

Son bol est court et très-rapide.

Yo 98. Angulatellus. Dup. hist.

Hub. sup. 8 - 10. - Zell. Isis, 18+7. - IIer.-Schf. $1^{\circ} 178$. - Ilejden. cat. 83.

I m mis te II a. Ilub. 364. - Guén. cat.

$\mathrm{S}$ u s p e c te $1 \mathrm{u}$ s. Zell. Isis.

Geniculeus. Haw.

In quin a t ellus. Steph. -- Wood. I5 I3.

Cette espèce, rare en divers lieux, est très-commune certaines années, autour de Lausanne, en août et en septembre (Lalı.). Assez rare près de Schüpfeı Rothb.), et de la Neıveville (Couleru).

Cette espèce ne craint pas les lienx ombrés, les taillis des montagnes et se trouve dans des endroits oì inquinat ellus ne paraît janais. La taille varie; le mâle ne diffère pas de la femelle.

X 99. Culmellus. Lin.

Treit. - Dup. - Wood. I501. - Guén. cat. - Her.-Sch. n 18:. - Heyden. cat. T4. - Zell.

Straminella. W. V. Hub. 49.

Striga. Haw.

Marginellus. Wood. 1500.

Extrèmement commun partout, sur les prairies et dans les taillis, dès le milieu de juillet. S'élève jusqu’aux $6000^{\prime}$ (Frey.).

$\mathrm{N}^{\circ}$ 100. Falsellus. W. V.

Ilub. 30. - Treit. - Dup. - Wood. I510. - Zell. Guén. cat. - Her.-Schf. n 184 . - Hryden. rat. 44.

A bruptella. Thunb.

Fréquent dans les environs de Lausaune, le long du Léman, dans les jardins et les plantages; fin de juillet et août. - Près de Soleure, assez rare Rothb.). L'Uto. près de Zurich; rare (Zeller). Malans (Amstein). La Neuverille (Couleru). 
$\mathrm{N}^{\circ}$ 101. Verellus. Zinck. II, 81.

Huh. sup. f. 137. - Her.-Schf. 1 $^{\circ}$ 18.5. Heyden. cat. 45.

Pris deu fois; en compagnie de falsellus, près de Paudex; très-rare (Lah.).

J'ai de la prine à croire que cette espèce ne soit pas une variétí de falsellus. M. Herrich-Schaffer la considère comme bonne espèce. Son vol, très-rapide et par bouds, est différent de celui de sa roisine.

$\mathrm{X}^{\circ}$ 102. Chrysonuchellus. Scop.

Treit. - Dup. - Zell. - Wood. 1508. - Guén. rat. -

Her.-Seld. n" I86. Heyden. eat. 4.2. Non W. V. new IIub.)

Gramella. Fab.

s. Campella. llub. 44.

Culmella. Hus. Schf. - W. V.

Espèce trìs-répaudue dans toute la Suisse, sur les pàturages hmmides et marécagoux: fin de mai et juin.

$\Gamma^{\text {to }}$ 10:3. Rorellus. Lin.

Treit. - Dup. - Zell. - Wood. 1509. - Her.-Scht

n $187 .-$ Guén. cat. - Heyden. cat. 40.

Craterella. Srop.

Linetella et lincatus. Fab.

Chrysonuchella. IIub. 43 .

Je cite cette espèce sur l'autorité de MI.I. Rothenbach et Conleru qui rout recueillie, f'un prìs de Sehüpfen, l'autre près de la Neurerille, an dire de M. Bremi; II. Rothenhach ne l'a point indiquée dans son catalogue et je ne l'ai point rue dans la eollection de M. Conleru. M. Bruand l'a trourée dans Je Doubs. Je l'ai des environs de Ileidelloerg.

$\mathrm{Y}^{\circ}$ 194. Ta $\mathrm{T}$ (niellus. Kullaw.

Inl). sup. f. 6. - IIer.-Schf. n" 193. - Ifeyden. cat. 66. Zell. - Dup. cat.

Combinellus. Dup. I 403. (non Treit. nec IHb. sup. f. 7).

Novellu s. Guén. cat.

Conlonellus. Dup. 273, 6. - Guén. cat.

Comnun sur tous les pâturages des Alpes et du Jura, dès la fin de juin au rommencément daont Lah., Rothb., Couleru, Bremi, Zell.). 
Cette espice a donné lieu à des confusions nombreuses, par suite de ses variations. Sa synonymic offre encore des incertitudes.

Combinellus Treit. (Ilub. sup. f. 7) est extrêmement roisin de ta eniellus, il en diflère cependant par des caractères suflisants. Son dessin est le mème à l'exception de la ligne centrale „ui est rarement apparente; de la ligne terminale formant un sinus plus arrondi et plus rapproché de la marge; des stries longitudinales blanches, plus saillantes. Le bord externe de l'aile est légèrement excavé ce qui fait paraître le sommet plus aign. La lịnne noire qui limite la frange est hien marquée dans $\mathrm{combinellus,} \mathrm{tandis} \mathrm{qu'elle} \mathrm{manque}$ dans taeniellus ou n'est indiquée que par quelques points; cette ligne fait mieux ressartir la ligne blanche de la frange sur laquelle ellé s'appuie. La première espèce a la tète, le rorselet et les palpes blancs; chez la seconde ils sont de couleur olive.

La synonymie de Heyden. est fautive à l'endroit de Duponchel, celle de Herrich-Scbieller l'est aussi. Guénée a crée une nouvelle espèce que rien ne justifie et Heydenreich l'a introduite dans son catalogue en reproduisant la confusion que Guénée voulait éviter. - Ci onlonellus, Dup., est à peine une variété de ta eniellus. M. Conlern qui l'avait fourui is Duponchel, m'en a adressé plusieurs exemplaires qui ne diffèrent en rien de cenx provenant des Alpes et sont à peine un peu plus foncés. On trouve aisément des iudividus qui représentent l'un et l'autre; j'en ai de plus pâles que la figure 5 de Duponchel, mais non dı plıs foncés que sa figure 6. Quant à simplon ellus Dup., voir à l u c tiferellus.

Après avoir comparé un très-grand nombre d'exemplaires, j'ai établi la synonymie précŕdente que je crois exacte. Au résumé, j’estime que petrificellus Dup. 1406 est une bonne espèce, fort différente de celle de llub. 47, et que co mbin ellus IV. V. n'est point identique avec $\mathrm{t}$ a e $\mathrm{n}$ iellus.

La femelle de taeniellus est plus rare que le mảle et un peu plus petite que lui. Ses ailes sont aussi plus étroites, coupées plus carrément à la marge, et par là plus aigües au sommet. Les deux raies transwerses sont volontiers plus rapprochées l'une de l'autre chez elle; mais on trouve des mâles qui ont le même dessin. La femelle est aussi moins foncée et ne donne pas la forme coulon ellus. Cette différence des deux sexes a peut-être été la cause de quelques erreurs.

$N^{\circ}$ 105. Aridellus. Thunb.

Hub. sup. f. 17, 18. - Iteyden. cat. 67. - Her.-Schl. n ${ }^{0} 196$.

- Zell. -

s Spuriella. Ilub. 471. 
Pedriolellus. Dup. 275, 6. - Guén. eat. - Heyden. cat. 68.

F a se elinel]u s. Zinck. - Hub. 368. - Dup. 272, 3. - Heyden. cat. 69. Jai reçu cette espèce de Mleyringen (Lah.). - Fort rare en Suisse.

$\mathrm{N}^{\circ}$ 106. Nargaritellus. Hub. 39.

Hub. Beitr. - Treit. - Dup. - Zell. - Wood. 1505. Steph. - Guén. cat. - Heyden. cat. 55. - Her.-Schr. $\mathrm{n}^{0} \stackrel{200 .}{ }$

Fréquent sur les prés marécageux du Jorat et des $\Lambda$ pes; moins dans le Jura; en juin et juillet (Lah.). Comınun près de Schüpfen (Rothb.), de Zurieh (Frey.).

La femelle ne diffère pas du mâle. Ne varie pas.

Je l'ai rencontré quelquefois sur les sapins; mais le plus sourent parmi les herbes.

$\mathrm{N}^{\circ}$ 107. Pinetellus. Lin.

W. V. - Treit. - Dup. 271, 3. - Wood. 1507. - Guén. cat. (exelus. synon. Hub.). - Hub. sup. f. 2. - Her.-Schf.

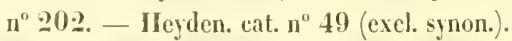

I'ai reçu cette espèce de M. Heuser de Burgdorf, qui l'avait prise dans les environs de rette ville. Tous les autres indiridus que j'ai vu et reçu sous ce nom, appartenaient à stenziellus, Treit. - Rare en Suisse. - Engelberg (Zell.). Schüpfen, de même (Rothb.).

Se distingue de conchellus W. V. (Stenziellus, Treit.) $\mathbf{1}^{\circ}$ par sa frange brune et brillante; 2" par sa taille plus petite; $3^{\circ}$ par sa couleur plus claire; $4^{\circ}$ par la ligne transverse, is peine visible, placée après la seconde tache argentée; cette ligne est coudée brusquement à l'extrémité de la tache et ne fait pas un sinus arrondi comme chez sa voisine.

\section{$\mathrm{N}^{\circ}$ 108. Mytilellus. Hub. 287.}

$$
\begin{aligned}
& \text { Treit. - Dup. 271, 6. - Guén. cat. - Her.-Schf. n } 203 . \\
& \text { - Ifeyden. cat. 52. }
\end{aligned}
$$

Assez rare dans les environs de Lausanne; une première fois en juin et une seconde en août (Lah.). - Près de Bienne, une fois (Rothb.). — La Neureville (Couleru). 
No 109. Conchellus. IV. V.

Ilub. 38. - Ilub. sup. f. 1. - IIer.-Schf. $n^{\circ} \mathbf{2 0 4 .}$

Sten zi ellu s. Treit. sup. - Zell. - Guén. cat. - Ileyd. cat. 48. - Dup.

P a up er ellus. Dup. descript. non fig. - (Non Treit.)

Commun sur les Alpes, à la fin de juin et en juillet Lah., Rothb., Zell.). Iescend quelquefois dans la plaine.

La femelle ne diffère pas sensiblement du mâle. La taille varie souvent. La couleur brune est plus ou moins marbrée de lauve. La seconde tache est quelquefois carrée, quelquefois munie d'une dent en arrière, le plus sousent elle est en forme de llamme; toujours arrondie du côté de la cûte, tandis que daus pi n e tell us elle est presque droite (voir pinetellus). La frange est plus ou moins épointée de brun à son extrémité, parfois entrecoupée inégalement de cette couleur, rarement toute blanche. La ligne transverse marginale est indiquée par une éelaircie qui en dessiue les contours arrondis.

Je n’ai jamais rencontré la variété à taches jaunes de Duponchel; je présume qu'elle ippartient à pauperellus Treit. et non à conchellus IV. V.

$\mathrm{N}^{\circ}$ 110. Myellus. Hub. 37.

Zell. - Guén. cat. - Her.-Schl. u²06. - Heyd. cat 50.

Conchellus. Treit.? - Dup. pl. 27I, f. $5^{\mathrm{b}}$.

Pinetella. Scop. - Knoch.

Cette espèce est assez également répandue en Suisse; mais partout rare. Je l'ai prise quelquefois dans les ensirons de Lausanne, au bord des bois, dans les taillis, en juin Lah.. A Schüpfen (Rothb.). Dans les environs de Zurich, sur l'Uto (Zeller), le Zurichberg, le Righi, l'Irchel (Bremi). Engelberg (Zell.).

La femelle est souvent deux fois plus grande que le mâle. Il n’est pas aisé de distinguri les petits individus de l'espece suirante. (Voir speculelus.)

$\mathrm{N}^{\circ}$ 111. Speculelus (alis). Hub. Verz.

$$
\begin{aligned}
& \text { Zell. - Guén. cat. - Hub. sup. f. 87. - Ileyden. cat. } 5 \text { I. } \\
& \text { IIer.-Schf. n } 207 .
\end{aligned}
$$

Pin e tella. IIub. 36.

Nyellus. Zinek.

Conchellus? Dup. 271, f. $5^{3}$.

Cette espèce étant sans cesse confondue avec la précédente je ne puis affirmer que ce que 
jai observé. Je l'ai prise quelıuefois sur les prairies dı Jorat, daus les licux humides, en juin et es juillet (Lah).

La figure citée de Duponchel appartient-elle bien à cette espèce, comme le pense Guénée: n'est-elle pas jlutôt une forme plus foncée de myellus?

Les individus que j’ai sons les yeux se distinguent de my ellus par les caractères suiıants: $1^{\circ}$ taille un peu plus petite; $2^{\circ}$ fond nuancé plus fortement de fauve clair; $3^{\circ}$ lrange entrecoupée de plus de blanc en face de la bande ceutrale; $4^{\circ}$ deuxième tache en forme de Hamme et non carrée; cette diflérence se voit surtout à l'angle interne et antérieur; $5^{\circ}$ ailes inférieures plus pâles; d’un blanc salc, luisant. - Les autres caractères ne sont pas constants. L’espace brum qui sépare les deux taches varie en largeur. La ligne blanche placée entre la marge et la seconde tache, est tantôt courbe, tantôt droite, tantôt parallèle an bord adjacent de la tache, tantôt s'en écarte en arrière et cela dans l’une et l'autre des deux espèces voisines.

La femelle de cette esprece a la taille dı màle et les ailes antérieures un peu phus étroites 'que lui. Il se pourrait cependant que tontes ces différences ne fussent pas spécifiques; il faut sur re point attendre de nowelles observations; mais les denx espèces étant rares, il est difficile de rassembler un nombre suffisant d'exemplaires. J'ai sous les yeux un individu femelle qui pourrait bien appartenir à une nouvelle espèce. Il diffère de speculellus frar l'absence de points marginanx, par sa ligne blanche terminale (troisieme tache) droite et fort étroite, nou parallèle aree le bord roisin de la seconde tache coupé aussi lui-mème en ligne droite. Les ailes inférienres sont moins enfumées et d’un blanc jaunâtre sur le centre et le bord interne. Par son facies il est plus rapproché de speculellus que de m jellus.

\section{$\mathrm{N}^{\circ} 112 . \quad$ Luctiferellus. Hub. 324.}

Treit. - Dup. 270, 4. - Zell. - Hub. sup. f. 21. - Gnén. cat. - Heyden. cat. 54. - Her.-Schl. n 209.

\section{Simplon cllus? Dup. pl. $273,7$.}

Pas très-rare sur les hautes Alpes centrales. Le St-Gotthard, le Simplon, la Grimsel, etc. Se prose sur les rochers luumides, le loug des routes, en compagnie de ra di ellis. Juillet (Lalı., Frè., Zell.).

Cette espèce varic passahlement. Les individus fanés prennent une teinte grisâtre on brunâtre. Les raies et les bandes sont sourent arortées ou rétrécies sur quelques points. Dans la figure de Ilub. (324) les taches blanches sont représentíes arec leur plus haut degré de développement; je dontais qu’il existat de pareils individus jusqu’à ce que j’en ren- 
contrai un semblable. La ligne transverse antémarginale s'oblitire souvent dans son milien; les taches blanches de la côte sont exposées a disparaître; l'espace noir qui sépare les deux taches est prarfois très-étroit, etc., etc.

Cette facilité à varier n’a fait peuser que simplon ellus Dup.. unique dans son genre, n'était quinne variété de luctiferellus. Il en a la forme et les dimensions. Peut-être est-il aussi une hybride de cette espèce et de ta en i ell us?

No 113. Pyramidellus. Treil.

$$
\begin{aligned}
& \text { Zell. - Dup. cat. - Guén. cat. - Hub. sup. f. 5. - Ileyd. } \\
& \text { cat. } 56 .
\end{aligned}
$$

Margaritella. W. V.

Cun e lus. Treit. sup.

A dam an tellus. Ginén. cat.

Assez rare dans les Alpes de la Suisse. Il a été pris çà et la sur le Jura par MII. Zeller at Rothenbach. M. Kriechbaumer l'a recueilli dans les Grisons. Je l'ai pris très-frais dans les sous-alpes du district d'Aigle à la fin de juin, au milien de juillet dans le val d'Hlier. Assez fréquent sur le Chasseral (Rotbb.).

Fulgi dellus, Hub., ne paraît pas exister en Suisse.

\section{$\mathrm{X}^{\circ} 11 /$. Radiellus. Hub. 325.}

Treit. - Dup. 272, 1. - Zell. -- Guén. cat. -- IIer.-Schl. $\mathrm{n}^{\circ} 212$. - Heyden. cat. 62 (non Wood.).

Pas fort rare dans les hautes Alpes, en juillet et en août. - Gemmi, Tzermatteı, St-fiottlard (Zeller, Amstein, Rothb., Lah., Frey.).

Aucun des caractères donnés par les auteurs ne peut servir à distinguer cette espèce de fulgidellus, à l'exeption peut-être de sa taille un peu moins forte, de sa couleur plus bromzée et de ses antenues dentellées plus fortement en dessous.

Les autres caractìres varient extrêmement. La largeur, la forme, l'étendue et la terminaison de la raie moyenne sont très-variables. La tête et le front passent de la couleur olivepàle au blanc-sale. Le bord postérieur des antérieures est ordinairement droit, mais aussi parfois un peu arqué. La côte est blanche dans quelques exemplaires. La frange est tantît blanche mouchetée de gris, tantôt grise mouchetée de blanc. Il importe de soumettre fulgidellus et radiellus à un nouvel exameı. Les descriptions de Treitschke et de Dupouchel, ainsi que les figures de Hubner et Duponchel, sont insuffisantes.

L'habitat de fulgidellus est trop dillérent de celui de radiellus pour admettre 
une seule et même espèce. La première se trouve dans les bruyères arides des environs de Brunswick, tandis que la seconde hante les rochers humides des plus hautes Alpes.

\section{11.5. Tristellus. W. V.}

Zell. - Dup. cat. - Guén. cat. - Hejden. cat. 88. - Her.Schf. n²15. - Wood. 1517-1522.

Culmella. IV. V. - Fab. - IIub. 50, 404-(morum) Wood. 15:0. A'puilellu s. Dup. hist. - Treit. - (var.) Hub. 52. - Wood. 1518. Heyden. cat. 89.

Palecla. Hub. 51. - Wood. 1519. - Curt. - Stepl.

Petrificella. Llaw. - Wood. 1517.

Fuscelinellus. Steph. - Wood. 1521.

Nigristriellus. Steph. - Wood. 1522.

Ferrugella. Thunb. - Exoletella. Illig.

Pratell a. Brahm. - Pascuella. IJub. Beitr.

Très-commun dans toute la Suisse sur les prés et les champs humides et le long des haies, en août et en septembre. Préfère les montagnes. La forme 404, Inub., est la plus fréquente; nigristriellus, Steph. (Hub. 51), l'est moins, ainsi que IHub. 50. A quilella (Ilub. .̆2) n’est pas commune. Duponchel 272 figure $5^{\text {et b }}$ l'est davantage. S'élève sur les Alpes.

$\mathrm{N}^{\circ}$ 116. Deliellus. Hub. 402, 403.

Treit. - Dup. - Zell. - Guén. cat. - Iler.-Schf. n² 214 . Heyden. cat. 87.

Je cite cette espèce d'après MII. Zeller et Couleru; le premier l'a recueillie sur le Balgrist [Zurich]; le second l'a prise aux environs de la Neuveville. Je ne l'ai vue nulle part en Suisse.

$N^{\circ} 117$. Selasellus (la). Hub. 405, 406.

Treit. - Dup. - Wood. 1515. - Guén. cat. - Heyden. cat. 90.

Pratellus. Lin.? - Zell. - Her.-Schf. $n^{0} 216$. 
Furcatellus? Zetter.

Obtusellus. Steph. - Wood. 1516.

Pas très-rare; un peu partout, en août (Lah., Bremi, Rothb.). - Sourent coníondu alvec tristellus.

$\mathrm{N}^{\circ}$ 118. Luteelus. W. V.

Treit. - Curt. - Dup. - Zell. - Guén. cat. - IIer.-Schf.

$11^{\circ} 217$. - Ileyden. cat. 92.

Och rella. IIub. 5.5.

Exoletella. Hub. 48. (non W. V.).

Tristis. Wood. 1512.

Convolutella. W. V. - Hub. Verz.

Commum en Valais et daus le Tessin, jusquau pied des glaciers, en juillet. - Au pied du Jura (Rothb.), à la Neuveville (Couleru), Bellinzone (Lah.).

$\mathrm{N}^{\circ}$ 119. Perlellus. Scop.

Hub. 40. - W. V. - Dup. - Zell. - Guén. cat. -- Her.-

Schf. $n^{\circ}$ 2 18. - Heyd. cat. 94. - Wood. 1485-1489.

Lithargyrellus. Wood. 148.5 .

Argentana. W. V. - Argentella. Fab. - Wood. 1488.

D e alb ana. Thunb.

Var. Argyreus. Ilaw. - Wood. 1486. - Dup. hist. $274,2^{b}$. I Ieyden. cat. 95 .

Arbustea. Haw. - Arbustorum. Wood. 1487.

Extrêmement commun partout en juillet et en août; principalement sur les prairies sèches.

La variété (argyreus), rałée de gris sur les nervures, n’est pas rare.

$\mathrm{N}^{\circ}$ 120. Rostellus. Lah. fig. 6.

Cette espèce est extrêmement voisine de W a ring to n ell us Staint.; mais s'un distiugue an premier coup-d'œil par l'ahsence de tout dessin et de toute strie ou raie sur les ailes supérieures.') Elle n'est pas rare sur les hautes Alpes du IIant-Valais et de l'Oberland bernois, où je l'ai recueillie et où l'a prise aussi M. Ott, de Meyringeu.

1. Sur quelques individus on aperçoil. en reflels plus clairs, le dessin de Wiaringtonellus; mais re dessin est forme de stries plus larmes el moins effiles. Du reste, ces rellels ne se prolontent point sur Ia lrange, comme dans l'espece anglaise. 
Sa taille est légèrement an-dessous de colle de perlellas; la coupre des ailes est la mème. Les palpes sont très-alongés, un peu recourbés en dessous, de la rouleur des ailes supérieures. Celles-ci sont d'un brun-olivàtre, métallique, très-brillant et uni. La frange est d'une conleur plus pâle, séparée du fond par une rangée de points blancs peu visibles, disposés sur les nervures ef aui n’existent pas toujours. La còte est souvent maryuée par une légère strie hlanche longitudinale.

Les inférieures sont en dessus d'un gris un peu siolacé et brillant, sans traces blanchàtres comme dans W a ringt on ellus. Leur frange est d'un gris-roux, limitée par une raie plus foncée très-fine; tandis que dans l'espèce voisine elle est blanchàtre du côté de l'aile et grise sur son bord lilre.

Le dessous des quatre ailes est entièrement d'un gris-plombé luisant, arec la frange rousse bordée par une ligne plus elaire et très-fine. On n’y aperçoit pas trace de stries ou de points blanes, comme dans $\mathbf{W}$ aring $\mathrm{t}$ onellus.

La femelle ne diflère pas du mâle.

$\mathrm{N}^{\circ}$ 121. L y thargyrellus. Hub. 227.

Treit. - Dup. - Zell. - Guén. cat. - Her.-Scht. $1^{\circ} 219$. IIeyden. cat. 98.

Lotella. Wood. 1481.

Niniosella. Wood. 1482.

Cette espèce est indiquée en Suisse par Herrich-Selrælfer. Jusqu'ici je ne l'ai pas observée dans ce pays et d'autres collecteurs ne l'ont pas recueillie, que je sache. - M. Bruand l'a prise dans le Jura français.

\section{P h y c i d e n. 'Zell.}

XIV. Gen. Pe m p elia. Hub. Verz. - Zell.

$\mathrm{N}^{\circ}$ 122. Carnella. Lin.

W. V. - Hub. 66. o. Treit. - Dup. - Zell. - Wood. 1478. - Guén. eat. - Iler.-Schf. $\mathbf{n}^{0} \mathbf{2} 31$. - Hejden. $n^{0} \stackrel{290 .}{ }$

Var. S angu in ella. Hub. 65. .. - Wood. 1479. - Steph. - Heyden. cat. 291 .

S emirubella. Seop.

Extrèmement commune sur tous les pâturages secs, principalement sur ceux des montagnes et des Alpes; fin de juillet et août. 
$\mathrm{X}^{\circ}$ 123. Perfluella. Zinck.

Zell. Isis. -- Dup. cat. - Guén. cat. IJeyden. n³07. Her.-Schf. ॥ $^{\circ} 237$. - Hub. sup. f. 49. 3.

D) ibaph hiella. Iiub. 472.

Du biella. 1)up. 280, 2. - Guén. cat.

Formosa. Wood. 1468?

Herrich-Schaffer cite perl'luella, Zinck., parmi les espèces suisses. Si dubiella, 1)ıp., lui appartient réellement, cette espèce a aussi été trouvée en Suisse par Y. Couleru. Je n'ai vu ni l'une, ni l'autre.

No 121. Subornatella. Dup. hist. pl. 284, f. 5.

Zell. Isis, 1846. — Guéu. cat. - Her.-Schf. n²39. - Heyl. eat. 300 .

S erpy ll e toru m. Zell. Isis, 1839. -- Hub. sup. f. 62. - Heyd. cat. 30 I.

Recueillie dans le Jura par M. Rothenbach. Lindividu que j'ai vu appartenait is la variété serpylle torum. Rare.

$\mathrm{N}^{0}$ 125. Adornatella. Treit.

Zell. Isis, 1846. - Hub. sup. f. 77, 78. - Dup. hist. 284, 4 ?

- IIer.-Schf. n 240 . - Heyden. cat. 302.

Obscura. Steph. - Wood. 1471?

Inscrip tella. Dup. hist. ?

Trouvée dans les environs de Schüpfen par M. Rothenbach. Je l'ai prise une fois pris de Lausanue, et M. Heuser m'en a envoyé deux individus des environs de Burgdorf.

Fuénée réunit dilutella, llub. 69, à cette espèce; si in seriptella, Dup., lui appartient en effet, ce rapprochement est fondé; ear la figure de Duponchel convient tout-àfait à adornatella, quoique Ileydeureich en fasse un synonyme de dilutella.

$\mathrm{N}^{\circ}$ 126. Ornatella. W. V.

Treit. - Zell. Isis. - Dup. - Guén. eat. - Her.-Schıl.

$\mathrm{n}^{0} 241$. - Heyden. cat. 299. - Wood. 147 ? ?

Criptella. Hub. 77. - Curt.

Cinerella. Đup. 284, 9 ?

Assez fréruente sur les páturages des sous-alpes exposés an midi; Sepey, vallée dev 
Ormonds; l'Etivaz, etc.; en juillet (Lah.). - Environs de Schüpfen: sur le Jura (Rothb.) Près de Burgdorf, pas rare, en août (Meyer). - Dübendorf; environs de Zurich, sur les jachères (Bremi). - Engelberg (Zeil.).

La femelle est plus petite et d'un brun plus foncé que le mâle. J'ai ıu un indiridu mâle diun gris olive-pale.

$y^{\circ}$ 127. Obductella. Fisch. v. Rösl. t. 85.

Zell. Isis. - Dup. sup. - Guén. cat. - Her.Schf. n² 24:2. IIeyden. cat. 294 .

Dilutella. Dup. hist. t. 279, f. 3.

Origanella. Srhleg.

J'en ai pris un exemplaire dans les environs de Lausanne et I. Couleru m'en a communiqué un second qu'il a recueilli a la Neureville. M. Bruand la prend aussi dans le Jura frauçais.

N 128. Carbonariella. Fisch. v. Rösl. t. 60.

Zell. Isis. - Dup. hist. t. X, 1. 292. - Guéu. cat. - Ileyden. cat. 304 . - Her.-Schf. u $^{\circ} \mathbf{2 4 4}$.

Spa dic ella. Ilub. 226 .

J an th in ell a. 1)up. hist. t. X, p. 235. t. 281, l. .2.

Fusca. IIaw. - Wood. 1683.

Assez fréquente sur les hautes Alpes centrales parmi les bruyères; en juillet. Simplon, près de l'hospice; Mleyenwand (Lah.).

Cette espèce se prend aussi, mais rarement, à la fin de juillet et en août, sur les montagnes et les collines sèches des cantons de Vaud, Neuchâtel et Berne. M. Mejer l'a recueillie pries de Burgdorf, M. Rothenbach près de Thun; je l'ai prise sur le Jorat parmi les bruyères. Les individus provenant de ces dernières localités diffèrent constamment de ceux des Alpes par leur couleur noir-foncé, par la seconde ligne (à peine visible) moins sinueuse et par la taille plus petite. Je n’ai pas pu découvrir d'autre différence.

La femelle est sourent plus petite que le màle et plus rare.

Y 129. Palumbella. W. V.

Treit.? - Zell. Isis. - Dup. hist. pl. 281, f. $1^{\mathrm{a}, \mathrm{b}}\left(\right.$ non $\left.1^{\mathrm{c}}\right)$. Guén. cat. - IIeyden. cat. 31 . - Her.-Schf. n²45. 
A Ib a riella. Hub. sup. f. 37 (non f. 36 , error.).

Var.? Contubernella. Hub. 72.

Cette espèce, rare en Suisse, a été prise dans les environs de Berne par M. Rothenbach et sur le Jura par M. Couleru. Dans l'Engadine, près de Samaden, 7000', fin de juillet (Frey).

XV. Gen. Ne phople r y x. Zell. - Hub. Verz.

$\mathrm{N}^{\circ}$ 130. Abietella. IV. V.

Treit. - Dup. - Zell. - Ratzeb. - Wood. 1472.

Guén. cat. - Her.-Schf. $n^{\circ} 2.54$. - ileyden. cat. 272.

Decuriella. llub. 74.

Sylvestrella. Ratz. et Schenk.

Var. Sple udide Il a. Maun. - Ilub. sup. f. 43.

Point rare sur les diverses espèces de pins, dont la chenille ronge les pires. Le Jorat, surtout dans les bois de pin sylvestre, au commencement de juillet. Le papillon est difficile à saisir là où les pins sont élevés, paree qu'il vole d'un arbre à l'autre (Lah). Près de Schüpfen (Rothh.) et de la Neuveville (Couleru). L’Ũto, près Zurich (Bremi).

$N^{\circ}$ 1:31. Roborella W. I.

Treit. - Dup. - Zell. Isis, 1846. - Wood. $\mathbf{1 6 8 0 .}$

Guén. cat. - Her.-Schf. n" 256. - Heyden. cat. 274.

5 Spissicella. Huls. 75. Spissicornis. Fab.

Leg a tell a. Haw. - Wood. 1682.

Cristella. Haw. - Wood. 1681.

Fréquente au pied du Jura dans les bois de ehène (Bruand, cat.. La Neuveville Couleru. Plus rare dans le reste de la Suisse; Burgdorf (Meyer); environs de Lausanne et de Schüpfen (Lah., Rothb.). Sur le Balgrist, près Zurich (Zell.).

$\mathrm{N}^{0}$ 132. Rhenella. Zinck.

Treit. - Zell. Isis, I846. - Fisch. v. Röst. 1. 29. - Hub.

P al u m bella. Ilub. 70.

sup. l'. 130. - Her.-Sehf. $\mathbf{n}^{0}$ 2.58. - - Ileyden. eat. 277.

Host ilis. Wood. 146r? (Her.-Schf.)

Je l'ai reçue de M. Couleru qui l'arait reeueillie aux environs de la Neureville (Lah).

H. Rothenbach l'a prise une fois près de Sehüpfen. 
Il m'est impossible de reconnaître l'espèce figurée par Fisch. 1. Rösl. dans la figure 200, 1 de Duponchel. Je ne puis done affirmer que l'espèce désignée par Guénée soit bien celle des auteurs allemands. J'ai tout lieu de croire que rhenella de Dup. (fig. $1^{\text {a }}$ ) est une autre espèce voisine d'e pely della (woir ter ebrella).

$\mathrm{N}^{\circ}$ 1333. Janthinella. Hub. 374, 375 (err. 274, 275).

Zinck. - Fisch. r. Rösl. t. 28. - Zetler. - Dup. t. 283, r. 8. - Frey. ält., Beitr. 108. - Hub. sup. f. 131 (var.) Guén. cat. - Her.-Schf. $n^{0} \geq 61$. - Heyden. cat. $28 \geq$.

Cette espèce a été prise dans quelques parties de la Suisse; mais toujours rarement et isolément. Dübendorf (Bremi). Schüpfen (Rothb.). Un exemplaire dans ma collection, venant de Burgdorf (Lah.).

No 134. Argyrella. W. I.

Hub. 64 , - Treit. - Dup. - Zell. - Guén. cat. - Iler.Sclif. $\mathrm{n}^{\circ} \geq 64 .-$ Heyden. cat. 283.

Argyreus (Cramb.). Fab.

Au Schanbühl près Burgdorf; fréquente en aoùt (Mejer). - Prés de Bienne (Rothb.); de la Neuverille Couleru): de Bellinzone, fin juillet (Lah.).

XVI. Gen. Hypo chalci a. Hub. Verz. Zell.

(Diosia.)

Yo 13.5. A uriciliella. Hub. $\mathbf{3 4 0 .}$

Zinch. - Treit. - Dup. - IIer.-Schf. 1" 268. - Heyden. cat. 255.

Var. Margin ell a e. Guén. cat.

Assez fréquente sur les pàturages des Alpes, vers le milieu de juillet (Rotbh., Lah.).

Marginella n’a pas été trourée en Snisse jusqu’ici. A part l’absence de jaunc sur li frange des supérieures, marginella ne peut se distinguer d'auriciliella. La teinte des inféricures est plus ou moins fuliginense dans l'une et dans l'autre sur les nombreux indiridus que j’ai observé. La coloration jaune de la frange des supérieures ne me paraît pas un caractère sùr; car la frange d'au r icili ell a rarie passablement. Le filet noir qui la limite en dedans peut manquer en tout ou en partie, tant aux supérienres qu'aux inférieures. Aux supérieures on voit parfois le noir gagner la bordure rers le sommet de l'aile, et même ça 
it lì dans son milieu. Le jaune empiète plus ou moins sur la marge de l'aile aux inlérieures e'n dessous, là où sur d'autres individus se voit un trait noir. Le violet métallique du corselet "I de la tête, se répand aussi plus ou moins sur la base des supérieures, sur les pattes et sur les palpes:

\section{(Oncocera. Stepl.. - Guén. - Plyycis. IIer.-Schli.).}

$x^{\text {ro }}$ 1:36. Dignella. Hub. $: 35$ (err. lignella).

Treit. - Zell. - Guén. rat. - Her.-Schl. $\mathbf{n}^{\circ} \mathbf{2} 70$. - Heyd. eat. $\mathrm{n}^{\circ} 250^{\mathrm{a}}$.

D i g nellus (Cramb.). Zinek, - Dup. cat.

M. Rothenbach indique eette espèce près de Bienne. Lindividu que j’ai su répond is la figure de Hubner.

$N^{\circ}$ 1:37. Germarella. Zinck.

Treit. - Zell. - Guén. eat. - IIuh. sup. f. 198. - IJer.Schf. $1^{\circ} 271$. - IIeyden. cat. 252.

Il el a nell a. Dus. sup. t. 60 (non Treit.).

Indiqué sur l'Irrbel, près Dättlikon [Zurich], par M. Bremi. L̈individu trouvé me paraît fort douteux (Lah.).

$\mathrm{N}^{\circ}$ 138. Ilicnella. W. Y.

Hub. 41, 58. - Treit. - Zell. - Dup. - Guén. cat. IIcr.-Schf. $n^{\circ}$ 273. - IJeyden. cat. 239. - Wood. I483.

Tristis, obscuratus et tetrix. Ilaw.

Var. B istrig ell a. Jup. hist. 277, f. 8. - Heyden. eat. 240.

"Fuliginella. Dup. hist. 27 7, f. 3. - Ileyden. cat. 256.

Cette espèce, partout assez rare, a été trouvée sur dixers points de la Suisse. Sur l'Irrhel, près Zurich (Bremi). - Près de Burgdorf (Meyer). - Dans les Grisons, près de Malan (Amstein). - Vallée d'Engelberg (Zell.) - Aux environs de la Newreville (Couleru). Je l'ai prise dans les environs de Lausanne et an-dessus d'Yrorme en juin (Lah.).

Bistrigella Dup. mérite à peine le nom de variété; c'est la forme la noins rare dans les lieux seess et ehauds. Les individus loncés qui ont volé quelque temps, prennent celle de fuliginella Dup. 
$\mathrm{N}^{\circ}$ 139. MeIanella. Treit. p. 146.

Zell. - Hub. sup. 72 , 73. - Viuén. cat. - Her.-Schi. $11^{\prime 2} 74$.

- Ileyden. cat. 238.

Giermarella. Dup. sup. 60.

Ocelle a. Wood. 1480.

Prise près de Thun par $\mathbf{M}$. Rothenbach.

$\mathrm{X}^{\circ}$ 140. Lignella. Hub. 57 (non 350 ).

Treit. - Zell. - Dup. - Hub. sup. C. 176. .. Guéll. rat.

Her.-Schf. $n^{\circ}: 275 .-$ Heyden. cat. $2.50^{\prime \prime}$.

Cette espèce, très-rare, est indiquée sur l'Irchel-Berg, près Zurich, par M. Bremi.

I. Bruand (catal.) la range aussi parmi les espèces du Doubs. - II. Dujonchel l'arait reçue de la Suisse. Tout ceci est assez douteux.

XVII. Gen. Z o p ho d i a. Hub. Verz.

(M e ga asis, Guén.).

I 14. Ripertella. Bdv.

Zell. - Hub. sup. f. 65, 66. - Guén. cat. - Iter.-Srht. $\mathbf{n}^{\circ} \mathbf{2 8 7}$. - Heyden. cat. $2 \mathbf{2} 8$.

Prodromella. Dup. hist. 27i, f. 1.

I. Anderegg élève cette espèce à Gamsen, dans le Haut-Valais, oì il l'a prise.

$\mathrm{N}^{\circ}$ 142. Compositella. Treit.

Dup. bist. 28:2, f. 2. - Hub. sup. f. 128. - Guén. cat.

Iler.-Schf. $\mathbf{3}^{\circ}$ 294. - Ieyden, cat. 217.

Jai pris un indiridu de cette espèce dans une maison à Lausanne, en juin. II. Bothenbach l'a priss près de Bienne, et M. Bremi à Dübendorf.

XVIII. Gen. I s a r t a. Zell.

(Chionea, Guén.)

Y 143. Alpicolella. Fisch. v. Rösl.

Zell. - Ilub. sup. f. 50, 51 . - Dup. cat. - Giném. cat.

Her.-Schf. $n^{\circ} \geq \mathbf{2 9 5}$. - Heyden. cat. $\mathbf{2} 34$.

II. Fischer de Röslerstamm indique cette espèce en Suisse, sur le Simplon; il l'arait sans doute reçue de II. Anderegg. - Je ne l'ai pas encore vue en nature. 
N" 144. A ethiopella. Dup. hist. 284, l. 3.

Guén. eat. - Ier.-Schf. $\mathbf{n}^{0} \mathbf{2 9 6}$. - IIerden. cat. ․ㅡㄹ.

Helve ticella. Zell. - Fisch. v. Rösl. - Ilub. sup. f. 5.5-5i.

Dup. cat.

Var. Honspessulal is (Ennych.). Dup. hist. - (Lella) Hub. sup. f. 52 . 54.

II isp a n ell a. Guén. (Her.-Sehf.)

J'ai pris cette espèce sur le col du Gotthard a la fin de juillet, volant assez nombreuse sur les pentes sèches exposées au soleil, le long de la route. Elle ne parait pas rare sur les hautes Alpes. M. Couleru l'a prise sur la Furca (Dup.).

XIX. Gen. A n c y losis. Zell.

$\mathrm{N}^{\text {ro } 145 . \quad C i n n a m o m e l l a . ~ D u p . ~ h i s t . ~ 279, ~} 4$.

Her.-Schf. $n^{\circ} 298$ (texte). - Heyden. cat. 26 6.

D il u tell a. Treit. - W. V. - Zell. - Hub. sup. f. 58 - 61. - Heyden. cat. 263 .

Trapezella. Dup.?

Dans les environs de la Neuverille (Couleru) et de Burgdorf (Ileuser). Assez rare.

Faut-il distinguer, arec Heydenreich, cinna no mella, Dup., de dil utella, Ilub. (i) ? - je ne le pense pas. Ilerrich-Schieffer réunit arec doute ces deux espèces. Des individus que j'ai regu de France sous le nom de cinnamomella, Dup., justifieraient rette réunion.

XX. Gen. Trachonitis. Zell.

No 146. Cristella. Hub. 76.

Treit. - Zell. - Guén. cat. - Ier.-Schl. n 300. - Hul. Sup. f. 206. - IIeyden. cat. 18:2. (non christell a, Frey.

Près de Malans, dans les Grisons (Amstein).

XXI. Gen. M y e I o i s. Zell.

Y 117. Rosella. Scopoli.

Zell. - Guén. cat. - IIer.-Schl. n' 302. - Ileyden. cat. 189.

Pudorella. IIub. 63, 318. - (alis.) W. V. - Treit. - Dup. 276,4.

Fort rare en Suisse. Je l'ai trourée une fois près de Lausanne, au Denantou, en juillet, sur une prairie lıumide. Près de Dübendorf, une fois (Bremi). 
N ${ }^{\circ}$ 148. Luridatella. Fisch. v. Rösl.

Iler.-Schf. n³ 306. - Ilub. sup. f. 22, 223. - II eyden. cat. 198.

Luridell a ? Schläg. - Guén. cat. - Ileyden. cat. 241.

Rufella. Dup. 277 , f. 6.

Tristrigella. Wood. 1473.

Advenella? Dup. 278, l. 2.

Impurella? Dup. 277 , f. 7.

Prise en abondance près de Sierre et de Raron, dans le Haut-Valais, par M. Meyer; 'n août.

$\mathrm{V}^{\circ}$ 149. Legatella. Hub. 71.

Treit. - Zell. - Guén. cat. - Hub. sup. f. 46 . - Her.-Selıf. $\mathbf{n}^{\circ} 307$. I Ieyden. cat. 202 . - (alis.) Ilub. Verz. nen W. V. - neque Dup. t. 284, f. 2 ).

Squalidella. Eversm.

M. Rothenbach l'a recueillie près de la Neweville. M. Couleru m'en a adressé un exemplaire de la Neuverille.

$\mathrm{N}^{\circ}$ 150. Advenella. Zinck.

Treit. - Germ. M. IX, 2I. - Zell. - Gún. cal. - Her.Schf. $\mathrm{n}^{0}$ 308. - Huh. sup. f. 201. - Heyden. rat. 20.5. - Wood. 1461.

Cette espèce rare a été recueillie près d'Aarberg, par M. Rothenbach.

$\mathrm{N}^{0}$ 15̄l. Suavella. Zinck.

Treit. - Zell. - Dup. sup. t. 60. - Guén. cat. -- Her.Schf. $\mathbf{n}^{0}$ 309. - Ilub. sup. f. 202.

Lega tella. Dup. hist. t. 284 , f. 2.

J'ai pris cette espèce me fois aux environs de Lansanne, dans une haie d'aubépine, le $\mathrm{I}^{\mathrm{er}}$ août. IIY. Rothenbach et Couleru l'ont aussi recueillie, le premier prìs d'Aarberg, le second à la Neurerille. — Zurieh (Frey.).

$\mathrm{N}^{\text {o } 152 .}$ Terebrella. Zinch. Germ. Mag.

Treit. - Zell. - Ilub. sup. f. 199. - Ileyden. cat. 200. IIer.-Schf. $n^{\circ} 312$.

R h e nella? Dup. hist. 280, $\mathbf{1}^{\text {a }}$.

Point rare dans les environs de Schüpfen où M. Rotlsenbach l'a recueillie. Je l'ai prise près de Lausanne, et Ml. Heuser près de Burgdorf. Chenille sur les pins. 
XXII. Gen. A c robasis. Zell. Isis, 1848.

$\mathrm{N}^{\circ}$ 153. Tumidella. Zinck.

(Alis) W. V. - Treit. - Dup. 280, f. $3^{\mathbf{a}}\left(\right.$ non $33^{\text {b) }}$. Z Zell.

- (ana) Wood. 1459. - Inb. sup. f. 45. - Iler.-Schl. $n^{0} 316$.

Ad renella. Steph.

Verrucella. Ilub. 73. - Ilaw. (non W. V.).

Dans les euvirons de Bàle (Imhoff), et de la Neuveville (Couleru).

$\mathrm{N}^{\circ}$ 154. Rubrotibiella. Mann.

Fisch. v. Rösl. t. 60. - Dup. sup. -- Guéı. cat. - Her.-Schll. $n^{\circ} 317 .-H e y d e n$, cat. 181.

Tumidan a. WV. V.

Un exemplaire, reçu de II. Couleru, confondu arec tumidella, élevé à la Neuveville (Lah.).

$\mathrm{N}^{\circ}$ 155. Consociella. Hub. 328.

Treit. - Zell. Isis, 1848. - Dup. t. 282, f. 4. - Hub. sup. f. 200. - Guén. cat. - Her.-Schf. n 318. - Heyden. cat. 178. - Wood. 1464.

T u midella. Dup. hist. $280,3^{\mathrm{b}}$.

F a scia. Haw. - Wood. 1460.

Fréquente dans les bois de chène, où la chenille vit en lamille. Hottingerwald, près Zurich (Bremi). La Neureville (Couleru). Les bords du lar de Bienne Rothl.). Lausaune (Lah.).

XXIII. Gen. Ny c tegretis. Zell.

$N^{*}$ 156. A chatinella. Hub. 451.

Zell. - Iler.-Schl'. n"327. -- Ileyden, eat. 187. - Dup. hist. 280,6 .

Près de Wan (Jura bernois), dans les lieux rocailleux, exposés au soleil (Rothb.), le 22 juillet 1852. La figure de Duponchel est trop chargée de couleur. 
XXIV. Gen. Hom a e o so ma. Curt.

No 157. Cribrella. Hub. 67..

Treit. - Dup. 285. f. 1. - Guén. cat. - IIeyden. cat. 192.

Cribrum (noctua.). W. V. Zell. -- Her-Schf, n³3:.

Cribriformis. Esp.

Cribrumell a. Hub. Beitr.

Cardui. Stph. - Wood. 1477.

M e dul a is. IHub. Verz.

In Platz, environs de Zurich, rare Vögeli, Bremi). Les prairies près de Bienute (Rothb.). La Neureville, au pied du Jura Couleru). Ensirons de Burgdorf, le 18 juin (Mey.). Les Grisons, Malans (Anstein).

\section{$\mathrm{X}^{n}$ 1.58. Flaviciliella. Mann cat.}

Heyden. cat. 19:3.

Découverte dans les environs de Burgdorf, par M. IIeuser. Ressemble à e ribrella arec le fond grisâtre et la frange jaune.

$N^{\circ}$ 159. Ceratoniella. Schmidt.

Fisch. v. Rösl. t. 56, 57. - Guén. cat. - Iler.-Schf. $n^{\circ} 335$.

- Ileyd'n. cat. 212.

Ceratonia e. Zell.

Cette espìce, dont la ehenille vit daus le fruit du Ceratonia siliqua, a été élerée a Zurich par M. Bremi; il la trousait dans les magasins des pharmaciens.

No 160. Oblusella. Hub. 215.

Fisch. 1. Rösl. t. 57. - Dup. t. 280, f. 4. - Zell. - Guén. eat. - Her.-Schf, n ${ }^{\circ} 336$. - Hedyen. cat. 1 i2 (non Treit. .

Les bois autour de Zurich; Dübendorf (Bremi). Environs de Bàle (Imhoff).

$\mathrm{N}^{\circ}$ 161. B inaevella. Hub. 38:3.

Treit. - Dup. 279, 6. - Eversm. - Guén. cat. - Her.Schf. no 342. - Hub. sup. f. 80. - Heyden. eat. 166. Wood. 1452 .

Var.? Petrella. Hub. sup. f. 81.

Nebulella. IIaw.

11. Bremi possède, dans sa collection, un individu, pris dans les environs de Zurich, qui 
se rapporte ì la figure 38.3 de Ihubuer, et II. Rothenbach a recueilli la variété petrella Her.-Schf.; l'une differe sensiblement de l'autre.

No 162. Convolutella. Hub. 34.

Zell. - Guén, cat. - Heyden. cat, 220. - Her.-Sshl. ॥" 345.

Grossulariella. Zinck. - Treit. Dup. Hub. Verz.

Prise au pied du Jura par M. Couleru; elle y est rare.

No 16:3. Boisduvaliella. Guén. cal. p. 81 .

Boisduval l'a rescue de la Suisse (sans doute d'Anderegg), qui l'avait recueillie en Valais Guénée).

$\mathrm{N}^{\circ}$ 161. Sinuella. Fab.

Zell. - Guén. cat. - Ileyden. cat. 16 i.

Elongella. Treit. - Dup. - Hub. sup. f. 124.

Ge m in a. Haw. - Curt. - Wood. 1475.

Var. Flavella. Dup. 284 , f. 6.

Cette espèce n'est pas rare dans les lieux secs et chauds de l'Europe centrale; I'réquente en Valais, en juillet, le long des vignes Lah.. .

XXY. Gen. E phesli a. Guén.

$\mathrm{X}^{\circ}$ 16.5. Elutella. Hub. 163.

Treit. - Dup. - Zell. - Guén. eat. - Her.-Schli. n 3ă8.

E I u t e a. Haw.

- Heyden. cat. 154. - Wood. 1454.

Rufa. Steph. - Wood. 14.55.

A blut alis. Ilub. Verz.

Cette espèce est commune dans tous les magasius de droguerie et d'épicerie. La larve attaque surtout les fruits secs. Le prapillon naît principalement en juillet et en aoìt.

XXVI. Gen. A ch roe a. Zell.

$\mathrm{N}^{\circ}$ 166. Alvearia. Fab. sup.

Stph. - Dup. sup. 60, 10. - Hub. sup. f. I 49. 1.50.

Wood. 1433. - Iler.-Sehf. n ${ }^{\circ} 360$.

Giris ell a. Fab. - Heyden. eat. 144. 
Cinereola (Bomb.). Hub. 91?

A lveariella. Guén. cat.

A lve a. Haw.

Cette espèce commune dans le Doubs (Bruand cat.), a été trouvée au pied du Jura par I. Conleru, de la Nenreville.

XXVII. Gen. Melis soblaptes. Zell.

(Melia. Curt.)

N 167. Inellus. IV. V.

Fab. - Treit. - Dup. - Zell. - IInb. sup. I. 151 .

Guén. cat. - Her.-Schf. n³62.-Hesden. cat. 142.

Wood. 1437.

Sociclla. Hub. 24.

U m brat e ll a. Treit. - Guén. cat.

Cetfe espèce est indiquée près de la Neuverille par M. Couleru; et à Coire par M. Bremi.

XXVIII. Gen. A pho ni a. Hub. Verz. - Zell.

$\mathrm{N}^{\circ}$ 168. Colonella. Lin..

W. V. - Hub. 23. - Treit. - Dup. - Her.-Sehf. $n^{\circ} 364$.

- Ileyden. cat. 139. - Wood. 143.5. - Fab.

Sociella. Lin. 5. - Fab. - Zell. - Guén. cat.

Tribunella. W. V. 5.- Hub. 22.

Assez fréquente, tont l'été, dans les maisons, près des ruches d'abeilles et dans les haies. Chenille dans les nids de bourdon.

XXIX. Gen. G a I e r i a. Zell.

$\mathrm{X}^{\circ}$ 169. M ellonella. Lin.

IV. V. - Fab. - Zell. - Heyden. cat. I38. - Her.-Schf. $1^{\circ} 365 .-$ Haw.

Cerella. Zinck. - Treit. - Ilub. 25. - Fab. - Dup. - Guén. cat. Curt. - Jlaw.

Cere a na. Lin. - Wood. 1434.

Plus rare que la précédente; les rochers et les maisons de campagne. - Les ruches d'abeilles au nord de l'Albis; surtout fréquente autour de Winterthour (Bremi).

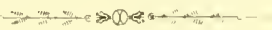




\section{TABLE IES MATIÈRES.}

\section{Table des genres, des espèces et des synonymes.}

derealis. Cosla .

Iglossi. Latr.

Angustalis. IV. I

Isopit. Treil.

Brumnealis. W. Y

Costalis. Fab.

Cuprealis. Hub.

Curlalis. II. I.

Erigalis. Fab.

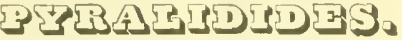

$\begin{array}{cc}N^{\circ} & \text { Page. } \\ 2 & 7 \\ - & 7 \\ 7 & 8 \\ - & 7 \\ 6 & 8 \\ 4 & 8 \\ 2 & 7 \\ 7 & 8 \\ 7 & 8\end{array}$

Farimalis. Lin.

Fimbrialis. W. V.

Glaucinalis. Ilub).

Glaucinalis. Lin.

Nitidalis. F.

pinguinalis. Lin.

Pinguis. F.

Pyrals. lin.
Page.

7

8

8

8

8

7

7

8

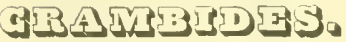

Abietella. IV. I.

Iblululis. Hub. Verz.

Abruplella. Thunb.

Achalinella. Hub.

A cirroka. Zell. .

I cuobasis. Zell.

Adamantellus. Guén.

Idornalella. Treil.

Advenella. Zinch.

Adverella. Dup.
A.

$\begin{array}{cr}N^{u} & \text { Page. } \\ 130 & 59 \\ 165 & 67 \\ 100 & 47 \\ 156 & 65 \\ - & 67 \\ - & 65 \\ 113 & 53 \\ 125 & 57 \\ 150 & 61 \\ 118 & 64\end{array}$

Advenella. Siph.

Aenealis. W. $\mathrm{I}$.

Aerealis. Hub.

Aethralis. Dup.

A elhiopella. Dup.

Agroteri. Sclir.

Ahenella. W. Y.

Albariella. Hub. sıp'

Alpesiralis. F. .

Alpicolella. F. I. R

Alpinalis. W. 1 .

Alpinalis, H.-S.
$\mathbf{N}^{a}$ Page.

15365

$28 \quad 18$

$34 \quad 27$

9) 11

14363

$-11$

$138 \quad 61$

$129 \quad 59$

$16 \quad 14$

11362

$49 \quad 25$

$.50 \quad 26$ 
Alpinalis, Dup.

Ilpinellus. Ilul.

llvearia. F.

Aleeu. Llaw.

Alceariella. liuén.

Ambigualis. IIı).

Ambigualis. Duj.

Ambiguella. Treit.

Iucipilella. Lah.

Axcruosis. Zell.

indereggialis. led.

Anellus. W, V.

Inguinalis. Hub.

Insulatellus. Ju!r.

Angustalis. Woud.

Angustellus. Wool.

Aphoxis. Ilub. Verz.

Aquilellus. Ilup., Treit. Mub.

Arbustea (orum). Haw.

Argentana. W. I'.

Argentella. Fab.

Irgyrella. W. V.

Argyreus. F.

irgyreus. lbup.

Asphodeliella. Mam

Irralis. Moh.

At'ulis. F.

Iuriciliella. Ilub.

Auriferclla. Ilul.
Arcualis. Ilul.

Argentalis. Fab.

Isarti. Zell.

$\mathrm{N}^{\circ}$ Page.

$53 \quad 27$

9546

$166 \quad 67$

166.68

16068

$76 \quad 36$

$83 \quad 39$

$71 \quad 31$

$86 \quad 41$

$-$

IB.

Barbella. Hul.

Bigutta. F.

Binaevella. Hub.

Bistrigella. llup.

Boisduvaliella. diuéu.

Borealis. Lefeb.

Botys. Lalr.

\section{('.}

Caespitalis. F.

Caespitella. Hul.
Campella. Ilub. . . 10

Carbonariella. F, i. li.

Caruealis. Tr.

Carnella. Jin.

Citicersta. Hub.

Caudellus, Wood.

Centuriella. Wr. Y.

Ceratoniae. Zell.

Ceratoniella. Schmidt.

Cercama. Lin.

Cerclla. Treil.

Cernssellus. W. Y. . . 9696

Cinlo. Zinck. . . . - .

Cinosea. Gitén. . . . . . 69

Chryscllus. Treil. . . . y'

Chrysonuchellus. Scop. . $\quad 102$ is

Chrysonuehelle. Hub. 1033 is

Chrysomuchella. W. I. . . . 93 4.5

Cinctalis. Treit. . . . (6) 29

Cincrulis. F. . . . 350

fineralis. Ilub. . . . . . 3t 20

Cinerella. 1)up. . . . . . 126

Cinereola. Ilul. . . . . 166 68

Cingulalis. Lin. . . . 21 215

Cinnamomella. 1)up. . . . 19.5 63

Cilralis. Porla. . . . . $38 \quad 2$

Clathralis. Ilub. . . . dil on

Crecinalis. Hub. . . . . 2' 16

Colonella. Liı. . . . . 168 68

Combinellus. 1)op. . . . . 10' 48

Comparalis. Hub. . . . ․ 10

Compositella. Treit. . . . 192 62

Conchellus, W. V. . . . 10! 51

Conchellus. Treit. . . . 1113 .51

Conchtlus. Dup. $\quad 111-51$

Comsaciella. Ilub. . . . 15.5 6.5

Consorta. Ilaw. . . . . 878

Contubernella. Hub. . . , 129 59

Convolutella. W. V. . . . 118 55

Comvolutella. Hub. . . . $162 \quad 07$

Corsicalis. lup. _ _ \& 11

Coulonellus. Dup. 10i is

Crinaus. Fab. - $\quad 12$

Crataegella. IIub. . . . . 84 40

Cratuegella. Stph. . . . 7i 36

$103 \quad$ is 
Cribrella. Ilub.

$N^{\circ}$ Page.

ribriformis. Esp.

Cribrum. W. V.

Cribrumella. IIul.

Criptella. IIub.

Cristella. II ub.

Cristella. llaw.

Crorealis. llub.

Culmella. W. V., Fab., IIub. . . 11.j

Culmella. Mıs, Schf. . . . 102

Culmellus. Lin.

Cuneelus. Treil. sup.

Cyanalis. Lah.

Crvaeda. Hul.

D.

Dealbana. Thunb.

lecrepitalis. F. v. R.

Jeliellus. Ilub. .

Dehunella. Guén.

Decuriella. Hub.

Dentalis. W. V.

Dibaphiella. IJub.

Jiguella. Ilub.

Dilutella. Dup.

Dilulella. Treil.

Dieersalis. I Iul).

Dubiellu. Hup.

Dubitalis. Hub.

Hubitella. Zell.

Jumelellus. Hub.

E.

Elongella. Treil.

Elutalis. Ilub. .

Eluter. Ifaw.

Elutella. Ilub.

Exwotrucul. Zell.

Ericellus. Wood.

Erosulis. Fab.

Erucalis. Hub.

Euborea. Cut'.

Exoletella. Illig.

Evoletella. Hub.

$\begin{array}{rr}119 & 55 \\ 68 & 33 \\ 116 & 57 \\ 83 & 39 \\ 130 & 59 \\ 10 & 12 \\ 123 & 57 \\ 136 & 61 \\ 127 & 58 \\ 145 & 63 \\ 51 & 26 \\ 123 & 57 \\ 79 & 37 \\ 85 & 11 \\ 89 & 13\end{array}$

165

16.)

-

90

6

jij

-

115

118
1 ror

Formosa. Wood. . . . . $123 \quad 57$

Frequentella. Stain. . . . . 8440

Fulgidellus. Hub. . . . . $113 \quad 53$

Fuliginella. Dup. . . . . 13861

Fulminams. Fab. . . . 1012

Furcatellus. Zet1. . . . . $117 \quad 55$

Fusca. Haw. . . . . 12858

Fuscalis. W. V. . . . . 35

Fuscalis. Hub. . . . . . . $\quad 56 \quad 28$

Fuscelincllus. Steph. . . . $115 \quad 54$

(i.)

Gilleria. Zell. . . . $\quad-68$

Gemina. Haw. . $164 \quad 67$

Geniculeus. Haw. . . . 98 fi

Ciermarella. Zinch. . . . . $\quad 13761$

Germarella. Dup. _ . . $\quad 139 \quad 62$

Glabralis. F. . . . 4324

Glabralis. Hub. . . . . 3521

Glabralis. Wood. . . . 1725

Granella. F. . 10248

Grisella. F. . . . . . $166 \quad 67$

Grossulariella. Zinch. . . . $162 \quad 67$

Guttalis. W. V. . . . 2216

II.

Haematalis. Hub. . . . . $27 \quad 27$

Helveticalis. Andg. . . . $18 \quad 18$

IIelreticella. Zell. . . . . 144 63 
llencya. Treit.

IIspanella. Guén.

Holosericealis. Hul.

Honteosona. Curt.

Horluellus. Hub.

Hostilis. Wood.

IIumeralis. Zell.

Ilyalinalis. Scbr.

Hybridalis. IJub.

IYpochalcit. IIub.
Lucliferellus. Ilub.

Luridatella, F. v. R.

Luridella. Schlg.

Lutealis. Dup.

Lutectis. Hub.

Lutcelus. Wood.

Luteelus. W. $\mathrm{I}$.

Lyllargyrellus. Ilub., Treit.

I.

Monticolalis. Khlw.

Leucophoealis. Jub.

Lignella. Hub.

Limbalis. Hub. .

Linetella. F.

Lileralis. W. Y.

Lithargyrellus. Wood.

Lolusella. Giuén.

Longipedalis. Wood.

Lotella. Wood.

Iucellus. Iler.-Schl.

14964

$131-59$

15164

$65 \quad 30$

$140 \quad 62$

61) 29

$103 \quad 48$

$31 \quad 19$

$82 \quad 39$

911

$121 \quad 56$

$92 \quad 44$
119
Manifestel\}a. F. v. K. $7: 3$

Manualis. Hub. .

Margaritalis. W. W.

Margaritella. WV. I.

Margaritellus. Hub.

Marginella. Guén.

Marginellus. Wool.

Melanella. Treit.

Mclanclle. D)up.

Nelissoblaptes. Zell

Mellonella. Jin.

Nercurella. Lin.

Mercurella. Dup.

Mereurii. $\mathrm{F}$.

Miniosella. Wood

Moestalis. J)up.

Monspessulalis. Duj.

Montana. Wood.

Montanellus. Wood.

Nuranella. Stjl.

Murinalis. F. V. R.

MYELOIS. Zell.

Jyellus. IJub.

Myellus. Zinck. .

Nytilellus. Ilub.

N.

Nebulella. Haw.

Nemausalis. Dup.

Nemoralis. Seop).

Nemorellus. Ilub.

Nephopteryx. Zell.

Nigralis. F. t61 66

$96 \quad 36$

6 11

$90 \quad 13$

- je

$19 \quad 15$ 
Nigralis. Schrk.

Nigristricllus, SIph.

Noctuella. W. V.

Novellus. Guén.

Nubilalis. Hub.

Numeralis. Ilub.

Nictegretis. Zell.

Nymphaealis. Lin.

Nymphacalis. W. V.

\section{0.}

Obduclella. F. v. R.

Oblitalis. Irup. .

Obscura. Siph. .

Obscuralus. Haw.

Obtusella. Ilub. .

Dbtusellus. Stply.

Ocellea. Wood. .

Ochealis. Wood.

Oehrella. Hub.

Octomaculalis. 'Tr.

Octonella. Zell.

Oertziella. Mess.

Olivalis. W: V.

Ovocers. Stph.

Opacalis. I)up., Hub.

Origanella. Schlg.

Ornalella. W. $\mathrm{V}$.

Ostrinalis, Hub.

P.

palealis. W. I.

Paleela. Hub., Curt.

Pallida. Curı.

Pallidalis. Hub.

Paludata. F.

Palumbella. W. V.

Palumbella. Ilub.

Pandalis. Ilub. .

Parella. Her,-Schf.

parella. Her.-Schf.

Parella. Zell.

Parietarialis. Mann

Pascualis. Lien.

Pascuella. Ilub. Heitr.

$\begin{array}{rr}N^{\circ} & \text { Page. } \\ 28 & 18 \\ 115 & 54 \\ 69 & 33 \\ 104 & 48 \\ 47 & 25 \\ 67 & 30 \\ - & 65 \\ 5 & 10 \\ 4 & 10\end{array}$

$\begin{array}{rr}127 & 58 \\ 11 & 23 \\ 125 & 57 \\ 138 & 61 \\ 160 & 66 \\ 117 & 55 \\ 139 & 62 \\ 46 & 25 \\ 118 & 55 \\ 22 & 16 \\ 81 & 39 \\ 82 & 39 \\ 68 & 32 \\ - & 61 \\ 57 & 27 \\ 127 & 58 \\ 126 & 57 \\ 25 & 17\end{array}$

Pascuellus. Lin.

Pascuum. F.

Pauperellus. 1)up.

Pedrialellus. Dup.

$-\quad 56$

Perlluella. Zinck. . . . $123 \quad 57$

Perlellus. Scop. . . . . 11955

Perplexella. F. v. k. . . . $72 \quad 34$

Petrella. Her,-Schf. . . . . 16166

Petrificella. Haw. . . . . 115 54

$\begin{array}{llll}\text { Phrygialis. Hub. . . . . } 14 & 13\end{array}$

Puycuex. Zell. . . . . . 56

Pnycis. Iler.-Schl. . . . . . - 61

Pinetalis. Zell. . . . . . . $53 \quad 27$

Pinctella. Hub. . . . . . 11151

Pinctella. Scop. . . . . 110 51

Pinelellus. Lin. . . . . $107 \quad 50$

Politalis. Hub. . . . . . $48 \quad 25$

Pollinalis. W. V. . . . . $12 \quad 12$

Polygonalis. W. V. . . . . . $51 \quad 26$

Porpliyralis. W. Y. . . . . $24 \quad 16$

Porphyralis. llub. . . . . . $\quad \begin{array}{lll}23 & 16\end{array}$

Potauogalis. Lin. . . . . 410

Fot amagalis. Hub. . . . . 50

Prietexlalis. llub. . . . . $48 \quad 25$

Pralalis, Zell. $\quad 53 \quad 27$

Pratella. Hlub. . . . . . . 8943

Pratella. Brahm. . . . . 115054

Pratellus. Zinck. . . . . 9043

Pratellus, Wood. . . . . . $\quad 8943$

Pratellus. Lin. . . . . . $117 \quad 54$

Pratorum. F. . . . . . 9043

Pradromella. Dup. . . . . . 14162

Prumalis. W. V. . . . . 650

l'udorella. Hub. . . . . . 14763

Pulveralis. llub. . . . . . $36 \quad 31$

Punctalis. W. V. . . . . . 9011

Punicaealis. W. V. . . . . 2316

Punicacalis. Hub. . . . . 25017

Punicalis. F. . . . . . 2316

Purpuralis. Lill. . . $\quad 25 \quad 17$

pygmacus, Slph. . . . . 9646

Pyralella. Ilub. . . . $\quad 85 \quad 41$

Pyralella. W. V. . . . 8140

Pyramidellus. Treit. . . . 11353

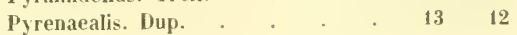


$N^{*}$ Page.

a.

Quedratella. Seop.

Quadrellus. W. V

\section{R.}

Radiellus. Ilub.

Ramulis. Hub

Remalis. F.

Repanulalis. IV. I.

Relicularis. Lin.

lihenella. Zinck.

Rhenelle. Dup.

lkhododendronalis. Dup.

Rippertella. Bdv.

Roborella. Wr. $\mathrm{Y}$.

Rorea. Haw.

Rorellus. Lin.

liosella. Seop.

Rostellus. Lah.

Rubrotibiella. F, v, R.

Rufa. Siph.

kufellu. Dup.

Rufimitrales. Ilub.

Rupestralis. 1lul).

Rupestralis. Ilub. Verz.

Rupicolalis. Huh.
$\$$

Saltalis. Hub.

Sambuealis. Dup.

Sambucalis. W. V.

sanguinalis. Lin.

Sanguinella. Hub., Stph.

Sasonellus. Zinch.

Scabralis. Evrsm.

Sciaphilella. laall.

Selasellus. Iluls.

Selenulis. Ilub.

Semirubella. scop.

Sericalis. llub.

Serpylletorum. Zell.

Silacealis. Ilub.

Silvellus. Hub.

$\begin{array}{rr}114 & 53 \\ 2 & 10 \\ 10 & 12 \\ 33 & 20 \\ 31 & 19 \\ 132 & 59 \\ 152 & 67 \\ 29 & 19 \\ 171 & 62 \\ 131 & 59 \\ 97 & 16 \\ 103 & 48 \\ 147 & 63 \\ 120 & 55 \\ 151 & 65 \\ 165 & 67 \\ 178 & 61 \\ 28 & 18 \\ 16 & 13 \\ 13 & 12 \\ 11 & 13\end{array}$

9

(j)

(1)

Simplonellus. Dup.

Simplonialis. Indg.

Sinuella. F.

Soeiella. llub.

Soeiella. Lin.

Sophialis. F.

Sordidalis. Hul.

Spalicella. Hub.

speculellus. llub. Verz.

Spissicella. Hub.

Spissicornis. F. .

Splendidella. Maun

Spariella. llıb. .

Squalidulis. F. v. R.

Squalidella. Evrsı.

Stachydalis. Zinek.

STEMA.

Stexoptertx. Guén.

Stenziellus. Treil.

Slicticalis. Lin. .

Stranentalis. Hub.

Straminclle. W. Y.

Stratiotalis. W, $\mathrm{V}$

Strige. llaw.

Strigella. F.

Suavella. Ziuch.

Subormatella. Mup.

Sudeticella. I)up.

Sulfurana. Mus. Schitl.

Suspectellus. Zell.

Syliestrella. Ratz.

T.

Taeniellus. Khlw.

Tegostosi. Zell.

Terebrella. Ziuck.

Terminalis. Wood.

Terrealis. Treil.

Telrix. llaw.

Tracilonitis. Zell.

Trapezella. Dup.

Tribunella. W. V.

Trigulla. Esp.

Trislellus. IV. I

Tristis. Wool.

$\begin{array}{rr}N^{\circ} & \text { Page. } \\ 112 & 52 \\ 13 & 12 \\ 164 & 67 \\ 167 & 68 \\ 168 & 68 \\ 58 & 29 \\ 26 & 18 \\ 128 & 58 \\ 111 & 51 \\ 131 & 59 \\ 131 & 59 \\ 130 & 59 \\ 105 & 49 \\ 52 & 27 \\ 149 & 69 \\ 45 & 29 \\ - & 11 \\ - & 33 \\ 109 & 51 \\ 56 & 28 \\ 57 & 29 \\ 99 & 17 \\ 3 & 10 \\ 99 & 47 \\ 93 & 45 \\ 151 & 64 \\ 123 & 57 \\ 79 & 37 \\ 29 & 19 \\ 98 & 57 \\ 130 & 59\end{array}$

104

is 10 64 
Trislis. Hall.

Tristrigella. Slph.

Tristrigella, Wood.

Tumidaue, W. I.

lumidella. Zinch.

Inmidellu. Dup.

i.

Cliginata. F

liginosellas. Zell

Limbratis. Hub.

Imbralella. Ilieil.

I'rtifalis, Liu.
$N^{0}$ Page.

$138 \quad 61$

8.) 11

1 is 61

$1.3 \quad 65$

1536.5

\$.5. 6.5

(i)

19

s8 12

(is 32

$167 \quad 68$

$32 \quad 20$
1.

No Page,

Valesielli. Duр.

Vandaliella. Her.-sidif. $\quad 8.3 \quad 39$

Varicgulis. F. . . . is 2 ?

Verbascalis. IV. I. .

Verbuscalis. Wood.

Verbascalis. Inub. $\quad 11 \quad 33$

Verellus. Zinch. . . . $101 \quad 48$

Verrucella. Hub. $\quad 1.53 \quad 6.5$

Verlicalis. lin. 位 24

Z.

ZopHodi. Hub.
Prefice, page 3 , ligue 12 , lisez: tranches

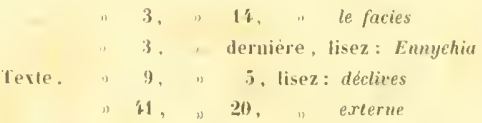

au lien de bruncties.

$$
\begin{aligned}
& \text { " les fucies. } \\
& \text { " " Eunychin } \\
& \text { " " dectines. } \\
& \text { " " postérieur. }
\end{aligned}
$$





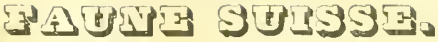

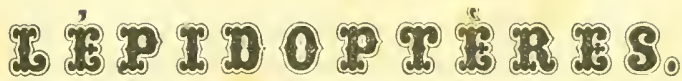

VI. Partie. Tortricides.

PAR

7. $\mathfrak{C}$. 㚼 $\mathfrak{e}$ la 


\section{PRÉ FA CE.}

Il n'est, chez les Lépidoptères, pas de groupe plus homogène que celui des Tordeuses. L'idée typique s'y reproduit avee une variété inépuisable. Les formes ne diffèrent les unes des autres que par des nuances délicates. Des transitions insensibles et entrecroisées lient les espèces entre elles et forment du groupe entier un tout lié et compacte. Quoi d'étonnant qu'avec cet ensemble d'espèces à la fois si voisines et si multipliées, les entomologistes systématiques aient été fort embarrassés d'établir des djvisions naturelles.

A ne voir que le groupe en général, l'isolant par la pensée de cenx qui l'entourent, on est tenté de n'en faire qu'un seul et unique genre limnéen, comme l’a fait Ilerrich-Schiffer. Les subdivisions dès lors s'expriment en sous-genres, et le classement donne une série que l'on peut commencer et finir où l'on veut. En reslant dans ce point de vue, l'habile entomologiste de Regeusbourg a classé (Révision de llubner) dans le senl genre Tortrix, près de 480 espèces européennes, qu'il répartit dans 33 sous-genres.

Guénée, dans son Index metlıdicus des lépidoptères d'Europe, dominé par le point de vue opposé et s’attachant ì tous les caractères quiil porrait saisir, fut conduit à exagérer les moindres différences de formes et construisil une répartition beaucoup plus compliquée. Le genre limnéen forma pour lui une grande division des lépidoptères nocturnes (sic) qui lui fournit 10 tribus. Chacune d’elles se subdivisa à son tour en genres dont le nomhre tolal s'élève à 69 . Entre ces deux extrêmes on trouverait dans les auteurs, ou lou construirait soi-même. des systèmes intermédiaires dont l'utilité serait fort problématique. lei encore il faut recoumaitre que Treitschke a le premier posé les bases des groupes naturels des Tordeuses et qu'on ne saurait les méconnaitre sans créer le dísordre. Je ue prétends point que lon ne puisse faire mieux que lui; je dis seulenent que pour faire une bonne dis- 
tribution, il fant se résigner à compléter on à améliorer celle quil a introduite. Ce rôle, malheureusement, ue satisfait pas tout le monde.

Faut-it maintenant faire des Tordeuses un genre, une tribu ou un ordre des Lépidoptères? - Ni l'un, ni l'autre. Ce n’est pas à l'oceasion d'ume fanne locale et de la faume d'un petit pays, que semblable question doit être résolue. Pour ne pas la préjuger, jen ferai simplement une famille; jamais, it me semble, la désignation ne fut mieux appropriée a l'ohjet; tant les Tordeuses forment un groupe uni, lić, compacte et distinct de tous les intres. Cette fanille se divisera natureltement en genres (non en tribus) et jadopte de préférence, pon les motifs que jai énoncés, ceux de Treitschke, tels que les a modifiés Iterrich-Schaller dans sa Révision de Ilubner. Il est donteux que l'on puisse faire mienx.

La famille des Tortmcunes (Tortricina), on le genre Tortrix de linné, fut confondu par Fabricius avec les Pyrales. Treitschke ! réunissail quelques genres qui en ont été successivement éloignés. Le genre IIalias, liussé par Duponchel et Guénée parmi les Tordenses, a élé réuni aux Nycteolides (Noctuelles) par HerrichSchäfer. Ses clrenilles, sans doute, ont les mèmes meurs; mais toutes les hurves qui pelotoment les fenilles sont loin d'appartenir anx Tordeuses, et mir grand nombre de celles qui en font partic n'en pelotonnent point.

Treitsclke avail encore, avec llubner, rapproché des Tordenses une petite tribn assez anormale, que l’on est généralement convenu de placer à côté des Bombyx et des llépiales; je veux parler des Cocliopodes de Boisduval, Ilelerogenea de Koch et de Treitschke, Limacodes de Duponehel. Quelle que soit la place qu'on lui assigne, toujours est-il qu’elle ne samrit fignrer parmi les Tortricides.

Vient ensuite Sarrothripa Ravayana des anteurs anglais, dont Treitschke fit nne Penthina, sans : attacher grande importance. Guence el Duponchel la laissent parmi les Tordeuses, tandis que Herrich-Schafler la réunit aux Nycteolides. Ce dernier rapprochement se fomle essentiellement sur la nervation des ailes; car sous le rapport des palpes et des organes de la locomotion on ne saurait l'éloigner des Tordenses, tandis que le dessin des ailes et les mours de la larve rappellent bon nombre des Plyycies.

Duponchel, dans son dernier ouvrage (Catalogue méthodiqne), rénnit anx Tordenses ipuclques genres qui il en laul encore éloigner.

Le genre $\mathrm{Xyl}_{\mathrm{J}}$ poda de Latreille, petit groupe que Linné l'énnissait aussi aux Tordeuses, Treitschke aux Pyrales, dont Guénce forma sa tribu des Anomala, a 
été rélégué par Ilerrich-Selıiffer ehez les Tineides. Cette place est sans doute la meilleure pour lui; la strncture de ses antennes l'y rattache de préférence.

Ladjonction aux Tortricides du genre Nola Steph. (Ræselia II.S.) ne fut pas une idée heurense de Buponchel. Il est évident que la place que Ilerrielı-Sehiffer lui a assignée parmi les Lithosies, à côté de Setina el de Nudaria, est bien mieux elowisie.

Il est encore trois genres, renfermant chaeun me seule espèee dont li place a été plus d’une fois contestée. Cheimatophila hyemana, Steph. (Diurnea, llaw.) avait déjà été rattaché aux Tordenses par llubner. I'autres le releguèreut parmi les Timeïdes. Le dessin et la forme des palpes sont cenx des Tordeuses, la nervalion est dillérente.

Il est plus diflicile d'assigner une place à Cuephasia punctulana W. V, et à Cheimonopliila gelatana (Exapate, llub.). Si lon sattache à la nervation, elle parle en faveur d'un rajprochement avec les Tordenses; si l'on doune plus d"importanee aux autres caractères, ces espèces resteront parmi les Tineïdes. Je préfère adopter ici la distribntion de llerrich-Sehäfier, quoiqque gelatana se trouve déjà enregistrée, dans la faune suisse, parmi les Tineïdes, par M. le prof. Frey 1).

Les Tortricides sont essentiellement une fanille europécnne. Les antres continents, antant quion peut le savoir, en fournissent un fort petit nombre. La majeure partie des espèces habite les climats tempérés. LEurope méridionale en compte moins que ses régions septentrionales. Il n’y a dès lors rien d’étonnant que la Suisse soit plus favorisće sous ee rapport quancun autre pays de TEnrope. Herrich-Schäller compte plns de 450 espèces européennes; en supposant quil faille en ajouter une vingtaine de nouvelles, nous aurions un total de 500 espèces. Le catalogue des espèees suisses que j’ai publié ,Bulletin de la Société vaud. des sciences naturelles, t. $V, N^{c} 39$ ) en énumère 307 , jen eompte actuellement 315, ce qui dome pour notre patrie 3 espèces sur 5 europécunes, proportion bien plus forte que celle fournie par la famille des Phalénides.

La distribution géographique par régions nous offrira, dans les limites du sol suisse, les mêmes résultats que I'Europe. Nos 31 jespèces en comprenneut 228 assez généralement répandnes sur tonte la partie tempérée de notre continent, 45 propres aux régions froides ou montagnenses et 42 exclusivement alpines. Ces der-

\footnotetext{
1) Die Tineen und Pterophoren der Schweiz v. P. II. Frey. Ziirich, $185 f$.
} 
nières pourraient encore se subdiviser en espèces méridionales et septentrionales, snivant qu'elles se trouvent dans les régions correspondantes des Alpes.

Quant à la fréquence, on observe que les espèces peu répandues sont plus nombreuses que les communes; celles-ci comptent environ une centaine d'espèces, tandis que 200 environ restent toujours restreintes à certaines localités. Une douzaine environ sont excessivement fréquentes et peuvent être envisagées à cause de la multiplication de leurs larves, comme des insectes nuisibles. Les espèces très rares sont beaucoup plus nombreuses. La fame du continent européen se tronve enrichie par le travail actuel de 16 espèces nonvelles.

Les 'Tordeuses ont toutes un vol court et pen élevé et sont peu exposées à être transportées an loin par les vents, loin des régions qu’elles préfèrent. Il résulte de là que, chez ces iusectes, il est assez lacile de constater l'influence des climats sur la distribution géographịue. Il existe sans donte quelques espèces qui vivent anssi bien dans nos plaines que sur les sommités élevées; mais elles sont en fort petit nombre et constituent plutôt unc exception. Dans la règle il existe ici, comme en général dans la faune et la flore suisse, une ligne de démarcation tranchée entre les Alpes proprement dites et les plaines ou les vallées. Cette démarcation n'existe point au même degré entre la plaine et les sous-alpes (montagnes). Ici les espèces de l'une des régions se retrouvent presque toujours dans l'autre; l'acclimation de l'une à l'autre n'est pas difficile. Les Alpes - et sous ce nom il ne fant entendre que la région inhabitée durant l'hiver, celle des paturages d'été - les Alpes, dis-je, ont leurs espèces particulières qui ne les quittent pas.

Cette confination de certaines espèces dans les régions élevées est bien moins une conséquence de l'existence, à ces hauteurs, de plantes qui ne vivraient pas ailleurs et serviraient de pâture aux chenilles - opinion généralement accréditće qu’un résultat des mœurs même de l'animal. Il a été créé pour les Alpes, ce ne sont pas elles qui l'on lait ce quili est. Sonstrayez-le, peu à peu, si vous le voulez, ì l'air pur et frais des hautes alpes, à leurs nuages et à leurs rosées, à l'ardent soleil du midi, à la température uniforme et fraîche du long hiver sous la neige, et vous anéantissez ses conditions d'existence, vous le tucz sans le modifier. Ces considérations ont quelque importance dans la détermination des espèces douteuses. Lorsque les caractères distinctifs de denx espèces sont peu tranchés, il est fort probable qu'elles sont distinctes, lorsque l'une habite les sommets des Alpes et l'antre leur pied. 
Plusieurs Tordeuses intéressent lindustrie agricole et forestière par les dégâts qu'elles commettent. Les larves, malgré leur petitesse, peuvent causer, par leur extrème multiplication, de véritables ravages. La chenille de Pomonan a fait tounber annuellement une quantité considérable de fruits (pommes, poires, noix, noisettes), dont la majeure partie est perdue pour la consommation. Celle de Pinicolana ravagea, en 1556, les forêts de mélèze des Alpes du midi de la France, et en 1857 celles du Valais. Les larves de Wahlbomiana, de Naevana, de Comitana, sans être aussi funestes, détruisent un grand nombre de bourgeons d'espèces forestières ou fruitières. Hais la plus nuisible de toutes est sans contredit Roserana, dont la chenille détrnit, certaines années, le quart, le tiers et jusqu’à la moitié de la récolte de quelques vignobles. Celui qui découvrirait un moyen sûr et expéditif de détruire sa larve mériterait une récompense nationale. Je ne parle pas des dégats causés à la vigne par Pilleriana, en France, et qui a été l'occasion d'une volumineuse publication 1), parce que, chez nous, cet insecte est inoffensif.

Il est à regretter que cette portion de la faune suisse ne soit pas accompagnée de planches, comme l'ont été les précédentes. L'essai que j’en ai fait ne m’a pas encouragé à continuer. Si j’ai mal réussi à reproduire des phalènes et des pyrales, qu'aurais-je obtenu pour les tordeuses? Un mauvais dessin est doublement fâcheux; son auteur se contente des figures qu'il domne et néglige les deseriptions caractéristiques: l'observateur qui le consulte ne s'attache qu'an dessin et se passe des descriptions, pour éviter la peine de les suivre. J'ajouterai qu'il serait fort à désirer pour le bien de la science que les lépidoptères fussent tous figurés dans un recueil unique, tel que celui de Hubuer, afun que désormais on fut obligé de s'en rapporter à lui senl pour la fixation des espèces.

Les individus originaux qui ont servi de base à mon travail resteront déposés au musée cantonal de Lausanne.

J'ai fait intervenir dans ce travail, autant quil était en mou pouvoir, la synonymie des auteurs français modernes, parce que plusieurs espèces propres à la France se retrouvent en Suisse, sans pénétrer en Allemagne. A plus forte raison devaisje faire mention, partout où cela pouvait être utile, du Catalogue des Lépidoptères $d u$ Doubs, que nous devons anx soins et à l'iufatigable activité de M. Th. Bruand, de Besançon.

1) Audoin, $\boldsymbol{y}$. Histoire des insectes nuisibles à la vigne, et particulierement te la Pyrale, etc. Paris, 18:2. 40. 
Je répèterai ici, sans craindre d'être fastidieux, les remerciments que je dois à MM. Rothenbach, instituteur à Schïpfen, Frey, professeur et G. Zeller, l'nn et l'antre à Zurich, puisque sans leur assistance désintéressée et bienveillante mon travail lut resté très incomplet. MH. (̇. Leresche, instituteur à Ste-Croix, J. Ott, collecteur à Heyringen, et Wullschlegel, instituteur à Oftringen, mont aussi fourni plusienrs renseignements importants.

Lausame, ce $1^{\text {er }}$ novenibre $185 \%$.

J. DE LI IIIRPE, D. II. 


\section{Tortricides (Tortricida).}

Insecte parfait. - Ailes supérieures trapézoïdes, plus ou moins écourtées, a còte forte et convexe; cachant les inférieures dans le repos. Inférieures pell développées, sans dessin, reployées en éventail dans le repos. - Téte mojeme, poilne; trompe rudimentaire ou nulle. - Antennes du màle, capillaires; rarement ciliées, plus rarement pectinées. - Palpes ordinaires simples, courts, recourbés en bas; dernier article fusiforme très-court: avant-dernier ordinairement pyramidal, poilu; palpes accessoires invisibles. - Corps court; thorax carré. - Pattes courtes, fortes, armées d'éperons. Insectes de petite taille.

Larve. - Chenilles verdàtres, lusifomes, obtuses aux deux extrémités, glabres on reconvertes de quelques poils implantés sur des verrues. 16 pattes égales. Un écusson lisse sur l'anus. Tète platte. - Vivant dans l'intérienr des végétaux ou sur tenrs feuilles roulées. Chrysalide ordinairement armée à l'anus d'une couronne d'épines et sur les anneatrs l'aspérités.

\section{Gen. Teras, Treit.}

Leptogramma, Teras et Dietyopteryx, Stph. Guen. - Glyphiptera, Steph.

1. Cristana, W. V.

Ilub. 176. - Treit. - Dup. 244. - Fröhl. - Hub. revis. $\mathrm{N}^{\circ}$ 1, - Hub. sup. f. 237, 25. - Heyd. C, 585.

Guén. Ind. - $\mathrm{N}^{\mathrm{o}^{5}} 1047-1062$, Wood. (Her.-Schfr.) Bruand Cat. $N^{\text {os }} 943,944,945$.

Sericana, Hub. 83. - Dup. 244.

Lefeburiana, Dup. 244. - Hub. sup. f. 26.

Ruficostana, Wood. - Ilub. sup. f. 27.

Combustana, Dup. 244.

Autumana, Hub. 247. (err. 274.)

Desfontainana, Fab. - Steph. 
Assez rare en Suisse. Mr. Rothenbach l'a prise à Schüpfen et à Langnau, M. Couleru dans les environs de la Neuveville, et II. C. Zeller près de Zurich, sur le Balgrist. Jo l'ai prise une fois près de Lausanne, dans un bosquet. Une variété très-rare a été élevée par M. le prof. Frey, à Zurich. - Environs de Bremgarten (Boll.). Apparaît en octobre et quelquefois au printemps.

2. Abildgaardana, Fab.

Fröhl. - Treit. - Dup. 244. - Hub. revis. No 3. - Heyden, C. 635. - Guén. Ind. - Bruand. C. 941.

Cristana, Hub. 55 (non 176).

Duplana, Fab.

Osbeckiana, Thumb.

Variegana, Fab. - W. V.? - Curt. - Wood. 1059. - Fröhl.

Blandiana, Ilub. verz. - Mus. Schif. sup.

Asperana, Lin. (non secund. Doubld.)

Borana, Steph.

Var. Nyctemerana, Ilub. 240.

IIub. revis. n 5. - Heyd. Cat. - Guén. Ind.

Asperana Fab. - Dup. 244. (non W. V.). - Wood. 1088. - Curt.

Briand. C. 942.

Var.? Permutatana, Dujs. - Guén. Ind. - H. S. f. 28.

La forme Abildgaardana est fréquente dans les environs de Lausanne, en aoùt et en septemhre, ainsi que près de Schüpfen (Rothb.); elle l'est moins près de Zurich (Zell., Frey). Nyetemerana a été prise près de Bâle (Imhoof), 'de Zurich(Zell.), de Schiipfen (Rothb.). Je l'ai prise deux fois, à la fin d'aoùt, près de Lausanne. Assez fréquente près de Zurich (Frey).

Un très-beł individu de la première forme, dans la collection de M. Frey, venant de Samaden, a été élevé de chenille par II. Pfaffenzeller sur le Mlespilus amelanchier. Je possède une variété nàle dans laquelle la moitié interne de l'aile r'offre qu'une tache noire au milieu d'un large champ blane pur; lá moitié externe est d'un fauve vif sans écailles foncées. Cette variété forme un passage à Nyctemerana.

Permutatana (et non Permutana) II. S. f. 28, n’a pas été jusquici trouvée en Suisse : je possède cependant un individu qui correspond parfaitement à la figure eitée; mais il ne répond pas aussi complètement à la description de Ilub. revis. Il est, du reste, fort 
probable que Permutatana n'est quine variété d'Abildgaardana dans une espèce si variable, il ne faut pas s'attacher à des caractères trop ininutieux de dessin et de forme de l'aile.

\section{Trist a na, Hub. 50 .}

Hub. revis. no 6 . - Heyden. C. n 614 (excl. synonym.). Curt.

\section{Logiana, Treit.? - Fiscl.. v. R.?}

Jai reçu de $\mathbf{I}$. Rothenbach un individu appartenant à cette espèce, pris dans les environs de Schüpfen.

Heydenreich confond Tristan a avec Logiana, Hub. 64, et Guénée l'envisage comme une variété de Proteana, Fisch. v. R. Elle ressemble aussi beaucoup à certaines variétés de Scabrana. Logiana, Hub. 64, est plus petite, a les ailes postérieures plus foncées et la hase des supérieures tachées de brun. Guénée et M. Bruand confondent F errugana, Erutana, H.S., et Tristana avec Proteana. Scabrana a les épanles plus saillantes et plus arrondies et la frange des supérieures grisàtre et non roıge-brun.

Tristana paraît fort rare partont.

1. Erutana, Hub. sup. f. 9, 19.

Ilub. revis. $11^{\circ} 8 .-$ Heydenr. C. 612 .

Logiana, Fisch. v. R. - Lin.? - Hub. 217?

Prise une fois dans les environs de Lausanne. H. Zeller la cite dans les environs de Zurich, et I. le prof. Frey mécrit qu'elle n'est pas commune en septembre et en octobre, près de cette ville. Il a trouvé la chenille sur le Viburnum lantana et l'a aussi élevée sur l'opulus. Je l'ai reçue de M. Th. Bruand sous les noms de Tristana et de Caledoniana provenant d'Angleterre.

\section{Favillaceana, Hub. 62.}

Fröhl. Treit. - Dup. 243. - Hub. revis. n" 9. - Hevd C. 609,610. - Gnén. Ind. - Curt. - Bruand. Cat. 938. -

Var. Lividana, Treit. - llub. sup. f. 270 (var.).

Logiana, Mus. Schif. - W. Y.?

Sparsana, W. V.

Sponsana, Fab.

Labeculana? Fr. n. b. 18, 3. - Wood. 1074, 1075. 107ti. 
II. Rothenbach a pris plusieurs fois cette espece dans les environs de Schupfen. Je lai recueillie dans le Jorat, au-dessus de Lausanne, sur le bord des bois de sapin, en septembre, où elle n'est pas rare. MII. Zeller et Frey la prennent aussi dans les environs de Zurich. où elle est rare.

Les figures de Duponchel sont mauvaises; celle de la femelle se rapporte à la variété figurée dans Hub. sup. f. 270 (lividana, Treit.); celle du màle est méconnaissable.

6. Schalleriana, Lin. faun. suec.

Fab. - Hub. 288, 289. - Treit. - Dup. 243. - Hub. revis. n 10. - Curt. - Heyden. C. n611. - Guéen. Ind.

Favillaceana, Zell. Isis. - Wood, 1055, 1087.

Labeculaua, Frey, n. b., 18?

Violaceana? Bruand, C. 955.

Les bois au-dessus de Lausanne en aoùt et en septembre; assez commune. - Envirous de Schiipfen (Rothb.), - de Zurich, où elle est pen commune (Frey).

La figure de Duponchel est mauraise; celles de Hubner ne valent guère mieux

7. Comparana, Hub. 284.

Treit. - Dup. 243. - Huh. revis. n 13. - Heyden. C. 626.-Guén. Ind.

Rufana, Wood. 1086, - Curt.

Logiana? Bruand, Cat. 956.

Plus rare que Schalleriana. Environs de Lausanne; en juillet et aoùt, dans les taillis Environs de Schüpfen (Rothb.), de Zurich (Frey).

La femelle, ainsi que dans Schalleriana, est ordinairement un peu plus grosse que le mâle. - Quoique voisines, ces deux espèces restent constamment différentes.

8. Rufana, W. V.

Hub. 178. - Hub. revis. $n^{6}$ 14. - Hub. sup. f. 20. -- Guén. Ind. - Heyd. C. 622.

Lucidana. Treit. - Heyd. Cat. 606.

Var. Densana, Frey. n. b. 18, 4. -- Heyden. Cat. 607.

Crassana, Dup. sup. 61.

Rubiginana, Khlw.

Similana, Wood. 1080.

Trigonana, Wood. 1084 .

Cette espèce rare a été recueillie dans les environs dEngelberg (Olten) par M. Wullschlegel. 


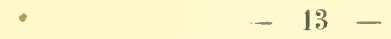

9. Ferrugana, W. V.

Treit. (partim). - Dup. 243. - Fisch. v. R. t. 23-25. Ilub). revis. ${ }^{\circ}$ 15. - Heyd. Cat. 616.

Var. Hub. sup. f. 407.

"Tripunctana. Dup, 243. - Hub. 129

Brachiana, Frey. n. h. 18, 2.

Gilvana, Fröhl.

Approximana, Fab. sup.

Conspersana, Fröhl.?

Wood. 10931095.

Ca et là dans les environs de Lansanne, au premier printenps et à la fin de l'été, dans lest taillis. Jai pris la variété claire (Tripunctana) en mars au bois de Sauvabelin et la variété ronge, en aoùt, à Paudex. Je ne saurais admettre une hibernation chez cette espece pas plus que chez Treveriana. - Environs de Zurich (Zell., Frey); de Schüffen (Rothb.). II. Heuser ma adressé un individu appartenant à la forme Tripunetana, pris daus les environs de Burgdort.

Ferrugana est souvent confondue avec Lythargyrana et avec Asperana, lorsfu'elle resèt une couleur de rouille. La coupe de l'aile a beaucoup de rapport. La concasité du bord externe, dans Ferrugana, porte, sur la frange, une teinte claire semblable it relle de Contaminana et qui ne se voit pas dans les deux autres espèces.

Labsence de dessin n'est pas toujours complète dans Ly thargy rana: quelques indivilus prortent sur la còte une tache triangulaire peu apparente. La découpure arrondie du hord externe d'Asperana la distingue à première vue des deux autres: la ligno de démar(ation de la frange est encore trés-caractéristique chez cette dernière.

Les synonymes français sont trop confus sur ce point pour les citer.

10. Adspersana, Hub. 259.

Fröht. Fisch. v. Rösl. - Dup. sup. 61.5. - Hub. revis. n 17. - Hub. sup. f. 406. - Heyd. C. 620. - Guín. Ind.Bruand, Cat. 959.

Ferrugana, var. Treit. - Dup.

Rufana, Fröhl, (exclus. synon.)

Subtripunctulana, Steph. - Wood.

Jai pris cette espèce à la fin de juillet et au commeneement daont, au-dessus der Lausanne, dlans une clairière, parni les herbes touffues. La femelle est difficile à saisir, parcer quielle vole pen et se jette à terre dès quion lapproche. 
M. Ie prof. Frey a élevé la ehenille en juin sur les Potentilles, le papillon naquit in juillet. Pas rare dans les clairières des bois, dans le canton de Zurich.

\section{Lithargyrana, Podev.}

Hub. revis. n 19. - supl. f. 23. - Heyd. C. no 624. - Vlus. Schif.

Rufana, IIub. 127.

Proteana, Guén. (partim).

J'ai recueilli un individu appartenant à cette espèce, à Paudex près Lausanne. 14. Rothenbach m'en a adressé un pris près de Sehüpfen. Rare partout.

\section{Quercinana, Man.}

Hub. sup. f. 21, 22. - IJub. revis. $\mathrm{n}^{\circ}$ 20. - Heyd. C. $\mathrm{n}^{\circ} 623$.

J'ai pris un individu de cette espèce sur les petits chènes qui bordent la route des Ormonts à Aigle, au mois de juillet, dans un endroit très exposé au soleil et chaud. Il répond parfaitement à la figure de H. S. et à sa description. - Je ne l'ai pas retrouvé depuis dans la mème localité.

13. Boscana, Fab.

Dup. 242. - Guén. Ind. - Heyd. C. 650. - Ilub. revis. $n^{\circ} 22$, Bruand, C. $n^{\circ} 937$.

Cerusana, Hub. 63. - Treit. - Curt.

Recueillie par M. le Dr. Imboof dans les environs de Bàle, et par M. Heuser près de Burgdorf.

14. Mixtan a, Hub. 215.

Treit. sup. - Dup. 261. sub permixtana (fig. mal.). - Ilub. revis. $\mathbf{n}^{\circ}$ 23. - Ileyd. C. 605. - Guén. Ind. - Curt.

Castaneana, Wood. 1114 .

lly emana, Haw.

M. Rothenbach mentionne cette espèce parmi celles quil a capturées dans les environs de Schüpfen. Il la prend au premier printemps sur les collines exposées au soleil et couvertes de bruyères.

La femelle est sensiblement plus petite que le mâle; la tache de la còte se dessine très légèrement sur le centre de l'aile en brun plus foncé. Le sommet des supérieures est aigu; le bord terminal, assez oblique, porte une frange large, qui le rend arrondi. 
Le centre des supérieures offre un gros point noiratre dont les écailles ne sont pas relevées. Treitsclke aura sans doute confondu cette espèce avec Erutana lorsqu'il parle Jécailles relevées en points et en lignes.

Les individus reçus d'Allemagne portent davantage de gris luisant sur les ailes et ont la frange de cette dernière conleur; ceux que j'ai reçus de $\mathbf{M}$. Rothenbach sont plus petits et ont la frange rouge-foncée.

Les uns et les autres portent quelques points très noirs à l'extrémité des inférieures, an dessous.

Je l'ai reçne de M. Bruand sous le nom de Tristana.

15. Umbran a, Hub. 59.

Fröhl. - Treit. - Dup. 243, 5.- Fisch. v. R. t. 35.- Hub. rev. $n^{\circ} 26$. - Heyden. C. n579. - Guén. Ind.

Radiana, Dup. 243, 6. (non Hub.). - Bruand, Cat. no 953 (non 947).

11. Rothenbach indique cette espèce dans les environs de Schüpfen. J'en ai pris un exemplaire dans les bois au-dessus de Lausanne, au printemps. - (Voir Abietana).

16. Maccana, Treit.

Hub. sup. f. 14-16. - Ilub. revis. ${ }^{e} 27$ - Heyden. C. 604 . Guén. Ind.

Dans les environs de Schüpfen; très rare. (Kothb.)

17. Scabrana, W. V.

Ilub. 169 (non 58). - Treit. - Dup. 243. - Hub. sup. f. 10-13. Ilub. revis. $1^{\circ}$ 28. - Heyden. C. 591. - Guén. Bruand. C. $946,947,948,949,950,951$.

Elevana, Fab.

Salebrosana, Kblw.

Iler.-Schäff. admet 10 formes:

1. Byringerana, llub. 61, 216. Hastiana et Leprosana, Fröhl. Coronana, Wood. 1072. - Abietana, Dup. 265, 2. - Ileidenr. Cat. 596, 597. - Spars ana, Fröhl. - Treit. (non W. V.). Dup. 243,1.- Ileyd. C. 595. - Sponsana, Fab.?

2. Reticulana et Favillaceana, Wood. 1078, 1075. - Wood. 1081? 1073? 1071? - Autumnana, Wood. 1070 (non Hub.).

3. Tota brunneo-grisea, obscura. 
4. Combustana, Ilub. 234 (non Dup.). Cristana, Fab. Frohl.

Wood. 1068. - Heyd. C. 592.

5. Centrovittana, Wood. 1067.

6. Albistriana, Wood. 1069.

7. Ramostriana, Wood. 1066. - Radiana, Hub. 177. - Heyd. C. 593. (non

Dup.). - Wood. 1065.

9. Divis an a, Hub. 198. - Heyd. C. 632.

9. Aquilana, Hub. 235. - Fröhl. - Heyd. Cat. 575. - Opacana. Llub. 334. -... Heyd. C. 603.

10. M a yrana, Hnb. 335. - Heyd. C. $59 \dot{4}$.

Pas fréquente en Suisse. M. Rothenbach l'a prise dans les environs de Schiplen sous la forme Seabrana ordinaire et Combustana Hub. -. M. C. Zeller a recueilli la lorme Aquilana, près de Zurich. Je l'ai reçue de If. Heuser, à Burgdorf. (V. Abietana).

18. A hietana, Hub. $275,276$.

Treit. - Fiseh. v. R. t. 34, 35. - (non Dup.). - Heyden. Cat. no 601. - Hub, revis, n²9. - Guén. (non Fröhl.).

Confixana, Jlub. 277. - Fröl.

Scabrana. Hub. 58 (rar.)

Wood. 1081, 1071, 1047, 1073?

Cette espèce n'est point très-rare dans les bois de sapin du Jorat, au-dessun de Lausanne, en septembre et oetobre; quelquefois en mars et avril.

Abietana, Scabrana et Umbrana sont très faciles à confondre par suite de leur ressemblance et de leur variabilité.

La première a les ailes supérieures un peu plus étroites que la seconde et compées plus carrément au bord externe, en sorte que l'angle interne s'arrondit plus brusquement. Dans Seabrana, le bord interne des mèmes ailes est légèrement convexe, ce qui faił paraître laile plus large; chez Abietana ce même bord est droit et un pen exeavé vers l'angle interne. - Umbrana se distingue des deux autres par le sommet de l'aile plus alongé et son bord externe faleiforme du eôté du sommet, largement arrondi du còté du bord postérieur. Les différences de dessin sont trompeuses et peu sùres.

19. Treveriana, Hub. 100.

Treit. - Dup. 242. - F. v. R. t. 25. - Guén. Ind. - Heydenr. C. $642 .-$ Hub. revis. $\mathrm{n}^{0} 31$. - (non IV. V.) 
Niveana, Fab. - Frohl.

Cerusa na, Dup. 264, 2? (non Ilub.)

Toutes les années, dans te hois de Sauvabelin, près de Lausanne, au premier printemps. Je l'ai prise en févies, en mars et en avril, suivant les années, toujours appliquéer contre léeoree blanche du trone des bouleaus.

Cerusana, Dup., appartient probablement a Boseana: Duponeleet sattache d'ailleurs is la distinguer de Treveriana.

20. Nebulana, Hub. 104.

Treit. - Dup. 242. - Hub. revis. n" 32. - IIerden. Cat. 640.

Fröhl. - Guén. Ind.

Var. Roseitlan a, Hub. 103. - Fröhl.

Cette espece a été prise par M. Rothenbach dans les emvirons de Schuiplen. Rare.

21. Literana, Lin.

W. V. - Fab. - llub. 88-91. - Frohl. - Treit. - Dup. 24?.

Ilul). revis. $\mathrm{n}^{\circ} 33$. - Heyden. C. 645. - Ciucin. Ind. Bruand, C. 933,934 .

M. Rothenbach possiede eette espèce prise dans les euvirons de Sethüpfen, oì elle est fort rare.

22. Asperana, W. V.

Mus. Schif.-- Treit. - Ilub. sup. f. 2, 3. - Ilub. revis. $1^{\circ} 35 .-$ Zetter. - Guén. hsd. - (non Dup.) - Bruand, C. 935.

Squammana, Fab. - Frohl. - I)up. 242, 2. - Wood. - Curt.

Squammulana, IIul. $92-94$.

Rom anana, Fab.

Tricolorana, Stepls. - Wood. - Haw.

Fulvomixtana, Wood. - Steph. - Curt.

Treveriana, W. V. - Mus. Schif.

Spectrana, Dup. 242, 4. $-\mathrm{N}^{\text {on }} 1099-1102$, Wood.

Var. Irrorana, ILub. 95-97. - Curt. - Wood.

Prise dans les euvirons de Schupfen par M. Rothenhach, par M. Zeller pris de Zurich. et par M. G. Leresche, au mois davril, au pied du Jura, au-dessus de Fontanezier, daus un bois de hètre.

Spectrana, Dup.. nappartient-il pas plutit ì Boseana? 


\section{Contaminana, Hub. 142.}

Treit. - Fröhl. - Dup. 244. - Guén. Inıl. - Huh. revis. n 38. Heyd. C. 575. - Wood. 1107-1109. - Curt. - Bruand, C. 960,961 .

Rosana, W. V.

Ameriana, W. V.

Var. Ciliana, Hub. 171. - Steph. - Dup. 265. - Ileyd. C. 576. - Curt.

n Rhombana, Inp. 244.

" Dimidiana, Hub. 299-300. - Fröhl. - Heyd. C.577.

"Obscurana, lon.

Pas rare, sur les chìnes, à la fiu daoùt et en septembre, aux environs de Lausamne. A Schiipfen (Rothh.) et pres de Zurich (Zell., Frey).

La forme figurée par Huh. sous son $n^{\circ} 142$ est la plus rare; Ciliana et Rhombana, Dup., sont plus communes; on en troure des deux sexes. de nai janais rencontré la teinte foncée (probablement exagérée) que Hub. doune à ses fignres 299-300. Les fig. de Ciliana et de Rhombana daus Dup. sont manvaises.

24. Caudana, Fab.

WV. V. - Fröhl. - Hub. 232. - Treit. - Dup. 244. - Gnén. Ind. - Fisch. v. R. 54, 55, 1, a. b. - Hnb. revis. $n^{\circ} 39$. Heyd. C.571. - Wood. 1106. - Curt. - Bruand, C. 962.

Oehracea, Steph.

Cette repéce n'est pas rare dans les haies du Jorat an-dessus de Lansanue, au mois d'aoùt et en septembre. Priss de Schiipfen (Rothb.), de Zurich (Frey). - (Voir Effractana.)

24 b. Var. Emargana, Fab.

Ilub. 233. - Dup. 244. - Fröht. - Fisch. v. R. t. 55, 1, c. d. -Guén. Ind. - Hub. resis. 39. - Heyd. C. n572. - Wood. 1103. - Curt.

Je l'ai regue de M. Rothenbach, qui la prise quelquefois ì Sebiupfen. - It. Zefler la recucillie aussi près de Zurich.

Les recherches de Fisch. v. Röisl. ne semblent laisser aucun doute sur la parenté d'Emargana et de Caudana et établir quilles appartiennent à une seule et mèmo espèce. M. Zeller, de Glogau, ne partage pas encore cette opinion. 


\section{Effraclana, Hub. 175.}

Treit. - Fisch. v. R. t. 55, 2. - Hub. revis. $1^{\circ} 40$, - Guin. Ind. Ilegrl. C. 574 - Wood. 1106. - Curt.

J'ai reçu de M. Rothenbach denx individus appartenant ì cette espece. II. G. Leresche la prise dans les environs de Ste-Croix.

Dans la distinction spécifipue dEffractana, IEmargaua ot de Camdana, il laut uniquement sen tenir à lexamen de la chenille. Emargana, si differente des dem autres par son dessin, nait de la mème chenilie yue Candana, au dire de Fisch. v. R.Jai recu de y. Her.-Scläff. un exemplaire typupue dEffract an a; jr l'ar comparé ì plusieur: Caudana que je possede, et a dautres Effractana, et il ne ma pas éte pessible de constater un earactere distinctif sùr pour les deux especes. Les stries transverses des ailes inférieures sont plus marquées dans Elfracta u a pue dans Ciandan a; mais on trouve des individus intermódiaires, et jen ai un sous les yeux tpui appartient à cette derniere espece par sa raye brune du bord interue des supérieures et présente des stries transwares trés prononcées. La raje brune dont je viens de parler, est-elle hicn un caractère spécifique? Ell, existe ou nexiste pils sur tes individus parfitement scmblahles il tous antres igards. Lorstu'elle nexiste pas clle est remplacé, chez les uns comme chez les autres, par une raye jaunàtre. On a dit que Ciandana seule portait des écailles jaunes sur le disque: j'en trouve plus ou moins sur tous les individus. La forme et la grandeur de léeliancrure, la longueur du bout crochu de laile supérienre. la couleur des franges, la distribution des teintes grises et rongeattes varient dans chagne indiridı, quil appartienne a $\mathrm{Cau}$ dana ou à Effractana. En somme, la distinction de nos trois espéces ne peut pas ìtre envisagée comme arrétéc et lemande de nouvelles recherches.

D'après ce que je viens de dire, ou conçoit que je regarde comme fort inutile tonte discussion sur la synonymie de Inumer dans létat dimperfection des figures guil nous a laissérs.

II. Gen. Lozotenia. Curl., Guén., Dup. elc. Tortrix, Treit. - Dichelia, Ampliysa, Oenoctra, Guén.

Ce genre se confond avec Sciaphila par Nubilana et Oxyacantlana et avec Tortrix, H.-S., par Steineriana et Dohrniana.

26. Ameriana. Lin.? - Fab.

W. V. - Trrit. et sup, - Ilup. 238. - Fisch. v. R. I. 43, 44. Zett. - Ilubn. revis. $n^{\circ} 41$. - Ileyd. C. n 49. 
¿ Pyrastrana, IIub. 124. - Guén. Ind. - Bruand, C. $n^{\circ} 909$.

¿ Rosana, Frölıl. 41. - Lin.? - Wood.861.

\& Congenerana, Ilub. 295. - Treit, - Dup. 238.

Podana, Scop. ?.

Fulvana, W. V. - Mus. Schif. - Hubn. Verz. - Curt.

Oporana, Wood. $\delta$.

Fuseana Stph. ?.

Gerningana Haw.

Assez fréquente dans les environs de Lausanne en juin et juillet; de mème près de Schijpfen et de Zurich (Rothb. - Zell., Frey).

La femelle est ordinairement plus grande que le mâle. La tache de l'espace margiual, chez celui-ci, est falciforme.

M. Frey a élevé la chenille sur le chèvre-feuille et moi sur le saule marecau.

27. Piceana, Lin. 299.

Treit. sup. X. Ill. p. 53. - Fisch. v. Rösl. t. 43. - Hubn. revis. n 42. - Guén. -- Heỳil. C. 4\%. - Dup. Cat.

¿ Xylosteana, Hub. $26 \dot{1}$ (non Dup.). - Fröhl.

§ Oporana, Lin. 292. - Fab. - Hub. 112. - Treit. - Fröhl. - Dup. hist. 261. - Frey, n. b. 48, 1. - Wood. 860. - Curt.

II ermannniana, Fab. - IV. Y. - Mus. Schif.

Dans les forèts de sapin de toute la Suisse; pas comnune. (Lah. - Rothb. - Zell.). Au milieu de l'été.

28. Xylosteana, Lin.

Fab. - W. V. - Mus. Schif. - Treit. - Fisch. v. R. t. 45. a. d. g. h. (non e.f.) - Frey. i. b. 49,3 . - Ilub. revis. $n^{\circ} 43 .-$ Guén. Ind. - Ileyd. C. n50. - Dup. 239. - Wood. 863. (non Ilulsn.). - Curt. - Bruand, C. 910.

\& Characterana, Hub. 125.

Obliquana, Wood. 865 . - Curt.

Assez commune sur les ehènes et autres arbres de nos forèts (Lah.); à Schiipfen (Rothb.); à Zurich (Frey). En juillet.

Le mâle est plus petit que la femelle. 
29. Crataegana, Hub. 107. ?

Treit. - Fröhl. - Dup. 238. - Ilub. revis. n 44. - Guén. Ind. Heyd. C. n $51 .-$ Bruand C. 911.

Roborana, Hub. $126^{\circ}$ ๖. - Dup. hist. 239. - Wood. 864.

Xylosteana, Fisch. v. R. t. 45, e, f. - Treit. sup. - Frey. ii. b. $48,4$.

Piceana, Fröhl.

Branderiana, Wood. 862 \&. - Curt.

Gerningana, Mus. Schif. (err.?)

Commune partout dans les forèts et les taillis au milieu de l'été (Lah.); de mème à Schüpfen (Rothb.) et dans les environs de Zurich (Zell., Frey).

Le màle est quelquefois de plus petite taille.

30. Lærigana, W. V.

Mus. Schif. - Treit. - Fisch. v. R. t. 11 et 98 . - Dup. sup. Hub. revis. no 47, - Guén. Ind. - - Heyd. C. 57. - Wood. 857. - Zell. Isis. 1839. - Hub. Verz. - Bruand, C. 913.

¿ Acerana, Ilub. 118. - Fröhl. - Wood. 869. - Dup. hist. 239. - Steph. Nebulana, Woot. 858 .

\& Oxyacanthana, Hub. (17. - Steph. - Dup. hist. 238. - Wood.875. Curt.

Variana, Fröhl.

Rosana, llub. 302.

Très commune à la fin de juin et en juillet dans toutes les haies (Lah. - Rothb. Zell. - Frey).

Le mâle, presque toujours un peu plus petit que la femelle, a l'extrémité des inférieures moins teintée de jaune que cette dernière. Quelquefois eette teinte disparaît en-dessus et ne se voit qu'en-dessous; dans ce cas-là on peut aisément confondre le mảle de cette espèce avec celui de Diversana. Chez quelques màles, le sonmet des supérienres se rapproche pour la forme de celui de la femelle.

31. Dumetana, Treit. sup.

Fisch. v. R. t. 20. - Guén. Ind. - Ilub. resis. n48. - Ileyd. Cat. 60.

Cratægana, Frey. n. b. 1. 48 . f. 4. 
Cette espèce n'est pas très-rare dans les haies au-dessous de Lausanne, à la fin de juillet et en aoùt. M. C. Zeller l'a aussi prise dans les environs de Zurich.

Ses palpes allongés et ses ailes fortement arquées sur la còte, la elistinguent an premier coup-d'ail.

\section{Gerningana, II. I.}

$$
\begin{aligned}
& \text { Mus. Schif. - Fab. - Fröhl. - Treit. - Dup. sup. 6f, - Iubn. } \\
& \text { mvis. u 49. - Hubu. sup. f. } 239 \text { ?, 414. - Wood. 8i6. - } \\
& \text { Guén. Ind. - Ileyd. C. 71. - Bruand, C. } 931 \text { b. }
\end{aligned}
$$

o Pectinana, Ilub. 105. - Curt.

Recueillie dans les envirous de Zurich par II. Zeller: sur le Jura pries de Ste-Croir et de Fontanezier, jar M. (i. Leresche, au mois d'aoùt. Ce lernier na pris que des màles. - It. le prof. Frey l'a fríquemment collectée dans lEngarline, prés de Sanaden $\left(5200^{\prime}\right)$, sur le col de la Bernina. Il ne l'a point rencontrée dans les embirons de Zurich.

Je dois à Jubligeance de $\mathrm{H}$. Bruand deus individus provenant d'Angleterre yui sont plus petits, plus colorés un brun à la base des supérieures, aux inférieures et en dessous: ils ont le sommet des supírieures plus aigu, lit baule jaune du centre plus uette et plus vive, séparée de la bande moyenne par un bord brun foncé; la frange plus foncée, mais non divisée comme dans Rubicundana. Hs coustituent érifemment une autre espece.

3:3. Consimilana, Treit.

Hul. revis. $n^{0} 50$. - (non Inbn.). - Hub. sup. f. $347,54 .-$ Heyd. Cat. $n^{\circ} 73$.

Semialbana. Guén. Ind. - Bruand. C. 919.

Modeeriana. Wood. 868. - Curt.

Pas trè-rare dans tes haies au-dessus de Lausanne, en juin. - M. Rothenbach la anssi recueillie près de Sehiipfen oì elle est très-rare.

Consimilana, Hlubn. 239, appartient il une autre espece.

31. Diversana, Ilub. 251, 。

Treit. - Dup. cat. - IIub. revis. $n^{\circ} 52$. - Ilub. sup. 50 et 51 8. 529. (non Dup. 265). - Guén. - Heyd. cat. n 88. Bruand, C. 930.

Acerana, Wood. 869 .

Trifascia na? Wood. 870. - Curt.

Viduana? Fröhl. 
Assez fréquente dans les environs de Lausanne, en juillet; plus rare à Schüpfen (Rothb.). Elevée plusieurs fois à Zurich par H. Frey.

Le màle est ordinairement de moitié plus peiit que la femelle.

35. Pilleriana, W. V.

$$
\begin{aligned}
& \text { Mus. Schif. - Fab. - Hub. } 172 \text { \%. - Treit. - Dup. 239. - } \\
& \text { Ilub. revis. n } 56 \text {. - IJub. sup. f. } 349 \text { (rar.). - Guén. Ind. - } \\
& \text { Heyıl. C. } n^{\circ} \text { 128. - Bruand, C. } 932 .
\end{aligned}
$$

Luteolana. IJub. 136 ठ. -- Wood. 1677.

Vitana. Fab. - Latr. - Audouin.

Cette espèce est fréquente daus la vallée d'Aigle, dans les prés marécageux, dans les taillis chauds, au bord des vignes, le long de la route des Ormonds; en juillet. On la prend quelquefois, mais rarement, clans d'autres parties dn vignol,le (A. Forel). M. Couleru l'a recueillie an pied du Jura dans des expositions chaudes.

Je ne l'ai jamais rencontrée dans les vignes elles-mèmes, mais plusieurs lois trèsloin d'elles. Jai quelıue lieu de croire que chez nous la chenille se nomrit de plantes très-diverses. Jamais en Suisse elle ne ravage les vignobles.

36. Grotiana, Fab.

Frohl. - Treit. - Duр. 239. - Fisch. v. R. 1. 11. f. 1. - IIul). revis. $n^{\circ}$ 58. - Guén. Ind. - Heyd. C. n'82. - (non Wood. $871)$. - Curt. - Bruand, C. 931.

Flavana. Hub. 133 (non 157, 258).

Oehreana. Wood. 872 .

Commune dans tonte la Suisse. S'ileve sur les hasses Alpes. Les haies et les pâturages; au milieu de l'été. - (Lab., Rothl., Zell.).

Jai trouvé une fois la chrysalide dans une feuille roulce de Laserpitium latifolium. - Varie très-peu.

37. Ochreana. Hub. 131.

Treit. - Inp. 113 i (non 1351). - Guén. Ind. - Hub, revis. n 59. - Heyul, C. n 86. (non Fröhl.).

Ochrealis (Pyral.) Hub. 47??

Grotiana, Wool. 871.

M. Rothenbach signale cette espèce près de Schinfen et II. C. Zeller près de Zurich. Jai pris le mále et la femelle dans les environs d'Aigle, a la fin de mai 1857 . 
Les imdividus que jai recueillis ne correspondent completement ui a la description de Treitschke, ni à la ligure de Duponchel, ni aux exemplaires reçus de Vienne. Leurs teintes sont heancomp plus vives. Le jaune est fortement parsemé de nuances ferrugineuses et le brun passe an noir-roux, foncé. Les ailes postérieures sont en-dessous diun noir fuligineux mat, surtout chez la femelle; cette mème teinte se reproduit anssi endessous an centro du disgue des infierienres, chez cette derniere. Les franges sont diun janne prononcé. La tache subapicale porte sur la còte le point clair indiqué par Frölich ; elle ne se fond proint aree la tache transverse, mais se borne a la toucher de sa pointe. La teinte ferrugineuse des taches ne se répand pas du còté de la marge externe, en sorte que l'aile se termine par un large espace diun jaune d'ochre vif, finement réticuli. La tache elaire de la còte a nue forme triangulaire et non ovoide; elle est anssi deux fois plus grande. Enfin à Aigle, le papillon paraît à la lin de mai et non en juillet, et hante, non les forits de sapin, mais les herbes Je long des chemins, non loin des vignes. Son vol est has, court et assez lourd. Jai lien de croire quil faudra plus tard en faire deux espices.

\section{Steineriana, Mus. Schil.}

Hub. 170. - Hub, revis, n62. - Hub. sup. f, 58 б. 58 ?.Dup. cat. (Sciaphila). - Heyden. cat. 72.

Gnomana, Curt.

Point rare dans les Alpes, parmi les hautes herbes et les taillis de rhododendron. A Meyringen, Ormont-Dessus, ete. (Rothb. - Zell.); la Sandalp (Glaris) à 6000'; lEngadine (Frey); an pied du Mont-Blane. - M. Boll a recueilli sur le Berglsalp (Glaris) des chenilles, vivant sur le Veratrum, qui ont fourni le papillon au cormmencement d'aoùt (Frey).

La femelle est plus rare que le màle; on la trouve difficilement parce quelle vole pen et se cache dans les herbes. Les individus de nos alpes ont généralement une couleur olivâtre plus foncée que la fig. 57 de II.-S. et que les individus provenant du Tyrol.

Yoisine de Dohruiana par la conformation de la femelle, dont les ailes sont étroites, terminées en pointe et souvent à deni avortées.

39. Dohrniana, Man. Catal.

IInl), revis. Nachtr. p. 156. - Heyden. C. 105.

Rogana, Guén. Inıl.?

Cette espèce est fort répandue dans les Alpes et le Jura, sur les pàturages, en jưillet. Parfois elle y est abondante; dautres anées elle est plus rare. La femelle est 
très-rare, parce qu'elle se eacle dans les lierbes et ne vole pas. Ie n'ai pu l'obtenir que par M. Mann. M. Frey a reeurilli aussi cette espece dans les Apes grisonnes.

l.e mâle varie beaucoup pour la taille ot pour la teinte des supérieures. La taille deseend jusqua celle de Rustican a o et peut arriver a cualer celle des grands molividus de Hinistrana. La tointe ollie toutes les nuances depuis le jame pale du laiton au wris-fanve. La forme des superieurs vario moins. La rote est arqué vers la racine de l'aile: mais olle lest moins que dans liburna na ; le reste de son étendue est légirement arque jusques vers le sommet ni quelpuefois alle se relieve un peu. Le somunet fait une légère saillie. Citant compe ì angle faiblement aigu. Le bort externe est un pen oblifune. La coulenr des posterieures est le gris páde ance des vergetures hlanchâtres,

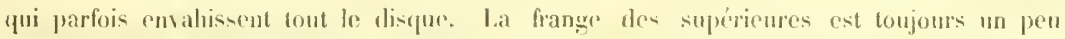
plus claire yue le fond. et celle dow infericures prestue blanche. Ino ligne grise de division la partage fort pròs de la marge. Le dessous des suprerieures ast plus ou moins gris-nuiratre. uni of luisant: relui dles inférienres blane-sale, luisant, aver une teinte jaunâtro vers l'extrómité.

Les antennes sont ciliées rn-dessous. dentieulées de chayur côté; lo dessus est formó alternativoment d'une rangíe de grosses écailles, larges et jaunâtres, suivie dime rangie de petites écailles noires. 'n sorte que we depuis dessus, lanteme paraît étranglée à charpue articulation.

Le pointillé des ailes niest pas frépuent; il est fort peu apparent lorspue la teinte des supérieures est claire, re qui lo distingue de celıi de Lusana.

La fenelle de Dohrniana a les ailes itroites, alongreses. en forme de fer de lan-

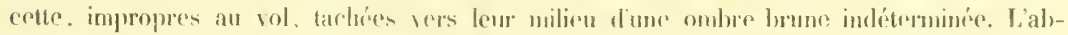
domen est fort gros at les inlériemes pen developproses.

(Voir Lusana, Scrophulariana of Vilumana).

10. Gnomana, Jin.

Fah. Treit. - Fisch. v. R. t. I1. - Dup. 239. - IIuL, revis.

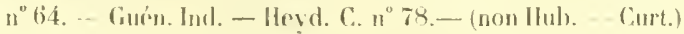

Coslana, IV. I. C.,5.

Livonana, Bull. Mose.

Jai recueilli cette espece dass da vallex de Tzernatten. en-ilessous du village de St-Nicolas, dans les taillis daulne. M. L. Zoller lindiepue dans les anverous ele Zurich.

Les individus que je posside ont tous la tache de la côte diun brun trìs-foncé, rn sorte que le papillon non itale porte une tache ronde foncén an centere ales ailes. Les 
stries et bandes sont beaucoup moins foncées. Les figures de Fisch. v. R. ne leur convienment pas à tous égards.

41. Costana, Fab.

Wood. 866. - Iluh. revis. $n^{\circ} 65 .-($ non Dup. - W. V.)

Gnomana, Hub. 131.

Vineulana, Treit. - Khlw.

Spectrana, Treit. - Huh. sup. f. 32. (non Dup.). - Guén. Ind. - Heydenr. Cat. $107 \%$.

Tres-rare en Suisse II. G. Leresche en a pris un exemplaire dans les environs de Lausame.

Costana Dup. est une espre voisine de Nisana. Sa Spectrana est très-probablement une forme de Parisiana.

42. I djunctana, Treit. sup. 59.

Fisch. v. R. t. 9.- Dup. sup. 61. - Ilub. revis. n 66. - Guén. Ind. - Ileyden. C. n53. -- Zett. - Bruand, C. 926.

Sineana, Frey. n. b. 18. 1.

Reticulana, Fröhl. - (non Hub.)

Laviceana, Dиј). 239, 4 .

Forsterana, Wool. 818. - Fab.

II. Couleru a pris cefte aspece dans les environs de la Neuvevilie et me l'a commusiquée. Rare.

Sur lindividu que je posside, les taches de la còte niesistent pas; celle alı bord interne ast seule risible.

\section{Obliterana, v. Heyd.}

Ilub. revis. " 67 . Ilub. sup. f. 60 ट, 361 \&. Heydenr.

$$
\text { C. } n^{\circ} 83 \text {. }
$$

Productana, Zell.

Unifaseiana, T)up. sup. (non flavina, 1333).-Guén. Ind.-Bruand, C.917?

Jai pris phusicurs fois cette espèce a Paudex près Lausanne, autour des charmilles. Insquici je nai pas pu saisir la fenelle. - Espèce néridionale.

Flavaua Dup. appartient i Neglectana, I1. S. - Consimilana, Iub. 239, ne peut ètre rattachice à Obliterana. 
Les deux taches lorunes qui marquent lextrémité des bandes, sur le borl interne. ainsi fue le prolongement de la bande moyenne sur la cote du còté de la racine de l'aile, font aisément distinguer cette petite espece de ses voisines. La forme alongíe de la tache du sommet est aussi caractíristinue. Un individu resu de M. Ifer.-Sehäffer est un peu plus grand que les miens et numis couvert de brun.

\section{Sorbiana, Hub. 11:3 .}

$$
\begin{aligned}
& \text { Fröhl. - Treit. - Dup. 238. - Fres. n. 1. 49, 2. - Guén. } \\
& \text { Ind. - Inub. mis. } n^{\circ} 69 \text {. Heyd. C. 5\%. - Bruand C. } 912 .
\end{aligned}
$$

Heparana, Mus. Schif.

$$
\text { Avellana, Steph. - Curt. }
$$$$
\text { Rosana, Sehwrz. - Roes. }
$$

Rare dans les environs de Lausanne. Prís de Schüpfen Rothl. : do Zurich (Zell.,. Au pied du Mont-Blane, parni les Rhododendron Lah..

45. Cerasana, Hub. 119.

$$
\text { Treit. - Dup. 235. - Wood. 454. - Hub. revis. } n^{\circ} 69 .
$$

Heyd. C. n 64. - Curt.

Avellana, Fröhl.

Ribeanae, var. Guén. Ind.

Pas fort rare dans les haies de coudrier au-dessus de Lausanne. en juillet. A Schupfen (Rothb.); Zurich (Frey).

Je n'ai jamais vu de transition entre cotte espece et kibeana.

16. Cinnamomeana, Treit.

Fisch. v. R. t. 9. - Dup. Cat. - Hub. revis. 110 70. - Guéu. Int. Heyd. C. $1^{\circ} 56 .-$ Wootl. 851 .

Cruceana, Hub. 120. - Froblıl.

II?parana, Wood. 850?

Les bois au-lessus de Lansanne, en juin "t juillet; assez ram. Pries do sehupfen (Rothb.), de Zurich (Zell.)

\section{Heparana, Degeer.}

Treit. 1)ıр. 238. - IIub. revis. n ${ }^{\circ}$ 2. - Guin. Inul. - Ileyd.

C. 55. - Ciurt. - non Wuod.). - Bruand, C. 914. 
Carpiniana, Ilub. 116.

Padana, Sehrk.

Rubrana, Bull. Mose.

Pasquayana, W. V. - F.?

Cinuamomeana, Wool. 851.

Commune dans les jardins les environs de Lausanne durant tout létó. Sehijpfen (Rothb.); Zurich (Zell. livey).

\section{Ribeana, Hub. 114.}

$$
\begin{aligned}
& \text { Fröl. - Treit. - Stoph. - Dup. 239. - Hub. revis. n" 73. - } \\
& \text { Guén. Ind. - Heydenr. Cat. 62. - Wood. 852. - Bruand, } \\
& \text { Cat. } 915 .
\end{aligned}
$$

Grossulariana. Wood. 853 .

Assez commune dans les jardins de toute la Suisse; en juillet. (Lah., Rothb., Zell., Froy).

Certains individus dont to réticule des ailes est peu prononcé, pewent passer facilement pour Corylana. La pointe jaunatre des inférienres sert à les distingner.

\section{Corylana, Fab.}

$$
\text { Fröh. - Treit. - Steph. - Dup. 23S. - (non Hub.). - Ilub. }
$$
revis. n 75. - Guén. Ind. - Ileyden. C. 61.- Wood. 855. Bruand, C. 916.

Avellana, Charp.?

Textana, Ifub. 115. (non 307).

Pasquayana et Oprorana, Mus. Selif.

Les haies et les taillis de toute la Suisse. Assez communo durant lété. Lah. Rothb. Zell. Frey).

50. Histrionana, Hub. $310,311$.

Treit. Fröht. - Dup. sup. 64. - Hub. resis. n7. - Guén. Ind. - Ileyd. C. 309. - Bruand, C. 929.

Asinana. Wood. 1000 .

Murinana, Iluh. 105. Heyd. C. 91.

Assez fréquente dans les bois de sapin au-dessus de Lausanne, en juillet et en aont. Près de Schüpfen (Rotlıb.). - Varie peu. 


\section{j1. Nubilana, Hub. 111 .}

Frey. n. b. 120, 1. - Wood. 1006. - Dup. sup. 62. - Heyd.

C. 305. Guén. Ind. - Ilub. revis, n 78. - Bruand, C. 1007.

Alniana, Mus. Schif.

IIybridana? Dup. hist.

Sociana, Gurn. olim.

Très-commune du 15 an 20 juin dans les environs de Lausanne, autour des haies daubépine. Vole en plein soleil et souvent en essaims. It est trés difficile de la prendre bien fraiche. Te mème dans les environs de Zurich (Zell. Frey).

La figure et la description de Duponchel pourrait aussi bien se rapporter à Virgaureana.

52. Nusculana, Hub. 98.

Fröhıl. - Treit. - 11u]. 255. - Itub. revis. $11^{0} 79$. - [lub. sup.

f. 53. - Guén. Inıl. - Ileyd. C. 307. - Bruand, C. 1006.

Yiduana, llub. 303 q.

Rare en Suisse. Bois de Sauvabelin au-dessus de Lausanne, sur les chènes. M. Rothenbach la recueillie anssi près de Schiipfen.

La figme de Dup. est fort mawaise.

53. Oxyancanthana, Man. (non Hubn. - Dup.).

Ifub. revis. n80. -- Ilub. sup. f. I61. - Ileyden. C. 306.

Je rapprote a cette espece deux individus, maile et femelle, que jai pris dans un bois taillis, an-dessus de Limsane, in mai.

La femelle répond parfaitement ì la ligure de H.-S., sauf une taille un peu plus lorte; mais ellr porte sur la crite deux doubles rechets distinets. La tiete porte une houpe de poils blanchàtres. Le sommet des supérieures coupé carrément et arrondli, ne permet pas de la rapprocher de seciaph. minorana. Les écailles noires et redressées sont en fort petit nombre.

Le màle est un pern plus grand que la femelle, son dessin est à peine distinct; mais la forme des ailes est la mème. L'anus porte un gros mouchet de poils. Le front est gris-clair. On apergoit en-dessous des rudiments de crochets. Les franges sont grispäle. Les antennes sont très-velues an-dessous et fortement ciliées sur les bords. Les tubercules de l'antenne sont gros et profondénent séparés par dess sillous. 
Jignore pourquoi M. Her.-Schäffer n'a pas reproduit cette espèee dans son Catalogue systénatique.

\section{Gen. Argyrolosa, Steph.}

Tortrix, Treit. Dup. - I ictyopteryx, Steph. Guén.

\section{Holmiana, Lin. 308.}

IV. V. - Fal. - Ilub. 39. - Fröhl. - Treit. - Dup. 241. Hub. revis. n $83 .-$ Guén. Ind. - Heyden. C. 153. - Wood. 874. - Curt. - Bruand, C. 964 .

Cà et là dans les haie's et les bosquets des environs de Lansame; de mème à Schijpfen et près de Zurich (Lah. Rothb. Zell. Frey).

55. Hoffmanseggana, Hub. 150.

Treit.- Dup. 241.- - Ilub. revis. n 86. - Heyden. Cat. nº 151.

Spixiana, Fröhl.

Cruciana, Wood. 873.

Convayan a, Fab. - Wood.1117.--Guén. Ind. - Bruand, C. 967.

Commune dans les haies et les bosquets, en juin. Près de Schïfen et de Zurich (Rothb. - Zell. - Frey). - Les teintes orangées passent du jaune au brun-foncé. M. Bremi a élevé lat ehenillr sur le cormier.

56. Lofflingiana, Lin. 305.

Fab. Fröhl. - Dup. 241. - Steph. - Hub. revis. n $87 .-$ Guén. Ind. - Wood. 1111. - Curt. - Bruand C. 963.

Plumbana, Ilub. 54. - Fröhl. - Dup. 241. - Treit. - Heyden. Cat. 147. Wood. 1110. - Curt.

Eetypana. Ilub. 190. - Ileyden. C. 148.

Les deux formes sont aussi commones lume que lautre dans les taillis et les bois des environs de Lausanne, en juin et en juilhet. - De mème près de Schüpfen et de Zurich (Rothb. - Zell.).

57. Bergmanniana, Lin. 307 .

Scop. - W. V. - Fal. - Jub. 340. - Fröhl. - Treit. - Dup. 241. - Hub. revis. n 88. - Guén. lnd.- Heỵden. C. 150.Wood. 1115 . Curt. - Bruand, C. 965.

Rosana, Ilul. 137. 
Assez commune dans les jardius et les bosquets, en juin et juillet. (Lah. - Rotbl.Zell.). - Varie peu.

58. Forskaleana, Lin. :301.

W. V. - Fab. - Ilub. 1 43. - Fröbl. - Treit. - Dup. 211. -

Woorl. 1112. - IluL, revis. n’ 89. - Gués. Ind. - Ileyden. C. I49. - Curt. - Bruand, C. 966.

Rare antour de Lansame, en juillet. (Iab.). - Moins rare dans les environs de Zurich. (Zell. Frey). - La bande foncér du centre se transforme quelquefois on une grande tache noiràtre oceupant le eentrer de laile du cóté du bord interne.

59. Rolandriana, Lin. 309.

$$
\begin{aligned}
& \text { Fab. - W. V. - Ilub. } 174 . \text { - Treit. - Dup. } 241 \text { - Guén. } \\
& \text { Ind. - Ilub. revis. } n^{\circ} 90 . \text { - Hub. sup.f. } 43,44 . \text { - Heyilenr. } \\
& \text { Cat. } 126 .
\end{aligned}
$$

Inopiana, Wood. 1159?

M. Rothenbach a recueilli cette espèce dans les Ilpes suisses.

La figure de Ilulner est mauraise; celle de Duponchel mécounaissable.

60. Bifasciana, Hub. heitr.

Wood. 1023. - Ilub. revis. n 91. - Ilub. sup. f. 62. - Ilejdr.

Cat. $216^{\circ}$. - Bruand, C. 939.

Apicalis, Westw.

Audouinana, Dup. 266, 5. - Dbłl. - Guén. Ind.

Jai pris deux fois cette espèce fort rave, daus les environs de Lausanne, en juillet. M. le professeur Frey l'a collectéc aussi dans les environs de Zurich, à la mêmo époque. La figure de Duponchel est fort grossière, mais sa description bonuc.

$$
\begin{aligned}
& \text { IV. Gen. Pty choloma, Wood. Guén. } \\
& \text { Tortrix, Treit. - Dup. }
\end{aligned}
$$

61. Leacheana, Lin. 301 .

Fröhl. - Fab. - W. V. - Ilub. 67. - Treit. - Dup. 241. -

IIub. revis, n 92. - Guén. Ind. - IIeyd. C. 123. - Curt. Bruand, C. 968 .

Obsoletana, Stepb. 
Pas commune en Snisse; preferer les montagnes; se trouve dans les Alpes. Pris de Schiipfen (Rothls.), de Zurich (Zell.). -- Juin et juillet.

\section{Gen. T o r I r i x, Wood.}

Tortrix, Anctor. partim.

Ce groupe est fable; Lusana et Rusticana se rapprochent de Cochylis of l'Euchromia; les autres passent ì Lozotenia.

62. Viridaua, Liu. 289.

W. Y. - Fal, - Llub. 156. - Treit. - Dup. 2 i0. - Fröhl. Iuls, revis. n 93. - Guín. Ind. - Iley den. C. 117. - Wood. 8\{4. - Bruand, C. 923.

Var. Suttueriana, W. V. (alis luteis.).

Extrèmement commune sar les chènes en juin; abonde surtout an pied des Alpes. dans les lisu chauds (Rothb. -. Tell.) - La variété ì ailes jannâtres est raı̀re; je la crois tout-it-fait accidentelle.

\section{Palleana, Mazz.}

Treit. - Huls. revis. u 9 4.- Hub. sup. f. 37. 38. — Guén. Ind. -Heyden. C. nं118.- Dup. Cat.

Flavana, Ilub. 157 ?

Cette espice a étí recucillic par. H. Rothenhach dans les Alpes du canton de Berne.Flavana, Ihuh.259, na pas enrore iti prise en Suisse.

61. Intermediana, Man. catal. - Hub. sup. f. 420, 121.

J'ai pris dens indisidus appartrnant ì cefte espece, lans les Alpes vaudoises, sur la montagne de l'trane $(6000 \%$ ), parmi les herhes, en juillet.

Extrimement voisine de Pallean a et ne sen dislingue que par ses inferiemrestein-

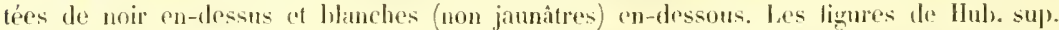
sont tros méliocres. Le sommet des supérieures est trop saillant et leur teinte est trop verdatre. Intermedian a fraîche est jaune-soufre; Palle a na est jaune-paille. Les inférieures sont bien arrondirs sur leur linbe ot non sinnenses vers le sommet conme la figure 420 lindique. 


\section{Rusticana, Treit. sup.}

Fisch. v. Rös. t. 10. f. 2. (exclus. cit. Hub. 102).- Dup. 240.(non Fab.) - Hub, revis. n 96. - Guén. - Heyd. C. $115 .-$ Curt. - Bruand, C. $923 \mathrm{~h}$.

Oehreaceana? Wood. 1113.

Helvolana, Hub. 301, ?.

Pulverana, Evrisiu.

II. Bruand la prend daus le Doubs, I1. Couleru dans le Jura bernois près de la Neuveville, et M. Rothonhach dans les Alpes bernoises. Assez fréquente dans les environs de Zurich, au printemps (Frey). Les bruyires. -- Le mile dilfere si fort de la femelle qu'il n'est pas surprenant qu'on ru ait fait deus espèces.

\section{Viburnana, IV. V.}

Fröb. - Treit. - Zell. 1s. 1846. - Dnp. sup. 61. - Hub. revis. 1"97.- Hul. sup. f. 45, ?. Guen. Ind.

๖. Unitana, Hub. 123.

. Rhombana, IV.Y. - Jus. schil.

Cette espere est tris-facile à confondre avee Dohrniana. Steineriana, Hub. 160, ne peut pas lui appartenir; jai sous les yeux des femelles le eette espéce qui répondent i la figure citée.

Viburnana est fort commune sur les pàturages du Jura a la fin de juillet; le màle vole te soir ef le matin en essaims sur les prairies des bords des bois. La femelle est plus diffieile it dícouvrir, elle se tient cachée dans llıerbe et stationne sur les feuilles basses. - Beaucoup moins frígurente dams les Apes, ou elle est remplacre par Dohrniana.

Le màle varie assez peu en Suisse: il est ordinairement diun faure terne et condré:

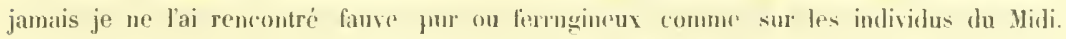
Dans les Apes on trouve des individus roux.

La femelle varie darantage, et pour la forme et pour la coloration. La forme lanecolée des supérieures exprimése dans lit figme 45 Hub. sup. est lextrime de sa, cheformation. J'en ai sous les yeux qui ne different du male, a ret igara, que par une moindre largeur des suprerieures et par le sommet un pen plus saillant. La couleur de la femelli"

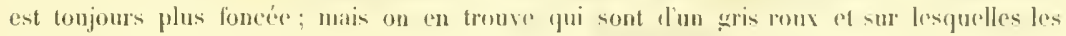
taches du disque ne se volent gur sous un eertain reflet de lumiere. Plus la couleur 
s'approche du brun rouge, plus ces taches se dessinent en brun foncé. Le réticule est habituel ehe\% les fenetles, moins chez le màle.

Dohrniana riest pas toujours si facile à distinguer de Viburnana. Je nai pas su découvir de diflérences dans les antennes. Les supérieures sont eouprés carrément dans la seconde et un peu obliquement dans la première; mais on troure des Dohruiana ayant ees mèmes ailes eoupées presque à angle droit. Cette dernière est toujours un peu

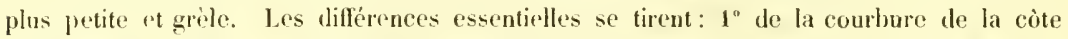
fortement prononéée aux épaules dans Viburnana; $2^{\circ}$ de la présence d'un rétieule qui eirez quelques Dohruiana est remplacé par un ponetué; $3^{\circ}$ de la eouleur des supérieures constamment et unformément moiratre dans Viburnana, tanclis que sa voisine les a plus ou moins vergettées de blanchatre et mème entièrement blanc-sâle; $4^{\circ}$ enfin de la taille ef de la configuration des femelles, plus petites et phlus étriquées que le mâle, dans Dobrniana; plus grandes et pius fortes que le màle dans Vihurnana.

Ces deux espéees, du reste, ne peuvent ètre éloignées l'ume de liautre, encore moins laire partie de deux groupes différents.

\section{Lusana, v. Heyd.}

$$
\text { Hub. sup. f. 330. - Hub. revis. u 99. - Heyd. C. } 127 .
$$

Jai pris un individu appartenant à cette espèee au baut du Col de Jaman, parmi les sapins, en juillet. II. Herrich-Sehälfer, à qui je l’ai présenté, a vérifí sa détermination. Je ne connais que le male.

Lusana est plus petite que Viburnana et Dobrniana auxquelles elle ressemble extrèmement. Ses ailes sont étroites, moins acumines que tlans Dohrniana et moins (arrées que dans Viburnana. Leur couleur est le jaune grisàtre, tirant sur le rosé. Cette dernière teinte provient diun pointilié très-lin, gris-rosé, brillant, qui reecour re toute la surface de laile sans y former de lignes distinctes, comme dans ses congénères.

Les inferieures sont gris de souris uni, avee la frange un pou plus claire. Le dessous est de la couleur du dessus, mais plus elair.

Les antemnes sont dentieulées sur les bords ot tris-villeuses en dessous.

Les tarses des postéricures sont lisses, renflés ret plus eourts que dans les espèces voisines.

68. Seropludariana, Hub. sup. ๔. 401, 405.

IIub. revis. n" 100 .

Lnicolorana, Dup. 240? 
Je rapporte, en liésitant. à cette espéce, un individu que j'ai sous les y ’ux, provenant du St-Gotthard. Ici encore je n’ai vu que le mâle. M. IJerrich-Schäffer, qui l'a exaniné, l'a déclaré très roisin de Scrophulariana.

La taille et la coupe des ailes sont celles de Viburnana. Le sommet est done moins aigu que chez Dohrniana. Cet individu differe de celle-ci: $1^{\circ}$ par la teinte brun-marron terne et uniforme des supéricures, sans points, ni róticule; $2^{\circ}$ par celle gris de fumée et unie, less inférieures, dont la frange est grisâtre et légèrement plus claire que le fond; 3n par le dessous d'un gris roux assez uniforme, plus foné aux supérieures.

Les tarses des postérieures sont lisses (ils sont poilus dans Viburna na ot Dohrniana); les antennes, fortement denticulées sur les còtés, sont très villeuses en dessous.

Du reste, à en juger par les figures de Ilub. sup., Scrophularian a se rapprocherait complètement, ¿̀ part la taille du mảle, de Viburnana telle qu'elle se présente dans notre Jura. La femelle offre une ressemblance frappante. La petitesse du mále s'expliquerait aisément par le fait de son éducation en captivité. La couleur est celle que présente habituellement eette espèce dans les régions moins chaudes.

VI. Gen. Lo p h o derus. Steph. Tortrix, Auctor.

\section{Ministrana, Lin. 286.}

$$
\text { W. V. - Fab. - Fröhl. -- Treit.- Dup. 240. - Guén. Ind.- }
$$

Ilub, revis. $n^{\circ} 101 .-$ Heyd. Cat. $n^{\circ} 100$. - Wood. 1038. Curt. - Bruand, C. 924.

Ferrugana, Ilub. 56. - Dup. 1309. pl. 261.

Var. Livoniana, Dup. Cat. Heyd. C. 106.

Servana, Fal).

Yar. Subfasciana, Wood. - Steph. - Curt.

Commune dans les hois de hêtre et les taillis de toute la Snisse, en juin. (Lah. - R Rthl. Zell.-Frey).

VII. Gen. Xanthosetia. Steph. Guén., etc. Tortrix. Treit.

70. Ha mana, Lin. 29).

W. V. - Ilub. 140. - Treit. - Dup. 25\%. - Hub. revis. nं 103. - Guén. Ind. - Ileyd. C. n 94. - Wood. 1157.Bruand, C. 1143. 
Cruciana, Fab.

Trigonana, W. V.

Var. Divers a n a, Fröhl. - Ilub. 139. - Wood. 1158. - lleyd. C. 95.-Curt.

Sulfurana, Mus. Schif. sup.

Assez commune dans les prés autour de Lausanne, en juillet. Autour de Schiipfen (Rothb.); çà et là près de Zmich (Zell. Frey).

71. Zoeg an a, Lin. 289 .

W. V. - Fal. - Fröhl. - Treit. - Ilub. 138. - Dup. 257.-

Woot. 1155. - Irub. revis. n'105. - Guén. Ind. - Heyd.

C. 96. - Curt. - Bruand, C. 1144.

II am ani, Clorck.

Var. Ferrugina, Wood. 1456 . - Ieyden. C. 97. - Curt.

Assez rare dans les prés des environs de Lausanne; juillet: de mème aux environs de Zurich (Zell. Frey). Phs rare dans le canton de Berne : le Jura, lOberland (Rothb.). - Les Alpes raudoises (Lab.).

VIII. Gen. Eu celia, IIub. verz.

Paedisca, Treit. - Dup. - Tryeleris, Guén.

72. Mediana, Fab.

W. V. - Fröhl. - Treit. - Hub. 179. - Dup. 263. - Hub. revis. $n^{\circ} 106$. - Hub. sup. f. 411. - Ginén. Ind. - Heyden. C. 378. - Zett. - Bruand, C. 1114.

A urana, Fab. 154. (non 22.) - Wood. 936. - Steph. - Curt.

A urantiana (rar.), Koll. - II.-S. 411.

Fulvana, F. sup).

M. Rothenbach a pris cette espèce dans les environs de sichüpfen. H. Vögeli, collecteur zélé, en a recueilli un exemplairo au Burghö̈lzli près de Zurich (Frey). M. A. Forel mien a remis un exemplaire collecté à St-Pré dlans le voisinage de Morges. - Très rare.

IX. Gen. Ablabia, Steph.

Aphelia, Curt. Guín. - Argyroptera, Dup. - Tortrix, Treit.

73. Gouana, Lin. 318.

Fab. - Fröhl. - Treit. - Dıp. 259. - Guén. Ind. - Ilub. revis. $\mathrm{n}^{\circ} 107$ - Ileyden. C. $\mathrm{n}^{\circ} 156$. - Bruand, C. 1145. 
Argentana, W. V. - Hub. 86,

Magnana, Ilub. 225, 226.

Margaritalis (Pyral.), llub. 48. - Dup.

Commune sur les pàturages humides des hasses montagnes en juillet. Préfére les lieux tonrnés au nord. (Lah., Frey, Rothb., Zell.). Descend jusqu'an bois de Sauvabelin, près de Lausanne (Lah.).

\section{Pralana, Hub. 227, 228.}

Frohl. - Treit. - Dup. 259. - Guén. Ind. - Hub. revis. no 110.

IIeyd. C. n 155 . - Bruand, C. 1147.

Quadripunctana, Haw. - Steph. - Curt.

Boreana, Zett. lapp.

Cantiana, Curt.

Osseana, Seop.

Extrèmement fréquente sur toutes les Alpes, en juillet et en aoùt; prófere les pâturages sees. (Lah. - Zell. Frey. - Rothb.).

La femelle diffire du mảe par ses ailes plus étroites et plus aigues; elle est aussi beaucoup moins commune. On la prendrait pour un insecte différent, n'était le pointillé distribué en rayons suivant la direction des nervures dont le thảle porte des traces.

Cette forme des ailes rappelle la díformation analogue que subissent les femelles de Steineriana, de Viburnana, de lohrniana, dlAlpicolana, etc, toutes espèces alpines. - Je doute toujours très-fort que la Pratana de Nornandie appartienne à la mème espèce (Guén. Ind.).

\section{Gen. Eupacilia, Wood. Sphateroptera, Guen. - Coceyx, Dup.}

75. N/picolana, Huh. 328, 329 б. Treit. sujp. - Dup. - Guenn. Ind. - IIub. revis. n¹19. llub. sup. fig. 395 9. - Heyd. C. 278.

Cia et la dans les hautes Alpres du Valais (Anderg.), de J lingadine (Zell., Frey), de loberland (Ott, Rothh.). Collectée sur lEngelberg, près dOlten, par H. Wullschlegel.

llibner a figuré deux mảles, et non les leux sexes comme l'estime Treitschke.

Lorsque les ailes sont un peu effacées, le dessin s'altère très-promptement et les stries décailles jaunes, noires et blanches sont difficiles à suivre. La partie externe de 
la còte est souvent très-marquée de taches blanches, qui s’étendent, dans quelques eas, sur le disque en forme de bandes semblables à celles dHistrionana. La taille varie souvent; les plus grants individus atteignent celle de Pratana, les plus petits eelle d'Ambiguana. Le mâle est très-fréquent daus les lieux qu'il habite; la femelle y reste toujours fort rare, parce qu'elle ne vole pas.

Se pose sur les rochers et les pierres; n'est pas facile a saisir (Frey).

La figure de Dup. est mauraise.

\section{Gen. Cochylis, Treit.}

Eupacilia, Stenodrs, Chrosis, Argyrolepia et Cochylis, Guen. - Tortrix, Treit. partim. - Euprecilia, Argyrolepia, Steph.

76. Decimana, W. $\mathrm{V}$.

Huls. 145. - Fröhl. - Treit. sup. - Dup. 263. - Guéu. Ind.

Hub.revis. n” 120. - Heyden. C. 134. - Bruand, C. 1137.(non Wood.).

Allionana, Fab.

Sur le Jura; assez rare. (Rothb.). - Sur le Chasseral, le 22 juin (Couleru). Près de Zurich, rare (Zeller). Quelquefois sur les Alpes; fin de juin; rare (Lah.). - Neyringen (Ott). En mai et juin sur une prairie au milien des bois de l'Uetliherg, près de Zurich (Frey).

77. Tesserana, W. V.

Trrit. - Dup. 258. - Guén. Ind. - Ilub. revis. $n^{\circ}$ 121. - Ilub. sup. f. 327. var. - Heyden. C. 133. - Wood. 1124. fig. 424. 425. - Bruand, C. 1138.

Tesselana, Hub. 144. - Curt.

Heiseana, F. - Froihl.

Gilvicomana, Fröhl.

Fréquente dans les environs de Lausanne, sur les pâturages exposés au soleil, tout l'été. Assez rare dans le canton de Berue: à Langnau, Aarberg, etc. (Rothb.) - Envi- . rons de Zurich (Zell.). - lo mảle est volontier, plus petit. - Deus générations (Frey).

78. Rutilana, Hub. 219.

Fröhl. - Guén. Intl. - Hub. revis. n 122.—Heyden. C. 136.I) up. Cat. - Bruand, C. 1142. 
Sanguinella, llaw.

Sur les paturages des Mpes en juillet. Jai pris un individu au pied de la Dent-duMidi (Bas-Valais) qui répront parlaitement à la ligure de IIubner, mais avec la frange pointillée de rouge. Deux autres individus, pris sur la Furea, ont les taches jaunes hien plus grandes que les rouges et la frange jaune dor. M. le prof. Frey l'a prise à Tzermatten et Y. Bruand dans le Jura, sur le Nont-d'or.

79. Aur ofasciana, Mann, Verhandl. d zool. bot. Ver. Wien, 1855. Valdensiana, Her.-Schiaf. nexe Schm. f. 33.

M. Pfilfenzeller a díconvert ecte nouvelle espèce sur les Alpes de l'Engadine en mème temps que $\mathrm{H}$. Ott, de Mleyringen, sur eclles de l'Oberland. Il. Iler.-Schäffer l'a dessince et clénommée daprès les individus tronvés par e'ux. Je n'ai un que deux màles.

Elle a le facies, la taille et le dessin dr Rutilana; mais elle en differe par la disposition des bandes brunes at par leur bordure métallique, brillante et hleuàtre.

Les supérieures portent trois loandes diun brun orangé at trois d'un jaune doré pur. Les trois premìmes sont situées l'une it la base, courte et peu visible, la seconde, au premier tiers de l'aile; celle-ci fait un coude en dehors et un léger sinus dı côté de la hase de l'aile; la troisième, plus large, placie aux deux tiers de l'aile, est eourbée en sens contraire de la précédente, de telle sorte que la baude jaune yui les sćpare se tronve étranglée dans son milieu. Des trois bandes jaunes, la premiere (basilaire) est plus étroite. Laile est terminée par un trait brun orangé qui sétend sur la frange; colleci est d'un janne orangé intense dans son milien. Chaque baude brune est linnitée, de part et lautre, par une rair métallique, bleuâtre et brillante qui en coure près du tiers. - Les inférienres sont noinatres: len frange est longne, marguce de fauve dans son milien.

le. dessmus est gris-noiratte ; an travers de hui se distinguent quelque peu les bandes jaunes du dessus. La frange tles suprérieures est mangée; celle des inférieures est grisblanchitre, ainsi que l'extrénití voisine du disques.

Trite it forselet loruns, anec quelques taches orangries. Alsdomen noiraitre terminé par quelques poils faures. Autennes brunes, fortement dentelées sur les lords et trèstomenteuses en dessous. L'extrémité des pattes est gris-fauve.

Mal. Maun et Lederer ont les premiers rerueilli cette espece sur les Alpes du Tyrol, dis 1844. La figure et la deseription que le premier entomologiste en donne, couvienuent parfaitement à l'espiece suisse. 
80. Zephyrana, Treit.

Dup. sup. 65. - Hub. revis. $n^{\circ}$ 126. - Hub. sup. f. 60 - if.-

Guén. Ind. - Heyd. c. 665 b. - Bruand, C. 1141.

Cembrella (Tin.) Mus. Schif.

Pas très-rare dlans les endroits chauds et sees des environs de Lausanne; sur la route d Aigle aux Ormonts, en juin. - Her.-Schäller en distingue cinq variétés. Jai observé la jaune et la grise, et jai de la peine à croire quelles ne forment pas deux espèces, comne l'aflirme II. Mann.

81. Perfusana, Fisch. . Rösl.

Hub. revis, n"127. - Ilub. sup. f. 24i, 245. - Guén. Ind. lleyden. C. 168.

Clatlurana, khiw.

Les pâturages des Alpes moyennes et inférienres, au borll des bois, en juin. Col de Jaman; Panex all-dessus d'Ollon.

Les individus que je possèdle n’offreut aueune trace des stries transverses indipuées sur les ailes inférieures dans les figures de Hub. sup.

\section{Baumanniana, Fab.}

W. Y. - Fröhl. - Treit. - Dup. 258. - Hub. revis. $1^{\circ} 129 .-$

Guén. Ind. - Heyd. C. 138. - Wood. 1154. - Curt. Bruand, C. 1140.

Hartmanniana, Clerck (non Lin.). - Hub. 146 (non 148). - Hub. beitr. I.

Lutosana, IHub. 200?

Avellana, Hus. Schif.

Commune sur les pảturages en juin; sélève sur les Alpes, ou elle parait i la fin de juin et en juillet. - Fréquente en Suisse. (Rothl), Zell.. Frey). - Varic pour la taille.

\section{Lutulentana, Her.-Sehäfr. n. Schm. f. 35).}

Javais déja trouś cette espèce au mois de juillet 1854 au pied de la Dent-du-Midi, dans le Bas-Valais; mais lindivilu que je pris alors était en trop mausais état pour le déterminer. En 1855 jai été plus hrureux et jai pu en collecter. is la mème éporgue, un autre au pied de la Tour dii, à $7000^{\prime}$ de hauteur.

Pour le dessin, la taille et le facies, cette espece ressemble à une Ba umanniana, extrèmemont fanée et effacée. Lindividu màle sur lequed cette description est prise étant très- 
frais, l'erreur n'est pas possible; dailleurs le dessin offre des différences. La couleur générale est le gris un peu verdàtre, marloré de stries brillantes presque argentées et de taches brun-jaunâtres, les unes et les autres pàles.

Le prenier tiers de l'aile supérieure qui se termine à la bande transverse est d'un brun olivatre à la base do laile et linité en dehors par deux traits irréguliers, argentés, éloignés vers la còte et se réunissant avant d'atteindre le bord interne. La bande moyenne, plus large que dans Banmanniana, est moins distinctement brisée dans son milieu; sa couleur est le gris roux un peu ferrugineux; un trait argenté irrégulier orne ses bords de part et d'autre. Deux taches carrées existent á l'extrémité de la còte; la plus externe est fort petite et séparée de sa voisine par un trait argentí (qui se dirige vers l'angle interue en formant un léger sinus et longeant la frange. La première tache carrée est aussi limitée en dedans par un trait argenté qui se dirige, parallèlement au précédent, vers l'angle interne et l'atteint. Ce trait sert aussi de limite à une grande tache triangulaire, de la mème couleur que la bande moyeme, qui existe entre elle et l'angle interne. Cette tache dans Baumanuiana est beaucoup plus petite et ne s'appuye pas sur le borl interne. La frange est d'un blanc sàle, divisée par un trait gris, interrompu ainsi que l'extrémité de la frange, par cinq mouchetures grises.

Les inférieures sont d'un gris de souris uniforne, avec la frange blanchàtre, divisée par un trait gris.

Le dessous est uniformément noirâtre, avec la frange blanchâtre et entrecoupée, aux supérieures. Les inférieures sont en-dessous conme en-dessus.

La tite et le corselet sont gris et les palpes de mème; leur dernier article se prolonge en pointe noire. Les antennes, noires en-dessus, sont fortement ciliées en-dessous. Labdomen est gris, terminé par un mouchet plus clair. Le dessous de l'aldomen et les pattes sont de la couleur des inférieures.

84. Smeathmanniana, Fab.

Fröhl. - Treit. sup. - Dup. 258. - Hub. revis. n 134. Guén. Ind. - Heydenr. C. 659. - Wood. 1151. - Curt. Bruand, C. 1131.

Fabriciana, Hub, 149. - Curt.

M. Rothenbach indique cette espèce prìs de Schüfen et M. Zeller dans les environs de Zurich. 
85. Rubigana, Treit.

Dup. cat. - Hub. revis. $n^{\circ} 135$. - Ileyd. c. 146.

Badia na, Fröhl. - Ilub. 147. - Wood. 1153. - Curt.

I. le prof. Frey mécrit que cette espèce u'est pas très-rare en mai et en juin dans les environs de Zurich, quoiqu'elle y soit peu répanduc.

86. Jucundana, Treil. sup.

Dup. 257. - Fisch. v. Rösl. t. 40. f. 2. - Ifub. resis. n 138. IIul. sup. f. 82. - Guéu. - Ileyden. C. 671.

I. Zeller a pris cette espece dlans te Tessin.

87. Rubellana, Mus. Schif.

Hub. 285. (non 286). - Treit. - Dup. 258. - Guén. Ind. Ifub. revis. n¹41. - Ileyd. c. 674. - Bruand, C. 1129.

Cilia n a, Fröhl.

Ciliella (Tin.), Ilub. 180.

Roseana, Woodl. 1142 .

Ruficiliana, Curt. - Haw.

Dilutana, Mus. Schif. - W. Y.

Permixtana, Ilus. Schif.

II. Rothenbach a recueilli cette espèce dans l'Oberland. - Je lai reçue des environs de Frauenfeld et lai prise dans les Alpes du Bas-Valais. Fréquente dans les environs de Zurich, où elle apparaît une première fois en avril et en mai, puis une seconde en juillet. Se prend aussi dans les Alpes de l'Engadine (Frey).

88. Dipsaceana, Fisch. v. Rösl.

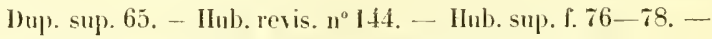
Guén. Ind.-Ieyden. C. 672 - Bruand, C. 1128.

Rubell an a, Ilub. 286, 297 .

Subroseana, Wool. 1143 . - Haw. - Curt.

Prise dans les euvirons de Schüpfen par .ll. Rothenbach. - Cette espèce varie par la teinte plus ou moins rongeàtre de l'extrémité des ailes; elle n'est pas facile à distinguer d'Epifinana, toujours plus påle sur le mème point. - Dans les environs de Zurich, au mois d'aoùt, je laai trouvée en abondance sur une place découverte au milieu des bois où croissait l'Arctium lappa (Frey). 


\section{Humidana, Fisch. v. Rösl.}

Ilub. revis. $n^{\circ}$ 146. - Ilub. sup. f. 86, 87. - Ileyd. C. n००10. Bruand, C. 1126.

Marginata, Steph. Cat.

Jai pris une fois cette espèce à l'embouchure du torrent à Paudex près Lausanne; mi-juillet.

90. Mussehliana, Treil. sup.

Ilub. revis. $n^{\circ} 147$. - 1lub. sup. f. $88-90$. - Dup. sup. 62. Guén. Ind. - Ileyden. C. 667.

Permixtana, Mus. Schif. - Ilub. 18\%.

Près de Zurich (Zell.). Au-dessus de Latisanue, dans un pré très-humide, mi-septembre; à Cour, au bord d'un marais, au-dessous de Lausanne, en juin. A Aigle, dans un pàturage sec, is la fin de mai.

Varie suivant les localités. Peut-être une ou deux espèces listinctes. Les individas recueillis en juin dans un marais sont inarbrés de fauve avec les taches claires peu brillantes. Ceux pris en septembre ont les clairs plus marqués et presque nacrés. Ceux diggle sont encore plus nacrés et ont les taches brunes de la còte nettement limitées, ce qui n'a pas lieu chez les premiers.

91. Phaleratana, Fisch. v. Rösl.

Ilub. revis. $\Pi^{\circ}$ 149. - Hub. sup. f. $8 \dot{4}, 85$. - Heyd. C. $n^{\circ} 670$.

Très-commune dans une for̀̀t près de Zurich, en juin (Frey, Zell.).

\section{Posterana, Hoffmsg.}

Hul. revis. n" 151. - Ileyd. C. 688, - Zell. lsis. 18.17.

Ambiguana, Treit. - Iluls, sup. f. 64.

Minorana, l'ritw. Ent. Zeit.

Variegana, W. I.?

Angustana, Ilub. 74 .

Fasciella? Don.

M. le prof. Frey la prise dans les environs de Zurich ou elle est rare. Lne fois pris de Sion, au milien d'anoùt (Lah.).

La ligure if de llibmer est bien inexacte. Carduana Guén. Cat. p. $5 \dot{f}$ me peut se rapporter i cette espece, puisque eet entomologiste dit «Il ohe n w a rtianae affinissina». 
93. Carduana, Zell. Isis. 1847, p. 741.

Hub. revis. n 152. - Ileyden. C. 718. - (non Guénée).

II ybridell a, (Tin.) Hub. 351. - Guén. Ind. p. 60. (excl. synon.). - Bruand, C. 1121.

D is solutana, Fisch. v. Rösl. - Heyd. C. n 659. - Ilub. sup. f. 83.

Prise plusieurs fois ì Paudex, près Lausanne, à la fin de juillet.

94. Pallidana, Fisch. v. Rüsl.

Zell. Isis. 184i. - Ilub. revis. n 154. -. Ilub. sup. f. 389. (non $65) .-$ (non Heyden.)

Impurana, Khlw. - Heyden. C. 682.

Dans lEngadine et aux environs de Zurich; pas rare dans cette dernière localité. (Frey). - Fréquente sur les pàturages des Alpes vers la fin de juillet. Les clairières des bois du Jorat au-ilessus de Lausanne. en mai (Lah.).

Vole en plein soleil autour des fleurs.

M. Je prof. Frey ma communiqué un individu extrèmement voisin de Pallidana, pris dans les environs de Zurich, à ce que je présume, que II. Herrich-Schäffer envisage comme une espèce différente. Si les caractères quill présente se reproduisaient sur d'autres individus, je me rangerais volontiers à l'opinion de ce Jernier; mais leur importance ne me paraît pas suffisante pour motiver une distinetion spécifique. Le facies, la taille et le dessin sont ceux de Pallidana, à lexception du tiers postérieur des supérieures. Dans Pallidana, cette portion de laile porte une tache transversale, marbrée, déchirée, semilunaire, d'un gris foncé mèlé de jaune, formant un sinus profond du côté de la base de laile. Dans ce sinus vient se loger une autre tache irrégulièrentent arrondie, d'un jaune pàle. Lindivida que possède M. Frey porte la tache semilunaire coupée perpendiculairement du còté de la base de laile et s'appuyant de ce côté contre une tache pàle, oblongue transversalement, qui n’en est séparée que par uu filet blanc. Dans Pallidana, la distance des deux taches est plus forte. Si cette dervière offrait moins de variations qu'elle n'en présente habituellement chez nous, joserais plus hardiment me prononcer sur ce point.

95. Purgatana, Treit. sup.

Hub. revis. n 156. - Hub. sup. f. 81. - Guén. Ind. - Dup. sup. 65 . 
Cà et là, toujours rare et isolée, dans les environs de Lausanne et d'Aigle, en mai et en juin, au milieu des herhages.

\section{Cruentana, Fröhl.}

Hub. revis. $n^{\circ} 159$.

Angustana, Treit. - Hub, sup. f.94. - Guén. Ind. - Heyden. C.693. Dup. 257. - (non Inbn.)

Sur les montagnes d'Aigle au milieu de juillet; rare. Près de Zürich, à la fin de juillet; rare (Frey),

97. Dubitana, Hub. 71.

Fröhl. - Treit. - Dup. 258. - Ilub, revis. $n^{\circ} 160$. - Guén. Ind. - Heydenr. C. 697. - Wood. 1138. - Curt.

Sur les Alpes randoises; la Furca; dans le nilieu de juillet; rare. M. Rothenbach la cite près de Sehüpfen et M. C. Zeller dans les environs de Zürich.

\section{Ambiguana, Fröhl. 112.}

Zell. Isis. 1847. p. 742. - Hubn. revis. $n^{\circ}$ 161. - Heydenr.

C. $686 .-$ (non Dup.).

Pallidana, Hub. sup. f. 65. (non 154).

Pumilan a, Hub. sup. f. 66. - Heydenr. C. 700, 701. - Kulılw.

Sodaliana, Wood.?

Jai reçu de M. Heuser à Burgdorf un exemplaire appartenant à cette espèce.

Ambigu ana, Duponchel, se rapporte ì Penthina simplana, Fisch, v. R.

99. Gratiosana, Lah.

Deux individus, l'un mâle et l'autre femelle, appartenant à cette nouvelle espèce, ont été pris, dans les environs de Lausanne, par M. G. Leresclie et par moi, an mois d'aoùt. - M. Iler.-Schäffer qui vit l’un d'eux, reconnut une espèce non dícrite.

Au prenier abord, ee petit cochylle se distingue à peine de Dubitana. dont il a le dessin et la teinte jaune enfumée. Cruentana lui ressemble encore darantage, mais sa taille est beaucoup plus forte. Sa taille est sensiblement plus petite que celle de Dubitana; semblable à celle de Zephyrana, Le fond des supérieures est d'un jaune påle, brillant, plus ou moins argenté; sur tui se dessinent des taches irrégulières d'un jaune-roux, dont la plus grande, arrondie, est placée sur le disque, au tiers externe de l'aile. Au centre et à l'extrémité se voient deux taches d’un gris foncé, nuancées de 
violet et tachetées du mème jaune roux. La tache médiane, cunćiforme, plus large et plus visible à la còte, se termine en pointe au bord interne. Sa partic interne est violacée; lexterne roussàtre, forme un angle saillant près de la còte; un point plus foncé existe à son ceutre et sur son bosd antérieur. - La tache terumale a la forme diun croissant, à coneavité interne. La partie concave porte un trait recoumé et alongé, se terminant en pointe effilée du cótí de langle posterieur et par une tache brune a la còte; cette demière tache, déchirée à son bord externe, est brun-foncé mèlé de fanve. Le soumet de l'aile est violet foncé, tacheté de gris. La frange est violette, milée de grisnoir et de roux. Le sommet de laile est très-arrondi. La còte est noiràtre dans toute sa longueur avec 3 à 4 taches blanches.

Les inférieures sont d'un gris un peu violet, sensiblement plus clair à la base, surtout chez la femelle. La frange est de la mème couleur, avec une ligne de division plus foncée.

Le dessous est d'un gris-roux plus foncé aux supérienres, surtout vers le sommet; marqué de vergetures aux inféricures.

La tète et les palpes sont blancs.

Il existe encore une autre espèce inédite que l'on pourrait confondre avec eelle que je viens de décrire; elle se distingue par sa taille plus forte, par la tache centrale plus grande et triangulaire, la terminale plus marbrée de clair et celle du disque, vers langle postérieur, brune, foncée. Je me suis abstenu de la décrire, quoique nouvelle, parce que je manque encore dindividus en bon état. Elle provient des environs de Sion, où je l'ai prise an mois d'aoùt.

\section{Roserana, Fröhl.}

Treit. et sup. - Dup. 257. - IIub. revis. n० 162. - Hub. sup. f. 93. - Heyden. C. 690. - Wood. 1288. - Bulletin de la Sociéti vaudoise, I. III, $\mathrm{n}^{\circ} 22$.

A mhiguolla. (Tin.) IIub. 153. - (ama). Guén. Ind.

Uvacana, Schenk.

Omphiaciana, Bruand cat. - (ella). Audoin, hist. de la Pyrale. - Bruand, C. 1122 .

Titisella, (Tin.) Bechst.

Cette espèce est extrèmement répantue dans tous les vignobles de la Suisse et $\mathrm{y}$ cause, certaines années, fle grands ravages. Sa larve vit sur les grappes. Deux appa- 
ritions; la première à la fin de mai et au commencement de juin; la seconde à la fin de juillet. La chrysalide, enveloppée d'une coque làche, est fixée sur les pierres et les morceaux de bois, quelquefois dans les débris de grappes desséchées. - Ne varie pas.

Pour plus de détails sur la synonymie et les mours de l'animal, consultez le Bulletin de la Société vaudoise des sciences naturelles, t. III, n²2, p. 41, et t. IV, n*36, p. 284.

Treitschke. en copiant l'article fourni par II. de Roser an Correspondensblatt wurtembergeois 1), a émis quelpues erreurs qui ont été reproduites ailleurs.

La première éclosion n'a pas lien dans le eanton de Yaud, mème dams les endroits les mieux exposés, en avril ; mais à la fin de mai et au conmeneement de juin, lorsque les bourgeons de la vigne ont de 3 à 10 ponces de longueur. De lá vicut que lorsque la lloraison est hàtive, la larve trouve les grains dija formés et les ronge un à un, faisant alors moins de mal au raisin.

Le seul moyen populaire de détruire cet insecte nuisille est celni recommandé par v. Roser: écraser la larve dans les grappes en lleur; on écrase aussi quelques chrysalides dans les paquets secs laissés par la larte. Rechereler les cocons sur les grappes en automne pour les détruire, comme le recommande linstruction badoise, pulliée à Constance en 181I, est parfaitement impraticable. L'emploi de feux allunés le suir au bord des vignes n'est pas moins inutile; l'imsecte ne vole pas de nuit, mais le matin et le soir, lorsquil fait chand; tout le jour si le temps est couvert.

XII. Gen. Ch e i mo no ph ila, Dup. (Tinei.) Lemmatophila, Treit. - Exapate, Zell. Guén.

101. Gelatana, (ella) Lin.

Fab. - IInb. 266. - Treit. - Dup. 287. - Frey. - Hub. revis. " 165. - Guén. Ind. (Tin.) - Ileyd. C. n² (Tin.). - Frey, Tineïd. helvet. p. 5 .

Gelata, Ilaw. - Fab. (Lithosia).

Congelatella, Clk.

Dans une haie au-dessus de Lausanne, à la fin doctolre et au commencement de novembre; assez frécuente. - Ilottingerlserg, près Zürich (Brenii).

Cefte espece se trouve déja inserite dans la Faune suisse par M. Ie frof. Frey qui la place parni les Tineides.

1) Correspondenzblatt des wirtemb. landwirthsch. Vereins. Vol. XVI; December 1829. p. 299. 


\section{Gen. Phtheocroa, Steph. Guén.}

Sciaphila, Treit. Dup.

\section{Rugosana, Hub. 82.}

Fröhl. - Steph. - Treit. - Wood. 1141. - Dup. 256. - Ilub. revis. $0^{\circ}$ 166. - Hejden. C. 257. - Guén. Ind. - Bruand, Cat. 1004 .

$\mathrm{V}$ - Albana, Don.

Prise une fois près de Lausanne, en juin; reçue aussi de la Suisse allemande. M. Frey l'a recueillie plusieurs fois dans l'Engadine; les échantillons qui en proviennent different quelque pen des autres.

La chenille vit sur la Bryonia dioiea.

\section{Gen. Sciaphila, Treit. Dup. Guén.}

Cuephasia, Steph.

103. Rigana, Treit. sup.

Bullet. mosc. - Hubu. revis. n 172 . - Heydenr. C. 651. Guén. Ind.

Modestana, Treit. (non Dup.)

IIorridana, Ilul. 327. - Dup. 256. - Fröhl.

Trifasciana, Fab.

II. Rothenbach l'a prise près de Schüpfen; je l'ai reģue de M. Anderegg.

104. Stramentana, Guén. Cat. p. 33. - Bruand, Cat. n 1018.

Cette espéce, qui parait propre à la France, a été prise sur le Jura, au Weissenstein, par M. Rotheubach. le 24 juillet 1856. Des iudividus, déterminés par Guénée et que M. Bruand m'a cotimuniqués, sont parfaitement identiques. Je n'ai vu que le màle.

La taille et le facies sont ceux J'Albulana dont elle est voisine: elle rappelle aussi Terreana et Plumbana, llub. 54. Le fond des supérieures est blanc-jaunatre; sur lui se dessinent un grand nombre de taches et d'atomes fauves. Ces taches sont plus nombreuses à la còte et brunes; à l'extrémité de l'aile il en existe souvent une plus grosse; les autres ont la forme de stries ondulées, transversales et irrégulières. La frange est de la couleur du fond et divisće par une ligne fauve. Les inférieures sont gris-de-fer uni et leur frange est blanc-sale, striée de gris. 
If dessous est entièrement gris-clair, marbrí de jaunàtre vers lextremité des supérieures et de blanchàtre au sommet des inférieures.

Palpes, tête et thorax de la couleur des supérieures. Abdomen eomme les inférieures. Inisant. Anus fauve.

105. Albulana, Treit. sup. III. p. 85.

Huh. sup. f. 190, 191. - Hub. revis. n 174. - Heydenr. Cat. $n^{\circ} 273$.

Hybridana, Jlub. 238. (non Treit.). Guén. Ind. - Bruand C. 1017.

Nodestana, Dup. 256, 6 .

Curvifasciana et Rectifaciana, Wood. - Curt.

Tres-commume en juin et juillet, aux environs de Lausanne, dans les taillis, les haies. "t sur les sapins. - De mème près de Schüpfen (Rothb.) et de Zurich (Zell.).

La vraie Hybridana de Treit. sup. n'a pas encore été prise en Suisse; un inctividu yue jai sous les yeux, provenant de Vienne, ne laisse aucun doute à cet égard. Il pst bien probable que Modestana Dup. appartient i notre especce, car elle est fréguente en France. Hybridana Hub. est douteuse.

Albulana a les landes claires, transversales, d'un blanc safe, pointillées datomes brums; les bandes foncées lauve-clair, passant quelquefois au noirâtre. La première bande blanche nest jamais plus claire que les autres, ni la seconde noire plus foncée, comme dans Hybridana. Le facies et la taille varient quelque peu; tantòt le blanc. tantòt le fauve dominent sur le fond. Le dessous des inférieures est plus ou unoins moucheté vers le sommet.

Jai reçu du Jura et de France une variété plus débile, dont le bline passe au grim de souris clair, et les bandes brunes sont moins fortement dessinées; comme il n'exister pas d'autres différences, je n'ai pu ladmettre comme espèce distincte.

\section{Bellana, Curt.}

Diurneana, Guén. Ind. - Heyd. C. 303.

Var. Penzianae, Jlub. revis. et auctor.

Penziana, Steph.

Pas rare dans les sous-alpes, le long des hois (Lah., Rothl., Zell.,. Samaden, flams lEngadine (Frey). - Je ne sache pas quelle ait etí prise juscuuici dans Je Jum, oin Penziana n'est pas très-rare.

C'est avec raison que H. Guénée a séparé cette espèce de sa voisine; mais je ue 
saurais établir le diagnostic dans les mêmes termes que lut. Aucun des caractères qu'il indique n'est constant, à part la couleur noire des taches et la teinte grisàtre des inférieures en-dessus. - J'en ai vn plus d'une dixaine d'exemplaires, tous semblables, et jai trouví quici le facies, comme en général dans les Sciaphiles, est le meillenr des earactères distinctifs, lorsquion possède un nombre suffisant dindividus.

Le màte diffère quelque peu te la femelle; il a les ailes inférieures parfois presque blanches - quoique jamais autant que Penziana - et chez lui les mouchetures du dessous de ces mèmes ailes manquent.

La coupe des ailes et leurs dimensions sont les mèmes dans les deux espéces voisimes. Les supérieures sont plus ou moins étroites, plus on moins coupées en biseau iII sommet, comme dans Wahlhomiana. Bollana a constamment les taches et les bandes transverses plus grandes et diun noir pur, tandis que Penziana les a brunes et plus petites. Leur distribution est, du reste, la mème. - La frange, sur quelques individus, est légèrement entrecoupée de grris, aux supérieures. Les inférieures, surtout chez les femelles, sont en-dessus d'un gris-noiràtre plus ou moins foncé; en-dessous elles portent souvent, rers le sommet, quelques vergetmes grisàtres. Labdomen est gris. annelé de blanc. Les palpes sont légèrement plus longs que lans Penziana; leur drrnier article est gris-noiràtre, avec l'extrémité blanche. (Voir Penziana).

\section{Penziana, Hub. 85.}

Fröhl. - Treit. - Dup. 256. - Hub. sup. f. 117, 118. - Hub. revis. $\mathrm{n}^{\circ}$ 176. - Guén. Ind. - Ileydenr. C. 299. - Wood. 996. - Bruand, C. 1016.

Octomaculana, Haw. - Curt.

Cette espèce ne paraît exister en Suisse que dans le Jura, où elle a été prise par III. Couleru, Rothenbach et Heuser.

La description de Treitschke ne se rapporte quà elle et non à Bellana. La figure de llubner (assez inexacte) lui appartient aussi exclusivement.

Le blanc tire un peu sur le roux, surtout aux inférieures et sur labdomen; daus $\rightarrow$ a roisine il est manifestement bleuàtre.

Herr.-Schäf. a ligmré les palpes de cette espèce dans ses tables sur la nervation Microlepiul. VII. f. 47. Le dernier article est gris-roux à la base et blanc au sommet: mais il est près de moitié moins alongé que dans la figure. Je présume que ce dessin a ité mis sur Bellana plutòt que sur Penziana. 


\section{Candidana, Lah.}

Les rhemins des vignes de Lavaux, à la fin de juin: on la trouve sur les horles it appliquée contre les murs du coté de lombre. J'en ai reçu un exemplaire pris dans les environs de Burgdorl prar M. Heuser.

Cette espèce, très-roisine de Styriacana, H.-Sch., se place entre Penziana et Chrysanthemana, pour le dessin. Sa taille et sa coupe sont celles de Wahlbomiana On ne peut la confondre quavec Styriacana, dont elle est très-roisine; elle sén distingu an premier coup-d'ocil par l'espace blanc, compris entre les denx premieres bandes noires, plus large surtout du côté llu bord interne. Les supérieures sont diun blanc de lait pur. semé de points gris; dans Styriacana le blanc tire un peu sur le bleu. Trois bandes grises traversent laile obliquement; la première (basilaire) nest hien nette que dans son milieu; la seconde, formant trois coudes, est élargie à la côte, étran glée (mais non interrompue comme dans Styriacana) à la cellule, puis étendue en forme dombre du còté de la urarge; elle est done plus large et plus marquée que dans sty riacana. La troisieme hande, vers le sommet, est constante, et se ternine en pointe vers les trois quarts de la frange; dans Styriacana elle manque ou nest que rudimentaire. La frange blanche, limitée en dedans par une rangée d'écailles noiros. plus ou moins visibles, est marquée de quelques taches gris-terne, peu visibles.

Le dessous des mèmes ailes est d'un gris roux, moucheté de gris plus foncé du côti du sommet. La côte est marquée de taches alternativement rousses et grises trèspeu prononcées; dans Styriacana elles sont plus prononcées.

Les inférieures sont luisautes, dun blane sale, teinté de roux sur le disque. La frange est diun blane teme. Styriacana a ces mèmes ailes plus rousses. ce qui fait paraitre la frange plus claire. Le dessous des inférieures est comme le desints.

La tète et le thorax participent de la couleur des supérieures, l'abdomen de celle dus inféricures. Les palpes et les antennes ne présentent rien de particulier.

La femelle est semblable au mâle, ordinairement un peu plus semé de grris. Canescana Guén. Ind. me parait plotòt appartenir à styriacana.

109. Incertana, Treit. sup. Ifub. sup. f. 121, 122. - Hub. mevis. n' 179. Guen. Ind. -
Heyd. C. $n^{\circ} 285$.

subjectana, Guén. Ind.?

Pas très-rare dans les haies des envirou- de Lausanne en juin of juillert. Environte schüpfon $\mid$ Rothb.. 
Je ne pense pas que le caractère indiqué par Treitsehhe, davoir un fond d'un blane prescque pur et les bandes transverses dun brun foncé, soit exclusif à cette espèce. On twouse des Wahlboniana qui loffrent aussi. La taille n'est pas non plus caractéristique ; ear de Minorana à Wahlbomiana, qui sont les deux extrèmes, on établit aisément une série croissante, au milieu de laquelle se trouve Incertana, et que f'on ne sait ì quel point interrompre. Le dessin et la coupe des ailes sont les mêmes que chez les espèces voisines; les variations y sont aussi fréquentes.

Si les chenilles sont différentes, comme le dit Treitschie, il ne reste pas de doute: mais on peut demander si cet auteur a réellement distingué les espéces qui forment ce groupe difficile. M. Iler--Schäffer méerit que son opinion nest pas non plus arrètèe sur (c) point embarrassant. Quoi qu'il en soit, Incertana se prend quelques semaines plur tard que Wahlbomiana dans les localités où celle-ci est fréquente; je lai d'ailleurs rencontrée habituellement sur les haies, tandis que sa voisine hante de préférence les arbres. Son vol est très-rapide et court.

Jo rattache à cette espèce uıe variété de mème taille, qui, sur un fond blane, pur. porte les bandes d'un noir foncé, plus marqué encore que dans la figure 121 de llub. sulp.

\section{Chrysanthemana, Goldg.}

Dup. sup. 83. - IIul. sup. 108 -111. - Ilub. revis. $\mathbf{1}^{\circ} 180 .-$ Heydem. C. ${ }^{\circ} 291$.

Tortricella. (Tin.) Huln. f. 11.

Alternella? (Tin.) W. V. (non Ilubn.) - (ana) Treit. t. 9. p. 1. - Woor. 1269. - Guén. Ind. - Bruand, C. 1014.

Pas rare sur les haies des montagnes; le Jorat au-dessus de Lausanne, 'n juin, parmi les noisettiers. - Les environs de Zirich (Zell.). - LEngadime (Frey).

Cette espèce se distingue aisément des précédentes et son caractère spécifique ne peut ètre mis en doute. - Les supérieures sont un peu plus arquées sur leurs deux hords que dans Wahlbomiana; la marge (bord externe) est aussi constamment plus arrondie. Le blanc est sale et tire sur le roux; on le remarque surtout sur labdomen qui est d'un blane jaunâtre. Les bandes foncées sont plus larges, moins anguleuses. quoiquion rencontre des individus dont le dessin est le mème que celui de Wahlbomiana Le rol est lourd et nirst pas en zig-zag rapides et brusques comme dans cette dernirre. L.es inférienres sont rnfin diun gris plus clair. 


\section{Wahlbomiana, Lin.}

Hub. 203. - Treit. - Dup. 256. - Ilub. revis. n 181. - Hub. sup. 107? 115, 116. - Guén. Ind. Heyden. C. n²89.Isis I8.6. p. 235 (chenilles). - Bruand, C. 1045.

Communan a. Itub. revis. n 182. - Ilub. sup. f. 113, 114. - Iley (l. C. 290. Alt icolana, Koll. - Hub. revis. no 183. - Hub. sup. 112. - Ilub. 203 (Wahlbomiana). - IJeyden. 287. - Bruaul, C. 1012 , 1013.

Interjectan a. llaw. - Curt.

Jaurais pu reunir encore aux trois especes de Her.-Sch., Incertana Treit. sup. 't Minorana Mann; car il est aisé de trouver des individus qui établissent une sérir contimue entre les cinc especes que je viens de nommer. Des observations suivies miengagent ecpendant à donner pour le moment le rang despèce à llinorana; je ne suis pas aussi sùr „Incertana. I]. Iler,-Schf. n'est d'ailleurs pas eloigné de ríunir nos cinı splèces en une seule. Virgaureana lui donne mème quelques doutes. Toutes varient pour la taille et le dessin; certaines differenees tiennent i l'habitat, dautres an sex". dautr's encore à des influences ignoríes. La coupe des ailes n'est pas mèmo exempte. de variations, en sorte que la distinction proposée par Her,-Schf. entre Communana et IVahlhomiana ne me parait pas admissible. Des individus recus de cet habile entomologiste ne me laissent pas de doute sur ce point.

Les femelles sont généralement un peu plus grandes, plus foncées et mieux dessinées. Le sexe ne paraît pas influer beancoup sur la forme de laile; les ailes étroites au sommet sont cependant un peu plus fréquentes ehez les femelles. Du reste, dans rette dernière appréciation il est trìs-facile d'ètre imluit en erreur par la manise dont linsecte est ítalé.

Lhabitat a une influence prononcée. Wahlbomiana se prent partout depuis les bords chauds de nos lacs jusque sur les sommets les plus élevés des Alpes. Dans les expositions chaudes les teintes sont plus claires ef le dessin moins net; c'est le contrairo pour les lieux élevés. Clest peut-ètre à linfluence du cliuat plutòt quä celle de lat mourriture quil faut attribuer la teinte plus foncée des individus recueillis dans les loois de sapin. Là où le sapin sarrète, le papillon se trouse sur les gemiéviors, ou mème sur de petits arbrisseaux (salix, rhammus).

Jignore si le genre de nourriture a quelque inlluence sur la couleur du dessin.

Wahlbomiana est en Suisse la plus répandue de toutes les Tordeuses. Les pramiers individus paraissent à la fin de nai et les derniers en juillot. Elle préfere les 
sapins et les pommiers dans le voisinage des bois; on la trouve certaines années par milliers sur le mème arbre. Dans les Alpes elle est tout aussi fréquente qua la plaine. Elle préfèe les arbres isolés et les bords des taillis.

La forme Communana H.-S. (ailes plus étroites et plus accummées) rst la plus répandue; Wablbomiana II.-S. n'est eependant point rare. On les prend ensemble.

Alticolana Koll. est propre anx Alpes. Elle ne dilfère des précédentes que par ses bandes d'un brun plus foncé et plus larges. Le fond gris est aussi chez elle plus couvert d'atomes bruns. On trouve, du reste, des individus parfaitement semblables dans les bois de sajuin de la plaine.

La forme et les dimensions des bandes et des taches varient a tel point que liun ne pent sy arrèter. (Voir Minorana et Incertana).

\section{Minorana, Mann.}

Hubn. sup. โ. 104-106. - Ilubn, revis. n 184. -- Ileydenr. C. 286. - (non Dup.). - Bruand, C. 1009.

Hinuseulana, Zell.

Assez fréquente dans les vergers en juillet; elle se pose de préférence sur les troncs des arbres. Quelquefois dans les Alpes. Apparaît après Wahlbomiana et Incertana. Commune dans les environs de Zurich (Frey).

A part sa petite taille, rien ne la distingue nettement des précédentes. Le dessiu est rarement net; on ne rencontre pas dindividus i fond blanchâtre, avec des landes brunes tranchíes. Les femelles sont un peu micux dessinées que les màles. Du reste. elle offre les mèmes variations de forme et de dessin que Wahlhomiana; mais ses habitudes et son vol sont constamment différents.

11:3. Virgaureana, Treit. sup.

Dup. sup. 62. - Ilub. revis. $n^{\circ}$ 185. - IIuh. - up. f. 102, 103.Guén. Ind. - Ileyd. C. 282. - Fisch. v. R. coll. - Bruand, C. 1011 .

Quetipues individus collectés au pied du Jura par M. Conleru. - Au pied des Alpes (Rothb.) - Environs d'Aigle et de Lausanne, près des vignes. dans les lienx abrités et chands. (Lah.) - Commune (?) autour de Zürich (Frey).

Il est improssible de distinguer cette espèce de Hinorana, si ce nest par sa couleur d'un gris-noir foncé et son dessin dès là mème fort peu apparent. I. Frey aura sans douto été induit en erreur par cette ressemblance. Mínorana se prend aussi plus 
tard contre le tronc des arbres fruitiers; Virgaureana dans les taillis parun les herbes.Des exemplaires reçns de M. IJer-Schf. peuvent anssi bien passer pour l'une que pour lantre espèce, à part la courhure plus marquée de la còte dans Virgaureana. La description et la figure de Duponchel conviennent anx deux espiees. - Les onglets de la còte ne sont pas blanes, comme le dit Dup.; mais gris, pâles, peu visibles.

Léducation des chenilles devrait, ce semble, lever bien des difficultés en fait de sciaphiles; mais malheureusement beaucoup de Tordeuses s'élevent mal en captivité et domnent naissance à des insectes rabougris et trompenrs.

\section{Pasivana. Hub. 99.}

IIub. sup. f. 100, 101. - Ilub. revis. $\mathrm{n}^{\circ}$ 186. - Guén. Ind.

Heyd. C. 292. - Bruand, C. 1010.

Var. Wahlbomianae, Dup. hist.

III. Rothenbach, Zeller et Frey indiquent cette espece en Suisse. Le second de ces messieurs la prend en juin dans les haies des environs de Zürich.

La figure 99 de Hubner est si imparfaite qu'on peut fort bien lappliquer it WahlLomiana de petite taille. Les individus que jai reçus de M. Mann portent un cachet que nont pas les diverses Pasivan a que jai pu voir. Celle prise par M. Rothenbach portait une tache fauve sur la cellule des supérieures. Ce caractère qui se rencontre ç̀ et lit chez les Sciaphiles, n'est pas spécifique.

\section{Derivana, Lah.}

Jaci pris un mâle de cette espèce, le 10 juin, sur les montağnes au-dessus diaigle. au milieu d'un essaim de Wahlbomiana qui séchappait dun sapin; son vol me la lit dlistinguer. La femelle m'a été communiquée par II. G. Leresche, qui l'avait recueillie an pied du Jura.

Cette espèce a la taille et le facies de Wahlbomiana: mais elle sen distingur nettement par le dessin.

La couleur des supérieures est le gris bleuâtre ou gr s the somris et non le gris plum ou moins brun de toutes les espèces voisines. La coupe de l'aile est aussi un peu diffŕrente: la còte forme une courbe égale et douce à partir de la racine de l'aile jusquà son sommet. Celni-ci est arrondi, de mème que le bord externe et langle interne, en sorte que laile entière a une forme elliptique différente de la forme pyramidale de Wah homiana et de ses voisines. La femelle présente cette forme plus marruée que le male; ses ailes sont aussi plus étroites. 
Le dessin est te mème que dans Chrysanthemana; les trois bandes sont larges et bien tranchées, anguleuses et sintreuses. Les bandes claires sont gris-bleuàtre, clair, les foncées sont gris-noirâtre. Ce qui distingue surtout cette espéce sont des rangées de points noirs très-rapprochés et formant autant de lignes, qui limitent toutes les bandes en dehors et en dedans. Quelques points noirs semblables existent sur le centre des bandes claires et foncées. La fentlle porte un point d'un beau jaune fauve sur la rp]lnle, an centre de la bande moyenne; ce point neviste pas chez le måle; il se rencontro parfois sur d'autres espèces.

La frange des supérieures est diun gris plus foncé que le fond, et limitér par une ligne noire. La còte est marquée vers le sommet par quatre taches plus claires. peu distinctes, divisées par un proint foncé.

Aux supérieures, le dessons est gris-brunâtre, uni; la còte est tachetée de blanc jannatre et de gris-noir; la frange est de la couleur du fond, avec deux traits de division jaunàtres (l'externe est très-fin) situés près de sa raciue.

Le dessus des infériemres est gris ardoisé plus foné vers le sommet et la marge. La frange est un peu plus claire, limitée par me ligne jaunàtre, suivie d'une grise. Cliez la femelle la couleur des inférieures est plus paile. Le Jessous des mèmes ailes est plus elair que le dessus et un peu luisant. Vers le somnet on remarque quelques monelwtures grises.

La tète, les paljes, les antennes et le corselet sont gris de ler. Les antennes dn màle portent un renflement à la base. Labdomen est de la couleur des inlérieures, luisant dessus et dessous.

11. Herrich-Schäffer, auquel j’ai présenté cette espèce, l'a déclarée nouvelle.

\section{Gen. Poecilochroma, Wood.}

Poedisca, Dup. Treit. Spilonota, Curt.

116. Parmatana, Fisch. v. Rös. t. 1-4.

Treit.- Dup. 254, 255. - Hubn. revis. $10^{\circ} 193$. Heydenr. C. 372 .

Solandria na, Lin. - Fab. - Guén. Ind. - Bruand, C. 1051.

$1^{\text {re }}$ var. Sylvana, Hub. 128. - Dup. 254, 3.

Communana, Wood. 1029. - Heyd. C. 374.

Parmatana, Hub. 254. 
Ratana, Ilub. 236. - Frohl. - Dup. 254, 4. - Heyd. C. 373.

Trapezana, Fab. - Wood. 1028.

$2^{*}$ var. Sordidan a, Ilub. 292. - Dup. 254, 6. - Heyden, C. 375.

3e var. Semimaculana, Hub. 48. - Dup. 254, 5. - Heyden. C. 376.

Solandriana, Deg. - Wood. 1027.

Semilunana, Fröhl.

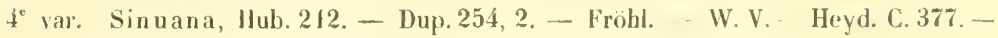
Mus. Schif.

Parmatana, Hub. 253.

Répandue dans toute la Suisse et mème sur les Alpes; on la trouve de préférence dans les bois taillis et les haies. Presque toutes les variétés ont été observées à Zurich, à Schüpfeı, à Lausanne et à la Neuveville.

il. Couleru, qui a fréquemment élevé cette espèce, mécrit que le mâle de la var Sordidana porte sur la côte un pinceau de poils; je lavais aussi observé de mon còté sur des individus très-frais. Je ne puis dire s'il est constant ou non, ni sil existe chez d’autres variétés. Aucun auteur ne parle, que je sache, de ce pinceau.

La synonymie des auteur's anglais est inextricable.

117. Melaleucana, Dup. 225, 2.

Hub. sup. f. 75. - Hub. revis. $n^{\circ}$ 194. - Heyden, C. 364.

II. Couleru, qui l'avait procurée à Duponehel, m'en a fait voir un individu dans sa collection. Il avait été pris dans les environs de la Neuveville. Sa taille est un peu au-dessous de Parmatana.

\section{Gen. Eu chromia, Steph.}

Tortrix, Treit.

Chacune des espèees qui composent ee genre appartient ì un genre difiérent dans les divers auteurs. Il n'est, il est vrai, pas très-caractérisé.

\section{Rosetana, Hub. 222.}

Treit. - Fröhl. - Hub. revis. n 195. - Guén. Ind. - Hẹd. C. 109. - (non Dup.)

Pas commune en Suisse. II. Zeller l'a prise dams les emvirons de Zirich: je l'a reçue de M. Meyer qui l'a prise à Burgdorf.

J'ai retranché le synonyme lig. 130 de Ilubner parce quo cette ligure répond evac- 
tement a une espèce que je crois distincte; il suffit de compares la coupe des ailes et spécialement celle du bord externe dans les deux figures de Hubner, pour se convaincre de leur différence (roir à Arenana).

Jai regu de M. Millière à lyon, sous le nom de Rosetana, une espèce extrimement voisine, mais qui en diffère non-seulement par la couleur dun rose vif, mais encore par les crochets costanx (peu apparents, il est rrai), qui n'existent pas dans Rosetin a. Je lenvisage comme la Rosaceana Schläg. et je la crois bonne espèce.

119. Irenana, Lah.

Rosetana, Hub. 130. - Dup. 240,8.

Jai observé cette espéce deux années de suite, en abondance, à lembonchure du torrent de la Paudèze dans le lac Léman; une première fois au milieu de juin, une seconde en aoùt. Elle volait en essaims, antour des Salix triandra, sur lesquels la chenille vit selon toute probabilité. Je n'ai pas pu décourrir encore colle-ci pour l'élever.

Valgré l'avis contraure de M. IJerrich-Schäfler, je ne puis y voir une simple variété de Rosetana. Il est vai que pom adojter un avis opposé à celui de cet habile entomologiste, jai dù roir un grand nombre d'exemplaires et par là m'assurer de la constance des caractères. Arenana est toujours plus petite que Rosetana: les plus petits individus de la seconde espèce égalent en grandeur les plus grands de la première. Le dessin des ailes est le mème de part et d'autre; mais la majeure partic des exemplairen d'A renana sont gris-olivattres on brunàtres avec des stries plus foncées, tandis que la couleur gris-rougeâtre est moins fróquente. Je n'ai pas encore vu des kosetana gris. Entin, et ce caractère est le plus signnificatif, le bord externe des supérieures est décidément arondi dans Arenana, comme lindiquent les figures citées de Inbmer et de Dup., tandis quil est droit dans la rraie Rosetana. Si nous joignons à cela les caracteres tires de Tluabitation, il est difficile de refuser a cette Tordeuse le rang despéce; la connaissance de la chenille lèvera tous les doutes.

120. Maurana, Hub. 122.

Treit. - Dup. 240, - Ilub. sup. f. 41. - Hub. revis. $1^{\circ} 197$.

Wood. 1033. - Guén. Ind. - Heyden. Cat. 89. - Bruand. C. 928 .

Viduana, Hub. 303. - Heyd. cat. 90.

J. Rothenbach indique cette espèce près de Schupfen. 


\section{Tussilaginana, Khlw.}

Ilub. revis. $n^{\circ}$ 198. - - Iluh. sup. f. 240. - Heyd. Cat. $11^{\circ} 10 ; 3$.

Grandaevana, Lien. - Zell. Isis. 1846. - Hub. sup. 348. - II'yd. C. n¹04.

Jai pris plusieurs fois eette espéce tans les vallées des Omnonts et du Val dilirrs, (n juillet. M. Rothenbach la posside venant des Npes. Je Jai reçue de Meyringen.

se trouve oxclusivement parmi les tonfles de Tussilago petasites et alba, jamais auprès de celles de T. farfara. Son vol est court et semblable à celui duu Crambe. Elle se pose sur les feuilles de tussilage et y reste immobile. La chenille vit probahle ment dans la tige de cette plante.

Ve varie pas. La fenelle est semblable au màle.

\section{Terreana, Treit.}

Dup. cat. - Huh. revis. n 199. - Heyden. C. 290.

Favillaceana, Hub. 255.

Rustic ana, Hub. 102 ?

Vulgana, Fr. - Guen. Ind.

Très-fréquente à la fin d'avitil et en mai, dans les forèts de hètre et de chène : sauvabelin, au-dessus de Lausanne. Pres de Schiiplen Rothl..). Je ne l'ai jamais apercure au milieu des sapins.

Guence en a fait, je erois avec raison, un geme spécial sous le nom de Triatodes.

12:3. Centrana, Hub. sup. f. $\mathbf{3 7 3 .}$

Hub. revis. $n^{\circ}$ 200. - Iley den. $11^{\circ} 169$.

II. le prof. Frey a collecté un exemplaire de eette espèce très-rare an mois de juillet, pres de Zurich, sur nue clairière du Ilottingerberg.

\section{Gen. Rhia cionia, Steph.}

Teras, Treit. Carpocapsa, Curt.

\section{Hasliana, IV. V.}

Clerck. - Ileyd. C. 653. - IIub. revis. n²01. Guén. Int.-Dup. eat.

II a stana. Hub. 186.

11. le prof. Frey est jusquici le seul qui ait collecte en Snisse rette usprece ratre il la prise sur lo Lægernberg pres de Baden, à la tin de juillet! 
XVIII. Gen. Ditula, Wood.

Paedisca, Treit. Dup. Guén.

125. Ophthalmicana, Hub. 51.

Fröhl. - Treit. - Dup. 254. - Hub. revis. n²02. - Guén.

Ind. - Heyden. 371. - Bruand, C. 1050.

Maeulana, Curt.

An mois de septembre sur la lisière des forêts de sapin, au-dessus de Lausanne; rare. - M. Heuser l'a prise dans les environs de Burgdorf; M. Rothenbach à Schüpfen et MN. Zeller et Frey près de Zurich, en aoùt et en septembre.

126. Corticana, W. V.

Hub. 209, 270. - Treit. - Dup. 255. - Hub. revis. n' 203. Communana, Curt.

Guén. Ind. - Heydén. c. 323. - Bruand, C. $104 \pi$.

Très-commune sur les troncs de saule marcean, an bois de Sanvabelin, au-dessus de Lansanne, en juillet. De mème près de Sehüpfen (Rothb.), de Zurich (Zell.). Elevée sur le chène par M. Frey.

127. Profundana, W. V.

Mus. Sehif. - F. - Fröhl. - Treit. - Dup. 253. - Fisch. v. R.

t. 62. - Hub. revis. no 204. - Heyd. c. 344. - Guén. Ind. Bruand, C. 1048.

Wellensian a, Hub. 237. - Wood. 882. - Dup. 255. - Curt.

Porphyrana, Hub. 26. - Dup. 264. - Steph.

Alphonsiana, Dup. 239. - Brrand, C. 1049.

Nubilana, Dup. sup. 62. (non alior.)

Point rare dans les bois et les taillis de chêne aux environs de Lausanne, en juillet et en aoùt. - Près de Schüpfen (Rothb.); de Zurich (Zell. Frey).

128. Oppressana, Khlw.

Treit. sup. - Dup. sup. 64. - Hub. revis. $\mathrm{n}^{\circ}$ 205. - Ilub. sup. f. 242. - Guén. - Heyd. e. 319. - Bruand, C. 1046.

Rare dans les environs de Lausanne; en juillet, dans les haies où croissent des anlnes et des saules. 
129. Achatana, W. V.

Fab. Nus. Schif. - Treit. - Fröhl. - Dup. sup. 64. - Iluh. revis. ${ }^{\circ}$ 206. - Guén. Ind. - Ileyd. c. 550.

Marmorana, Hub. 25. - Fröhl.

Cacale an a, Mus. Schif.

H. le prof. Frey l'a prise dans les environs de Zurich et M. Rothenbach près de Schuipfen. Assez rare en Suisse.

\section{Pinicolana, Zell.}

Hub. revis. n²07. - IIub. sup. f. 384, 385. - Ileyd. C. 433.

Grise a na, flub. 135 (non Treit.) - Heyden. C. 129.

Diniana? Guén. Ind.

II. le prof. Frey a trouvé communément cette espèce près de Samaden dans l'EIgalline; elle y vit sur les aroles (Pinus cembra) et sur les mélèzes (P. larix). Varie beaucoup (Frey).

Je laai recueillie ẹà et là isolément dans les Alpes du district d'Aigle. Très-commune dans le Valais. - Dans les étés 1856 et 1857 elle était fort abondante dans les environs de Lonesche, où elle abîma la végétation de forèts entières, ainsi que l'a olsservé II. Davall, fils, inspecteur forestier. - M. Bellier de la Chavignerie mécrit qu'en 1856 la larve ravagea les forèts de mélèze du département des Basses-Alpes, au point de canser le vives inquiétudes à l'adıninistration forestière.

La Ggure 113 de Hubner ne peut se rapporter qu'à cette espèce; des individus reçus du Midi de la France la reproduisent parfaitement. Ceux de la Suisse sont ordinairenent un peu plus grands.

La chrysalide est brune et porte sur ehaque anneau deux rangs circulaires d'aspérités dont les pointes sont dirigées en arrière. Son extrémité annale est garnie de courtes ipines.

Pinicolana varie beaucoup sans jamais perdre sa couleur grise. Certains individus portent sur le bord interne une grande tache triangnlaire et blanchàtre semblable à celle de Sinuana var. Parmatana. La plupart ont le fond entièrement gris, moucheté de 'quelques taches irrégulières; lorsque la tache basilaire apparaît nettement, on les prendrait pour une forme grise de Ratana var. Parmatana; en général, cette espèce est extrimenent voisine de Parmatana et sons plusieurs rapports. - La tache basilaire forme sur le disque un angle plus aigu que dans Parmatana. La tache obligue du 
milieu de laile est rarenıent dessinóe. Les croehets de la còte sont assez visibles. tandis que dans Parmatana ils ne le sont pas toujours. - Quelques individus portent des nuanees lorunàtres sur le milieu de laile; d'autres ont le fond mélangé de hlane; d'autres sont d’un gris pointillé uniforme: d'autres ont les deux tachen noires; dantres nnfin les (nil brunàtres.

La taille varie tout autant chez les máles que chez les lemelles.

La larve differe de celle de Parmatana. Voici sa description: Longneur th 10 a 12 millimètres; diun vert presque noir; tète et anus noirs; pattes noires; 4 vermes dorsales noires sur chaque annean, les 2 antérieures plus rapprochées; 3 verrues dorsales sur l'avant-dernier anneau, disposées en triangle; 2 verrues latérales sur chacpue anneau: un poil sur chaque verrue; deux rayes pâles sur la ligne des stigmates : le '2e et $12^{*}$ anneau noir, chagriné; 4 soies brunes au-dessus de l'anus.

Le dessin est rarement aussi net que le représentent les tigures de H.-S.

131. Ratzeburgiana, Saxes.

Hub. sup. f. 333 . - Hubn. revis. n"208. - Heydenr. c. 326. Bruand, C. $1047^{\text {h. }}$

Abietis, Zell.

Abietisana, Fröhl. - Hejd. c. 2 ['7.

Tenerana, Dup. 266. - (non Ilub.).

Errana, Guén. Ind.

Fuscana, Khlw.

Recueillie par M. Zeller dans les environs de Zurieh, sur le Jura par IJ. Th. Bruand. Ie lai recue de $\boldsymbol{~ M . ~ R o t h e n b a c h ~ q u i ~ l a ~ p r i s e ~ p l u s i e u r s ~ f o i s ~ d a n s ~ l e s ~ e n v i r o n s ~ d e ~ S c h u p f e n : ~}$

Le 12 juillet appliquée sur le trone des sapins (Rothb.). Environ de Lausinne.

La femelle est micux dessinéc que le màle.

XIX. Gen. Seric or is, Treit. Dup. Guén. Ortbotaenia, Stepl. Curt.

\section{Trifoliana, Hub. sup. f. 372 .}

Hub. revis. $n^{\circ} 210$. - Ileyd. cat. $1^{\circ} 252 .-$ Bruand. C. $916^{\text {b" }}$ ?

II. Rothenbach a capturé cette espèce rare près de Schüjfen, le 22 juillet 1856 ! Je nai vu de lui qu'une femelle. Cet individu est plus foneé et plus grand que ceux provenant d'Allemagne. - M. Wullschleger l'a prise aussi près d'Aarburg. 
1:3:3. Striana, W. V.

Treit. - Fröhl. - Dup. 255. - llub. revis. n²13. - Guén.

Ind. - Ileyden. C. 249. - Bruand, C. 1001.

F a seiana, Curt.

Rustieana, Hul, 66 (non 102).

q F aseiolana, Huh. 260. - Frihl. - Treit. - Guén. Ind.

Commmne sur les prairies un peu sìches, jusque dans les Alpes, en juillet et auût. Pils aussi fréquente dans les environs de Zmich (Zell. Frey). - Commune dans le canton we Berne (Rothb.). - Varie fort peu.

\section{Zinckenana, Frölıl.}

Treit. - Dup. 247. - Ihub. revis. n²16. - Heylen. C. 209

Schulziana, Fab. - Zett. - Guén.

Pinetana, Ilub. 57. - Steph.

Ahrensiana, Hub. $337,338$.

Benthleyana, Curt. - Wood.

Pas tres-rare dans les Alpes centrales; Meyringen; loberhasli; le Gotthard, etc. Rothl. - Zell. - Lah.).

La conlenr brune des taches s'effaee vîte par le vol. et le blanc en devient plus saillant. -. Varie pour la taille.

\section{Sudetana, Standf. Zeitschrift f. Entom. 1849.}

Hub. sup. f. 272 . - Hub. Pevis. n०217. - Heyd. c. 212.

Lan giana, Iler.-Sch. olim.

Bistrigana, Wood. 1123.

Prise dans les environs de Burgdorf par H. Heuser. - Jusquici on he la connaissait 'ure dans le Riesengebirge.

\section{Rejectana, Lah.}

La riche eollection de M. Rothenbach m'a fourni eette espece inedlite et curiense it plus dun égard: l'époque et le lien exaets de sa provenanee me sont inconnus. Je niai vu que le màle.

La taille et la eoupe sont celles de Striana; par son facies clle se rapproche de sudetana; mais elle se tlistingue de toutes ses voisines par une légere éehancrure qui existe en-dessous du sommet arrondi des supérieures, sans quilil y ait cependant de point 
dintersection sur la marge. Cette échancrure rappelle le genre semasia, oì peut-ìtre elle devrait prendre place à còté de Citrana.

Quoiquil en soit, Rejectana se distingue aisément a ses ailes supérieures dim yris fauve uni et sans aucun dessin. La còte est uniformément et légèrement arquée dès la hase; vers le sommet elle porte trois erochets, jaunàtres, simples, assez espacés, dont linterne est plus gros. Le sommet de l'aile est arrondi, ainsi que l'angle postérieur.

Les inférieures sont du mème gris que les supérieures, mais saus teinte fauve, sauf ruelque peu vers le sommet.

Le dessous est entièrement du mème gris, mn peu luisant, avec quelques taclıes fauves peu marquées vers le sommet des deux ailes.

La tête, le thorax et l'abdomen sont gris; mn mouchet de poils fauves termine l'abdomen.

137. Siderana, Treit. sup.

Hulı. sup. f. 203. - Hub. revis. n² 218. - Guén. Ind. - Ileyd. C. 218. - Dup. sup. 79. - Bruand, C. 996, 997?

Astrana, Guén.?

Jai regu cette espèce de M. Ott à Meyringen et de II. Heuser à Burgdorf. Très-rare en Suisse. Il est difficile de comprendre pourquoi II. Guénée sépare Siderana de Dup. de celle de Treit., sous le nom d'Astrana. La figure de Hul. sup. ne differe en rien le celle de Dup.

138. Textana, Hub. 307-30y.

Fröhl. (non Dup.). - Guén. Ind. - Heyden. C. 219.

II. Mejer de Burgdorf m'a communiqué cette espèce prise dans les environ de sa demeure. II. Rothenbach l'a collectée sur les Alpes bernoises et dans les environs de Schüpfen sur une prairie humide. Je lai vue une fois en juillet, dans le Val-dlliers en Valais. II. le prof. Frey a élevé le premier la chenille trouvée, au premier printemps, dans le cour de la Scabiosa arvensis. aux environs de Zurich: il a pris le papillon en liberté au mois d'aoùt.

139. Pictana, Lah.

Je ne possède malheureusement quiu seul individu femelle de cette espèce unique par son facies; elle est si complétement différente de tout ce que nous connaissons que ses droits à prendre rang parmi les espèces nouvelles ne sauraient ètre mis en doute. J'ai pris cette Tordense sur une sommité assez élevée (5500') du Val-d'lliers (Bas-Valais), 
au-dessus du village de Champéry, au mois de juillet 1854; elle voltigeait au milieu des genièviers, des myrtils et des rhododentrons en compagnic de Charpentierana.

De loin, la taille et le dessin rappellent Demarniana et à quelques égards Immundana; mais labsence de points d'intersection de la frange, sous le sommet des supérieures, s'oppose à tout rapprochement avec les genres Paedisca et Grapholita. 11 existe bien une espròce d'écusson formé par deux lignes métalliques, descendant de la còte pour se réunir vers l'angle postéricur; mais cette apparence se retrouve dans certaines Sericoris. Il y a plus, les taehes brunes et blanclies du bord de la marge s'étendent à la frange comme dans ce ternier geure; ce qui me déternine à la placer à còté de Textana dont elle a ruelques-uns des earactères.

L'aile supérieure se subdivise en quatre ehamps. Le premier est formé par lespace basilaire, noir, conpé carrément et parsemé de quełques écailles d'un rose vif. Le second est représenté par une bande blanche, étroite à la còte, plus large au bord interne, portant 3 ì 4 stries noiràtres qui en obscurcisseut la blancheur. Un point très-noir occupe la còte sur cette bande. Le troisième champ est eclui tle la bande médiane. Celle-ci, derechef étroite à la côte, élargie vers le bord postérieur, est dirigée très-obliquement du milieu de la côte à l'angle abslominal. Elle est d'un brun très-foncé, mais fortement sablée décailles roses et fauves, ce qui la fait paraître brune. Après cette bande survient lespace terminal, de forme triangulaire, dont le dessin est plus compliequé. Sur un fond rosé on distingue de nombreux points métallicques, blenàtres, qui forment audevant de l'angle abdominal une espèce d'écusson circulaire. Une ligne rosée, pointée de noir, traverse l'écusson d'arant en arrière. Au-dessis de lui se voient plusieurs points noirs rassenblés en lignes longitudiuales. La marge est maryuée de deux points blancs vers le sommet et d'un vers l'angle postérieur.

La frange est noirâtre, mouchetée de trois ou quatre points blancs, divisée par un trait blanchàtre, très-fin. Le sommet est à peine arrondi. La còte est marquée de einq crochets; le premier (dès la base), le second et le troisième sont doubles; ces deux-ci peu distincts; le quatrième et le cinquième sont simples; entre cux la còte est noiràtre.

Les inférieures sont d'un noir brum, avee la frange plus claire.

Le dessous des supérieures est brun, nuancé de rose, surtout vers la inarge. Les crochets de la côte y sont visibles. Le dessous des inférieures est semblable au dessus, mais plus pàle; un ou deux points noirs se voient an bord antérieur près du sommet.

La tète, les palpes et le thorax sont noirs. Les pattes sont annclées de blanc-rosé. 


\section{Schafferana, Mann cat.}

Iluh. sup. f. $144,145,-$ Heyd. c. 210.

Furfuran a, Haw. - Hub. revis, n²19.

Lateritiana, Khlw.

Cette espèce, découverte d'abord dans le Tyrol par M. Mann, a été prise dans les Alpes bernoises par M. Rothenbach, et dans le Jura, au Mont-d'Or, par I. T. Bruand.

\section{Spuriana, v. Heyden.}

Ilub. sup. f. 334 . - Ilub, revis. $n^{\circ} 220$. - Ileyd. c. 327.

II. le prof. Frey en a collecte un individn màle sur le sommet du Celeriner, dans l'Engadine, près de Samaden, à environ 7000 pieds.

\section{Porphyrana, Koll. (non Hub. - Dup.)}

\section{Heyden. Cat. 211.}

Jai reçu un exemplaire de II. Mann, provenant du Grossglockner, puis un second de II. Ott, pris dans les environs de Meyringen. Tous deux sont màles. Celui de rOberland est sensiblement plus grand et privé de fauve sur le disque.

La taille et la forme sont celles dlrriguana. La couleur générale est le gris-noiràtre très-mélangé de blane argenté, de violet, de noir et d'un peu de fauve vers l'extrémité.

Cette espèce est difficile à décrire. Ses caractères essentiels sont:

$1^{\circ}$ Liespèce basilaire et la bande médiane diun brum foncé, striés, situés obliquement et occupant les deux tiers internes de laile. Ś’parés par une bande plus claire, très-ombrée de gris. lers le hord interne, la bande médiane est confuse. - $2^{\circ}$ Quatre crochets doubles, placrís sur la moitié externe de la còte, envoient du còté de l'angle anal autant de lignes blanches métalliques, qui se reproduisent sur la marge, en avant de langle, par quatre lignes blanches qui traversent la frange grise. Ces lignes sont séparées par quatre points noirs. - $3^{\circ}$ Une trace brume oblique part du sommet de laile et se dirige vers le nilieu de la bande transverse; olle est quel|uefois marquée de traits noirs sur les nervures qui se reproduisent sur la bande transverse. Cette trace est en outre traversée par les traits blanes partis, des crochets, qui prennent à son nivean une teinte violette et plombée. - $4^{\circ}$ Le sommet de l'aile est brun; la frange au-dessous de lui est blanche, síparie de la marge par une ligne noire. L'angle anal est blanc. $5^{\circ}$ Les inférieures sont d'un gris violacé, pàle, avec la frange blanche. - $6^{\circ}$ Le dessous est entièrement d'm gris luisant. - Lextrémité de la còte des supérieures porte 
une trace blanche, la frange des mêmes ailes est entrecoupee de gris et te blane. Un petit trait blane linite la frange vers le sommet des inféricures. - $7^{\circ}$ Tète et corselet noiràtres, semés de poils roux ou gris. - Antennes de même couleur, annelées de blanc. Moucliet anal fauve.

Se rapproche surtout de Spuriana.

143. Micana, Treil.

Hub. revis. n $221 .-$ Ilub, sup. f. 243 . - (non llub. - W. Y.Dup.) - Hey d. C. 233 ? — Bruand, C. 99.4 ?

Stibiana, Guén. Ind. ?

M. C. Zeller indique cette espece dans les environs de Zurich et M. Rothenbach près de Schuipfen. Je n'ai pas encore pu la voir.

144. Metallicana, Hub. 68.

Ilub. revis. n" 222. - Guén. Ind. - Heydenr. C. 213. - Dup. 246? - Bruand, C. 991?

Netallana, Fröhl.

Nebulosana, Zett.

J'ai reģu cette espèce de M. Rothenbach qui la prise dans les environs de Schijpfen, et de II. Ott de Heyringen. Rare.

Il m'est impossible de reconnâtre l'espéce aetuclle dans la figure le Duponchel. Sa description s'applique plutòt à Metalliferana.

145. Conchana, Hub. 106.

Treit. - Dup. 246? - Hubn. revis. n² 223, - Guén. Ind. Ileyden, C. 232, - Bruand, C. 987.

Rivellana, Fröh. - Fab.
Undulana, Steph. - Wootl. 1014. - Curt.

Très-commune sur les prés humides et montagneux en juillet et en aoùt, dans toute la Suisse (Lah. Rothl. Zell.). - S'bleve sur les hautes Alpes. (Frey, Boll.).

Ne varie pas. La femelle est volontiers plus petite que le mâle et moins fréquente que lui, comme dans presque tontes les sericoris communes.

La figure de Dup. se rapporte plutòt à Lucana; sa description est insuffisante.

146. Giganteana, Hub. sup. f. 23:3.

Ilub. revis, $n^{\circ} 224$. - Heyden. C. 222, 
Fulgidana, Guén. Ind. - Bruand, C. 995.

Textana, Dup. 246 f. 8 (non Uubn.).

llelveticana, Dup. eat.

Jai recueilli cette espèce en juin, dans les environs de Lausanne, au Signal et dans la vallée de Montmeillan. Elle est assez fréquente près de Villeneuse, au pied de la montagne, dans les bois de chàtaigniers, à la mème époque. I. Rothenbach l'a collectée dans les Alpes bernoises et auprès du signal du Weissenstein au-dessus de Soleure. MN. Zeller. Bremi et Frey l'ont aussi prise dans les environs de Zurich (Uetliberg); N. Boll près de Bremgarten et M. Wullselılegel près d'Aarburg.

La figure de Ilubn. sup. pourrait induire en errenr; nos exemplaires suisses sont beaucoup plus nuancés de vert, de violet et de brun. En les voyant, on eomprend plus aisément l'erreur de Duponchel; car il existe une certaine ressemblance entre la figure grossie de T extana Hub. (f. 308) et notre Giganteana.

La femelle ne diffère pas du màle. On trouve des individus plus petits et plus pàles, dans les deux sexes, qui se raprrochent de la figure de llub. sup.

\section{Cespitana, Hub. 214, 215.}

Treit. - Dup. 246? - Hubn. revis. n²25. - Guén. Ind. fleyd. e. 237. - Bruand, C. 986.

Var. Fla vipalpana, Fisch. v. Rösl. - Hub. sup. f. 216, 217. - Ileyd. c. 238.

Cette espèce est l'une de celles qui disparaissent certaines années et reparaissent abondamment dans d'autres. Je lai prise aux environs de Lausanne, sur les prairies maigres, une première fois à la fin de mai et une seconde à la fin de juillet et en aoùt. A Aigle dans le marais, en juin. Commune. - M. Rothenbach la cite près de Schuipfen et $\mathbf{N}$. Zeller près de Zurich. I. Frey la eollectée près de Samaden dans l'Engadine. Quoique fréqquente dans les Alpes, elle préfère les expositions chaudes; aussi est-elle très-commune dans le canton du Tessin et en Valais.

Jai pris la var. Flavipalpana dans les environs de Bellinzona où elle est commune sur les pentes arides. Elle ne differe que par ses teintes plus brillantes et son fond micux marbré. La couleur jaune des palpes existe plus ou moins sur tous les individus à couleurs plus elaires.

Cespitana varie souvent pour la taille; on trouve des individus des deux sexes de moitié plus petits que dautres. Le dessin est aussi plus ou moins narbré d'olive, de brun, de roux, de fauve, d'orange et de violet. La tète est tantòt fauve, tantòt grise.

La figure de Duponchel reproduit plutòt Rurestrana. 


\section{Olivana, Treit.}

Ilub. revis. $n^{\circ} 226$. - IIub. sup. f. 218, 219. - Ileyd. c. 234.(non W. V.)

Mic an a, Iłub. 28. - Guén. Iud. - (non W. V.) - Dup. 246, 5. - Bruand, C. 994 .

₹ Stibiana, Guén. Ind.?

Commune sur les prés tourbeux des montagnes et des Alpes. Le mále est plus grand que la femelle et ses couleurs sont moins tranchées. Alpes d'Aigle, de Chamonix, etc. Le Jorat au-dessus de Lausanne et de Lutry. Les Alpes bernoises (Rothb.). Cà et là autour de Zurich (Frey).

C'est à tort que Treitschke rapproche Troelıilana (Ilub. 313, 314 Fröhl.) d'Olivana, et que tous les entomologistes l'ont copié. J'ai vo dans la collection de M. Rothenbach Trochilana provenant d'Allemagne; ce n'est point une Olivana. Les figures de IIubner sont reeonnaissables. L'espéce la plus voisine est Cespitana. La bande obligue de la marge externe se termine sur le disque par une tache quadrilatère, là où Cespitana en porte une ronde. Troehilana se rapproche aussi de Palustrana; mais sa bande moyenne est plus foncée et le sommet de l'aile est chez elle presque noir.

Ja femelle dO livana est plus petite que le mâle; ses conleurs sont plus brillantes et plus tranchées; les bandes elaires sont chez elle plus larges, plus visibles et presque blanches; les bandes foneées sont brunes et semées de points blanes. Elle est moins commune que le màle.

Les figures que Duponchel donne de Mic ana ne peuvent se rapporter quài Olivana.

Jai regu de II. Ott un individu màle sur lequel les taches et stries métalliques sont en trés-petit nombre et où la couleur fauve pàle des bandes claires est janne, avee lextrémité de l'aile plus claire. Cette variété est toujours reconnaissable aux points blanchàtres, très-saillants, de lis còte.

\section{Palustrana, Lienig.}

$$
\text { Zell. - IIub. revis. } n^{\circ} 227 \text {. }
$$

D is ertana, Fisch. v. Rösl. - Ilub. sup. f. 213-215. - Ileyd. C. 235.

H. Zeller a recueilli cette espèce dans les Alpes; jai pu la comparer aux figures de Ifub. sup. 


\section{Umbrosana, Zell.}

Frey, 4. 318. - Dup, sup. 79. - Hubn. revis. n० 229. - IJubn. sup. f. 207, 208. - Guén. Ind. - Ileyden. C. 223.

\section{Gris e istriana, Bruant, C. 993?}

Les taillis humides du bois de Sanvabelin près de Lausanne; une premiere fois à la fin de mai et en juin, une seconde en juillet et en aoùt. II. Rothenbach l'a rapportée des Alpes bernoises. - Rare, quoique assez fréquente dans les lieux quielle habite.

Cette espèce ne peut ètre confondne quavec Lacunana; mais elle est plus grande, a les supérieures plus larges, n'a pas de points ou de lignes brillants. Les deux bandes claires qui limitent la tache oblique de la marge sont sillonées de stries qui partent des crochets de la còte. - La frange des inférieures est grande, blanchàtre dans toute son étendue.

\section{Lacunana, W. Y.}

$$
\begin{aligned}
& \text { Mus. Sehif. - Dup. sup. - . Jub. revis. n² } 229 . \text { - IJub. sup. } 84 . \\
& \text {-Guén. Ind. - Heyd. C. } 225 . \text { - Bruand, C. } 989 .
\end{aligned}
$$

Decussana, Zett.

Vieinana, Mus. Podev.

Var?? Il e ba na, Guén. Ind.?

Très-conmune partout, dans les prés et les taillis; sur les Alpes. - Mai et juin. Conmune près de Zurich (Frey).

La fig. 65 de Ilub. ne peut lui ètre appliquée; elle reproduit trop hien Urticana. Ilerbana Guén. nest probablement quiune variété de Lacunana. Dans les lieux secs et chauds on tronve des individus dont l'extrómité des supérieures est de conleur olive et fes inférieures blanchàtres, surtout à la racine de l'aile. Dans les Alpes, au contraire, linsecte est plus gros, plus lourl et plus fortement coloré; les bandes deviennent presque noires; les points métalliques brillent comme des perles violettes ou argentées. On pourrait prendre aisément ces individus pour une nouvelle espèce se rapprochant de Mic a na. La femelle diflere pen clu maile.

\section{Rurertrana, Fisch. v. Rösl.}

Dup. sup. 84. - Ilub. revis. n 230. - Ilub. sup. f. 211,212 . -

$$
\text { Guén. Ind. (excl. synon.). - Heyd. c. 227. - Bruand, C. } 989 .
$$

Rare. J'ai pris quelıuefois cette petite espece sur les pàturages sees de Puidoux, an-dessus de Chexbres, en mai. 
Ne peut guères se confondre quavec Laeunana; mais elle est plus petite, a le sommet des supéricures un peu arromdi, tandis que chez sa voisine il est à angle vif.

153. Urticana, Hub. (65.

Treit. - Dup. 246. - Ilub. revis. n² 231, - Hub. sup. f. 209, 210. - Guén. Ind. - Ileyd. c. 224. - (non W. Y.). Curt. Bruand, C. 992.

L a c un ana, (partim) W. V.

Undana, Fab. - Fröhl.

Undulana, Mus. Schif.

Monet ana, Treit. sup.

Commune en juin dans les haies des environs de Lausanne; en juillet dans les Alpes. Reparait à la fin d'aoùt autour de Lausanne. Environs de Schiipfen (Rothh.); de Zurich (7ell., Frey).

Ne varie guères qu'en prenant une couleur plus ou moirs brune, aux supérieures. La femelle est moins fréquente, un peu plus petite ef a pour l'ordinaire les postérieures plus foncées.

\section{Venustana, Hub. $\mathbf{3 2 6}$.}

Frohl. - Ilub. revis. n'232. - Iluk. sup. f. 187, 188. - Heyd. c. $17 \%$.

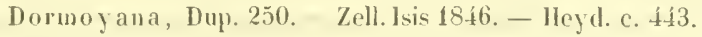

Grapholitan a, Guén. Ind. - Bruand, C. 985.

lmplicana, Khlw. - Hejul. c. 239.

Un marais tourbeux, an centre du bois de Sauvabelin, au-dessus de Lausanne, fin de juin et juillet; rare. - Pres de Schiijfen (Rothb.); de Zurich (Zell). Sur rUethiberg au milien des bois de sapin (Frey). - Euvirons dEngelberg (Olten) en juillet (Wtlsch.).

Difficile à saisir à canse de sa petitesse et de son vol rapide.

155. Luc a na, Guéll. Ind.

IHuls. revis. " ${ }^{\circ} 234$. - Hejol. c. 229. - Bruand, C. 999.

Lucivagana, Lieng. - IIub. sup). f. 335-337.

Charpentierana, Hul, 281?

Nic an a, Dup. 2⿺6. 3?

Pas rare dans le Jorat au-dessus de Lausanne, en juillet. On la rencontre aussi 
dans les basses Alpes. - Environs de Zurich, à la fü de juin; peu rare (Frey). - Dès le commencement de juin, au pied de la montagne, près d'Aigle.

156. Bipunctana, Treit.

Fröhl, - Hubn. revis. n² 236. - Ilubn. sup, f. 230 . - Heyd. c. $241 .-$ Dup. cat.

Charpentierana, Zell. - (non Ilub. 281.).

Trouvée dans les Alpes par MM. Zeller et Rothenbach. - Près de Tzermatten (Frey). An Pleswald (Meyer). Cà et là dans les Alpes, parmi les rhododendrons et les herbes; pas rare; juillet.

Il r'est pas toujours facile de distinguer cette espece de Charpentierana (voir cette dernière). La femelle est plus petite, et ses teintes blanches sont plus tranchées. Chez le màle les ombres violettes et brunes de lextrúmité de laaie sont très-faibles et rarement apparentes. - Les erochets de la còte sont très-distincts, dessous comme dessus, et presque toujours doubles. Le dernier, à partir du sommet, se place à l'extrénité de la première hande transverse blanche ou existe un point noir it la còte qui divise la bande. Le second est sur la bande médiane transverse et plus petit que les autres. Les troisièmes, quatrièmes et cinquièmes sont gros et très-visibles, séparés par deux points noirs desquels partent des lignes dombre qui parcourent la tache blanche du bout de l'aile. Le sommet de l'aile est formé par un gros point noir sous lequel la frange est blanche, entrecoupée de noir.

Le front porte, dans les individus très-frais, un petit mouchet blanc; le sommet des palpes est de la mème couleur. La ligne noire qui linite la frange aux supérieures n'est jamais coupée par les points blancs qui l'abordent.

Jai récolté, dans la vallée de St. Gervais, au Nant-Bourant, nne variété que l'on prendrait aisément pour une espièce particulière. Chez elle la bande moyenne s'unit ì la tache terminale par une ligne de mème conleur, dirigée directement en travers. De part et dautre de cette ligne existent dès lors deux taches blanches ellyptiques, traversées dans le sens de leur longueur par un trait noir. Les deux extrémités de la frange et langle abdominal restent blancs. La bande blanche basilaire est aussi divisée par un filet noir.

\section{Irriguana, Zell.}

llub. sup. f. $424,425 .-$ lleyd. cat. n 214 .

I. Rothenbach la recueillie dans les Alpes de l'Emmenthal et M. Frey dans les 
environs de Samaden (Engadine). - Jai sous les yeux un individu collecté par M. Rothenbach qui ressemble parfaitement à ceux expédićs par M. Mann. (Yoir Mendosana),

\section{M endosana, Lah.}

Jai vu plusicurs individus appartenant aux collections ile M. Rothenbach et de M. le prof. Frey; jen possède aussi trois. Tous ont éte pris dans les Alpes. Ce nest qu'après un minutienr examen que je me suis dócidé ì en faire une espèee, tant elle ressemble à plusicurs autres bien connues. M. Herrich-Schiffer penchait à la rattacher à Irriguana; je ne saurais partager cette opinion; elle se rapproelse davantage, à mon avis, de Palustrana, dont on ne pourrait mème la distinguer si l'on devait s'en tenir aux figures de cette dernière (H.-S. f. 213-215). Elle offre aussi quelque ressemblance avec Turionana?, lorsqu'elle prend une teinte rouillce; l'éclat métallique des raies blanches la fait bientòt distinguer d’elle. Olivana $q$ pourrait déjà plus difficilement ìtre confondue avec elle.

La teinte générale est le lorun fauve, tirant tantôt sur le brun foné, tantòt sur la couleur de rouille, tantôt sur l'olivâtre. Sur cette teinte se détachent plusicurs bandes transverses, étroites, blanches, avec íclat métallique, divisées par des stries brunes qui les teruissent. L'éclat argenté est surtout marqué sur la tache oblongue de langle anal des supérieures. La còte est marquée de 5 crochets blanes, correspondlant aux lignes blanches; les denx premiers (a partir de la base) sont doubles, les trois derniers sont quel puefois simples; le quatrième n'abontit pas turjours a une raie blanche. L'espace basilaire est limité par une raie blanche qui vers le bord abdominal se confond parfois avec les bandes hlanches voisines. Le reste de laile est pareouru transversalement et obliquement par des filets blanes conlluant les uns avec les autres. Vers la marge et sous le sommet existe une tache brune, plus grosse que les autres, qui aboutit diune part à celle du sommet, de l'autre envoie un ramean au bord interne. Dn reste, ce dessin varie passablement sans jamais arriver à celui dririguana.

Les antérieures sont étroites; la côte est droite; le sommet assez aigu et le bord externe coupé obliquenent. La frange est blanche, entrecoupée de gris au sommet et vers langle anal.

Les inférieures sont d'un gris roux, avee la frange blanchâtre. - Le dessous est gris-brun, un peu marbré; une tache grise existe au sommet des infirieures. La frange est limitée par un trait blane. saillant surtout aux supérienres. Les erochets blanes et les taches de mìme couleur de la frange se voient nettenent en dessous. - Mouchet anal blanc. 
Irriguana s'en distingue: $1^{\circ}$ Par l'absence de teintes rouillées. $2^{\circ}$ Par le tiers externe des supérieures souvent effacé. $3^{\circ}$ Par la còte plus droite, surtout vers le sommet. $4^{\circ}$ Par sa frange grise (et non blanche) mouchetée de blanc. $5^{\circ}$ Par sa bande médiane formant use demi-lune à une concavité interne, avee un angle à l'opposite, élargie vers le bord interne et retrécie vers la còte. - Dans Il endosana, la bande médiane, mal dessinée pour l'ordinaire, est de forme pyramidale, large à la eòte, étroite vers le bord postérieur et déchirée sur les flancs. $-6^{\circ}$ Par ses crochets grisàtres. $7^{\circ}$ Par la teinte gris-noir des inférieures. $8^{\circ}$ Enfin par le monchet anal gris.

Ilendosana pourrait anssi itre confondue arec Trochilana, Fröhl.; mais chez celle-ci les teintes fauves sont d'un brun foncé et les blanehes sont enfunées, moins semées d'atômes et sans éclat métallique.

Schacferana reproduit eneore le dessin de notre nouvelle espèce; mais eclle-ci n'offre pas les points noirs prononcés, placés au centre des bandes brines et formant par leur continuité une espèce de trace longitudinate, entrecoupée.

La femelle ne diffère pas du màle.

\section{Trifasciana, Zell.}

Hub. revis. n²38. - Hub. sup. f. 184. - Heyden. c. 43.

Artemisiana, Zell. - Ileydl. c. 243.

Bieinctan a, Dup. sup. 90. - Guén. Ind. - Ileyd. c. 39.

Marmorana, Koll.

Cette espèce assez rare a été prise dans le Jura, anx environs de la Neuveville, par M. Conleru. M. Bruand l'a récoltéc aussi dans le Doubs, M. Rothenbach près de Bienne.

La chenille a été tronvée par M. Rothenhach sur l'Allium oleraceum; elle est verte et se loge dans les eapitules de fleurs. Elle a atteint toute sa croissance au commencement de juillet; le mois suivant le papillon éelot d'une petite chrysalite verdàtre dont la tète est brune et le dos couvert daspérités. Au monent de l'éclosion, la chrysalide prend une teinte violette (Rothb. in litt.).

160. Charpentierana, Treit.

Dup. 246. - Hub. revis. n 239. - IIubu. sup. f. 201. - Ileyd. C. 240.

Inter ruptana, Fröhl. - Guén. Ind.

Assez fréquente à la fin de juillet sur les pàturages inférienres des Alpes du haut 
Valais; Simplon; Tzermatten; les Alpes vaudoises çì et là. Sur le Mühlebachilp (Glaris) (Frey). - Vallée de l'Oberhasli (Ott.).

La femelle, plus rare, est un peu plus petite que le inâle et ses teintes foncées sont plus vives. Les croehets de la còte sont incomplets; celle-ci n'est inarquée que de trois points noirs; mais en y regardant de près, on tronve: $1^{\circ}$ Tout près du somnet, un crochet double presque toujours caché sous les teiutes violettes qui ombrent l'extrémité de l'aile. $2^{\circ}$ Un autre erochet simple ou clouble, rapproché du précédent, placé au bord de la tache noiràtre du sommet et quelquefois couvert par elle. $3^{\prime \prime}$ Les deux crochets suivants, placés sur la tache blanche, sont larges et simples; leurs intervalles, réduits à deux points noirs, n'envoient aucune ligne d'ombre. - En dessous, les crochets sont peu marqués et très-enfumés. Un bon earactère se tire de la ligne noire de démarcation de la frange des supérieures, qui, en dessous, est pour lordinaire coupée par les taches blanches de la marge qui entreeoupent aussi la frange. Le bord externe des supérieures est légèrement plus oblique que dans Bipunctana. (Voir Bipunetana).

\section{Gen. Notocelia, Steph.}

Aspis, Treit. Guén. Curt. - Aspidia, Dup. - Spilonota, Steph.

161. Udmanniana, Lin.

Ilus. Schif. - W. V. - Fab. - Fisch. v. R. t. 91. - Hub. revis. n²40. - Dup. cat. - Guén. Ind. - Hleydenr. c. n²58. -Bruand, C. 983. - Curt. - Wood. 1026.

Solandriana, Frey, ï. b. - Treit. - Dup. 245. (non Lin.).

A elu at ana, Ilub. 49.

Rubiana, Scop.

En juin et en juillet dans les taillis, un peu partout, quoique nulle part fréquente. De même dans les environs de Zurich (ZelI. Frey), de Schüpfen (Rothb.).

XXI. Gen. Coccyx, Treit. Dup.

Retinia, Guén. - Orthotenia, Steph. Curt,

162. Comitana, W. V.

Treit. - Mus. Schif. - Dup. 247. - Hubn. revis. $n^{\circ} 241$. Curt. - Wood. 1131. - Heyd. c. 188. - Bruand, C. 1072.

Ilereynana, Fröhl. (non Treit.) — Guén. Ind. 
Pice ana, Hub. 72. - Zett. - (non Lin.).

Abietana, Steph.

Très-commune partout oì il y a des sapins, dès la fin de mai en juillet, suivant les altitudes (Zell. Rothb. Frey). - Dans les Alpes comme dans la plaine.

16:3. Proximana, Metzn.

IJub. revis. " 242 - IIub. sup. \{. 127. - Heyd. e. 189.

Jai pris une fois la femelle dans les bois de sapin au-dessus de Lausanne: M. Rothenbach a recueilli le mâle jrìs de Sehiipfen; jai reçı un individu màle, collecté par $H$. G. Leresehe, dans le Jura; II. Zeller l'a aussi rencontrée dans les environs de Ziirich.

Partont isolée. Se prend en mème temps que Comitana. Sa rareté au milieu d'essaims de eette dernière semble justifier sa rualité d'espéce.

161. Nigricana, Mann.

Hub. revis, " 243. - Hub. sup. f. 138. - Heyd. c. 202.

Dans les bois de sapin du Jorat, au commencement de juillet; pas rare. De mème pris de Zurich (Zell., Frey) et de Schuipfen (Rothb.). Les exemplaires bien frais sont rares.

16.5. Nana, Treit. sup.

$$
\begin{aligned}
& \text { Ratzl. - Dup. sup. 66. - Hubn. revis. n²44. - IJubı. sup. } \\
& \text { f. 129. - Guén. Ind. - IJeyden. c. 203. - Bruand, C. } 1067 .
\end{aligned}
$$

Jai recueilli un exemplaire de cette petite espèce dans les bois du Jorat, en aoùt.

166. Clausthaliana, Ratzb.

Hubn. revis. n² 246. - Inß). sup. f. 131. - Guén. Ind. Dup. eat.

Hercyniana, Treit. - Bechst. - Heỹden. e. 187. - (non Fröhl.).

Pas très-rare dans les bois de sapin du Jorat, à la fin de juin. Je l'ai aussi reçue des environs de Burgilorf. II. Rothenlsach l'a eollectée du 10 au 30 juillet.

Varic quelque peu; parfois le fond est complétement noir, sablé irrégulièrenent de blane; chez d'autres individus les atòmes blancs forment une bande transverse au tiers de l'aile et une deni-])ande oblique aux deux tiers; ces derniers ressemblent quelque peu à Ulmana $\%$. La frange porte parfois un ou deux points blanes en dessous du sommet.

Nos individus ont rarenıent autant de blanc que la figure 131 1I. S. et le noir y est très-foncé. 


\section{Resinana, (ella) Lin.}

Fab. - Fröhl. - Treit. - Dup. 247. - Ilub. 29. - Iful. revis. $n^{\circ}$ 248. - Guén. Ind. - Heyd. c. 178. - Bruand, C. 1075.

11. C. Zeller l'a prise dans les environs de Zurich; jai pu massurer de son iılentité. 168. Fuligana, Hub. 109.

Treit. - Dup. sup. 62. - Ilub. revis. n² 249. - Ilub. sup. f. 332. Heyd. c. 337 . - Curt. - Brrand, C. 1102.

Cáa et là sur les prés sees, en juin et en aoùt; plus rare dans les environs de Lansanne; plus commune dans la vallée dlAigle. Environs de Schüpfen (Rothb.); de Zurich (Frey).

Lidentité des individus recueillis autour de Lausanne et de eeux reçus d'Allemagne ne miest point encore démontrée. La place de cette espèce me praraît aussi mal choisie, elle a plus de rapport arec eertaines Penthina quavec les Coecyx.

169. Arbutana, Hub. 195.

Lin. - Fröhl. - Hub. revis. n²50. - Zett. - Guén. Ind. Ileyden. C. 113. - Dup. eat. - Bruand, C. 1000.

Reeueillic Uans les bautes Alpes de l'Oberhasli, en aoùt, par M. J. Ott, de Meyringen. Fréquente dans l'Engadine (Frey).

On rencontre quelques individus plus pàles; cette décoloration est due aux influences atmosphériques.

Spadiceana llub. 312 me parait se rapprocher davantage de Mercuriana.

170. Senecionana, Hub. 263.

$$
\text { Fröhl. - Guén. Ind. - Hejden. n¹12. }
$$

II. Imhof, professeur à Bàle, ma communiqué obligeamment un exemplaire bien couservé de cette espèce, pris dans les environs de Bàle. Lindividu répondait parfaitement à la figure de Hubner. Malheureusement jignorais alors que cette espèce n'était guc̀re connue que par la figure de llubner et la eitation de Fröhlich, en sorte qu'après avoir constaté son identité, je la retoumai à son propriétaire sans la décrire.

Il est difficile de dire à quel groupe it convient de la réunir. L'absence de dessin ne permet que des conjectures. La forme et les dimensions sont celles de Daldorfiana; mais il n'existe pas de point d'intersection an-dessous du sommet des supérieures. La couleur jaune des supérieures, avec la frange et les inférieures grises me semblent établir une certaine analogic avec les Cochyles. 
La figure de Ilubner est très-exacte. - Herrich-Schäfer, n'ayant pas vu l'espèce en nature, ne la mentionne pas dans sa Revision; mais dans sa Table synoptique il penche à n'y voir quune variété de la femelle de Rusticana. Ce rapprochement, qui ne repose que sur une certaine analogie de coloration, est tout-à-fait inadmissible.

\section{Buoliana, W. V.}

F. - Fröhl. - Treit. - IIubn. revis. n 251. - IItubn. sup. f. 49 (err. 94). - Guén. Ind. - Heydenr. c. 186. - Ratzb. Bruand, C. 1074.

Gemmana, IIub. 269. — Wood.1127. - Dup. 217. -- Curt.

Xylosteana, llub. 154.

Espèce partont assez rare en Suisse; gè et là dans les bois des montagnes. M. Rothenbach l'a prise dans les environs de Langnau.

172. Turionana, Hub. $220,221$.

Ratzl. - Wood. \{126. - Hu!nn. revis. n²52. - Guén. Ind. -

Resinella, Curt.?

Heyd. e. 181. (non Dup.).

Dans les environs de Bàle (Imhof). Près de Schüpfen (Rothb.). Je l'ai'reçue de Meyringen. - Partout rare.

173. Pudendana, Fisch. r. Rösl.

Hub. revis. n' 253. - Itub. sup. f. 149, 150. — Ifeyd. c. 182.

Lateritiana, Khlw.

Pinivorana, Zell. Isis. 1846.

H. Rothenbach a collecté cette espèce près de Seliupfen.

174. Duplana, Hub. 229, 230.

Treit. - Hub. revis. n 255 . - Guén. - Heyden. c. 184.

Spadiceana, Dup. 263. (non Hub.).

Posticana, Zett.

Jai reçu cette espèce de H. Andereggg qui T'arait recueillie en Valais. M. Rothenbach en a pris plusieurs individus près de Sehüpfen au premier printemps. M. Frey la prise près de Bremgarten, au printemps, et moi, à la mème époque, près de Lausanne, sur les pins.

La femelle a les ailes un peu plus larges et plus fortement dessinées que le mále. 


\section{Squalidana, Fisch. v. Rösl.}

llub. revis, no 256. - Heyd. c. 336 .

Nubilana, Mus. Podev.

Var. Remy anae, Ilub. sup. f. 276. - Heyd. cat. 339.

I. le prof. Frey a collecté, dans les environs de Zurich, un individu appartenant très-probablement à cette espèce. M. Wullsclılegel note cette espèce parmi celles quil a recueillies à Engelberg près dolten.

176. Metalliferana, Fisch. v. Rösl.

$$
\text { IIub. revis. 258. - IIub. sup. f. 204. - Heyden. c. } 215 .
$$

Scorian a, Guén. Ind.?

Metallicana, Dup. 246,6, - Bruand, C. 99 I.

Cette jolie espèce riest pas rare sur les hautes Alpes et sur le Jura, ì la fin de juillet et au commencement d'aoùt. Elle préfere les moraines de glaciers, les lieux pierreux, et sole en plein soleil, le matin. - Enzeindaz, la Furka, etc. Les luantes Alpes (Zell.); le pied du glacier de l'Aar; IEngadine (Frey). Au-dessus de Ste-Croix (Jura) (Leresche).

M. G. Leresche a obtenu cette espece de chrysalides quil avait trouvées sous les pierres dans le mois de juillet. Lestrémité de l'aile est ordinairement d'une teinte plus claire que sa base; on trouve dans les Alpes des individus entièrement noirs arec les handes jaunes l'une égale intensité.

La figure de H.-S. est prise sur un petit individu.

177. UImana, Hub. 278 ๖.

Dup. 264. (non 242). - Hubn. revis. n² 261. - Guén. Ind. IIeyd. c. 275. - Bruand, C. 1060.

Areolana, Ituh. 279 \&. - Dup. $264,6$.

Assez fréquente dans les haies du Jorat et des montagnes, en juin et juillet. Près de Schiipfen (Rothb.); de Zurich (Zell.). Pas rare sur lUetliberg, au commencement de juillet (Frey).

Duponchel figure la fenelle sous le nom dUlmana et le male sous celui d'Areolana.

178. Schreibersiana, Hub. 306 .

Fröhl. - Treit. - Dup. 258. - Guén. - Ilub. revis. n 263. Ileyal. c. 712 . 
Lediana, Hub. 151. - W. V. - Mus. Sclif. (non Lin.). - Bruand, C. 1117.

Cette espèce a été collectée par M. Imhof dans les environs de Bàle. Dans le Doubs (Bruand).

179. Andereggiana, Hub. sup. f. 416.

Guén. Ind. p. 27. - Hub. revis. n 266.

III. Boisduval et Fisch. v. Rösl. ont reçu chacun un exenplaire de M. Anderegg. Je ne l'ai jamais vue. Très-voisine de Botrana.

180. Fischerana, Fisch. v. Rösl.

Hub. revis. n 268. - Ilubn. sup. f. 313, 314. - Guén. Ind. -

Heyden. c. 717 . - Treit.

Permixtana, IIul). 75 (non 187). - (non W. V.) - Dup. 263. - Bruanl, C. 1115 .

Cá et là dans les environs de Lausanne; rare. Le 6 juin, sur les sapins (Rothb.). Cette espèce est souvent confondue avec Botrana, W. V. (Reliquana, Hub. verz.).

XXII. Gen. Penthina, Treit.

Penthina et Aspidia, Dup. - Ditula, Penthina, Antithesia, Spilonota et Pardia Guen.

181. Salicana, Lin. (ella).

Hus. Schif. - W. Y. - F. - - Fröhl. - IIub. 11. - Treit.-

Iup. 245. - Fuessli. - Guén. Ind. - Heyd. c. 15. - Wood. 895. - IIub. revis. n² 269. - Curt. - Bruand, C. 976.

Partout commune en juin et juillet dans les haies, les taillis. Elle se pose sur les feuilles et ressemble parfaitement alors à une fiente d'oiseau.

De mène dans les cantons de Berne (Rothb.) et de Zurich (Frey, Zell.).

182. Inundana, W. V.

Mns. Schif. - Hul. 60. - Fröhl. - Treit. - Hub. revis. n² 270. Guén. - Heyden. C. 304. - Dup. cat.

Cette espèce rare a été élevée par M. Couleru et prise par Mr. Rothenbach dans les environs de Schiipfen.

183. Acutana, Treit. sup.

Frey. - Dup. sup. 64. - Hubn. revis. $n^{\circ} 271$. - Ilubn. sup. f. 179, 180. - Guén. Ind. - Ileyd. c. 12. - Frey. n. B. 342. 
Elutana, Dup. 1335. t. 264.

Profanana, Wood. 1047.

Semifasciana, Steph. - Curt. - Haw.

Liturana, Khlw.?

Une fois dans les environs de Lausanne. Reçue de M. Heuser à Burgdorf. - Environs de Schiipfen (Rothb.). - Rare.

\section{Hartmanniana, Lin.}

$$
\text { Fab. - Treit. - Dup. 245. - Treit. - Guén. Ind. - Iubn. }
$$
revis, no 272. - Hub. sup. f. 162 (var.). - Bruand, C. 969.

Line a n a, L'harp. - Mus. Schif.

Seriptana, Hub. 110. - Fröhl. - Steph. - Curt.

Liturana, Khlw.

Les baies au-dessus de Lausanne, à la fin de juin; pas très-rare. Environs de Schipfen (Rothb.).

Si elle se prend aussi en septembre, comme l'affirme Herrich-Schäffer, elle fait denx apparutions par an.

185. Picana, Fröhl. 101.

Guén. Ind.

Corticana, Hub. 13.

Capreana, var, auctor. - Dup. pl. 245 f. 4. b.

Fasciana, Charp.

Cette espèce n'est pas très-rare dans les haies et les taillis du Jorat, au-dessus de Lausanne, en juin.

Guénée a, selon moi, parfaitement raison de distinguer cette espèce de Capreana Inub. 250 et de sa Lencomelana. Ses earactères les plus saillants sont: $1^{\circ}$ Les supérieures en tout sens plus arrondies; $2^{\circ}$ leur base marquée de deux taches blanches $3^{\circ}$ la deuxième bande d'un noir foncé, marbrée de brun, très-irrégulièrement coupée sur ses denx bords. 4 l'espace terminal, d'un blane de lait pur, semé de quelques traits gris, obliques, d'un ou deux petits points noirs, est ruarqué sur la còte de trois petites dents. $5^{\circ}$ La frange des supérieures d'un blane sale en dessous, entrecoupée de gris-noir au centre. $6^{\circ}$ Les inférieures d'un roux clair.

La tète ef le thorax ne sont pas entièrement noirs comme le dit Guénée; car les palpes portent plusieurs poils blancs, et la collerette est grise. 
Cette espèce se rapproche surtout de Ifartmanniana, aussi Treit. lenvisageait-il comme une variété de cette dernière.

186. Capreana, Hub. 250).

$$
\text { Treit. — Guén. Ind. }
$$

Capreana, var. Auctor. - (non Dup.).

Jai sous les yeux un très-bel exemplare mâle de cette espèce, pris dans les environs de Lausanne, en juillet, je erois. Il répond parfaitement à la figure de Jluhner.

Guénée doute que cette espèce soit différente de sa Leucomelana. A mes yeux elle se rapproche davantage de Variegana que d'elle. La taille, le facies et la coupe des ailes sont les mèmes.

L'espace basilaire est brun, pointillé de blanc sale, arrondi et irrégulier sur son bord. La première bande est d'un blanc terne fortement chargé datômes bruns et gris. La seconde bande (médiane) est bistre, tachée de brun foncé et marbrée de gris, de blanc et de violet. Ses deux bords sont très-irréguliers; l'interne forme un angle très-saillant au centre et denx sinus de part et d'antre de langle; l'externe, un sinus d'un blane pur au centre, auprès duquel se trouvent deux points d'un brun très-foncé. Le brun de la bande moyenne se prolonge un peu le long de la còte. L'extrémité de l'aile est blanche et parcourue par deux à trois ondes plombées, au milieu desquelles se placent en groupe 4 à 5 points noirs. La còte ne présente pas de crochets. La frange est grise, limitée par un trait gris suivi d'un blanc; son angle interne est blanc pur.

Les inférieures sont brunes en dessus et sensiblement plus elaires à la base. Leur frange est rousse et grise vers l'angle internc.

Le dessous est entièrement roux avec les inférienres plus claires. La còte présente 4 à 5 points blancs. La frange des supérieures est rousse, bien limitée par un trait brun précédé et snivi d'une ligne blanche. La frange des inférieures est comme en dessus.

Comme on le voit, cette espèce ne peut se rattacher ni à Pieana, ni à Leucomelana.

\section{Ochroleucana, Hub. 30 4.}

Fröhl. - Treit. sup. - Hub. revis. n 274. - Heyden. c. 24.Guén. Ind. - Dup. sup. 61. - Bruand, C. 972.

Gentianeana, Dup. 245. 7. 
Point rare, en juin et juillet, dans les jardins des environs de Lausanne, où la chenille vit sur les rosier's. Environs de Schiipfen (Rothb.).

188. Variegana, Hub. 14.

Treit. - Dup. 245. - Mus. Schif. - llubn. revis. n²75. Guén. Ind. - Heyd. c. 18. - (non W. V.). - Bruand, C. 970.

Poecilana, Fröhl.

Tripunctana, Wood. 888. - Steph. - Curt.

F asciana, llub. larv.

Cynoshana, Mus. Lin. (fide Bru.) - Don.

Commune dans les environs de Lausanne, en juin. De mème près de Zurich (Zell. Frey) et de Schüpfen (Rothb.).

189. Pruniana, Hub. 15.

$$
\begin{aligned}
& \text { Fröhl. - Treit. - Dup. 245. - Zell. - Hub. revis. n॰276. - } \\
& \text { Guén. Ind. - Heydenr. c. 19. - Wood. 890. - Bruand, } \\
& \text { C. } 971 .
\end{aligned}
$$

Fasciana, Scop. - Zinc.

Pullana, Wood. 891.

Extrèmement commune en juin, dans toutes les haies d'aubépine. (Zell., Rothb.).

Varie pen, sauf par l'extrémité de l'aile plus ou moins couverte de brun et de noir. Je n'ai pas encore observé la variété teintée de couleur chair dont parde Treit.; n'appartiendrait-elle pas à Dimidiana?

On tronve sur les Alpes une variété dont l'espace terminal de l'aile est entièrement blanc, à l'exception dune tache grise au centre de eet espace et du sommet noiràtre; on ne peut en faire une espèce distincte, car on retrouve sur elle des traces de l'un des caractères spécifiques de Pruniana, caractère qui se tire de deux petits points blancs situés, l'un à côté de l'autre, aux deux tiers antérieures du bord interne (marge). Ces deux points, plus visibles en dessous qu'en dessus, correspondent sur la frange, à deux traits blancs ou bleuàtres qui ne divisent pas la ligne d’intersection, et sur le disque, à deux traits bruns ou noirs (visibles seulement sur les individus fortement teintés) qui divisent d'abord une ligne plombée, puis parfois la tache brune du centre de l'espace blanc terminal. Dans les individus des Alpes dont nous avons parlé, les points blancs et la ligne plombéc existent seuls. Le sommet de l'aile reste toujours noir. 


\section{Sauciana, Hub. 305.}

Frölıl. - Zell. - Hubn. revis. n² 279. - Ilubn. sup. f. 181. Guén. Ind. - Ileyden. c. 22. - (non Dup.).

Marginana, Wood. 892.

Langerana, khlw.

Cette espèce n'est pas rare sur les hautes Alpes; je l'ai prise à la Mayenwand, sur le col du Gotthard, près de l'hospice; au pied du Mlont-Blanc; je l'ai reçue des Alpes de l'Oberhasly. M. Rothenbach lindique près de Sehïpfen et M. Zeller près de Zurich; n'y a-t-il pas ici erreur?

Sauciana habite surtout parmi les Rhododendron dans le voisinage de lAlnus viridis, sur lequel il est probable que vit la chenille. Je lai aussi prise sur le Rhamnus alpinus.

La partie blanche de l'aile est parfois teintée de fauve, sur les individus frais; dans ce cas sa partie foncée prend une coloration rousse; lorsque le blane est pur, cette teinte passe au violet. Les caractères tirés de labsence et de la présence des points noirs et blanes sur l'extrémité de l'ailc, de la coloration noiràtre plus ou moins étendue du sommet accompagnée de points noirs divers, de lexistence d'un gros point noir au centre du bord externe de la bande moyenne et se détachant sur le blane, - ne sont pas constants. On ne peut la distinguer sùrement de Pruniana que par ses ailes plus étroites, plus arrondies au sommet; par sa frange entièrement gris-foné et par les caractères indiqués pour Pruniana.

\section{Gentianana, Fröhl.}

Treit.? - Frey, n. b. 120, 2. - Ilubn. verz. - Hubn, revis. n²81. - Guén. Ind. - IIeyd. c. 29. - Bruand, C. 974.

Pas fort rare dans les lieux montagneux au-dessus de Lausanme, en juin. - Environs de Schüpfen (Rothb.), de Zurich (Zell., Frey).

Je ne puis admettre, pour cette espècc, la figure 12 de Ilubner. J'ai sous les yeux une Ochroleucana teintée de gris-rose qui reproduit parfaitement cette figure. Nos individus dépassent rarement la taille de Sellana et lui ressemblent beaucoup (comme le dit aussi Guénée), à part les inférieures entièrement dun brun-noir. Il est probable que Duponchel a été induit en erreur par la figure de Ilubner en donnant à Ochroleucana le nom de Gentianeana. 
192. Sellana, Hub. 3331 .

Fröhl. - Hub. revis. n²82. - Hub. sup. f. 183. - Guén. Ind. Heyden. c. 30. - Bruand, C. 975.

Var. Gentiananae, Treit. - Zell. - Evers. - Heyden.

Sauciana, Dup. 262.

Tenebriosana, Khlw.

Oblongana, Wood. 593. - Haw. - Curt.

Assez fréquente sur les prés des environs de Lausanne et de la vallée d'Aigle; une premiere fois en avril et mai, une seconde en juillet. Environs de Zurich; vallée d'Engelberg (Frey).

La figure de Duponche] n'est pas tries-bonne.

198. Betul etana, Zell.

Hub. sup. f. 251, 252. - Haw. - Hub. revis. n 283.

Leuconelana, Guén. Ind. p. 18.

Capraeana, Dup. pl. 245. f. 4. a.

Sororeulana, Zett.

Je possède deux individus de cette espèce dont une femelle très-frat̂che, prise en aoǹt, au bois de Sauvabelin, près Lausanne.

Quoique H. Herrich-Schäffer envisage lindividu dont il est ici question, et qui lui a été soumis, comme une Capreana (Picana), je ne puis me ranger à cet avis.

L'époque de l'apparition et très-dilférente, puisquil a été pris le 10 aoùt, tandis que Capreana rapparaît plus après le milieu de juillet. Il y a plus, Betuletana se rapproche davantage de Pruniana et surtont de Variegana que de Picana. Les ailes supérieures sont un peu plus étroites que chez ees dernières; le bord terminal est coupé presque droit et le sommet moins arrondi que dans Picana. Les deux tiers basilaires sont marbrés de brun bistre et de violet, avee quelques points noirs et blanes; les premiers sur la còte, les seconds sur le centre. Le tiers externe forme un triangle dont le còté interne est bien moins irrégulier que dans Picana. Son còté antérieur est à peine marqué de $3-5$ petits crochets donnant naissance à autant de lignes plombées, peu marquées, qui descendent sur le disque en se recourbant et s'élargissant. La frange est d'un blanc sale entremèlé de gris, et limitée par un trait gris peu prononcé qui ne s'appuie pas sur une rangée de points noirs, conme chez Variegana.

En-dessous, les supérieures sont d'un brun bistre, avee la moitié de la còte et la marge blanches; lextrémité de la frange est mouchetée de gris. 
Aux inférieures, le dessus est brun, plus clair à la racine; la frange est blanche et le dessous blanc-sale assez luisant.

La ligure de Duponchel est nauvaise, quoiqu'elle indique bien les lignes plombées de la portion blanche et la dent qui se voit sur cette portion vers le milieu de la bande moyenne.

Les figures de llub. sup. ne répondent pas entièrement aux individus que jai sous les yeux. Elles sont diun tiers plus petites, beancoup plus sablées de clair sur la partie foncée et tachées de noir et de brun vers le sommet; ce qui n'existe pas chez moi.

194. Dealbana, Fröhl.

Ilubn. revis. $n^{\circ}$ 288. - Guén. Ind. - Heydenr. c. 38. - Dup. cat. - Bruand, C. 979.

Minorana, Fisch. - Treit. - Fisch. v. Rösl. t. 22. - Dup. 265.

Neglectana, Dup. sup. 89 ?

Commune dans les environs de Lausanne en juillet, partout où croissent les aunes. Environs de Schüpfen (Rotlıb.), de Zurich (Zell. - Frey).

La figure de Duponchel est assez mauraise.

195. Aceriana, Mann.

Fisch. v. Rösl. - Hub. revis. n² 289. - Hub. sup. f. 350. Guén. lud. - Dup, sup. 76. - Heyd. c. 41. - Zell. Isis. 1847. - Bruand, C. 978.

Pas rare dans les haies le long du torrent de la Paudèse, près Lausanne, en juillet.

196. Suffusana, kiuhlw.

Dup. sup. 83. - Hlubn. revis. $n^{\circ} 290$. - Hubn. sup. f. 178. Guén. Ind. - Ileyd. c. 35. - Bruand, C. 980.

Cynosbana, Dup. hist. 245. (non Treit.).

Communana, Guén. olim. (non Hub. revis.).

Funerana, khlw.

Trimaculana, Wood. 899. - Haw. - Curt.

Commune dans les haies des environs de. Lausanne, en juin. De mème près de Zurich (Zell., Frey).

197. Incarnatana, Hub. 191.

Fröhl. - Dup. 264. 4. - Wood. 966. - Guén. lnd. - Hubn. revis. $n^{\circ} 291$. 
Amoenana, Ilubr. 248. - Dup. sup. 84. - Ileydenr. c. 34. - Guén.? Bruand. C. 973.

M. G. Leresche a pris une fois cette espèce en septembre, près de Lausanne. Près de Schüpfen (Rothb.).

198. Roborana, W. V.

Treit. - Mus. Schif. - Zinck, - Ilub. revis. n 294. - Zell. Heyd. e. 33.

Cynosbana, Fab. - Guén. - (non Dup.). - Bruand, C. 981.

Aquana, Ilub. 17. - Wood. 898. - Curt.

Commune partout, dans les jardins et les haies, en juillet. De mène près de Schüpfen (Rothb.), de Zurich (Zell., Frey).

199. Tripunctana, W. $Y$.

Mus. Schif. - Fröhl. - Hubn. revis. n 295. - Guén. Ind. Bruand, C. 982.

Cynosbana, Treit. - Steph. - Zell. - Fuessli. - Wood. 897. - Heydenr. c. 32. - Curt. - Haw. (non Dup.),

Ocellana, Ilub. 18. - Dup. 245.

Assez commune dans les haies des entirons de Lausanne, à la fin de mai et en inin. De mème près de Zurich (Frey) et de Sehupfen (Rothb.).

200. Ocellana, W. V.

Mus. Schif. - Treit. - Zinek. - Ilubn. revis. n 296. - Hubn. sup. f. 37 1. - Guén. Ind. - Heyd. c. 36. - Bruand, C. 977.

Luseana, F. - Fröhl. - Dup. 245.

Comitana, Hub. 16. - Wood. 900. - Curt.

Var. Grisescens, Guén.

Assez rare dans les environs de Lausanne, en juin; les bois du Jorat. - Rare près de Sehripfen (Rothb.). - Envirous de Zurich (Frey).

La figure de Dup. est trop grande.

201. Simplana, Fiscl. v. Rösl. t. 22.

Hub. revis. n² 298. - Guén. Ind. - Heyd. c. n 44. - Dup. cat.

Ambiguana, Dup. hist. 262. (Cochylis).

Oppositana, kllw.

Espèce très-rare. Près de Zurich (Frey). Environs de Burgdorf (IIeuser), de Schüpfen (Rothb.). 
202. Servillana, Dup. hist. 262. 6.

Hub. revis, n²99. - Hubn. sup. f. 98. - Guén. Ind. - Heyd. c. 696 .

Nubilosana, Fiscl.. v. R.

J'ai collecté un individu appartenant à cette espèce rare, dans les environs de Lausanne, en juin 1849.

XXIII. Gen. Pa edis ca, Treit. Dup.

Ephippiphora, Guén.

203. Similana, W. V.

Mus. Schif. - Treit. - Fisch. v. Rösl. t. 63. - Ilubn. revis. n³01. - Guén. Ind. - Jleỵd. c. 345. - Dup. cat. — Bruand, C. 105 t.

Seutulana, Dup. 253.

Stroemiana, F. - Curt.

\& Fluidana, W. V. - Mus. Schif.

\& Asseclana, Iub. 19.

Bimaculana, Don.

Les prairies au milien des bois, en mai et juin, sur l'Uetliberg près Zurich. Assez commune certaines années (Frey). Environs de Zurich (Zell.), de Schüpfen? (Rothb.). Une fois, au pied de la montagne, sur des pàturages secs, près de Villeneuve, en juin (Lah.); près de Berne et sur l'Engelberg (Olten), (Wullschlegel).

204. Mendiculan a, Treit. sup. III., p. 87.

Hul, revis. n³02. - Hub. sup. f. 146. - Guén. Ind. - Heyd. cat. $\mathrm{n}^{\circ} 279$.

q Cinerosana, Zell.?

J'ai recueilli cette espèce près de l'hospice de la Grimsel, volant sur les bruyères; le long de l'Aar en remontant son cours. Y. Ott m'en a fait parvenir un exemplaire des environs de Meyringen. Je l'ai prise aussi dans les Alpes vaudoises, au-dessus du glacier de Plan-Névé.

Treitschke rapproche avec raison cette espèce de Quadrana. Comme chez celle-ci, la femelle est plus petite, a les ailes plus étroites, plus nettement dessinées, avec les taches plus foncées et sans mélange de couleur rouille. Les crochets de la côte sont 
au nombre de quatre, et non de deux; "mais les deux derniers (du còté de la base) sont très-rapprochés et peu distincts. Le dessin est celui de Quadrana.

La figure de Hub. sup. fut sans doute prise sur un individu effacé par la vetusté. Sa taille est celle des plus grands indivilus. La tache carrée du bord postérieur n'atteint pas le bord antérieur; elle est de couleur brun-foncé, ainsi que la suivante, et se dessine nettement sur le fond gris voisin. Les teintes rouillées du mâle, dispersées sur le centre de l'aile, napparaissent pas dans cette figure; il en est de mème du pointillé blane du fond et d'une petite ligne blanche placée le long de la marge au-dessous du sommet de l'aile. - Si mes individus n'enssent pas passé sous les yeux de M. Herr.Schälfer, jaurais admis une discrsité d'espíce. Nayant pas sous les yenx d'exemplaires venus de Ilongrie, je ne puis décider la question.

20j. Confusana, Fisch. v. Rösl. collect.

$$
\text { Man. cat. - Ileyd. c. } 335 .
$$

Leucophoeana, Khlw. ?

M. Frey a recueilli un exemplaire sur le Zürichberg, à la fin de juillet. Je ne l'ai pas vu, mais il a été déterminé par M. Herr.-Scháffer.

llepaticana n'a pas encore été prise en Suisse.

206. Scutulana, W. V.

Mus. Schif. - Fisch. v. Rös. t. 64. - Treit. sup. - Hubn. revis. n 309. - Ilub. sup. P. 228. - Guén. Ind. - Ileyd. c. 346.(non Dup.) - Bruand, C. 1053.

\& Profundana, Hub. 21.

Trigeminana, Pflugiana, Semifuscana, Wood.?

II. Rothenbach a collecté cette espèce près de Schïpfen, H. Zeller dans les environs de Ziirich et M. Frey dans I'Engadine, près de Samaden.

207. Dissimilana, Treit. sup.

Fisch. v. R. 1. 62. - Hub. revis. $1{ }^{\circ} 310$. - Guén. Ind. - lleyd. c. 363 (non Dup.).

Similana, flub. 41. - Dup. 254.

Stroemiana, Wood. 903.

Cá et là dans toute la Suisse; habite les hois taillis, en juillet. Près de Schüpfen (Rothb.); de Zurich (Zell., Frey); d'Olten (Wullschlegel). 


\section{Demarniana, Messing.}

Fisch. v. Rösl. t. 65. - Hub. revis. $n^{0} 31$ f. - Ilub. sup. f. 232.

Plugiana, F.

- Guén. Ind. - Heyd. c. 351.

Indistinetana, Khlw.

Trouvée une fois au bois de Sauvabelin près Lausanne, en juin.

209. Delitana, Fisch. v. Rösl. t. 65.

Dup. sup. 64. - IJubn. revis. $n^{\circ} 312$. - Guén. - Heydenr. C. 328 .

IV. Herr--Schïffer a reçu de la Suisse cette espèce rare.

\section{Couleruana, Dup. hist. n 125i, 1. 25:3, 8.}

Guén. Ind. - Ileyd. cat. 341. - Bruand, C. 1052.

Riseana, Ilub. revis. $n^{\circ}$ 313. - IJub. sup. f. 186. - IJeyden. c. 652.

Cette espèce, propre au Jura, a été fréquemnent élevée par II. L. Couleru de la Nenveville ef par M. Bruand. Sa chenille vit en mai sur le Teucrium montanum. Linseete parfait apparait à la fin de juin et en juillet. - M. Rothenbach l'a collecté au pied du Jura, sur les pentes arides. Je l'ai pris, une fois, sur le Nauremont, près Lassaraz. M. Herr--Sehäfer a reconnu sa Riseana sur des individus provenant de H. Couleru, que je lui ai présentés.

La figure de Dup. est bonne; celle de Ilub. sup. l'est un peu moins et indiquée trop petite. Les erochets sont doubles et au nombre de 5 ; le $4^{\text {e }}$ correspond à la bande transverse grise, le $5^{\mathrm{e}}$ à l'espace blane qui sépare la base de la hande transverse. Le point sous le sommet est aussi double et très-marqué. La frange au-dessous de lui est tantôt grise, tantòt hlanche nuancée de roux, tantòt tachée de roux dans son milien. L'écusson est souvent entièrement blane et confondu avec l'espace blane voisin; quelquefois il est gris, toujours appuyce en debors contre une tache grise. Il porte 4 à 5 points noirs, rangés en ligne et surnontés par une petite tache de mème conteur ordinairement irrégulière, d'autres fois en forme de point. La tète et les épaulettes sont blanches; celles-ci sont tachetées de noir.

211. Monachana, Fisch. v. Rösl. I. 5:3.

Guén. Ind. - Ilub. revis. $n^{\circ} 314$ - Ileyden. c. 318 .

Crenana, IJub. 242. - Dup. cat. - Eves. - Guén. Ind.

Melaleucana, Khlw. (non II.-S.). 
M. Frey a obtenu deux individus femelles de chenilles qu'il avait récoltées sur le Salix angustifolia, au mois de juin, pres de St-Moritz, dans les Grisons. L'insecte parfait naquit en aoùt.

212. Graphana, Treit. sup.

IJub. revis. $n^{\circ} 315$, - Jubn. sup. f. 439. - Heydenr. c. $321 .-$ Guén. Ind.

Pierettana, Dup.

Vulpinana, Fröhl.

Alfiniana, Kuhlw.

M. Rothenbach possède cette espece dans sa collection; mais il ne pent affirmer quelle ait été prise en Suisse.

213. Poecilana, Guén. Ind. p. 4:3.

Hub. revis. $n^{\circ} 317$. - Hubn. sup. ๔. 226. - Heylenr. c. 356. Bruand, C. 1059 .

Jai pris deux fois cette espèce dans les taillis an-dessus de Lausanne en juin, et une fois, en juillet, sur les montagnes d'Ollon (vallée d'Aigrle). Espèce rare; jusqu'ici recueillie en France.

214. Cirsiana, Zell., ent. Zeit. 1843 .

Hub. revis. $n^{\circ} 318 .-$ Hul. sup. f. 225. - Heyden. C. 350.

Clualybeana, Fisch. v. R. (olim). - Guén. Ind.

Scutulana, var. Fisch. v. R. t. 64. f. 6.

Dans la vallée de l'Etivaz. M. Frey l'a collectée dans l'Engadine et M. Rothenbach sur le Jura, au Weissenstein, le 24 juillet. - Ne se rencontre qu'isolément en juillet, sur les pentes gazonrées des Alpes exposées au soleil. Son vol est lourd.

215. Brunichiana. Lin.

W. V. - Fröhl. - Treit. - Dup. 243. - Fisch. v. R. t. 65.

IIub. revis. n 319 . - Guén. Ind. - Ileyd. c. 355. - Bruand, C. 1055 .

Simploniana, Dup. 248. - IIeyd. c. 264.

Profundana, IJub. 21 ?

Quadratana, Eversm.

Rusticana et Sticticana, Wood. - Curt.

Jacquiniana, Mus. Schif. 
Très-commune partout où eroît le Tussilago farfara; les vallées des Alpes; juin et juillet. De mème près de Schiipfen (Rothb.), de Zurich (Frey).

La figure de Simploniana, dans Dup.. ressemble plus à la nature que celle de Bruniehiana.

216. Luctuos ana, Dup. hist. 1242. 252, 4.

Guén. Ind. - Hul, revis. $n^{\circ} 321$. - Ilub. sup. f. 227. - Heyd. c. 352 .

Cnicolana, Zell. Is. $1847 ?-$ Ileyd. c. 353.

II. Heuser a pris eette espece rare dans les environs de Burgdorf, et MI. Frey en a collecté quelques exemplaires au mois de juillet, sur le bord des bois de l'Uetliberg, près Zurich. - La figure de Dup. est trop harbouillée de noir.

XXIV. Gen. A p he li a, Steph. - Curt.

Phoxopteryx, Treit. Dup. - Leptia, Guén.

217. Lanceolana, Huh. 80 .

Treit. - Dup. 253. - Guén. Ind. - IIubn. revis. n 323. Heyd. e. 540. - Bruand, C. 1019.

L a ne a n a, Fröhl.

Egenana, Wood.990. - Haw. - Curt.

Eg e s t a n a, Wood.992.- Ilaw. - Curt.

Plag ana, Wood. 993. - Haw. -- Curt.

Expallidana, Wood. 994. - Ifaw. - Curt.

Var. Dibelian a, Ilub. 272. - Dup. cat. - Ileyd. c. $5+1$.

Var. Sign a n a, Podev. -- Ilub. sup. f. 317. - Ileyd. eat. 542.

Commune sur les pàturages maréeageux des Alpes et des montagnes, là où croissent les jones. Varie beaucoup pour le dessin. Deux apparitions dans les environs de Lausanne; une première en mai et une seconde en aoùt. En juillet dans les Alpes. Près de Schüpfen (Rothb); de Zurich (Zell.); IEngadine (Frey).

\section{Gen. S e masia, Steph.}

Carpocapsa, Curt. - Grapholita, Treit. Dup. - C a toptria, Guén.

218. Infidana, Hub. $296-298$.

Treit. - Dup. 262. - Fröhl. -- Guén. - Ileydenr. c. $38+$ a. Hub. revis. 326.

Cette espèce a été collectée dans le Hant-Yalais, par M. Zeller. - Rare. 
219. A bs ynthian a, Hub. 34 .

Treit. - Hub. revis. $n^{\circ} 328$. - Heyd. c. 402 . Z Zett.

Pupill a n a, Lin. - F. - Dup. 248. - Guén. - Wood. 985. - Curt.

M. Anderegg élève eette espéce dans le Ilaut-Valais; je lai reçue de lui.

220. C i t r a n a, Hub. 185.

Fröhl. - Treit. - Dup. 257. - Hubn. revis. 329. - Heydens. c. 386. - Guén. Ind. - Bruand, C. 1113.

Cà et là sur les prés des environs de Lausanne, en juillet; assez rare. Environs de Schiipfen (Rothb.).

221. In c a n a, Zell. Isis, 1846.

Hub. sup. f. 298,299 . - Hub. revis. n' 335 . - Ileyd. e. 394. Guén. Ind.

M. Rothenbach m’a présenté deux indivilus appartenant à eette espèce et qu'il avait recueillis dans les environs de Schüpfen et pris dabord pour Wimmerana.

La larve vit sur l'Artemisia eampestris dont elle ronge les tiges.

222. Caecimaculana, Hub. 27.

Fröhl. - Treit. - Dup. 249. 5, a. — Guén. Ind. - Hubn. revis. $n^{\circ} 336 .-$ Bruand, C. 1112.

Kollariana, Mann. - (non Hub. 323, 324). - Hub. sup. f. 295.

Les pentes arides le long du torrent de la Paudèse, près Lausanne; une première fois en juin et une seconde en aoùt. Environs de Schiipfen (Rothb.).

M. Heydeureich, dans son catalogue, a confondu Kollariana, Mann, avee Kollarana, IJub. 323, 324, qui est Strobilana, Lin.

22:3. Conterminana, Fisch. v. Rösl.

Guén. Ind. - Hub. revis. n 338. - Hub. sup. f. 297. - Heyd. c. 392 .

Caecimaculana, var. Dup. 249.5 , b.

M. Couleru a collecté cette espèce dans les environs de la Neuveville et II. Zeller près de Zurich.

\section{Decipiana, Lah.}

II ohenwartiana, Guén. Ind.? - Auctor.

Dans la vallée d'Aigle, sur les pentes chaudes, au pied des montagnes d'Ollon, en juillet. 
J'ai aussi reçu cette nouvelle espèce de Lyon et de Besançon sous le nom de IIohenwartiana, avec laquelle elle avait été confondue jusquici. Cette confusion est très-aiscee, puisque Decipiana tient le milieu entre elle et Jaceana, qui elle-mème est souvent aussi réunie aux précédentes. Ces trois espèces restent cependant parfaitement distinctes, comme je vais essayer de le prouver.

La taille et la coupe des ailes antérieures de Decipiana (enverg. $18 \mathrm{~mm}$.) est la mème que celle de Jaceana; mais ses teintes sont d'un bruı olivatre et non fauve. La tache claire du bord interne est aussi moins marquée, plus alongée et plus étroite,

Les ailes inférieures du mảle sont parfois à moitic blanches, tandis que celles de la femelle sont presque noires avee quelques vergetures grises. Dans Jacean a elles sont d'un gris uniforme dans les deux sexes. La frange des mènes ailes dans la première est blanche, grise ou noiràtre, suivant que le foncé ou le clair domine sur le fond.

L'écusson de Decipiana est d'un blane jaunàtre, de forme quadrilatère, marqué de trois traits noirs. Les traits sont souvent remplacés par des points. Le jaune de l'écusson se répand sur la frange voisine.

Les doubles crochets de la còte an nombre de cinq, sont bien dessinés, marqués faiblement de reflets métalliques parfois presque nuls. Les deux premiers crochets, a partir de la còte, sont assez espacés et séparés par des taches en forme le larges virgules de la couleur du fond; les deux derniers sont contigus. Dans Jaceana la cotte paraît entièrement d'nu fauve clair, striée de lignes fauves foncées, également distantes entre elles.

La partie antérieure des ailes inférieures de Decipiana est en-dessous plus foncée que le reste du disque et porte vers le sommet deux ou trois points noirâtres. Jace ana a les inférieures d'un gris fauve clair, uniforme.

La tête est blanche dans Jaceana et fauve dans Decipiana.

Notre nouvelle espece est hien plus facile à distinguer de Ilohenwartiana. La taille et la largeur des ailes de cette dernière, ainsi que la disposition des crochets de la côte, suffisent pour empècher la confusion.

Ibiceana Koll. se rapproche aussi beaucoup de I)ecipiana: mais chez elle la frange est grise nuancée de clair dans son centre et sur son extrème bord; les taches du bord interne n'existent pas; la còte porte un liséré blanc étroit sur lequel se dessinent une sćrie de stries brunes et grises, très-rapprochées, ne formant pas de doubles crochets distincts. 


\section{A spidicana, Hub. 256 .}

Treit. - Fröhl. - Dup. 249. - Ilubn. revis. n 339. - Guén.

$$
\text { Ind. - Heyden. c. } 395 \text {. - Bruand, C. } 1106 \text { ? }
$$

Var. Nebritana, Ilub, sup. f. 241.

Fréquente aux environs de Lausanne, en mai et juin, dans les taillis et le long des hois, sur les coteaux exposés au soleil. Environs de Schüpfen (Rothb.), de Zurich (Zell., Frey). - Reparaît en aoùt.

226. Hohenwartiana, W. V.

$$
\text { Mus. Schif. - Treit. - Dup. 249. - Hub, revis. } n^{\circ} 340 \text { - Hub. }
$$$$
\text { sup. f. } 296 \text { ? - Heydenr. c. 388. - Bruand, C. } 1 \text { II1 ? }
$$

Pupillana, Hub. 20.

Antiquan a, Frobl.

II o netulana, Hub. 257.

Fulvan a, Curt. - Steph.

Ca n a, Wood. 9s7. - llaw.

Cum u l a a, Guén. Ind.?

J'eu fréquente sur les prairies sèches des environs de Lausanne et d'Aigle, en juin, puis une seconde fois 'n aoùt. - Environs de Zürich (Frey), de Schüpfen (Rothb.).

Cette espèce est fort difficile à caractériser, ainsi que ses voisines. Comme elle est très-souvent confondue avec elles et que le dessin ne peut que fort difficilement reproduire les caractères spécifiques des unes et des autres, il ne faut pas attacher une grande importance à la synonymie.

Hohenwartiana se distingue au premier coup-d'ceil, comme l'indique Treitschke, par la plus grande largeur proportionnelle des ailes antérienres qui mesnrent 20 à 21 millimètres. Le dessin est tellement confus et variable que l'on ne peut s'y attacher. La teinte générale est le brun clair, mèlé de gris et de faure. Il en est de même de la coloration des ailes postéricures qui sont plus on moins blanchàtres, bordées de gris dans le màle et grises dans la femelle. - Le caractère essentiel se tire de la disposition des crochets de la còte. Ils sont d'un blanc sale avec un faible reflet argenté; leur nombre ne peut ètre déterminé, attendu qu'ils forment une série de stries alternativement blanches, fauves et grises; les lulanches sont plus marquées. Les stries nées de ces crochets divers vout, en formant quelques sinuosités, se réunir vers la marge, entre l'écusson et le sommet de l'aile.

L'écusson est peu visible, mal détermiué, circulaire, quelquefois d'un jame clair, 
ordinairement de la couleur dı fond et marqué de deux petits traits ou points, souvent invisibles. Lorsque l'écusson est clair, la frange voisine l'est aussi; sans cela elle est grise. La marge est limitée par un petit trait foneé, quelquefois à peine marqué, formant dans son milieu un léger simus à concavité externe.

La tète est plus on moins blanche, ainsi que les palpes. Liextrémité des ailes postérieures, en-dessous, porte toujours 2 à 4 vergetures grises.

La figure 296 de Hub. sup. n’appartient pas à notre espèce, la coupe des ailes est trop diflérente. Dans ces espèces difficiles la disposition des erochets peut seule donner des indications sùres.

227. J a cean a, Zell.

Hub. revis, $1^{\circ} 3+1$. - Heyd, cat. 389 .

Quelquefois dans les environs de Lausanne sur les prairies séches, en juin et en juillet. - Environs de Zurich (Zell.).

Cette espèce est réellement différente de Hohenwartiana: $1^{\circ}$ Par sa taille beaucoup plus petite (enverg. 17 millimitres). $2^{\circ}$ Par sa tache arrondie, d'un blanc roux au milieu du bord postérieur qui fait ressortir lespace basilaire fauve et plus foncé. Dans la vraie Hohenwartiana l'espace basilaire n'est pas distinct. 3" Par ses crochets fauves, bien nets, très-rapprochés, au nombre de 5 , jamais brillants ou métalliques. $4^{\circ}$ Par ses ailes antérieures plus étroites, taillées plus sensiblement en biseau sur le sommet, parce que le bord externe est plus oblique. La frange est fauve, linitée en dedans par un

pointillé brun ou pourpre foncé que Treitschke attribue à Hohenwartiana. $5^{\circ}$ Par son écusson fauve clair, de forme ovoïde ou plutòt earrée, marqué de deux traits noirs peu visibles, avec un ou deux très-petits points entre eux. Le liséré métallique externe de l'écusson est irrégulièrement sinueux, de manière à former une dent du côté du centre de l'écusson, entre les deux traits noirs; cette disposition n'est pas toujours très-visible. $6^{\circ}$ Par un seul point noir sur la frange du sommet des inférieures, en-dessous, point qui manque quelquefois.

\section{A emu lana, Schläg.}

$$
\text { IHub. revis. } n^{\circ} 342 .- \text { Heyd. eat. } 406 .
$$

Latiorana, Hub. sup. f. 312.

Modestana, Bruand, C. 1108?

Je possède deux exemplaires de cette espéce, pris dans les environs de Lausanne. Ils sont beaucoup plus petits qu'Hohenwartiana (13 à 14 millimètres) et portent sur 
l'écusson 3 à 5 stries noires, très-prononcées. Les écussons sont plus grands el plus rapurochés de la marge que chez celle-ci. Les crochets de la eòte, au nombre de 5 , sont bien dessinés et régulièrement espacés; ce qui nest pas le cas dans sa roisine.

\section{Laharpana, Rothenbach in litteris.}

Au premier instant, cette espece nouvelle me parut tellement voisine dibieeana que je ne pensai pas à len sépares; un examen phus attentif ma décilé à l'admettre. Jamais infument préféré la dédier à lentomologiste zèlé et obliggeant qui en a fait la déconverte et m’en a coummniqué plusieurs exemplaires; mais sur ce point il s'est montré inflexible. - Laharpana a été prise sur une clairière couverte de bruyères, pres de Schüpen, du 12 au 24 juiltet. Voici sa description :

Les ailes antérieures sont narduces dans leur longueur do trois bandes; nne antérieure étroite, le long de la còte, un peu plus claire que la suivante et séteignant souvent vers lo milieu de laile; sur elle se dessinent les crochets en blanc jaunàtre. Une moyenne diun brun bistre, se fondant en arant avec la bande précédente, assez bien distincte de la suivante en arriere. Le hord postérieur de cette bande coupe la partic suprérieure de l'écusson au niveau du trait noir antérieur et étend un peu sa teinte foncée sur la frange. La troisiome bande on postéricure, plus claire que les deux autres, est lavée de gris et de fauve clair. Elle occupe sur la marge les deus tiers de lécusson; yuelquefois elle se recouve de brun vers la racine de l'aile et se confond alors ave la bankle moyenne.

L'écusson est carré, limité en dedans et en dihors par deux bordures à reflets métalliques sourent peu distinctes. Son centre est blanc ou blanc-jaunàtre narqué de deux, quelquefois de trois points noirs. Le trait central manque ordinairement on n'est représenté que par un point. La teinte claire se place entre les deux traits.

La frange est grise, un peu brune dans sa moitié anterieure, plus claire et mouchetée de blanc dans la postérieure, linitée en dedans par une ligne pointillée grise.

Les crochets sont au nomlore de trois on quatre, distincts, doubles, bien espracés, d'un blanc sale, courts. - Des lignes lrumes, quelquefois nétalligues, s'en détachent pour se diriger au-dessus de l'écusson. Les trois premiers crochets sont bien visibles, le quatrième s'efface sourent, le cinguième est i peine indiqué.

Les ailes postérieures sont dun garis de souris uniforme, légirement teinté de roux ver: les bords. Chez la femelle elles sont plus foncées. Leur frange est plus claire, luisante. 
Le dessous des ailes est entièrement gris-roux, plus foncé chez la femelle, avee les franges teintées de fauve. Trois crochets fauves se dessinent faiblement à la còte.

Front et palpes gris. Tète et corselet fauves. Abdomen noiràtre. Anus fauve clair. Pattes grises.

Laharpana se distingue dlbiecana: $1^{\circ}$ Par sa taille un peu plus faible; la première a 15 millimètres d'envergure et la seconde 17 à 18 . 2 $2^{\circ}$ Surtont par les crochets de la còte qui dans celle-ci représentent une sẻrie de points noirs et bruns, rajproehés les uns des autres sur un fond blane assez pur. 30 Par ses franges rousses en dessous et non pas grises. $4^{\circ}$ Par l'absence de taches blanches et noires à l'extrémité des ailes postérieures en dessous.

Une confusion aree Modestana, Jaeeana, Deeipiana ou Aemulana n'est pas possible, avec un peu d'attention.

230. Hypericana, Hub. 2:3.

Fröhl. - Treit. sup. - Dup. 250. - Ilubn. revis. n ${ }^{\circ} 345$.

Guén. Ind. - Heydenr. eat. 400. - Wood. 981. - Bruand, C. 1110 .

Petiverella, W. Y.

Assez fréquente sur les bords du lae Léman et les collines arides; en juillet. Probablement deux générations, l'une en mai, l'autre en juillet. Commune près de Zurich (Frey, Zell.). Environs de Schijpfen (Rothb.).

Dans les Alpes le papillon ne paraît qu'en jnillet et prend des teintes beaucoup plus pàles, en sorte quon le confond aiscment avec liohenwartiana, si l'on n'y fait attention.

J'ai trouvé la chenille sur la Scrophularia canina et l'ai élevée aree eette plante. Il paraît qu'elle vit sur divers végétaux.

XXVI. Gen. Rox a n a, Wood. Steph.

Car proc a psa, Treit. Dup. - M elodes, Guén.

231. Arcuana, Lin.

$$
\text { F. - W. V. - Treit. - Dup. 248. - Hub. 33. - Hub. revis. }
$$

346. — Guén. - Ifeyden. e. 266. - Wood. 973. - Curt. Bruand, C. 999.

L ambergiana, Seop.

Assez fréquente dans les taillis de noisettier de toute la Suisse, en juin. (Lah, Rothb. - Zell. - Frey). 


\section{Gen. Carpocapsa, Treit. Dup. Guén.}

\section{2:32. Pomonana, Lin.}

$$
\begin{aligned}
& \text { W. V. - F. - Hubn. 30. - Treit. - Dup. 248. - Frobl. - } \\
& \text { Inb. revis. } 348 . \text { - Guén. Ind. - Ileydenr. c. } 259 \text {. Wood. } \\
& \text { 974. - Curt. - Bruand, C. 1079. }
\end{aligned}
$$

Très-fréquente dans tous les vergers en juin. La larve vit dans les pommes, les poires, les noix et les noisettes; elle en sort en septembre, lorsque les fruits tombent. Le meilleur moyen de la détruire est de recueillir les fruits verreux dis quils tombent, pour les utiliser de manière à détruire la larve.

\section{2:3:3. Fag̨igla ndana, von Heyden.}

Hub. revis. n³ 349. - Inub, sup. f. 157-160. - Heyd. c. 261. Grossana, Woot. 976. - Curt. - Ilaw.

M. Rothenbach a collecté cette espèce dans les environs de Schipfen et M. Heuser prés de Burgdorf.

\section{O bes ana, Lah.}

Je ne connais de cette espèce que la femelle, ap'partenant à II. Rothenbach. Cette Tordeuse a été prise dans les environs de Langnau par un de ses amis, je ne sais it quelle époque.

Le facies est celui de Resinana avec laquelle on la confondrait aisément; mais elle s'en distingue: $1^{\circ}$ Par l'existence d'un écusson irrégulier et qui n'en a pas lapparence ordinaire. $2^{\circ}$ Par son dessin moiré sur toute l'étendue de l'aile et non distrilmé par' bandes transwerses. La taille et la coupe sont du reste celles de Resinana.

Laile supérieure est d'un gris blanchâtre, luisant, non métallique, sur lequel se dessinent une foulr dondulations transrersales brunes-foncées, nuancées de bruı clair. Vers le milieu de l'aile les ondulations sont plus rapprochées que vers la base. An tiers externe le fond blanchâtre plus dégagé forme une espèce d'écusson pyramidal dont le sonnmet arrive à l'angle anal. Le centre de cet écusson est occupé par une tache d'un brun plus foncé, alongée, irrégulière, qui envoie un filet à la còte, un autre à l'angle posténieur et deux autres à la marge.

Celle-ci porte une ligne de points bruns contigus, à laquelle succirde la fiange de couleur brune, mèlée de gris.

Le sommet de l'aile est arrondi et d'un lırun fauve. La côte porte deux petits crochets blanchàtres en regard de l'écusson. 
Les inférieures sont dun brun-noir uniforme, avec la frange presque blanche.

Le dessous est lrun, irrégulièrement moucheté de blanc-sale.

La tète, le thorax et l'abdomen sont bruns.

L'existence d'un rudiment d'écusson place cette espèce entre Pom onana et Resinana; je prefere hi assigner une place dans le genre Carpocapsa.

2:35. Splendana, Hub. 31 .

Fröhl. - Treit. - Dup. 248. - Guen. - Hub. revis. n $350 .-$ Heyd. C. 260. - Wood. 975. - Curt. - Bruand, C. 1077.

Aphalana, Khlw.

Furvana, Mus. Sibif.

II. Rothenbach a collecté cette espèce à Wimmis, près Thoune, le 4 juillet. Je lai prise une fois à Paulex, en aoùt.

\section{2:36. Su c c ed ana. Fröhl.}

Treit. -- Dup. 251. - Guén. Ind. - Hubn. revis. n"352. Heyd. c. 417. - Bruand, C. 1107.

Asseclana, Hub. 194.

Subocellana, Wood. 970.

II. Rothenbach a pris cette espèce au pied du Jura. H. G. Leresche l'a collectée dans les environs de Fontanezier, au pied du Jura. Je l'ai trouvée commune parmi les bruyères au-dessus de Rovéréaz, près Lausanne, en juin. Forèts d'Aighle. Jusque sur les hautes Alpes au pied de la Dent-du-Midi. Pas rare partout où croît la bruyère. Près de Würenlos, dans la vallée de la Limmat, autour les genèts (Frey).

XXVIII. Gen. Grapholita, Treit. Dup.

Opadia, Endopisa, Stigmonota, Dichrorampha, Pyrodes, Guén. Ind.

237. Daldorfiana, Fab.

Fröhl. - Hub. revis. n 355. - Wood. 1119.

A urana, Hub. 22.

Khediana, Lin. (ella). - Treit. - Dup. 250. - Heyd. c.442. - Guén. Ind.Curt. (excl. syn. Ilub.). - Bruand, C. 1104.

Pas rare dans les environs de Lausanne en avril, mai et juin; dans les maisons, les haies, les jardins. Elevée par M. Frey de feuilles desséchées recueillies en automne. Près d'Aarberg (Rothb.). 


\section{Fulvifrontana, Zell.}

IIubn. revis. n 356 . - Heyd. c. 445 . - Ilubn. sup. f. 258 (non 282). - (non Guén.?).

Parvulana, Fröhl.

Gernarana, Fisch. v. Rösl. - Dup. sup. 63?

Je lai reçue de M. Neyer qui l'avait recueillie dans les environs de Burgdorf. M. Rothenlach l'a collectée aussi près de Schuipfen.

239. Coecana, Fisch. v. Rösl.

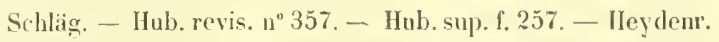
c. 453 .

Jai pris deux fois cette espèce dans les environs de Lausanne, en jnillet.

210. Plumbatana, Zell. Isis 1816.

Ilub. revis. 11" 358. -- Ilub. sup. f. 321. - Ileyd. c. 515.

M. le prof. Frey a pris cette espèce dans les environs de Zurich et dans I'Engadine.

Très-roisine de Blepharana: les crochets de la còte sont plus distinets, blancs et au nombre de cinq. Les deux premiers sont contigus, les autres assez espacés.

241. Biepharana, killw.

Hub. revis. ${ }^{\circ} 359$. - Hub. sup. f. 197, 198, - Heyd. c. 536.

Zachana, Treit. - Dup. 250. - (non Hubn.). - Heyd. c. 455.

Ulicana, Guén. Ind. - Bruand, C. 1096.

Ulicetana, Kurt.

Germana, Fröhl.

Var. Monticolana, Jann. - Ileyd. c. 454.

Très-conmune sur les pàturages de toute la plaine, des montagnes et des Alpes, surtout en mai et en juillet.

Monticolana Mann est une variété très-foncée quil ne faut pas confondre avec la Monticolana de Guén. et de Dup., qui est Mercuriana. La couleur do fond varie du jaune-olive au brun-noir; la tache du bord interne est rarement visible et senlement sur des individus très-frais.

212. Bugnionana, Fisch. v. Rösl.

Dup. sup. 548, 83. - Ifub. revis. n 360 . - Hub. sup. f. 126,Ilumilana, khlw. Guén. Ind. - Ileyd. c. 174 . 
Cette espèce n’a point été découverte dans les environs de Lausanne par M. Bugnion; elle habite exclusivement les pàturages des hautes Alpes. Environs de Meyringen. M. Fisch. v. R. lavait probablement reģue de 1. Anderegg.

\section{1:3. Chavanneana '), Lah.}

Je n'ai vu qu'un individu màle de eette espèce, provenant, je crois, de Meyringen. Sa taille et son facies sont eeux de Gruncriana, le dessin est eelui de Bugnionana; elle differe de l'une et de l'autre par des earactères bien marqués. - Le bord externe des supérieures porte, vers l'angle interne, quatre points noirs eomme ecs dernières. Le dessin est plus marqué que dans Bugnionana, mais le fond est d'un gris pâle qui n’a rien de brillant comme chez elle. - La tache quadrilatere du bord interne est bien marquée comme dans Quadrana et Bugnionana, tandis qu'elle est indistincte dans Gruneriana. Les croehets de la còte, doubles, bien marqués, d'un blanc argenté et au nombre de 4 à 5 dans cette dernière, sont peu marqués, très-espacés, ternes et au nombre de 3 à 4 dans Chavanneana. Dans Bugnionana ils ne se distinguent pas du fond et sont la terminaison des lignes plombées. La frange des supérieures dans celle-ci est d'un gris de souris brillant, un peu plus pàle en dedans. Dans les deux autres espèces elle est limitée par un trait blanc très-saillant; mais Gruneriana porte un point blanc très-visible interrompant la frange au-dessous dı sommet de laile; il n'existe pas dans sa voisine.

Les ailes inférieures sont d'un gris uniforme dans Bugnionana; grises sur la marge et blanches à la racine dans Gruneriana; dun blanc sale uniforme dans Charanneana. En dessous la première est uniformément gris de fer; la seconde présente des teintes plus elaires vers la racine des inférieures, et la troisieme est brune aux supérieures et gris-clair aux inférieures.

Il est eneore d'autres différences de détail que je passe sous silence.

\section{Gruneriana, Mann cal.}

Hub. revis. n 362 . - IIub. sup. f. 261, 262. - Ileyd. c. 457 . Bruand, C. 1100 .

Var. Distinctana, Man. - Heyd. c. 467 ?

J'ai collecté une fois cette espèce dans les clairières des bois de sapin, au-dessus

1) Jai dédié cette espèce a mon ani M. le prof. Chavannes, qui le prenier, de concert avec M. Bugnion, a étudiè et collecté avec soin les Lépidoptères des environs de Lausanne et des Alpes voisines. 
de Lausanne, en juin. H. Rothenbach l'a recueillie aussi sur les sapins, le 19 juillet. (Voir Chavanneana).

245. Mi crogrammana, Guén. Ind. p. 54.

$$
\text { Ilub. revis. 365. - Hub. sup. f. 291. - Bruand, C. 1109? }
$$

Debiliana, khlw.

II. Th. Bruand a colfecté cette rspèce dans le Jura. Espèce suisse douteuse.

24t. Ustulana, Ilub. 231 (non Treit.).

Ilub. revis. n³68. - Guéı. Ind. - Heyd. c. 564. (excl. syn.).

Albersana, Bruand, C. 1105 ?

1I. Zeller et Frey collectent cette espèce très-rare, dans les environs de Zurich, sur les buissons de coudrier, en juillet et aoùt. - Dans l'été de 1855 elle était commune en certains endroits (Frey).

Treitschhe rectifie Iui-mène (suppl. III., p. 120) lerreur quil avait commise en associant sou Ustulana (Uepupana) à celle de Ilubner: pourquoi la reproduit-on encore?

Ustulana ressemble beaucoup à Albersana; le fauve de l'extrémité de l'aile s'étend davantage du còté du sommet dans celle-ci et du còté de langle interne dans celle-lia. Du reste, laspect violacé des trois quarts internes de l'aile est le mème. Les crochets de la côte sont différents; ils sont au nombre de 4 dans Ustulana, également espacés, igraux et enfumés de violet. Celle-ci porte un point blanc, subapical, qui ne divise pas la ligne marginale; Albersana en est dépourve.

217. Tenebrosana, Fisch. v. Rösl.

Ilub. revis. n 369 . - Ilubn. sup. f. 292. - Guén. - Ileydenr. c. 419. - Dup. sup.? - Bruand, C. 1083.

Jai reçu cette espèce de M. Meyer à Burgdorf qui lavait prise dans les emirons de son domicile et dans lOberthal. Je l'ai collectée daus les environs de Lansanne en mai et en juin. V. Rothenbach l'a aussi prise dans les environs de Schüpfen.

Ordinairement confondue arec Neluritana.

248. Nebritana, Treit.

Dup. 250. - IInb. revis. n॰ 3\%0. - IIub. sup. f. 234. 241, t.Guén. Ind.? - IIeyd. c. 447. - Bruand, C. 1081?

Pisana, Guén. Ind. p. 48?

Rare daus les environs de Lausanne. Jai élevé la chenille trouvée sur les fleurs du Colutea arborescens. - Euvirons de Schüpen (Rothb.). 
Il est prohable que Guénée a pris Tenebrosana, quil n'avait pas encore vue, pour Nebritana et formé sa Pisana avec la vraie Nebritana. La deseription de sa Pisana est d'ailleurs trop vague pour assurer le diagnostic.

249. Gemmiferana, Treit. sup.

$$
\text { Ilub. revis. n³ 32. - Ilub. sup. f. 294. - Guén. Ind. -- Heyd. }
$$

$$
\text { c. } 45 \text { 2. - Dup. cat. }
$$

Zachana, Hub. 243.

Jai pris quelquefois cette espèce le long du torrent de la Paudèze, près Lausanne, parmi les aulnes. - Le petit nombre dindividus et en mauvais état que jai collecté ne me permet pas d'affirmer fislentité de cette espèce et de colle de Hubner.

250. Funebrana, Treit. sup. (excl. synon.).

Dup. sup. 66. - Ihub. revis. n 373. - Guén. Ind. - Ileydenr. c. $455 .-$ Bruand, C. 1080.

En mai, sur les pruniers, à Paudex près Lansanne. Près d'Aarberg (Rothenb.). Environs de Zurich (Zell.).

251. A cuminataua, Schläg.

$$
\text { IIub. revis. } n^{\circ} 374 \text {. Ileyd. c. } 459 \text {. - Zell. Is. } 1846 .
$$

Acutana, Schläg.

Germana, Fisch. v. R. - Fröhl.

Germarana, Treit. - Huh. sup. f. 282. - Heyd. c. 450. - Dup. sup.63.Bruand, c. 1092 ?

Nigricana, F. - Frohl. - Curt.

Caliginosana, Dbld. cat.

II. Rothenbach a recueilli cette espèce dans les environs de Schipfen.

252. Ligulana, Hub. sup. f. 279.

$$
\text { Hub. revis. n³75. - Ileyden. c. n" } 493 .
$$

II. C. Zeller a collecté dans l'Engadine un individu appartenant à cette espèce qui reproduit parfaitement la ligure de Hub. sup. Je lai regue de II. Ott qui l'a prise dans le Ilaut-Talais, en juillet 1857 .

Cette charmante espéce mérite une mention particulière. Sa taille est celle d'Alpinana. Les supérieures ont le fond olive-bronzé avec un rellet brillant. Sur ce fond se dessinent un grand nombre de lignes métalliques, sinueuses, à rellets plombés et irrisés. Les crochets, au nombre de 5 . sont simples, très-marqués; les 3 preniers (à partir du sommet sont espacés; les deux derniers rapprochés. 
La marge porte 5 à 6 gros points noirs; entre les 2 antérieures se place un point blane (point subapieal); en face de ce dernier point la frange forme un petit sinus et sa ligne de démareation est interromjue.

Les franges sont grises avee des reflets mótalliques brillants et chatotants. Une grande tache jaunàtre, irrégulière sur les bords, marquée d'un point gris, qui sétend en monrant an-delia du milicu de laile, se voit i son bord interne.

Le dessous est gris-noiràtre; les crochets jaunâtres y sont bien marqués et la marge a des reflets violets.

Tète grise, avee des poils jaunàtres. Palpes sécuriformes, alongés; jaumes à la base, noirs au sommet.

La fig. 279 de Ilub. sup. n’est pas prarfaitement exacte. Les crochets de la côte y sont figurés trop longs; ils forment de simples points blanes d'ou partent des lignes plombées. Celles de ces lignes qui forment l'écusson se détachent des crochets qui surmontent la tache blanche. Le point subapical est blanc pur et non plombé; il niexiste pas de erochet au sommet mème qui lui donne une forme occulce. Enfin les stries ondulées convrent toute la racine de l'aile.

Les dimensions de linsecte sont près du double de celles indiquées: plus grand que Blepharana.

25:3. Alpestrana, Fisch. v. Rösl. col.

Fröhl. - IIub. revis. n 376. - Zell. Ent. Z. 1543. - Ileydenr. c. $466 .-$ Bruand, C. 1103 ?

Ifontanana, Fisch. v. Rös. (olim). - Dup. sup. 83. - IJubn. sup. f. 193. Guén. - Ileyden. c.53\%.

I. Le prof. Frey a collecté cette espéce dans I'Engarline près de Samaden, où elle est commune.

254. Plumbagana, Treit.

Dup. sup. 66. - Ilubn. revis. n 377 . - Ilubn. sup. f. $289 .-$ Guén. Ind. - Heyd. c. 460. - Bruand, C. 1098.

Strobilana. Dup. 1223. 250, 4.

Cinerana, Ilub. 211. - Wood. 946.

Angulosana, khlw.

Scintilullana, Gún. (test. H. S.) - Bruand, C. 10tit.

Près de Samaden, dans l'Engadine, à la fin de juillet; commune (Frey). - Environs de Scliüpfen (Rothb.). 
25.5. Caliginosana, Treil. sup.

Frey, 324. - Dup. sup. 63. - Ilub. revis. n 379. - Hub. sup.

f. 263. - Guén. - Heyd. c. 470. - Bruand, c. 1099.

Collectée près d'Aarherg par M. Rothenbach.

256. Argyrana, Hub. 46.

Iluhn. revis. n 331 . - Ilubn. sup. f. 192. - Fisch. v. Rös]. Lien. - Zell. Isis. - Guén. Ind. - Ilejdenr. c. 484. - (non Treit. nec Dup.). - Bruand, C. 1071.

Lathyrana, Dup. 25I. (uon Ilub.).

Melaleuc ana, Fröhl.

Atromargana, Haw.

Recueillie dans les environs de Bàle par. II. Jmhof. J'en possède un exemplaire pris dans les environs de Lausanne.

257. Kochiana, Hub. sup. f. 236 .

Heyd. c. $\mathrm{n}^{\mathrm{n}} 435$. - Hul, revis. $\mathrm{n}^{\circ} 383$.

Prise dans les environs de Burgdorf par M. Heuser.

258. Janthinan a, Dup. 1207. 2/8, 1. - Guén. Ind.

In cisan a, Fisch. v. Rösl. - Hub. revis. n 334. - Ilub. sup. f. 173. - Heyd. c. 476 .

J'ai pris une fois cette espèce dans les emvirons de Lausanne, sur des pentes sèches et arides, en juillet. M. Rothenbach l'a aussi collectée près de Schüpfen.

259. Woeberiana, W. V.

Fröhl. - Treit. - Dup. 249. - Hubn. revis. n 388 . - Ilubn. verz. - Guén. Ind. - Ileydenr. c. 262. - Curt. - Bruand, C. 1062.

Ornatana, Ilub. 32.

Pas très-rare dans les haies de condrier aux environs de Lansanne, en juin. Environs de Schiipfen (Rothl.).

260. Composana, Fab.

Fröhl. - Dup. 25̌1. - llubn. revis. n 389. - Guén. Ind.

Curt. - Bruand, C. 1088.

Gundiana, Ilub. 42. - Treit. - Wood. 932. - Ilub. sup. f. 28t. - Curt. 
Commune sur les haies d'aubépine en mai et juin, dans les environs de Lausame. Environs de Zurich (Zell.), de Sehüpfen (Rothb.).

261. Corollana, Hub. 282.

Frölıl. - Ilu!b. revis. n 390. - Hub. sup. โ. 235. - Guén. Ind. Ileyd. c. 498. - Dup. eat.

Environs de Lausanne, en avril, sur les sapins, le long du lac. Rare.

262. Loderana, Treit.

Dup. sup. 83. - Koll. - Hub. revis. n³ 392. -- Ileyd. e. 496.

Sehrankiana, Fröhl. - Guén. Ind. - Heydenr. e. 509. - Bruand, C. 1086, 1087.

Lathyrana, IIub. 207. - Hub.revis. 265. - Heyden. C. 507.

Dorsana, Dup. 1234. 251. (non Hub.).

Eleg antana, Fröhl.

Includana, Khlw.

Assez commune dans les taillis herbeux du bois de Sauvabelin, parmi les orobes, en avril et mai. Environs de Zurieh (Zell., Frey), de Schüpfen (Rothenb.).

\section{6:3. Fissana, Fröhl.}

Zell. - Dup. 264. - Hub. revis. $n^{\circ}$ 393. - Bruand, C. 1085.

Divisana, Dup. (fig.).

Dorsana, Treit. (II.-Schf.).

Près de Baden, auf der Lägern, en juin, assez fréquente; pas encore trouvée dans les environs de Zurich (Frey).

Lexemplaire de Fissana yue jai reçu de M. Herrieh-Sehäffer ne laisse aucun doute sur sa valeur comme espèce.

\section{Diffusana, Fisch. $、$ Rösl.}

$$
\text { Ilul). sup. f. } 284 \text {. }
$$

Assez commune, en juin, sur les elairières des bois du Jorat, près de Lausanne, dans les lieux où croissent les orolres et les vesces. - Dans le Jura; rare (Rothb.).

Jai sous les yeux un exemplaire de Diffus an a reçu de M. Ierrich-Schäffer qui ne differe en rien des nòtres.

Dorsana, Hub. 36 , tel quil est figuré dans H.-S. f. 287, n’a pas eneore été trouvé en Suisse. La synonymie de eette espèce et des roisines est fort olsseure. 
26.5. Coniferana, Saxes.

Ilub. revis. $n^{\circ} 395$. - Ileyd. c. 503.

Separatana, Fisch. v. Rösl. - Ilub. sup. f. 32?. - Ileyd. c. 512.

Demissana, Fisch. v. Rösl. - Heyd. c. 506.

11. Rothenbach en possède un exemplaire quill a collecté en Suisse. Très-rare.

266. Coronillana, Zell.

Ilub. revis. n 397 . - Hub. sup. f. 286. - Hę̧d. c. 494.

Pusillana, W. V.

Argyrana, Treit. (non Hub.).

Zelleriana, Kha. - Heyd. c. 349 ?

I. Rothenbach a recueilli cette espece dans les environs de Schüpen. II. Frey croit lavoir aussi collectée dans les environs de Zurich, en juillet.

267. O robana, Treit.

IInb. revis. nº 401, - Ilubn. sup. f. 288. - Guén. Ind. - Heyd. c. 518 .

y. Rothenbach possiede cette espèce provenant de Langnau!

268. Jungiana, Lin.

Fröhl. - Dup. 264, 8. - Treit. - Hub. revis. no 402. - Ileyd. c. $520 .-$ Curt.

Lunulana, W. Y. - Ihub. 3j.--Guén. Ind. - Wood. 920.

Dorsana, Fab.

Negerleana, Fröht.

Concinna, Steph.

Assez rare; prise une fois dans les environs de Lausanne et reçue de H. Heyer de Burgdorf.

269. Interruptana, Khw.

Fisch. v. Rösl. - Hub revis. 405, - Ilub. sup. f. 280. - Heyd. eat. 501.

Dors an a, Hub. 36 ? - Ratzb.

lıqu in at ana, Dup. 251, 9. (Jungiana). - (non Ifubn.).

Jungian a, Guén. Ind. (exel. synom.).

Lunulana, Bruand, C. 1084?

Rare. Une fois près de Lausanne dans les clairières des bois secs; une autre fois près de Tzernatten, sur les hautes Alpes. 
Lindividu pris sur les hautes Alpes est de forte taille. - Guénée n’ayant pas vu IInterruptana de Khlw, a reconnu lerreur de Inp., mais a inserit cette rspèce sous le nom de Jungiana. La ligure de Dup. est très-reconnaissable.

\section{Geniculana, Lah.}

Je lai recucillie une fois dans les environs de Lausanne, au milieu des Jroussailles tris-exposées au soleil, an mois de juin 1549. M. Rothenbach l'a aussi prise clans les environs de Schüpfen.

Cette espèce, dont je nai vu que deux femelles, se ropproche beancoup dinterruptana, et je naurais pas osé l'en séparer si la femelle de cette derniire n'en restait pas hien distincte.

Comme on ne peut la confondre fuase Interruptana, dont elle a la taille, je me Jomerai à la distinguer de celle-ci. $1^{\circ}$ Interruptana a le fond les ailes din gris noiràtre, tandis que Geniculana présente une teinte générale noir-brun, foncée, fuligineuse; cette différence est surtout marquée aux ailes inférieures. $2^{\circ}$ La premire a les crochets de la còte et la tache du bord interne d'un blanc pur; la seconde les a légèrement jaunitres. $3^{\circ}$ Le quatrieme croclset, a partir du sommet, toujours double dans Interruptana, se prolonge jusquà l'écnsson en formant une ligne blanche coutés au milieu; dans lautre espèce, du troisione crochet part une ligne plombée, métallique, peu visible, qui se rend à lécusson. $4^{\circ}$ Les crochets sont un pen différents dans Ies deux espèces; ils sont au nombre le 5 dans Interruptana; le second et le troisieme, a partir du sommet, sont parfois simples et rapprochés; les autres sont doubles. Ge uiculana a 4 crochets doubles; les trois premiers sont espacés de façon a représenter plutòt 6 crochets simples et courts, également distants les uns des autres. $5^{\circ}$ La frange des postérieures est d'un blane sale, grise à la base dans Interruptana; elle est dun hlanc jaunàtre arec la base noire dans Geniculana. $6^{\circ}$ Le dessous est noirbrun dans la seconde et gris daus la première.

La tache du bord interne varie igalement dans les denx esjèces; tantôt elle décrit une demi-lune et tantòt elle est condíe, tantòt elle est diviséc par une petite strie et tantòt elle ne l'est pas.

II serait possible que Dorsana Ilub. f. 36 se rapportât à cette espiece. En tout cas, la dénomination de Dorsana ayant été appliquée à plusieurs espèces differentes, devait c̀tre mise de còté. Elle se rapproche aussi beaucoup dinquinatana; mais ses franges sont blanches, et il nexiste pas de pincean de poils noirs sur les inferieures du mále. 
271. Spiniana, Fisch. r. Rösl.

Dup. sup. $370.66,-$ Hub. revis. ${ }^{\circ}$ 406. - Hub. sup. f. $267 .-$ Guén. Ind. p. 45. - Ileyd. c. 478. - Bruand, C. 1061.

Alb uginan a, Guén. Ind. p. 4 4.

Argyrana, Dup. 1330. 263. (non Jubn.).

Lathyrana, Treit. - (non Dup.).

Spinetorum, Zell.

Prise dans les environs de la Neuveville par M. L. Couleru, et de Schüpfen par II. Rothenbach.

272. Ephippana. Hub. 246.

Dup. 251. - Inb. revis. 407. - Guén. Ind. - Heyd. c. 483.-

Curt. (non Fal. nee Bruand).

Populana, Fröhl. - Fal. - Curt.

Sticticana, Fab.

Trigonana, Steph.

M. Zeller possède un individu pris dans les envirous de Zurich. H. Rothenbach l'a aussi collectée dans les environs de Schüpfen.

La figure et la description de Dup. sont exactes.

\section{Trauniana, W. V.}

F. - Hub. 38. - Dup. 251. - Treit. - Hubı. revis. nº $411 .-$ Jubn. sup. f. 303. - Guén. Ind. - Heyd. c. 532. - Wood. 930. - Curt. - Bruand, C. 1090.

Cette espéce rare a été prise une fois en septembre, dans les environs de Zurieh, par H. Zeller.

271. Petiverana, Lin. (ella). F. - Fröhl. - Treit. - Dup. 251. - Guén. Ind. - Ilub. revis. 414. - Ileydenr. c. 524. - Wood. 919. - Curt. - Bruand, C. 1095 .

Conwayana, F.

Montana, Scop. - Hub. 37 .

Assez conmune sur les jachères, le long des haies et des clemins, dès la fin de mai jusqu'en juillet. On la trouve fréquemment dans les $\mathrm{A}_{\mathrm{p}}$ es. Vole souvent en essaims, au soleil. De mème près de Schüpfen (Rothb.), de Zurich (Zell., Freỵ). 


\section{Alpinana, Treit.}

Dup. 252. - Hubn. revis. $\mathrm{n}^{\circ} 415$. - Hubn. sup. f. $155,156$. Heyd. c. 527 .

Petiverana et Politana, Mus. Schif.

Politana, W.Y. - Guén. Ind. - Bruand, C. 1093.

Simpliciana, Strigana et Atropurpurana, Wood. 921, 923, 924.

Assez rare; près de Lausanne, en juin, sur les champs de trèfle. Dans les Alpes (Rothls.).

Jai vn sonvent dans les Alpes des essaims de Petiverana sans y rencontrer une Alpinana. Près de Lausanne je l'ai trourée mélangée avec sa voisine.

L'espèce est donteuse. Cependant toutes les Alpinana que jai recueillies ont une taille plus forte, les ailes un pen plus alongées et la tache du borl interne très-oblique, d'un jaune fort pàle. Les crochets de la còte sont aussi moins marqués.

276. Angustana, Hub. 205 (non 20\%).

Treit. - Dup. 261. - Hobn. revis. 417. - IInlsn. sup. I. 362 Guén. - Ileydd. c. 437. - Bruand, C. 10.45.

Coruscana, Fröhl.

Point rare sur les basses Alpes et le Jura; le long des haies et sur les pâtnrages, à la fin de juin et en juillet. Elle se prend aussi dans la plaine, oì elle est moins fréquente. Environs de Schüpfen (Rothl.), de Zurich (Zell.), dans l'Engadine (Frey). - Ne varie pas.

277. Mercuriana, Fröhl. 170.

Ilub. 322. - IIul. revis. 418. - Iub. sup. f. 322. - Heydenr. cat. 440 .

Monticolana, Man. cat.-- Dup. sup. 83. - Guén. Ind. p. 46.

Pustulana, Ilub. 208?

J'ai collecté une fois cette espèce sur la montagne d'Enzeindaz, au pied du Diableret, en aoùt. I. Alfred Rothenbach l'a prise sur la Gemmi, M. Zeller dans les Alpes et H. Ott dans le IIant-Valais.

Mann a anssi donné le nom de Monticolana à une variété de Blepharana.

278. Loxiana, Fisch. v. Rösl.

Hub. revis. n 419. - Ilub. sup. f. 323. - Ileyd. c. 516.

Luctiferana, Khlw. 
Ochsenlieimeriana, Zell.

M. Heyer ma envoyé, des environs de Burgdorf, un indiridu appartenant à cette espèce.

279. Strobilana (ella), Lin.

F. - W. Y. - Treit. - Ratzb. - Zett. - Ifubn. 70. - Hubn. revis. $n^{\circ} 421$. - IIub. sup. f. 254. - Guén. Ind. - Ileyden. cat. 198. - Wood. 9f\%. - (non Dup.) - Curt.? - Bruand, C. 1064 b.

Kollar ana, Fröhl. - Ilub. 323, 324.

Fraternana, Curt. - Wood. 915.

Iloffmansegana, Wood. 1116.

Le bois des sous-alpes; fréquente à la fin de juin. Commune dans les environs de Zurich (Zell., Frey). Le Jorat au-dessus de Lausanne en arril et mai; rare. Environs de Burgdorf (Heuser).

280. Pactolana, Khlw.

Zell. - Hub. revis. no 422. - Hub. sup. f. 253. - Heyd. c. 50 ?. - Bruand, C. 1089.

Dorsana, Ratzb.

Stagnana, Hub. 193.

M. Rothenbach a collecté cette espèce rare dans les environs de Sclrüpfen.

281. Cosmophorana, Treit. sup.

Ratzl. - Dup. sup. 63. - Ifubn. revis. no 424. - Ilubn. sup. f. 325. - Guén. Ind. - Heyd, c. 513. - Bruand, C. 1063.

Dalecarliana, Zett. - (non Guén.).

11. Rothenbach possède cette espèce, collectíe dans les environs de Langnau.

282. Scopariana, Hub. sup. f. 324.

Hub. revis. $n^{\circ} 425$. - Heyd. c. 544 .

Latly yrana, Treit. - Zell. - Hub. 207.

Collectée dans les environs de Lausanne, en juin, par M. G. Leresche. - Je l'ai reçue de II. Th. Bruand sons le nom de Cosmophorana Guén. 


\section{Gen. S y n d e m is, Hubn.}

Eriopsela, Guén. - Sciaphila, Treit. Dup.

283. Vacciniana, Fisch.

Zell. - Ilub. revis. n² 427. - Hub. sup. f. 206. - Ileyd. c. 200.

M. le prof. Imbof a recueilli cette espèce dans les environs de Bàle. - Espèce suisse douteuse.

\section{Ericetana, Zell.}

IHub. revis. 428. - IIub. sup. f. 136. - IIeyd. c. 558. - Bruand, C. $1003^{2}, 1003^{3}$.

Flexulana, Dup. 1359. 265. - Fröhl.?

Virgatana, Fisch. v. R. (olim).

M. Rothenbach a collecté cette espèce en juin dans les environs de Sehüfen. M. Frey l'a prise au Miblebach et sur la Berglialp, volant autour des rhododendrons, au commencement d'août, à 5000 pieds de hauteur.

285. Cuphana, Fisch.

Fisch. v. R. - Dup. sup. 338. - Hubn. revis. $n^{\circ} 430 .-$ Hubn. sup. f. 134. 135. - Guén. Ind. - Hejdr. c. 271. - Bruand, C. 1003.

¿ Stagnana, W. V. - Mus. Schif.

\& Rivulana, W. Y. - Hub. 184.

Extersana, Metzn.

Caricana, Guén. Ind. p. 29. - Bruand, C. 1002.

Commune en arril et mai sur les prés marécagreux de tout le Jorat. Séléve sur les Alpes. De mème près de Zurich (Frey) et de Schiupfen (Rothb.).

Le màle differe beaucoup de la femelle. On peut sans erainte rattacher à cette espèce Caricana, Guén., car c'et entomologiste parait avoir été assez mal renseignó sur Cupliana et Quadrana.

286. Quadrana, Hub. 22:3.

Fröhl. - Treit. … Dup. 255. - Ilubn. revis. n 433. … Itubn. sup. f. 137, 256. - Guén. Ind. - Heyd. c. 267.

Dans les environs de Zurich, au printemps; rare. LEngadine près de Samaden (Frey). LOberland, Frutigen, la Gemmi (Rothb.). Au bois de Sauvahelin, en mai, parmi les bruyères (Lal.). 
Les individus pris aux environs de Lausanne sont d'un gris de fer presque uni; le dessin se distingue à peine sur le fond.

La figure de Dup. est méconnaissable; celle de la femelle que donne Herr.-Schäff. (f. 256) ne répond pas à nos individus; ceux-ci ont moins de roux, les crochets le la còte blancs, les points et les strics de lécusson mieux marqués.

XXX. Gen. S teg a n o t y cha, Steph. Curt.

Grapholita, Treit. Guén. Dup.

287. Freyeriana, Fisch. v. Röst. t. 51.

IIuh. revis. no 436 . - Guén. Ind. - Heyd. c. 423. - Bruand, C. $1035,1036$.

Campoliliana, Mus. Schif.

Talpana, khlw.

M. le prof. Frey a recueilli deux exemplaires de cettc espèce rare, sur l'Uetliberg, en juillet.

288. Campoliliana, Treit.

Dup. 251. - Hul). revis. $n^{\circ} 437$. - Ilubn. sup. f. 269. -- Guén. Ind. - Ileyd. c. 422. - Bruand, C. 1039.

Equitana, Fröhl.

Assez fréquente dans les taillis herbeux des environs de Lausanne, en juillet. De même près de Zurich (Frey, Zell.), de Schüpfen (Rothb.).

289. Immundana, Fisch. v. Rösl. t. 533.

Tisch. - Hubn. revis. 439. - Dup. sup. 66. - Guén. Ind. Heyd. c. 316. - Bruand, C. 1044.

Succedana, Mus. Schif.?

Deux fois dans les environs de Lausanne, en juin. La tache blanche du bord interne niexiste pas toujonrs et l'espèce parait varier autant que Frutetana.

290. Raman a, Lin.

IIub. revis. n 439 . ('non Treit. - Dup.).

Triquetrana, Huls. 280, 336. - Fröhl. - Treit. - Dup. 249. -- Heyd. c. 45. Paykulliana, Guén. Ind.

Près de Schüpfen (Rothb.). Une fois près de Lausanne, dans un bois, en juin. Un exemplaire dans les curirons de Zurich (Frey). 
291. Siliceana, Hub. 196.

Treit. - Dup. 249. - Hub. revis. $n^{\circ} 440 .-$ Heyd. c. 411.

Var. Petrana, Hub. 210. - Dup. 249. - Fröhl. - Heyd. c. 412.

Var. Decorana, Ilub. 265. - Treit. - Dup. sup. 62.

Nis a n a, Lin. - Scop. - Guén. Ind. - Bruand, C. 1029.

Ericetana, Dup.

Point rare en Suisse sous ses diverses formes; environs de Lausanne en avril et mai et une seconde fois en juillet et en aoùt. - Schüpfen (Rothb.), Zurieh (Zell., Frey).

A Lausanne, la forme Petrana est de beaucoup la plus ordinaire durant l'été. J'ai collecté la var. Decorana près de Glaris, en juillet.

M. Bremi n'éerit avoir élevé la ehenille sur les prêles (Equisetum).

292. Naerana, Hub. 261, 262.

Fröhl. - Treit. - Dup. sup. 255. - Ilub. revis. n 442. - Hub. sup. f. 275. - Ileyd. e.552 - Wood. 964 . - Guén. Ind. Bruand, C. 1034 .

Commune dans les taillis dı Jorat et sur les arbres fruitiers, tout l'été. Il est probable qu'elle fait deux apparitions dans les localités chaudes, puisqu'on la trouve une première fois en mai et une seconde en aoùt. Sur les Alpes. Dans les bois des montagnes d'Aigle la chenille s'attache surtout aux extrémités des branches du houx dont elle ronge le bourgeon.

29:3. Abiegnana, Fisch. v. Rösl.

Hubn. revis. ${ }^{\circ} 445,-$ Ilubn. sup. f. 128. - Dup. sup. 83. Guén. Ind. - Heydd. c. 195. - Zell. Ent. Z. 1849.

Pygmaeana, Fröhl. 217.

Jai recueilli un individu appartenant ì cette espèce dans les bois du Jorat. M. Rothenbach la aussi collectée dans les environs de Schüpfen, les preniers jours l'aoùt.

Nos individus ne répondent pas complétement à ceux de Hub. sup.; ils n'ont pas la ligne de division des franges recourbée au niveau du point subapical, ni les ailes blanchàtres à la base; ils ont l'éeusson elair au centre, bordé dune ligne plombéc.

294. Pygmaeana, Hub. 69.

Treit. - Dup. 252. - Ilub. revis. 446. - Guén. Ind. - Wood. 1136. - Heydd. c. 192. (non Fröhl.).

Dans les environs de Schüpfen (Rothb.). Rare. 
295. Frutetana, Hub. 293, 294.

Treit. - Dup. 255. - Fiseh. v. Rösl. 1. 52, 53. - Hubn. revis. n 448. - Guén. Ind. - Iteyd. c. 315. - Bruand, C. 1043.

Commune contre le trone des arbres, dans les bois, en juin et juillet. Pas rare dans les environs de Zurich (Zell., Frey); de la Neuveville (Couleru).

Varie beaucoup pour la taille et le dessin. Certains individus sont complétement noiràtres.

La figure de Dup. est mauvaise.

296. Rubiginosana, Fisch. v. Rösl.

Hub. revis. no 449. - Hub. sup. f. 185. - Ileyd. e. 314.

Cette espèce rare a été prise une fois par M. Frey dans les environs de Zurich. M. J. Wullschlegel en a aussi capturé un individu màle sur l'Engelberg (Jura) en juillet.

297. Penkleriana, W. V.

Hubn. revis. $n^{\circ} 450$. - Guén. Ind. - Heydenr. c. 425. - Dup. 253, 5 (fig.) - Bruand, C. 1032.

Mitterbacheriana, Treit. (excl. syn.). - Dup. 253.5. - Fisch. v. Rösl. t. 19, 20. - Zell. - Wood. 972.

Tenerana, W. V. - Mus. Sehif. - Hub. 183. - Fröbl. - (non Dup.).

Oblitana, Dup. sup. 63.

Pas rare dans les haies et les jardins des environs de Lausanne, durant l'été. Environs de Schüpfen (Rothb.); de Zurich (Frey, Zeller).

298. Minutana, Hub. 73.

Treit. - Dup. 250. - Hubn. revis. $\mathbf{n}^{\circ} 451$. - Ilubn. sup. f. 174, 175. - Guén. Ind. - Ileyd. e. 432. - Bruand, C. 1030.

Environs de Schüpfen (Rothb.). Quelques taillis autour de Lausanue; rare. Environs de Zurich (Zeller).

299. Lithoxylana, Fröhl.

Dup. 250. - Zell. - Ilub. revis. ${ }^{\circ} 452$. - Ilub. sup. f. 176, 177.

Ulmariana, Zell. (olim). - Dup. sup. 83. - Guén. Ind. - Ileyden. e. 430. Bruand, C. 1031.

Var. Stannana, Guén.

Quelques haies dans les environs de Lausanne; assez rare. Environs de Zurich (Zell.). Il n'est pas aisé de distinguer cette espèce de la précédente au moyen des figures de 
Hub. sup. L'angle que forme sur le disquo l'espace basilaire varie de dimension dans les deux espèces. Le dessin varie aussi. Le meilleur caractère se tire de la ligne de division des franges.

J'ai regu de Lyon la variété Stanana et ne l'ai pas encore vue en Suisse.

XXXI. Gen. Phoxopteryx, Treit. Guén. Dup. Anchylopera, Steph.

300. Mitterpachiana, W. V.

F. - Fröhl. - IIubn. 192. - Hubn. revis. n 457. - Guéı. Heyd. c. 565. - Dup. 250, 8 (fig.). - Brnand, C. 1027.

Penkleriana, Treit. - Dup. 250. (texte). - Fisch. v. R. t. 21.

Subuncana, Wood. 948 . - Curt.

Assez fréquente dans les taillis des environs de Lausanne, à la fin de juin et en juillet. De mème près de Schüjfen (Rothb.) et de Zurich (Frey, Zell.).

301. Deras a na. Hub. 206.

Fröhl. - Treit. - Dup. 1327 (non sup.). - Ilub. revis. nº 458.

- Guén. Ind. - Heydenr. c. 568. (excl. synon.). - Bruand,

C. 1026.

Recueillie dans les environs de Schüpfen par H. Rothenbach.

La figure de Ilubn. est mauvaise; la tache basilaire ne ressemble pas à colle de Comptana; la teinte grise qui entoure cette tache se fond souvent arec elle. La figure de Dup. parait ètre copiée sur celle de Ilubner.

302. Badiana, W. V.

Treit. - Dup. 253. - Zell. - Hubn. revis. $n^{\circ} 459$. - IIeydenr. c. 567 .

Lund a na, F. - Fröh. - Steph. - Curt. - Guén. Ind. - Bruand, C. 1025.

Corylana, Hub. 53. - Mus. Schif.

Bo eberana, l.

Commune dans les haies des montagnes et des AJpes en juin et juillet. Je l'ai recueillie jusques sur les pàturages des Alpes, loin de tout coudrier. Environs de Zurich, de Schipfen (Rothb.). Deux générations (Frey).

Parmi les nombreux individus que jai recueillis, jen ai un sons les yeux qui se distingne par quelques caractères insuffisants pour justifier une espece distincte, à moins guils ne se reproduisent sur plusicurs individus dune manière constante. Chez lui, la 
tache basilaire forme un angle presque droit du còté de la côte. en sorte que les deux ailes rapprochées portent sous le corselet une tache foncée quadrilatère, au lieu diune tache ovoïde. Les crochets de la còte sont confondus à leur base dans une ligne blanejaunattre sur laquelle les stries brunes se détachent comme les dents d'un peigne. Les inférieures sont d'un noir tirant sur le brun, et leur frange, d'un blanc jaunitre, présente à la base une ligne de division bordée par deux lignes jaune-clair. Le dessous est brunfoncé uniforme.

Le dessin ítant it tous autres igards exactement celui de Badiana, il faut, avant de se prononcer, recueillir sur ce point de nouseaux renseignements.

303. Lyellana, Curt. cat. 1829. - Wood. 952.

Je n’ai collecté jusquici cette espèce que sur les pàturages élevés (6000 pieds) situés an pied de la Tour-d'Ai, en juillet. M. Ott liı aussi recueillie dans le Haut-Yalais, à la mème époque. - Je dois sa détermination ì I. Ilerrich-Schäffer.

Ressemble beaucoup à Badiana, mais s'en distingue: $1^{\circ}$ par ses teintes brunes tirant sur le gris-noir; $2^{\circ}$ par le sommet des supérieures alongé en bec; $3^{\circ}$ jar l'angle plus alongé que forment les deux lignes métalliques en se rencontrant du côté de la frange; $4^{\circ}$ par la tache du bord interne plus uniformément arrondie et ne formant pas bosse du còté lu bord antérieur; $5^{\circ}$ par labsence de tout éclat métallique sur les deux lignes obliques du disque. Sa taille est un peu plus forte que celle de Badiana et de Myrtillana. La femelle ne diffère pas du mìle.

304. Myrtillana. Treit. p. 215.

Dup. 253. - Hub. revis. n 460. - Iubn. sup. f. 316. - Guén. Ind. - Ileyd. c. 369. - Bruand, C. 1024.

Rare en Suisse; une fois dans les bois du Jorat, en juin. H. Rothenbach l'a aussi collectée près de Schüpfen et M. C. Zeller près de Zurich.

La dent que porte en avant la tache du hord interne n'est pas assez prononcée dans la figure de Duponchel. Cette espèce a sans doute été confondue aree la suivante; voil à celle-ci pour le diagnostic.

305. Dentana, Lah.

Jai reçu deux fois cette espèce de $\mathrm{M}$. Ott à Heyringen. Je l'avais prise d'abord pour une grande variété de Myrtillana; mais un examen plus complet m’a détrompé. Elle est d'un tiers plus grande que M yrtillana, un peu plus mème que Comptana, de la taille de Quadrana ${ }^{\prime}$, à laquelle elle ressemble extrèmement pour le coloris. 
Le fond des supérieures est gris mćlangé dle blanchâtre, avee une grosse tache noirâtre a la base et une bande transverse hrunàtre, très-irrégulière, brisće dans son milieu, située aux deux tiers externes. Le sommet est brun-noir, aussi fortement crochu que celui de Comptana (très-peu dans Myrtillana).

La tache basilaire dun noir brun, s'étend jusquà la moitié du bord interne (plus courte dans Myrtillana) et forme en avant une dent. Le hord externe de cette tache est conpé dabord obliquement sur le centre de laaile, puis perpendiculairement au bord interne, du còté de ce bord, en sorte que ces deux coupures forment un angle obtus, vif, et non point un bord undulé, arrondi, comme dans Myrtillana.

La bande transverse est formée de deux taches brunàtres ou bistres, l'une antéricure, l'autre postérieure, irrégulières et séparées par un trait blanc, oblique, dirigé du còté dı sommet. Lantérieure, placée an milieu de la còte, est plus on moins ovoïde on quadrilatère. La postérieure, en forme de demi-lune ou de virgule, se termine en pointe en se recourbant du còté du somnet, tandis que par son autre extrémité elle s'appuie sur le loord postérieur, tout près de l'angle abdominal. Cette dernière tache enferme dans Sa concavité un écusson arrondi (retréci et alongé dans Myrtillana), gris-blanchàtre comme le fond, marqué en dehors d'une tache arrondie, brune on grise, sans traits ni marques.

La còte, légèrement arquée, est diun blanc sale dans sa première noitié et marquée de 5 à 6 points noirs, fort petits; entre la tache du milieu et le sommet elle porte 4 crochets doubles, hien espacés, placés sur un fond blanc et n'envoyant pas de lignes prolongées du còté de la marge. (Dans Myrtillana les crochets sont plus rapprochés et rarement divisés). La frange est entierement d'un blane terne, linuitée par iquelques petits points noirs, wais non par un trait, comme dans Myrtillana.

Les postérieures sont dun gris-clair uniforme, avec la frange blanchàtre à sa racine et grise ì son extrémité. Dans Myrtillana les inféricures sont d'un gris rou:

Le dessous est entièrement diun gris de fer, plus clair anx inféricures, avec les crochets indiqués. Myrtillana a ces teintes rousses et plus claires.

Palpes, tìte et corselet gris (blanes dans Myrtillana). Abdomen plus clair; mouchet anal un peu clair; pattes grises, annelées ì leur extrémité.

306. Complana, Fröhl. 2 价.

Dup. sup. 68. - Ilulon. sup. f. 318. - Huln. revis. $n^{\circ} 461 .-$

Ileyd. cat. 560. - Guén. Ind.

Obtusana, Bruant, C. 10032 2 ? 
II. le prof. Frey a pris cette jolie petite espèce, à la fin de juillet, près de Baden; il l'a aussi reeueillie dans lEngadine.

\section{In comptana, Lah.}

J'ai trouvé cefte espèce fréquente sur la sommité et les pàturages de la montagne de Chanx-de-Mont, au pied de la Tour-dAï, en juillet, à 7000 pieds d'élévation. Malheureusement un petit nombre d'individus était bien conservé.

Elle a le dessin, mais non le faeies de Comptana, avee laquelle senle on peut la confondre, soit par sa petite taille, soit par la ressemblance extrème du dessin. Elle en diffère eependant par des earaetères tranchés et qui en font une espèce distincte. La taille est eelle de Badiana de petite dimension, avec les ailes plus étroites. La couleur du fond est le brun veiné de blanc pur: Comptana est d'un brun rouge, et ses stries blanches sont moins prononcées. La tache de la base, d'un brun presque noir, oecupe plus du tiers de l'aile; elle se termine par un bord arrondi auquel succède un ruban blane qui sétend obliquement d'un bord à l'autre de l'aile. Ce ruban est gris-sale, pointillé de brun dans Comptana. Le bord antérieur est séparé de la tache basilaire par une bande diun blane roux, sur laquelle se dessinent six points noirs. Ces points sont plus petits dans Comptana. Le ruban blane qui borle la tache basilaire se contourne en $\mathrm{S}$ en se dirigeant vers le bord externe de laile et eireonserit vers l'angle anal une petite tache brune, en forme de virgule, placée au centre d'un écusson arrondi, lequel reste séparé de la frange par un trait noir vif. Le ruban blane est divisé par une ligne brune dans toute sa longueur. Comptana n'offre pas cette ligne de division, et son liséré en $\mathrm{S}$ est gris, limité par deux traits blancs, fins. L'angle apical est brun, alongé, acuminé, recourbé en serpe; au-dessous de lui la frange est blanche, divisée par un trait noir. Dans Comptana il est moins alongé, en sorte que le sommet dépasse fort peu le bord de la frange. Les denx tiers externes de la còte portent 4 doubles erochets très-prononcés. Chaque crochet est divisé par un trait presque noir; ils sont également distants les uns des autres et séparés par des taches brunes. Le quatrième crochet donne naissance à un filet brun qui se dirige vers la marge en passant au-dessus du ruban blanc, sans le toucher. Dans Comptana les. erochets sont moins espacés entre eux, en sorte qu'ils se présentent plutòt eomme 8 erochets simples, síparés par des points et mème fondus les uns dans les autres vers le sommet. - La frange, à lexeeption de sa portion antérieure, est rousse teintée de blane du eòté du disque.

Les inférieures sont d'un gris pàle, uniforme, un peu luisant, avee un trait noir qui 
limite la frange un peu plus foncée que le fond. Un large trait blanchàtre se voit à la base de la frange.

En dessons, les supérieures, fun brun elair luisant, portent sur la còte le dessin du dessus et le long de la frange le trait noir de séparation. Les inférieures, diun blane sale luisant, ont le sommet brunâtre. Comptana a le dessous des supérieures uniformément brun et la côte blanchàtre, sans dessin; les inférieures sont d'un blane roux.

Tète ornce d'une houpe de poils fawes; palpes sécuriformes, l'un gris roux; troisième article invisible. Corselet brun; abdomen gris-noir; anus fauve; pattes blanchìtres, amıelées de noir.

308. Unguicana, Fab.

$$
\begin{aligned}
& \text { Treit. - Fröhl. - Dup. } 252 \text { (texte). - IIuhn. revis. n० } 462 .- \\
& \text { Guén. Ind. - Ileyden. c. } 559 . \text { - Bruand, C. } 1021 .
\end{aligned}
$$

Falcan a, Hub. 78.

Ericeana, Dup. 252. 9.

Conmune sur les bruyères en mai; fin de juin et juillet sur les Alpes, où elle s'élève avec la bruyère. Environs de Schijpfen (Rothb.).

309. Uncana, Hub. 76.

$$
\begin{aligned}
& \text { W. V. - Fröhl. - Treit. - Dup. } 252 \text { - Inb. revis. } 1^{\circ} 463 .- \\
& \text { Guén. Intl. - Ileydenr. cat. } 556 . \text { - Wood. } 95 \text { z. - Bruand, } \\
& \text { C. } 1022 .
\end{aligned}
$$

M. Ie prof. Frey a recueilli cette espice à la fin de juillet, dans les environs de Samaden (Engadine).

310. Flucligerana, Fisch. v. Rösl.

Hub. revis. $1^{\circ} 464$. - Ilub. sup. f. 319. - IIegd. c. 545.

Uncana, var., Guén. Int.

Crenana, Dup. 1245. 252, 7. (non lluh.), - Heyden. c. 557.

Collectée dans les environs de Schiipfen par M. Rothenbach et au pied du Jura par II. L. Couleru.

\section{Cuspidana, Treit.}

$$
\begin{aligned}
& \text { Dup. sup. 66. - Huhn. revis. } n^{\circ} 465 .- \text { Iluhn. sup. } 38 .- \\
& \text { Guén. Ind. - Ileyd. c. } 555 .
\end{aligned}
$$

Une fois près de Zürich (Frey).... Environs de Schipfen (Rothlo.). 


\section{Harpana, Hub. 77.}

Hub. revis. $n^{\circ} 467$.

Rama na, Treit. - Fröhl. - Ileyden. c. 548? - Bruand, C. 1028 ?

Indiquée assez fréquente dans les environs de Zurieh, par M. Frey; n’a pas été observée ailleurs en Suisse.

313. Siculana, Hub. 79.

Fröhl. - Treit. - Dup. 252. - Hubn. revis. n 468. - Guén.

lnd. - Heydenr. c. 546. - Bruand, C. 1020.

A picella (Tin.), IV. V.

Reçue de M. L. Louleru, à la Neuveville; prise une fois sur le route des Ormonts, au-dessus d'Aigle. Pas rare dans les environs de Zurich (Frey).

XXXII. Gen. Che i mathophila, Steph.

Le m mat ophila, Treit. Dup. - Tortricodes, Guén.

314. Hy emana, Hub. verz.

Fröhl. - Guén. Ind. - Hub. revis. n 469. - IIub. sup. f. 123, 124. - Heyd. c. 293. - o IHub. 267.

Hyemella (Tin.), Treit. - Dup. sup. 67.

o T ortricella, Ilub. 11.

\& Alternella, Dup. cat. (non W. V.).

Nubilea, Wood. 1269.

Fort commune partont, au premier printemps, sur les chènes. La femelle est plus rare que le màle. 


\section{TABLE DES MATIËRES.}

\section{Table des genres et des espèces.}

NB. Les noms en letlres majuscules indiquent les genres; la premiere colonne de chifres les numéros des especes et la seconde les pages.

\begin{tabular}{|c|c|c|c|c|c|c|c|c|c|}
\hline & & & & & & & & $\mathbf{N}^{0}$ & Pages. \\
\hline & & & & & Alpinana . & . & . & 275 & 111 \\
\hline & & & $\mathbf{N}^{\circ}$ & Pages. & Alteruella & . & . & - & 52,122 \\
\hline Abiegnana & . & . & 293 & 115 & Alticolana . & . & . & - & 53 \\
\hline Abielana & . & . & 18 & $15,16,76$ & Ambiguana & (ella) & . & 98 & $43,45,46,87$ \\
\hline Abielis & . & . & - & 62 & Ameriana . & - & . & 26 & 18,19 \\
\hline Abielisana & . & . & - & 62 & Amoenana & . & . & - & 87 \\
\hline Abildgaarda & ana & . & 2 & 10 & AMPHISA & . & . & - & 19 \\
\hline ABLAB'A & . & . & - & 36 & АХCHYLOPEB & & . & - & 117 \\
\hline Absynthiana & & . & 219 & 93 & Andereggian & & . & 179 & 80 \\
\hline Acerana & . & . & - & 21,22 & Angulosana . & & . & - & 105 \\
\hline Aceriana & . & . & 195 & 86 & Angustana . & . & . & 276 & 111 \\
\hline Achaiana. & . & . & 129 & 61,75 & Anliquana . & . & . & - & 95 \\
\hline Acuminalane & & . & 251 & $10^{\prime}$ & INTithesla . & . & . & - & 80 \\
\hline Aculana & . & . & 183 & $80,10^{\prime} 1$ & Aphalana . & . & . & - & 100 \\
\hline Adjunctana & . & . & 42 & 26 & APnelia & . & . & - & 36,92 \\
\hline Adspersana. & & . & 10 & 13 & Ipicalis & . & . & - & 31 \\
\hline Aemulana & . & . & 228 & 96 & Apicella & . & . & - & 122 \\
\hline Affiniana & - & . & - & 91 & Approximan & & . & - & 13 \\
\hline Ahrensiana & . & . & - & 63 & Aquana & . & . & - & 87 \\
\hline Albersana & . & . & - & 103 & Aquilana & . & . & $\sim$ & 16 \\
\hline Albisiriana & . & . & - & 16 & Arbutana & . & . & 169 & 77 \\
\hline Albuginana & . & . & - & 110 & Arcuana & . & . & 231 & 98 \\
\hline Albulana & . & . & 105 & 49 & Arenana & . & : & 119 & 58 \\
\hline Allionana & . & . & - & 38 & Areolana & . & . & - & 79 \\
\hline Alniana & . & . & - & 29 & Arqentana & . & . & - & 37 \\
\hline Alpestrana & & . & 253 & 105 & Irgyrana . & . & • & 256 & $106,108,110$ \\
\hline Alphonsiana & & . & 一 & 60 & Argyolepya & & . & - & 38 \\
\hline Alpiculana & & . & 75 & 37 & ARGYROPTER & & . & - & 36 \\
\hline
\end{tabular}




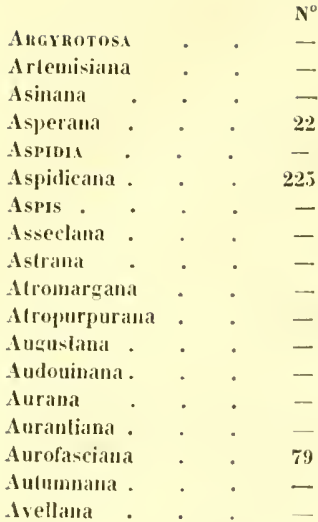

IB.

Badiana . . . 302

Baumanniana . . $8: 2$

Bellana . . . 106

Benthleyana. . . -

Bersmantiana . . 57

Beluleliana . . . 193

Bicinclana . . . -

Bifasciana . . . 60

Bimaculana . . -

Bipuncidna . . . 156

Bistrigata . . . -

Blaurliauna . . -

Blepdarana . . 2'11

Boeberilla . . .

Borinti . , . -

Borealla . . . -

Boscina . . . 13

Brachiana . . . -

Branteriana . . -

Bromichiana . . 215

Bugniona . . . 212

Buoliana . . . 171

Byringerana . . -

C.

Cacaleana . . . -

Caecimaculana

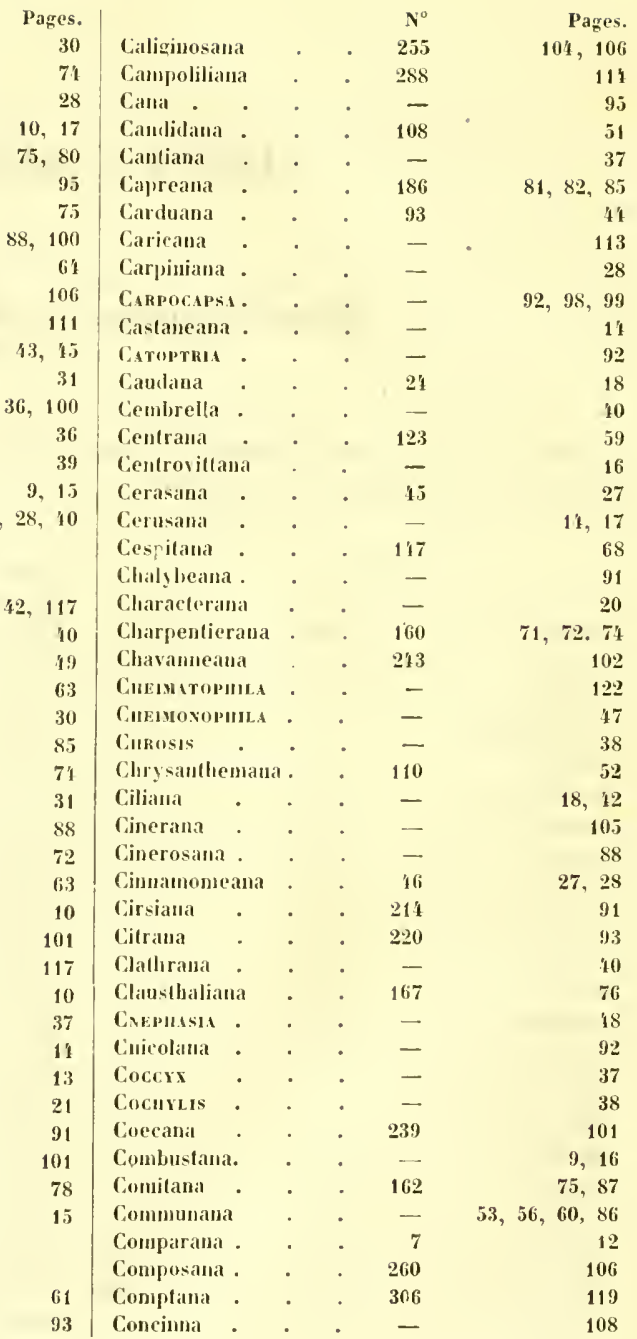




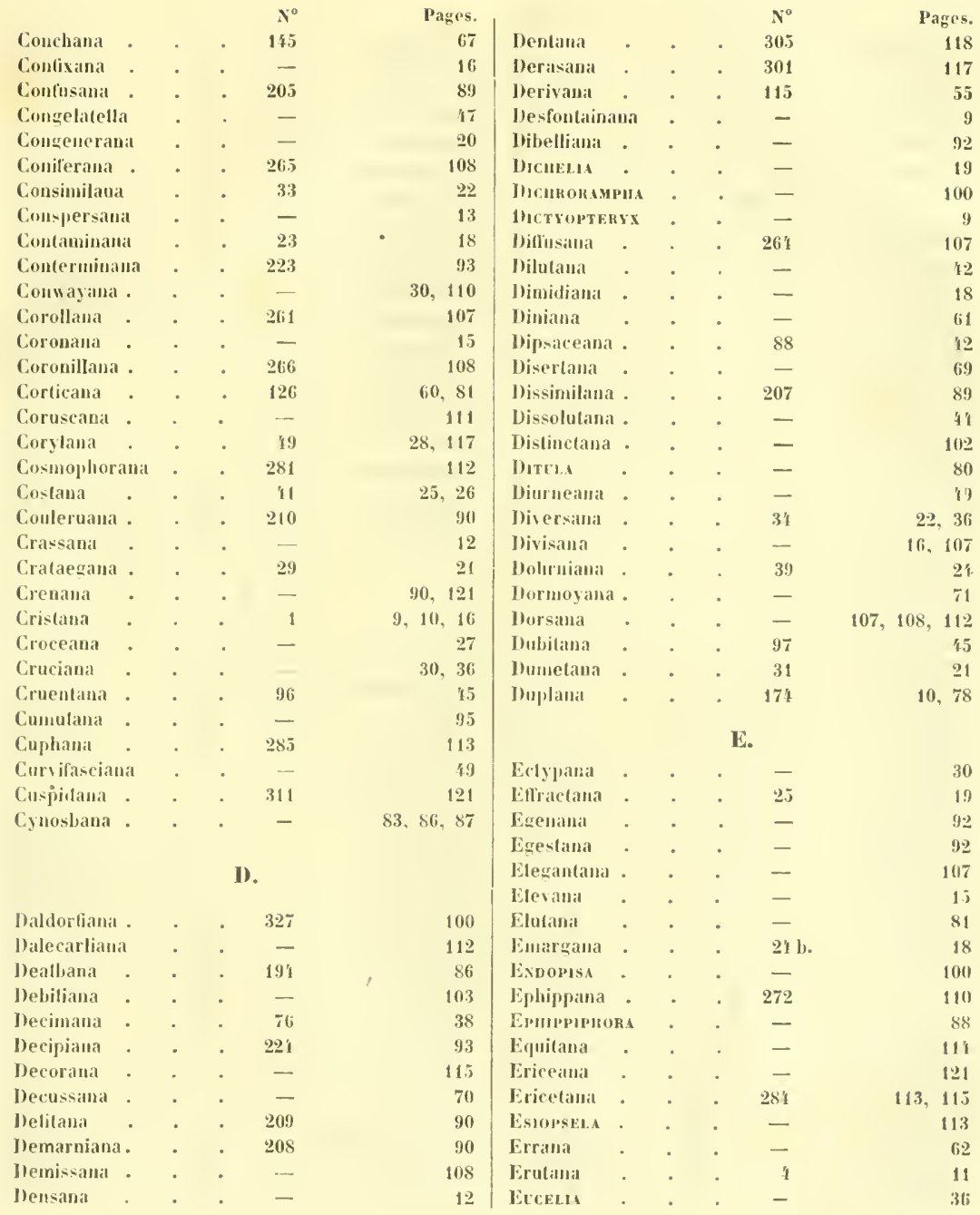




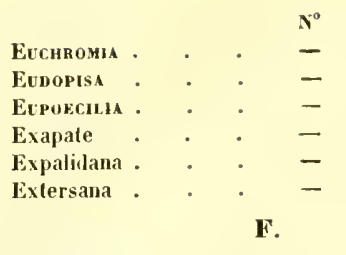

Fabriciana . . . -

Fagiglandana . . 233

Falcana . . . -

Fasciella . . . -

Fasciolana . . . -

Favillaceana . 5

Ferrugana - . 9

Fischerana . . 180

Fissana . . . 263

Flavana . . . -

Flavipalpana . . -

Flexulana . . -

Fluctigerana , . 310

Fluidana . . . -

Forsterana . . . -

Fraternana . . . -

Freyeriana . . . 287

Frutelana . . 295

Fulgidana . . . -

Fuligana . . . 168

Fulsana . . - -

Fulvomixtana . . -

Funebrana . . . 250

Funerana . . . -

Furfurana . . . -

Furvana . . . -

Fuscana . . . -

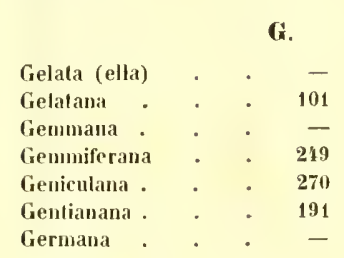

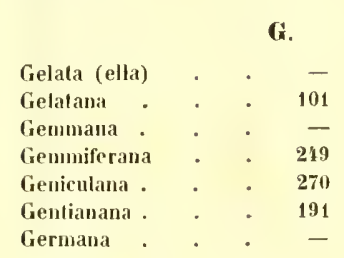

Fasciana . . . -

Forskaleana - $\quad 58$

Fulvifrontana . . 238

\begin{tabular}{r|} 
Pages. \\
57 \\
100 \\
37,38 \\
47 \\
92 \\
113 \\
\\
41 \\
99 \\
121 \\
81,83 \\
43 \\
63
\end{tabular}

$11,12,15,59$

$13,3.5,36$

80

107

23,32

68

113

121

88

31

26

112

111

116

68

77

$20,36,95$

101

17

104

86

66

100

20,62

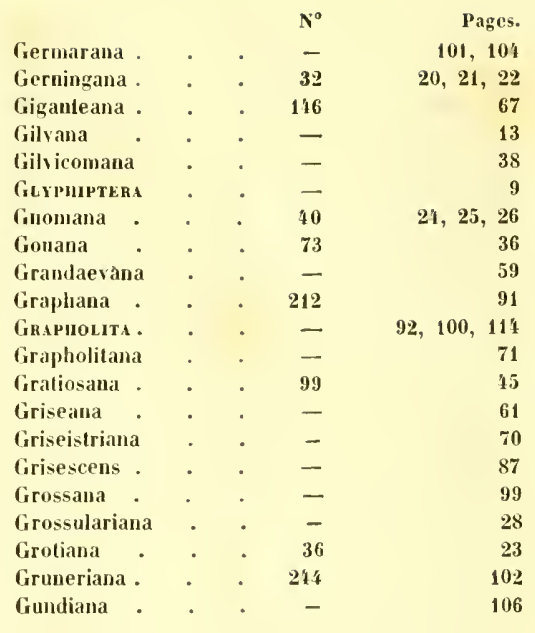

II.

Hamana . . . 70

312

Harlmanniana . . 18'

Hastana . . . -

121

Heiseana . . . -

lletreticana. . . -

Helvolana . . . -

Heparana . . . 47

Herbana . . . -

Hercyniana . . . -

Ifermanniana . . -

Histrionana . . . 50

Hothmanseggana . . 55

Hohenwartiana . . 226

Holmiana . . . 51

Horrilana . . . -

llumidana - . 89

IIumilana . . : -

35,36

121

40,81

59

15,39

38

68

33

27

70

75,76

20

28

30. 112

93,95

30

48

43

101

29, 19

41

11, 122

98 


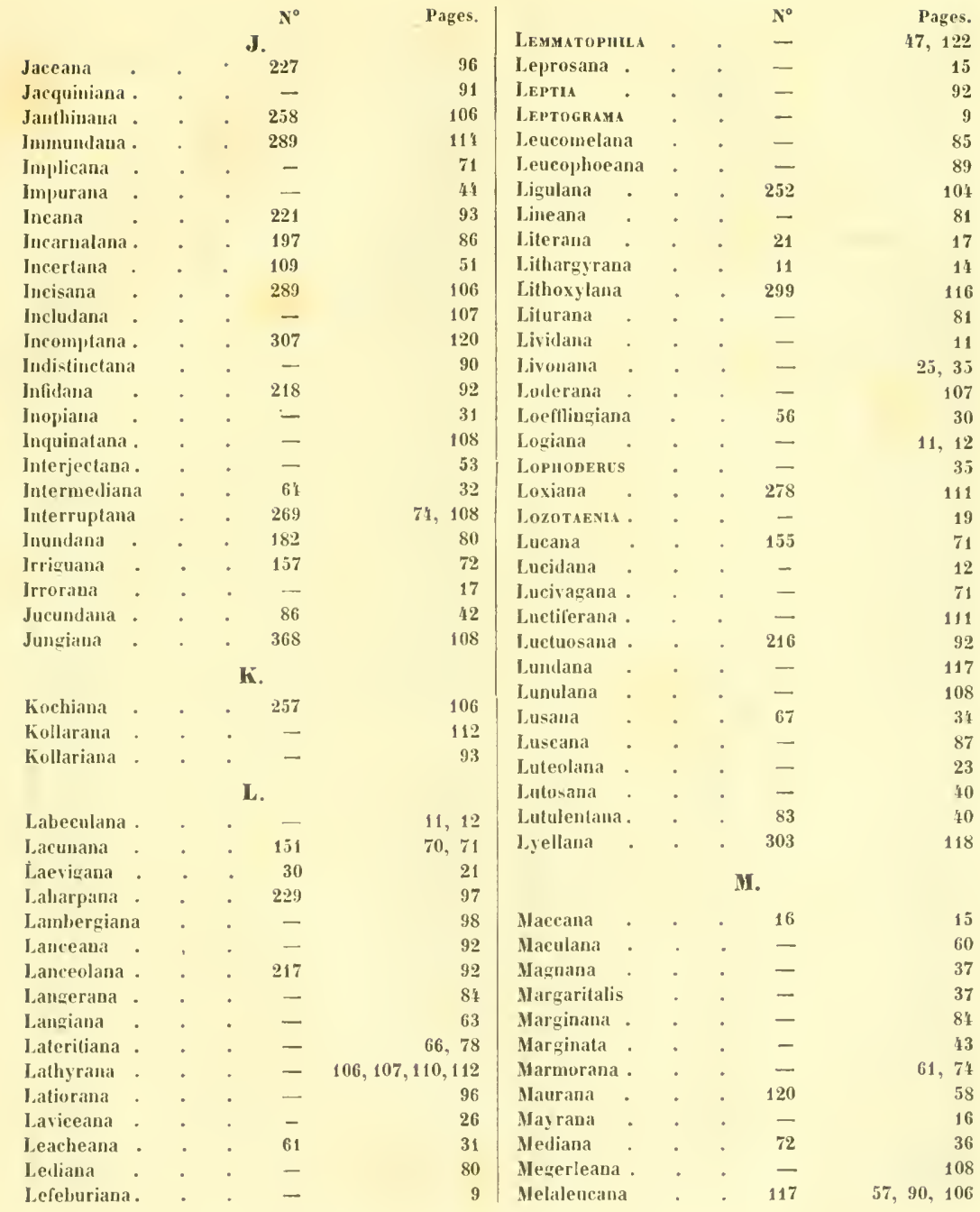




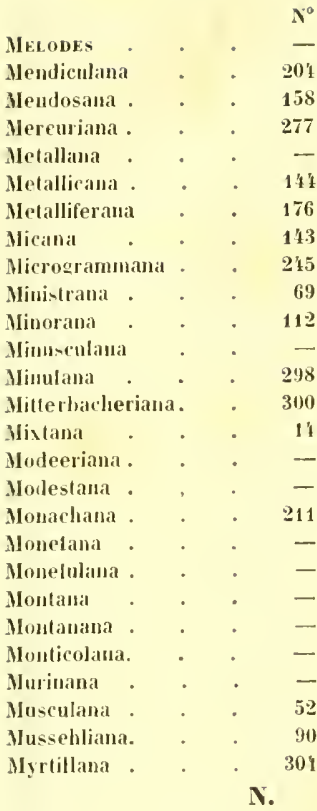

Naesana . . 292

Nana. . . 165

Nehritana . . 2's

Nebulanat . . 20

Sebulosina. . . -

Neglectana. . . -

Nigricana . . 164

Nisana. . . -

Niveana . . . -

Notocell . . -

Nulitana . . 51

Nubilea . . -

Nubilosana. . . -

Niclemerana . . -

o.

Obesana . . . 23 !

Obliquana. . . -

Oblitana . . -

\begin{tabular}{|c|c|c|c|c|c|}
\hline Pages. & & & & $x^{0}$ & Pages. \\
\hline 98 & Obliterana . & . & • & 43 & 26 \\
\hline 88 & Oblongana. & . & - & - & 8; \\
\hline 73 & Obscurana & . & . & - & 18 \\
\hline 111 & Obsoletana & . & . & - & 31 \\
\hline 67 & Oltusana & . & . & 306 & 119 \\
\hline 67,79 & Ocellama & . & . & 200 & 87 \\
\hline 79 & Ochracea & . & . & - & 18 \\
\hline $67,69,71$ & Ocliraceana & . & . & - & 33 \\
\hline 103 & Ochreana & . & . & $3 \bar{T}$ & 23 \\
\hline 35 & Ocliroleuean & na & . & $18 \overline{7}$ & 82 \\
\hline $13,57,86$ & Ochsenheime & aeriana & & - & 112 \\
\hline 54 & Octomaculan & & . & - & 50 \\
\hline 116 & OENECTRI. & . & . & - & 19 \\
\hline 116,117 & Olisana & . & . & 118 & 69 \\
\hline 1't & Omphiaciana & & . & - & 16 \\
\hline 22 & Oplatcana & . & . & - & 16 \\
\hline $48,19,96$ & Or.sis & . & . & - & 100 \\
\hline 90 & Oplithalmica & ana. & . & 125 & 60 \\
\hline 71 & Oporana & . & . & - & 20,28 \\
\hline 95 & Oppositima . & . & . & - & 87 \\
\hline 110 & $0_{\text {prressana }}$ & . & . & 128 & 60 \\
\hline 10.5 & Ornatani & . & . & - & 106 \\
\hline 101,111 & Orobana & . & - & 267 & 108 \\
\hline 28 & ORTHOTAEN & & . & - & 62 \\
\hline 29 & Osbechiana & . & . & - & 10 \\
\hline 43 & Osseana & . & . & - & 37 \\
\hline \multirow[t]{2}{*}{118} & Oxyacanthat & ma & . & 53 & 21.29 \\
\hline & \multicolumn{5}{|c|}{ P. } \\
\hline $\begin{array}{r}115 \\
76\end{array}$ & Pactulana & . & . & 280 & 112 \\
\hline 95,103 & Paulana & . & . & $\bar{n}$ & 28 \\
\hline 17,21 & Palleana & . & . & 63 & 32 \\
\hline 67 & Pallidana & . & - & 94 & 11. 45 \\
\hline 86 & P'alustranal & . & . & 149 & 69 \\
\hline 76,101 & Р'хина & . & . & - & 80 \\
\hline 115 & Parmatana & . & . & 116 & 56.57 \\
\hline 17 & Parsulina & . & . & - & 101 \\
\hline 75 & Pasic ana & . & - & 114 & 5.5 \\
\hline $29,60,79$ & Pasquilyana & . & - & - & 28 \\
\hline 122 & Payhulliana & . & . & - & 114 \\
\hline 88 & l'ertinana & . & - & - & $2 \cdot 2$ \\
\hline \multirow[t]{3}{*}{10} & Peihleriana & a. & . & 297 & 116,117 \\
\hline & Pextmisi & . & . & - & 80 \\
\hline & Penxiana & . & . & 107 & 49,50 \\
\hline 99 & Perfirsana & . & . & 81 & 10 \\
\hline 20 & Permixtana & & - & - & $42,43,80$ \\
\hline 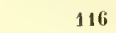 & Permutatana & & . & - & \\
\hline
\end{tabular}




\begin{tabular}{|c|c|c|c|c|c|c|c|c|c|c|}
\hline Petiverana & & & $\mathbf{N}^{o}$ & Pages. & Pyrastrana & . & . & . & $\begin{array}{l}N^{\circ} \\
-\end{array}$ & $\begin{array}{r}\text { Pages. } \\
20\end{array}$ \\
\hline $\begin{array}{l}\text { Petiverana } \\
\text { Petiverella }\end{array}$ & . & . & 27 & 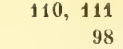 & Pyinones & . & $\dot{0}$ & ${ }^{\circ}$ & - & $\begin{array}{r}20 \\
100\end{array}$ \\
\hline Petrana & . & . & - & 115 & \multirow{2}{*}{\multicolumn{6}{|c|}{ a. }} \\
\hline Ptlugiana & . & . & - & 89,90 & & & & & & \\
\hline Phaleratana & & . & 91 & 43 & Quadrana & . & . & . & 286 & 113 \\
\hline l'HOXOPTERY & & . & - & 92,117 & Quadratana & & . & $\cdot$ & - & 91 \\
\hline Phtueucroa & & . & - & 48 & Quadripunct & tinna & . & ${ }^{\circ}$ & - & 37 \\
\hline Picana. & . & - & 185 & 81 & Quercinana & . & . & . & 12 & 14 \\
\hline Piceana & . & . & 27 & $20,21,76$ & \multirow{2}{*}{\multicolumn{6}{|c|}{ R. }} \\
\hline Pictana & . & . & 139 & 64 & & & & & & \\
\hline Pierettana & . & . & - & 91 & Radiana & . & - & . & - & 15,16 \\
\hline Pilleriana & . & . & 35 & 23 & Ransana & - & . & . & 290 & 114,121 \\
\hline Pinetana & . & . & - & 63 & Ramestrian & & - & . & - & 16 \\
\hline Pinicolana & . & . & 130 & 61 & Ratana & . & . & . & - & 57 \\
\hline Pinivorana & . & , & - & 78 & Ratzehurgia & ana & . & . & 131 & 62 \\
\hline Pisana & . & . & - & 103 & Reclifascian & & . & . & - & 49 \\
\hline Plagana & . & . & - & 92 & Rejectana & . & . & . & 136 & 63 \\
\hline Plumbana & . & . & - & 30 & Remyana & . & . & . & - & 79 \\
\hline Plnmbagana & & . & 2.54 & 105 & Resinand & - & . & . & 167 & 77 \\
\hline Plumbalana & & . & 210 & $10 t$ & Resinella & . & . & . & - & 78 \\
\hline Podana & . & . & - & 20 & Retixia & . & . & . & - & 75 \\
\hline Puecilana & . & . & 213 & 83,91 & Reticulana & . & . & . & - & 15,26 \\
\hline Poecilocuro & вома & . & - & 56 & Rhediana & . & . & . & - & 100 \\
\hline Poendsca & . & - & - & 36,56 & lomiacjonia & . & - & . & - & 59 \\
\hline Politana & . & . & - & 111 & Rhombana & . & . & . & - & 18,33 \\
\hline Pomonana & . & . & 232 & 99 & Ribeana & . & . & - & 48 & 27,28 \\
\hline Populana & . & . & - & 110 & Riøana. & . & & . & 103 & 18 \\
\hline Porphyrana & & . & 142 & 60,66 & Riseana & . & . & . & - & 90 \\
\hline Posterana & . & . & 92 & 43 & Rivellana & . & . & , & - & 67 \\
\hline Posticana & . & . & - & 78 & Rivolana & . & . & . & - & 113 \\
\hline Pratana & . & . & 71 & 37 & Roborana & . & . & . & 198 & 21,87 \\
\hline Productana & . & . & - & 26 & Rogana & . & - & . & - & 21 \\
\hline Profanana & , & . & $-\infty$ & 81 & Rulandriana & a. & . & . & 59 & 31 \\
\hline Profundana & $\therefore$ & . & 127 & $60,89,91$ & Romauana & . & . & . & - & 17 \\
\hline Proleana & . & . & - & 14 & Rosana & . & . & . & - & $18,20,21,27,30$ \\
\hline Proximana & . & . & 163 & 76 & Roscidana & . & . & . & - & 17 \\
\hline Pruniana & . & . & 189 & 83 & Roseana & . & . & . & - & 42 \\
\hline Ptycholoma & & . & - & 31 & Roserana & . & . & . & 100 & 46 \\
\hline Pudendana & . & . & 173 & 78 & Rosetana & . & . & . & 118 & 57,58 \\
\hline Pullana & . & . & - & '. & Roxaxa & . & . & . & - & 98 \\
\hline Pulverana. & . & . & - & 33 & Rubellana & , & . & . & 87 & 42 \\
\hline Pumilana & . & . & - & 45 & Rubiana & . & . & . & - & 75 \\
\hline Pupillana & . & . & - & 03,95 & Rubigana & . & . & . & 85 & 42 \\
\hline Purgatana & . & . & 95 & 41 & Rubiginana & . & . & . & - & 12 \\
\hline Pusillana & . & . & - & 108 & Rubiginosan & & . & - & 296 & 116 \\
\hline Pustulana & . & . & - & 111 & Rubrana & . & . & . & - & 28 \\
\hline Pygmeana. & . & . & 294 & 115 & Rufana & . & . & . & 8 & $12,13,11$ \\
\hline
\end{tabular}




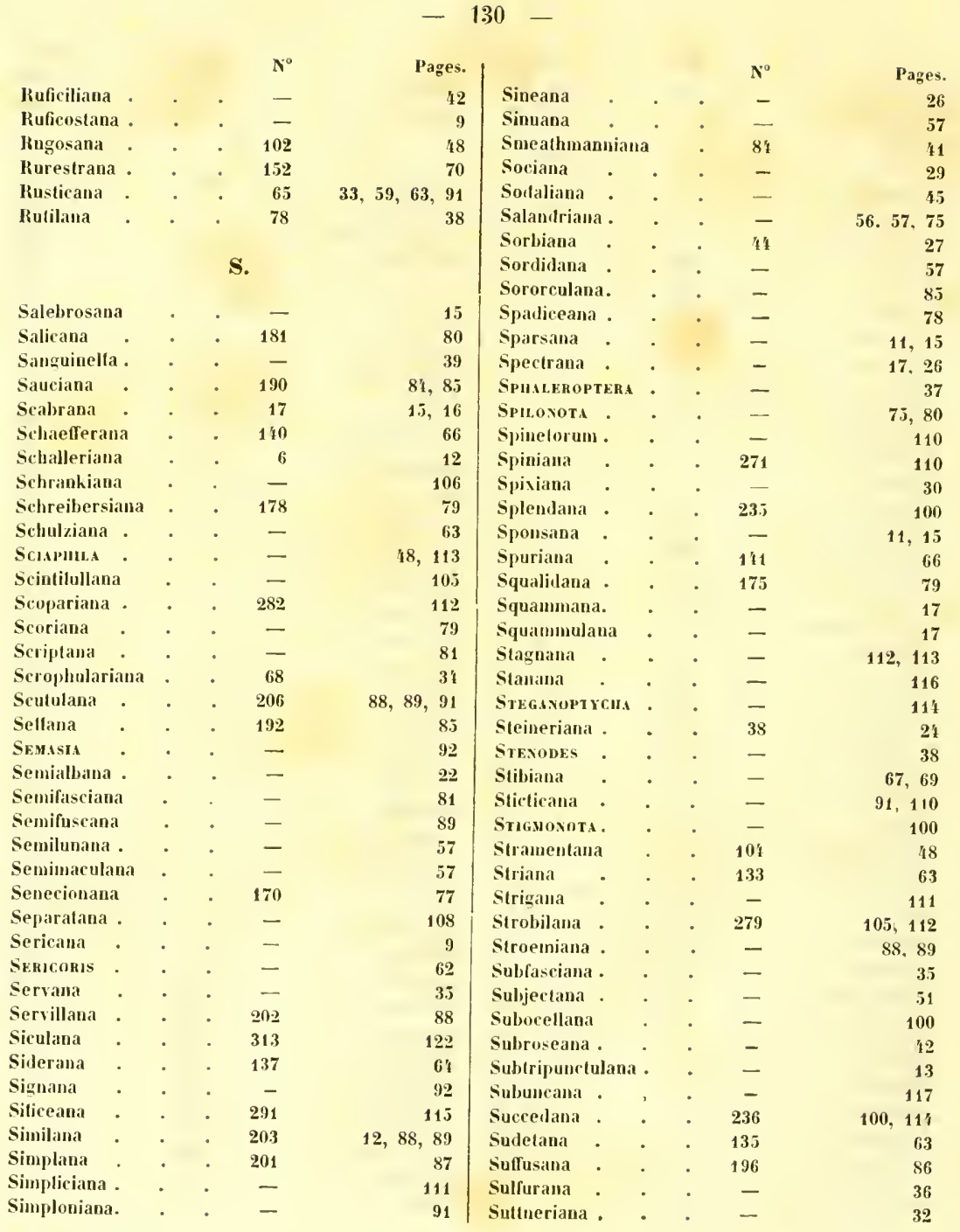




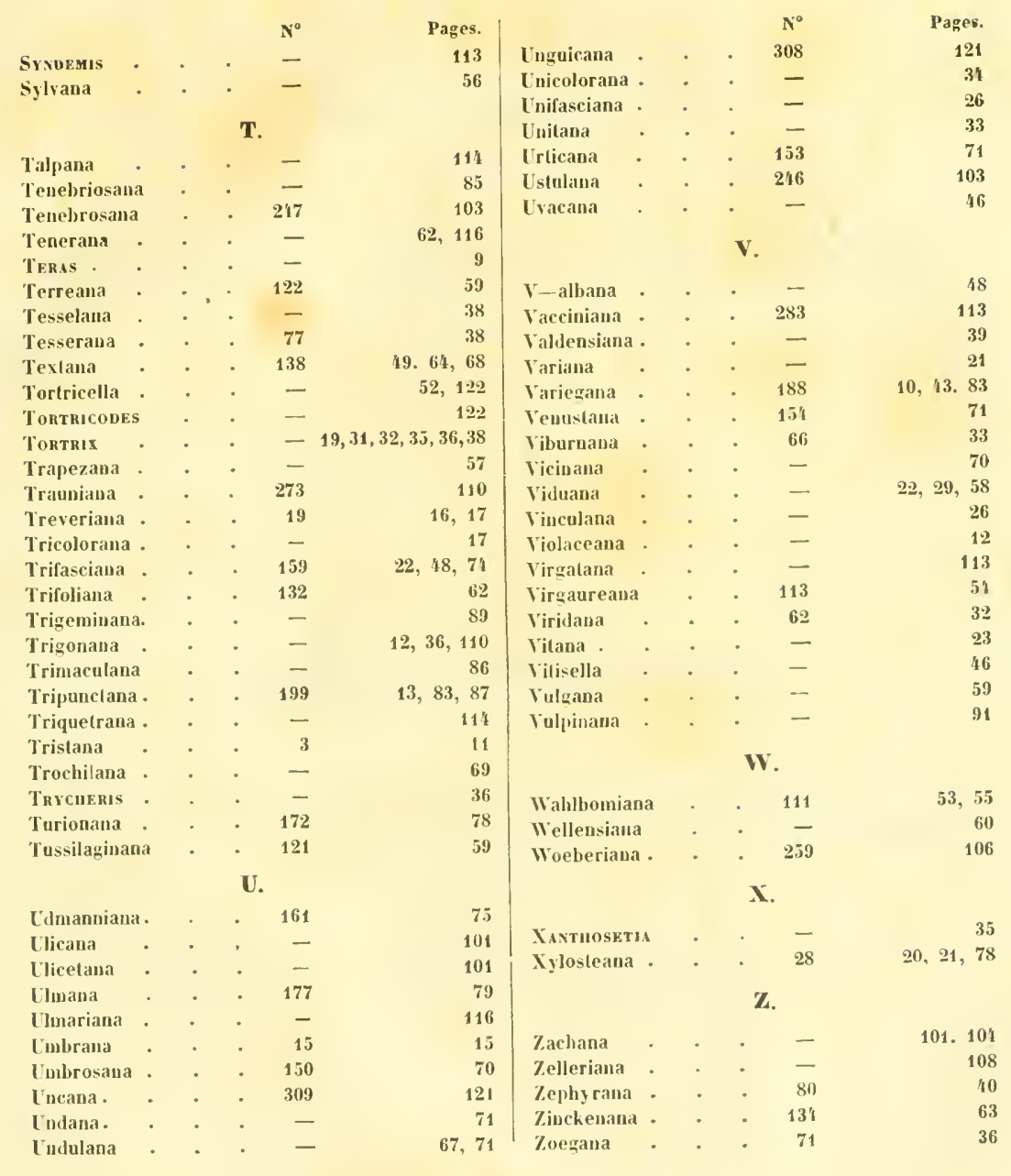




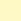




\section{SUPPLÉMENTS}

A LA

\section{FAUNE DES LÉPIDOPTÈRES SUISSES.}

(Phalénides, Pyralidides. Crambides et Tortricides.)

Par

J. C. De la Harpe, D. M.

\section{'TROISIL̀ME SUPPLĹMENT'}

AUX

\section{PHALÉNIDES DE LA FAUNE SUISSE.}

(Voir: Nouveaux Mémoires de la Société helvétique des sciences naturelles;

Vol. XIII. p. 146, et Vol. XIV.) 


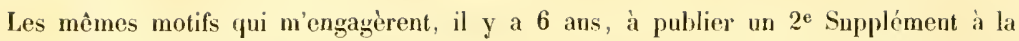
Faune Suisse, pour les Phalénides, me font un devoir d'en domer anjourd'hui un $3^{\mathrm{e}}$ et dernier. Je le fais suivre des corrections et adjonctions devenues nécessaires pour les familles des Crambides et des Tortricides.

Les $N^{o s}$ indiqués pour les Phalénides sont ceux du Catalogue qui termine le $2^{\mathrm{d}}$ Supplément; je n’ai pas eru devoir les changer encore une fois.

Le nombre des phalènes suisses s'élève actuellement à 363 espèces; celui des Pyralidides et Crambides à 198, et celni des Tortricides à 323 .

Lausanne, Mai 1863. 


\section{5. b. Porrinaria. Zell.}

Ilub. sup. f. 566. - Heyd. Cat. 63.

Var. viridata. Guén. hist. gén. t. IX. p. 347.

Assez fréquente sur toutes les Alpes et à toutes les hauteurs, en juillet. Les pâturages humides.

Les caractìres tirés de la coloration de la còte, des pattes antérieures, du dessous du thorax et du front, destinés à la distinguer de viridaria, me paraissent fort peu sùrs. Plus on s'élève sur les Alpes, plus la teinte verte de l'insecte devient sombre; les tarses des pattes antérieures sont alors noiràtres et le front gris foncé, uè̉é de rougeàtre; la còte presque noire. A mesure que l'on descend, ces teintes foncées disparaissent; dans les sous-alpes et les montagnes basses, le desious du thorax se montre blanchàtre, grisàtre, le front plus fauve et la còte pàle. Enfin, au pied des Alpes, les teintes rouges de ces mèmes parties sont plus ou inoins évidentes.

La taille et les villosités du corps ne signifient rien, parce que, dans toutes les espèces cosmopolites, la taille diminue et les villosités augmentent avec l'altitude.

Jai reçu de M. Mann, de Vienne, des exemplaires de porrinaria parfaitement semblables à ceux collectés dans nos Alpes. Je ne saurais non plus les séparer de viridaria mème comme sariété et je me range à l'opinion de M. Guénće qui reste dans le doute jusquà preuves positives de la validité de l'espèce.

\section{Laevigaria (ta). W. V.}

Hub. 74, 331 .

Renularia. Ilub. 331? - Faune suisse, 2e Suppl. 27. b.

Ayant pu me procurer des individus bien frais de renularia Hb., je me suis convaincu qu'ils ne différaient en rien de sa lacvigata, fig. 74. - Ces deux figures sont du reste assez peu exactes, la fig. 331 est préférable: je pense donc qu'il convient de les réunir définitivement sous une mème dénomination. La couleur jaune nanquin que jarais notéc était le résultat de la rétısté et l'absence d'ombre inédiane un accident.

Jai reçu rette espèce de Meyringen et je l'ai prise dans les environs de Lausanne. 


\section{$-4-$ \\ 18. Bischoffaria. Hub. 586-588.}

Après avoir comparé à plusieurs reprises les fig. de Hubner avee divers exemplaires de cette espèce, j’étais demeuré convaineu que j’avais sous les yeux l'espèce figurée: je l'étais beaucoup moins qu'elle fut réellement distinete d'incanaria var. grisescens. En voyant la teinte enfumée se produire sur des màles, j'en concluais que ces individus devaient ètre séparés d'incanaria, puisque, dit-on, elle ne se montre que ehez la femelle. Tous mes doutes séelaireirent au unois de juin (30) 1858, lorsque je pris, dans une maison de Lausanne, la véritable Bischoffaria que je n'arais pas vue jusques là. Comme elle est voisine d'ineanaria et qu'elle appartient au mème groupe, je dois la caractériser de manière à éviter désormais toute confusion. L'individu pris était heureusement un mâle très frais.

Les figures de Hubner n'en donnent pas nne idée très exacte; en tout eas, elles s'appliquent mieux à elle qu'à ineanaria var. grise. Je n'hésite lonc pas à les rattacher à l'individu que j'ai sous les yeux. La taille et le dessin sont les mèmes dans les deux espèces. Ineanaria varie dans sa teinte, du blanc an blane sâle et au gris de fumée. La femelle est plus souvent foncée que le mâle. Chez elle les teintes elaires de la frange, de la marge, de la ligne fulgurale et de la bande médiane s'obscureissent comme les foncées, en sorte que les individus gris ont une coulenr générale tirant sur le roux qui rend les clairs pen apparents. Les dentelures des trois lignes transverses, marquées de noir sur les nervires, restent en tont cas bien visibles.

Dans Bischoffaria la distribution des teintes est différente. La couleur générale des supérieures est le gris de fer presque noir, sous lequel disparaissent entièrement les dentelures des lignes transverses. Les parties elaires telles que la frange, les points marginaux entre les nervures, la ligne fulgurale et le pourtour des gros points discoïdaux, sont d'un blanc pur très saillant, ceqni morlifie entièrement le facies de l'insecte.

La strueture des antennes et des tarses postérieurs est la mène de part et d'autre.

11 est encore d'antres caractères distinetifs. $1^{\circ}$ Bischoffaria a le front noir, le sinciput blane pur, le collier presque noir et les épaulettes blanches. Le thorax et l'abdomen sont noirâtres et ce dernier est annelé de blane; toutes choses qui n'existent pas dans incanaria. $2^{\circ}$ Les teintes les plus foncées, dlans la $\mathbf{L}^{\mathrm{re}}$, sont placées le long de la eòte, sur la marge des 2 còtés de la ligne fulgurale et à la base de l'aile; dans la $2^{\text {de }}$, la marge seule présente des nuances plus obscures. $3^{\circ}$ Les points blancs placés entre les nervures, sur la marge, sont gros et très saillants dans Bischoffaria; ils 
sont à peine visibles dans sa voisine. $4^{\circ} \mathrm{La}$ frange est très distinctement entrecoupée à sa base de gros points noirs chez celle-là; ils sont à peine visibles sur la variété grise de celle-çi. $5^{\circ}$ Le dessous de Bischoffaria est beaucoup plus elair que dans incanaria var. enfumée, comme on le voit dans la fig. 587 de IIubner. Désormais la confusion ne sera donc plus possible et Bischoffaria occupera enfin la place qui lui revient.

\section{0. b. Litigiosaria. Bdv. Gener. 1899.}

Dup. sup. IV. 54, 5. - Ilub. revis. 30. - Hub. sup. f. 303, 304.

Guén. hist. nat. t. IX. 859. - Heyden. Cat. 642. - Stauding. Cat. Geom. 40.

Nitidularia. Dentsch. Insect.

Je n'ai pris en Suisse que la femelle. Une fois, en aoùt, sur la rive du lac, près Lausanne.

C'est arec raison que Boisd. compare cette espèce à cacspitaria, Bork., car elle ressemble ì la femelle de cette espèce et beaucoup moins à straminaria ou à immutaria. Le sommet plus aigu du antéricures et l'absence de fourcau aux tarses postérieurs, chez le màle, l'en distinguent suffisamment. Ce dernier caractère la rapproche de commutaria. Rare partout et encore confondue souvent avee d'autres espèces. -

Litigiosaria 99. de mes Contributions à la l'aune de Sicile n'est pas celle de Guénée, ni celle que je décris iei. Est-ce bien celle de Rambur? Les points marginaux sont chez elle situés sur la frange et non à sa racine comme chez celle de Boisd.

21. b. Subversaria. Iah.

Jai reçu cette nouvelle espece de Mr. Rothenbar.h. Je n'en ai vu qu'un exemplaire mâle. On ne peut la rapporter à inornata Haw., dont la frange est limitée par un filet (Guén.), ni à maritimata qui n'a de points marginaux que chez la $q$. Voir pour la distinguer à deversaria, seule espèce arec laquelle on puisse la confondre.

๑.). Deversaria. 'Treit. - Guén. hist. gén. t. 9. p. 5.

Depuis la publication du $2^{d}$ Suppl. je n'ai point appris que suffusaria Treit. ait été trouvée en Suisse; mais en revanche Mr. Rothenbach a pris, près de Schüpfen, une 
Acidalia plus voisine de deversaria que de suffusaria, quil n'est point facile de distinguer d'elles et que je nomme subversaria.

La caractéristique de ces 3 espèces est malajsée si l'on se borne au dessin, parce qu'il n'est pas constant. Certaines lignes s'effacent ou s'expriment plus fortement suivant les individus; d'autres s'écartent, se rapprochent ou s'infléchissent un peu dilféremment. La position du point discoidal rélativement à la ligne transverse mediane varie aussi.

Dans les trois espèces les tarses, le front uoir, les antennes et la taille sont semblables.

La couleur des individus frais est différente; suffusaria est de conleur jaunenanquin pàle; deversaria est jaune-paille avee une nuance olive; lespèce de Mr. Rothenbach n'a pas cette dernière teinte.

Les points du centre des ailes sont petits et peu marqués, sur les supérieures. dans deversaria et suffusaria; ils le sont un peu mieux aux inférieures. Dans l'espece nouvelle ils sont gros, très marqués aux 4 ailes en dessous, et triangulaires.

La marge des ailes présente les meilleurs earactères distinctifs. Dans desersaria la base de la frange est marquóe par une séric de petits points noirs placés vis-à-vis des nervures, en delsor's de la ligne de démarcation. Suffusaria noffre pas ces poiuts, mais une ligur de démarcation fine et noire. bien marquée. subversaria porte une série de points alongés, triangulaires et contigus, à la place de la ligne de démarcation.

Guénće vit dans inornata Haw., degeneraria et suffusaria, trois variétés d'une même espèce; je crois quil est dans l'erreur. Les points marginaux peuvent disparaître on se prononcer, mais non se changer en ligne ou en lımules. N'ayant pas iu inornata en nature, je ne puis en parler.

\section{Ochraria. IV. V.}

C'est beaucoup moins de perochrearia quil importe de distinguer ochraria que de rufaria. La taille de la première reste toujours inférieure et les lignes transverses plus ondulées sont aussi plus régulièrement espacíes; la frange est toujours un peu plus foncée que le fond et souvent limitée par une rangée d'atomes noirs.

Le dessin et la couleur d ochraria sont jdentiquement les mèmes que cenx de rufaria. En dessous, ce mème dessin diffère dais les denx espèces; tandis que celui de la seconde est formé de lignes fauses sur un fond clair, semé d'atomes de la mèmo couleur et de quelques points noirs; celui d'ochraria est formée de lignes presque noires et le foud est recouvert d'un grand nombre dlatomes de la mème couleur. 
Les franges diffèrent aussi lans les 2 espèces; chez rufaria elle est limitcel par un trait fauve peu apparent en-dessus et en-dessous. Ce mème trait est brun, très apparent, surtout en-dessous, et formé de lignes placées bout à bout, séparées par les nervures, dans lautre espèce. Les individus bien dessinés d'ochraria présentent quelques points bruns sur les franges, surtout aux inférieures en-ilessous, que rufaria ne porte jamais. Nais le caraetère essentiel se trouve dans les antennes du màle; rufaria les a à peine denticulées sur les bords et pubeseentes en-dessous; tandis que eclles d'ochraria sont fortement ciliées, ont les tubercules bien séparés et portent un poil brun à chaque artienlation ou dentelure du bord.

31. b. Antiquaria. Her.-Schf. Hub. revis. $4 \%$.

Hub. sup. f. 340,341 б - IJeydenr. Cat. 669.

Sylvestraria. Dup. hist. 17t. 8, (non 7). - Guén. hist. génér. t. 9. p. 452. et Gallorum.

q Macilentaria? Ramb. faun. Andal. Bdr. 1876. - Juls. sup. f. 230, 231.

Jai recenilli cette espèce à Rovéréaz, près Lausanne, dans les près, au milieu des bois, an mois de juin 1859. La femelle y est moins fréquente que le màle. Le papillon vole dans lherbe et se suspend aux ehaumes. Macilentaria Ramb. appartientelle réellement à la femelle? e’est ce que je ne puis décider sur ume figure, elhez des espèces aussi difficiles à distinguer. Voir à sylvestraria an sujet de la confusion qui existe aree notre espèce.

Antiquaria ressemble pour la taille, le facies et le dessin, à remutaria; son aspect soyenx est plus prononé encore; mais les lignes transverses sont plus nombreuses et mieux maripuées et le bord des postérieures porte une légère échancrure là où renıtaria offre un angle saillant. Cette espèce étant encore pen connue en Suisse 't en Allemagne et confondue arec d'autres, il consient d'en donner une description complète.

La couleur générale est le jaune-paille pàle, très sali par des atomes noiràtres et par 4-5 lignes transverses d'un jaune-nanquin. La couleur des lignes se répand sur le disijue le long de la eòte. Les atomes noir's sont dispersés sur toute la surface des ailes, mais plus nombrenx sur les lignes.

Ailes antérieures. Còte légèrement arquée, surtout vers le sommet. Celui-ci un peu aigu. Bord externe presque droit, sarrondissant à langle abdominal. 5 lignes 
transverses bien marquées: la $1^{\text {re }}$, à partir de la racine de l'aile, est arquée; la $2^{\text {de }}$ plus large, est plus ou moins ondulée et droite; la $3^{\mathrm{e}}$ parallèlle à la précédente est généralement plus saillante et plus ondulée; la $4^{\mathrm{e}}$ est de rechef plus large, surtout du côté de la còte, droite et ondulée; l'espace clair qui la sépare de la $3^{\mathrm{e}}$ est étroit; la 5e, séparée de la précédente par une ligne claire formée de lunules, est peu prononcée. 8 points noirs, souvent effacés, sont placés sur la base de la frange, à l'extrémité des nervures. La frange unie et de la couleur du fond, est séparée de lui par un trait noiràtre très fin et parfois imperceptible; ce trait unit les points noirs les uns aux antres par autant de petits ares.

Le dessous des antérieures reproduit le dessin du dessus, mais très modifié. Sur un fond gris-charbonné, on observe: $1^{\circ}$ Un espace gris uni occupant la racine de laile jusques à son milieu. Un petit point noir, invisible en-dessus, se place vers le bord externe de eet espace. $2^{\circ}$ Une large bande moyenne plus claire. $3^{\circ}$ Un trait noirittre, bien marqué, dentelé en dehor's, sur chaque nervure. $4^{\circ}$ Une ombre transierse claire, puis une charbonnée. $5^{\circ}$ Une ligne de lunules claires, se détachant sur l'extrémité noircie de l'aile. Les nervures sont dessinées en brun; la frange claire et luisante contraste avec le fond chargé datomes noirs. Un trait noir, portant les points noirs du dessus, sépare la frange du disque.

Ailes postérieures semblables aux antérieures; arec les lignes transverses continuant celles déjà décrites à l'exception de la $1^{\text {re }}$ qui manque. Un très petit point discoidal sur l'espace basilaire, très près de la $2^{\text {e }}$ ligne. Bord externe portant un léger sinus en face de la cellule. Frange comme aux antérieures.

Le dessous des postérieures continne le dessin des antérieures et n'en différe que par l'espace basilaire moins charbonné, par la $3^{e}$ ligne très sinueuse et par l'espace clair, médian, plus large. Le bord est bien marqué par un trait foncé, angulenx.

Palpes très courts, fauses à l'extrémité; front lause; collier de mème couleur. Antennes du màle faures, unies et luisantes en-dessus, garnies en-dessous d'une double rangée de forts cils, disposés deux à deux, noirs à la base et divisés ell pincean transparent à leur sommet. Tubercules gros, détachés, saillants et ciliés. --

Thorax fauve clair; abdomen uni, blanc-jaunâtre; anus plus clair, dépassant le bord des postérieures. - Ventre légèrement fauve; pattes noirâtres en-dessous; tarses de la dernière paire sans renflement, ni foureau, ni pinceau de poils; armés d'une paire de forts éperons.

La femelle est un peu plus petite que le màle, porte les ailes antérieures un peu plus pointues et les postérieures plus étroites. 
Par l'absence de foureau aux tarses des postéricures cette espèce se rapproche de commutaria, mais elle s'en distingue à d'autres égards.

Mr. Guénée lui conserve la dénomination de Duponchel quoiqu'elle repose sur une erreur. Loin d'éclaircir par là la synonimie on l'embrouille encore davantage, ainsi que eelle de sylrestraria Bork. actuellement fixée définitivement.

3:. b. Tulpinaria. Mann. Cat.

Hub. sup, f. 473,474 . -

N'est point une variété de rusticaria et doit prendre rang parmi les espèces suisses. Les tibias des pattes postérieures portent 2 éperons dans l'un et l'autre sexe, ce qui n'a pas lieu dans rusticaria. (Iler.-Sehf.)

33. Confinaria. F. V. R.

Lisez Confirmaria, et Ileyden. Cat. 693.

34. Commutaria. Treit. sup.

Ajoutez encore aux synonimes.

Simplaria. Fr. n. b. 594, む 1, \% 2.

Nitidaria. Bdv. 1904. (Guén.)

Gypsaria, Bdv. 1905. (Guén.)

3S. Sylrestraria. Bork. - Treit.

Innutata. Guén. hist. gén. 9. p. 498.

La fig. 97 de Ilubner reproduit mieux la syliestraria de Dup. prar ses bords externes droits que eelle de Itub. sup.; cependant elle porte les 4 points discoidaux qui nexistent pas dans l'autre. Sa fig. 94 est straminaria: voir dans Treitschke vol. 6, $2^{\text {e }}$ p., p. 30 i le dédale créé par Huhner sur ce point.

La description de Borkhausen n'est pas evacte à part ce qu'il dit des 4 points et de langle obtus du bord postérieur des inférieures. Les stries ne sont pas presque lroites; du reste il n'a vu (pu'un exemplaire.

Treitselıke déerit mieux que Bork. la rraie sylvestraria. Les fig. 103-105 de Hub. sup. sont très exactes, il faut done s'en tenir à elles et à la description de Treitsehke, et ne pas retouner en arrière aux descriptions incomplettes et si sousent contestées des anciens auteurs. 
Quant à la fig. 8 pl. 177 de Duponchel j'ai pu m'assurer qu'elle appartient à antıquaria. Her.-Schf. (II. S. f. 340, 341), espèce assez répandue en France et que j'ai aussi recueillie dans nos environs. Des individus reçus de MIVrs. Bruand et Bellier-de-la Chavignerie, comme appartenant à la sylvestraria de Dup., ne laissent aucun doute à cet égard. Mr. Her.-Schäffer a d'ailleurs constaté qu'ils appartenaient bien à son antiquaria (roir cette dernière espèce).

\section{Caricaria. Hub. sup. f. 553, 554 .}

Depuis la publication du 2e Suppl. aux Phalénides jai recueilli dans les marais de la vallée dAigle, à la fin de juin et en juillet, plusicurs individus appartenant à cette espèce. Elle y vole parmi les jones et se pose anssi sur les arbustes bas. Les caractères qu’elle présente dans cette dernière localité différant à quelques égards de ceux que m'avaient offerts les individus de Zurich, je dois modifier ma deseription.

La blancheur des ailes supérieures, dans l'état frais, n'est pas parfaite, car elles sont saupoudrées légèrement d'atomes noirs très petits, dispersés sur la moitié externe du limbe et surtout sur les 4 rayes transverses. Celfes-ei ne sont cependant point produites par l'accumulation de ces atomes, car elles sont brunàtres. Les points du disque sont généralement visibles aux inférieures; tandis qu'ils sont petits aux supérieures où ils manquent souvent.

Le dessous des ailes supérieures à la base, et mème sur le disque, est souvent enfumé au point de masquer entièrement le point central.

La frange est large, luisante, sans points ni lignes, avec un léger reflet jaunàtre.

Les rayes transverses, ordinairement peu marquées, sont jaunâtre ou brunìtres: la $3^{\text {e }}$ (à partir de la base) est parfois noire et alors très marquée dessous et dessus.

La femelle est volontiers un peu plus petite que le màle et d'un blanc de lait plus pur.

\section{Cerusaria. Lah.}

Depunctata, Guén. hist. gen. 9. p. 500.

La dénomination de Guénée pourrait ètre adoptée définitivement avec avantage. 
49 b. Imitaria. Hub. 51.

Treit. - Dup. - Hub. revis. 73. - Heyden. Cat. 638.

Jai pris cette espèce méridionale près de Lugano, appliquée sur les rochers, en eptembre ( $2^{e}$ génération).

50. Aureolaria. Fab.

Je dois à l'obligeance de Mr. Bellier de-la-Chavignerie quelques exemplaires de cette espece provenant de Fontainebleau et qu'il m'affirme ètre la flaveolaria de Bdv. Il faut done ajouter aux synonimes d'aureolaria.

Flaveolaria, Bds. $N^{\circ} 1861$

et le tracer an $\lambda^{\circ} 49$ de la fanne suisse.

51. Flaveolaria. Hub.

La femelle est un pen plus petite que le màle, avec les antérieures plus pâles et plus aigues au sommet. Vole peu et lourdement; reste rare.

52. Auroraria. Hub.

J'ai collecté cette espèce dans les marais de la vallée d'Aigle en juillet 1855.

60. Schaefferaria. Lah. Faun. helv.

Subpunctaria? llub. sup. f. 415.

Cette espèce est indiquée dans Hub. sup. Nachträge (p. 135), sous le nom de ruficiliaria. Guénée ayant désigné une espèce du Canada, voisine d'albicillaria, par le mème nom, la désignation de Her.-Schf. ne pouvait subsister. Plus tard ce dernier adoptant ma cerusaria (Acidalia) a transporté, je crois, le nom de subpunctaria, qu'il avait donné à celle-ci (fig. 311-313), à ma Schaefferaria. - Gyraria, Treit. n'est pas notre espèce.

61. Pupillaria. Hub. 69.

Gyraria. llub. 434.

Se montre sans nul doute dans la Suisse transalpine: essentiellement méditerranéenne. Je rapporte gyraria, Huls. 434 (non 543) à cette espèce, après avoir fait de vains 
efforts pour l'en sépares spécifiquement. La couleur des atomes et du fond, les points centraux et ceux de la marge, la teinte de la frange, la ligne ponctuée, langle médian des postérienres, offrent des variations, comme le fait d'ailleurs remarquer Guénée.

Quant à Schaefferaria elle ne peut se rapporter à aucune figure de Ifubner ou de Duponchel et doit rester distincte. Je regrette que Mr. Guénće ne laie pas vue.

\section{Petraria. Esp.}

Bois de Sauvabelin en juin; ahondante au milieu des arbustes d'une clairière marécageuse, entourée le chènes et de hètres.

\section{Ulmaria. Fab.}

Prise dans les sous-Alpes vaudoises, en juin.

\section{Capreolaria. W. V.}

Dans les Apes l'écelosion d'automne est parfois retardée jusqu'au printems et linsecte parfait apparait en juin.

\section{Serotinaria. Hub.}

Ajoutez aux synonimes.

Freyer n. 1. เ. 600 . f. 2.

Varie du gris brun, entremôlé de fanne, au janne sàle tacheté de gris. - On peut la confontre avec dilucidaria très chargée d'atomes foncés. L'absence totale de faune sur les ailes, le sommet un pen arondi des supérieures, le manque d'atomes en dessous et de festons à la marge des inférieures, chez cette dernière, serviront à les distinguer.

11\%. Mendicaria. Hub. sup. f. $491-493$.

$$
\text { Dilucularia, Kerferst. (non Frey. n. b. t. } 570,1,2 . \text { ) }
$$

Les antennes sont d'un gris-pàle endessus; le front est brun. La ligne de points das inférieures forme une courbe uniforme. Les lignes transserses sont plus espacées que chez dilucidaria. Le fond des ailes est blanc enfumé. L'ombre antémarginale est nulle ou peu marquée. 


\section{Dilucidaria. W. V.}

IJub. sup. f. 494, 495.

Ajoutez à la description: Antennes blanches au dos. Front blanc. Fond constamment de couleur blane-blenàtre. Ligne de points des postérieures formant un angle à son milieu. Onbre anténarginale presque toujours lien marquée, surtout chez le mâle.

119. Spurcaria. Fam. helv.

$$
\text { Caelebaria. II. S. f. 421, } 507 .
$$

M. R. Zeller una fait voir un exemplaire de la caelebaria, provenant du Tyrol. qui se rapporte évidemment à ma spurcaria. On adoptera la dénomination que I'on préférera, peu mimporte. Cette espèce est très roisine d'operaria. Lorsque je la rapprochais de Zelleraria je n'avais vu que la femelle de celle-ci; le màle lui assigne une tout autre place.

\section{Zelleraria. Frey.}

Jai pu voir deux individus, màle et femelle, provenant de la haute Engadine. Cette espèce forme avec Andereggaria un petit grompe distinct.

Le mâle est beaucoup plus grand que la femelle, à per près comme furraria. Il est uniformément gris perlé avec deux rayes rapprochées, presque parallèlles et un point discoïdal, peu visible, entr'elles. La raje externe forme un angle aigu, vif, sous la còte; elle est à peine dentelée. Les inférieures sont partagées par une raye dentelée, 2 fois recourbée. Une lunule discoidale. Corselet recouvert de longs poils blanchàtres.

\section{Operaria. Hub.}

Cette espèce est réellement suisse. M. Zeller l'a prise dans les hantes alpes d'Engelberg et de IEngadine; Mr. Rothenbach près de Langnau. Jai collecté la femelle sur le mont Joly, en juillet, audessus de St. Gervais (Faucigny) à $7000^{\prime}$; elle porte des rudimens d'aile marqués de 2 bandes transverses noirâtres. -

Le front est gris-roux. Les barbillons des antennes sont spatulés, ainsi que chez celebaria qui en est voisine. 


\section{Pullaria. Hub.}

Ajoutez aux synonimes:

flub. sub. f. 500,501 \&. -

On rencontre dans le Jura des individus presque entièrement blanc-jaunàtres et sans dessin.

124. Meyeraria. Lah. Faune suisse.

A mbiguaria. Faun. suis. $2^{\text {e }}$ Suppl.

Ayant pu me procurer 2 individus très frais l'un ó, pris à Lavey à la fin de Mai 1857, l'autre $q$, capturé à Meyringen, en juin mème année, j’ai soumis cette espèce à un nouvel examen. Mr. R. Zeller l'a collectée sur les Alpes du Celerina. La fig. pl. 186, de Dup. offre quelque analogie de teinte et de dessin; mais elle est trop peu exacte pour servir de point de départ. Les fig. 379,380 et 381 de llub. sup., beaucoup plus exactes, ne peuvent en aucune façon lui ètre apliquées; ainsi done Meyeraria doit subsister distincte d'ambiguaria, Hub. sup.

Voici du reste quels sont les caractères distinctifs de Meyeraria. Elle ressemble plus à mendicaria qu'à dilucidaria; mais les antennes du mâle sont filiformes. Sa teinte grise n'a pas l'aspect bleuàtre et luisant de la $2^{\text {de }}$, ni le pointillé grossier et brun de la $1^{\text {re }}$. La couleur du fond est le blane sâle fortement recouvert par un pointillé gris-lle-fer. Dans l'un des màles que j’ai sous les yeux la teinte générale est formée de la réunion de trois pointillés, un blanc pur, un gris clair et un gris foncé, outre le pointillé noir des stries et des taches.

Les antennes, chez le mâle, sont filiformes, sans cils ou villosités, et portent des tubercules presque carrés et bien distincts les uns des autres. Les tarses postérieurs, dans le mème sexe, sont courts et très renllés. Le front est gris; de la couleur de la tète. Les 4 taches oculées dı disque sont grosses et bien dessinées. Les points marginaux sont petits. Le dessous est d'un gris roux, finement pointillé; enfumé parfois aux antérieures.

La femelle ne diffère du mâle que par ses teintes un peu plus claires.

La figure de la faune suisse est mauvaise ei ressemble plus à dilucidaria $\$$, qu’à Meyeraria (Collection R. Zeller.) 


\section{Glaucinaria. Hub.}

Freyer. n. B. 593. f. 2 et 3.

Le fond est quelque fois d'un beau jaune paille.

128. Variegaria (ta). Dup. t. 184. f. 4 (non- 185, f. 5).

llub. sup. f. 503, 504. - Heydenr. Cat. 137. - Guén. hist. t. $9 . N^{\circ} .473$.

Mucidata. Frey. ii. Beitr. 125, 3. - Faun. suisse. $2^{\text {e }}$ Suppl.

Confondue jusqu'ici avee mucidaria Ilub. 148. - Ilub. sup. f. 502. - Cette espèce toujours rare, a été prise par Mr. Wullschlegel dans les environs d'Olten. Je l'ai aussi reçue de Sicile.

Elle se distingue de mucidaria par plusienrs caractères; mais il sulfit de mentionner les antennes filiformes, non pectinées, chez le màle. Les tubercules de l'antenne sont étroits, alongés en còne, rapprochés les uns des autres et recouverts d'un duret fin.

\section{Crepuseularia. W. V.}

$$
\text { Defessaria. Fr. n. B. t. } 510 \text {, f. } 1 \text {, }
$$

n’est quiune mauraise variété de cette espèce.

\section{Strigillaria. Esp.}

Cette espèce est bien suisse; je l'ai prise le $10^{\mathrm{e}}$ juin 1858, au pied des montagnes d'Ollon (Vallée d'Aigle) dans les taillis. Mr. Meyer l'a récoltée à le mème époque près de Viège. La var. brune (Hub. G. 540, 541) l'a été par Mr. Wullschlegel dans les environs d'olten.

166. Hipocastanaria. Hub.

Mr. Rothenbach a collecté plusieurs fois cette espèce dans les environs de Schüpfen, du 20 mai jusqu'en septembre. 


\section{Bombycaria. Bdv.}

Cette espèce existe aussi dans la haute Engadine; Mr. Hnateck, de Sils, l'élève chaque année.

Le corps de la femelle est revètu d'un duret noir et de longs poils blanes. Les rudimens d'ailes blanes, sont particulièrement relus.

\section{Venetiaria. Hub.}

\& Hub. sup. f. 444 .

\section{4. 'Torvaria. Hub.}

llorridaria, Hub. 149 n'est pas une $q$, mais un t (Treit.). - La femelle est rare et a les supérieures encore plus arondies au sommet que ne les représente la fig. 368 de Hlub.

L'angle de la $2^{\text {d }}$ ligne transrerse n'existe pas toujours quoiqu'en dise Treitsehke.

\section{4. b. Alticolaria. Man. Cat. 1856.1)}

Guén. hist. génér. IX. p. 320.

Cette espèce, fort rare, déconterte pour la première fois en Tyrol par Mr. Mann, a été récoltée dans les Alpes, an-dessus de Meyringen par Mr. J. Ott, auquel nous derons plusieurs espèces rares ou nouvelles. Je n'ai vu que le màle.

Son lacies et sa couleur sont ceux de Torvaria, et non de trepidaria, arec laquelle elle a peut-ètre été confondue. Sa taille est un peu inférieure. Le noir foncé des quatre ailes a un éclat soyeux. La còte des supérieures est fortement arquée à sa base. Les lignes transterses sont an nombre de trois et se dessinent en noir mat; la première (dìs la base) est arquée, la seconde rapprochée de la précédente, forme denx coudes, l'm vers son milieu, l'autre vers le bord postérieur; entrelles existe un point

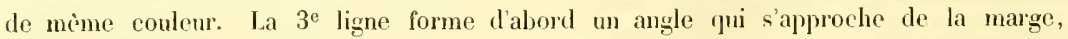
puis deux ondulations jusqu'a l'angle abdominal où elle se termine. La frange des supérieures est limitée par un trait foncé et par trois on quatre taches chatoyantes vers le sommet.

Les inferrieures portent deux bandes chatoyantes qui occupent la moitié de l'aile

1) Voir le Bultetin de la Société vaudoise des sciences naturelles. T. V, No . 40. p. 228. 
et sont séparées par une bande d'un noir mat, ondulée. La marge est limitée par un trait noir, brisé, dessinant un sinus en face de la cellule (comme dans les Gnophos). La frange est d'un noir fuligineux.

Le dessous des 4 ailes est d'un noir luisant. Un gros point existe sur la cellulle des antérieures. La marge des 4 ailes est borlée par une large bande jaunitre, bien tranchée, bordée de noir foncé du côté interne et divisée en avant par un trait noir qui s'évanouit en filet et vient toncher la marge en face de la cellule. Le trait noir qui limite les franges est ici très marqué.

Les cuisses, le corps, les palpes et la tête sont couverts de longs poils noirs. Les antennes sont liliformes, noires.

Cette espèce intermédiaire entre les Gnophos et les Psodos, genres très voisins, pourrait motiver une fusion les deux.

181. Plumularia. Frey.

Rheticaria. Faune suisse.

Je restitue à cette espèce son nom primitif puisque M. Lederer et Guénée ont modifié celui de Boisduval ( $\mathrm{Nr}$. 1940) et l'ont remplacé par coneoloraria.

Jai reç de la baute Engadine cette espèce, lien distincte de purpuraria.

Ajoutez aux synonymes :

Hıb. Sup. f. 475-477. - Guén. hist. génér. t. 10, p. 173.

188. Erutaria. Bdv.

N'est pas très rare dans nos Alpes: Au Val-d'lliers en juillet; au Creux-de-Champ, vallée d'Ormont dessus, mi-juillet; dans les clairières des forìts de sapin. l'ai pris deux fois des individus màles sur lesquels les ailes inférieures n'existaient pas. Il faut ajouter anx symonymes.

Nebulosaria. Heydenr. Cat. 362.

Cambricaria. Curt. - Leder. - Guén. hist. génér. t. 9, p. 440.

196. Succenturiaria. Lin.

J'ai regu de Mr. J, Ott à Meyringen la variété brune figurée par Hubner (f. 247), ainsi que la variété grise non figurée dans Hübner. 
19S. Modicaria. Hub.

Ajoutez aux synonymes:

Semigrapharia, Bruand, Cat. du Doubs.

Jai reç, sous ce nom de Mr. Brwaud, une variété plas pàle et plus jaunàtre, figuréc au Nr. 537 de Ilubn. suppl. $1 \mathrm{mp}$ urata, Ihuln. 347, reçe aussi de France et de Sicile.

206. Obrutaria. Fisch. v. Rösl. Collection.

IIuls. Sup. f. $145,146$.

Pimpinellata, IIub. coll.

Piperata. Wood.?

Majoraria. Faune suisse, Nr. 1956, $2^{\text {me }}$ suppl. f. 2.

II. Jäggi, de Berne, l'a prive dans les environs des bains du Gurnigel, en juin.

Je nhésite pas à réunir cette enpèce à eelle que jai décrite sous le nom de majoraria, quoique les exemplaires un peu altérés) recus de Mr. Herrich-Schäffer m'aient dabord conduit à les sćparer.

Obrutaria, majoraria et arecutharia sont peut-ète, autant de variétés de la mème espèce; l'rumen de la question exige des individus très frais et nombreux. Mr. Staudinger rémit helveticaria à areeutharia: je ne puis partager cette opinion. Ilelvelicaria, reçe de Andereggr, a les antérieures plus allongées en forme damande, et les postérieures à peu près dépourvues de desin.

\section{8 b. Distinctaria H. S. f. $162,163$.}

Javais admis eette espèce, dans mon $1^{\text {er }}$ suppléntent, au nombre des e-pèces suisses. Plus tard, métant convaincu que les individus auxyuels je donnais ce nom appartenaient a cast igaria, jai dì les retrancher du Catalogue. Naintenant un nouvel exemplaire, ‘ue je tiens de Mr. Ott a Mejringen, exige que je rétablisse eette espece. C'est d'ailleurs l'avis de Mr. Herrich-Sehıiffer, qui possède des échantillons semblables venus de Laponie.

Lindivilu femelle que jai sous les yeux se rapproche extrèmement de castigaria; aussi me contenterai-je de signaler les caracteres qui l'en distinguent. $1^{\circ}$ La teinte générale est le gris pur, ardoisé sur les clairs, sans mélange de fance comme chez castigaria. $2^{\circ}$ Les 4 points discoïdaux sont fornés par un trait noir, gros aux supérieures, petit aux inférienres. 3 La moitié interne de l'espace marginal, élargie à la côte', se rétrécit brusquement el depuis la cellule ne forme plus qu'un étroit ruban. - - ${ }^{\circ}$ Une 
dent noire à la còte au-devant du point discoüdal. 5 Hoitié interne de laile à peine dessincee, sablée de gris. $6^{\circ}$ Dessous phus clair, sans teintes faures.

213. Valerianaria. Hub. 395.

Doit ètre conservée comme espèce. Un individu recueilli à Meyringen par Mr. J. Ott réproduit parfaitement la fig. 395 de Hubn. el ne peut être confondu avec Begrandaria.

213 b. Laqueraria. Fisch. v. R.

Ilub. révis. Nir. 67. - Hub. sup. f. 181, 182. - Heydenr. Cat. Nr. 511 .

Subumbrata. Treit.

Intricata, Treil. in litt - Fisch. V. R. (olim).

Facies de pusillaria. - Les vergers près de Schupfen (Rothb.).

23:3. Seripturaria. W. V.

Placidaria. Frey. N. B. 600. 3.

239 b. Lapidaria. Hub. 324.

Guén. II. 435. - Ilub. sup. ^. 286, 287.

Trouvée une fois dans les environs dOlten, sur le Jura. par Mr. Wullschlegel!

250 b. Zumsteinaria. Lah. Mittheil. der schweiz. entom. Gesellsch. Nr. 1. p. 24.

Cette espèee, entièrement nouvelle, a été découverte par Mr. Meyer-Dur, dans les environs de Viège (Haut-Valais), du 30 mai au 20 juin I 861.

Je ne puis réproduire ici la description que jen ai donnéc dans les a Mit theilungen " de la Société suisse d'entomologie.

Liespèce dont elle se rapproche le plus me paraît être aptaria, quoique son colorit et son aspeet général soient fort differrens. 


\section{Arctaria. Lien.}

Albimacularia. Frey. n. b. 534, 5.

Toniata. Steph. - Guén. hist. génér. t. 10. p. 293.

Assez fréquente aux Plans (Alpes de Bex) dans les bois de sapin des Sous-Alpes, en juillet.

La femelle est ordinairement un peu plus grande que le màle; celui-ci porte à ranus un mouchet de poils bruns. Sur quelques individus la bande moyenne s'élargit en dedans par la coloration en brun d'une bandelette transierse, limitée elle-mème par une ligne grise qui fait partie du premier ruban. Dans ce cas le point discoïdal se place au milieu de la bande moyenne et non sur son bord. Ces individus ressemblent à spadicearia.

255. Laetaria. Lah. Faune suisse.

Hub. sup. f. 555 .

La figure de Ilub. sup. est trop pàle. Sur les individus frais la marge a une teinte verte beaucoup plus prononcée et la bande moyenne est plus noire. La figure que j’ai donnée sous le nom de Kollariaria est plus exacte, quoique ses teintes soient un peu trop tranchées. - Les basses Alpes, en juin.

266. Minoraria. Treit.

Une variété recueillie dans les Alpes passerait aisément pour une espèce nouvelle, tant elle differe des individus ordinaires. Chez elle les deux rubans blanes sont partagés par un trait gris prononcé, et ombrés de fauve. La bande mojenne est fortement tachée de gris et de noir. Trois dents de la ligne fulgurale sont marquées en noir, en face te la cellule. Les postérieures sont très enfumées. Il résulte de cette augmentation de teintes foncées un aspect sombre très différent du facies ordinaire.

2\%. Funeraria. Hub. 260.

Ajoutez aux symonymes:

Ilub. Sup. (Nachträge) f. 542.

Cette figure est trop couverte de noir.

Mr. Guénée fait erreur lorsqu'il réunit funeraria à Iristata Lin. Funeraria 
(H. S. f. 542) est commune sur nos Alpes et n'est point nouvellement trouvée; elle n'était que confondue aree tristata. La distinction des deux espèces est par trop aisée. Celle de hastata et de hastulata l'est moins, quoique tout aussi assurée, si l'on a de bons exemplaires sous les yeux.

281. Decoloraria. Hub.

Frey. n. b. $600,1$.

Consentaria. Frey. n. b. 486.

282. Hydraria. F. v. R.

Mr. Wullselılegel la aussi collectée prés d Olten.

286. Lotaria. Bdv.

Ajoutez aux synonymes:

Hub. Sup. I. 455. ‡.

Le sommet de l'aile est trop obtus dans cette figure.

287. Ablutaria. Bdv. Index Nr. 1626.

ILub. Sup. f. 382. 383. -- Dnp. Cat. - Zell. Ent. Zeit. 1849. p. 212. Olivaria. Dup. hist. pl. 183 (non ILubn.).

Salicata. Guén. hist. gén. t. 10. p. 283 (non Hub. Sup.).

q Muscosata. Leder. (non Donzel). - Ileydenr. Cat. 383.

Ferraria? Ilub. Sup. f. 398. - Hub. revis. Nachtr. - Heydenr. Cat. 372.

q Il ispalata? Ramb. Catal. pl. XXII. C. 7.

Podevinaria Hub. revis. Nr. 136. - Hub. Sup. f. 250. - Heydenr. Cat. 382.

Bullet. de la Soc. vaud. des scienc. nat. Nr. 40. p. 227.

Afin de faire disparaittre la confusion qui règne sur l'une des espèees les plus répandues en Europe, il importe tout dabord de revenir à la dénomination de Boisduval à laquelle Dup. rapporte la figure très reconnaissable qu'il en donne sous le nom d'Olivaria. Je l'ai reçue d'Autriche comme muscosata, Leder., de France sous le 
nom dablutaria, Bdv., de Mr. Herrich-Schäfer lui-mème sous celui de Podevinaria: de Palerme et des Alpes, etc, conservant partout ses earactères distinctifs, très reconnaissables et foumissant les mèmes variations. Si l'on veut établir deux variétés malgrí les nombreuses transitions qui existent, il faut prendre celle ou lo gris foncé domine (ferraria) pour l'une et muscosaria, Leder. pour celle qui est tointe en jaune, mais cutte demiere ne comprendra gnères que des femelles.

Aux caractires tirés des antennes et de la marge, on peut joindre celui que donne le pointillé blane, éparpillé sur les nervures et visible surtout sur le deuxième ruban. en dehors de la bande moyenne.

Mr. Guénce rapporte à ablutaria, Bdr. I'infidaria, Lah. faune suisse (llavicinctata, Dup. hist.), qui en est très différente; puis il ra'tache Podevinaria, II.S. à salicata, W. V. (qui n'est point celle de IIub. sup.), anci que ferraria, II. S. De la sorte, lobscurité s'accroit au lien de diminuer. - Pourouoi toujours ressusciter la salic ata de IIubn. que chacun conçoit ì sa maniere?

\section{Nobiliaria. Hub. Sup.}

Les individus provenant d'Autriche et figurés par II. S. sont généralement plus pàles due ceux de IEngadine: ceux-ci sont fortement teintés de couleur olive, mélangée de jaune et de brun. Chez eux les postérienres sont quelquefois presque entièrement noiràtres, awe la frange jaunàtre, bien entrecoupée. Le point discoïdal des supérieures. lorsqu'il existe, est jaune-safran.

29:. Caesiaria. Hub.

Cette espèce, ne varie en Suisse que dans certaines limites. Je n'ai pas encore pu trouver le passages entre elle et llavicinctaria, Hub. Les quelques ombres safranées que l'on observe sur certains individus restent loin du jaune orangé vif de cette dernière Il existe d'ailleurs des dilférences constantes, telles sont: $1^{\circ}$ la teinte blanchàtre, uniforme, des postérieures en-dessus, sur laquelle se détache en gris un point discoüdal bien marqué, deux lignes marginales, ordinairenent à peine visibles et une légère ombre grisàtre sur la marge, dans quelques individus. $2^{\circ}$ En-dessous, les antérieures sont constamment cufumćes, avec deux taches jaunàtres allongées à la còte et la bancle moyenne parfois désignée par un trait externe. $3^{3}$ Les postérieures, du mème còté. sont blanchàtres, pointillées de gris, et du reste semblables à lautre face. $4^{\circ}$ Les 4 points discoidaux bien marqués. 
J'ai reçu de M. Wocke une fllavicinctaria, IIub. provenant des Alpes de Norrège yui n'est autre que la varićté jaunàtre de caesiaria et en reste bien distincte.

\section{9:3. b. Annosaria (ta). Ketterst. f. lap.}

Je ne posiéle yuiun seul individu de cette remarquable espèce; il a été pris dans lOberland par Mr. J. Ott. Je l'ai rapporté à l'espèce de Zettersted sur l'autorité de Mr. Staudinger.

Son dessin, sa configuration et la coupe de ses ailes la rapprochent de caesiaria. Elle sen distingue au premier coup-d'oei] par la teinte noire générale des antérienres, sur laquelle se drssinent finement en blane pur: $1^{\circ}$ quelques petits points placés sur la racine des nervures dans l'espace basilaire, sans s'éteulre sur la bande médiane, yu ils délimitent à son bord interne; $2^{\circ}$ deux lignes de points placés aussi sur les nervures et indiquant is la fois la limite externe de la bande médiane et la place du deuxième ruban; $3^{\circ}$ la ligne fulgurale formée de cherons aigus clans sa moitio antérieure, de lumules at de points dans sa moitié postérieure; $4^{\circ}$ quelques traits ef points situés sur l'extrénité des nervuren. La frange est blanchàtre, divisée par ume ligne noiràtre et entrecoupée par de petites taches de cette dernière couleur, au niveau des nerrures.

Les mèmes ailes, en-dessous, sont d'un gris noiràtre aree deux taches blanchàtres à la còte vers le sommet, les chevrons de la ligne fulgurale faiblement indiqués, l'extrémité blanche des nervures et li frange d’un blane sâle, entrecoupée de points noirs.

Les postéricures sont gris de fer en-dessus, plus foncées du còté interne; traversées, au-delì de leur milieu, par deux bandes maculaires blinchàtres, parallèes à la marge, dont la postérieure est plus prononcée. Un gros point discoïdal gris est rapproché de la racine do l'aile. Nervures marjuées en blanc tout le long de la marge. Une ligne noire, entrecompé par les nervures, limite la frange. Celie-ci est semblable à celle des superieures; mais arec une ligne de division à peine indiupée. Bord alulominal noir marpué de deux points blanes rers sa terminaison. En-dessous les mènes ailes ont l'espace basilaire gris de fer, marqué d'un point discoüdal: eet espace est limité par un trait plus foncé qui le sépare diune bande post-médian, lavée de clair en dedans, ombrée de gris en delors. L'espace marginal est blanchitre, de la couleur de la frange: la ligne de points noirs et les intersections de la lrange y sont prononcées.

Antennes filiformes, annelées de blane. Paljes courts, noirs nn avant, blant-jaunàtres en dessous. Gorge blanche. Front noir foncé. Double collier jaunàtre. Corselet noir, semé de poils blancs, surtont en arrière. Abdomen noir, annelé dle blanc en-des- 
sns; jaunâtre en-dessous. Pattes noires en-dessus, blanc-jaunàtres en-dessous. Mouchet anal noir; jaunàtre à son extrémité.

Cette phalène est tellement différente de caesiaria par sa couleur noire mouchetée de blanc pur que je n'hésite pas à la considérer comme une espèce distincte: elle se rapproche d'ailleurs davantage, par plnsieurs caractères, de llavicinctaria que de caesiaria.

Mr. Staudinger de Dresde m'affirme que dans le nord caesiaria varie (ce qui n'a pas lieu dans nos Alpes) au point de fournir des individus entièrement blancs et d'autres presque noirs. Ce fait extraordinaire mérite un examen plus attentif. L'on sait assez que les climats froids disposent les Lépidoptères à revètir des couleurs foncées. Dans le nord verrait-on chez ces insectes tantòt la prédominance du noir, tantòt celle du blane? Cela serait possible. Dane ce cas il faudrait attribuer ces modifications à des causes différentes et voir si, peut-ètre, le noir est le résultat des longs hivers et le blanc des longs jours d'été agissant sur les larves. Si cela est, les variétés noires et les variétés blanches du nord doivent apparaitre les unes an commencement, les autres à la fin de la saison 1).

\section{Flavicinctaria. Hub. 354 .}

Frey. n. B. fig. 504 et larva.

La figure de Ilubner est assez exacte, à part, le milieu de la bande médiane trop clair, le point discoïdal des antérieures trop prononcé et celui des postérieures rarement visible et dans ce cas-là très peu marqué.

Il est fort difficile de distinguer cette espèce de caesiaria, et cependant la différence de moeurs trahit une différence spécifipue -; la première fort commuse sur la zône moyenne des Alpes, dans le voisinage des sapins, se pose toujours contre les rochers et dans les cavernes; la seconde, très rare partout, excepté dans I'Engadine, se cache dans les grandes herbes des hantes Alpes.

J'ai pu me procurer plus d'nne douzaine d'individus bien conservés et leur examen n'a fait 'lue confirmer ma première opinion. Indépendamment des teintes d'un jaune orangé vif qu'on ne rencontre jamais chez caesiạia, on peut noter comme différences constantes: $1^{\circ}$ Laspect des postérieures en-dessus, dont l'espace basilaire est constam-

1) Conf. Bulletin de la Soc. vaud. des scienc. nat. Nr. 47. p.388. - Contributions à la faune de sicile. 
nent plus foncé que le reste de l'aile, la marge plus ou moins enfumée et séparée de cet espace par un liséré plıs clair. L’angle abdominal de ces mèmes ailes porte le long de la frange une éclaircie. $2^{2}$ L'absence de point discoüdal, à part quelques exceptions où il se montre faiblement. $3^{\circ}$ Le dessous des quatre ailes assez uniforménent grisjaunàtre, légèrement moins enlumó aux postérieures; aver la moitié interne plus foncée rt le hord externe de la bande moyenne du dessus marqué sur les yuatre ailes par une ombre noire diffuse. $4^{\circ}$ Le pointillé gris du dessous est plus prononcé que dans caesiaria. $5^{\circ}$ L'absence de points discoídaux du mème còté aux antérieures, dans tous les cas.

L’intensité du gris qui forme le fomel varie beaucoup; j’ai vu des indivilus ò̀ la bande moyeune et la marge sont presque noires, à l'exeeption des taches jaunes et de la ligne fulgurale; il en est d'autres ou le gris de fer disparaìt presque entièrement.

29t;. Infidaria. Faune suisse.

Cette espece bien plus facile à distinguer de flavicinctaria, Ilub. que ne l'est caesiaria, est cependant sans cesse confondue avec la première. - Les caractères tirés de la frange sont les plus surs. Aux antérieures, en-dessus, elle est blanche, sans ligne de division, sans trait, ou points à la base, et plus on moins entrecoupée de gris. Endessous, aux mèmes ailes, mème disposition; pour l'ordinaire elle est limitée par un bord blane, étroit, inégalement renflé de slistance en distance qui lui donne un aspect festonné. Aux postérieures, sur les tleux faces, ce bord blanc est ordinairement plas saillant et la frange en parait mieux festonnée encore. Le trait noir qui limite la frange, divisé par l'extrémité blanche des nervures, manque ici dans tous les cas.

297. b. Frustraria (ta). 'Treit. VI. 2. p. $50 .-$ TII. p. 218.

Dup. sup. t. IV. pl. 71. - Boisd. 1774.

Frey. n. b. 54, 2. - IIub. sup. f. 205, 206.

Ileydenr. C. 396. - Guén. list. t. X. Nr. 1368.

Muscosata. Donz. An. Soc. entom.

IIub. Gey, 595 (mala) - (non Leder.).

Mr. Rothenbach la prise près d'Ausernier (Jura) en juin 1846. Je l'ai reçue de Mr. Leresche, provenant du Jura vaudois, et de Mr. Ott, collectée à Mryringen. La haute Engadine. Elle est identique dans ces diverses localités, quoique plus ou noins colorée en vert et nébuleuse. I uscosata Donz. n'est pas mème une variété. 
299. Incultaria. Hub. sup.

$$
\begin{aligned}
& \text { Ajoutez aux synonymes: } \\
& \text { Ilub. sup. f. } 456 \text { \%. }
\end{aligned}
$$

300. Achromaria. Lah. Faune suisse.

$$
\text { Saxicolata, Leder. (Stauding.). }
$$

Très voisine de nebularia II. S. 370, 371. Je n'hésiterais mème pas à les réunir si dans ces figures la frange était entrecoupée, le $2^{\mathrm{e}}$ ruban restait bien séparé de l'espace marginal, la ligne fulgurale était indiquée vers le sommet seulement et à peine visible, enfin, si les inférienres étaient plus étroites et moins dessinées. La couleur légèrement jaunàtre d'aehromaria la fera tonjours aisément distingner de toutes les variétés de nebularia.

304. Sororaria. Hub.

Hub. sup. n. Schmett. f. $52,53$.

Tacetaria? Frey. 640,2 . (H. S.)

Je l'ai reçue de la haute Engadine. Les individus provenant du Labrador sont identiques.

308. b. M unitalia (ta). Hub. 346.

IIub. revis. Nr. 168. - Hub. suf. f. 53. - Guén. hist. t. II. Nr. 409. - Ileydenr. Cat. Nr. 542. - Stand. Cat. Nr. 491.

Arcticaria. Germ. faun. ins. - Zetter. ins. lapp.

Collinaria. Jetzn. ent. Zeit.

Jai reçu cette espèce de la haute Engadine. Elle paraît fort rare en Suisse; mème dans les liautes Alpes.

309. Bipunctaria. Bork.

Gachtaria. Frey. n. B. 594, 595, est à peine une variété plus foncée.

316. Silacearia. W. V.

Ajoutez aux synonymes:

Frey. n. B. f. 587. 


\section{6. b. Capitaria. Her.-Schf.}

H. S. Dentsch. Ins. 165. pl. 3. - Hub. sup. f. 460. - Heydenr. Cat. Nr. 550.

$$
\text { Balsaminata. Frey. n. B. 588. - Zell. }
$$

Très rare en Suisse; jen ai reçu de Mr. Ott un exemplaire femelle pris dans l'Oberland bernois.

La ehenille très voisine de celle de silacearia en reste cependant distincte (Dr. Rössler; in Jahrb. des Ver. f. Naturk. Ilerzogth. Nassau v. XII. p. 387).

Lindividu, fort bien conservé, que je possède répond parfaitement à la caractéristique donnée par ller. Schf.

Cette espèce étant peu répandue dans les collections et souvent avariée, jajouterai quelques détails fournis par lindividu que jai sous les yeux. - La taille est un peu au-dessous de celle de silacearia; mais la eoupe des ailes est identiquement la mème. Les antennes au lieu d'ètre annelées de blane le sont de jaune clair. Les palpes sont de la mème couleur arec très peu de noir à la base en dehors. Le front est entièrement jaune, tandis que dans silacearia les antennes et le front sont grisàtres, tachetés de noir.

L'espace radical est noir foncé, coupé carrément. Le fer ruban forme une ondulation dans son milieu et non une pointe externe saillante. La bande médiane est d'un noir pur, à peine étranglée dans son milieu. Les nervures ne se dessineut point en elair, ni sur elle, ni sur le Ier ruban, ni sur lespace radieal. Le $2^{\mathrm{e}}$ ruban, fort étroit, est. bordé, rers la còte seulement, de deux fers de flèche, dont lantérieur se confond avec une tache costale noire: les autres fors dr fleche sont fort petits. Liextrémité des nervures, en dehors dı $2^{\text {e }}$ ruban, sont saillantes et colorées en jaune jusques à la frange. La couleur jaune se répand sur lespace terminal du côté de l'angle anal et sur les elairs de la frange. Aux inférieures en-dessus la mime teinte jaune existe sur l'extrémité des nervures et sur la frange entière. La ligne de lunules y est également arrondie et festonnée, ce qui n'a point lieu dans silacearia.

Le dessous est d'un gris pointillé, jaunitre, avec le dessin moins prononé que chez sa voisine. L'abdomen est couleur de rouille en-dessus, un pen rosé en-dessous. Les pattes sont anrelées de brun et de fauve clair à leur extrémité et noiràtres sur les euisses, en-dessus. 


\section{1\%. Reticularia. W. V.}

Le màle de cette espèce présente une particularité que je ne trouse citée nulle part et qui pour les anateurs de classifications lui vaudrait l'hommeur de motiver un genre à part. Il existe chez lui, vers l'articulation de l'aile antérieure et en-dessous, un gros pinceau de poils noirs, dirigé du còté du disque et inséré très près du bord interne. Ces poils sont roussàtres à leur hase et reposent. lorsque les ailes sont ployées, sur une tache jaunitre visible sur le disque de l'aile inférieure et sur une autre semblable à la face inférieure des antérieures. Evidemment iei la couleur du pincean à déteint des deux parts sur la face correspondante des ailes.

318. b. Coraciaria (ta). Hubn. 278.

Var. psittacariae. Faune suisse.

Miata. Lin.? (Guén.) - Guén. hist. génér. t. 10. p. 460. pl. 13 f. 2.

Bulletin de la Soc. vaud. des seienc. nat. Nr. 40. p. 223.

On sera surpris que je rétablisse maintenant e oraciaria comme espèce, après avoir affirmé sur le ru d'un individu (2e supplément), qu'elle était une varjété de psittacaria, prouvée par de nombreuses transitions. Lindivilu yue jarais alor's sous les yeux était bien en effet une sariété de celle-ei et lorsque jeus la uraie coraciaria sous les yeux, c'est lui qui m'a convaincu de l'existence de deux especes distinctes. Les individus marbrés de blanc qui appartiennent à psittacaria restent complètement différents de coraciaria, et réciproquement les formes plus foncées de coraciaria(j'en ai sous les yeux) ne passent point à psittacaria. La taille, la coupe des ailes et le dessin diffèrent toujours.

La figure de Hubner est certainement mauvaise, mais beaucoup moins qu'on ne l'a affirmé; elle reste très reconnaissable. Celle de Guénée est très bonne, mais prise sur un petit individu.

Je ne puis réproduire ici tout ce que jai dit dans le Bulletin de la Soc. raudoise sur la distinction de coraciaria, de frustraria et de psittacaria.

Coraciaria fait en Suisse deux apparitions, une en mai et juin. l'autre en aoùt et septembre. On la prend surtout dans les vallées an pied des Alpes, où elle se pose de préférence contre les rochers. - Meyringen, Aigle, Schuipfen. Guénée lui a restitué la dénomination de Linné; je doute fort que la synonymie gagne en clarté à ces réparations tardives et souvent hazardées. 
319. Firmaria. Treit.

Cette espèce est positivement suisce. Mr. Alf. Rothenbach l'a price en aoùt, en septembre et en octobre près de Schüpfen. Jai recueilli une femelle à Aigle, au mois de mai. Mr. Meyer-Dür J'a enfin collectée au printemps daus les environs de Viège (Valais), où elle n'est point rare, en compagnie d'obeliscata var. variaria. Firmaria a donc deux gémérations comme variaria.

328. b. Simularia (ta). Hub. :345.

Ifulb. revis. $\mathrm{Nr}$. 194.

Gencata. Feisth. An. ent. 1834. - Borsd. 1732. - Hub. sup. f. 396.

Recueillie une fois en Valais par Mr. R. Zeller, entomologiste Zuricois.

330. Aemularia. Hub. 448.

Ajoutez aux synonymes:

Her:-Schf. neme Sehmett. f. $49,50$.

Mr. Iler--Schiffer malfirme que testaceata $\mathrm{Hb}$. 338. reste distincte de tersaria aussi bien que daemularia. 


\section{PREMIER SUPPLGMENT}

\section{AUX \\ PYRALIDIDES ET AUX CRAMBIDES \\ DE LA FAUNE SUISSE.}

(Voir: Noureaur Mémoires de la Société helvéticue des seiences naturelles;

Vol. XIV. p. 7.)

\section{Pyralidides. Her.-Schf.}

․ Cuprealis. Hub.

Je J'ai reçue de Mr. Couleru qui l'avait capturée daus les environs de la Neuvevilke.

Crambides. Her.-Schte

Genre Duponchelia. Zell. Isis.

(Epicorsia. Hub. verz. - Mascia. Guén.)

2. b. Cilialis. Hub. f. 119.

IInb. sup. f. 60 ?

Acutellus? Eversm. - Guén. Pyral.

Dans les marais d'Aigle, fin de juin et juillet; rare. 4 lndividus; 3 t. 1 q.

Très soisine de Catalaunialis, quoique bien distincte d'clle. Celle-ci a le sommet des antéricures allongé, aigu, presipue falciforme; les stries longitudinales blanches, plus nombreuses et plus marquées.

Mr. Her.-Schïller, qui a ru mes individus, les croit identiques avee sa figure 60 , quoique l'apparence soit différente. 
En tont cas l'espèce que jai sous les yeux est exactement celle de Hubner (f. 119). On ne peut s'y méprendre. On doit cepenlant reprocher ì cette figure le sommet des supérieures trop aigu, les palpes trop courts: l'abdomen (du màle) trop court aussi (il dépasse le bord des postérienres de toute la longuenr du mouchet anal); lalssence de trace médiane sur les inférieures et enfin langle abdominal des supérieures trop arrondi.

La fig. 60 de llub. sup. differe bien darantage. Les palpes sont figurés plus longs; l'angle abdominal des antérieures est encore plus effacé que dans la fig. de IIuln., i] n'existe pas trace de la deuxième ligne transverse aux antérieures: les lignes intercostales jaunes n'y paraissent pas non plus; celles des nerrures sont différentes: les inférieures sont uniformément jamàtres et non traversées par un trait gris et ombrées de la mème couleur sur la marge et au centre; enfin l'abdomen est plus long.

Les différenecs qui la séparent de catalaunialis (1. 56, 57) sont plus marquées encore, surtout en-dessons. - Je penthe it croire qne cilialis Hub. 119, doit former une espèce distinctr et conserver son nom, et que celle de Hub. sup. doit garder la dénomination dEversman jusyua preure du contraire. La description de Treitschke est insuffisante pour éclaircir la question. Celle de Her.-Sehf. parle des franges diun blancsale et grises, des lignes costales un peu plus claires, des palpes denx fois plus lomgs que la tète, du dernier article les palpes long et linéaire; toutes choses qui sont différentes dans la mienne.

\section{Flammealis. W. V.}

J'ai reçu de la France occidentale une variété entièrement d'un noir bistré. Le dessin est le mème et les taches blanches de la frange tris saillantes.

\section{Atralis. Hub. $2 \%$}

IJubn. Beitr. Th. 3. t. 2. f. k. - Treit. - IInb. revis. Nr. 23.

A Lausanne; une fois, dans un jardin. en juillet.

Ce qui est dit datralis Hub. dans la faune se rapporte à nigralis, en retour il faut transporter à atralis, IIuh. 27 ce qui est dit de nigralis, Hub. 26 ( $\mathrm{Nr}$. 19 de la faune).

13. Pyrenaealis. Dup.

Guén. hist. gén. t. 8. p. 153.

Le màle differe quelque pen de la femelle par sa taille plus faible. - Oberlami bernois $(\mathrm{Ott})$. 


\section{Rupicolalis. Hub.}

Guén. hist. gén. t. 8. p. 154 .

Ajoutez aux caractères qui la distinguent d'holosericealis; antennes, chez le màle. simplement pubeseentes, tandisque dans sa roisine elies sont plutòt ciliées (Guén.). La différence est peu prononcée, mais réelle cependant.

16. Alpestralis. F.

Guén. hist. génér. t. 8. p. 158.

J'en ai ru un grand nombre d'exemplaires; car elle n'est pas rare. La femelle ne diffère pas sensiblement du màle; si ce n'est pas une taille souvent un peu plus forte. Les marbrures azurées sont parfois si nombreuses qu'elles semblent former la couleur du fond sur laquelle le noir se dessine en points et en taches. Le dessin rappelle celui des Eudorées; sur certains individus on distingue les deux raies transverses, la tache en $x$ et les trois taches de l'espace marginal. Les franges sont d'un blane azuré pur sur les individus très frais, mais entrecoupées aux antérieures. Les postérienres portent souvent en-dessus, près du milieu de la marge, 2 a 3 petites taches azurées et sur leur bord antérieur une tache plus grande, indécise, blanchàire.

\section{Helveticalis. Anderegg.}

Jai reçu quatre exemplaires recueillis par Mr. J. Ott sur les hautes Alpes de l'Oberland bernois. Ces individus sont plus corsés et plus robustes que ceux de Mr. Anderegg: ce qui tient, peut ìtre, à ce que ces derniers sont élevés artificiellement.

Cette espèce se reconnaît aisément à ses ailes blanchàtres en-dessous, marquées d'un gros point noir discoidal aux supérieures et d'une ceinture noiràtre sur la péripherie des quatre ailes. Leśs ailes portent, sur le distue et en-dessus, des écailles bleuàtres dispersées, très nombreuses à l'état frais.

\section{8. b. Conspurealis. Lal.}

Six exemplaires, pris dans les hautes Alpes de lOberland berno's, mont été adressés par Mr. J. Ott. Mr. de Heyden m'écrit quil 'en possède un exemplaire $q$ pris à la Bernina dans l'Engadine. En aoùt 1860 jai collecté un individu $q$, au pied du PetitIeurran près des neiges qui dominent le glacier des Irartinet, à $5000^{\prime}$. L'insecte vole au soleil et se cache sous les pierres lorsqu'on cherche à le saisir. 
Tient le milieu entre holveticalis et rupicolalis, très voisin de nevadalis Stgst. (v. Heyd.). Taille plus forte, $25^{\text {mas }}$. Noirâtre, nuancé vaguement de gris soyeux olivâtre; sans dessin. Ailes antérieures oblongues, sommet arrondi, eôte droite; épaules moins saillantes que chez rupicolalis. Quelques taches bleuàtres diffuses sur le disque. Une tache jaunàtre aux $2 / 3$ externes de la còte donne naissance à la deuxième transverse à peine indiquée, sinueuse, finement éclairée de gris. Un trait gris et quelques taches claires indiquent la place de la première transverse au $1 / 3$ interne de l'aile. Un gros point noir, mal limité sur la cellule. Frange grisàtre, faisant suite au fond; blanchàtre a l'extrémité. Inférieures noiràtres, nuancées de clair avec des reflets chatoyants et soyeux chez les individus très frais. Un trait indistinct, pâle, continue la denxième transverse et limite un large espace plus foncé à la marge. Dessous d'un gris de souris uniforme et luisant. Le point discoïdal des antérieures peu apparent. Un trait circulaire clair indique la place de la deuxième transverse. Còte légèrement plus éclairée. Poils jaunâtres épars près de la racine des inférieures.

Palpes labiaux de la longueur de la tête, épais, écailleux, recourbés en bas; troisième article noir en-dessus, nu. lalpes accessoires relevés en forme de potit pinceau. Trompe visible. Antennes du mâle filiformes, non pubescentes. - Corselet carré, noirâtre, semé de poils gris. - Abdomen annelé de blanc sâle. Pinceau anal, chez le $\delta$, très long, noir dessus, blanc dessous.

Femelle plus massive que le màle et mieux dessinée.

19. b. Alb of ascialis. Treit.

$$
\text { Hub. sup. f. 19. - Frey. ä. b. } 84 \text {. }
$$

Mr. Wullschlegel, entomologiste zélé, a recueilli quelques exemplaires sur l'Engelberg, près de Olten. Rare dans la localité même qu'elle habite.

\section{1. b. Vittalis. Lah.}

Ce n'est pas sans hésiter que je me suis décidé à séparer eette espèce de cingulalis à laquelle elle ressemble extrêmement. Il suffit d'indiquer les caractères qui l'en distinguent pour la déterminer. - Deux individus pris sur les hantes Alpes de Bex, en juillet. - Taille un peu plus forte que celle de cingulalis; ailes supérieures un peu plus allongées, coupées légèrement en bizeau à l'extrémité, en sorte que le sommet est plus aigu, quoique encore légèrement arrondi, et l'angle abdominal est tout à fait émoussé. 
La raise transverse est plus large, d'un blanc un peu terne ou un peu roussi. La frange porte un très petit liseré blanc à son extrémité.

Les pattes, la gorge, le dessous des palpes, sont d'un blanc plus ou moins roux. Les cuisses et une partie des tarses sont nuancés de noiràtre. Racine des antérieures, en-dessous, avec des vergetures fauves, très prononcées.

\section{Punicaealis. W. V.}

Cette espèce varie beaucoup plus peut-ètre que purpuralis. J'ai sous les yeux des individus provenant de Sicile, qui sont dun rouge sang clair, sur la marge, tandisque le disque et la racine des ailes sont couverts de l'or le plus pur. Leurs inférieures se divisent en deux espaces, l'un interne, plus large, entièrement jaune d'or, traversé par un filet brun arqué et marqué d'une tache cellulaire de mème couleur; l'autre externe rouge brunàtre, portant une trace jaunâtre parallèle à la marge. Un caractère indiqué dans la figure 103 de II.-S. suffira pour distinguer cette espèce de toutes ses voisines: la tache postérieure de la bande maculaire est traversée, d'avant en arrière, par un filet brun qui n'est autre chose que l'extrémité de la ligue coudée. Ce caractère est plus constant chez le o que chez la $q$; mais il sert à déterminer la forme de la ligne coudée tout autrement disposée dans cette espèce que dans les autres. Ici, en effet cette ligne quitte la côte en passant entre les deux taches antérieures et décrivant un petit sinus autour de la denxième tache jusqu'à son côté interne d'où elle se dirige en ondulant, droit au bord interne en partageant la dernière tache. - Jen ai vu un grand nombre.

24. Porphyralis. W. V.

Collectée dans le Hasli par Mr. J. Ott.

25. Purpuralis. Lin.

De toutes les espèces voisines c'est celle qui présente le plus d'incertitudes. Après en avoir examiné un grand nombre je suis resté convaincu quil faut, de deux choses l'une, ou bien réunir sous cette espèce, avec Fisch. v. Röslerst., les grandes variétés d'Italie, les petites des Alpes et leur adjoindre ostrinalis, ou bien former, outre purpuralis, trois espèces séparées. Je me rangerais à cette dernière opinion si javais pu examiner un assez grand nombre d'individus étrangers à la Suisse. Mais en admettant cette deuxième alternative, à laquelle des quatre espèces conservera-t-on le nom de 
purpuralis? Je donnerais la préférence à la plus répandue dans IEurope centrale. Les figures de Hubner et de Duponchel sont trop ineorrectes pour servir de types; cependant Hub. f. 35 paraît avoir été prise sur celle dont je parle et pourrait désigner purpuralis. La grande espèce du midi n’a pas été figurée: celle des Alpes paraît avoir fourni la figure 105 de H.-S. - Ostrinalis est figurée H.-S. f. 107 et 108.

En attendant que ess difficultés soient résolues, je désignerai la forme méridionale et plus grande par eoceinalis; celle des Alpes, plus petite, par puralis, avee Mr. de Heyden. Nous aurons ainsi:

A. Purpuralis, offrant pour caractères principaux : $1^{\circ}$ Une coloration rouge-brun, terne, plus vive sur la marge est vers le milieu de l'espace moyen. Le brun passe au noir près de la eôte, au sommet de l'aile et autour des taches. Les nervures sont souvent marquées légèrement en brun. Le rouge des inférieures est plus recouvert de brun-noir. 2 Les antérieures plus larges que dans puralis et ostrinalis; un pen moins que dans coceinalis. $3^{\circ}$ Leur bord externe droit, du côté du sommet assez aigu, est arrondi à l'angle interne. $4^{\circ}$ La troisième tache (postérieure) formant une demilnne, tridentée en dehors. $5^{\circ} \mathrm{La}$ bande transverse des postérieures du mème jaune que les taches des antérieures. $6^{\circ}$ Les lignes antémarginales très peu prononcées, surtout en-dessus, n'étant guères représentées que par quelques points jaunes disposés en ligne courbe vers l'angle postérieur. Le dessous varie beaucoup; les taches claires y sont moins étendues que dans les autres variétés. Le jaune de la base aux supérieures est très rarement visible.

B. Coecinalis se rapproche plus que les deux autres de purpuralis par ses formes et sa taille plus forte. Quelques individus sembleraient mème former le passage entre celle-ci et phoenicaealis, F. v. R. J'en ai reçu d'Allemagne une sous ce dernier nom. Ce qui distingue surtout cette variété est l'extension que prennent toutes les taches et les lignes d'un jaune d'or pur. La bande maculaire fait un angle moins prononcé arec le bord abdominal et l'espace marginal est sensiblement d'une égale largeur dans toute son étendue. Les lignes antémarginales sont fortement exprimées par un trait jaune, parallèle à la marge qui est droite. La tache postérieure devient presque arrondie. Le jaune apparaît fortement à la base des quatre ailes. Les franges sont jaunàtres. Le pourpre vif des ailes porte fort peu de brun aux supérieures. Cette variété ne se trouve en Suisse que dans le Tessin.

C. Puralis se distingue beaucoup mieux de purpuralis, moins bien d'ostrinalis; cependant on rencontre de petits individus de la première qu'il est à peu près im- 
possible de séparer. Ses traits distinctifs principaux sont une taille constamment plus petite, avec les supérieures un peu plus étroites et coupées plus carrément sur le bord externe. Le rouge des ailes a une teinte violette qui dans ostrinalis passe au rose. Les ombres noirâtres de la côte, du pourtour des taches et du disque des inférieures sont très prononcées. La tache jaune de la base disparaît plìs ou moins sons le noir.

Un trait fin, vif, droit, borde presque constamment la frange des quatre ailes. La bande maculaire est plus maigre, ses taches sont plus petites et se tonchent quelquefois (dans ostrinalis elle se confondent); sa couleur jaune est plus pâle, surtout aux postérieures. La direction de cette bande est sensiblement oblique et l'espace marginal est plus large en arrière qu'en avant. Cette disposition est encore plus prononcée dans ostrinalis. La tache postérieure de la bande est étroite; elle forme un croissant tridenté plutôt qu'une demi-lunc. Le bord externe des franges est blanc.

Cette forme est fréquente dans les Alpes.

D. Ostrinalis, Hub. 113 ne peut-ètre distingué de puralis que dans ses formes exagérées où le jaune des bandes devient presque blanc, où le rouge passe au rose vif, où le bord externe et la bande maculaire s'inclinent encore plus sur la direction de l'aile et où les taches se fondent les unes dans les autres. La taille reste constamment plus faible. Les ailes inférieures sont noires; les antérieures presque sans mélange de cette dernière couleur; les franges sont blanches à l'extrémité; le filet antémarginal comme dans la précédente.

Je l'ai reçue de l'Engadine et l'ai recueillie en mai dans les environs de Sion.

\section{Aenealis. W. V.}

Recucillie par M. Meyer-Dür, dans les environs de Viège, Haut-Valais.

\section{Fuscalis. W. V.}

J'ai collecté, près d'Aigle, sur une pente très chaude, une variété assez remarquable. Son dessin est plus vif et plus varié. La marge des supérieures est plus foncée que le disque, et la ligne claire qui borde la deuxième raye transverse en dehors est très marquée. Aux inféricures la marge est d'un gris-foncé uni; la ligne claire et la raie qui lui succède sont fortement marquées. Le réste de l'aile est d'un gris-fauve, clair. La coupe des ailes ne permet pas de la confondre avec terrealis. 


\section{5. b. Deceptalis. Lah.}

Quoique très voisine de fuscalis, je n'hésite pas un instant à la considérer comme espèce différente. On ne peut la eonfondre avee terrealis dont les supérieures ont toujours le sommet allongé et aigu. Les caractères qui la distinguent de fuscalis la séparent d'ailleurs aussi de terrealis. - J'ai reçu denx exemplaires mâles, de Mr. Ott, de Meyringen.

Deceptalis a complètement le facies de fuscalis, aussi faut-il y regarder de près pour distinguer l'un de l'autre. Les dimensions, la coupe des ailes, la teinte générale est la mème; le dessin est seulement moins prononcé dans le premier.

A la face supéricure on peut noter comme caractères distinctifs de deceptalis: $1^{\circ}$ Les dentelures de la deuxième ligne transverse moins aigues, moins prononcées, moins éclairées de jaunàtre, en dehor's. $2^{\circ}$ L'angle rentrant de la mème ligne, près de la còte des supérieures, peu marqué. $3^{\circ}$ Une rangée de très petits points bruns, sur la marge, à l'extrémité des nervures, placée sur le bord d'un trait jaunàtre. $4^{\circ}$ Deux points discoïdaux aux inférieures (lorsqu'ils sont apparents).

Les différenees des deux espèces sont plus saillantes à la face inférieure; on y note: $1^{\circ}$ Les inférieures beaucoup plus elaires et blanchàtres que les supérieures: ce qui n’a pas lieu dans les deux espèces voisines. $2^{\circ}$ Deux points discoïdaux très prononcés et petits, aux inférieures. $3^{\circ}$ La ligne transrerse des mèmes ailes formée d'une rangée de points noiràtres, contigus, et non d'une ligne brunàtre continue. 4 Une rangée très saillante de points noirs, limitant la frange des quatre ailes, placés sur un fond plus clair jaunâtre. Chez fuscalis et terrealis ces points sont remplacés par un trait peu marqué.

Ces différences suffisent pour distinguer nettement la nouvelle espèce. Il est fort probable qu'elle existe déjà quelque part dans les collections, confondue avec fuscalis.

36. Pulveralis. Hub.

Se prend en abondance dans les marais de la vallée d'Aigle, en juillet. Les femelles m'ont paru plus fréquentes que les mâles.

\section{Flavalis. W. V.}

Assez fréquente sur les pâturages sees et chauds du pied du Jura (Rothb.).

Aux caractères qui la différencient de citralis on peut ajouter l'apparence oculée de la tache réniforme. 
Du reste, ayant reçu d'Allemagne des cinctalis sous le nom de flavalis, j'ai quelque lieu de croire que plusicurs entomologistes prennent la première pour la deuxième et par suite eitralis pour la vraie flavalis, qu'ils connaissent peu.

46. b. Rubiginalis. Hub. 79 .

Fisch. v. R. t. 27. - Dup. 218. - Treit. - Hub. revis Nr. 86. - Guén. hist. t. 8. p. 363. - Heydenr. Cat. 90.

Le 28 juillet, entre Faido et Giornico (Rothb.). - Espèce méridionale: je l'ai reçue de Sicile.

49. Alpinalis. IV. V.

Au pied du Bernina (Rothb.).

Les figures 175 et 176 de Hubn. doirent ètre rapportées à monticolalis.

50. Monticolalis. Khlw.

Alpinalis, Hub. 175, 176. - Ilub. sup. 7-10.

Certains individus màles ont les $3 / 4$ antérieurs des inférieures d'un blane un peu sale, sur lequel se dessinent les nervures en gris. Dans alpinalis ces nervores paraissent peu, le blanc est plus pur et le bord abdominal est couvert de gris. Il y a plus; sur le bord externe de la partie blanche chez la première se voit un trait gris, mal limité, séparant une légère ceinture blanchàtre, fondue sur ses bords avec le gris. Alpinalis n'a jamais cette bande blanchàtre, et l'espace blanc est nettement terminé.

\section{Manualis. Hub.}

A été prise par Mr. Ott dans les Alpes bernoises (Rothb.).

53. b. Donzelalis. Guén. Deltoïd. Nr. 392. 500.

Sororialis, v. Heyd. Graubündn. Jahresber. 1858-1859.

J'ai vu, dans la collection de Mr. Millière à Lyon, l'espèce de Guénée, identique avec celle recueillie par Mr. de Heyden lui-même dans les Grisons et que j'ai sous les yeux.

Elle est extrèmement voisine de nebulalis et non de rlododendronalis et au premier abord n'en parait qu'une forme fanée par le soleil. L'individu provenant des Alpes françaises est plus grand que les nòtres, mais son dessin est de mème, à peu près nul. 
La teinte générale de Donzelalis est constamment plus claire. Les ailes, d'un blanc sale en-dessus, sont en-dessous largement ombrées de noirâtre, le long de la côte, tandis que chez nebulalis cette partie est précisément la plus claire. Enfin nebulalis porte sur les antéricures les deux taches ordinaires que sa voisine ne présente pas ou presque pas.

La femelle, comme dans nebulalis et d'autres espèces alpines, a les ailes plus courtes, coupées droit à la marge et par conséquent plus aigues au sommet.

58. Sophialis. Fab.

Frey, n. b. 600, 4, 5 (fig. mala).

\section{Clathralis. Hub.}

A Martigny, sur le rocher de la Bàtia, en juillet. Dans le Haut-Valais; environs de Viège (Meyer).

\section{Numeralis. Hub.}

Son existence en Suisse est douteuse; sous ce nom se cache probablement decrepitalis ou olivalis var. leucophaealis, Ilub.

64. b. Decrepitalis. Fisch. v. Rösl.

Hub. revis. Nr. 126. - Hub. sup. f. 67, 68. - Guén. hist. t. 8. Nr. 519. - Heyden. C. 64.

Rare dans nos Alpes. Je l'ai reçue de Mr. Ott à Meyringen. La femelle est parfois presque entièrement blanche et sans dessin. Le mâle ressemble quelque peu à olivalis; mais ses ailes acuminées l'en distinguent sur-le-ehamp. Le point discoïdal des postérieures est aussi double. Aux mêmes ailes la bande brune marginale est moins bien déterminée.

\section{4. c. Elutalis. W. V.}

Hub. sup. f. 16. - Fisch. v. Rösl. 92. f. 1. - Heyden. C. Nr. $1^{105^{\mathrm{b}}}$. - Staudinger Cat. Nr. 190.

Albidalis. Hub. 118.

Lutealis. Haw.

Institialis. Curt. - Steph.

Actialis. Guén. - Dbl.

Haut-Yalais; environs de Viège; assez commune, fin de mai et commencement de juin (Meyer-Dür). 


\section{Olivalis. W. V.}

Je n'ai pas encore rencontré des individus qui se rapprochent de la figure 61 (umbralis) de Hubn. : tous ceux que j'ai vu sont beaucoup plus marqués de blanc.

Il serait à souhaiter que nous eussions enfin une bonne figure de cette espèce, qui reproduisit l'un de ses caractères essentiels, déjà noté par Fabricius et par Haworth, celui de porter deux points cellulaires aux inférieures.

69. G. Eudorea. Curt. - Dup.

(Scoparia. Haw. - Guén.)

J'ai du revoir complètement et à plusicurs reprises ce genre difficile et dont la synonymie devient de plus en plus embarrassée. J'ai publié dans le journal entomologique suisse (Mittheil. der sehweiz. entomol. Gesellschaft, Nr. 4) un synopsis des espèces européennes, auquel je renvoie les entomologistes embarrassés.

\section{Centuriella. W. V.}

Corrigez aux synonymes l'erreur suivante:

Numeralis. Zetters.

\section{Ambiguella. Treit.}

Je n'ai pas vu de rraie ambiguella avec la tache en $x$ ombrée de faure; ces individus-là appartiemnent ou à ingratella, et ils ont alors le sommet moins aigu quambiguella, ou à mereurella qui a l'aile plus large. Atomes gris de fer, fond bleuàtre.

\section{Manifestella. H.-S. f. 104.}

Graecella. Gn. - Cembrae. Haw. Steph. - Zelleri. Wocke.

Un exemplaire pris dans les Sous-Alpes d'Aigle et d'autres provenant des environs d'Olten (Kothb.), me permettent d'asseoir son diagnostic.

La taille est plus forte que celle d'ambiguella; le fond pâle et grisàtre est surchargé d'atomes roux; la tache en $x$ est à peine ombrée de la mème couleur, parfois mème elle disparait; les deux autres sont effacées. La ligne transverse interne est arquée, l'externe, très oblique, est brisée dans son milieu et peu denticulée; l'une et l'autre à peine bordées de clair. Sommet aigu; marge droite, fort peu oblique. La taille, la simplicité du dessin et la teinte générale rousse la font aisément distinguer. 


\section{Asphodeliella. Man.}

De nouveaux individus en petit nombre, il est vaai, sont venus confirmer mon diagnostic.

Facies et taille d'ambiguella; atomes roux; fond blanc pur; marge coupée carrément; sommet arrondi; taches ombrées de jaune; large bande rousse, unie, sur le troisiène espace.

75. Phaeoleuca. Kell. Lin. 1846. - H.-S. f. 204. Sciaphilella. Faune helv. Nr. 75.

Mr. Rothenbach l'a retrouvée sur les bords du lac de Bienne, au-dessus de Thouanne, appliquée contre les rochers; du 17 juin au 13 juillet.

\section{Mercurella. Zink. - Treit. - H.-S. f. 108.}

Pas toujours facile à distinguer d'ingratella et de conicella; il faut ici s'en tenir aux individus bien frais.

\section{6. b. Conicella. Lah.}

En parlant de mercurella (fanne suisse) j'ai dit que chez certains individus la bande moyenne se retrécissait beancoup vers le bord interne. Ces individus, provenant du pied des Alpes d'Aigle, n'étaient pas fort bien conservés. Depuis lors jai pu en obtenir de très frais et $\mathrm{j} y$ ai reconnu une espèce distincte.

Il suffit de la différencier de mercurella à laquelle elle ressemble pour la taille et le dessin. Dans conicella la còte est droite dans ses 3/4 internes, dans mercurella elle est ceintrée. La bande moyenue de la première a la figure d'un entonnoir par le fait du rapprochement les lignes du còté du bord interne. On peut ajouter que conicella a les atomes fauves tandis que sa voisine les a le plus souvent gris, et qu'enfin le troisième espace est strié longitudinalement de traits noiràtres qui sont rares et rarement marqués dans l'autre espèce.

\section{7\%. Ingratella. F. v. R. - Zell. Limn. p. 283. f. 5.}

Cette espèce est bien suisse, seulement elle est difficile à distinguer. Elle se place entre dubitalis dune part et les petits individus de mercurella de lantre.

Espèce à étudier sur les exemplaires suisses qui paraissent diflérer de ceux d'Autriche: Sous-Alpes de I'Oberland, ete. 
78. Octonella. Zell. Isis 1839.

Parel]a. H.-S. f. 119, 120 (non 100, 101, 102).

Ilautes Alpes de l'Engadine et du Haut-Valais. Jnillet.

Jai déjà dit qu'il importe de restreindre cette espèce aux figures 119 et 120 de H.-S. si l'on reut sortir de la confusion qui existe sur ce point et la distinguer une fois de valesialis. Jai ru trois exemplaires, un màle et deux femelles. L'une des femelles est sensiblement plus grande que l'autre.

La teinte du fond est le blanc enfumé, tirant sur le brun. Cette teinte est tellement reconverte de noir (surtout chez la femelle) qu'elle n’apparaît qu'en dedans de la première transverse, en dehors de la deuxième et clair-semée sur lespace marginal. Les trois taches du disfue disparaissent ordinairement sous les atomes noirs. - L'espace moyen, assez large, forme un coude prononcé au milieu de son bort externe; ce dernier est finement denticulé. - La frange est d'un blanc enfumé et précédée d'une série de points noirs. L'aile antérieure est étroite comme celle dambigualis, arec la còte presque droite; son sommet est sub-aigu et sa marge sensiblement oblique.

La femelle a cette aile plus aigue au sommet et parfois plus courte et plus étroite, coupée plus obliquement, avec les deux points internes bien marqués, comme lans suleticella?.

Les ailes postérieures sont d'un gris foncé arec la frange jaunàtre. - Le dessous est uniformément gris ardoisé, luisant et porte l'ombre d'une bande plus claire vers ses $2 / 3$ externes. Ia tite, les palpes et le corselet sont noirs semés décailles blanchàtres; les palpes accessoires, très visibles, forment un pinceau blanc à l'extrémité. L'abdomen en-desisus est brun, annelé de blanchàtre.

78. b. Imparella. Lah.

Cinc exemplaires de la haute Engadine; quatre màles, une femelle. - On pourrait pent-ètre lui appliquer la figure 101, II.-S., parce que le dessin ressemble à celui de valesiella (muranella); je préfére cependant le réserver pour cette dernière, répandue en Allemagne sous le nom de parella.

Il faut avoir sous les yeux plusieurs individus frais pour la distinguer soit doctonella, Zell., soit de murana v. valesiella. La femelle, plus petite, arec le sommet des antérieures plus aigu, ressemble à celle de sudeticella, n'était le parallélisme des deux lignes transverses chez impar ella. 
Dilfère de murana par ses ailes plus étroites et d'un bleu azuré très prononcé; la frange des antérieures est en outre presque entièrement blanche. Le bord externe est sensiblement moins arrondi et plus obliquement coupé. Le dessin offre peu de différences à part les lignes transverses plus distantes.

Octonella a d'ailleurs le fond des ailes jamàtre et la bande moyenne presque entièrement noire. Chez imparella, celle-ei est à peine bordée de bleuâtre de part ('t d'antre, et son bord externe très denticulé, forme, non un angle, mais un sinus court et prononcé. Ailes étroites, allongées, terminées en biseau. Dessin peu saillant; des trois espaces, le moyen fortement saupoudré dlatomes gris de fer', se détache en foncé sur les deux autres qui sont d'un gris bleuâtre. Une tache triangulaire noiràtre au milien du bord externe. La tache en $x$ circonserit parfois deux petits points blancs; les deux taches internes, punctiformes, bien visibles, isolées et éloignées de la ligne interne. Postérienres gris-bleuàtres, un peu enfumées au sommet. Dessous uniformément grisperlé.

80. Muranella. Curt. - Stph.

$$
\begin{aligned}
& \text { Delphinat alis. Guén.: } \\
& \text { Valesialis. Dup. } 229,3 . \\
& \text { Parella. Zell. - Guén. - H.-S. f. 101, } 103 .
\end{aligned}
$$

Jai dù comparer un grand nombre d'exemplaires prosenant de diverses contrées de l'Europe pour parvenir à définir nettement cette espèce qui se trouve confondue dans les collections avec toutes celles de sa taille qui s'en rapprochent pour le dessin. C'est elle yui est habituellement yendue par les marchands de Vienne sous le nom de parella, Zell.

Elle présente trois variétés passant les unes aux autres par tous les intermédiaires possibles. Sa taille varie quelque pen et se montre surtout développée chez la var. valesialis, Dup. (non Zell.). Dans le nord elle est parfois d'un tiers plus petite. La coloration grise ou noire varie à tous les degrés et par sa teinte et par son abondance. Le dessin et la coupe des ailes restent identijues.

Lorsque le fond blanc-bleuatre n'est recouvert que de quelques taches grises et reste partout bien visible, elle fournit la var. delphinatalis, Guén., qui se retrouve sur les pàturages méridionaux de nos Alpes et avait été recueillie par Mr. Guénée dans les environs de la grande Chartreuse. - Parella des marchands de Vienne et murana des 
Anglais s'appliquent généralement aux individus bien recouverts de gris de souris ou de gris de fer. Ce sont les plus fréquents dans nos Alpes. Dans eelles de Norwège et de l'Allemagne orientale le gris passe au noir.

Dans nos hautes Alpes du Valais et des Grisons le gris devient noir pur, ce qui rend d'autant plus saillant le pointillé et les lignes elaires d'un blanc-bleuâtre, ainsi se forme valesialis, Dup.

La dénomination anglaise étant la plus ancienue doit prévaloir; celle de Dup. appliquée à oetonella, et parella à trois ou quatre autres, doivent disparaître.

82. Pallida. Stph. - Wood.

La dénomination anglaise, plus ancienne, doit être préférée à celle bien plus barbare de II.-S.

83. Vandaliella. H.-S. 157 (Pyral.).

Resinella. Guén. Hist. t. 8. Nr. 563.!

Synonime déterminé par un individu reçu de Mr. Guénée lui-mème.

83. b. La etella. Zell. Linn. p. 298. f. 8.

Hub. sup. f. 115. - Guén. hist. t. 8. Nr. 560 ? - Heyd. C. 116. Albatella, Fisch. v. R. - Ileyd. C. 119.

J'ai collecté cette jolie espèce au milien de l'été daus les bois de sapin au-dessus de Lausanne. Mr. Wullschlegel l'a prise dans les eusirons d'Olten.

On ne peut la confondre quavec vandaliella dont elle est très roisine; mais sa blancheur franche et ses taches moins nombreuses, d'un noir pur, la font bientòt reconnaître. Très différente de erataegella pâle; celle-ei conserve toujours un fond sablé de gris, a la deuxième ligne infléehie sur deux points et non en forme de sinus unique, ne porte pas de points blanes en avant et en arrière de la tache en $x$, et enfin a la frange divisée et grise.

85. b. Petrophila. Standf. Std. E. 7. 1848.

Vesuntialis? Guén. hist. 1. 8. n. 551.

Erralis? Guén. id. id.

Taille et lacies de dubitella, sans trace de fauve et avec les ailes un peu plus 
étroites. Fond blanc-azuré; dessins gris de fer. Cinq exemplaires pris par Mr. G. Leresche dans les environs de Ste-Croix.

Còte presque droite; bords externe et interne légèrement arrondis; sommet à peine obtus. Espace basilaire blanc-blenàtre; articulation marqué de noir; première ligne droite. formant un angle vif, dans son milieu, du còté de la raeine de l'aile. Lspace moyen fortement recourert d'atomes gris-noirs; sur lui se dessinent en noir: $1^{\circ}$ les deux taches internes punctiformes; l'antérieure parfois légèrement pupillée; $2^{\circ}$ les nervures sous forme de réticule Iongitudinal; $3^{\circ}$ la tache en $x$ souvent marquée d'un point blanc. - La deuxième ligne finement denticulée et simplement coudée, se dirige du point de courbure directement et obliquement vers le bord interne. Troisième espace comme le premier; une ombre noiràtre, élargir à son milieu, occupe toute la longueur de la marge; les nervures sont iei marquées en gris. Frange blanche, luisante, plus ou moins entrecoupée de gris et divisée près de sa racine par un trait noir intrecoupé de blanc. Inlérieures enfumées en-dessus, luisantes, marquées d'une trace transversale peu visible et d'une ombre foncée sur le bord. - Dessous entièrement gris-roux, uni, luisant, plus foncé aux supérieurè. - Femelle semblable au mâle ou un pen plus petite avec l'oviducte saillant. Cette description est prise sur les individus de notre Jura. Les exemplaires de petrophila, communiqués par Mr. le Dr. Wocke, différent des nòtres par leur dessin très charbonné sur toute la surface de l'aile.

86. Ancipitella. Faune suisse.

Truncicolella. Staint.

Jai vu plusieurs individus des deux sexes, tous identiques. Elle se rapproche d'ambiguella et des grands individus de crataegella; on la prend aisément pour la première elfacée; mais elle en diffère 1 par son fond grisàtre uni, sur lequel ne se dessinent que les denx lignes, les trois points et une ombre de tache marginale; $2^{\circ}$ par sa còte également ceintrée; $3^{\circ}$ par l'ombre fauve et légère de la taclie en $x$; $4^{\circ}$ par l'espace moyen plus élargi, tandis que le terminal est plus étroit; $5^{\circ}$ par ses antérieures sensiblement phus larges; $6^{\circ}$ par les postérienres pellucides, pâles, sans traits, ni vergetures. La frange, blanchàtre. est clivisée par un trait gris. La deuxième ligne transverse, denticulée, forme une courbe uniforne à convexité externe. Tout l'animal est frèle et délicat, ce qui seul le fait distinguer de manifestella. Sa ressemblance avec crataegella lorsqu'elle est foncée et de grantle dimension, a fait penser que ce pourrait bien être la m ereurella de Lin., ce qui nous importe assez peu maintenant. 
88. b. Uliginosellus. Zell. Bresl. Z. t. 3. f. 8.

J'ai trouré cette espèce assez abondante dans les marais d'Aigle, au bord du Rhòne, vers le milien de juillet, en mème temps que paseuellus, et mèlée avec hui. La comparaisen d'un nombre suffisant d'exemplaire ma convaincu de ses droits à former espèce à part. Aux caractères déjà indiqués il faut ajouter : $1^{\circ}$ Une taille constamment inférieure à celle de pascuellus avec les ailes proportionnellenent plus larges; $2^{\circ}$ les inférieures plus blanches et d'un blane de lait enfumé; le monchet anal du màle de la mème couleur; $3^{\circ}$ le bord externe des supérieures à peine infléchi dans son milieu; la portion antérieure de ce bord est beaucoup moins saillante que dans pascuellus et la postérieure est moins arrondie; $4^{\circ}$ entre la ligne brisée antémarginale et la marge, n'existe pas d'espace bleudtre sur lequel se dessinent les points marginaux; ceuxci placés sur une bandelette jaune ou blanche, sont plus gros, plus saillants et rapprochés de la ligne antémarginale; $5^{\circ}$ la noitié interne de cette dernière ligne légèrcment arquée et non droite; $6^{\circ}$ enfin, la bande longitudinale, argentée et ensiforme, plus large, tonche à la còte dans sa moitié interne. Dans pascuellus elle s'en rapproche insensiblement et n'atteint la eòte quà sa base.

Les teintes sont généralement plus pàles que dans pascuellus. La femelle ne differe pas du màle. Le papillon voltige toujours au milieu des joncs et des graminées. Par l'extrémité des antérieures il se rapproche extrèmement de pratellus et par sa bande argentée et pure, de paseuellus.

La femelle de pratellus, lorspu'elle est blanche, se rapproche beaucoup d'uliginosellus; mais ses postérieures sont noiràtres et sa bande ensiforme marquée en long d'un ou deux traits bruns. Cette bande porte souvent une trace fause dans uliginosellus, tandis que dans pascuellus elle n'en a aucune.

\section{2. b. Scirpellus. Lah.}

Je n’ai pu collecter justu'ici que trois individus, un màle et deux femelles, appartenant à cette espèce très rare dans la localité mème qu'elle halite. Les marais d'Aigle, en juin et juillet, avec paseuellus, uliginosellus, cilialis, aureliellus, ete.

Il est aisé de la caractériser en la comparant à pascuellus d'une part et à hortuellus de l'autre; les antérieures sont terminées à la manière de la première; lo disque reproduit celui de la deuxième, mais laale est plus étroite et plus allongée. Taille $25^{\mathrm{mm}}$. Toici du reste sa description. 
Tête, corselet et palpes de coulenr gris-jaunàtre, avec des rellets métallipues semblables à cenx du laiton poli. - Palpes trais, aigus, allongés, recourbés légèrement endessous, deux fois plus longs que la tète, recouverts de longues écailles, subulées. Palpes accessoires de moitié pluss courts, formant un monchet sécuriforme, appliqués sur les précédents. Antennes sétiformes, brunàtres, lisses dessus, à peine pubescentes endessous. Ailes antérieures ètroites, allongées, très lisses, brillantes, d'un jaune mélangé de gris arec des stries métallipues couleur de laiton. La còte est presque droite dans ses $3 / 4$ internes et recourbée légèrement vers le sommet. Le bord externe porte une échancrure prononcée à angle sif. Le bord interne est presque droit, arrondi vers la base. Sommet très aigu. Angle anal très arrondli. Le disque, sur un fond olivàtre clair, porte un grand nombre tle lignes costales disposées en ésentail, a éclat métallique plombé et bordées d'écailles noires près de leur extrémité. La couleur fauve du fond n'apparaît yuientre les lignes costales. Le long de la còte se voit une bandelette longitudinale de la conleur des nervures, qui remplace la bande nacrée de pascuellus. La ligne transserse antémarginale est coudée brusquement, ì angle presque droit, en regard de la cellule; sa teinte est plombée, métallique. Sa moitić antérieure limite un triangle fause sur lequel est inserit une ombre brune, plus ou moins marqué et en forme de 1 . Cette onbre sappuie en dehors sur un petit trait blane qui la sépare de la frange; en dedans sur un trait fauve. La moitié postéricure de cette ligne est séparée de la frange par un espace demi-éliptique, gris, pointillé de noir, bordé de fause, sur lequel se dessinent trois à quatre points noirs, allongés et nersulaires. La frange, jaune de laiton très brillant, porte un petit trait blanc à sa limite, le long du triangle apical.

Les antérieures sont en-dessous entièrement d'un gris enfumé, aree la frange plus claire, d'un gris mèlé de fauve.

Inférieures grisitres des deux còtés en dehors, blanchàtres en rledans, avee la frange d'un blane sàle.

Abdomen entièrement gris-fause; mouchet anal plus elair. Palpes ordinaires.

96. b. Aureliellus. Fisch. r. R. t. 89, 1. Ilub. revis. Nrr. 176, - Heiden. Nr. 77.

J'ai collecté quatre individus appartenant a cette espèce, deux femelles au mois de

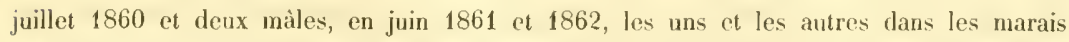
d'Aigle où elle est rare. A en juger d'après mes exemplaires les figures de Fischer v. 
Rösl. ne sont pas complètement exactes; le mâle, fig. 1, $a$, est trop nuancé de fause, il a les antérieures coupées trop carrément au sommet et les antennes capillaires: la femelle fig. 1, $c$ (aussi bien que le màle), porte les deux lignes transverses trop rapprochées; l'interne est légèrement arquée, presque droite; l'externe forme un coude très prononcé à ses $2 / 3$ antérieurs et un sinus près de l'angle anal. Les ailes sont un peu plus étroites et la tache des inférieures, sur le màle et sur la femelle, plus foncée. Cette tache est formée par un bouquet de poils fauses légèrement soulevés.

\section{0:. Rorellus. Lin.}

Il n'est point prousé que cette espèce existe réellement en Suisse. Les exemplaires de Mr. Rothenbach appartenaient it angulatellus.

\section{Myell us. Hub.}

J'ai reçu de l'Eugaline une variété d'un brun-verdàtre foneé qui ne se distingue des antres que par ce caractère. Le bord interne sur l'un des individus, porte une bandelette blanche sui partie de la racine de laile, s'éteint arant son milieu; cette bandelette est divisée, par une tache de la couleur du fond, en regard de la première intersection de la bande centrale.

\section{Luctiferellus. Hub. 324.}

$$
\text { Treit. - Her.-Sebf. Nr. } 209 .
$$

Lorsque jenregistrai cette espèce je navais sous les yeux que luctuellus. II.-S., f. 21, dont un seul exemplaire, un peu plus nuancé de blanc, se rapprochait de la figure de Hubner, sans eependant ètre identique. Dès lor's jai pu me proeurer plusieurs exemplaires du vrai lıctiferellıs. Il existe éridemment ici deux espèces distinetes.

Luctiferellus porte, sur un fond brun-noir, les taches blanches beancoup plus grosses et plus nombreuses, dessous et dessus. On rencontre des luctuellus qui ont les taches médianes plus déreloppées: mais en-dessous le blanc n’apparait qu'en légères teintes, vers le sommet des inférieures. La frange blanche, ou blanchàtre, des inférieures, le sineiput et le dessus des palpes de la mème couleur, le blanc plus ou moins saillant du dos, la distinguent encore mieux de lietuellus.

Cette espèce parait, en Suisse, plus rare que luctuellus; elle habite aussi les hautes Alpes centrales. Sa taille est rolontiers un peu plus forte. Les exemplaires très marqués de blanc en ont parfois des vergetures sur les postérieures.

Simplonellus Dup. n’appartient ni à cette espèce, ni à la suirante. 
112. b. Luctuellus. Hub. sup. f. 21 .

Dup. 270,4 .

Luctiferellus, var. Treit.

Heeriellus, Heyd. ined.

Cacuminellus. Zett.

Le fond brun-noir de cette espèce est ordinairement mélangé de nuances fauves, surtout le long de la marge. La frange des antérieures est parfois entrecoupée de petits traits blanes et linitée de la mème manière. Aux inférienres les franges ne sont jamais blanches; le dessous est quelquefois nuancé légèrement de cette couleur. Jamais de trait blane sinueux le long de la marge aux mèmes ailes en-dessus, comme on en rencontre parfois dans luctiferellus. Tète, palpes et corselet bruns ou gris-foncé.

113. b. Fulgidellus. Hul). 365. 366 (265, 266).

$$
\text { Treit. - Dup. 272, 2. - Ilub. revis. Nr. } 211 .
$$

Recueillie en Suisse. H. Benteli m'a présenté un individu pris sur la Gemmi.

Fulgidellus se distingue de radiellus, avec lequel seul il est possible de le confondre, par la teinte d'un brun pur; par la ligne longitudinale fortement dentelée en scie en arrière, accompagnée de deux autres plus petites très nettes; par la frange brune divisée en deux nuances et entrecoupée nettement en regard des lignes argentées; par le bord externe arrondi, coupé plus carrément, avec le sommet moins aign; enfin, par les postéricures blanchàtres avec des vergetures brunes vers le sommet.

114. b. Furcatellus. Zett. ins. lap. Laponicellus. Dup. - Guén.

Radiellus. Curt. - Steph. - Wood.

Radiolellus. H.-S. f. 4.

Cette espèce que jai recue de Mr. Mann sous le nom de Ledereriellus, prise en Tyrol, se collecte aussi dans l'Engadine, où elle n'est pas très rare. Plus petite que radiellus. Tète, palpes, corselet, ailes supérienres d'un brun-rougeàtre foncé. Ligne longitudinale élarugie à son extrémité, digitée, d'un blanc sale. Franges, ailes postérieures, dessons des quatre ailes et abdomen, gris-foncé un pen rongeàtre.

La figure de Ilubner sup. a si mal réussi sur l'exemplaire de l'ouvrage que j’aj consulté '[u'il niest pas possible de constater lidentité. 


\section{Deliellus. Hub.}

Lindication de Mr. Zeller, de Zurich, est erronée; l'individu signalé se rapporte à tristellus. - En retour j’ai trouvé cette espèce assez abondante sur les collines arides et très chandes des environs de sion, en septembre. Comme elle est mal décrite dans Treitschle et ordinairement confondne avec la var. paleella (IIub. 51) de tristellus, il est bon d'indiquer ses caractères distinctifs: Deliellus est d'un jaune-paille pur, jamais teinté de jaune-souffre, ni d'orangé. - Sa còte est constamment droite ou mème légèrement concave ce qui fait paraître laile plus étroite. Ses nervures blanches sont de moitié plus ténues que celles de tristellus. Les points de la marge peusent manquer dans l'une et l'autre espèce. Deliellus ne porte jamais de denxième raie transterse à peu de distance du bord externe.

120. Rostellus. Faune helr.

Mr. de lleyden méerit que cette espéce existe aussi dans l Engadine, où il l'a collectée. Mr. Herrich-Schäffer la figurée plus tard sous le nom de monochromellus (Hub. sup. f. 164. Pyral.). La teinte de cette figure est trop pàle et trop grise.

\section{1. b. Zinckenella. Treit. XI. 1.}

ZelI. Is. 1846. - Hub. revis. Mr. 230.

Etiella Treit. X. 3. - F. v. Rösl. t. 29. - Dup. sup. 278, 5.

Cette espèce méridionale a été prise dans le Ilaut-Valais, à Viège, par. Mr. MeyerDuir.

\section{2:3. Perfluella. Zinck.}

Mr. Rothenbach a collecté cette espèce dans les environs de Schuipfen. Ses individus sont plus rouges que la figure de Hub. sup.., surtout à la base de l'aile.

129. Palumbella. W. V.

Un très grand individu sur les Alpes de Bex, en juillet, à 5000 '.

137. Germarella. Dup.

Modifier les synonymes:

Melanella. Treit. (non Dup.). 


\section{Melanella. Dup. sup. t. 60 (non 'T'reit.).}

Je l'ai regue de Ste.-Croix, recueillie par Mr. G. Leresche.

\section{Aethiopella. Dup. Cat.}

Que na-t-on laissé de côté les errements de Duponchel à loccasion de cette espèce si mal figurée et si imparfaitement décrite, dans son ourage, quili est impossible, sans recourir a dautres sources, de dire sil avait devant les yeux celle-ci ou sa roisine alpieolella, Zell. J'ai mòme lieu de croire que lorsquil décrivit son Ennychia monjpessulalis il avait devant lui cette dermière espece et quien l'appellant plus tard Phycis aethiopella il entendait par la celle à la quelle nous conservons cette dénomination.

Aethiopella, auquel on aurait dù conserver la désignation dhelveticella donnéc par Zell., habite exclusivement, parait-il, les hautes Alpes. Elle se distingue Jien moins par le dessin du dessous des ailes que par sa teinte gris-noiràtre, visible surtout aux infrieures. Ses ailes sont plus spatulées, plus étroites il leur extrémité. La deuxième liqne transverso décrit un sinus très visible dans son milien. Mais ce qui la caractérice surtont est l'exintence, sur les individus frais, de poils d'un beau violet, dispersés sur le disque des supéricures. sur le corsclet, à lextrémité des palpes et surtout à lextérieur des tarses. Alpicolella présente quelquefois des écailles le la même couleur sur le disque des ailes, mais jamais sur les tarses.

144. b. Alpicolella. Zell. Isis.

$$
\text { Hub. sup. f. } 50, \text { วิ1. }
$$

IIahite de préférence les expositions rocailleuses et chaudes des Apes valaisannes. Mr. Staudinger la rapportéc dEspagne.

Les inférieures sont d'un noir fuligineux foncó. Leur frange est grise, émarginéc de blanc et quelquefois entièrment blanche i liriception d'un filet noir il leur bave. Les supéricures ont le troisieme espace terminal) noir-uni et pur, les deux transverses incertaines et lo disfue semé de petites éeailles blanches, arrondies, formant un vai tapis perlé. Jamais d'éeailles violettes sur les antennes, les palpes et les tarses comme sa boisine. 
145. Cinnamomella. Dup.

Assez fréquente dans les lieux ehands, surtout au premier printemps. Le Mauremont; les vignes de Lavaux et d'Ollon. Bienne, dans les lieux sees: premier juillet; Sehüpfen : 24 septembre (Rothb.).

15\%. Oribrella. Hub.

Au pied de la montagne à Villeneuve.

158. Flaviciliella. Man.

Zell. - II.-S. Zusätze p. 148. f. 144.

Collection Rothenbach. - Regue aussi de IEngadine.

Gen. XXIV. b. Anerastia. Hub. - Zell.

164. b. Lotella. Zinck.

Treit. - Dup. 283, 6. - H.-S. f. 90-92. - Zell. Is. 1848. Staud. Cat. Nr. 534.

Miniosella. Zinck. - Steph.

Pulverella. Hub. 454.

Recueillie par Mr. Benteli dans les environs de Berne. Toujours rare.

165. b. Interpunctella. Hub. 310.

Dup. 60, 280, 5. - Ilub. revis. Nr. 359. - Ileyil. Cat. Nr. 161.

Prise dans les environs dolten par Mr. Wullschlegel. - Excessirement fréquente dans les magasins de droguerie à Lyon, où sa larve dévore les fruits secs. 


\section{Catalogue synoptique}

\section{des Pyralidides et des Crambides suisses.}

\section{Pyralidides}

Aglossa.

$$
\begin{aligned}
& \text { Faune. Suppl. } \\
& \text { No. No. }
\end{aligned}
$$

1. Pinguinalis . . . 1. -

2. Cuprealis . . . 2. 2

\section{Asopla.}

3. Farinalis . . . 3. -

4. limbrialis . . . 4. -

5. Glaucinalis . . 5. -

Pyralls.

6. Brunnealis . . 6. -

7. Angustalis . . 7 . -

\section{Crambides.}

Calaclysta.

1. I.emnalis. . . . 1. -

Duponchelia.

2. Cilialis . . . - $2^{\mathrm{h}}$

Tegostoma.

3. Comparalis . . $2 . \quad-$

1. Stratiolalis . . 3.

5. Potamogalis. . . 1. -

6. Nympliaealis . . 5. -

Agrotera.

7. Nemoralis . .
Endotricha.

Faune. Suppl. No. No.

Faune. Suppl.
No. No.

8. Flammealis . . 7. 7

29. Purpuralis . . 25. 25

-. v. Coccinalis . . - 25

—. v. Puralis . . . - 25

\section{Stenlat.}

9. Carnealis . . 8.

10. Punctalis. . . . 9. -

Cynacda.

11. Jenlalis . . 10. -

llercyna.

12. Atralis ... 11.

13. Pollinalis . . . 12 -

14. Pyrenaealis. . 13. 13

15. Rupicolalis . . . 11. -

16. Holosericealis . . 15. -

17. Alpestralis . . 16. 16

18. Andereggialis . 17. -

19. Helveticalis . . 18 . -

20. Conspurcalis . - $18^{\text {l, }}$

\section{liotys.}

21. Nigralis . . . 19. -

2.2. Albofascialis . . - $19^{\text {b }}$

23. Inquinalis . . 20. -

24. Cingulalis . . . 21 . -

2.j. Villalis . . - $21^{\mathrm{h}}$

26. Octomaculalis . . 22 -

27. Pnnicatealis. . . 23. 23

28. Porphyralis . . 21. 2 !
-. v. Ostrinalis . . - 25

30. Cacspitalis . . 26.

31. Sanguiualis . . 27.

32. Aenealis. . . 28. 28

33. Rhododendronalis 29.

31. Murinalis . . . 30 .

35. Literalis. . . 31.

36. I'rticalis . . . 32 .

37. Repandalis . . 33 . -

38. Terrealis. . . 31.

39. Fuscalis . . . 35. 35

40. Deceptalis . . - $35^{\text {b" }}$

41. Pulveralis . . . 36. 36

42. Crocealis . . 37. -

43. Cifralis . . . 38 .

41. Flasalis . . . 39. 39

15. Hyalinalis . . . 40.

46. Pandalis . . 11.

17. Verticalis . . 42 -

18. Lancealis . . . 43. -

19. Sambucalis. . . 11. -

50. Stachydalis . . 45. -

51. Verbascilis . . 16.

52. Rubiginalis . . - $46^{\mathrm{h}}$

53. Silacealis . . 17.

54. Praetextalis. . . 48.

5.. Alpinalis . . . 49.49 


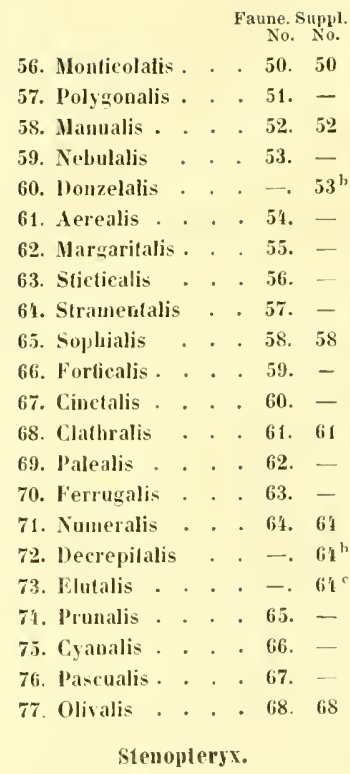

78. Hybridalis . . . 49. -

\section{Eudoren.}

79. Cesituriella . . 70.

80. Ambiquella . . 71. 71

81. Perplesella. . 72. -

82. Mauife-tella • . 73. 73

83. Isphodeliella. . 71. 71

81. Phaeolenca. . 75. 75

85. Mercurella . . 76. 76

86. Conicella . . - $76^{\text {b }}$

87. Ingratella . . 77. 77

88. Oetonella . . . 78.78

89. Imparelia . . . $78^{\text {b }}$

90. Suleticella . . 79.79

91. Muravella . . . 80. 80

92. Pallida . . . 82. 82
Faune, Suppl.
No. No.

Faune. suppl.

93. Vaudaliella . 83.

94. Laetella . . - $83^{\mathrm{b}}$

95. Crataegella . 84. -

96. Duhitella . . . 85.

97. Petrophila . . - $85^{\mathrm{b}}$

98. Ancipitella . . 86. 86.

\section{chillo.}

99. Forficellus . . 87 . -

\section{(rimbus.}

100. Pascuellıs . . 88 . -

101. Cligmosellus . . - $88^{\mathrm{h}}$

102. Dnmetellus . . 89. -

103. Pratellus . . 90. -

101. Adipellus . . 91. -

105. Lucellus . . . $92-$

106. Scirpellus . . - $92^{\mathrm{b}}$

107. Horfuellus . . . 93. .

108. Saxonelius. . 94. -

109. Alpinellus . . 95. -

110. Cerumellus . . 96.

111. Aureliellus . . $-96^{\text {br }}$

112. Inquinatellus . 97. -

113. Angulatellus . 98.

111. Culmellus . . 99. -

115. Falsellus . . 100 . -

116. Verellus . . 101. -

117. Chrssonuchellus 102.

118. Rorellus . . $103 \quad 103$

119. Taeniellus . . 104. -

120. Aridellus . . 105

126. Nargaritellus . 106.

122. Pinetellus . . 107 . -

123. Nytilellus . . 108 .

121. Conchellus. . 109. -

125. Nyellus . . . 110.110

126. Speculellus . .111. -

127. Luctiferellus . . 112. 112

128. Luetuellus . . - $112^{1}$

129. Pramidellus . . 113.
130. Fulgidellus . $-113^{\mathrm{b}}$

131 Radiellus . 11\%. -

132. Furcatellus . - $11 \mathrm{t}^{\mathrm{b}}$

133. Trivtellus . . 115 . -

131. Deliellus . . . 116116

135. Selasellus . . 117. -

136. Luteellus . . . 118.

137. Perlellus . . 119. -

138. Rostellus . . 120. 120

139. Lythargyrellus . 121 . -

Pempelia.

110. Zinchenella . - . $121^{\mathrm{b}}$

111. Carnella . . . 122. -

112. Perlinella . . 123. 123

113. Subornatella . 124. --

114. Adornatelia . 125.

115. Ornatella . . 126 . -

146. Obductella. . 127. -

117. Carbonariella. . 128.

118. Palumbella . . 129. 129

\section{Nephopleryx.}

119. Ahietella . . 130 . -

150. Roborella . . 131 . -

151. Hhenella . . 132 -

152. Janthinella . . 133.

153. Argyrella . . 13i. -

Ilypochalcia.

151. Auriciliella . 135 . -

155. Hignella . . . 136 . -

156. Germarella . . 137. 137

157. Ahenella . . 138 . -

158. Melinella . . 139. 139

159. Lignella. . . . 140. -

Zophodia.

160. Rijertella . . 141.

161. Compositella . 112 - 


\begin{tabular}{|c|c|c|c|c|c|}
\hline \multicolumn{2}{|c|}{ Asarta. } & \multicolumn{2}{|c|}{ Aerobatsis. } & \multicolumn{2}{|l|}{ Anerastlit. } \\
\hline & $\begin{array}{l}\text { Frune Suppl. } \\
\text { No. No. }\end{array}$ & & $\begin{array}{l}\text { Faume. Sappl. } \\
\text { No. No. }\end{array}$ & & $\begin{array}{l}\text { Faune. Suppl. } \\
\text { No. No. }\end{array}$ \\
\hline 162. Alpicolella . & $.113 . \quad-$ & 173. Tumidella. & . . 153. - & 185. Lotella &.$-164^{13}$ \\
\hline 163. Aethiopella & $.14 \% .141$ & 174. Rubrotibiella & . . 154. - & Ephes1la. & \\
\hline $\begin{array}{l}\text { 161. Helveticella } \\
\text { Ancylos }\end{array}$ & . $-141^{13}$ & $\begin{array}{l}175 \text { Consoriella } \\
\text { Nyctemre }\end{array}$ & .15 .5 & $\begin{array}{l}186 \text { Elutella. . } \\
\text { 187. Interpunclella }\end{array}$ & $\begin{array}{l}165 .- \\
. \quad-165^{\mathrm{h}}\end{array}$ \\
\hline $\begin{array}{r}\text { 165. Cinuanomella } \\
\text { Trachoui }\end{array}$ & $\begin{array}{l}.145 .145 \\
\text { 11s. }\end{array}$ & $\begin{array}{l}\text { 176. Achatinella } \\
\text { Homaeoso }\end{array}$ & $\begin{array}{l}.156 .- \\
\text { ma. }\end{array}$ & $\begin{array}{l}\text { Achroea. } \\
\text { 188. Alvearia . . }\end{array}$ & .166. \\
\hline $\begin{array}{l}\text { 166. Cristefla } \\
\text { Myeloi }\end{array}$ & $.146 .-$ & $\begin{array}{l}\text { 177. Cribrella } \\
\text { 178. Flaviciliella }\end{array}$ & $\begin{array}{r}.157 .157 \\
. \quad 158.158\end{array}$ & Helissoblapt & tes. \\
\hline 167. Rosella . . & . 147. & 179. Ceratoniella & $.159 .-$ & 189. An & 167. \\
\hline 168. Luridatel!a & 148. & 180. Obfusellá . & . $.160 .=$ & Aphonia. & \\
\hline 169. Legatella. & $.149 .-$ & 181. Binaevella . & . . $161 .-$ & 190. Colonetla & .168. \\
\hline 170. Advenella. & 150 & 182. Couvolulella & . . 162 - & & \\
\hline 171. Suave!Ia & .151. & 183. Boisduvaliella & $163 . \quad-$ & rial. & \\
\hline 172. Terebrella & . 152. - & 18'. Simuella. . & . . 164. - & 191. Mellonella. & $.169 . \quad-$ \\
\hline
\end{tabular}




\section{PREMIILR SUPPLÁNIENT}

\section{AUX \\ TORTRICIDES \\ DE LA FAUNE SUISSE.}

(Voir: Nouveaux Mémoires de la Société suisse des sciences naturelles;

Tome XVI. p. 9.)

\section{Abildgaardana. Fab.}

Jai recu cle Mr. L. Conleru une famille entière de cette espèce dont il avait fait l'éducation.

La forme ordinaire (Hub. 55) était de beancoup la plus nombreuse et variait surtout par la pureté plus ou moins mariuée du blanc de l'espace basilaire et par la couleur plus on moins fauve on brune de lespace terminal. La forme nyctemerana (Hub. 240) comptait quelques représentants dont lespace basilaire était faure.

Un seul individu màle représentait la var. permutatana, Dup. avec cette différence que toutes les portions claires étaient d'un roux fauve, sur lequel se dessinaient en brun-noir, la tache interme et la tache costale; celle-ci semblable, pour la forme, à celle d'erutana, II.-S.

Une variété plus curieuse encore, et linverse de la demière, a été prise à Olten par Mr. Wullschlegel. Les ailes antérieures sont d'un noir velouté avec des rellets violets et de nombreux points d'un noir mat. Une petite tache rouillée existe à langle anal et une grande tache triangulaire, tronquée en arrière, d'un beau blanc-jaunàtre, occupe le milieu de la côte. 
4. Erutaua. Hub. snp. f. 9, 19.

Mr. Herrich-Schäffer m'écrit que tristana, Hub. 50 et logiana, II.-S. f. 24. IJub. 64, ne sont que des variéiés derutana. Tristana doit donc disparaître de notre catalogue. Cette fusion met un terme ì un désordre qui allait croissant.

9. Ferrugana. W. V.

Je dois ajonter que les antéricures chez cette espèce sont plus ou moins réticulées et portent rolontiers un point noir au dessus du centre. Sur cing individus de lythargyrana je n’ai pu déeourir ni réticule, ni points.

An lieu dasperana lisez: adspersana.

10. Adspersana. Hub.

Mr. G. Leresche a pris la femelle dans les environs de ste-Croix; elle reproduit parfaitement la figure 406, Ilub. sup. - LEngadine.

3). Pilleriana. W. V.

Ahonde, en juillet, dans les buissons de ronce, au bord des rignes, à Ollon, et cependant on napperegoit pas trace de son passage dans les vignes elles-mèmes.

37. Ochreana. Hub. 134.

Mr. Meyer l'a recueillie abondamment dans les enviroms de Viege (Ilaut-Valais). Ses individus sont semblables aux miens. Un grand màle arait les ailes coupées plus obliquement et plus étroites, la tache costale isolée et le facies de Viburnana o. - C'est par erreur que cette espèce est indiquée dans les mnirons de Schüpfen.

37. b. Strigana. Hub. 141.

Treit. - Dup. - Fröhl. - Guén. - Hub. reris. Nr. 60.

Ginomana. IIus. Sehif.

Yole en juin sur les pentes chaudes, parmi les taillis des environs dollon (Lah.).

39. Dohrniana. Nan.

Steineriana. llub. sup. I. 419.

La distinction de Dohrniana et de Viburnana est à peu près impossible, pour le màle; la femelle scule, lort difficile ì découvir, est totalement différente. Lorsqu'on 
rencontre des individus màles à ailes étroites, aree une légère ombre cuircée an centre, on peut dire avee certitude que lon a sous les yeux Dohrniana. Ces exemplaires sont rares. Faut-il encore rattacher à rette espéce tous ceux qui sont pointillés? En tout cas ceux dont la tète est recouverte d'une houppe faure, plus elaire que les ailes, doivent ìtre placés parmi les viburnana. Il ne mest pas possible d'en faire, avec Mr. Wocke (Catal. de Lepidopt.), une mime expèce que Lusana et Steineriana. Lus ana a les antéricures plus obtuses et plus étroites. La tache eostale, le poli luisant des antérieures et la blaneheur de la moitié externe des postérieures dintinguent suffisamment Steineriana. La figure 419 de llub. sup. appartient à Dohrniana; je possède des individus pareils.

\section{Arjunctana. Treit.}

Regue de l'Engadine. Alpes bernoises (Rothb.). La femelle differe queliue peu du mâle; elle est plus grande, plus faure; ses ailes sont plus réticulées et les postérieures ont une teinte unie plus foncée.

\section{Obliterana. v. Heyd.}

Une femelle, que j’ai pu me procurer, est d'un brun-fauve luisant, plus foncé que dans la figure $36 \mathrm{I}$, H.-S., sans ancune trace de dessin.

46. b. Vulpisana. Frey.

Inb. revis. Nr. 71. - Hub. sup. f. 34 .

Jai recueilli un individu mâle de cette espèce dans les marais d'Aig̣le, en juin 1861.

Sa couleur est le rouge-brun foncé; elle ne porte aueun dessin visible; on découvre seulcment quelques réticules sur les supérieures; la marge et la còte sont de couleur rouille foncée. Les antérieures sont beaucoup moins robustes que chez hcparana.

\section{Rolandriana. Hub.}

C'est par erreur que cette espèce est indiquée en Suisse. 


\section{Flavana. Hub. 258 (non 15\%).}

Hub. revis. Nr. 95. - Heyd. Cat. Nr. 120. - Dup. catal.?

leterana. Frobl, - Guéll. ind.

Intermediana. Ilub. sup. - Fanne helv. Nr. 64.

C'est cette espèce-ci et non palleana, Treit. qui se prend dans les Alpes jusquà 6000 pieds de hauteur, en juillet. Elle y est constamment jaune pàle, arec les postérieures parfois entièrement charbonnées en-dessus, comme daus la figure de IIubner. Je ne lai jamais observée dans la plaine.

Palleana doit done disparaitte de la faune suisse pour faire place à flavana, Hub. 258. et intermediana ne peut ìtre distinguée spécifiquement de cette dernière, comme l'indique arec raison le catalogue de MH. Staudinger et Wocke. La réunion de Il avana, Hub. 157, à palleana Treit. comme variété, n’est pas admissible daprès les exemplaires que jai sous les yeux.

65. Rusticana. Treit.

Je l'ai recue de Ste.-Croix (Jura), prise par Mr. Leresche, et de IEngadine.

67. Lusana. v. Heyd.

Pas fort rare, en juin, sur les basses Alpes. Montagnes d'Ollon et du Val-d'liers.

68. Scrophulariana. Hub. sup.

Sa présence en Suisse niest point établie; elle ne paraît ètre dailleurs qu'une forme de viburnana due à la nourriture et à la provenance septentrionale.

\section{Mediana. Fab.}

La var. a urantiana, Koll. - II.-S. f. 411 - a été prise dans les environs de Viège par Mr. Meyer.

75. b. Diproltana. Treit. sup.

$$
\text { Ilubn (Tin) I. 345. - Treit. (Tin.) - Dup. sup. - Gnén. ind. - }
$$
Heyl. Cat 167. - Ilub. revis. Nr. 117.

Zebralla. Dup. pl. 260.

Margaritana. Woorl. 1121. - Steph.

Mr. Rothenbach eo lecte cette espree dans les environs de Schïpfen et me l'a communiquée. 


\section{7\%. T'esserana. W. V.}

Jai reçı de Mr. G. Leresche à Ste.-Croix, la varicté II.-S. fig. 327.

\section{Rutilana. Hub.}

Le mile dilfère volontiers de la femelle par ses ailes plus allongées oi la couleur rouge s'étend sur le disque aux dépens des trois bandes james. La première en partant de la hase se réduit quelquefois à un gros point, irrégulièrement découpé, qui n'atteint pas les bords de l'aile. La deuxième soblitore dans son milieu; elle est alors remplacée par deux points, dont lantérieur seul touche constamment a la còte. La troisieme derient un gros point entièrement cerné de rouge et de forme triangulaire. La frange reste d'un jaune pìle entremélé de queldues écailles faures, rarement limitée par un petit trait rouge. Chez la femelle les postérieures sont plus foncées ef le dessous plus enfumé.

79. Valdensiana. H.-S.

$$
\text { Aurofasciana. Man. - Faunc suisse. }
$$

Alin de ne pas confondre cette espece arce anrofasciana, Iaworth, il convient dadopter la dénommation de Mr. Herrich-Schäffer.

8:. Ванй апniana. Fab.

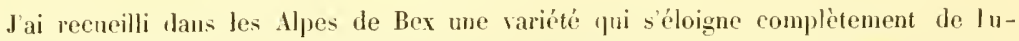
tulentana. L'intervalle des taches lormes est entierement d'un jaune-souffre uni, sans points bruns ni roticules; les points brillants sont rares et les sspaces clairs plus étendus.

83. Deutschiana. Zett. ins. lapon. 1. !81.

Wocke, Catal. Nr. $74 \%$.

Lutulentana. Her.-Sehäf. n. Schm. f. 35. - Fame helv. Nr. 83.

Je dois à l'obligeance de Mr. Wocke un exemplaire de lespice de Zetterstedt dans lequel je retrouve celle que jai décrite dlans la faune helsétique, sous le nom de lutulentana, If-S. - Celle de Doverfjelıl (Norvègo') ne differe des individus de nos hautes Alpes que par des teintes brunes plus chaudes et plus prononcées sur les bandes, qui la rapprochent davantage de Baumanniana var., citée plus laut. Dans les Sous-Alpes nous retrouvons la forme de Noriège.

Ne finira-t-on pas par trouser tous les passages des unes aux autres? 


\section{Rubigana. Treit.}

Je l'ai prise dans les Alpes de Bex, en juillet, à $3500^{\prime}$.

\section{Rubellana. Mus. Schif.}

Assez fréquente sur les Alpes et le Jura. La femelle est généralement plus petite et plus grise. La taille varie beaueoup. La ligne de division de la frange est très prononcée, surtout vers l'angle anal.

88. Dipsaceana. Fisch. v. Rösl.

Alpes de Bex, assez fréquente, en juillet. Ste-Croix (Loresehe).

88. b. Richteriana. Zell.

Fiseh. v. Rosl. t. 40. 1. 3. Frey, n. B. 324, 4. - IJul, revis. Nr. 145. - Heyden. Cat. Nr. 679. - Wocke, Cat. Nr. 756. Ruficiliella. Wood.

Un individu màle, dans les vignes de sion, en septembre 1862.

Cette espèce varic beaueoup pour la taille, à ce quil parait. Mr. Herrich-Sehäfer trouve la figure de Finch. ı. Rösl. trop grande; jai sons les yeux une femelle provenant d'Angleterre yui est beaucoup plus grande qu'elle. L'exemplaire pris i sion a la taille indiquée par Fiseher; tandisqu'un troisieme provenant d'Allemagne est plus petit.

\section{Pallidana. Fisch. v. Rösl.}

J'ai sous les yeux un grand individu lemelle, qui eliffere de pallidana à quelques ígards. - La trite et les palpes sont fauves clair et non blane pur. Le troisième article des palpes plus saillant est aussi plus roux. Le foud des antérieures est jaune-souffre elair et non blanc. La racine est fortement sablée de gris. La tache dorsale est mal limitée et porte, en dedans, une trainée grise qui se prolonge du tòté de la racine, sur le bord postérieur. La tache anté-apicale, earactéristique de pallidana, allongre, recourbée, allant de la còte jusques près de langle anal, est ici beancoup plus courte et conposéc de tleux taches jaunàtres, saupondrées de gris et contigues. Le sommet est légèrement rosé.

Les postérieures sont noiratres et fortement charhonuées en-dessous. Est-ce encore une espèce distincte? 
96. b. Flaviscapulana. Fisch. v. Rösl.

Ilub. revis. Nr. 264 (Coceyx.) - Hub. sup. f. 97. - Ifeyden. Cat. 711.

Gilvicomana. Zell. 1s. - Wocke, Cat. Nr. 779.

Une fenclle recueillie par Mr. G. Leresehe et déterminée par Mr. Her--Schäl.

96. c. Sanguisorbana. Hub. sup. f. 41:.

Se rapproche beaucoup de lespèce précédente et je n'aurais pu l'en distinguer sans les individus reçus de Breslau et collectés par Mr. le Dr. Wocke. Un màle collecté aussi, à Ste.-Croix, par Vr. G. Leresche. - La figure de H.-S. est outrée et peut induire en erreur; jai eependant sous les yeux des individus très frais.

9\%. Dubitana. Hub.'

La figure if de Hlubner pourrait mieux se rattacher à a mbiguana qu'à dubitana

98. Ambiguana. 'Treit.

Je I'ai prise plusieurs fois dans les Alpes et l'ai reçue du Jura (Leresehe). La plupart des individus ont les postérieures marbrées et pointillées en-dessous, vers leur extrémité.

100. Roserana. Fröhl.

Prise près de Schiupfen par Mr. Rothenbach; là n'existent pas de vignes roisines. -. Commence à se répandre dans les vignobles de la Frauce.

10:3. Riguana. Treit.

Reçue aussi de l'Engadine, où elle n'est point rare. N'existe pas dans les environs de Schiipfen.

\section{Stramentana. Guén.}

Plusieurs exemplaires recueillis sur le Weissetistein par My. Yüller et Jaggy.

La femelle est semblable au màle.

Chez deux inlivilus le fond, au lien d'ètre jaunàtre ou roussàtre, est entièrement gris-jaunàtre. Il est alors entièrement couvert d'atomes et de filets gris. Sur ce fond 
se distinguent cependant la tache carrée du milien de la còte et la tache déchirée du sommet. L'aspect général est celui de Nematoph. pilella. Celte espíce n'a du reste aucun rapport avec albulana, Treit.

106. Diurneana. Guén. Index. méthod. p. 32.

Bellana. Faune suisse p. 49.

Penziana. II.-S. f. $117,118$.

De crainte de faire une fansse application du synonyme de Curtis, je prefere adopter la dénomination de Guénée; quoique je ne puisse en faire autant des earactères distinctifs quil donne à cette espèce-ci et à penziana. - La figure de IJubner est trop mauvaise pour sy arrèter. Celles de Hab. sup. sont prises très probablement sur diurneana.

Jai un un grand nombre d'individus appartenaut a cette espèce-ci, venant tous des Alpes; jen ai vu un peu moins de lespece voisine pris dans le Jura et dans la haute Engadine. De tous les caractères indigués deux seuls paraissent se maintenir; diurneana a le blanc des antérieures tirant sur le blenàtre, les postérieures un peu grisàtres et les taches noiratres plus nombreuses. Dans penziana le blanc est très pur, mat, les taches plus noires et moins nombreuses et les postérieures blanches en-dessus. Les caractères tirés de la forme des ailes, de la taille, de la couleur de l'anus, despalpes, des vergetures des ailes postérieures en-dessons, sont inconstants. Si l'on fait une mème espèce de Wahlbomiana et de commnnana, II.-S., je ne wois pas que la distinction entre diurneana et penziana puisse se maintenir.

114. b. A brasana. Dup. sup.

Hub. revis. Nr. 190. - Hub. sup. f. 99. Zell.

I'ai collecté à Sion (Talais), au mois de mai 1861, un individu répondant exactement à la figure et à la description de Her.-Schäf. - Lugano (Mey.).

L'intervalle des denx premières baudes était légèrement indiqué anx antérieures, par une nuance plus claire; quelques points noirs étaient dispersés sur le disque. - Prise et élevée sur l'ombilicus dans les environs de Lyon par Mr. Millière.

115. Derivana. Faune suisse.

Jai rẹu encore quelques individıs appartenant à cette espèce qui présentaient les caractères indiqués, à part la tache jaune discö̈lale que je n'ai plus observée. 
115. b. Cretaceana. Hub. 318.

Dup. - Fröhl. - Ilub. revis. Nr. 192. - Heyden. Cat. 46.

Mr. Mejer a collecté cette espèce dans les environs de Viège (Valais), en juin.

118. Rosetana. Hub. 222.

Mr. Wullschlegel a pris, dans les environs dolten, la variété ou l'espèce, dans laguelle jai cru reconnaitre la rosaceana, Schlig. Sa couleur est le rove vif, carmin; la còte est marquée des crochets indiqués. Jai tont lieu de la croire espèce bien distincte.

119. A renana. Fame suisse.

Je lai retrouvée dans les marais d'Aigle, en juin, et Mr. Wullschlegel l'a prise dans les emirons dolten. Sa teinte reste tonjours gris fauve, sa frange rouillée, sa taille plus petite et sa marge arrondie.

120. Ha urana. Hub.

M. Meyer, de Burgdorf, a collecté cette espèce dans les environs de Viège (Valais). Collection Jaggy.

121. 'T'ussilaginana. Khlw.

Se prend aussi dans le Jura. - Ste-Croix (G. Leresche).

132. ๖. Antiquana. Hub. 213, 214.

Guén. ind. - Jeyd. Cat. 251. - Dup. cat. - Hub. revis. Nr. 209.

Divellana. Hub. 339. - Ileyd. Cat. Nr. 208. - Dup. Cat.

Quatrimaculana. Steph.

Les Sous-Alpes de Bex; en aoùt.

13:3. b. - Mygindana. W. V. - Fab.

Ilub. 181. - Dup. Cat. - Ileyd. Cat. 248. - Guén. ind. Hul), revis. 214. - Staud. Cat. 833.

Flammeana. Hub. 321. - Heyd. Cat. 180. - Dup. Cat. - Guén. ind. Cruentana. Zell.

Undatana. Bullet. Mose.

Formosana. Curt.

Fulvipunctana. Haw. - Steph.

Les hautes Alpes: lOberland bernois; la Grimsel; la haute Engadine, et c. 
Les figures de IJuhner sont liune ef lantre manvaises. - La femelle a pour l'ordinaire les ailes antérieures plus étroites, avec le bord externe plus oblique et le sommet plus aigu. En tout cas le sommet reste plus arrondi que dans arbutana. Les postérieures sont aussi plus pàles que chez cette dernière, mais elle s'en distingue surtout par un grand nombre d'écailles blanches, dispersées sur la moitié exterue de l'aile. Ces écailles sont distribuées en bordure le longr des veines rouges et forment une sorte de lizéré le long de la marge, eu dedans de la ligne brune qui borde la frange.

Hygindana ne saurait ètre séparée d'arbutana à layuello elle ressemble extrèmement, ni de rosetana, larenana et de rosaceana, dont elle est tout aussi voisine.

La teinte violette du fond est souvent aussi prononcée à la base que sur la marge externe; le pointillé blane donne seul à la teinte générale une apparence plus claire.

136. Rejectana. Lah.

Cette espece se place à còté d’alpinana (roir $275 \mathrm{~b}$ ).

13\%. b. Astrana. Guén. index.

Bruand. Catal. 996.

Siderana. Dup. sup. 507 (non Treit.).

Cette espèce remarpuable se prend dans les environs de Pontarlier (Jura). Mr. G. Leresche l'a aussi récoltée au-dessus de Ste-Croix, aux Granges. La chenille vit sur le polygonum bistorta, dont elle attaque les épis en fleurs; on la trouve en juin.

Ne peut être confondue qu'avee siderana, Treit. ainsi que l'a fait Duponchel. Elle s'en distingue immédiatemeut par ses points orangés plus visibles: deux de ces points sont partieulièrement remarquables; lun, situé au tiers interne de la còte, est de forme semi-lunaire; lautre, en regard du premier, sur lo bord interne, est allongé.

L’angle anal est orangé. La frange des inférieures l'est aussi à l'exeeption d'uu point noir au sommet de l'aile. La tète, le eorselet, les antennes et le dessus des palpes sont tachés de jaune orangé.

\section{Pictana. Lah.}

Cette espece doit disparaître; elle appartient à la femelle de sublimana, assez différente du nàle, comme je l'ai reconnu plus tarl (ıoir Nr. 215 b). 


\section{Micana. Treit.}

Ir. Rothenbach ma communiqué un individu femelle pris dans le Jura.

Sa taille est celle de rurestrana. Le sommet de l'aile, la bande moyenne et la base sont noiràtres, séparés par deux bandes jaunes. Quelgues points brillants sur le disque.

Le màle, que jai vi plus tard, ne porte des atòmes noirs quà la base de l'aile; le reste est fauve avec de nombreux points brillants. Sa taille est plus forte.

\section{Metallicana. Hub. 68.}

Parait assez fréguente sur les hasses Alpes, en juin. Chanupéry, dans le Val-d'Iliers (Bas-Valais). - LEngadine, ote.

\section{8. b. 'Trochilana. Fröhl.}

Je crains fort de me présenter ici arec une troisiome ou quatriime esperce sous la dénomination de trochilana. Les figures 313 et 314 de llub. sont bien mausaises pour baser sur elles un diagnoutic; cependant elles s'appliquent 'n tout cas mieux à celle que jai sous les yeur urü Couleruana. Les citations des anteurs sont encore plus incertaines. Duponchel en fait une olis ana, Treit.; les teinters jaunes de la figure de Ilub. pritent à ce rapprochement. Herrich-Schäfer penche pour hepaticana: opinion qui me parait fort douteuse. IIeydenreich suit Duponchel. Wocke (Catal. Nr. 924́) doune tont is fait ì ganche lorspuil en fait une Couleruana. Pour lui domer la dénomination de Fröhlich je m'appuie sur la vue d'un individu reçu sons ce nom d'Allemagne, prar Mr. Rothenkach, et provenant, je crois, de Freyer. Quoiquil soit, vo'ci en quelques mots sa description. Laspeet général est celui de bipunetana, Treit. n'étaient le brillant argenté des bandes claires, la tache oblique de la marge (chez trochilana) et les déchirures de la bande moyenue du còté de l'angle abdominal.

Le dessin se rapproche particulierement de celui de lacunana; un peu moins de cespitana et dumbrosana. Elle se distingue te la dernière par sa tache oblique marginale terminée sur le disque en massue quarrée, et de lasant-dernière par labsence de toute teinte rouillée et par son sommet plus aigu. Je la comparerai de préférence ¿ lacunana. Celle-ci porte des points métalliques bleuàtres que trochilana ne présente pas; ses ailes sont d'ailleurs plus larges et plus obtuses au sommet; ses bandes claires plus ombrées de stries transiorsales; son espace basilaire moins uniformément 
noir et tranché; sa tache oblique plus longue et non recourbée an sommet en forme de crosse, etc., etc. Trochilana a l'espace basilaire brun, nettement tranché, arec une dent au centre, conme bipunetana, et des stries noires peu visibles. Le premier ruban blanc ent semblable à celui de bipunetana avec quelques reflets argentés et quelques points bruns. La bande moyenne reprodnit parfaitement celle de lacunana, moins les points métalligues violets et avec la tache du bord externe plus petite, comme dans cespitana.

La tache oblique de trochilana śest à peine détachée de la marge qu'elle se recourbe en dedans sous forme de crosse et se termine par un épatement quadrilatère, entouré de blanc argenté. Sous ce rapport on la rapprocherait d'urticana, mais la crosse n'est pas arrondie et étranglée. Le sommet est un peu allongé é aign; le bord externe un pen oblique et l'angle interne blanc. La frange est blanchàtre arec quelyues dentelures noiratres at point de contact de la tache oblipue. Sommet noiràtre. - Postérieures d'un gris de fer, uniforme sans nuance de roux ou de clair. Palpes noiràtres, avec un peu de blanc sale en-dessus.

L'étroitesse de ses ailes, qui la rapproche de lucana, l'a fait aisément distinguer de ses analogues.

Je l'ai reçue de la haute Engadine.

150. Umbrosana. Zell. Isis.

C'est par erreur que j’ai indiqué les Alpes bernoises parmi les localités qu'elle habite.

158. Il endosana. Lah.

Cette espèce doit encore disparaitre et rentrer dans palustrana Nr. 149. Des individus foncés appartenant à palustrana, recueillis près de Gastein et que Mr. Herrich-Schiffer ma communiçués, sont identiques avec mendosana, prise dans nos Alpes.

165. Nana. Treit.

Mr. Rothenbach la collectée dans les environs de Schüpfen et Mr. G. Leresche près de Ste.-Croix (Jura). 


\section{5. b. Rufimitrana. Fisch. v. Rösl.}

Ilub. revis. Nr. 245. - IIub. sup. f. 139. - Heyden. Cat. 244.

Un individu femelle, parfaicement conservé, a été pris sur les pins, par Mr. Meyer, dans les environs de Viege (Valais).

C'est arec quelque trésitation que je ratlache l'insecte que jai sous les yeux à celui figuré et dlécrit par Mr. Iler.-Schäf; mais je ne saurais à quel autre le réunir. Le dessin dillère de la figure 139, H.-S., par l'extrémité des antérieures bien arrondie, par l'al-sence de tache noiratre en forme de virgule, a la marge, et par les crochets non distimets du fond.

Palpes faures; troisième article invisible. Tïte surmontée diune houppe fauve. Antennes fauses à la bace, grisàtres vers le sommet. Espace basilaire des antérieures grisàtre, tacheté de noir et limité par une bande rouillée en forme dare, faisant angle à son centre du còté de la marge. Expace moyen large, entièrement gris; trois filets gris, tachetés le famse, le sillonnement en travers sur un fond blanc, luisant, presque argenté. Bande moyenne étroite, rouillée, pointillée de noir, envoyant de son centre $m$ filet fance is la tache sulsapicale. Celle-ci est d'm faus vif, très irrégulière, et porte quelques rares points noirs sur ses bords. Une ligne argentée la sépare de la bande moyenne; deux à trois taches de mème aspect se logent entre ses rameaux. Sommet faure, très arrondi. Còte maryure de quatre crocheti peu apparents, qui sont la terminaison des lignes argentées. Un trait noir limite la frange brune, luisante. Un point blanc, peu sisible, sour le sommet, ne divise pas la ligne de la frange.

Postérieures d'un gris brunàtre plus foncé vers le sommet, semées d'écailles noires. Frange grise à l'extrémité antérieure, blanchàtre vers l'angle postérieur. Ligne de limite très marquée en-dessous, bordée de part et cl'antre d'un liseré blanchàtre.

\section{Arbutana. Hub. 195.}

Nos exemplaires ressemblent tellement à mygindana, à part la taille, quil est difficile de les en distinguer; en tout ras y a-t-il plus de dulférence entre arenana et rosetana, qu'entre les deux premières.

\section{Squalidana. Fisch. v. Rösl.}

Prise dans les environs de Lausanne. 


\section{9. b. Botrana. W. V.}

Ilub. reris. 267. - Hub. sup. f. 315. - Guén. ind.

Vitis a a Jacq. Misc.

Reliquana. Hub. Verz. (non f. 75).

En mai, dans les environ de Viège (Valais). Heyer de Burgdorf.

\section{Gentianana. Fröl.}

La còte est rouillée à son extrémité et ses crochets sont bruns.

193. b. Lapideana. Fisch. v. Rösl.

Hub. revis. Nr. 28i. - Hub. sup. f. 246. - Heyden. Cat. 28. Sudetana. Khlw.

Un exemplaire en 1858, près the Lausanne, dans un bois de hètre.

20"4. b. 'T'urbidana. 'Treit. sup.

Dup. sup. 64. - Guén. ind. - IIubı. revis. Nr. 304. - IJub. sup. f. 245 . - Heyden. Cat. 343. - Staud. Cat. 960.

Zelleriana. Schläg.

Fuscana. Khlw.

Terreana. Mus. Poder.

En abondance sur une prairie humide au milieu des tussilages, près de Burgdorf, en mai 1862 (Meyer-Dir).

La figure de Duponchel est an-dessous de tonte critique; celle de Ifub. sup. est peu reconnaissable, en tout cas inexacte; heureusement que la description de Treit. est parfaite. - Linsecte a unc coulenr fanse sombre.

206. Scutulana. W. V.

Mr. Rothenbach m'écrit qu'il ne l'a pas recueillie près de Schüpfen.

212. Graphana. 'T'reit.

Ne peut ètre jusiqu'ici enrégistrée parmi les espèces suisses. 
215. b. Sublimana. Fisch. v. Rösl.

Iub. revis. Nr. 320. - Heyd. Cat. 361. - Hul). sup. f. 229.

J'ai reçu cette espèce de l'Engadine. Le màle differe sensiblement de la femelle; tandis que le premier rappelle Brunnichiana, la deuxième (pour la plupart des exemplaires du moins) ressemble à ulmana. Jai donné une description très détaillée de celle-ci sous lo nom de pictana (fame Mr. 139). Il est des individus de ce sexe chez lesquels la tache du bord interne s'éteint en majeure partie avant darriver à la còte. Quant an màle, il est sulfisamment caractérisé daus la description qu'en donne IferrichSchäffer.

Les sommets gazonnés des hautes Alpes; toujours rare.

220. Citrana. Hub. 185.

Bords du Léman et vallée d'Aigle seulement.

2.21. I n c a na. Kell.

Les individus de Mr. Rothenbach ont l'écusson earré; ceux de Mr. Herrich-Schäffer lont arrondi.

22:3. Conterminan a. Fisch. v. Rösl.

Je l'ai prise quelquefois dans les environs de Lausanne. Il faut rapporter ì cette espèce ce que jai dit de jaceana, faune suisse Ni. 227.

2.4. Decipiana. Lah.

Espèce à retrancher. La comparaison d'un grand nombre d'individus m'a couraincu de l'insuffisance des caractères qui doivent la distinguer de Ifohenwartiana. (Voir à cette dernière.)

205. Aspicliscana. Hub.

Varie beancoup pour la taille et la largeur des ailes. Le màle a fréquemment la base des inférienres plus ou moins blanche. Je ne lai pas observé chez la femelle qui les a toujours plus foncées. Cette dernière est souvent plus petite, avec les antérieures proportionnellement plus larges.

J'ai reçu de lEngadine la variété figurée sous le Nr. 24 (nebritana) de II.-S. Les 
postérieures, che\% le màle, ne sont pas constamment blanches it la base; mais le milieu lles antérieures ent strié longitudinalement de vergetures de cette couleur, sous forme de bande transverse irrégulière, sous largelle ressort la couleur noire du fond en traits longitudinaux. Cette varićté peut aisément passer pour une espèce différente.

\subsection{Hohenwartiana. IV. I.}

Expèce très variable. Sa taille peut atteindre (dans les Alpes surtout) celle de W'ahllomiana. Les ailes inférieures, chez le màle, sont puelquefois entierement blanchàtres. Les crochets sont tantòt très nots, tantòt embrouillés et peu visibles. Dans les Alpes le brun du discue est plus foncé. La tète est tantòt blanche, tantòt fauve. Le sommet des inférieures porte souvent quelques vergetures blanches, moins prononcées que dans Aspidiscana. Jamais on ne distingise, comme dans celle-ci, une bande brune se prolongeant entre le quatrième et lo einuluième double-crochet.

Monetulana. Ilub. 25\%, appartient trè probablement is Aemulana.

Pupillana, Ilub. 20, représente parfaitement l'expèce telle qu'elle se rencontre ordinairement. Je possède des indisilus \& qui se rapprochent de Her.-Schäf. f. 296; je n'ai cependant jamais vu la tache interne aussi tranchée, ni narynée tle traits bruns, et surtout pas le bord externe aussi oblicuement coupé.

\section{Jaceana. \%ell.}

Ne peut ètre consersée. Les individus que je rattachais à cette e-pèce appartiennent í conterminana.

2:28. A emulana. Schläg. (non Hub. Ter\%.).

Cette espece est plus roisine dispidiscana que de Hohenwartiana. Lécusson est étroit, ‘un jaune assez vif.

2.29. Laharpana. Rothl.

Trois indivilus que jai regus de mon ami Mr. Rothenbach étaient mèlés avec inc a na, Zell. - Il convient de mieux préciser le diagnostic. Le facies est celni de Ilohenwartiana arec l'envergure de $16^{\mathrm{mm}}$. Pour la forme et la coupe des ailes, elle reproduit ibiceana, asec une taille inférieure à la sienne. Conme cette ternière elle a la còte blanchàtre, dans sa partie consacrée anx crochets: une bande longiturlinale d'un 
brun-fawe sur le centre dn disque, et une semblable plus claire tout le long du bord interne. L'écusson est blanchàtre, carré, bordé de deux lignes plombées très courtes et coupé à son bord autérieur par l'ombre brune du eentre. - Les erochets servent particulièrement is la distinguer d'ibiceana; ils sont doubles, au nombre de quatre, le dernier, du èté de la racine de l'aile, est souvent peu visible; les trois premiers sont ordinairement bien espacés: leur couleur est le blanc-lauve. Dans incana les crochets sont blanes, indistincts i partir des deux premiers; dans ibiceana ils le sont encore plus et toute la côte est inarquée de points bruns.

Les ailes inférieures du màle sont blanchàtres à la base; celles de la femelle, et leur bord, dans le màle, sont d'un gris-brun.

La frange des supérieures se divise en deux parties. l'interne grise, pointillée; l'externe fauve, unie. Celle des inférieures est d'un blane sale.

Du reste la description concorde avec celle cjue jai dounée dans la faune.

229. b. 1 biceana. Her.-Schäf. Syst. Verz. 1861.

Un individu dans les marais d'Aigle, en juillet.

La distinetion des trois bandes longitudinales est moins prononcée que dans Laharpana; du reste la taille est plus forte $\left(19^{\mathrm{mm}}\right)$; les ailes sont proportionnellement plus étroites et V'écusson plus petit. moins distinct. Deux caractères la distinguent surtout des espèces voisines. $1^{\circ}$ Ses erochets blanchàtres indéterminés (pour l'ordinaire) formant une série dle traits bruns et plombés, s'étendant aux $2 / 3$ externes de la eòte et quelquefois jusques à sa racine; $2^{\circ}$ cinq à six points bruns sur le bord postérieur des supérieures (ils peuvent manquer). Un ou deux traits noirs, sur l'éeusson fause-clair et bordé de deux lignes plombées brillantes. Bord externe des supéricures un peu évidé, ce qui rend le sommet plus saillant.

233. Fagiglandana. Zell.

Environs de Ste-Croix (G. Leresche).

234. Obesana. Lah.

Espèce à retrancher: e'est la femelle de Resinana Nr. 167.

235. Splendana. Hub.

Enıirons d'Olten (Wullschlegel), de Lausanne; sur les chènes (Lah.). 


\section{Fulvifrontana. Zell.}

Ajoutez aux synonymes :

Germmana. Ilub. f. 47.

\section{Chavanneana. Lah.}

Cette espèce doit disparaitre; elle n'est que la lemelle de Bugnionana, tonjours assez différente du male. Il conviendrait de la figures.

\section{Grineriana. H.-S.}

Les Alpes de Bex, en juillet. Rare.

Var: distinctana, Man. An Wylerfeld prè Berne (Jaggi).

246. Ustulana. Hub.

Près de Berne (Rothb.).

248. Tebritatla. 'Treit.

Excl. : Synon. Hub. sup. f. 241.

Na pas été prise aux environs de schiipfen.

2) 1. A cum inatana. Schläg.

Sur IAmend, à Thoune (Jaggi); pas réeoltée pas Mr. Rothenhach.

25:3. Alpestrana. F. V. R.

In individu pris le 31 juillet, sur I Almend, pres Thoune, diflère sensiblement de la ligure 193. 11.-S. - Taille d'une petite blepharana. Fond gris foné, fortement rouillé, comme daus aemninatana. Bord externe phus oblique que chez blepharana, mais moin que sur la pricédente. Sommet arrondi; sinus sons-apical sans point hlane. Ciny ì six points noirs le long de la marge; lantérieur au sommet. Moitié interne le la frange blane-jaunàtre, comme dans Gruneriana. - Tache du bord postérieur peu apparente: large en arrièe, très ollique dı ecoté de la racine de laile; striée de gris. Espace basilaire allongé en pointe jusfrues sur le milieu du disque et maryué de stries ondulies. Crochets de lextrémité de la eòte simples, blanes; les antres plombés. Lignes plonbées brillantes. Eeusson orale, sans taches, ni marepes. - Tite portant une houppor 
de poils gris qui s'avance sur les palpes; ceux-ci sont jaunàtres, avec le dernier article ténu, allongé.

\section{Plumbagana. Treit.}

N’a point été prise dans les environs de Schüpfen.

254. b. Cacaleana. Man.

Hub. revis. Nr. 378. - Ileyd. Cat. 472.

Mr. Rothenbach la collectée dans les Alpes. Non figurée dans Hub. sup.: la fig. 264 citée est curvana.

266. b. Foeneana (ella). Lin.

$$
\text { Treit. - Dup. - Hub. revis. Nr. 400, etc. }
$$

Pflugiana. Fab.

Tibialana. IIols. 40.

Eusirons de Viège (Valais), en juin (Meyer).

g\%o. Geniculana. Lah.

Cette espèce n'est pas fondée sur des caractères suffisants. - Intcrruptana varie beaucoup, ainsi que j’ai pu m'en assurer, et geniculana n'est qu'une variété femelle. La teinte genérale est plus on moins grise ou brune; la strie blanche de l'écusson tantòt à peine marquée, tantòt longue et prononcée. Le voisinage de l'écusson est parfois semé d'écailles lauves. La frange passe du gris de fer au blanc pur et sa ligne tlintersection est plus on moins marquée.

275. b. Rejectani. Lah. Faune suisse Nr. 136.

Un individu regu de lOberland et mieux conservé ma permis de préciser la place que cette espece doit oceuper.

Les cing points noirs qui bordent la marge de laile, comme dans blepharana, la rapprochent de eacaleana et d'alpinana. Ces points sont petits et sitnés très près do la frange. Un point d'intersection jame à peine maryué existe au-dessous du sommet. Celni-ei est arrondi et saillant comme dans cacaleana. Aueune trace le lignes plombées, ni de dessin, à part quelques légères ondulations sur le disrgue. Cinq crochets 
à la còte, jaunàtres, espacés, pen marquús, quelques-uns doubles. Le disque est finement marbré, de couleur gris-bronzé dans sa moitié externe et grisàtre dans l'interne. Pas de tache au bord interne, mais une très faible éclaircie.

276. Augustana. Hub. 205.

Souvent plus petite, plus ıniformément foncée, avee les dessins peu marqués, dans les hautes Alpes.

278. b. Vigeliana. Hub. sup. f. 195.

Flexana. Zell. e. Zeit.

Capturée par Mr. Rothenbach dans les environs de Schüpfen.

284. Ericetana. Zell.

Le Ilaut-Talais (Jaggy). Fréqunente dans l'Engadine.

La femelle est un pen plus petite et a les ailes plus étroites.

286. Quadrana. Hub.

Les femelles prises dans les Alpes reproduisent parfaitement la fig. 256 de Ilub. sup.

990. Ram a na. Lin.

Paykulliana. Fab.

Fimbriana. Woot.

Na point été prise prèès de Schiipfen.

29). Siliceana. Hub.

Mr. Rothenbach possìde une superbe variété noire, marlorée gil el lì de violet; avee les doubles crochets blancs très saillants. L'écusson moitié noir et moitié blanc.

Mr. Wullseblegel en a collecté une autre entièrement grise asec une grosse tache noire an bord interne.

298. Minutana. Hub.

L̈indication de Mr. Rothenlach est erronée. 
303. Lu una. Lah. ').

Lyellana. Fanne suisse (non Curt.).

Mr. Herrich-Schiffer mécrit que Lyellana Curt. n'est que badiana; il devient done nécessaire de changer la dénomination de cette nourelle espèce.

Assez répandue dans toute la région moyenne des Alpes digigle, de Bex, et du BasValais; sur les pentes gazonnées, en juillet.

De nouvelles captures miont permis de déterminer plus exactement les caractères yui la distinguent de badiana, seule espéce avec laquelle on puisse aisément la conlondre. $1^{\circ}$ La taille de Luana est généralement un peu plus forte; du moins chez le màle. $2^{\circ}$ La teinte générale des foncés est le gris-noir et non le gris-bistré. Cette différence so remarque surtont sur la tache interne, sur les postérieures et sur toute la surface inférieure. 3 La partie blanche (interne) de la còte ne porte jamais de points bruns. 4 Elle se prend exchisivement dans les herbages touffus; balliana est commune dans les haies de noisetier. Les autres caractères sont plus variables; cependant la pointe aigue, formée par la rencontre des deux bandelettes obliques et dirigée du còté du sommet, est généralement plıs allongée; le sommet de l'aile l'est aussi davantage.

304. Myrtillana. Treit.

Le Jura (et non les environs de Schiipfen), Rothb. L'Engadine, etc.

305. Dentana. Faune suisse.

Espèce à retrancher. Mon erreur provenait de quelques individus recus d'Allemagne sous le nom de myrtillana et qui appartiennent peut ètre à une espéce encore indéterminée.

\section{Incomptana. Faune suisse.}

D'autres exemplaires plus frais ne font que confirmer mon diagnostic.

Les caractires essenticls de cette espèce, comparée à comptana, sont: la taille plus grande; la teinte d’un brun noirâtre; les ailes plus étroites et plus acuminêes; l'écusson wale et non arrondi, en srande partie couvert par une grosse tache et un trait semi-cireulaire; lespace basilaire mal limité et irrégulièrement anguleux sur le disque:

1) L, uan, nom de la montagne oủ je la pris pour la première fojs. 
une intersection noiràtre, sur la frange, an-dessous du sommet croehu et de la double intersection blanche.

308. Unguicana. Fab.

La femelle est volontiers plus foncée, mienx dessinée, sans mélange de brun.

:30. Fluctigerana. F. r. R.

Au Gurnigel (Jaggi).

312. Нагpana. Hub. $8 \%$.

Ramana. Dup. - Guén. - Fröhl. (non Lin.).

Laetana. Fabr.

Commune. - Environs de Lausanne, dans les taillis humides (Lah.). Sehüpfen idem. Rothb.). Juillet. 


\section{Enumération systématique}

\section{des Tortricides suisses.}

Les nombreux changements que ce supplément apporte à la série des espèces m'obligent à la remanier eu entier afin d'éviter un désordre trop complet. La première colonne de chiftres, après le nom d'espèce, désigne le numéro de la faune, la denxième celui du supplément.

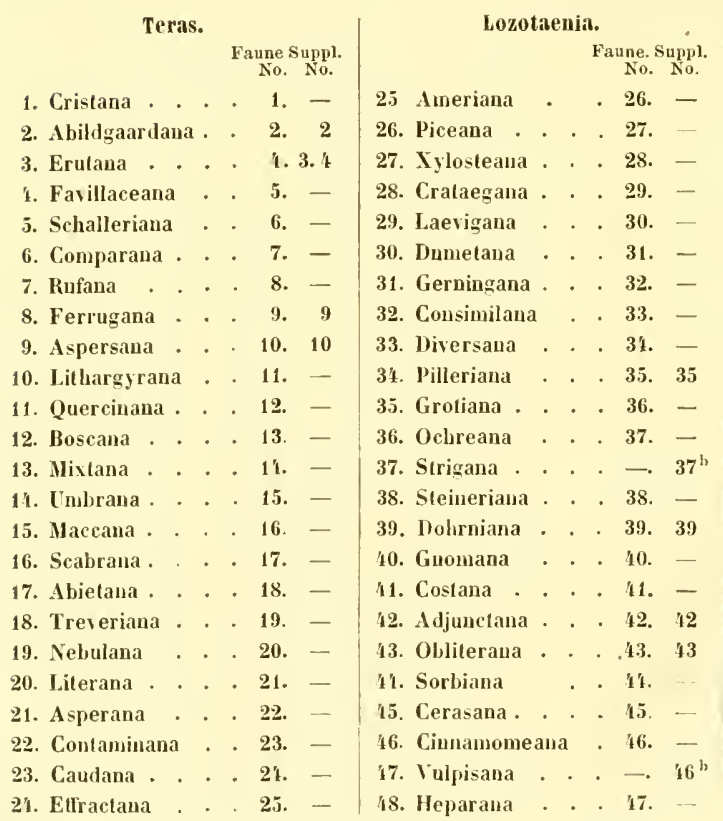
Faune. Suppl No. No.
49. Ribeana . . . 48.
50. Corylana . . . 49.
51. Hytrionana . . 50. -
52. Nubilana . . . 51. -
53. Musculana . . 52
54. Oxyacantbana. . 53 . -
Argyrotosa.

55. Ilolmiana . . . 51.

56. Hoftmanseggana . 55 .

57. Joeflingiana . . 56

58. Bergmanniana . . 57.

59. Forskaleana . . 58 .

60. Bifasciana . . 60.

\section{Ptycholoma.}

61. Leachiana . . 61. -

Tortrix.

62. Viridana . . . . 62.

63. Flavana . . . - 63. 64

67. Rusticana . . . 65. 65

65. Viburnana . . 66.

66. Lusaua . . . . 67,

Lophoderus.

67. Ninistrana . . 69. 
Ianthosetia.

Faune. Suppl. No. No.

68. Hamana . . . 70 . -

69. Zoegana. . . 71. -

Eucella.

70. Mediana . . . 72. 72

Ablabla.

71. Gouana . . 73.

72. Pralana , , 74.

Eupoecllia.

73. Alpicolana . . 75.

74. Dipollana . . - $75^{\mathrm{b}}$

Cochylls.

75. lvecimana . . 76. -

76. Tesserana . . 77. 77

77. Rutilana . . . 78. 78

78. Valdensiana . . 79. 79

79. Zephyrana . . . 80 . -

80. Perfusana . . 81. -

81. Batumanniana . . 82. 82

82. Deulschiana . . 83. 83

83. Smeathmamniana . 8\%. -

8\%. Rubigana . . . 85. 85

85. Jucundana . . . 86 . -

86. Rubellana . . 87. 87

87. lipsaceana. . . 88.88

88. Kichteriana . . - $88^{\text {h }}$

89. Humidana . . . 89.

90. Musseheliaua . . 90.

91. Phaleratana . . 91. -

92. Posterana . . 92.

93. Carduana . . . 93. -

9'. Pallidana. . . 94. 94

95. Purgatana . . . 95.

96. Cruentana . , 96. -

97. Hlaviscapulana $-.96^{h}$

98. Sanguisorbana . - $96^{\mathrm{c}}$

99. Hubilana. . . 97. 97
Faune. Suppl. 100. Ambiguana . . 98. 98

101. Gratiosana. . . 99. -

102. Roseraua . . . 100. 100

Chelmonophllat.

103. Gelatana . . 101. -

\section{l'htheochroa.}

10\%. Rugosana . . 102 .

\section{Sciaphlla.}

105. Rigana . . . 103. 103

106. Stramentana . . 104. 101

107. Albulana . . . 105. -

108. Diurneana . . . 106. 106

109. Penziana . . 107. -

110. Candidana . . 108.

111. Incertana . . 109.

112. Chrysanthemana 110 . -

113. Wahlbomiana . 111. -

114. Minorana . . 112. -

115. Virgaureana . . 113.

116. Pasivana . . 114.

117. Abrasana . . - $114^{\text {h }}$

118. Derivana . . 115. 115

Poecilochroua.

119. Cretaceana $. \quad-115^{b}$

120. Parmatina . . 116.

121. Melaleucana . . 117. -

\section{Enchroula.}

122. Rosetana . . 118, 118

123. Arenana . . . 119. 119

121. Maurana . . . 120.120

125. Tussilaginana . 121. 121

126. Terreana . . 122.

127. Centrana . . 123.

\section{Ris iaciouia.}

128. Hasliana . . 12'1. -
Ditula.

Faune. Suppl.

129. Ophthalmicana . 125, -

130. Corticana . . 126 . -

131. Irofundana . . 127.

132. Oppressana . . 128. -

133. Achatana . . . 129 .

134. Pinicolana . . . 130. -

135. Ratzeburgiana . 131 .

Sericoris.

136. Trifoliana . . 132. -

137. Antiquana . . $-132^{b}$

138. Striana . . . 133.

139. Mygindana . - $133^{h}$

140. Zinckenana . . 134. -

111. Sudetana . . . 135. -

112. Siderana . . 137.

143. Astrana. . . . $137^{\text {b }}$

14'. Textana - . 138. -

115. Schaefferana . . 140. -

146. Spuriana . . . 141. -

147. Porphyrana . . 142. -

148. Micana . . . 143. 143

149. Netallicana . 14t, 141

150. Conchana . . 115. -

151. Giganteana . . 146 .

152. Cespitana . . 147. -

153. Olivana . . . 148.

15\%. Trochilana . - $148^{\mathrm{h}}$

155. Palustrana . , 149, 158

156. Umbrosana . . 150. 150

157. Lacunana . . 151. -

158. Rurestrana . . 152. -

159. Urticana . . 153.

160. Venustana . . 15\%. -

161. Lucana . . . 155. -

162. Hipunclana . . 156 . -

163. Irrignana . . . 157. -

161. Trifasciana . . 159. -

165. Charpentierana 160. 
Sotocella.

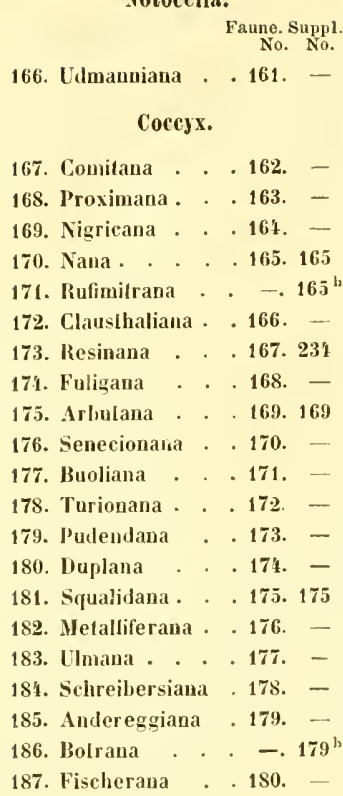

Pentlina.

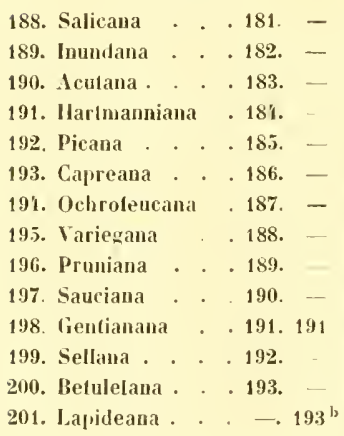

Faune. Suppl. No. No.

202. Dealbana . . 191.

203. Aceriana . . 195 -

204. Sullusana . . . 196.

205. Incarualana . . 197.

206. Roborana . . 198.

207. Tripunclana . . 199.

208. Ocellana . . 200. -

209. Simplarra . . . 201.

210. Servillana . . 202 .

Poedlsea.

211. Siniland . . 203.

212. Mendiculana . $20 \%$.

213. Turbidana. . - $201^{h}$

214. Confusana . . 205.

215. Scutulana . . 206. 206

216. Dissimilana . .207.

217. Demarniana . . 208.

218. Deliland . . . 209 .

219. Couleruana . . 210 . -

220. Monachana . . 211.

221. Poecilana . . 213.

222. Cirsiana . . 214.

223. Brunichiana . . 215 .

221. Sublimana . . . $215^{13}$

225. Luctuosana . 216

\section{Apleclia.}

226. Lanceolana . . 217.

Semasia.

227. Inlidana. . . 218.

228. Alsyntiana . . 219. -

229. Cirana . . 220.220

230. Incana . . . 221, 221

231. Caecimaculana . 222.

232. Conterminana 223. 223

233. Aspidiscana . . 225. 225

23 t. Hohenwartziana 226.221 .226

235. Lenulana . . 228. 228

236. Jaharpana . . . 229. 229
Faune. Suppl. No. No.

237. Ibiceana -. $229^{\prime \prime}$ 238. Hypericana . 230

\section{Roxalla.}

239. Armana . . 231. C'arpoeapsa.

2'i0. Pomonana . . . 232. 2'11. Fagiglandana . . 233. 233 2'12. Splendana . . 235. 235 2'13. Sunedana . . . 236.

\section{Grapholtta.}

214. I)aldorfiana . . 237. 215 Fulvifronlana . . 238. 238 216. Coecana . . 239. 217. Plumbatana . . 2'0. 2's. Blepharana . . 2'it. 2'9. Bugnionana . . 2'2. 213 250. Fruneriana . 2'1t. 214 251. Microgramnana . 2'15. 252. Istulana . . 2'16. 2'16 253. Tenebrosana . . 247.

251. Nebritana . . 218. 248 255. Gemmiferana. . 219. 256. Funebrana . . 2.50. 257. Acuminalana . . 251. 251 2.58. Ligulana . . . 252. 259. Alpestrana . . 253. 253 260. Plumbagana . . 251. 251 261. Cacaleana. . - $251^{\mathrm{h}}$ 262. Caliginosana . 25.j. 263. Argurana . . 256. 265. Kochiana . . 257. 26.5. Janthinana. . . 258. 266. Woeberiaua . 259. 267. Composana . 260. 268. Corollana . . 261. 269. Lołerana . . . 262. 270 . Fissanal . . . 263. 271. Iiflusana . . 264. 
Fanne. Suppl. No. No.

272. Coniferana . . 265.

273. Coronillana . 266.

27 t. Foeneana . . $-266^{\mathrm{b}}$

275. Orobana . . 267. -

276. Jungiana . . 268.

277. Iulerruplana . 269. 270

278. Spiniana . . 271. -

279. Ephippana . .272. -

280. Trauniana . . 273.

281. Petiserana . . 27i. -

282. Alpinana - . 275. -

283 Rejeclana . 136. $275^{\mathrm{h}}$

284. Augustana . . . 276. 276

285. Mercuriana . 277 . -

286. Loxiana . . 278.

287. Vigeliana . $-.278^{\mathrm{h}}$

288 Strobilana . . 279.

289. Jactolana . . 280 . -

290. Cosmophorana . 281. -

291. Scopariana .

282.
Syndemis.

Faune. Suppl. No. No.

292. Vacciuiana . . 283.

293. Ericelana . . 28\%. 284

291. Cuphana . . .285. -

295. Quadrana . . 286. 286

\section{Steganopijclia.}

296. Freyeriana . . 287.

297. Campoliliana . . 288.

298. Immundana . . 289.

299. Ramana . . . 290. 290

300. Siliceana . . . 291. 291

301. Naevana . . . 292.

302. Abiegnana . . 293.

303. Pyguaeana . 294.

$30 \%$. Frutetana . . 295.

305. Rubiginosana . 296.

306. Penkleriana . 297.

307. Minutana . . 298. 298

308. Litloxylana . 299.
Phoxopteryx.

Fatune. Suppl.

309. Millerpachiaua , 300.

310. Derasana . . 301.

311. Badiana . . 302 .

312. Luatıa (Lyellana) 303. 303

313. Myrtillana . 301.301.305

31 i. Complana . 306.

315. Ineomplana . 307. 307

316. Unguicana . . 308. 308

317. Incana . . 309.

318. Flucligerama . . 310. 310

319. Cuspidana . . . 311.

320 Harpana . . 312. 312

321 . Siculana . . 313 .

Cheintatophila.

322. Hyemana . . 31\%. - 






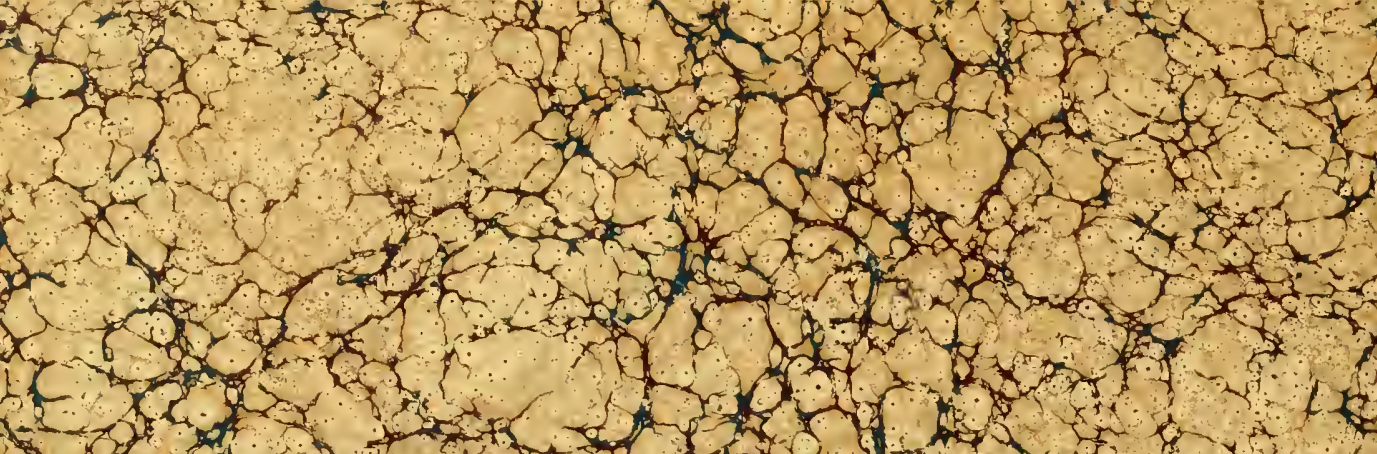

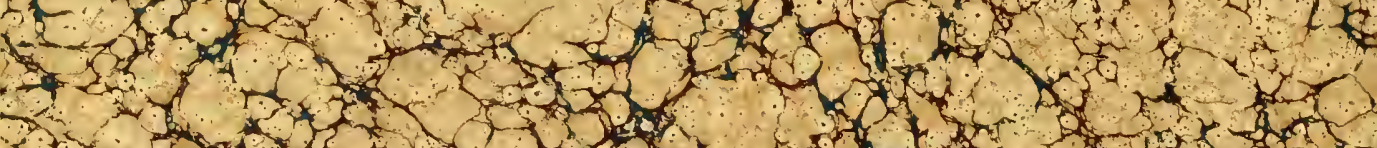

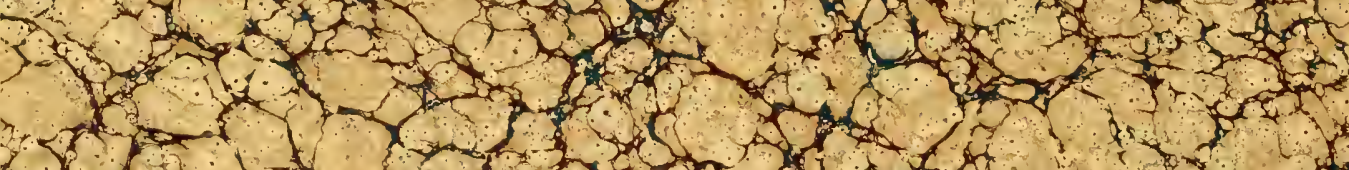

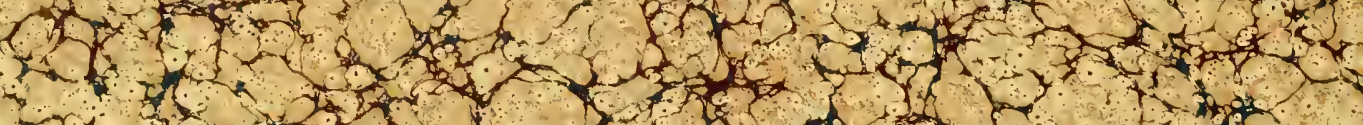

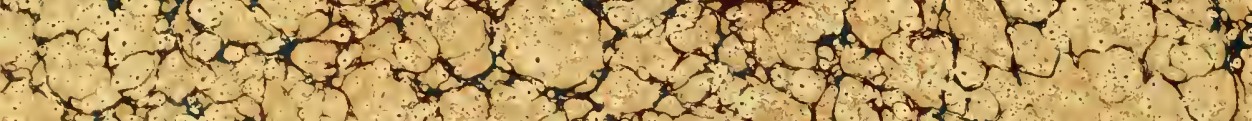

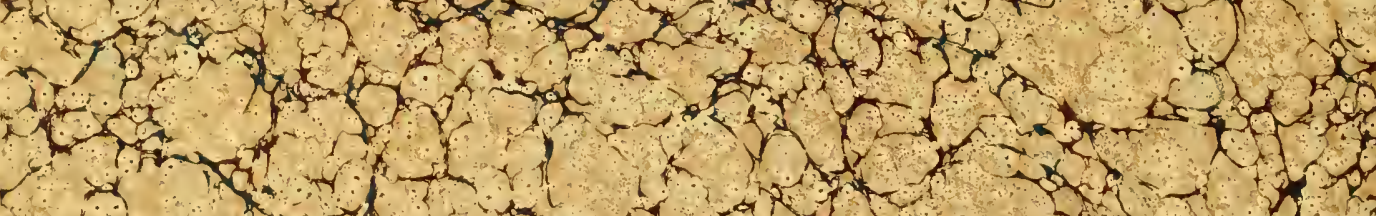

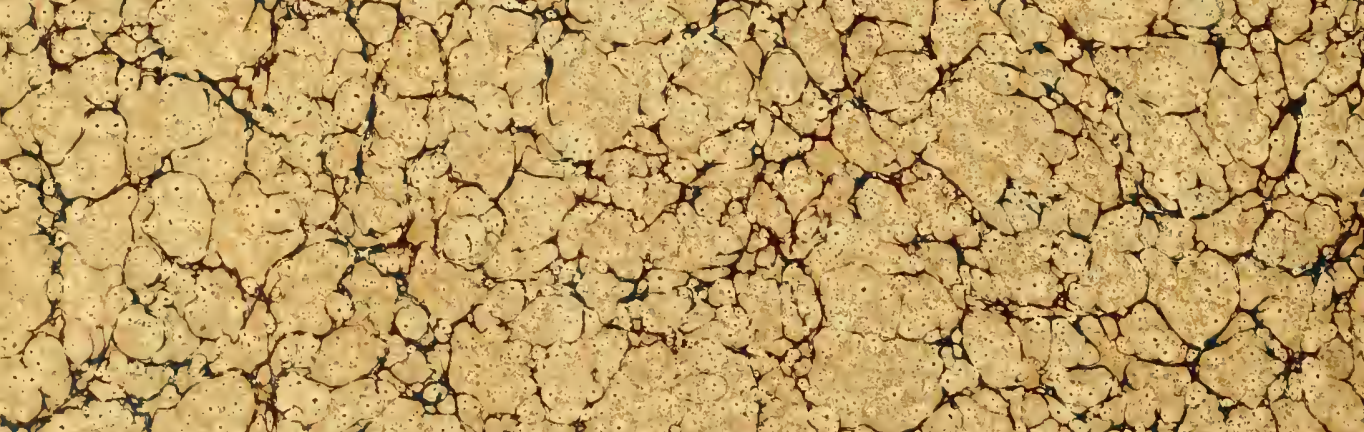

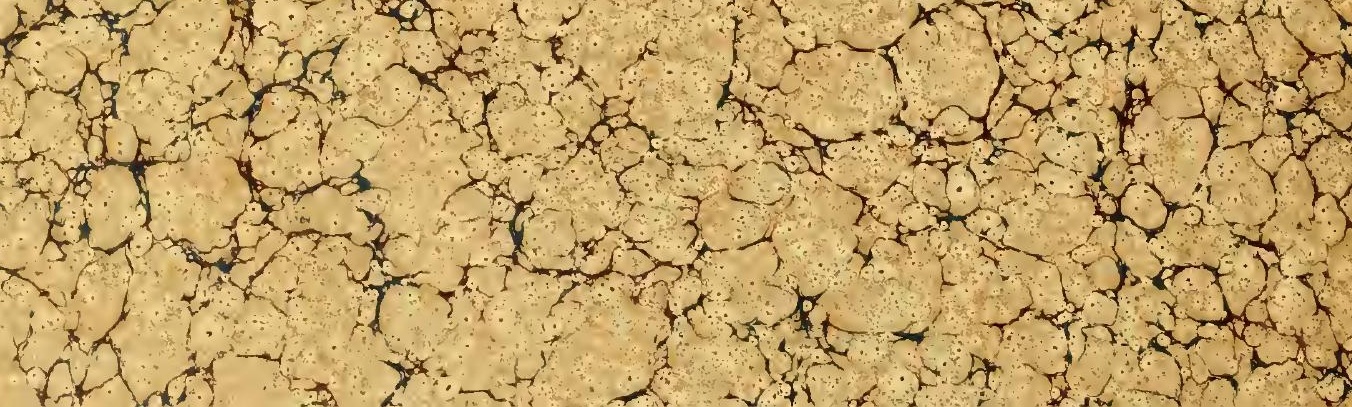


F.

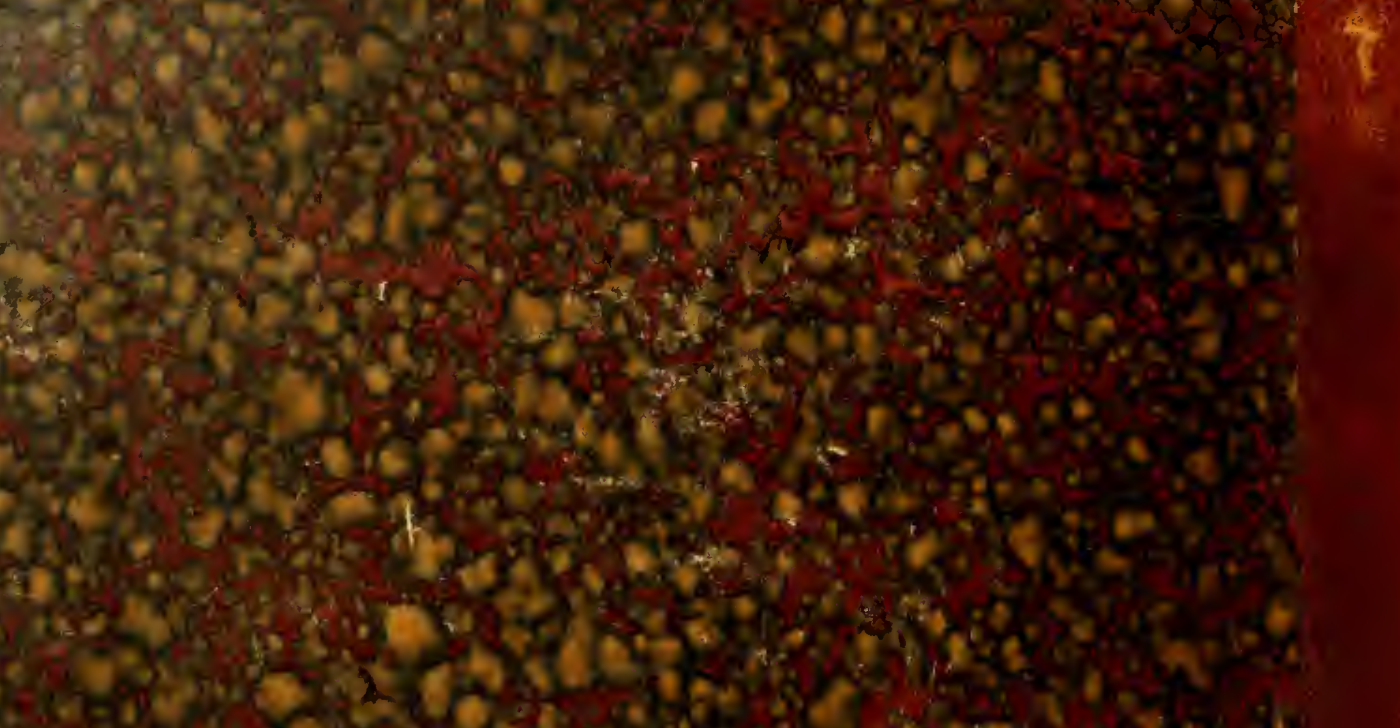
3050

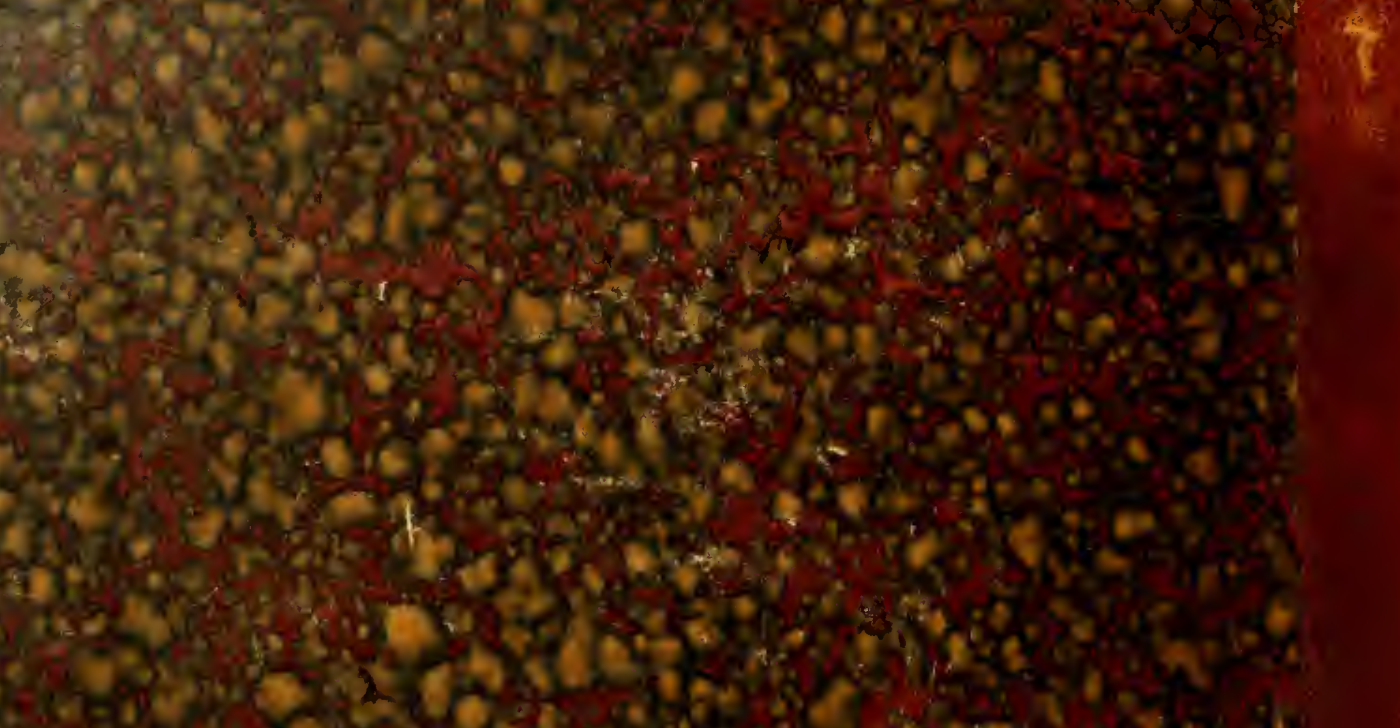

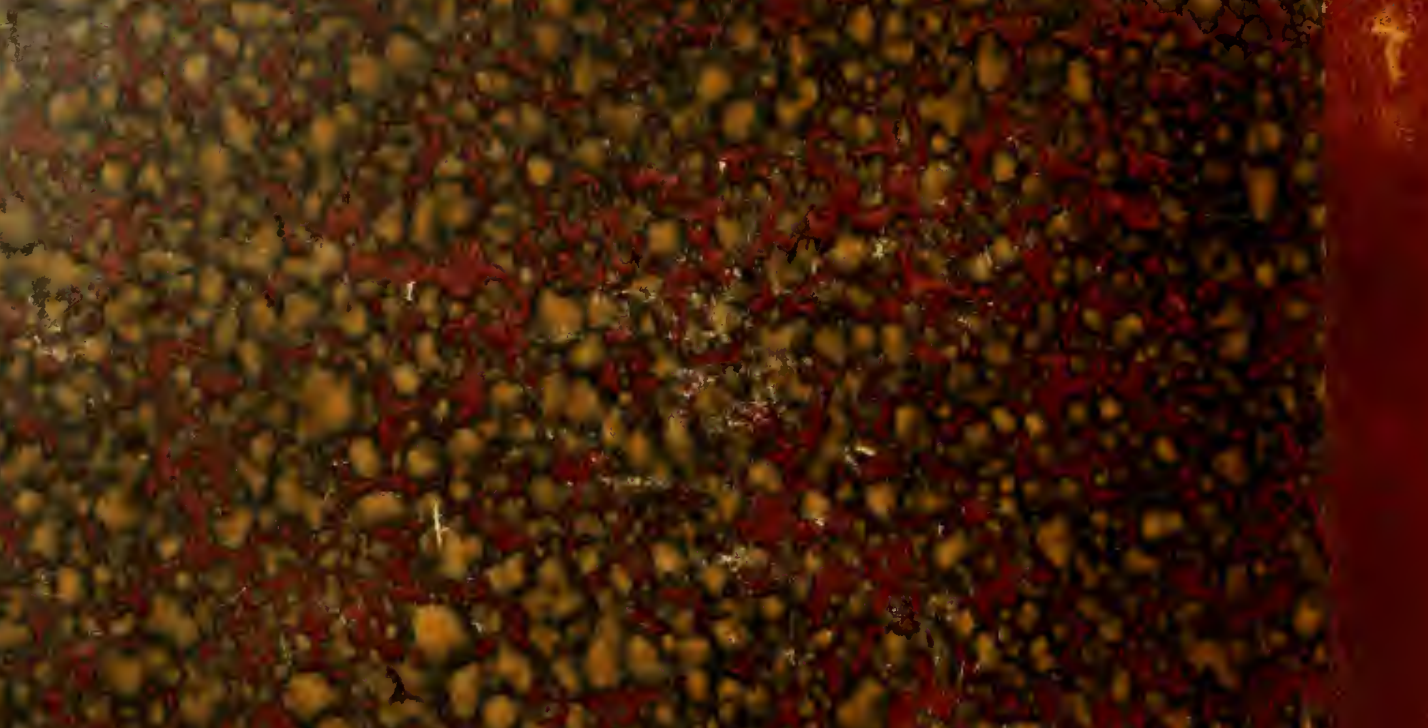

(2)

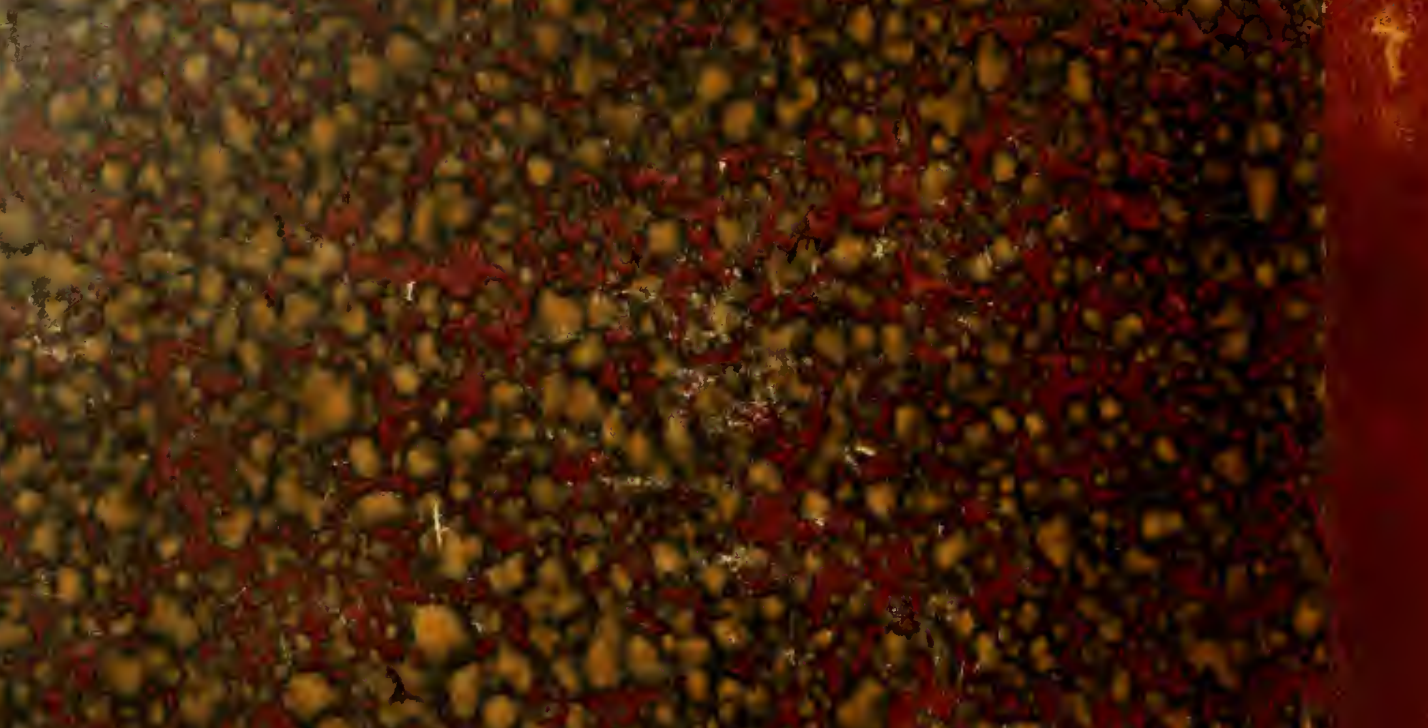

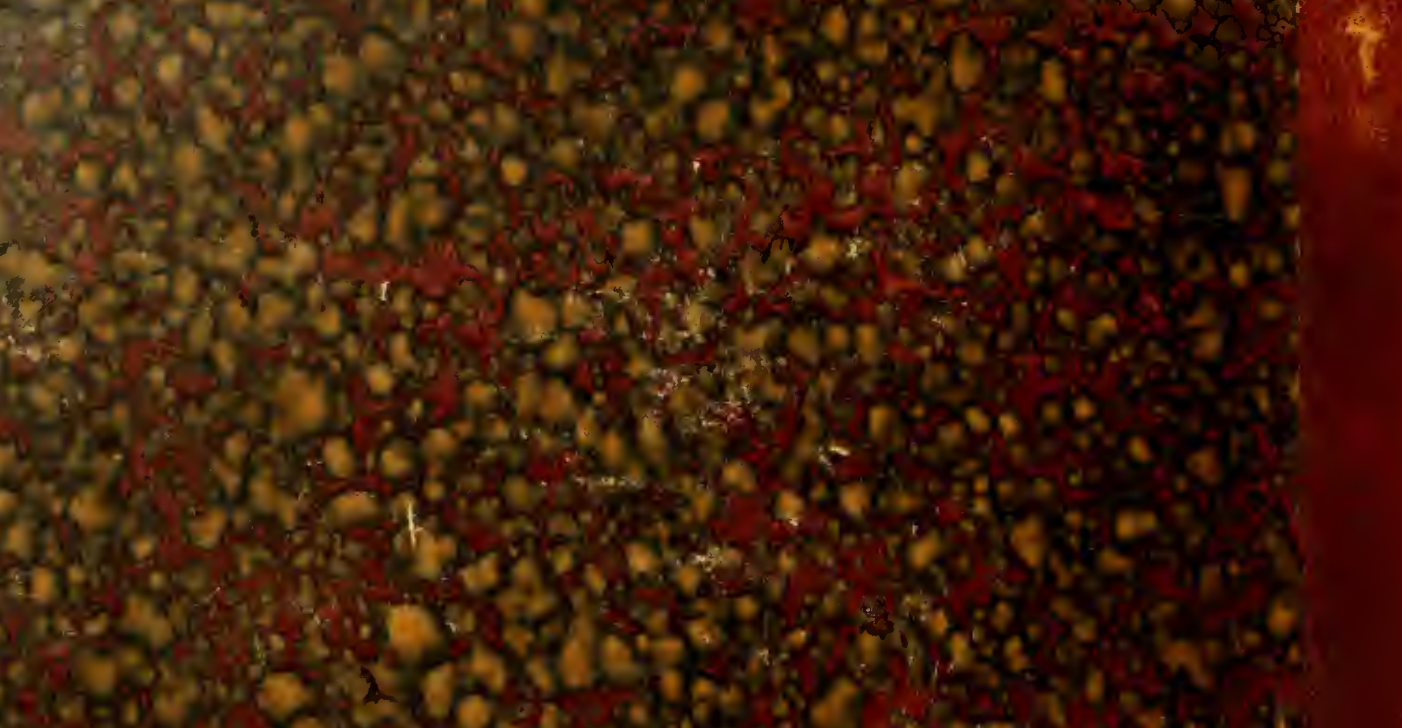
20030

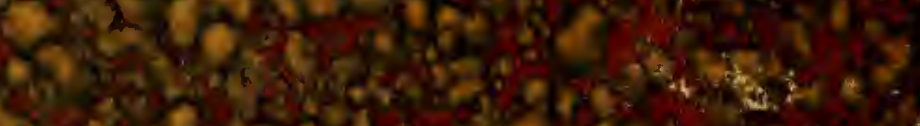

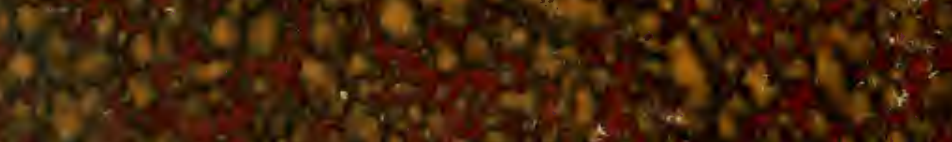
20.

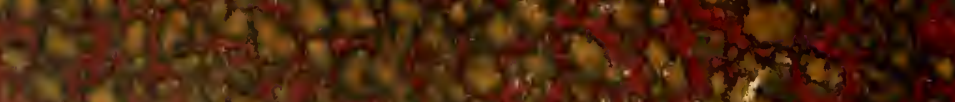

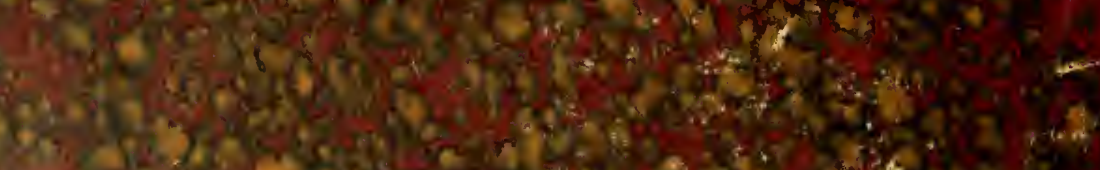

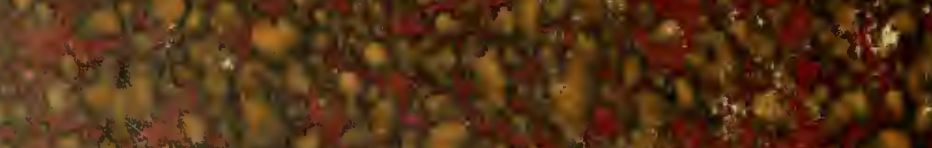

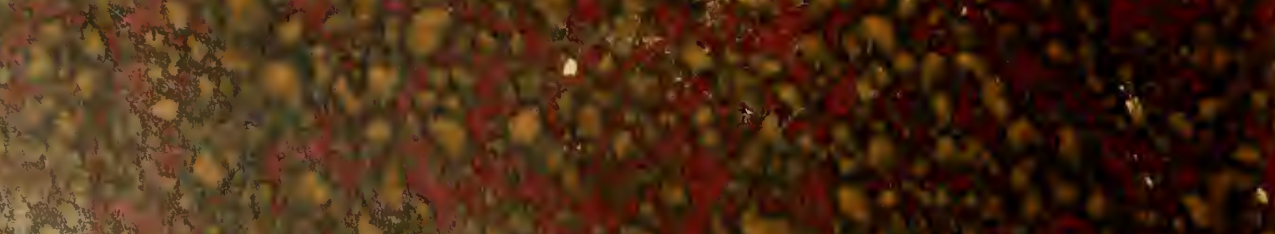

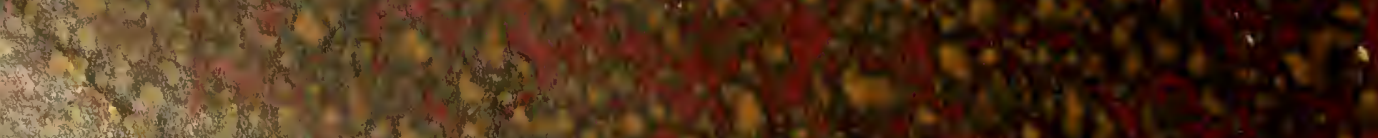

